\title{
Flow and Transport at the Las Cruces Trench Site: Experiment IIb
}

Manuscript Completed: May 1997

Date Published: July 1997

Prepared by

J. Vinson, NMSU, P. J. Wierenga, UA,

R. G. Hills, NMSU, M. H. Young, UA

Soil and Water Science Department

University of Arizona

Tucson, AZ 85721

Subcontractor:

Agronomy and Mechanical Engineering Departments

New Mexico State University

Las Cruces, NM 88003

R. Cady, NRC Project Manager

Prepared for

Division of Regulatory Applications

Office of Nuclear Regulatory Research

U.S. Nuclear Regulatory Commission

Washington, DC 20555-0001

NRC Job Code L1286

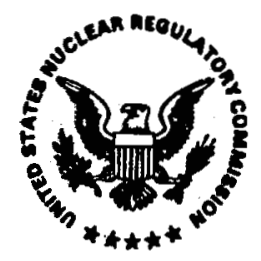

DISTRIBUTION OF THIS DOCUMENT IS UNLIMITED 


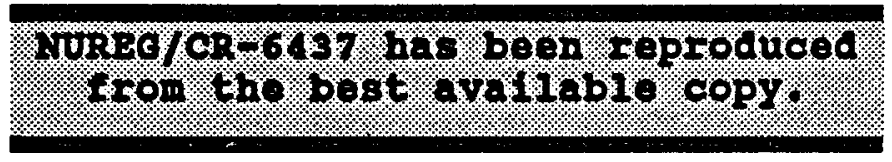




\section{DISCLAIMER}

This report was prepared as an account of work sponsored by an agency of the United States Government. Neither the United States Government nor any agency thereof, nor any of their employees, make any warranty, express or implied, or assumes any legal liability or responsibility for the accuracy, completeness, or usefulness of any information, apparatus, product, or process disclosed, or represents that its use would not infringe privately owned rights. Reference herein to any specific commercial product, process, or service by trade name, trademark, manufacturer, or otherwise does not necessarily constitute or imply its endorsement, recommendation, or favoring by the United States Government or any agency thereof. The views and opinions of authors expressed herein do not necessarily state or reflect those of the United States Government or any agency thereof. 


\section{DISCLAMIER}

Portions of this document may be illegible in electronic image products. Images are produced from the best available original document. 


\begin{abstract}
Three water flow and solute transport experiments were performed as part of a comprehensive field trench study near Las Cruces, New Mexico to test deterministic and stochastic models of vadose zone flow and transport. This report presents partial results from the third experiment (experiment IIb). Experiments II $a$ and $b$ were conducted on the North side of the trench, on a plot $1.22 \mathrm{~m}$ wide by $12 \mathrm{~m}$ long, perpendicular to the trench. The area was drip irrigated during two time periods with water containing a variety of tracers. The advance of the water front during the two irrigation episodes was measured with tensiometers and neutron probes. Solute front positions were determined from soil solution sampling through suction samplers and from disturbed sampling.
\end{abstract}

ABSTRACT

The results from experiment IIb show predominately downward water movement through the layered unsaturated soil, as evidenced from neutron probe data and gravimetric sampling. Tritium plumes were only half as deep and half as wide as the water plumes at 310 days after the beginning of experiment IIb. Chromium, applied as $\mathrm{Cr}$ (VI), moved as readily as, and similar to tritium, but there was a loss of mass due to reduction of $\mathbf{C r}$ (VI) to $\mathrm{Cr}$ (III). Chloride and nitrate, initially present at high concentrations in the soil solution, were displaced by the low concentration irrigation water, resulting in chloride and nitrate concentration distributions that looked like negative images of the tritium distributions. The extensive data presented should serve well as a data base for model testing. 


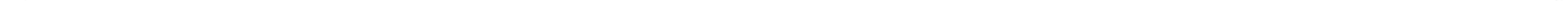




\section{CONTENTS}

\section{Page}

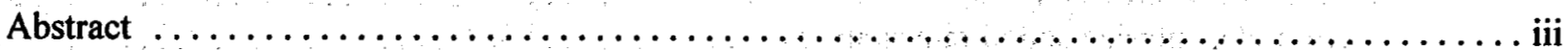

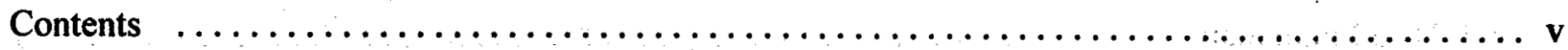

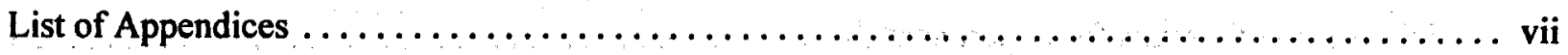

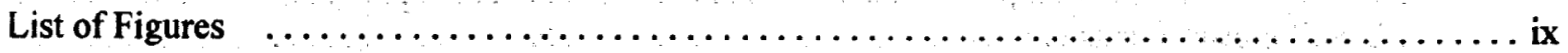

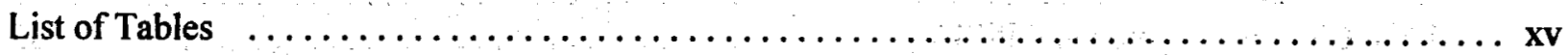

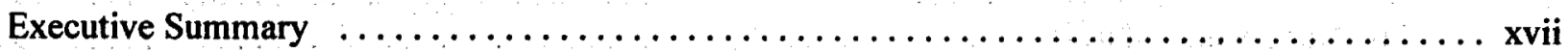

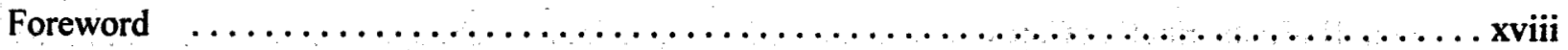

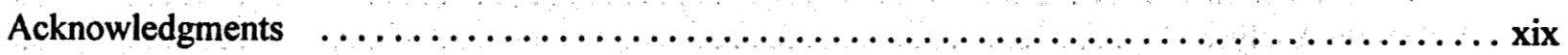

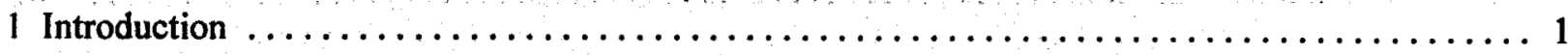

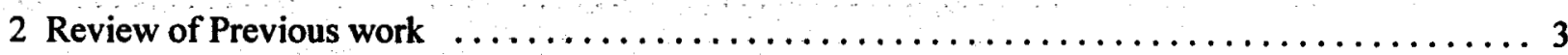

2.1 Site Description and Characterization $\ldots \ldots \ldots \ldots \ldots \ldots \ldots \ldots \ldots \ldots \ldots \ldots \ldots \ldots$

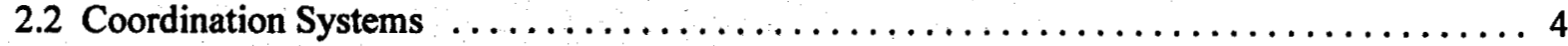

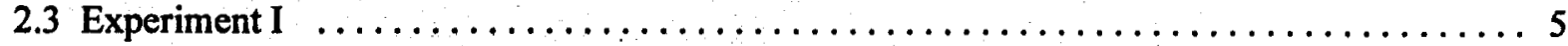

2.4 Experiment I Redistribution Cores $\ldots \ldots \ldots \ldots \ldots \ldots \ldots \ldots \ldots \ldots \ldots \ldots \ldots \ldots \ldots$

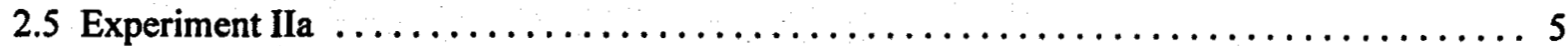

3 Experiment IIa Redistribution Cores $\ldots \ldots \ldots \ldots \ldots \ldots \ldots \ldots \ldots \ldots \ldots \ldots \ldots \ldots \ldots$

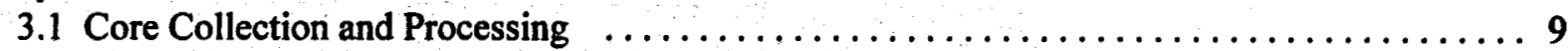

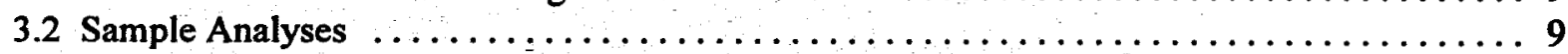

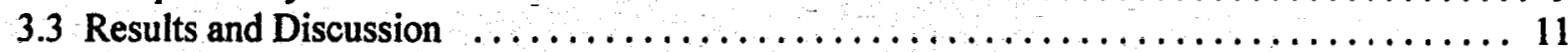

4 Experiment Ilb Water and Solute Application $\ldots \ldots \ldots \ldots \ldots \ldots \ldots \ldots \ldots \ldots \ldots \ldots \ldots \ldots \ldots \ldots \ldots \ldots$

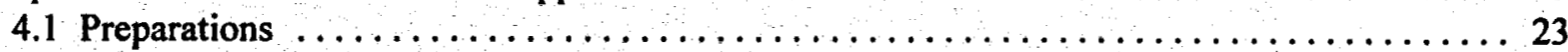

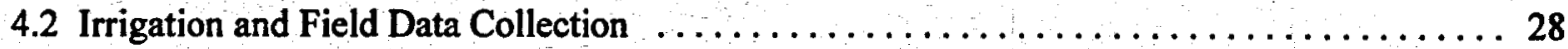

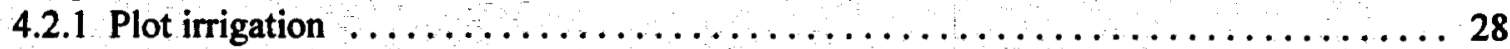

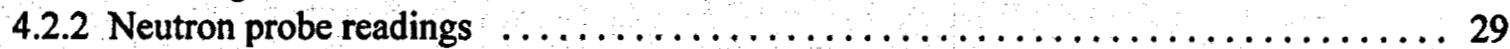

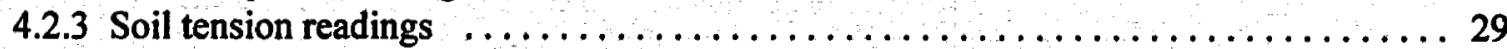

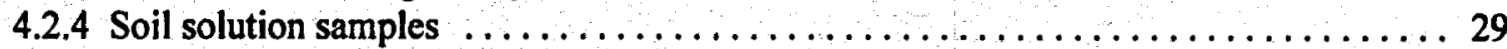




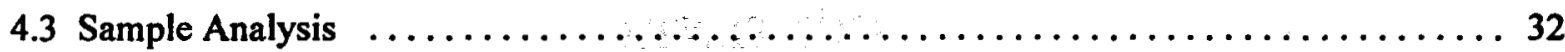

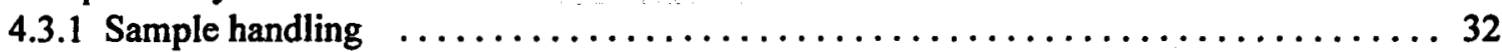

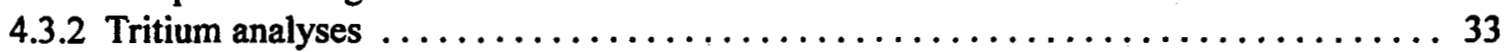

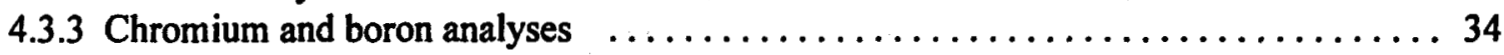

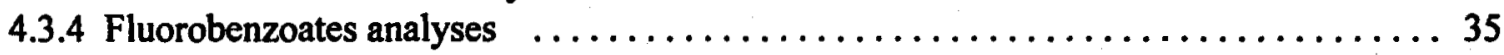

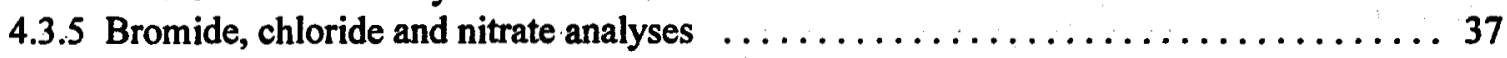

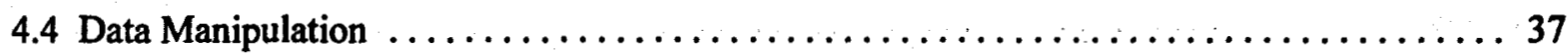

4.5 Discussion of Results for Experiment IIb $\ldots \ldots \ldots \ldots \ldots \ldots \ldots \ldots \ldots \ldots \ldots \ldots \ldots \ldots$

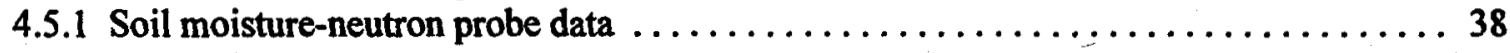

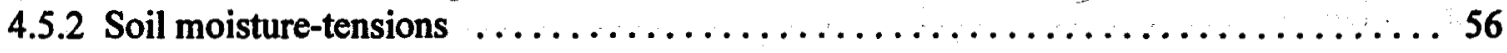

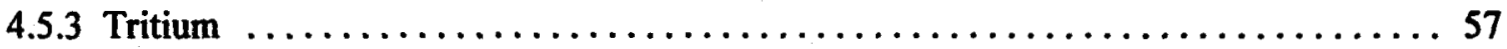

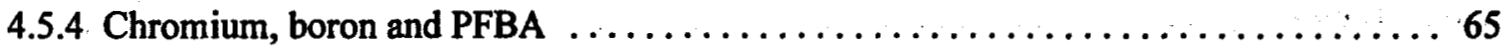

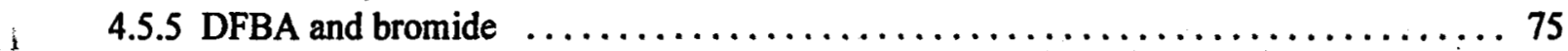

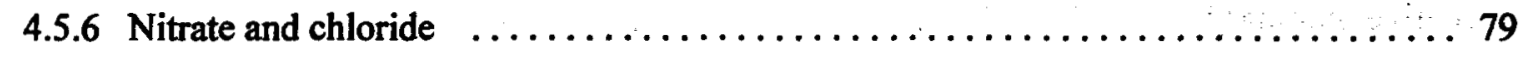

5 Experiment IIb Redistribution Cores $\ldots \ldots \ldots \ldots \ldots \ldots \ldots \ldots \ldots \ldots \ldots \ldots \ldots \ldots \ldots \ldots$

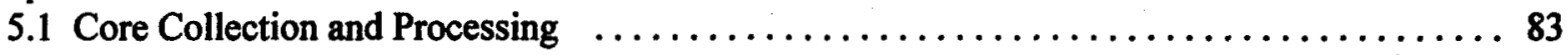

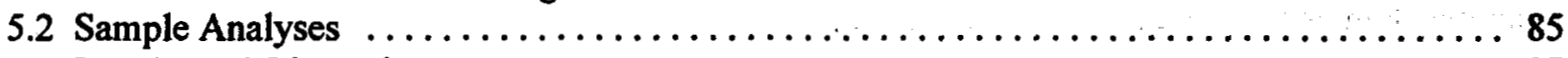

5.3 Results and Discussion $\ldots \ldots \ldots \ldots \ldots \ldots \ldots \ldots \ldots \ldots \ldots \ldots \ldots \ldots \ldots \ldots \ldots \ldots \ldots$

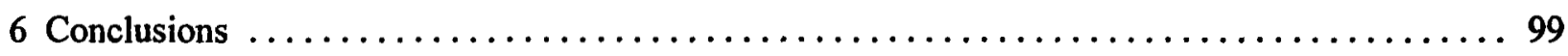

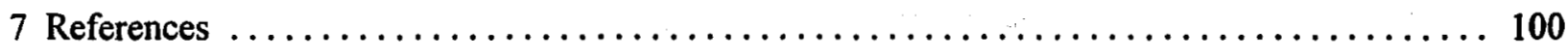




\section{APPENDICES}

Appendix A: Experiment IIb Data Base Tables $\ldots \ldots \ldots \ldots \ldots \ldots \ldots \ldots \ldots \ldots \ldots \ldots \ldots 10 \ldots$

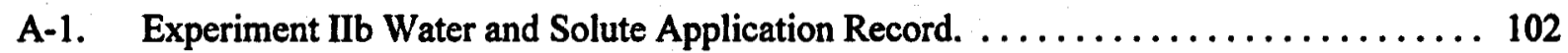

A-2. Example of Neutron Probe Soil Moisture Data. $\ldots \ldots \ldots \ldots \ldots \ldots \ldots \ldots \ldots \ldots \ldots$

A-3. Experiment IIb Tensiometer Soil Moisture Data. .................... 105

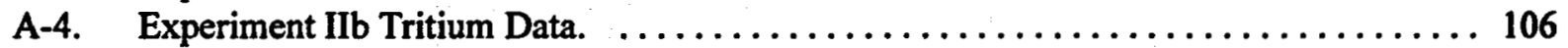

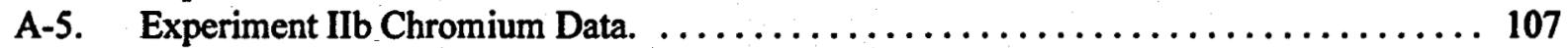

A-6. Solute Concentrations: PFBA, DFBA, Br, NO3, and Cl. . . . . . . . . . 108

A-7. Experiment IIb Redistribution Cores (Baseline Data). $\ldots \ldots \ldots \ldots \ldots \ldots \ldots \ldots \ldots$

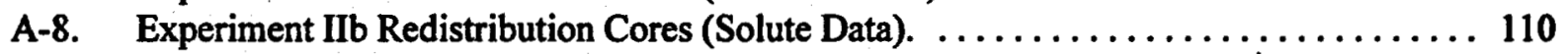

Appendix B: Experiment IIb Extension. $\ldots \ldots \ldots \ldots \ldots \ldots \ldots \ldots \ldots \ldots \ldots \ldots \ldots \ldots \ldots \ldots$

B-1. Experiment IIb Dripper Uniformity Test. $\ldots \ldots \ldots \ldots \ldots \ldots \ldots \ldots \ldots \ldots \ldots \ldots$

B-2. Tritium Scintillation Cocktail and Aliquot Volume Adjustments. $\ldots \ldots \ldots \ldots \ldots \ldots 119$

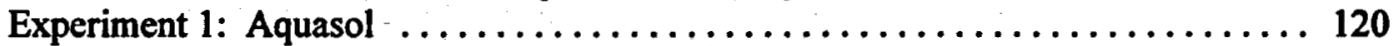

Experiment 2: Aquasol and Ready-Safe Cocktails .................. 123

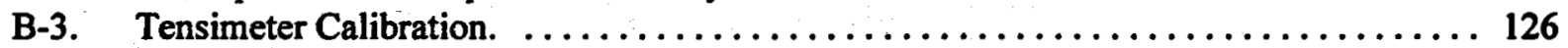

B-4. Comparison of NMSU and University of Arizona Laboratory Results. $\ldots \ldots \ldots \ldots \ldots 129$

B-5. Experiment IIb Forms for field data collection. $\ldots \ldots \ldots \ldots \ldots \ldots \ldots \ldots \ldots \ldots \ldots$

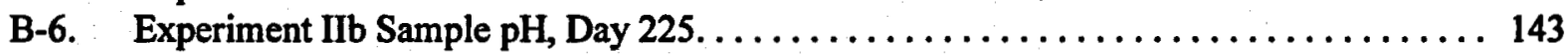

Appendix C: Miscellaneous Appendices $\ldots \ldots \ldots \ldots \ldots \ldots \ldots \ldots \ldots \ldots \ldots \ldots \ldots \ldots \ldots$

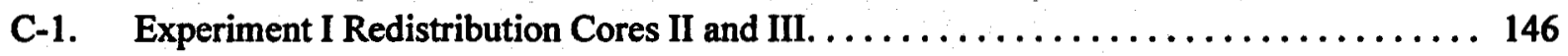

C-2. "A" Profile Pre-Leaching Chloride and Nitrate. . . . . . . . . . . . . . . . 162

C-2a. "A" Profile Pre-Leaching Chloride and Nitrate. $\ldots \ldots \ldots \ldots \ldots \ldots \ldots \ldots \ldots \ldots$

C-2b. "A" Profile Pre-Leaching Chloride and Nitrate. $\ldots \ldots \ldots \ldots \ldots \ldots \ldots \ldots \ldots \ldots$

C-2c. "A" Profile Pre-Leaching Chloride and Nitrate. $\ldots \ldots \ldots \ldots \ldots \ldots \ldots \ldots \ldots \ldots \ldots \ldots$

C-2d. "A" Profile Pre-Leaching Chloride and Nitrate. $\ldots \ldots \ldots \ldots \ldots \ldots \ldots \ldots \ldots \ldots \ldots$

C-3. Evaporative Loss From $20 \mathrm{ml}$ Glass Scintillation Vials. $\ldots \ldots \ldots \ldots \ldots \ldots \ldots \ldots \ldots$

C-4. Facilities Maps of Trench Site and Ranch Headquarters. $\ldots \ldots \ldots \ldots \ldots \ldots \ldots \ldots 173$

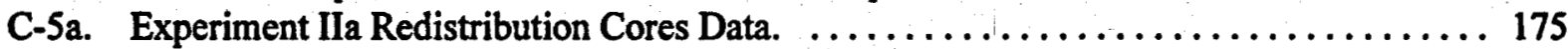

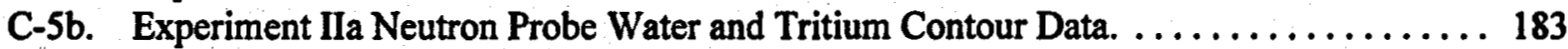

C-6. Construction of Narrow-Profile Ceramic Devices. $\ldots \ldots \ldots \ldots \ldots \ldots \ldots \ldots \ldots \ldots$

C-7. NRC Trench Wall Ceramic Device Installation. $\ldots \ldots \ldots \ldots \ldots \ldots \ldots \ldots \ldots \ldots \ldots$

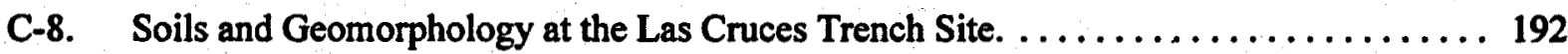

C-9. Chronology of Las Cruces Trench Site Experimental Events. $\ldots \ldots \ldots \ldots \ldots \ldots \ldots 195$

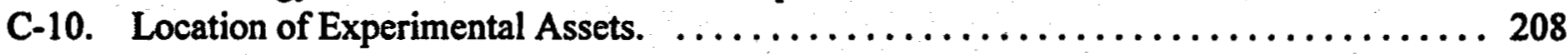




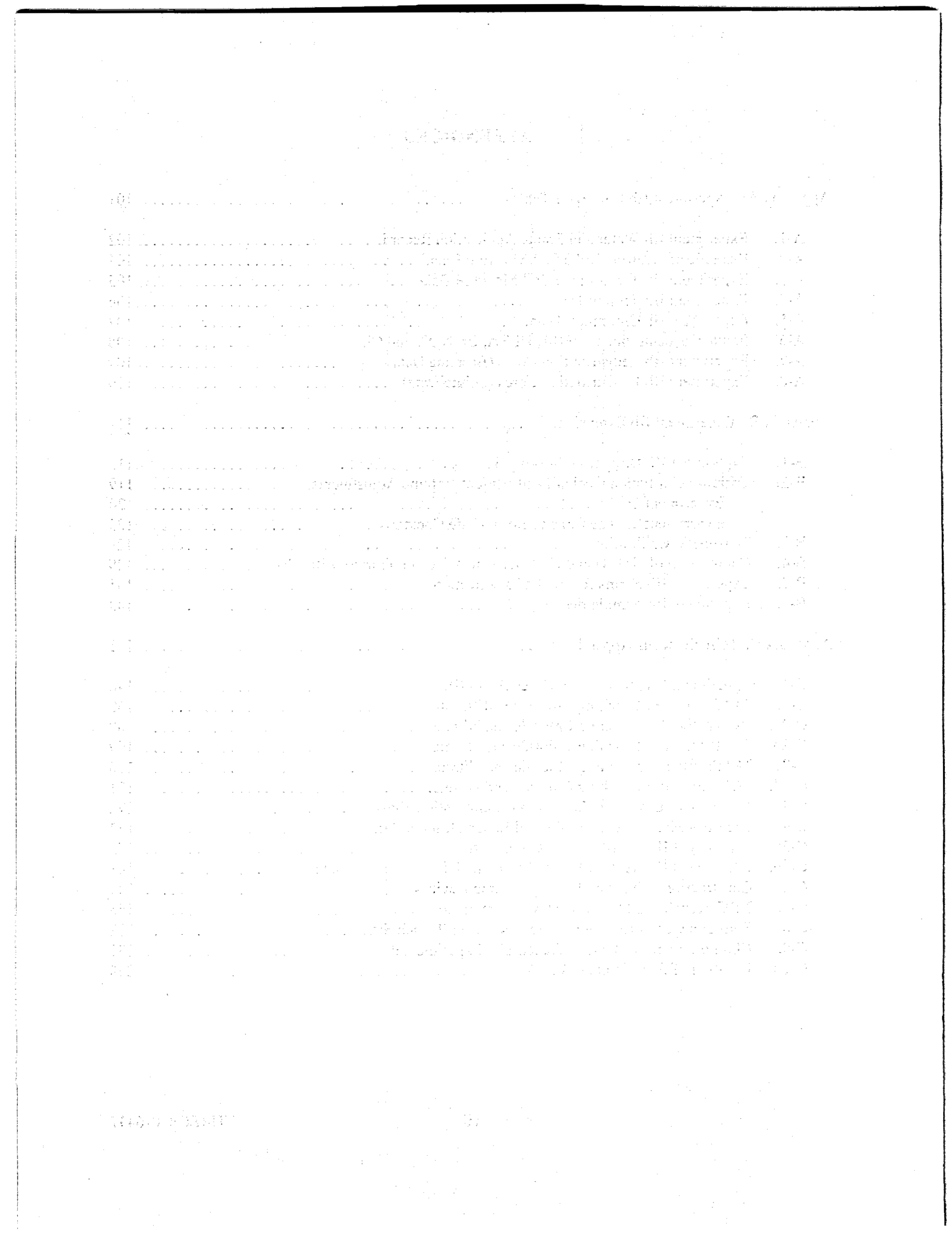




\section{FIGURES}

1 Timeline for Las Cruces Trench Site experiments. Shaded area denotes experiments described in this report.

2 Location of Las Cruces Trench Site within the state of New Mexico and within the county of Dona Ana.

3 Overhead view of trench, experimental irrigation plots and origins points for $(X, Y)$,

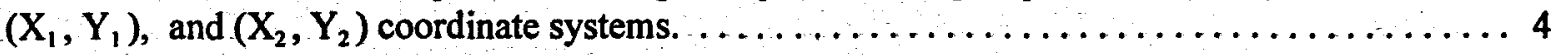

4 Plot 2 irrigation system of emitters and header lines. $\ldots \ldots \ldots \ldots \ldots \ldots \ldots \ldots \ldots \ldots \ldots$

5 Installation and use of tensiometer device through trench wall. $\ldots \ldots \ldots \ldots \ldots \ldots \ldots \ldots \ldots$

6 Installation and use of solution sampler device through trench wall $\ldots \ldots \ldots \ldots \ldots \ldots \ldots \ldots \ldots 8$

7 Location of experiment IIa soil cores. $\ldots \ldots \ldots \ldots \ldots \ldots \ldots \ldots \ldots \ldots \ldots \ldots \ldots \ldots \ldots \ldots \ldots \ldots \ldots \ldots \ldots$

8 Experiment Ila neutron probe volumetric moisture contours at $Y_{2}=6 \mathrm{~m}$ on days 78 and 655. Boxes are added to show similar regions of the profile which were sampled by soil cores at $Y_{2}=5 \mathrm{~m}$ on day 661 .

9 Experiment IIa soil core volumetric moisture contours at $Y_{2}=5 \mathrm{~m}$ on day 661 .

Gravimetric soil core moisture is converted to volumetric moisture with an estimated

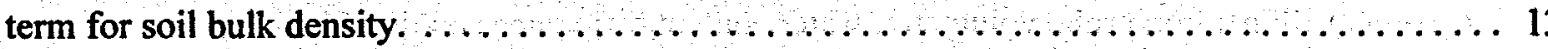

10 Experiment IIa neutron probe volumetric moisture contours at $Y_{2}=10 \mathrm{~m}$ on days 78 and 655. Boxes are added to show similar regions of the profile which were sampled by soil cores at $Y_{2}=9 \mathrm{~m}$ on day 661 .

11. Experiment Ila soil core volumetric moisture contours at $Y_{2}=9 \mathrm{~m}$ on day 661 .

Gravimetric soil core moisture is converted to volumetric moisture with an estimated term for soil bulk density $\ldots \ldots \ldots \ldots \ldots \ldots \ldots \ldots \ldots \ldots \ldots \ldots \ldots \ldots \ldots \ldots \ldots \ldots \ldots \ldots \ldots \ldots 16$

12 Experiment IIa relative tritium $(\mathrm{C} / \mathrm{Co})$ contours at the trench face $\left(\mathrm{Y}_{2}=0.5 \mathrm{~m}\right)$ on days 78 and 429 . Liquid samples collected by solution samplers.

13 Experiment Ila relative tritium contours from soil cores at $Y_{2}=5 \mathrm{~m}$, day $661 \ldots \ldots \ldots \ldots \ldots 18$

14 Experiment IIa relative tritium contours from soil cores at $Y_{2}=9 \mathrm{~m}$, day 661 .

15 Experiment IIa relative bromide $(\mathrm{C} / \mathrm{Co})$ and solution chloride $(\mathrm{me} / \mathrm{L})$ contours from soil cores at $Y_{2}=5 \mathrm{~m}$, day 661 . 
16 Experiment Ila relative bromide $(\mathrm{C} / \mathrm{Co})$ and solution chloride $(\mathrm{me} / \mathrm{L})$ from soil cores

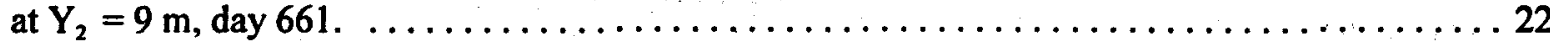

17 Survey of plot 2 experimental apparatus illustrating locations of neutron probe access tubes, tensiometers and solution samplers relative to irrigated area and plot vapor barrier.

18 Schematic of trench face illustrating placement of tensiometers and solution samplers relative to the area of soil directly beneath irrigated area (shaded) $\ldots \ldots \ldots \ldots \ldots \ldots \ldots \ldots \ldots$

19 Schematic of pumphouse plumbing system showing delivery route of irrigation water from either 2500 gallon storage tank or $\mathbf{3 0 0}$ gallon treatment tank to plot 2 emitter headers.

20 Experiment IIb totalizer record. Plotted application rates are calculated as mean application from one observation to the next. Aberrations from normal irrigation rates are noted.

21 Schematic of neutron probe access tubes in relation to plot 2 , pondliner cover and the

22 Experiment IIb neutron probe volumetric water content on day 0 for a plane through $X_{2}=0 \mathrm{~m}$ along the length of plot 2 , and for a plane through $Y_{2}=2 \mathrm{~m}$ parallel to the trench face.

23 Experiment Ilb neutron probe volumetric water content on day 0 for planes through $Y_{2}=6 \mathrm{~m}$ and $Y_{2}=10 \mathrm{~m}$ parallel to the trench face

24 Experiment IIb neutron probe volumetric water content differences on day 31 for a plane through $X_{2}=0 \mathrm{~m}$ along the length of plot 2 , and for a plane through $Y_{2}=2 \mathrm{~m}$ parallel to the trench face. Values expressed as differences from day 0 water content.

25 Experiment IIb neutron probe volumetric water content differences on day 31 for planes through $Y_{2}=6 \mathrm{~m}$ and $Y_{2}=10 \mathrm{~m}$ parallel to the trench face. Values expressed as differences from day 0 water content. Small circles in plots indicate missing data.

26 Experiment IIb neutron probe volumetric water content differences on day 73 for a plane through $X_{2}=0 \mathrm{~m}$ along the length of plot 2, and for a plane through $Y_{2}=2 \mathrm{~m}$ parallel to the trench face. Values expressed as differences from day 0 water content.

27 Experiment IIb neutron probe volumetric water content differences on day 73 for planes through $Y_{2}=6 \mathrm{~m}$ and $Y_{2}=10 \mathrm{~m}$ parallel to the trench face. Values expressed as differences from day 0 water content Small circles in plots indicate missing data.

28 Experiment IIb neutron probe volumetric water content differences on day 126 for a plane through $X_{2}=0 \mathrm{~m}$ along the length of plot 2, and for a plane through $Y_{2}=2 \mathrm{~m}$ parallel to the trench face. Values expressed as differences from day 0 water content. 
29 Experiment IIb neutron probe volumetric water content differences on day 126 for planes through $Y_{2}=6 \mathrm{~m}$ and $Y_{2}=10 \mathrm{~m}$ parallel to the trench face. Values expressed as differences from day 0 water content Small circles in plots indicate missing data.

30 Experiment IIb neutron probe volumetric water content differences on day 525 for a plane through $X_{2}=0 \mathrm{~m}$ along the length of plot 2, and for a plane through $Y_{2}=2 \mathrm{~m}$ parallel to the trench face. Values expressed as differences from day 0 water content . Small circles in plots indicate missing data. ............................ 48

31 Experiment IIb neutron probe volumetric water content differences on day 525 for planes through $Y_{2}=6 \mathrm{~m}$ and $Y_{2}=10 \mathrm{~m}$ parallel to the trench face. Values expressed as differences from day 0 water content Small circles in plots indicate missing data.

32 Experiment IIb neutron probe volumetric water content on day 525 for a plane through $X_{2}=0 \mathrm{~m}$ along the length of plot 2 , and for a plane through $Y_{2}=2 \mathrm{~m}$ parallel to the trench face Small circles in plots indicate missing data $\ldots \ldots \ldots \ldots \ldots \ldots \ldots \ldots \ldots \ldots \ldots \ldots$

33 Experiment IIb neutron probe volumetric water content on day 525 for plane through $Y_{2}=6 \mathrm{~m}$ and $Y_{2}=10 \mathrm{~m}$ parallel to the trench face. Small circles in plots indicate

34 Experiment IIb neutron probe wetting front in a plane through $Y_{2}=2 \mathrm{~m}$ parallel to the trench face.

35 Experiment IIb neutron probe mass balance over time.

36 Experiment IIb soil tension contours at $Y_{2}=0.5 \mathrm{~m}$ from trench face for days $0,7,14$, and 33 of the experiment. Median tension values are shown within individual plots

37. Experiment IIb soil tension contours at $Y_{2}=0.5 \mathrm{~m}$ from trench face for days $47,61,72$, and 79 of the experiment. Median tension values are shown within individual plots.

38 Experiment IIb soil tension contours at $Y_{2}=0.5 \mathrm{~m}$ from trench face for days 112,147 , 281 , and 525 of the experiment. Median tension values are shown within individual plots.

39 Experiment Ilb soil tension front at $\mathrm{Y}_{2}=0.5 \mathrm{~m}$ from trench face. Front delimited by tensions less than or equal to $100 \mathrm{mb}$.

40 Experiment IIb soil tension front at $\mathrm{Y}_{2}=0.5 \mathrm{~m}$ from trench face. Front delimited by tensions less than or equal to $300 \mathrm{mb}$.

41 Experiment IIb tritium contours at $Y_{2}=0.5 \mathrm{~m}$ from trench face for days $0,33,47$, and 70 of the experiment. Concentrations as $\mathrm{C} / \mathrm{Co}$ normalized for experiment IIb input.

42 Experiment IIb tritium contours at $Y_{2}=0.5 \mathrm{~m}$ from trench face for days $79,112,281$, and $\mathbf{5 2 5}$ of the experiment. Concentrations as $\mathrm{C} / \mathrm{Co}$ normalized for experiment IIb input. 
43 Experiment IIb tritium breakthrough curves at $X_{2}=0.25 \mathrm{~m}$ and samplers at various depths. Concentrations as $\mathrm{C} / \mathrm{Co}$ normalized for experiment IIb input. Isolated symbols boxed to the right of the plots indicate day 525 concentrations. Vertical dashed lines indicate the end of plot irrigation.

44 Experiment IIb tritium breakthrough curves at $X_{2}=0.75 \mathrm{~m}$ and samplers at various depths. Concentrations as $\mathrm{C} / \mathrm{Co}$ normalized for experiment IIb input. Isolated symbols boxed to the right of the plots indicate day 525 concentrations. Vertical dashed lines indicate the end of plot irrigation.

45 Experiment IIb tritium breakthrough curves at $\mathrm{X}_{2}=1.25 \mathrm{~m}$ and samplers at various depths. Concentrations as $\mathrm{C} / \mathrm{Co}$ normalized for experiment IIb input. Isolated symbols boxed to the right of the plots indicate day 525 concentrations. Vertical dashed lines indicate the end of plot irrigation.

46 Experiment IIb chromium contours at $Y_{2}=0.5 \mathrm{~m}$ from trench face for days $30,70,112$, and 525 of the experiment. Concentrations as $\mathrm{C} / \mathrm{Co}$.

47 Experiment IIb chromium breakthrough curves at $\mathrm{X}_{2}=0.25 \mathrm{~m}$ and samplers at various depths. Concentrations as $\mathrm{C} / \mathrm{Co}$. Vertical dashed lines indicate the end of plot irrigation.

48 Experiment IIb chromium breakthrough curves at $\mathrm{X}_{2}=0.75 \mathrm{~m}$ and samplers at various depths. Concentrations as $\mathrm{C} / \mathrm{Co}$. Vertical dashed lines indicate the end of plot irrigation.

49 Experiment IIb chromium breakthrough curves at $X_{2}=1.25 \mathrm{~m}$ and samplers at various depths. Concentrations as $\mathrm{C} / \mathrm{Co}$. Vertical dashed lines indicate the end of plot irrigation.

50 Experiment IIb chromium and tritium breakthrough curves at $X_{2}=0.25 \mathrm{~m}$ and samplers at 0.5 and $1.5 \mathrm{~m}$ depths. Tritium curves shifted to the left by 29 days to normalize pulse applications at the soil surface. Concentrations expressed as $\mathrm{C} / \mathrm{Co}$

51 Experiment IIb PFBA contours at $Y_{2}=0.5 \mathrm{~m}$ from trench face for days 30,47 , and 70

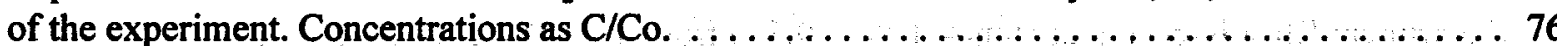

52 Experiment IIb DFBA contours at $Y_{2}=0.5 \mathrm{~m}$ from trench face for days 47 and 70 of the experiment. Concentrations as $\mathrm{C} / \mathrm{Co}$.

53 Experiment IIb bromide contours at $Y_{2}=0.5 \mathrm{~m}$ from trench face for days $14,30,47$, and 70 of the experiment. Concentrations as $\mathrm{C} / \mathrm{Co}$ are normalized for experiment IIb application of the solute.

54 Experiment IIb nitrate ion contours at $Y_{2}=0.5 \mathrm{~m}$ from trench face for days $14,30,47$, and 70 of the experiment. Concentrations as $\mathrm{mg} / \mathrm{l}$ of soil solution.

55 Experiment IIb chloride ion contours at $Y_{2}=0.5 \mathrm{~m}$ from trench face for days $14,30,47$, and 70 of the experiment. Concentrations as $\mathrm{mg} / \mathrm{l}$ of soil solution. 
56 Location of experiment IIb soil cores relative to irrigated plot, earlier experiment IIa soil cores and nearby neutron probe access tubes.

57 Experiment Ilb redistribution cores volumetric water at $Y_{2}=5 \mathrm{~m}$ compared with neutron probe volumetric water at $Y_{2}=6 \mathrm{~m}$.

58 Experiment IIb redistribution cores volumetric water at $\mathrm{Y}_{2}=9 \mathrm{~m}$ compared with neutron probe volumetric water at $Y_{2}=10 \mathrm{~m}$.

59 Experiment IIb neutron probe volumetric water content differences on day 310 for planes of access tubes at $Y_{2}=6 \mathrm{~m}$ and $Y_{2}=10 \mathrm{~m}$ from the trench face. Values expressed as differences from day 0 water content.

60 Experiment IIb redistribution cores tritium contours at $Y_{2}=5 \mathrm{~m}$ from the trench face. Concentration expressed as $\mathrm{C} / \mathrm{Co}$.

61 Experiment IIb redistribution cores tritium contours at $Y_{2}=9 \mathrm{~m}$ from the trench face.

Concentration expressed as $\mathrm{C} / \mathrm{Co}$.

62 Experiment IIb redistribution cores chromium contours at $Y_{2}=5$ and $9 \mathrm{~m}$ from the trench face. Concentration expressed as $\mathrm{C} / \mathrm{Co}$.

63 Experiment IIb redistribution cores chloride and nitrate contours at $Y_{2}=5 \mathrm{~m}$ from the trench face. Concentration expressed as me/L of soil solution.

64 Experiment IIb redistribution cores chloride and nitrate contours at $Y_{2}=9 \mathrm{~m}$ from the trench face. Concentration expressed as me/L of soil solution.

65 Experiment IIb redistribution cores chloride ion vs nitrate ion scatter plot for combined $Y_{2}=5 \mathrm{~m}$ and $9 \mathrm{~m}$ observations. Concentration expressed as me/L of soil solution. 


\section{TABLES}

I Irrigation regime and tracers applied during experiment $\mathrm{IIb} \ldots \ldots \ldots \ldots \ldots \ldots \ldots \ldots$

2 Analytical, equipment and software used for analysis of the fluorobenzoates $\ldots \ldots \ldots \ldots \ldots$

3 Mobile phase characteristics and gradients for analytical events of January $7-9,1991 \ldots \ldots$

4 Retention times for benzoates, bromide and nitrate $\ldots \ldots \ldots \ldots \ldots \ldots \ldots \ldots \ldots \ldots$

5 Standard solutions for bromide, chloride, and nitrate analyses $\ldots \ldots \ldots \ldots \ldots \ldots \ldots$

6 Mean water contents $\left(\mathrm{cm}^{3} / \mathrm{cm}^{3}\right)$ and mean water content differences $\left(\mathrm{cm}^{3} / \mathrm{cm}^{3}\right)$ for each of 4 observational planes at days $31,72,126$, and $525 \ldots \ldots \ldots \ldots \ldots \ldots \ldots \ldots \ldots \ldots \ldots$

7 Mean tensions on days $\mathbf{7 0}$ and 72 for the three center tensiometer locations at each of six depths 


\section{EXECUTIVE SUMMARY}

The U.S. Nuclear Regulatory Commission (NRC) has been directed by Congress in the Low Level Waste Policy Act of 1980 to develop regulatory guidance and assist the individual states and compacts in siting and assessing future low level radioactive waste (LLW) disposal facilities. Technical siting and performance requirements have been issued by NRC in 10 CFR Part 61 for use in LLW sites. These technical criteria encourage LLW disposal in the unsaturated zone (above the regional water table) and also require that the site be capable of being modeled. Conceptual models that describe two- and three-dimensional flow and solute transport in spatially variable hydrogeologic systems have been developed. However, the number of well controlled field studies to test and validate these models is limited. Therefore, NRC funded a validation study to develop an accepted laboratory and field data base suited to test deterministic and stochastic flow and transport models. The experimental plan for this study was developed by investigators at The University of Arizona, New Mexico State University, Pacific Northwest Laboratory, and Massachusetts Institute of Technology in cooperation with NRC.

The experiments were performed on a site located in southern New Mexico, 24 miles northeast of Las Cruces. Three experiments were performed on the sides of a $26.4 \mathrm{~m}$ long by $4.8 \mathrm{~m}$ wide by $6.0 \mathrm{~m}$ deep trench. This trench was constructed to determine the variability in subsurface hydraulic properties, and to gain access to the soil below the plots for installation of tensiometers and solution samplers. A total of three experiments were conducted at the Las Cruces Trench Site. The results of experiment I and most of the results of experiment IIa were presented in Wierenga et al., 1990. The present report contains some results from experiment IIa and all results from experiment IIb. For experiments IIa and IIb a $1.22 \mathrm{~m}$ by $12 \mathrm{~m}$ strip, perpendicular to the trench wall, was uniformly irrigated using a carefully controlled drip irrigation system. During and after the first irrigation, the movement of water and the transport of tritium, bromide, chloride, and nitrate were measured by neutron probe, tensiometers, solution samplers, and core sampling. The results were presented in an earlier report. The analyses of the core samples taken after experiment IIa are presented in this report.

Following experiment IIa, the same strip was irrigated a second time with water containing tritium, boron, chromium, bromide, and two fluorobenzoic acids (experiment IIb). The results of this latter experiment constitute the main part of this report. They show (especially the water flow data obtained with the neutron probe, and the tritium data from core sampling) fairly regular downward and lateral flow of water and solute. Even though the soil was heterogeneous, there was no preferential flow observed at this arid soil site.

The water flow and tritium data presented here has been used to test a variety of models (Hills and Wierenga, 1993). 


\section{FOREWORD}

This technical report was prepared by The University of Arizona under a contract with the Waste Management Branch of the Office of Nuclear Regulatory Research (FIN L1286). This report presents experimental methods, procedures, and data from the third Las Cruces Trench experiment.

Reference herein to any specific commercial product, process, or service by trade name, trademark, manufacturer, or otherwise does not necessarily constitute or imply its endorsement, recommendation, or favoring by the United States government.

NUREG/CR is not a substitute for NRC regulations, and compliance is not required. The approaches and/or methods described in the NUREG/CR are provided for information only. Publication of this report does not necessarily constitute NRC approval or agreement with the information contained herein. 


\section{ACKNOWLEDGMENTS}

The authors wish to acknowledge the support from the U.S. Nuclear Regulatory Commission and the U.S. Environmental Protection Agency. The advice of Dr. Lynn Gelhar and Dr. Dennis McLaughlin from the Massachusetts Institute of Technology, Dr. Glendon Gee from Battelle Pacific Northwest Laboratories, and Mr. Tom
Nicholson and Dr. Ralph Cady from the U.S. Nuclear Regulatory Commission with the design and execution of the experiment is gratefully acknowledged. Finally, this project could not have been done without the help of David Hudson, Warren Strong, Ricardo Carrasco, and David Lopez. 


\section{Introduction}

The Low Level Policy Waste Act of 1980 was sinacted by the Unites States Congress to guide the regulation and development of low level waste disposal sites. As a result, the U.S. Nuclear Regulatory Agency has required that such sites "shall be capable of being characterized, modeled, analyzed, and monitored" (10 CFR, Part 61, Subpart D). These requirements have created a demand for flexible computer models that are useful for predicting water and solute movement through undisturbed and spatially variable soils and geologic features. Many models have emerged with claims to satisfy these needs. But due to the difficulty of conducting well-controlled field-scale experiments, few data sets exist to provide spatially descriptive soil characterization data and observations of water and solute movement in the field; thus, many of these models are inadequately validated.

The Las Cruces Trench Site experiments were begun to provide a solid set of data for model validation. A $6 \mathrm{~m}$ deep trench was excavated in 1986 to provide experimental access to large horizontal and vertical soil faces. As the excavation work progressed, the site was extensively sampled and characterized. A roofed structure was constructed within the trench with several levels of access to exposed soil faces. In 1987 the first water flow and transport experiment was performed in an area adjacent to the south side of the trench (Plot 1).

Afterwards, in early 1989 a single deep soil core was extracted near the center of the plot to describe the redistribution of applied water and solutes. The methodologies of the first irrigation experiment were improved upon, and in 1988 a second experiment was performed on an area adjacent to the north side of the trench (Plot 2).
In the spring of 1990 a series of $3 \mathrm{~m}$ deep soil cores was taken from transects in Plot 2 to describe the redistribution of water and solutes applied during the second irrigation (experiment IIa). About that time two additional deep cores were taken from Plot 1 . In late summer of 1990 a third water flow and transport experiment (IIb) was started in plot 2. Finally, in 1991 a second series of soil cores, to 6.3 meter depth, was collected from Plot 2 to describe solute and water redistribution following the two irrigation experiments there. A timeline illustrates the chronological relationships between these various experiments and projects at the trench (Figure 1).

In the late 1980's the project became part of INTRAVAL, an international consortium to test and validate geosphere models. INTRAVAL participants agreed to model the third leaching experiment based on information obtained during previous experiments and trench site characterization work. Participating modelers were given initial and boundary conditions and the events of water and solute application during the third irrigation experiment. Experimental results were withheld from the modelers until model predictions were completed, thus creating a "blind validation" of a variety of stochastic and deterministic models.

The Las Cruces Trench experiments were designed by scientists at The University of Arizona and New Mexico State University in cooperation with Glendon Gee of Battelle Pacific Northwest Laboratories and Lynn Gelhar of the Massachusetts Institute of Technology. Participating modelers represent a variety of institutions, including Batelle Pacific Northwest Laboratories, New Mexico State University, Massachusetts Institute of 
Technology, The University of Texas, and the Center for Nuclear Waste Regulatory Analyses.

This report describes several experiments performed at the trench site since the second irrigation experiment. The purpose of the report is to present a comprehensive summary of the data collected since the end of experiment $\mathrm{Ila}$, and since the first deep core collected from Plot 1. Experiments previous to those discussed here are detailed in Wierenga et al (1988, 1989, 1990 and 1991). Comparisons of observed data with model predictions are provided in Hills and Wierenga (1993) and will be published elsewhere.

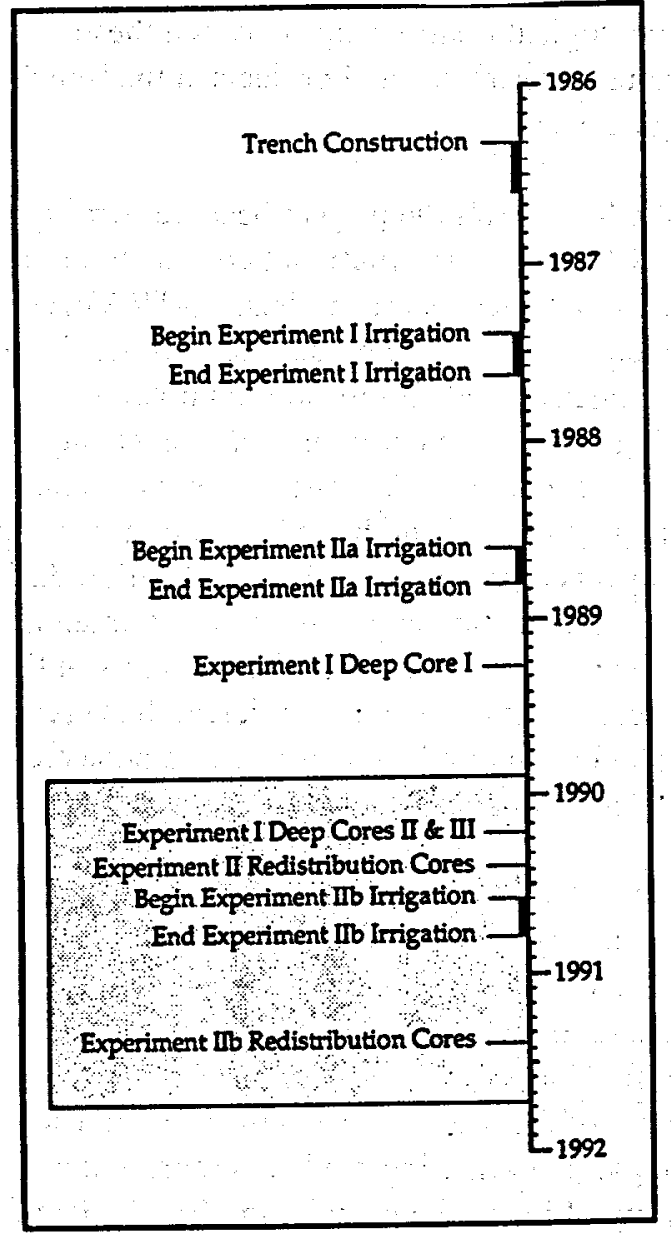

Figure 1. Timeline for Las Cruces Trench Site experiments. Shaded area denotes experiments described in this report. 


\section{Review of Previous Work}

\subsection{Site Description and Characterization}

The Las Cruces Trench Site is located on the New Mexico State University College Ranch about $40 \mathrm{~km}$ northeast of Las Cruces, New Mexico. It is at an elevation of about $1357 \mathrm{~m}$ on the eastern Piedmont slope of Summerford Mountain, the northern most peak of the Dona Ana Mountains (Figure 2). The climate in the area is characterized as arid, with the boundary between the arid (less than $25 \mathrm{~cm}$ rain per year) and semiarid considered to be at an elevation of $1524 \mathrm{~m}$. Average annual precipitation at the site is $23 \mathrm{~cm}$ with an average Class A pan evaporation of $239 \mathrm{~cm}$ per year (Wierenga et al., 1987). Soils at the site consist of well-drained coarse loamy Typic Haplargids which overlie a sequence of well-defined geomorphic surfaces exposed in the top $6 \mathrm{~m}$ (Gile and Grossman, 1979). Additional discussion of the geomorphology and soils at the site is presented in Appendix C-8.

In the spring and summer of 1986 a $26.4 \mathrm{~m}$ long, $4.8 \mathrm{~m}$ wide and $6.0 \mathrm{~m}$ deep covered trench was excavated in undisturbed soil to provide experimental access to large vertical soil flanks, to collect soil samples and undisturbed cores for laboratory characterization and to allow in-situ measurement of soil hydraulic properties. During excavation nine major soil horizons were identified in the vertical profile. Centered within each of these horizons a 25 meter horizontal transect was established along the north side of the trench with $\mathbf{5 0}$ sampling stations located on $50 \mathrm{~cm}$ intervals. Each successive transect with depth (and each sampling station) was located directly below the transect above. Beginning with the first transect at the soil surface three stations were selected which roughly divided the length of the trench into thirds. These stations were sampled continuously in $13 \mathrm{~cm}$ increments from the surface to $6 \mathrm{~m}$ depth. With this sampling scheme a total of 594 undisturbed cores and 594 soil samples were collected to gather statistical information about soil morphology and hydraulic properties at the site. These data are discussed in detail by Wierenga et al. (1988, 1989, 1990).

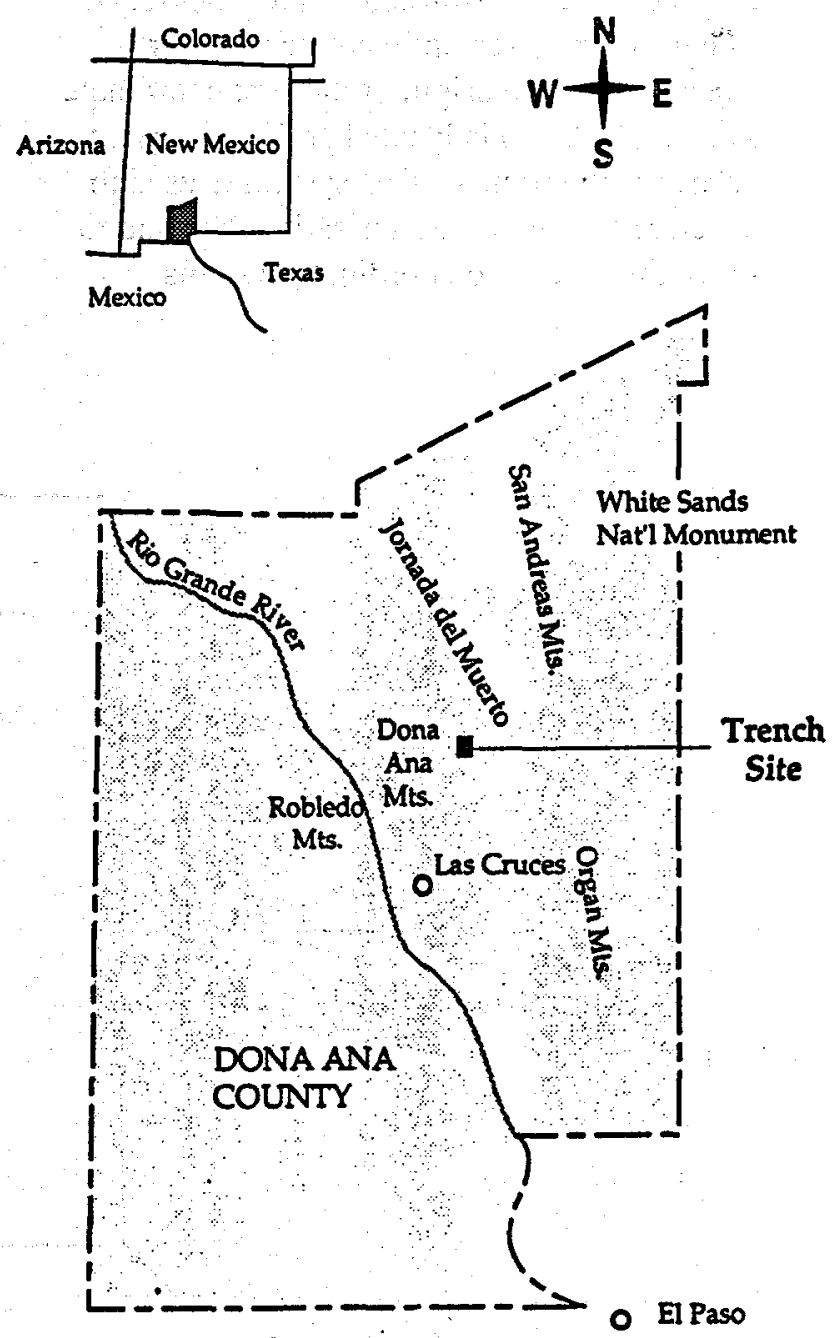

Figure 2. Location of Las Cruces Trench Site within the state of New Mexico and within the county of Dona Ana. 


\subsection{Coordinate Systems}

Three coordinate systems are used to describe the locations of the neutron probe tubes, tensiometers, solutions samplers, and irrigated areas relative to the northwest corner of the trench (Figure 3), a method first described by Wierenga et al., 1990. In each system the $X$ axes are parallel to the north wall of the trench, which run roughly east-west; the $Y$ axes are perpendicular to $X$; and the $Z$ axis describes depth from the soil surface, always positive with depth. Each $\mathrm{X}$ and $\mathrm{Y}$ axis is located with respect to the undisturbed wall of the trench rather than any structure emplaced after excavation. The origin of the first coordinate system $(X, Y, Z)$ is located at the northwest corner of the trench. This system is used to describe the site characterization data and to unify the other two coordinate systems to a common point. The $\mathrm{X}$ axis is parallel to the north wall and positive to the east. The $Y$ axis is horizontal and positive to the north. The $Z$ coordinate describes depth from the soil surface. The second coordinate system $\left(X_{1}, Y_{1}, Z_{1}\right)$, used to describe plot 1 , originates on the south wall of the trench at the center of the plot 1 irrigated area. The $X_{1}$ axis is parallel to the south wall of the trench and is positive to the west. The $Y_{1}$ axis is perpendicular to the $X_{1}$ axis and positive to the south. The $Z_{1}$ axis is depth from an imaginary plane that is level relative to the natural slope of the terrain, a $2.5 \%$ west slope. $Z_{1}$ is at 0 meters where $X_{1}=2.0 \mathrm{~m}$. Due to the $2.5 \%$ slope, the distance to the surface from $Z_{1}=$ 0 is given by:

$$
\text { Distance }(\text { meters })=\left(0.025 \times \mathrm{X}_{1}\right)-0.05
$$

The third coordinate system $\left(X_{2}, Y_{2}, Z_{2}\right)$, used to describe plot 2 , originates on the north wall of

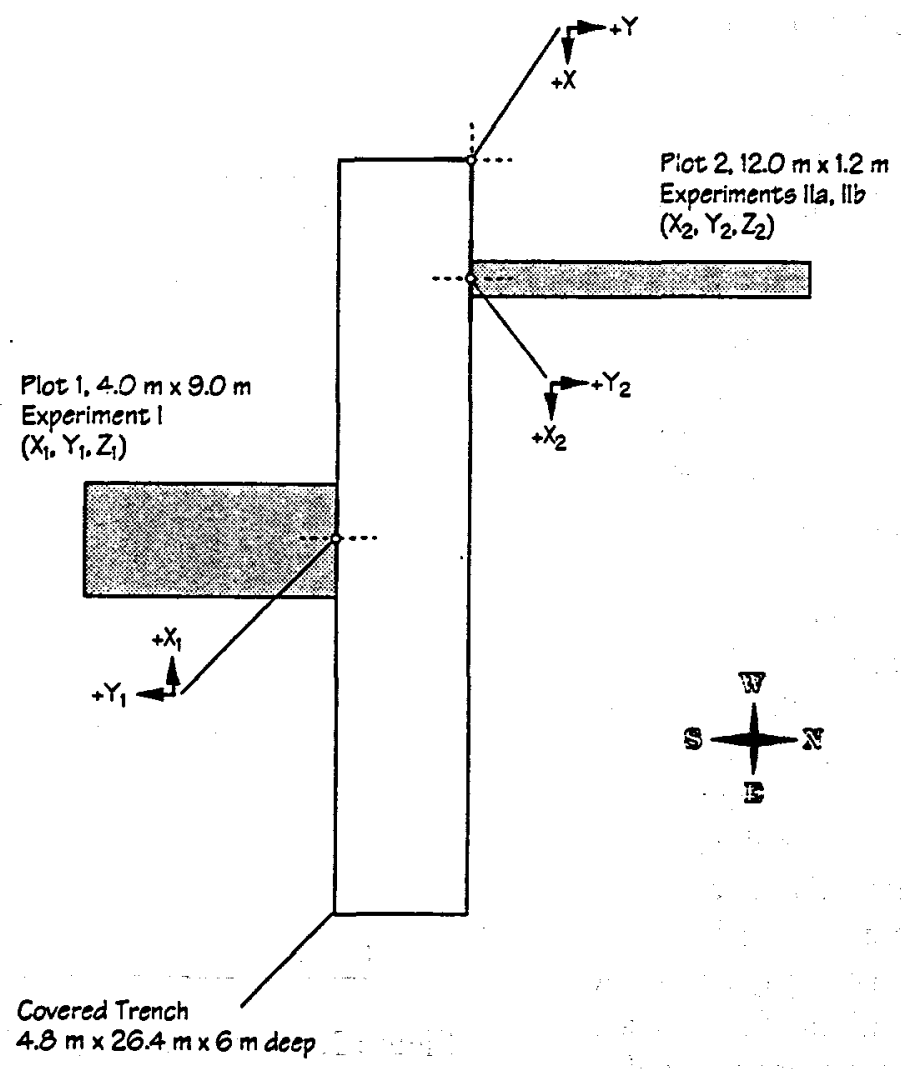

Figure 3. Overhead view of trench, experimental irrigation plots and origination points for $(\mathrm{X}, \mathrm{Y}),\left(\mathrm{X}_{1}, \mathrm{Y}_{1}\right)$, and $\left(\mathrm{X}_{2}, \mathrm{Y}_{2}\right)$ coordinate systems. 
the trench at the center of the plot 2 irrigated area. The positive direction of its axes is similar to the $X, Y, Z$ coordinates. Note that the $X_{2}$ and $X_{1}$ axes are not parallel because the north and south walls of the trench are not exactly parallel. The coordinates for the corners of the trench and experimental plots, neutron access tubes, tensiometers and solution samplers are given in Appendix C-10.

\subsection{Experiment I}

In the summer of 1987 the first irrigation experiment was performed on an area adjoining the south side of the trench (Plot 1). A covered $4 \mathrm{~m}$ wide by $9 \mathrm{~m}$ long area was irrigated by a water application system composed of a closely spaced grid of drip emitters and precision controlled water application pumps. Water was applied four times a day with an average surface flux of $1.82 \mathrm{~cm} \mathrm{~d}^{-1}$ for 86 days; the first 10 days with tritium-containing well water and the final 76 days with non-traced water. Water flow was monitored by neutron probe and by tensiometers. Ceramic cup solution samplers were embedded $50 \mathrm{~cm}$ deep into the face of the trench to draw samples for tritium analysis. Further descriptions of this experiment are found in Wierenga et al. (1990).

During the first experiment the soil walls of the trench were exposed to allow visual tracking of the wetting front. Eventually a portion of the south wall was weakened by the applied water and collapsed into the trench, abruptly ending any further data gathering from tensiometers and solution samplers. The fallen debris was cleared from the floor of the trench and an engineered shoring structure was constructed to prevent further cave-ins. This structure featured heavy wooden lagging, a packed soil backfill and a plastic vapor barrier between the backfill and the undisturbed soil wall.

\subsection{Experiment I Redistribution Cores}

On April 6, 1989, 594 days after the last irrigation of plot 1 , a $14.74 \mathrm{~m}$ deep borehole was advanced near the center of the $4 \mathrm{~m}$ by $9 \mathrm{~m}$ irrigated area. A $5.08 \mathrm{~cm}$ diameter split-spoon sampler was used to collect continuous soil cores in $61 \mathrm{~cm}$ intervals. Core sub-samples were analyzed for water content, tritium, chloride ion, electrical conductivity and soil morphological features.

Another pair of cores was collected from plot 1 nearly a year later, on March 16, 1990, 938 days after the last irrigation. The locations of all three boreholes are:

$\begin{array}{cccc}\text { Borehole } & \mathrm{X}_{1}(\mathrm{~m}) & \mathrm{Y}_{1}(\mathrm{~m}) & \mathrm{Z}_{\text {total }}(\mathrm{m}) \\ & & & \\ \text { I } & -0.46 & 4.67 & 14.72 \\ \text { II } & 2.02 & 4.95 & 15.22 \\ \text { III } & 4.00 & 5.00 & 15.19\end{array}$

The purpose of boreholes II and III was to supplement the data collected from the center-of-plot borehole with data from the edge of the irrigated area and 2 meters outside of the area, while remaining near the center of the plot with respect to $Y_{1}$. A 6" hollow-stem CME rotatory drill was used to collect continuous soil cores in $152 \mathrm{~cm}$ long sections. The core samples were analyzed for volumetric water content, tritium, chloride ion, and nitrate ion. The methods and results of this work are described more completely in Appendix C-1.

\subsection{Experiment IIa}

In early 1988 a second irrigation area, $12.0 \mathrm{~m}$ long and $1.2 \mathrm{~m}$ wide, was sited on the north side of the trench (Plot 2). A covered metal structure was fabricated to support a leveled irrigation system for uniform water application to the plot 
area. The irrigation system design was similar to that used in experiment $\mathrm{I}$, consisting of a closely spaced grid of drip emitters (Wade Manufacturing, Fresno, CA, p/n 62-2). For this experiment individual emitter lines were $1.2 \mathrm{~m}$ long with 4 emitters spaced on one-foot centers. Eighty of these emitter lines were connected in parallel on alternate sequence to one of two separate header pipes along the $12 \mathrm{~m}$ length of the plot (Figure 4).

Water supply to the headers was obtained through solenoid control valves which merged to a single buried supply line which in turn was supplied by a precision-metered pumping system located in a separate building.

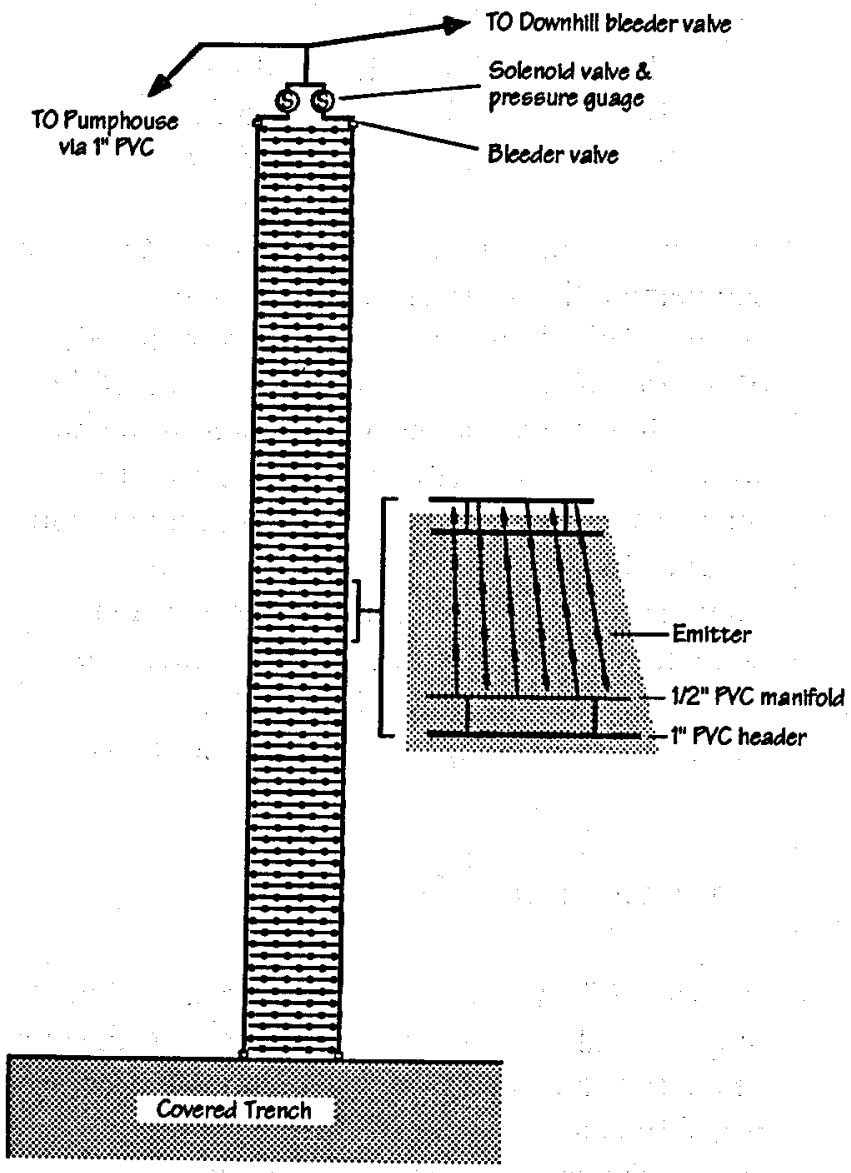

Figure 4. Plot 2 irrigation system of emitters and header lines.
Pre-irrigation levels of soil nitrate and chloride near plot 2 were analyzed from a single vertical transect of samples collected at the trench face. The purpose of this work was to identify natural distributions of these ions before experimental disturbance. These data are presented in Appendix C-2.

The first plot 2 irrigation (experiment IIa) began on August 8, 1988. Well water was applied twice a day (once a day through each header line) resulting in an average surface flux of 0.43 $\mathrm{cm} \mathrm{d}^{-1}$ over the irrigated area. Two solute tracers were added to the irrigation water during the first 11.5 days $(719.5 \mathrm{~L})$ of irrigation: 71.95 $\mathrm{mCi}$ of tritium and $575 \mathrm{~g}$ of bromide as potassium bromide. After a total of 75.5 days (4721 L), irrigation was discontinued, beginning a redistribution phase of the experiment. Water and solute movements were monitored during redistribution until the beginning of the next experiment, IIb.

The advance of the wetting front was tracked by neutron probe and tensiometer measurements, and the solute front by solution sampler collections of in-situ soil solution. Prior to irrigation a total of 37 aluminum, 5.08 $\mathrm{cm}$-diameter neutron probe access tubes were emplaced within and adjacent to the irrigated area, each one $6 \mathrm{~m}$ deep. Another 6 access tubes were added on April 10, 1989 (day 246 of experiment IIa) making a total of 43 access tubes for the experiment. Readings were taken from $0.25 \mathrm{~m}$ to $6.0 \mathrm{~m}$ depth in $0.25 \mathrm{~m}$ increments.

A regular grid of tensiometers and solution samplers was installed along the face of the trench below the irrigated area. These devices were installed at a $10^{\circ}$ angle through the wooden and backfill materials used to support the trench wall. The $Z$ - coordinates and lengths of the drilled holes were adjusted so that the ceramic cups would be placed at the targeted soil depths $\left(Z_{2}\right)$, in a plane at $Y_{2}=0.5$ meters from the undisturbed trench wall. 
Tensiometers were constructed with ceramic cups rated for 1-bar air entry values (Soil Moisture Equip. Corp., Santa Barbara, CA, $\mathrm{p} / \mathrm{n}$ 652X04-B1M3) and $1 / 4$ " nominal clear schedule 40 PVC pipe (U. S. Plastics, Lima, $\mathrm{OH})$. The construction of these devices is described in Appendix C-6. A few days before arrival of the wetting front tensiometers were filled with water and then sealed with septum stoppers for easy tension measurement with a hand-held pressure transducer (Figure 5).

A solution sampler was constructed and installed in the trench wall very much like a tensiometer. A vacuum-operated apparatus was inserted within the PVC pipe of the sampler to draw soil solution into the ceramic cup and then transfer that solution to a sample vial (Figure 6).

The irrigated plot and surrounding area were covered with a UV resistant reinforced 36 mil chlorinated polyethylene pond liner (Watersaver Co. Inc., Denver, CO., PO. Box 16465, 602-966-6892, model CPER 036) to prevent evaporation from and rain water infiltration into the soil surface. This pondliner extended approximately $X_{2}= \pm 6$ meters on the plot flanks and from the face of the trench to about $Y_{2}=18$ meters.

The methods and results of experiment IIa are detailed by Wierenga et al., 1990 . That report discusses results obtained through May 24, 1990 (day 655), one day before the first collection of $3 \mathrm{~m}$ deep soil cores from the plot.

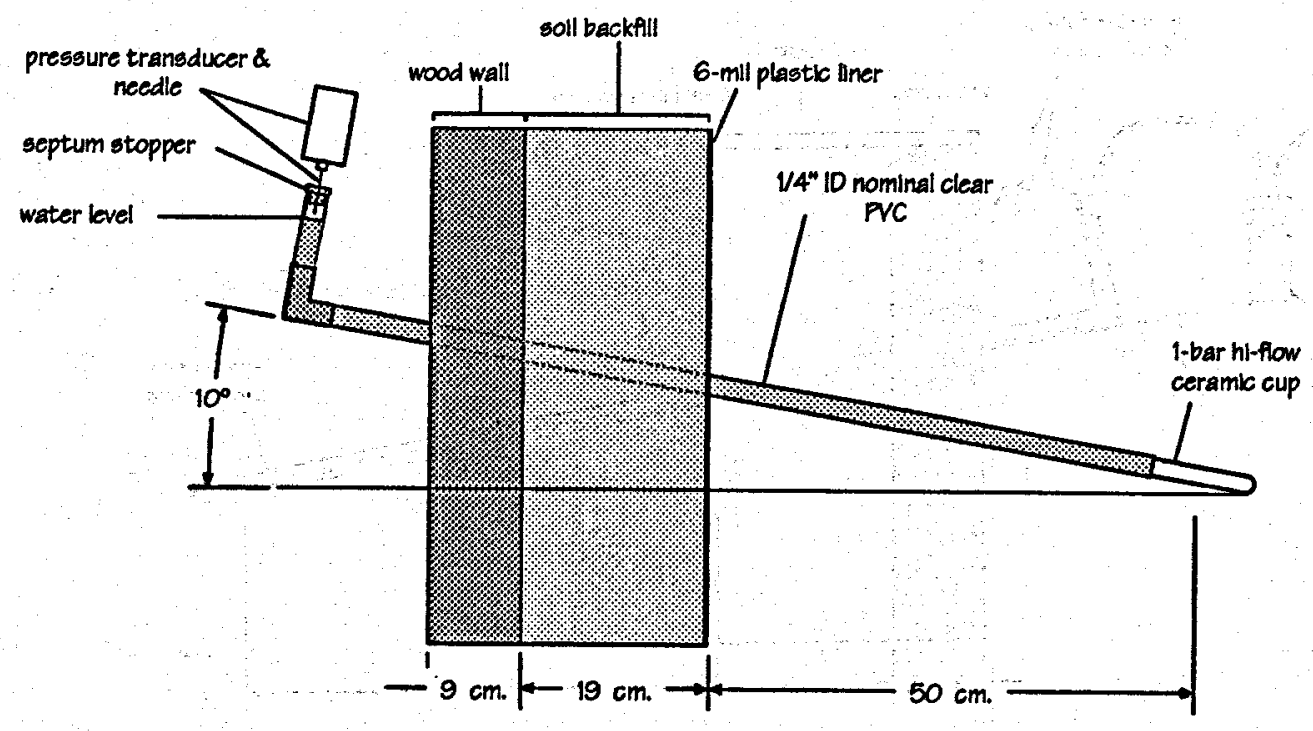

Figure 5. Installation and use of tensiometer through trench wall 


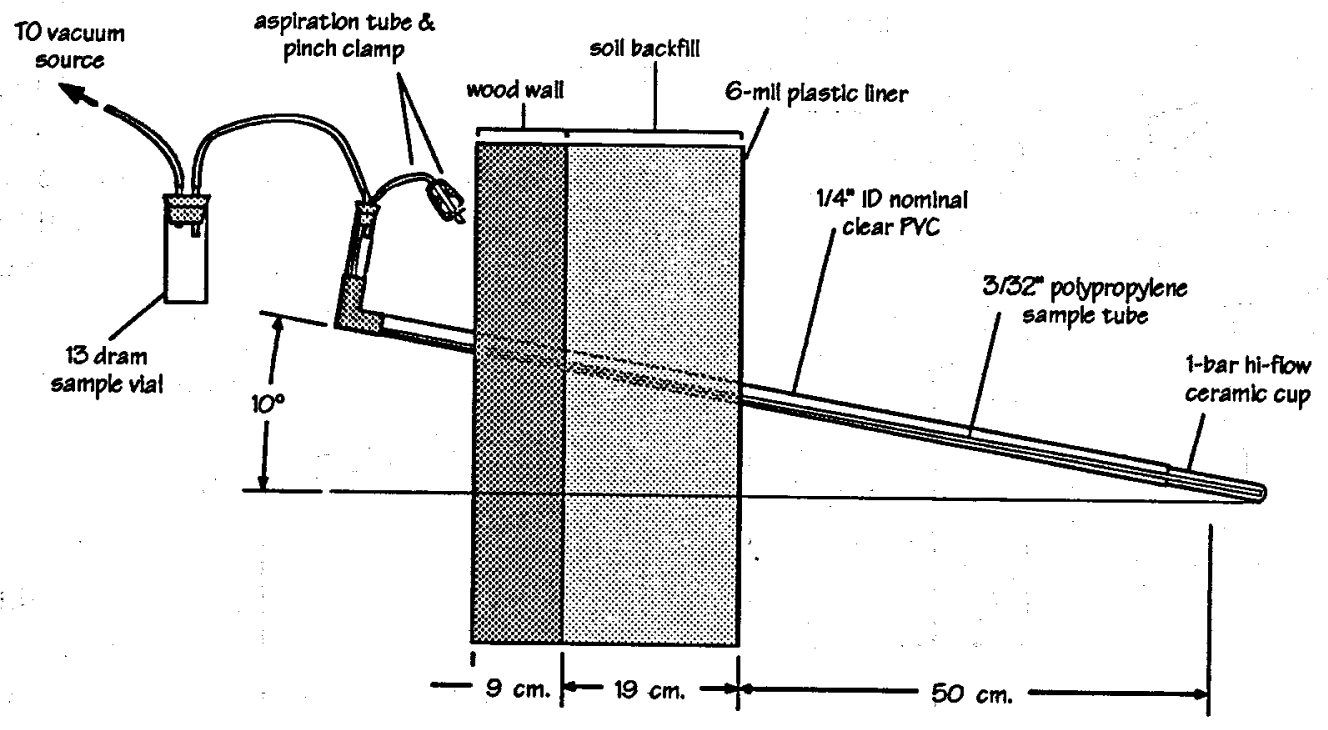

Figure 6. Installation and use of solution sampler device through trench wall. 


\section{Experiment IIa Redistribution Cores}

\subsection{Core Collection and Processing}

On May 30, 1990,586 days after the last irrigation of experiment Ila, a series of soil cores was collected from within plot 2 . Cores were taken during a two-day period from two transects bisecting the irrigated plot at $Y_{2}=5.0$ and 9.0 meters from the trench face. Boreholes were made on $0.5 \mathrm{~m}$ centers along each transect at $\mathrm{X}_{2}=-1.5,-1.0,-0.5,0,0.5,1.0$, and $1.5 \mathrm{~m}$ to a depth of 1.4 to $1.8 \mathrm{~m}$. Forty-two days later a second set of core samples was collected from the same boreholes to extend sampling depth to $3.0 \mathrm{~m}$ (Figure 7). Altogether a total of 214 soil core samples from 14 boreholes were analyzed.

One inch diameter hollow-stem soil sampling tubes (6' and 10' long) and a $13 \mathrm{~kg}$ drop hammer (Soil Moisture Equipment Corp., Santa Barbara, CA) were used to collect the cores. Beginning at the soil surface the sampling tube was hammered $10 \mathrm{~cm}$ into the soil and then removed from the hole. This first soil core was retained for analysis. A second soil core representing the next $5 \mathrm{~cm}$-depth of soil was discarded. A third $10 \mathrm{~cm}$-long soil core, centered at $20 \mathrm{~cm}$-depth, was retained. Thereafter, the sampling tube was hammered into the soil in $10 \mathrm{~cm}$ increments to the bottom of the borehole. The consequent soil cores were alternately disposed or retained in sequence. Each retained core was placed in a tared aluminum soil can which was then capped, weighed and sealed with two to three layers of Parafilm ${ }^{\circ}$ wrap (American National Can, Greenwich, CT) to prevent evaporation. The samples were protected from direct sunlight while in the field and then taken to the laboratory for refrigerated storage.

Before processing, samples were brought to room temperature, unwrapped, and reweighed to determine any evaporative loss during storage. The sample was mixed and any non- representative coarse fragments were weighed and discarded. Samples were then divided into two sub-samples, one to determine gravimetric water content and the other to make a soil solution extract for chemical analysis. The portion used to measure water content was oven-dried at $105^{\circ} \mathrm{C}$ for 24 hours before re-weighing.

The sub-sample used to make a solution extract was placed in a tared plastic beaker and weighed. An amount of deionized-distilled water equal to about one-fourth of the fresh soil mass was added to the container, weighed again with the container and then mixed with a spatula for about one minute. The rim of the beaker was sealed with a close fitting acrylic plate and allowed to stand for 20 minutes before extraction. The soil paste was stirred briefly once more during the standing period.

A Buchner funnel apparatus was used to extract the paste samples. Each ceramic funnel was fit with a $5.5 \mathrm{~cm}$-diameter disk of Whatman's \#42 ashless filter paper and placed in a rack which was connected to a common vacuum source, about $\mathbf{4 2 5}$ mbar strong. During filtration the rim of the Buchner funnel was covered to reduce evaporation. The effluent was collected in clean 13 dram polypropylene vials which were capped and then wrapped with Parafilm ${ }^{\circ}$ for refrigerated storage.

\subsection{Sample Analysis}

The extracts were analyzed for tritium, chloride ion, and bromide ion. For tritium $0.5 \mathrm{ml}$ aliquots of sample were mixed with $5.0 \mathrm{ml}$ of Aquasol universal liquid scintillation cocktail (Dupont New England Nuclear, Boston, MA) in $8 \mathrm{ml}$ "Mini Poly-Q" scintillation vials (Beckman Instruments, Inc., Fullerton, $\mathrm{CA}$ ) and run with a 


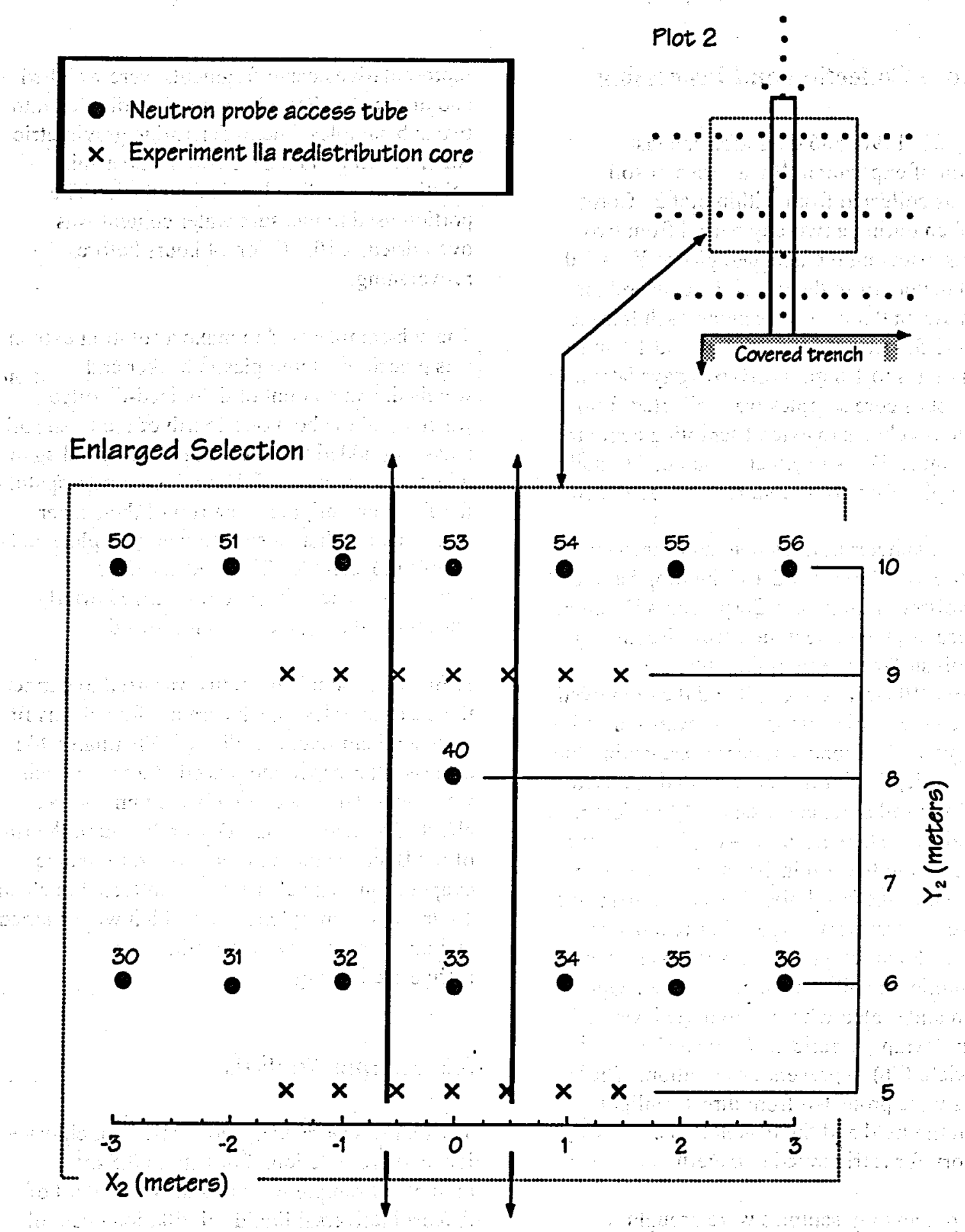

Figure 7. Location of experiment IIa soil cores. 
Beckman model LS1800 scintillator in "autorun" mode. Raw counts were taken for 10 minutes within a counting window of $0-400 \mathrm{~nm}$. Since relative tritium content was sought rather than absolute concentrations no quenching factors or standards were used in the counting routine. Tritium background was taken from vials prepared with well water in place of extract.

Chloride was analyzed with a Buchler model6 4-2500 chloridometer (Buchler Instruments, Inc., Ft. Lee, NJ). The instrument was used in high sensitivity mode. A series of five standard readings were taken with $0.1 \mathrm{ml}$ aliquots of 100 $\mathrm{me} / \mathrm{L} \mathrm{NaCl}$ standard to make a one-point calibration. This calibration was updated with two new standard readings every 15 samples. Five background-correction vials were prepared with distilled-deionized water. Sample titrations were performed with 0.10 to $0.50 \mathrm{ml}$ volume aliquots, depending on the concentration of the sample.

Bromide was analyzed with an Orion model 901 bromide ion electrode (Cambridge, MA). The single junction reference electrode was an Orion model 900100 filled with a saturated solution of $\mathrm{AgCl}$ (Orion, model 900001). Measurements were taken to the nearest millivolt using a Beckman model $\phi \mathrm{pH} / \mathrm{mV}$ meter. A calibration curve was constructed by reading 4 standard $\mathrm{NaBr}$ solutions containing 12.5, 125.0, 437.5, and $1250.0 \mathrm{mg} / \mathrm{L}$. These standard readings were taken at the beginning and at the end of a run of analyses. An average of the two sets of standard readings was used to construct the curve.

Bromide analyses with an ion selective probe have tended to yield suspect data. We found that electrode performance deteriorates quickly when a new electrode is used continuously with a soil solution matrix. Though a consistent calibration curve can be made with a series of bromide salt standards, selectivity between bromide and chloride ions in a strong and complex solution matrix is compromised.
Therefore, the bromide electrode results should be viewed critically.

Gravimetric water content values from the soil core samples were converted into volumetric water units by using an estimated term for soil bulk density. The bulk density was taken from soil cores collected during the original trench characterization work, near the plane at $Y_{2}=0 \mathrm{~m}$. In that work 9 major morphological horizons were distinguished along the trench face from 0 to $6 \mathrm{~m}$ depth. At the center of each horizon a transect was established along the length of the trench with 50 equidistant sampling stations. Soil cores from each station were evaluated for bulk density (Wierenga et. al., 1987, 1988, and 1989). A mean value of bulk density for each depth was calculated from these 50 measurements in the transect.

Experiment IIa soil core sample density was estimated directly from these means by depthwise linear interpolation.

\subsection{Results and Discussion}

The moisture and solute data for the Experiment Ila redistribution soil cores are presented in tabular form in Appendix C-5a. The volumetric water contents obtained from the soil core gravimetric samples (day 661) at $Y_{2}=5$ and 9 meter planes are compared to the neutron probe water contents at $Y_{2}=6$ and 10 meters, respectively. Figure 8 shows neutron probe water at $Y_{2}=6 \mathrm{~m}$ for day 78 , three days after the last irrigation of experiment IIa, and for day 655,6 days before the soil core samples were collected. The day 78 neutron probe data show that the wetting front was found between 2 and $3 \mathrm{~m}$ depth, with less than $3 \mathrm{~m}$ of lateral movement. By day 655 the wetting front had dispersed to become mostly indistinct. The day 655 distribution of water in the profile is very similar to that found in the $Y_{2}=5 \mathrm{~m}$ plane of soil core samples (Figure 9). The layer of relatively high moisture content found about 0.5 $\mathrm{m}$ deep at $Y_{2}=5 \mathrm{~m}$ is also found at $Y_{2}=6 \mathrm{~m}$ by 

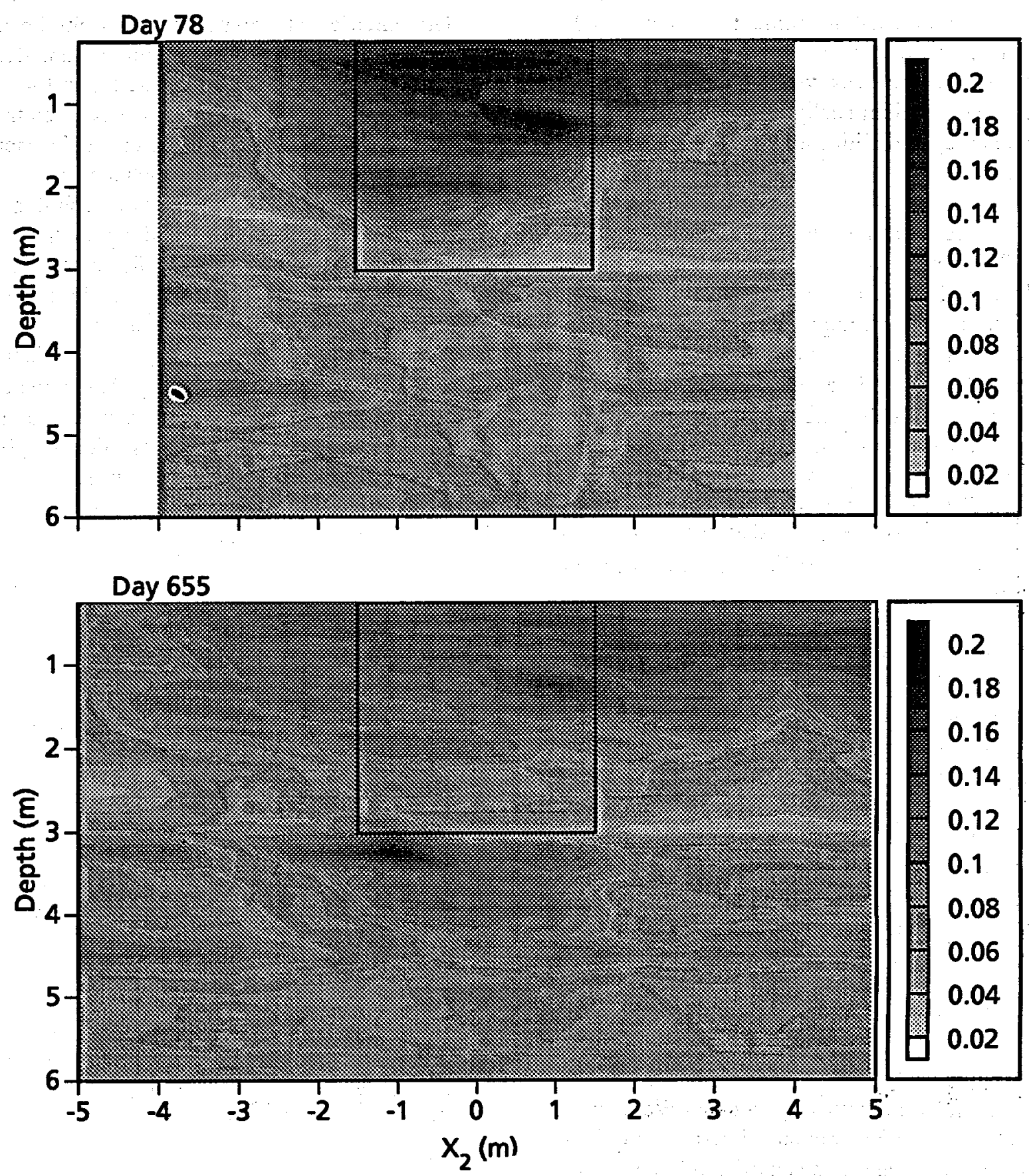

Figure 8. Experiment IIa neutron probe volumetric moisture contours at $\mathrm{Y}_{2}=6 \mathrm{~m}$ on days 78 and 655 . Boxes are added to show similar regions of the profile which were sampled by soil cores at $\mathrm{Y}_{2}=5 \mathrm{~m}$ of day 661 . 


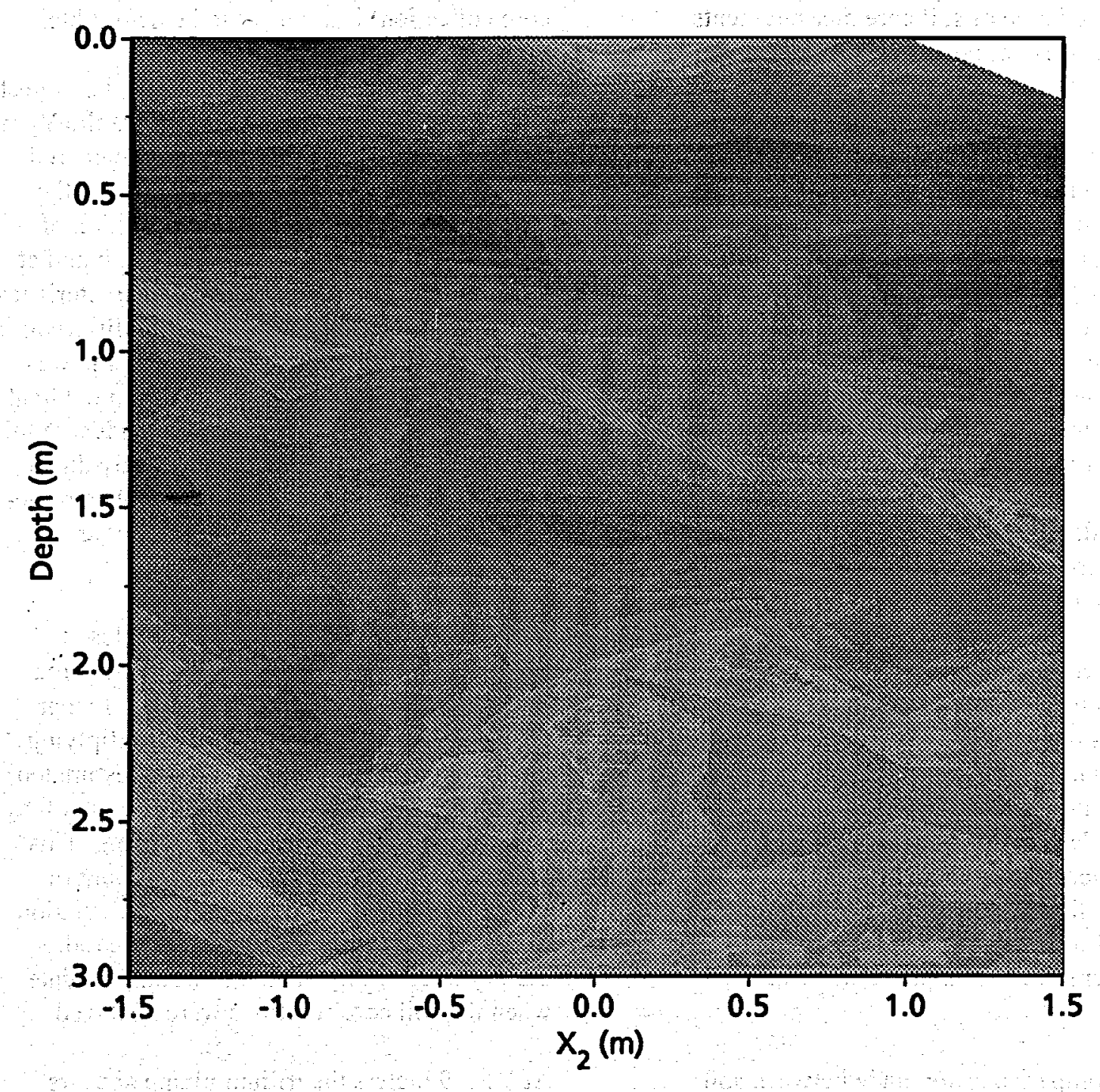

$\begin{array}{llllllllllll}0.02 & 0.04 & 0.06 & 0.08 & 0.10 & 0.12 & 0.14 & 0.16 & 0.18 & 0.20\end{array}$

Figure 9. Experiment Ila soil core volumetric moisture contours at $\mathrm{Y}_{2}=5 \mathrm{~m}$ on day 661 : Gravimetric soil core moisture is converted to volumetric moisture with an estimated term for soil bulk density. 
the neutron probe. Note that the horizontal and vertical scales in Figures 8 and 9 are different. Figure 8 shows the full range of neutron probe measurements at $Y_{2}=6 \mathrm{~m}$ to illustrate the extent of the wetting front. In this figure the more limited area of soil core measurements (of Figure 9) is delineated by the boxed areas.

The total water content determined by neutron probe measurement was compared to that measured gravimetrically from the soil cores (with an estimated soil bulk density term). This comparison was restricted to portions of the profile in common with both methods of measurement, i.e., from $-1.5 \mathrm{~m} \leq \mathrm{X}_{2} \leq 1.5 \mathrm{~m}$ and to $3 \mathrm{~m}$ depth. Within these confines the $\mathrm{Y}_{2}=6 \mathrm{~m}$ neutron probe plane held an average $33.4 \mathrm{~cm}$-long column of water versus about 32.4 $\mathrm{cm}$ measured in the $Y_{2}=5 \mathrm{~m}$ soil core samples.

Lateral redistribution of applied water obtained from neutron probe data is more dramatic at $Y_{2}=10 \mathrm{~m}$ (Figure 10). Soil core water distribution at $Y_{2}=9 \mathrm{~m}$ appears less similar to neutron probe water, though areas of higher moisture content in the $+\mathrm{X}_{2}$ direction, between 0.5 and 1.5 meters depth, correspond to both locations (Figure 11). Note that the soil core contours represent a two-fold increase in horizontal sampling density compared to the neutron probe measurements. The average depth of the water column is similar between the measurement planes; $31.3 \mathrm{~cm}$ of soil-core water at $Y_{2}=9 \mathrm{~m}$ and $31.4 \mathrm{~cm}$ of neutron probe water at $Y_{2}=$ $10 \mathrm{~m}$.

Tritium comparisons are made between soil core sample planes at $Y_{2}=5$ and 9 meters and the plane of solution sampler placement at the trench face, $Y_{2}=0.5 \mathrm{~m}$ (Figures 12, 13, and 14). At the trench face the tritium plume is contoured from data that represents only $61-66 \%$ of the solution sampler grid, i.e., from 27 to 29 individual solution samplers. By contrast each soil core transect provides 107 samples for contour construction. For comparison with soil core transects, tritium at the trench face is contoured from day 429 tritium data since collections of solutions after that day represented fewer samplers and would lead to poorer contour resolution. Tritium redistribution between day 429 and day 661 (soil core collection) is assumed to be negligible.

The horizontal location and shape of the largest body of the tritium plume is similar at the $\mathrm{Y}_{2}=$ $5 \mathrm{~m}$ and $0.5 \mathrm{~m}$ measurement planes, centered about $\mathrm{X}_{2}=0.5 \mathrm{~m}$ from the center axis of the plot. The highest observed concentration of tritium at $Y_{2}=0.5 \mathrm{~m}$ was $27 \%$ of input and at $Y_{2}=5 \mathrm{~m}$ was $23 \%$. But in other characteristics the behavior of the plume was quite different; in terms of vertical displacement the plume was found at least $0.5 \mathrm{~m}$ deeper at $Y_{2}=5 \mathrm{~m}$. Greater horizontal displacement is also evident at the $5 \mathrm{~m}$ transect, though this may be partly due to the higher resolution and more complete representation of the profile with the soil core samples.

Tritium recovery from the core samples at $Y_{2}=5 \mathrm{~m}$, was about $110 \%$ of the tritium mass originally applied to the soil surface. Planar recovery of tritium was found by multiplying individual observations of $\mathrm{C} / \mathrm{Co}$ by (estimated) volumetric water content and the domain of $\mathrm{X}_{2}$ and $Z$ dimensions for each observation. This product was divided by the total amount of tritium applied at the surface. The calculation of this mass balance accounted for the radioactive decay of input tritium through the date when the soil core solutions were analyzed.

At $Y_{2}=9$ meters the tritium plume appears more centered under the irrigated area and with vertical displacement more like on the trench face. Horizontal redistribution is more like the plume represented at $Y_{2}=5 \mathrm{~m}$. The peak observed concentration was about $26 \%$ of input. Samples at $Y_{2}=9 \mathrm{~m}$ recovered about $134 \%$ of tritium applied to the soil surface in that plane of measurement. This high recovery figure may be due to several factors. The values for bulk density, used to find volumetric water content, 

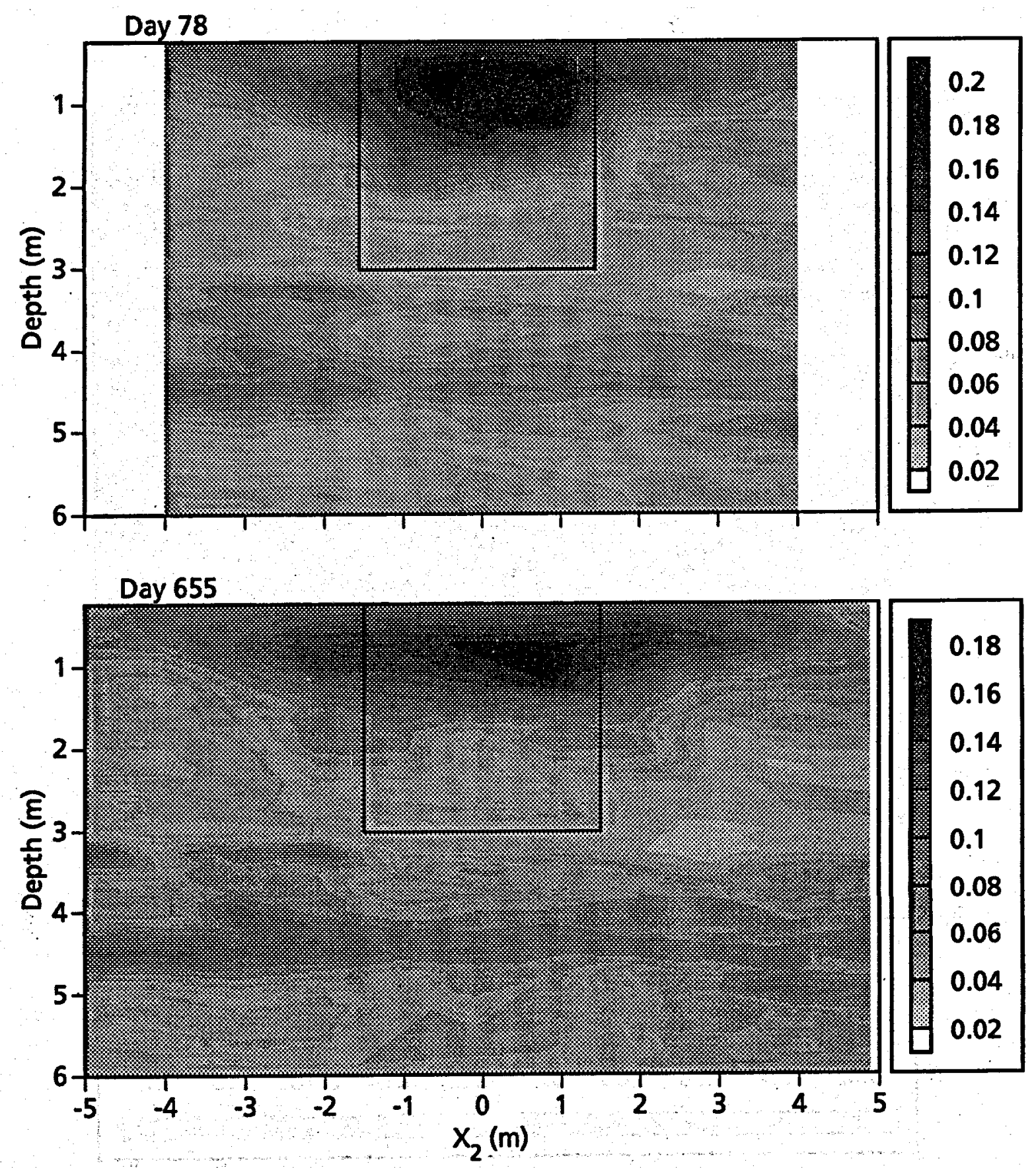

Figure 10. Experiment Ila neutron probe volumetric moisture contours at $Y_{2}=10 \mathrm{~m}$ on days 78 and 655. Boxes are added to show similar regions of the profile.which were sampled by soil cores at $Y_{2}=9 \mathrm{~m}$ on day 661 . 


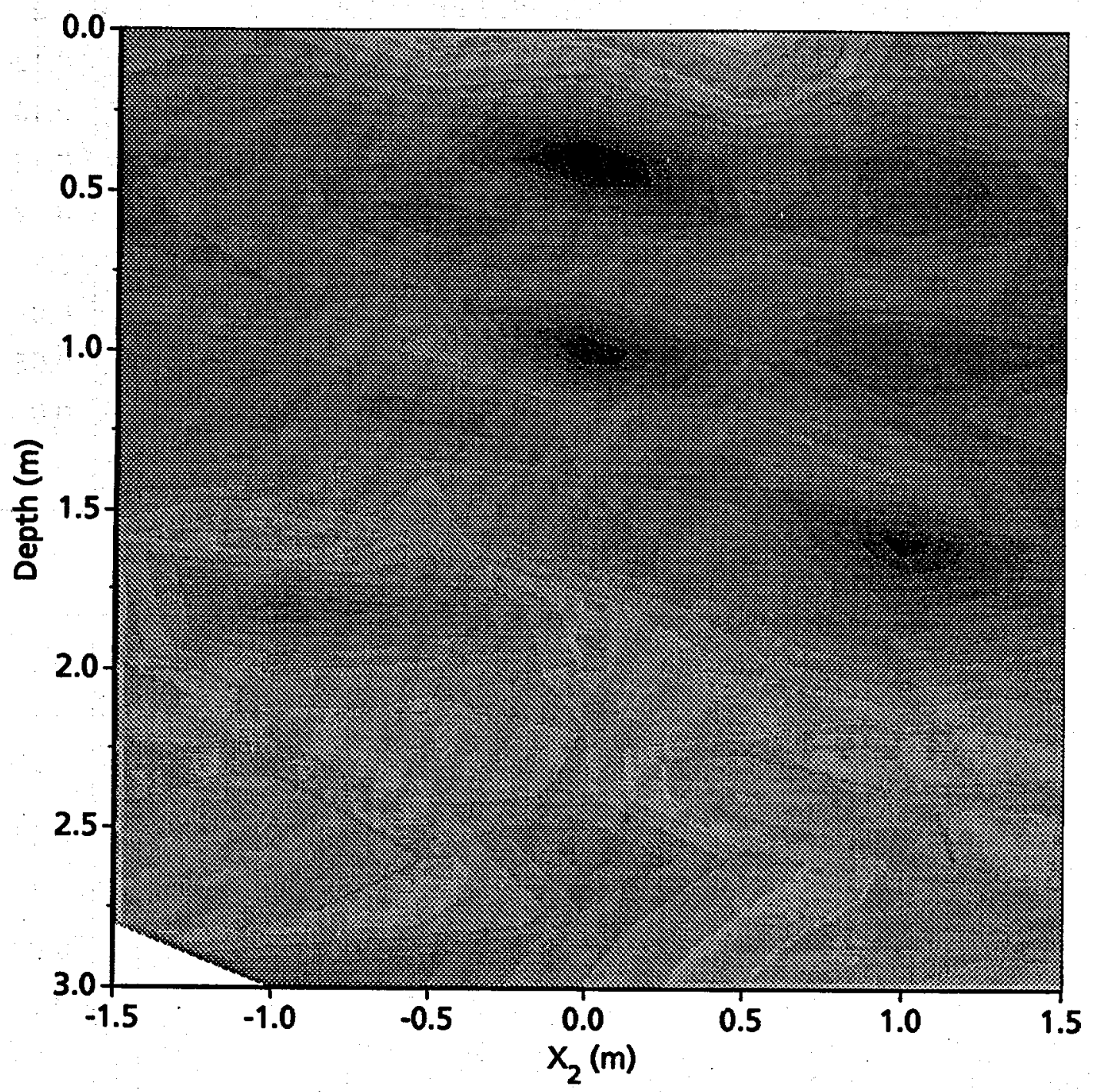

$\begin{array}{llllllllll}0.02 & 0.04 & 0.06 & 0.08 & 0.1 & 0.12 & 0.14 & 0.16 & 0.18 & 0.2 \\ \square & & & \end{array}$

Figure 11. Experiment IIa soil core volumetric moisture contours at $Y_{2}=9 \mathrm{~m}$ on day 661 . Gravimetric soil core moisture is converted to volumetric moisture with an estimated term for soil bulk density. 

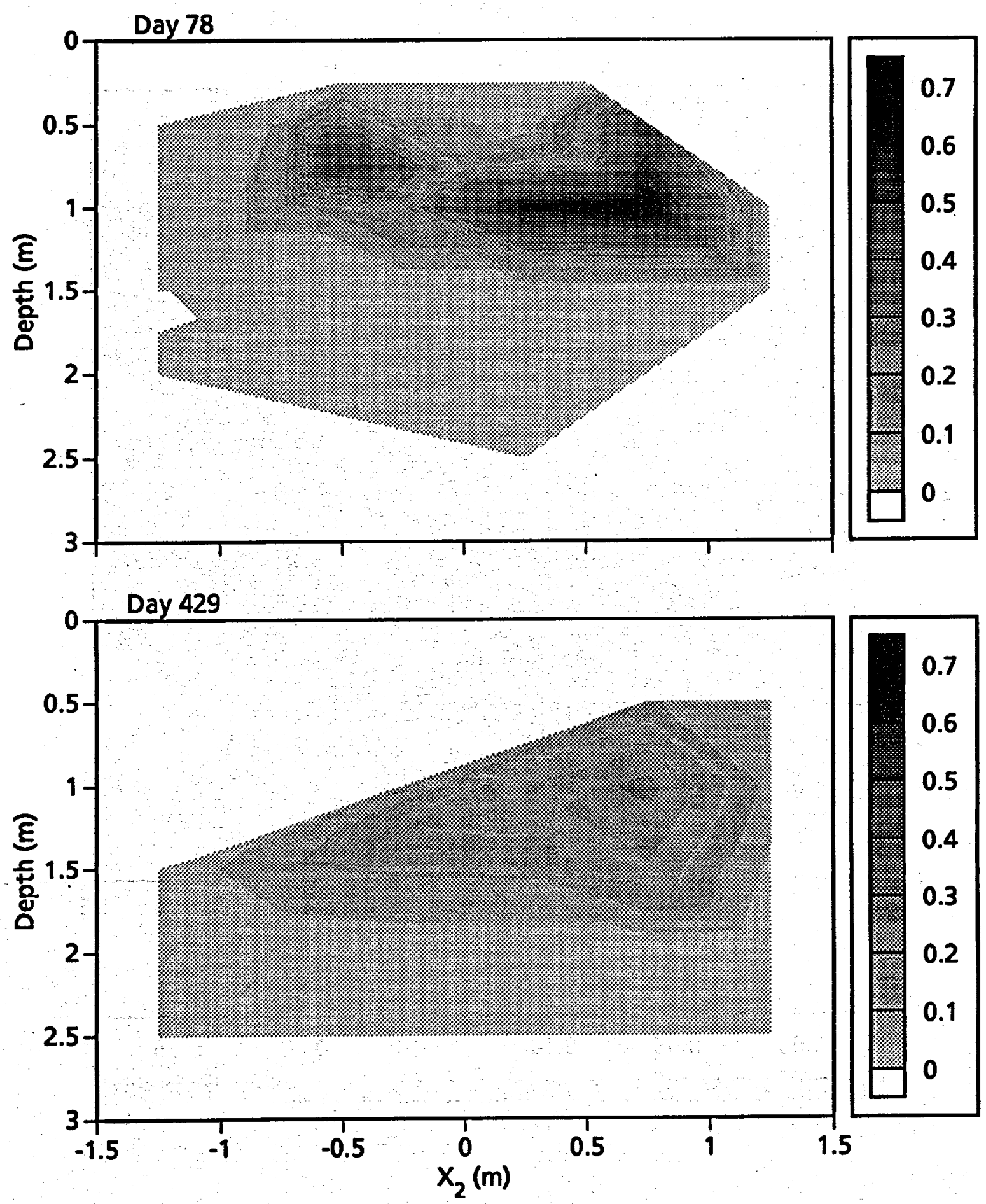

Figure 12. Experiment IIa relative tritium $(\mathrm{C} / \mathrm{Co})$ contours at the trench face $\left(\mathrm{Y}_{2}=0.5 \mathrm{~m}\right)$ on days 78 and 429. Liquid samples collected by solution samplers. 


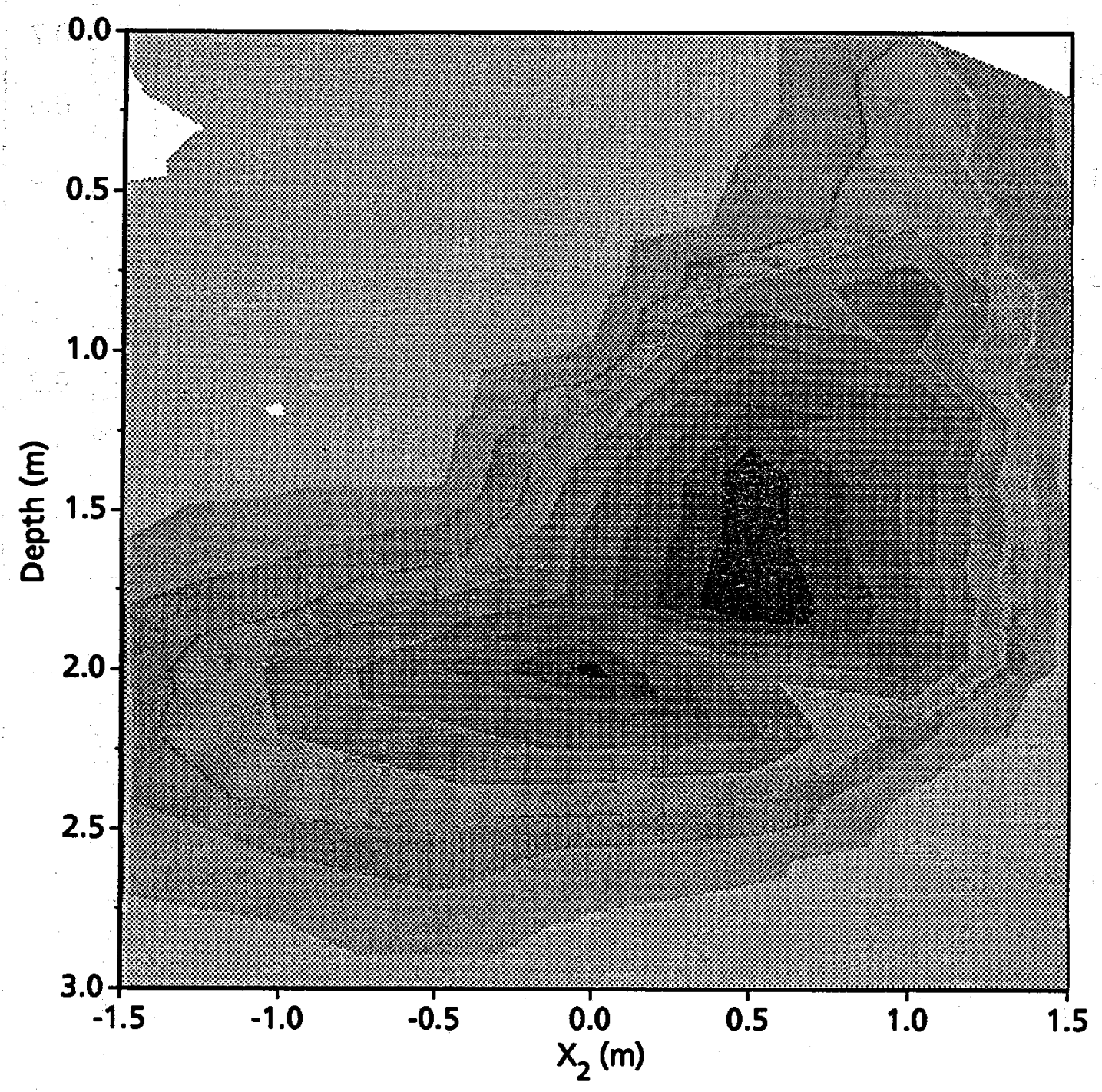

\begin{tabular}{|llllll|}
\hline 0.00 & 0.05 & 0.10 & 0.15 & 0.20 & 0.25 \\
\hline & & & & & \\
\hline
\end{tabular}

Figure 13. Experiment IIa relative tritium contours from soil cores at $Y_{2}=5 \mathrm{~m}$, day 661 . 


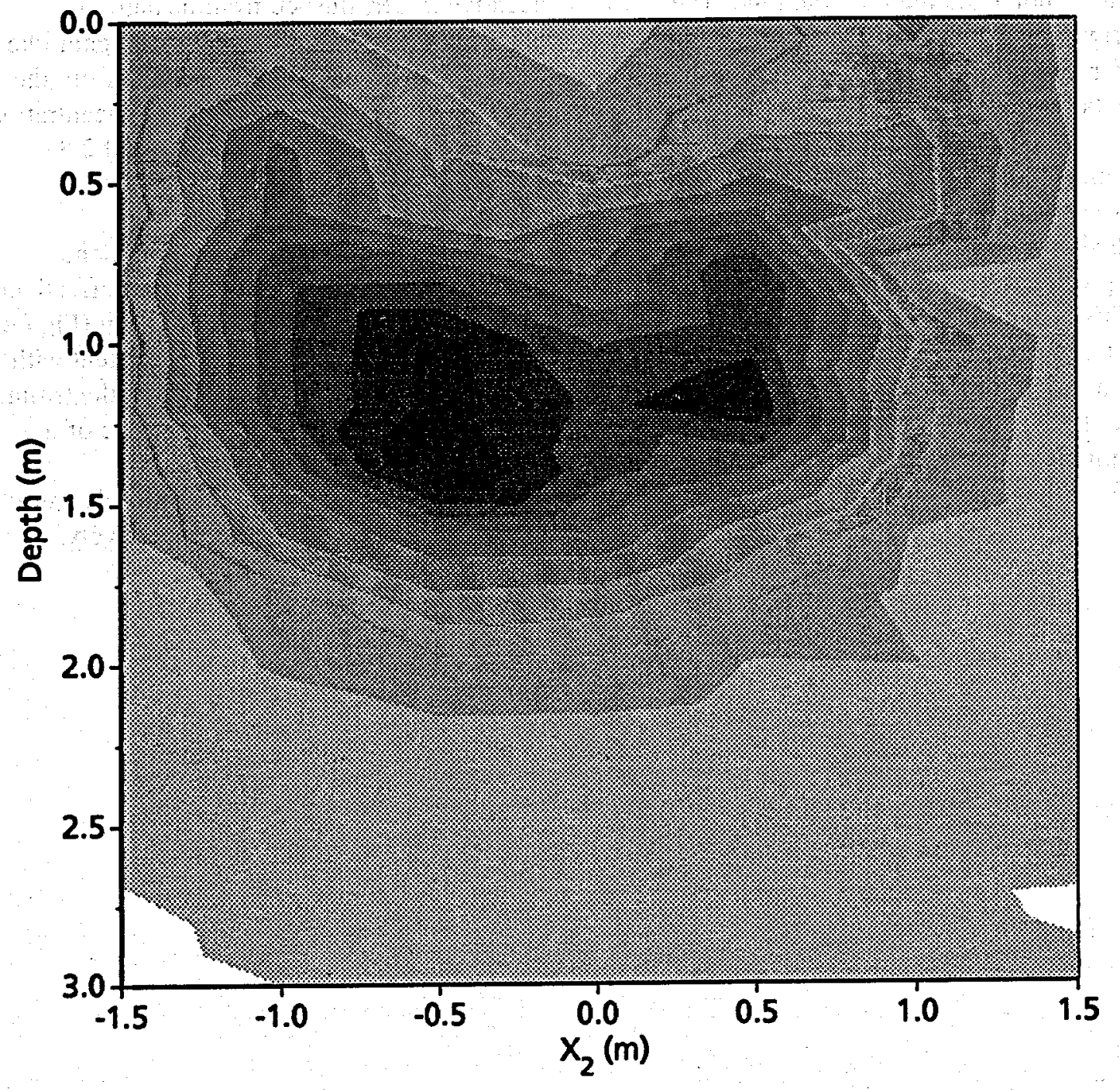

\begin{tabular}{|llllll|}
\hline 0.00 & 0.05 & 0.10 & 0.15 & 0.20 & 0.25 \\
\hline & & & & & \\
\hline
\end{tabular}

Figure 14. Experiment IIa relative tritium contours from soil cores at $Y_{2}=9 \mathrm{~m}$, day 661 . 
may be overestimated, since they assume that soil properties found at the trench face extend uniformly into the length of the plot.

Also, samples of the original applied tritium solution were not available for counting. The counts originally generated by the scintillation counter for that solution were assumed constant (less counts for decay) over time.

Bromide and chloride results from the soil core samples (day 661) are considered together.

Figure 15 shows that bromide is largely absent from the profile at $Y_{2}=5 \mathrm{~m}$ to about $1.5 \mathrm{~m}$ depth. This is due to irrigation with $27.5 \mathrm{~cm}$ of bromide-free water, following a $4.9 \mathrm{~cm}$ pulse of bromide in the first 11.5 days of experiment IIa irrigation. Like tritium, a portion of the bromide plume on the $+X_{2}$ side of the plane is divided from the main plume. The highest observed bromide concentration was 0.84 of input at $1.8 \mathrm{~m}$ depth, $\mathrm{X}_{2}=-0.5 \mathrm{~m}$.

Chloride was not applied as a solute tracer so results can be treated as though the ion was a negative tracer, flushed from its natural placement in the profile. Chloride, again like tritium, was more effectively eluted from the $-\mathrm{X}_{2}$ side of the plot. The highest concentration of chloride $(36.9 \mathrm{me} / \mathrm{L})$ was found at $2.8 \mathrm{~m}$ depth, $\mathrm{X}_{2}=1.5 \mathrm{~m}$.

Compared to data for the $Y_{2}=5 \mathrm{~m}$ plane, bromide and chloride ions were less effectively moved through the profileat $Y_{2}=9 \mathrm{~m}$ (Figure 16). This behavior was again consistent with tritium results. The leading edge of the tritium plume dovetails with the trailing edge of the eluted chloride plume. The highest concentrations of bromide and chloride were $\mathrm{C} / \mathrm{Co}=0.99$ and $38.2 \mathrm{me} / \mathrm{L}$, respectively. 

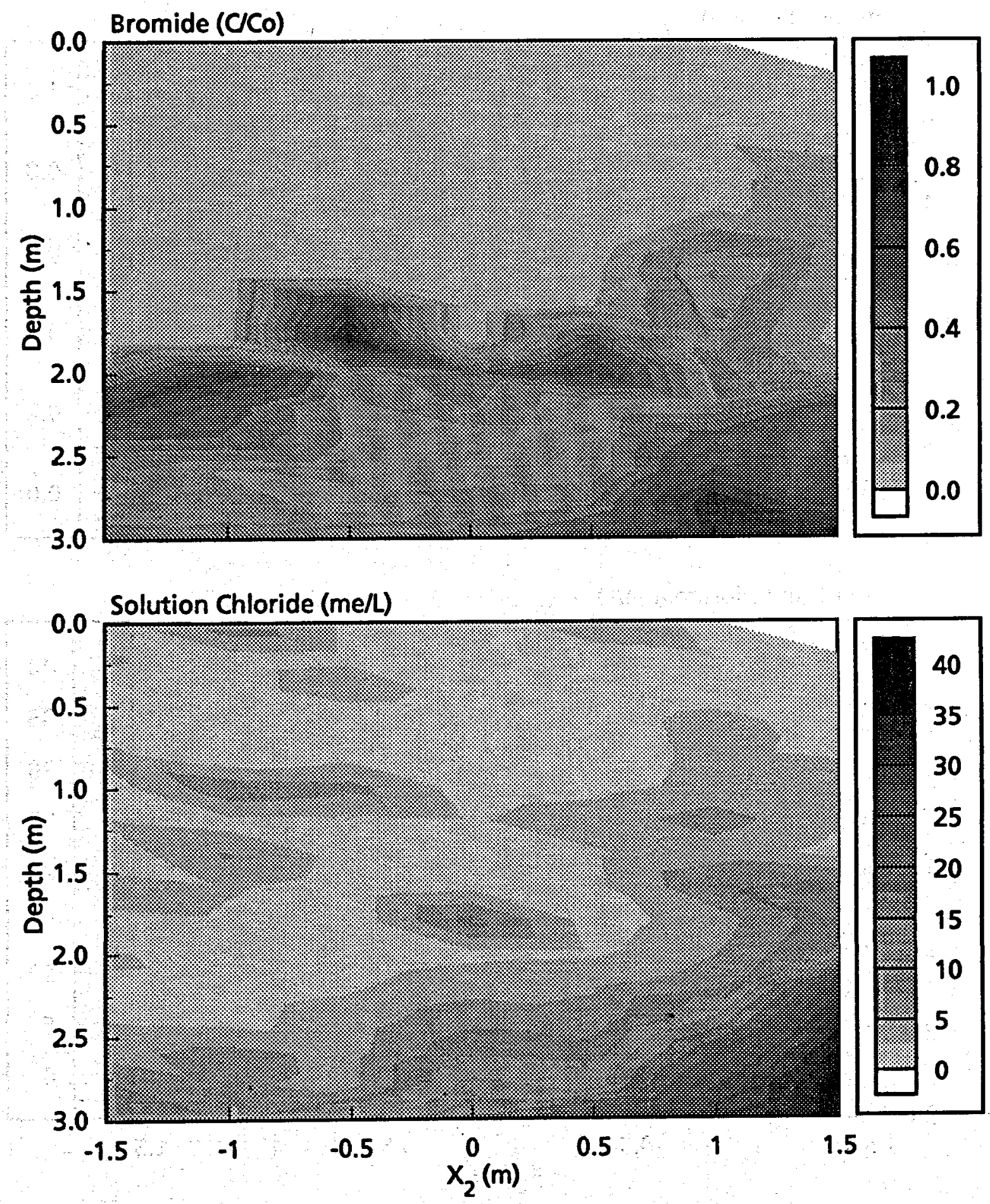

Figure 15. Experiment IIa relative bromide $(\mathrm{C} / \mathrm{Co})$ and solution chloride $(\mathrm{me} / \mathrm{L})$ contours from soil cores at $Y_{2}=5 \mathrm{~m}$, day 661 . 

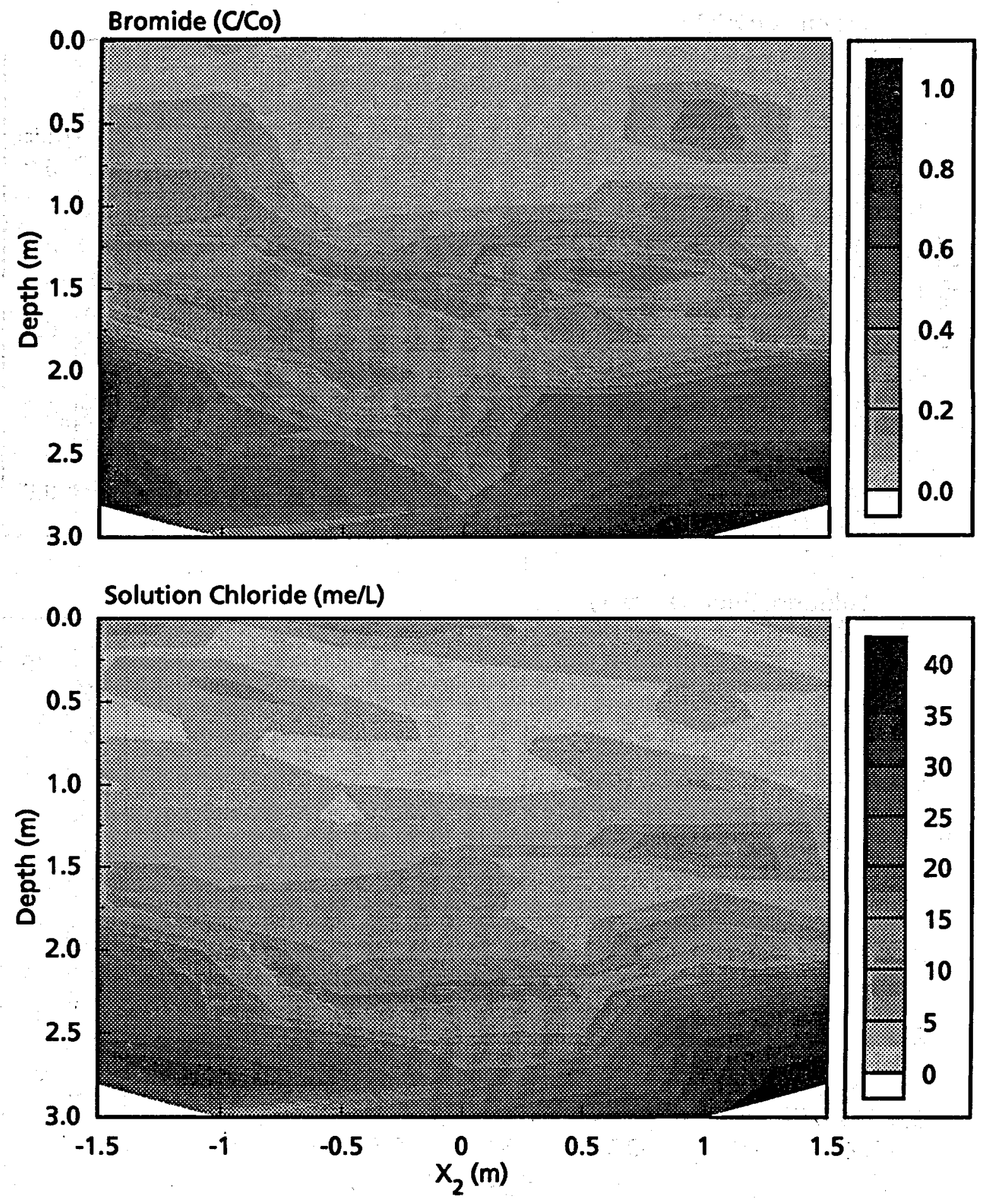

Figure 16. Experiment IIa relative bromide $(\mathrm{C} / \mathrm{Co})$ and solution chloride (me/L) from soil cores at $Y_{2}=9 \mathrm{~m}$, day 661 . 


\section{Experiment IIb Water and Solute Application}

\subsection{Preparations}

A second water flow and transport experiment on plot 2 (experiment IIb) was begun on August 1,1990, following the last water application of experiment IIa by 649 days. For both experiments the same experimental apparatus was used, with the exception of a number of improvements and additions. Figure 17 illustrates the location of data collection devices; neutron probe access tubes, tensiometers, and solution samplers relative to the trench and plot 2 .

The plot pondliner and plot cover were modified for improved performance as a vapor barrier.
The area surrounding the irrigated plot which was covered by pond liner material was expanded. Pondliner pieces were added to the east and west sides of the plot which added an additional $1.8 \mathrm{~m}$ of lateral coverage. Existing pondliner was carefully checked for holes and defective seams, and then repaired or replaced. In experiment IIa a total of 12 tensiometers were installed through the plot cover at $Y_{2}=$ $2.5,5.5$, and 6.5 meter planes. These tensiometers yielded questionable data and created problematic leaks in the cover. These tensiometers were removed prior to Experiment Ilb. New devices were added to expand lateral coverage from $\mathrm{X}_{2}=-2.25$ to 2.25 meters and to the bottom of the trench, with the last row of devices installed at $6 \mathrm{~m}$ depth.

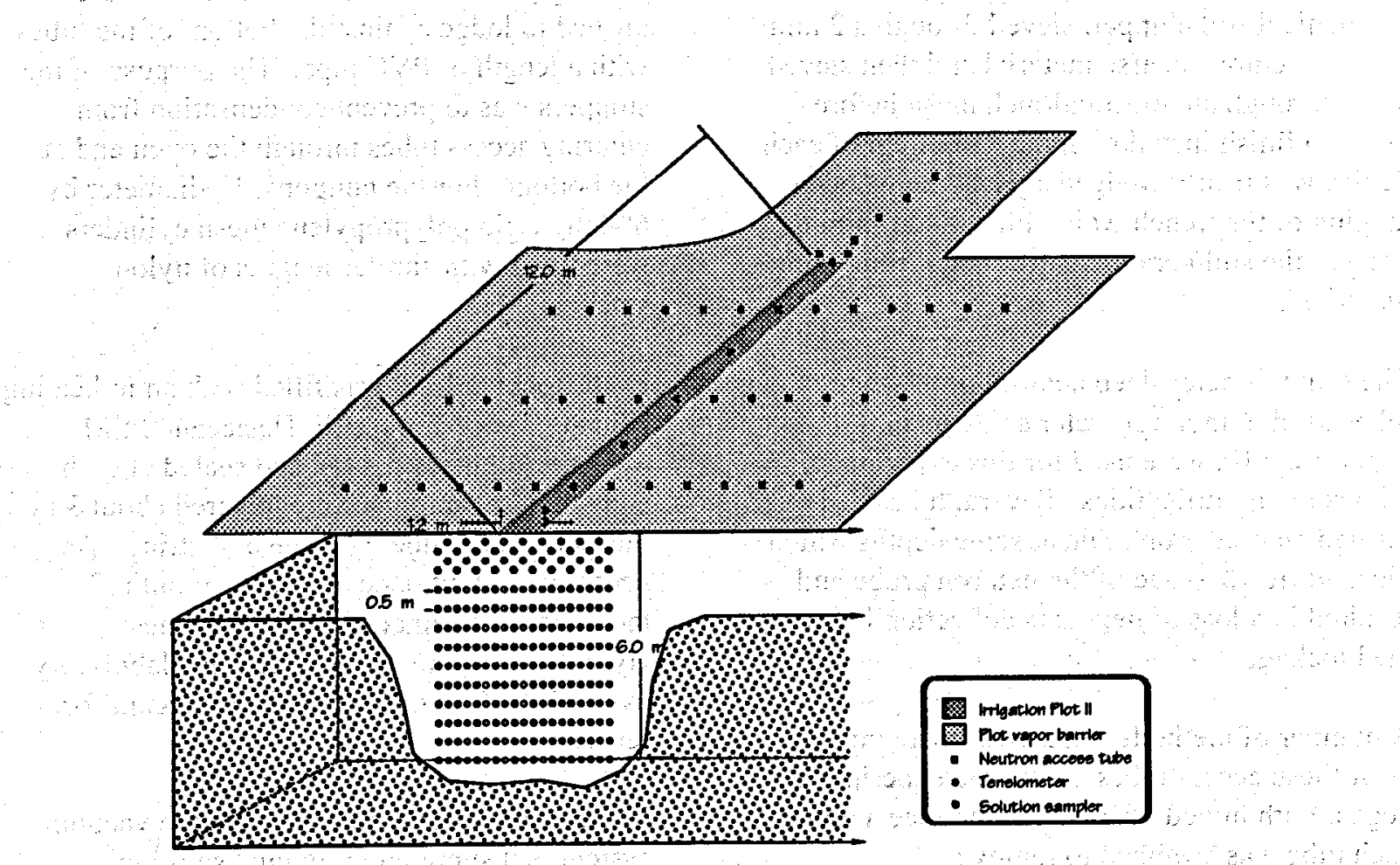

Figure 17. Survey of plot 2 experimental apparatus illustrating locations of neutron probe access tubes, tensiometers and solution samplers relative to irrigated area and plot vapor barrier. 
Some devices were placed offset from the regular grid to avoid structural obstacles (trench walkways and vertical H-beams). In all a total of 108 tensiometers and 120 solution samplers were emplaced to monitor water and solute movement at $Y_{2}=0.5 \mathrm{~m}$. The location on the trench wall and the identification of each device by row and column is illustrated in Figure 18. In this figure the identification of each device is found by adding the row number to the column number.

During installation (or reinstallation) a native mud slurry was injected at the distal end of each drilled hole just before seating the device (Appendix C-7). This slurry was used to improve soil contact with the ceramic cup. Soil used for the slurry was collected from the trench face at several depths in the profile to approximate the target depth of the ceramic device. This material was first chipped with a mechanical soil chipper, sieved through a $2 \mathrm{~mm}$ mesh to remove coarse material and then sieved again through an 80 strand/inch mesh before use. To finish installation the PVC stem of each device was firmly wedged against the wooden lagging of the trench wall with a metal shim to prevent the soil/ceramic cup contact from being disturbed.

The same 6-meter-deep neutron probe access tubes used to monitor water contents in experiment IIa were used for this experiment, with some modifications. Several tubes had formed an oxidation scale at some depths which blocked free passage of the neutron probe and resulted in a loss of new data collection below the blockage.

A number of methods were used in attempts to clear these access tubes before the experiment began, with mixed results. About once a month each tube was swabbed to remove accumulated scale and moisture from within the tube. The swab was made with a $25^{\prime}$ length of
PVC pipe with a cloth swath bound tightly to one end for a snug fit within an access tube. Problem tubes were scoured with a tight-fitting metal bristle brush to free the scale. This brush was either rotated by hand or with a power drill throughout the depth of the tube.

Some obstructions were not cleared by these treatments. The neutron probe was returned to the manufacturer for modification. The aluminum wall of the probe body was milled from an original $4.73 \mathrm{~cm}$ outside diameter by 0.050 " to $4.48 \mathrm{~cm}$ diameter. This step proved helpful for some access tubes, though not all. Neutron probe counting performance was not changed by this procedure.

To reduce moisture condensation, each tube was fit with a size 10 rubber stopper and a water-absorbent tampon. The rubber stoppers fit snugly within the access tubes and were pushed to lodge against the bottom of the tubes with a length of PVC pipe. The purpose of the stoppers was to prevent condensation from entering access tubes through the open end at the bottom. For the tampons, 1"-diameter by 6"-long rigid polypropylene mesh cylinders were lined with similar lengths of nylon stocking.

Each stocking liner was filled with an indicating silicone desiccant (T.H.E. Desiccant ${ }^{\star}$, EM Science, Gibbstown, NJ), and sealed at both ends. These tampons were lowered about $3 \mathrm{~m}$ into the access tube by a piece of string. As necessary, about once or twice a month, tampons were replaced with fresh ones. Hydrated tampons were placed in a laboratory oven at $60^{\circ} \mathrm{C}$ to dehydrate the desiccant for reuse.

In previous experiments, leaks in the vacuum system that supplied individual solution samplers, were often problematic. For this experiment a new vacuum system was 


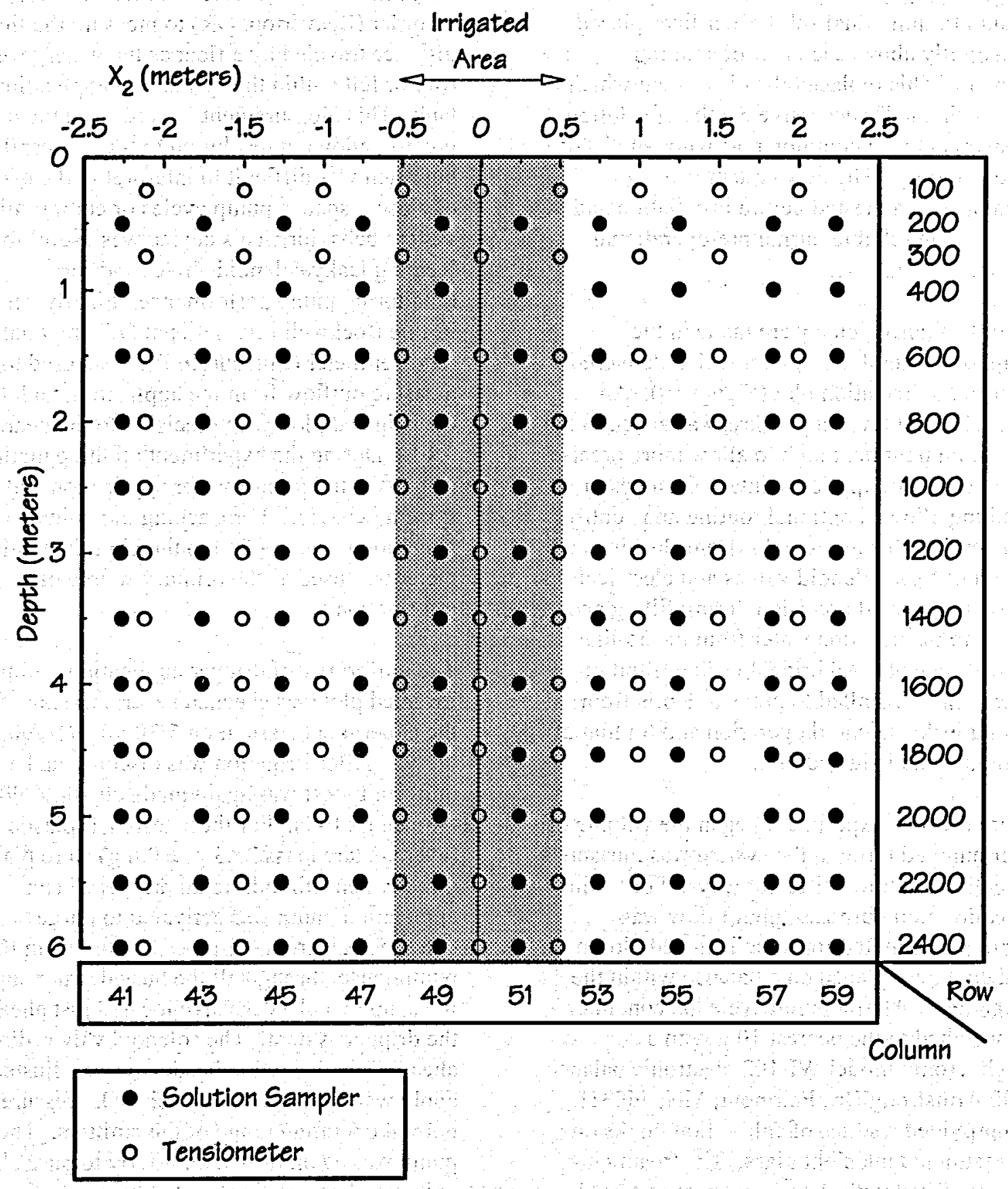

Figure 18. Schematic of trench face illustrating placement of tensiometers and solution samplers relative to the area of soil directly beneath irrigated area (shaded). Individual devices are identified by row + column designations, indicated by boxed areas to the right and bottom of the figure. 
constructed of $1 / 2$ " schedule 80 PVC pipe and reinforced ports that connected to individual samplers. A vertical main header supplied vacuum to individual sub-header lines placed horizontally above each row of solution samplers. This replaced the old system which "daisy- chained" successive depths of solution samplers below a common port with lengths of Tygon tubing. The new system was extensively leak tested before installation and proved quite reliable during pre-operational tests and normal use.

Several enhancements were made in the pumphouse plumbing system which delivered water to the irrigation plot (Figure 19). An external liquid level sight glass was added to the 300 gallon treatment tank to allow more precise measurement of applied solutes. Changes in plumbing allowed optional routing and control of water flow for improved system flushing and cleaning. New solenoid valves and electrical controls improved precision during filling and discharge of irrigation water from the 35 liter application tank. Additional in-line filtration devices were installed to prevent debris from causing leaky solenoid operation and fouling of emitters at the irrigated plot.

Before the new experiment began the volume of water pumped through the system was measured and calibrated three different ways. For testing and calibration purposes, pump flow was temporarily shunted from the irrigated plot to a tared container placed on a balance within the pumphouse. After a pump cycle the container was weighed to the nearest $10 \mathrm{~g}$ with a Weigh-Tronix model WI-102 electronic balance (1000 Armstrong Dr., Fairmont, MN, 56031). This provided a series of calibration marks on the treatment tank sight glass. The treatment tank was filled with successive pumping cycles of known volume. Interpolation between these marks allowed confident estimation of tank drainage volume during draining or filling to the nearest $0.5 \mathrm{~L}$. This calibration was typically used to calculate the amount of concentrated solute to add when refilling the treatment tank with tracer solution. The second method of calibration used a Stevens model 68 water stage recorder (Beaverton, $\mathrm{OR}$ ) to measure the linear distance traveled by a float as the water level rose or fell within the cylindrical application tank. This measurement proved to be the most precise, allowing resolution to the nearest $0.1 \mathrm{~L}$, but often was difficult to interpret in the event of closely spaced pump cycles or compromised system behavior. This device was useful for locating leaky solenoid valves and for monitoring pump performance. Finally, an in-line Rockwell International 5/8" inlet gallon totalizer meter (Pittsburgh, PA) was used to measure outflow from the application tank to the irrigated plot, with precision to the nearest $0.4 \mathrm{~L}$. During the experiment all three methods were used to monitor water application and system behavior. For tracking the volume of irrigation water applied to the plot, the totalizer meter was used as the primary source of information.

The uniformity of dripper application within the irrigated plot was checked several times before the experiment started; on $5 / 30 / 90,7 / 16 / 90$, and $8 / 1 / 90$. After irrigation was discontinued a final uniformity test was again made on 10/24/90 (Appendix B-1). For these tests $1 \mathrm{~L}$ plastic cups (average tare mass $25.3 \mathrm{~g}, \pm 0.6 \mathrm{~g}$ ) were placed directly beneath individual drippers. The application pump was activated to purge entrapped air from the pipes leading from the pumphouse, through all the buried water supply lines, up to a bleeder valve located just ahead of the dripper system. The solenoid valves directly ahead of the irrigation headers were adjusted to apply water from 10 to 14 psi back pressure, the normal operating range of the emitters. The pump was again turned on briefly to purge both irrigation headers through the bleeder valves located at both ends of each header and then finally reactivated for one or two pumping cycles per header to partially fill the plastic cups. Each cup was then weighed to the nearest $0.1 \mathrm{~g}$ to ascertain individual dripper output. 


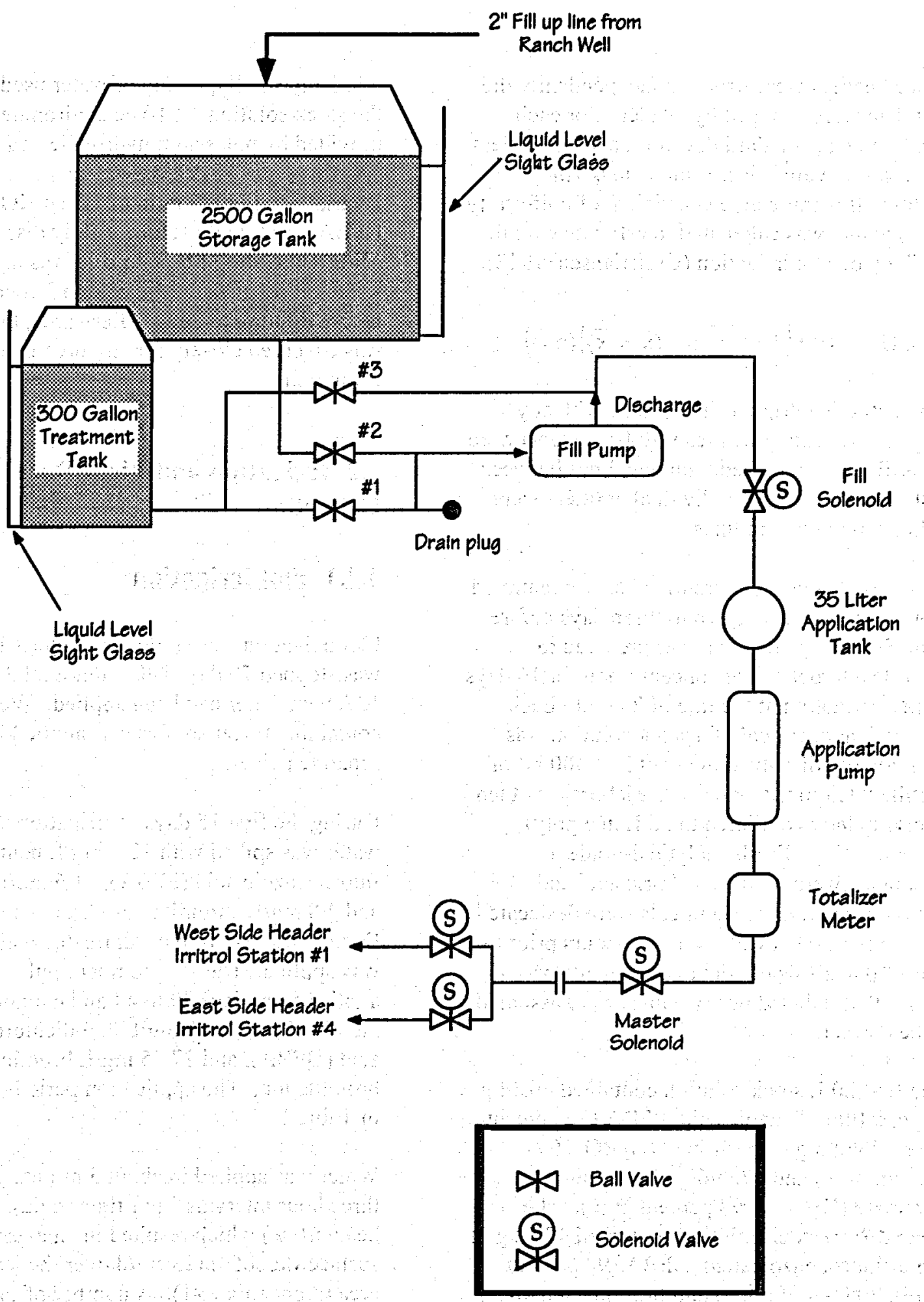

Figure 19. Schematic of pumphouse plumbing system showing delivery route of irrigation water from either 2500 gallon storage tank or 300 gallon treatment tank to plot 2 emitter headers. 
Since headers were irrigated independently the test data were grouped by header. For each header mean, standard deviation and coefficient of variation values were calculated. An additional parameter, a coefficient of uniformity (CU) value, was calculated to estimate overall efficacy of plot irrigation (Christiansen, 1942):

$$
C U=100\left(1-\left(\sum \operatorname{abs}(x-\bar{x})\right)(n \bar{x})\right)
$$

Any defective dripper lines (those with any dripper exceeding the mean by 10 percent) were identified and replaced with new.lines between tests. In some cases individual emitters were spliced into existing lines.

Tracer solutions were premixed as concentrated stock solutions about two to three days before use. Sufficient chemical was prepared to provide a target solute concentration for 16 days of plot irrigation at the rate of $2 \mathrm{~cm} / \mathrm{d}$. Each component chemical of a stock solution was individually dissolved in about $200-400 \mathrm{ml}$ of distilled deionized water in the laboratory, then commingled and diluted to $20 \mathrm{~L}$ in a polypropylene jug. Fresh analytical-grade chemicals were used. The boric acid and potassium chromate chemicals were desiccated in the presence of $\mathrm{CaCO}_{3}$ for 72 hours prior to use. No analysis of container contents was performed; labeled purity values were assumed to be correct.

The first $20 \mathrm{~L}$ stock solution contained $46.54 \mathrm{~g}$ of pentafluorobenzoic acid, PFBA (99 percent pure), $289.93 \mathrm{~g}$ of boric acid $\left(\mathrm{H}_{3} \mathrm{BO}_{3}, 99+\right.$ percent pure) and $87.796 \mathrm{~g}$ of potassium chromate $\left(\mathrm{K}_{2} \mathrm{CrO}_{4}, 98\right.$ percent pure $)$. The second $20 \mathrm{~L}$ stock solution contained $47.02 \mathrm{~g}$ of 2.6 difluorobenzoic acid, DFBA (98 percent pure), $205.9 \mathrm{~g}$ of potassium bromide and 460.8 $\mathrm{mCi}$ tritium (as $10 \mathrm{mCi} / \mathrm{ml}$ laboratory stock). The DFBA was difficult to dissolve in the relatively small quantity of water used to make the stock solution. A basic environment, provided by potassium hydroxide, was needed.

By trial titrations, a ratio of $1.3 \mathrm{eq} \mathrm{KOH} / \mathrm{eq}$ DFBA was found useful for fully dissolving this acid. This ratio of base to acid was used to successfully dissolve the acid in 3 liters of water before diluting to 20L. Afterwards, this stock was observed closely for any precipitate formation.

\subsection{Irrigation and Field Data Collection}

\subsubsection{Plot irrigation}

Plot irrigation was started on August 1, 1990. It was stopped 70 days later after a total pulse of $127.4 \mathrm{~cm}$ water had been applied. Well water containing tracer solutes was applied in two separate pulses.

During the first 15 days of irrigation the well water was spiked with $12.3 \mathrm{mg} / \mathrm{L}$ pentafluorobenzoic acid (PFBA), $11.6 \mathrm{mg} / \mathrm{L}$ boron and $4.8 \mathrm{mg} / \mathrm{L}$ chromium as chromate (VI) ion. During the next 14 days untreated well water was applied. The second tracer pulse was applied from days 29 to 44 and contained 0.10 $\mathrm{mCi} / \mathrm{L}$ tritium, $10.3 \mathrm{mg} / \mathrm{L} \mathrm{2,6}$ dichlorobenzoic acid (DFBA), and $27.75 \mathrm{mg} / \mathrm{L}$ bromine as bromide ion. The application periods are shown in Table 1.

Water was applied in about 5 minute pulses on three hour intervals (four times a day to each header line) which resulted in an average surface flux of $1.825 \mathrm{~cm} / \mathrm{d}$ over the irrigated area (Appendix A-1). A number of system malfunctions occurred during the 70 day irrigation period that required attention. These difficulties are illustrated in Figure 20. 
Table 1. Irrigation regime and tracers applied during experiment IIb.

\begin{tabular}{|c|c|}
\hline Irrigation Days & $0 \ldots \ldots 10 \ldots .20 \ldots . .30 \ldots .40 \ldots .50 \ldots .60 \ldots . \ldots 70$ \\
\hline Well water & $* * * * * * * * * * * * * * * * * * * * * * * * * * * * * * * * * * * * * * * * * * * * * * * * * * * *$ \\
\hline & $0-70$ \\
\hline Boron, Chromium, \& PFBA & $* * * * * * * * * *$ \\
\hline Tritium, Bromide, \& DFBA & $\begin{array}{c}* * * * * * * * * * * * \\
29-44\end{array}$ \\
\hline
\end{tabular}

\subsubsection{Neutron probe readings}

Neutron probe readings were taken at $0.25 \mathrm{~m}$ depth increments with a CPN model 503DR neutron probe (Martinez, CA). During plot irrigation and through day 75 probe readings were taken three times per week; the 29 access tubes nearest to the irrigated plot were read on two of those days, all 43 tubes on the third. After day 75 the frequency of neutron probe readings was gradually reduced commensurate with the rate of water redistribution. Readings were taken twice per week through day 103 , once per week through day 154 , once every two weeks through day 225 , once a month through day 345 , then not again until days 408 and 525 . After day 103 all access tubes were read on a given day. On February 7 and 8, 1991 (days $190,191)$ an additional 6 access tubes were installed to extend neutron probe coverage by an additional $1 \mathrm{~m}$ on either side of the plot, to $X_{2} \pm 6 m$ at the $Y_{2}=2 \mathrm{~m}, 6 \mathrm{~m}$ and $10 \mathrm{~m}$ planes (Figure 21 and Appendix C-10).

\subsubsection{Soil tension readings}

Soil tensions were measured and solution samples were collected 3 times per week during irrigation. This frequency was reduced after irrigation was discontinued, generally to match the days when neutron probe readings were taken.
Soil tensions were measured to the nearest millibar with a hand-held pressure transducer (Soil Measurement Systems, Tucson, AZ). A backup transducer was used occasionally. The operation and calibration of these units was checked periodically against a regulated laboratory manometer (Appendix B-3). Tension was measured through a rubber septum which sealed the exposed end of the tensiometer. The air column within the stem of the device was measured to the nearest $1 \mathrm{~cm}$ to correct the raw tension reading for water head from the visible water level to the center of the ceramic cup. If the water level within the stem dropped below the septum by more than $3 \mathrm{~cm}$ the tensiometer was refilled with distilled-deionized water. This operation was performed at least 1 day before taking measurements to allow the device to come to equilibrium with soil moisture. This equilibration period was extended to several days during the redistribution phase of the experiment. In very dry soils ( $>800 \mathrm{mb}$ ) tensiometers were left unfilled to prevent cavitation and unnecessary water additions to the profile. The progress of the wetting front was estimated and tensiometers filled for use just before its anticipated arrival.

\subsubsection{Soil solution samples}

Soil solution samples were taken on the same days as tension readings. Solution samplers 


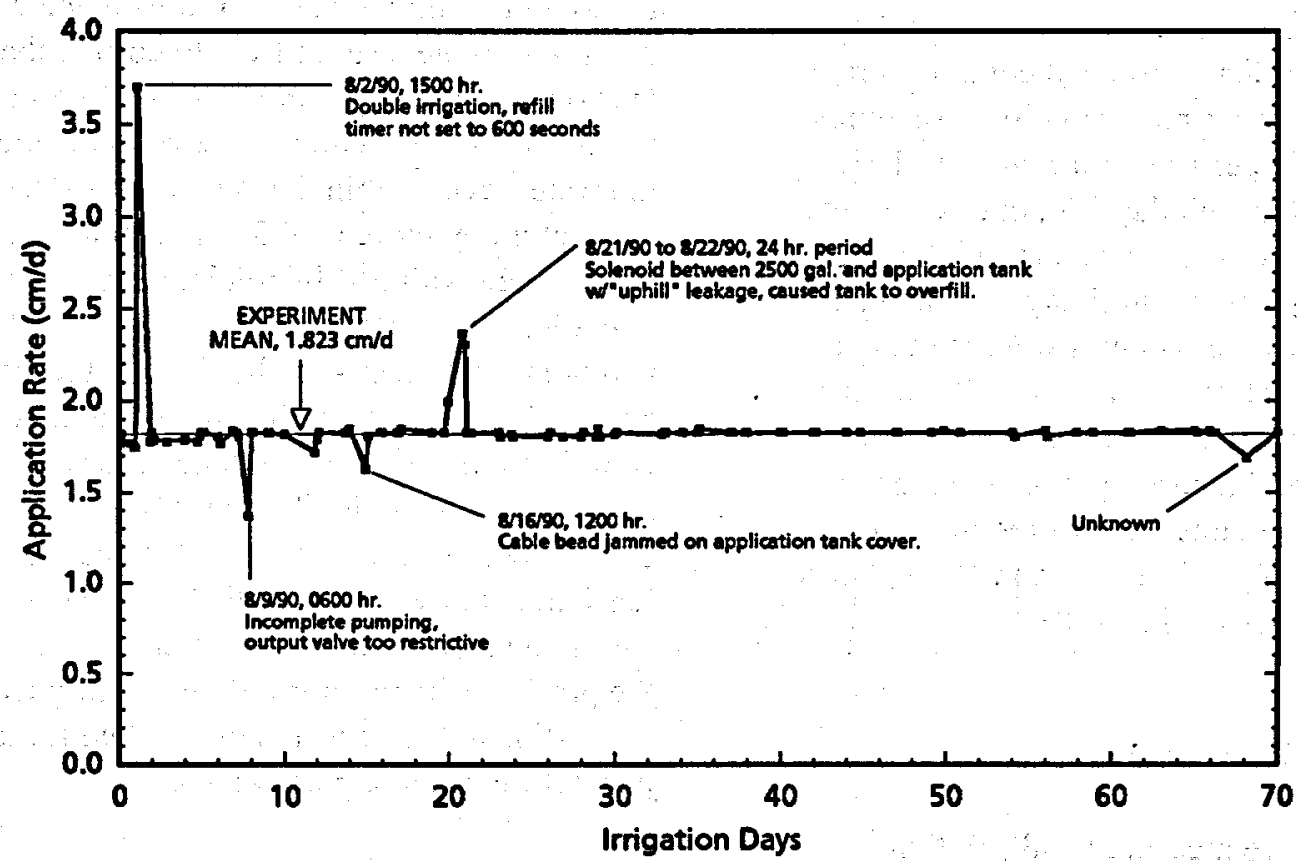

Figure 20. Experiment IIb totalizer record. Plotted application rates are calculated as mean application from one observation to the next. Aberrations from normal irrigation rates are noted. 


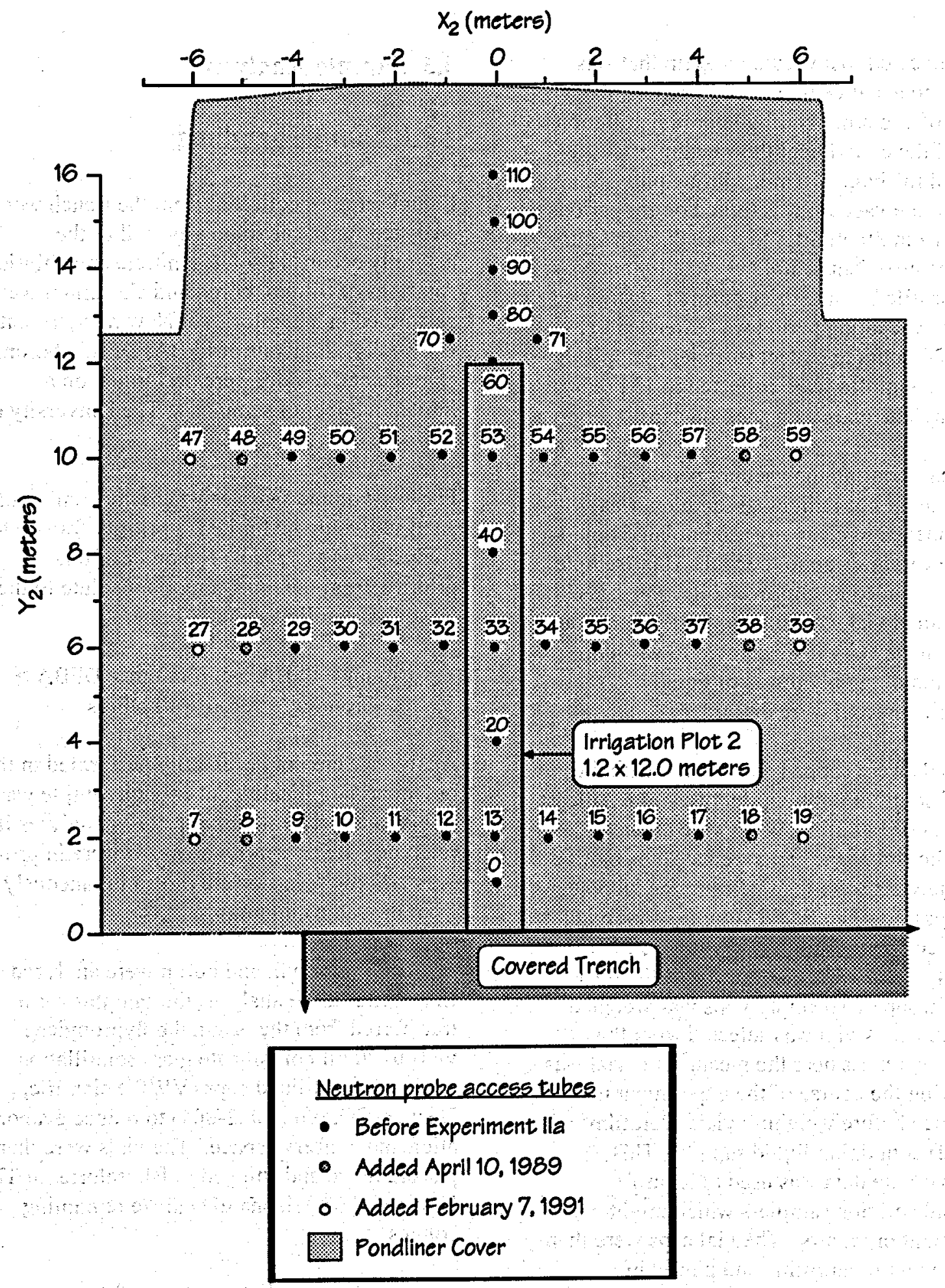

Figure 21. Schematic of neutron probe access tubes in relation to plot 2, pondliner cover and the covered trench. 
were connected to a vacuum system that was plumbed to a source regulated to supply about $200 \mathrm{mb}$ of vacuum. During later redistribution stages of the experiment the vacuum was increased to about $450 \mathrm{mb}$. During plot irrigation the vacuum was turned on for periods ranging from $\mathbf{3 0}$ minutes to more than an hour to collect sufficient sample volume for analysis. As the profile became drier during redistribution, the evacuation period was extended to as long as $\mathbf{2 4}$ hours to collect sufficient sample volume. Samples were collected in 13 dram polypropylene vials with snap-on caps.

Periodically during the vacuum period the air-inlet tubing of individual samplers was briefly opened to clear any accumulated liquid from the ceramic cup to the vial. This "sample pumping" tended to improve collection rates. If an individual vial was filled to more than about 75 percent capacity it was closed to vacuum. Vials containing only traces of liquid ( $\leq 0.1 \mathrm{ml}$, approx.) were discarded.

Filled vials were capped and labeled with the date and solution sampler identification. At least two layers of Parafilm ${ }^{\oplus}$ wrap were applied around the rim of the cap to reduce evaporative loss. The vials were then taken to the laboratory for processing. The liquid volume of individual samples collected through February 1, 1991 (day 184) was estimated. For this estimate a random group of 10 empty vials was weighed on a balance. A vial was selected from the group with a mass near the mean. This vial was used during the course of the experiment to tare the balance before weighing vials (Parafilm removed) containing liquid sample. This sample volume data was used to identify individual solution samplers which might need replacement or repairs. The vial caps were then rewrapped with Parafilm ${ }^{\circ}$ and placed in refrigerated storage.

\subsection{Sample Analysis}

\subsubsection{Sample handling}

Liquid samples collected from the trench were analyzed for tracer solutes as well as the naturally occurring solutes, nitrate and chloride. Analyses of tritium, boron and chromium were performed in laboratories at New Mexico State University. Analysis of PFBA, DFBA, boron, chloride and nitrate, were performed on a limited number of samples at The University of Arizona.

For low-volume samples where the number of analyses would be limited, a priority for solute analysis was assigned. The order of this priority, from the most important solute to the least was as follows:

$$
\begin{gathered}
\text { tritium }>\text { PFBA }>\text { chromium }>\text { DFBA }> \\
\text { bromide }>\text { boron }>\text { all others }
\end{gathered}
$$

Analyses were not necessarily performed in this order chronologically, but enough sample was reserved to perform higher priority analyses if runs for a lower priority analyzes were in progress. Some solutes were run simultaneously from the same aliquot.

Tritium, chromium and boron were analyzed first. After these analyses the samples were transferred from the original polypropylene vials to $20 \mathrm{ml}$ borosilicate glass scintillation vials, with foil-lined caps (VWR Scientific, Denver, CO, p/n 66022-004) to reduce evaporation and conserve space. The vials were then packed in ice and shipped to laboratories at The University of Arizona to analyze remaining solutes.

During the redistribution phase of the experiment the volume of liquid sample 
collected by solution samplers began to decline. These samples were diluted with water to increase volume and extend the number of analyses which could be performed with a limited original volume. Beginning with the samples collected on February 15, 1991 (day 198) individual polypropylene vials were weighed (less cap) to the nearest $\mathrm{mg}$ before use. The vial's mass was written on the side of the vial with a permanent marker. With mass known the mass of the liquid sample within a vial could be determined with $0.001 \mathrm{~g}$ precision. This data was used to calculate appropriate sample dilution prior to chemical analyses. The sample dilution was limited to no more than $5 x$ the original volume, to ensure that the detection limit of the least sensitive analyses would not be exceeded for the highest $(\mathrm{C} / \mathrm{Co})$ concentration of solute.

Long holding times between sample collection and chemical analyses were common. It was necessary to protect samples from chemical degradation and evaporative loss as best as possible. Before any analysis proceeded samples were removed from the refrigerator and brought to room temperature to prevent condensation on the vials and temperature effects on the analyses. After any dilution, chemical analysis or liquid transfers between containers, the vials used for storing samples were wrapped with Parafilm ${ }^{\star}$ and returned to refrigerated storage. In a simple laboratory trial it was found that

Parafilm ${ }^{\circledR}$ wrap greatly reduces evaporative losses when liquid samples are stored for long periods (Appendix C-3).

\section{4,3,2 Tritium}

Tritium was analyzed with a Beckman LS- 1800 liquid scintillation counter (Beckman

Instruments Inc., Fullerton, CA). Single replications of solution samples were prepared for scintillation by pipetting $0.1-0.5 \mathrm{ml}$ aliquots of sample to $5 \mathrm{ml}$ of scintillation cocktail into
Beckman 8 ml Mini Poly-Q vials. Each vial was capped, marked to identify its contents, and then placed in a rack with other vials. When the entire rack was filled it was vigorously shaken to mix the sample with cocktail to form the desired gel. Vials were then transferred to a counting rack compatible with the counter. The counter was programmed to run with a singlechannel counting window of $0-400 \mathrm{~nm}$ and no internal standard. Counting time per vial was set to a maximum of 10 minutes. Vials with early counts of less than 16 counts per minute, an approximate background count, were terminated within 1 minute of the routine.

At the beginning of an analytical run a series of five background vials and five tritium input sample vials was counted. Background vials were prepared with $0.5 \mathrm{ml}$ aliquots $(500 \mathrm{ul}$ Eppendorf pipettor, Brinkman Instruments, Inc., Westbury, NY, $\pm 0.6 \%$ error) of well water in place of solution sample in the scintillation vials. The input vials were prepared with $0.5 \mathrm{ml}$ aliquots of traced well water, collected from the 300 gallon solute tank on $9 / 4 / 90$. This collection was refrigerated in a capped $3 \mathrm{~L}$ glass bottle, sealed with Parafilm ${ }^{\star}$. The input vials were used to track the consistency of tritium scintillation for the same solution over time.

The aliquot volumes used to prepare scintillation vials was a factor in counting results. The preferred aliquot size was $0.5 \mathrm{ml}$ ( 500 ul Eppendorf pipettor), whenever sufficient sample volume was available. Since many samples were well below this preferred volume, $0.1,0.2,0.3$, or $0.4 \mathrm{ml}$ aliquots $( \pm 1.0 \%$ error) of sample were used instead (adjustable Eppendorf pipettor, 1-100 ul). As aliquot size is reduced counts become less quenched.

Therefore, a sample counted with $0.1 \mathrm{ml}$ added to a scintillation vial will produce more than $0.1 / 0.5=20 \%$ of the counts as when $0.5 \mathrm{ml}$ of sample is used. However, this quenching characteristic is linear with aliquot volume and was characterized for this experiment 
(Appendix B-2). A correction factor was used to correct raw counts for various aliquot volumes.:

Two different scintillation cocktails were used for samples collected at different times in the experiment. All samples collected through day 184 were prepared with Aquasol universal LSC, a xylene based product manufactured by Dupont New England Nuclear (p/n NEF-934, Boston, MA). After day 184 samples were prepared with Ready-Safe (Beckman Instruments, Inc.), a newer cocktail formulation with significantly higher counting efficiency than xylene based cocktails (Klein and Gershey, 1990). ReadySafe was less expensive, more safely handled, and proved to mix more readily with sample to form a gel than did Aquasol. Since Ready-Safe yields higher counts than Aquasol, and has a different volume/quenching characteristic, an experiment was performed in the laboratory to correct for substitution effects (Appendix B-2). Tritium data (Appendix A-4) is expressed as relative tritium concentration:

$$
\begin{aligned}
& \text { Relative Concentration }=\frac{C}{C_{0}} \\
& =\operatorname{Diln} \frac{\left[\left(C^{\prime}-B k g\right) K_{y c}\right]}{C_{0}^{\prime}-B k g} \times 0.622
\end{aligned}
$$

where $\mathrm{C}$ is the adjusted input concentration; $C^{\prime}$ is the observed raw counts per minute for a sample; Bkg is the background counts per minute on a given day of analysis and $\mathrm{K}_{\mathrm{yc}}$ is the correction factor for aliquot volume, specific to a cocktail. Diln is the dilution factor for a sample.

$\mathrm{C}_{0}$ is the concentration of tritium applied as tracer to the plot, expressed as raw counts per minute $(17,142)$. To find this value 9 samples of solution were drawn from the $\mathbf{3 0 0}$ gallon solute tank on different days during the 15 day period of tritium application. Six replicate scintillation vials were prepared from each of these collections, counted normally and then averaged. The constant 0.622 is used to normalize data to the concentration of tritium applied during experiment IIa, 27,574 counts per minute (Hills and Wierenga, 1991).

Much of the tritium analysis for samples collected through day 184 was repeated to correct procedural errors made during the original analyses. Many of these samples had been incorrectly mixed before counting.

Scintillation counts are very sensitive to gel homogeneity. When an aliquot of sample is added to cocktail and capped the vial must be vigorously shaken with an up and down motion, especially when using a more viscous cocktail such as Aquasol. This mixing motion was inconsistent between technicians and was often performed with a less effective side to side motion. This resulted in an insufficiently homogenized gel and counts which were more variable between vials, i.e., lower than normal.

When the correct nature of the problem was identified a decision was made to reanalyze tritium for all the samples collected through day 184 using the more effective mixing procedure. For many samples this reanalysis involved a dilution step to obtain sufficient volume. Original data was retained for samples with good original data or where liquid sample was no longer available for re-analyses.

\subsubsection{Chromium and boron}

Chromium and boron were analyzed simultaneously with a Perkin-Elmer model 6000 inductively coupled plasma instrument (ICP) and a model 7500 integrated computer (Norwalk, CT). The linear working range given by Perkin-Elmer for this instrument is from 0.02-300 $\mathrm{mg} / \mathrm{L}$ for chromium and from $\mathbf{0 . 0 5 - 3 0 0}$ $\mathrm{mg} / \mathrm{L}$ for boron, with a detection limit of 0.004 and $0.002 \mathrm{mg} / \mathrm{L}$, respectively. In practice, the detection limits were found to be much higher 
for this machine; $0.029 \mathrm{mg} / \mathrm{L}$ for chromium and $0.130 \mathrm{mg} / \mathrm{L}$ for boron.

The ICP was programmed to measure spectral emission of chromium at $267.7 \mathrm{~nm}$, then to switch the monochromator wavelength to $249.77 \mathrm{~nm}$ for boron. The instrument was calibrated with a series of three solutions; $a$ standard containing $10 \mathrm{mg} / \mathrm{L}$ chromium and $10 \mathrm{mg} / \mathrm{L}$ boron, a water blank, and a second standard to check for reproducibility. This calibration procedure was repeated after every 15 samples.

Samples were transferred to $15 \mathrm{ml}$ polystyrene conical tubes (Becton Dickinson, Lincoln Park, $\mathrm{NJ}$ ) and run in groups of $\mathbf{5 0}$ samples (including calibration tubes). The run would begin after allowing the machine at least $\mathbf{2 0}$ minutes to warm up and produce a stable plasma field, confirmed by successful calibration.

Both elements are expressed in the data as relative concentrations, $C / C_{0}$, where $C$ is the observed concentration of the element in the sample $(\mathrm{mg} / \mathrm{L})$ and $\mathrm{C}_{0}$ is the input concentration (mg/L).

\subsubsection{Fluorobenzoates}

High Performance Liquid Chromatography instruments (HPLC) at laboratories of the Agricultural Research Service, U.S.
Department of Agriculture in Tempe, Arizona, and at the Department of Soil and Water Science at The University of Arizona were used to analyze the fluorobenzoic acid tracers.

Sample analyses were performed during two periods: samples analyzed between January $7-9,1991$ were collected on days $0,14,30,47$, and 70; those analyzed between September 23-25, 1991 were collected on days 127 and 310.

Fluorobenzoic acid tracers are best analyzed using High Performance Liquid Chromatography (HPLC). The reason for this is interference from nitrate and bromide ions under UV light at the optimum wavelength for benzoic acid tracers (i.e. $205 \mathrm{~nm}$ ). Bowman (1984a) showed that nitrate and bromide became excited under UV energy at $205 \mathrm{~nm}$ wavelength, and that HPLC methods allow the separation of different fluorobenzoates from other anions. Analytical equipment used during the two analyses periods differed slightly (Table 2). The column used for these analyses was a Regis Rexchrome S5-100-SAX 5 mm Reverse Phase HPLC column. The column length was $250 \mathrm{~mm}$ with an internal diameter of $4.6 \mathrm{~mm}$.

This is the same type of column used by -Bowman (1984a), except that the packing material, which has been improved over the years, differs slightly.

Table 2. Analytical equipment and software used for analysis of the fluorobenzoates.

\begin{tabular}{||l|l|l|}
\hline Date & Jan. 7-9,1991 & Sept. 23-25, 1991 \\
\hline Pump & Waters 510 & Waters 510 \\
\hline Detector & Waters 490 Multi-wavelength & Waters 480 Multi-wavelength \\
Sampler & Waters 710 Wisp & Perkin-Elmer ISS 100 \\
Software & Waters Maximum 820 & Waters Maxima 820 \\
\hline
\end{tabular}


The assays used for both analyses were modified from Bowman (1984a, b). The gradient system (Table 3 ), used a potassium phosphate $\left(\mathrm{K}_{2} \mathrm{PO}_{4}\right)$ mobile phase adjusted to a

$\mathrm{pH}$ of approximately 2.8 , and acetonitrile. The detector wavelength was kept constant at

$205 \mathrm{~nm}$. After the sample was injected into the fluid stream, the percentage of acetonitrile was increased (ramped) to enhance the separation of the constituents in the sample. Once the benzoates and bromide were released from the column material, the acetonitrile was decreased to the original concentrations, thus allowing the column to return to equilibrium for the next sample. The increases and decreases of acetonitrile in the mobile phase were optimized to improve separation.

Because of significant interference of nitrate and bromide with the benzoic acid tracers, and because each column has slightly different adsorption characteristics, the molarity and $\mathrm{pH}$ of the mobile phase had to be adjusted to improve the separation of the constituents, while maintaining an acceptable analytical time.

Table 3. Mobile phase characteristics and gradients for analytical events of January 7-9, 1991.

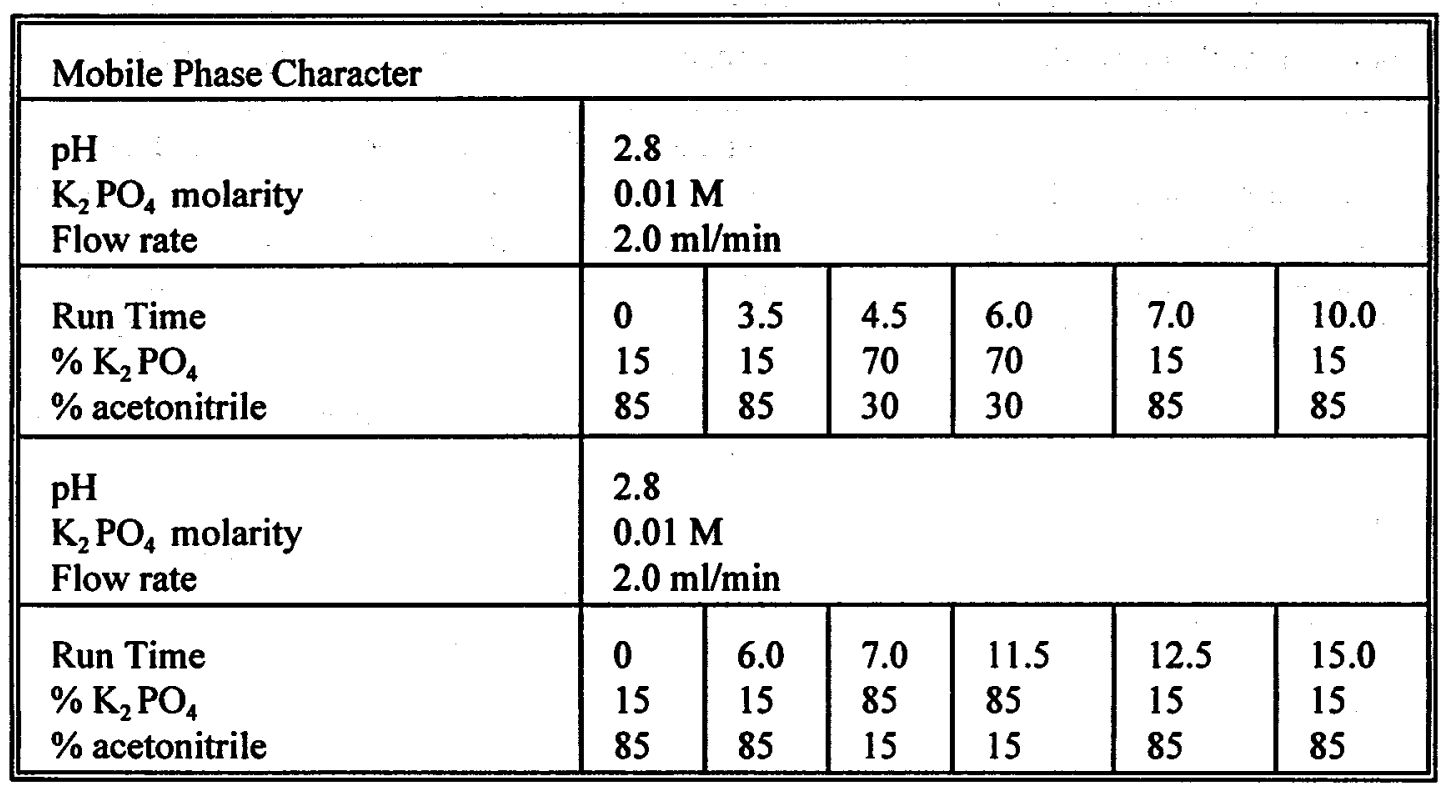

Retention times were (Table 4) also found to vary significantly between analytical events. A possible reason for this could be the change in columns. Although each column is manufactured with the same packing material, slight variations between columns can cause retention times to vary. It is noted that retention times did drift during the analyses. This was caused by changes in the column's ability to retain the anions. As the column ages, the retention characteristics decrease, leading to shorter retention times. The computer program which operated the HPLC (Water Dynamic Solutions, Ventura, CA) was programmed to allow for $10 \%$ drift. Larger drifts indicated a problem with the column, in which case the column was cleaned. 
Table 4. Retention times for benzoates, bromide, and nitrate.

\begin{tabular}{||l|c|c|}
\hline Event & 1 & 2 \\
\hline DFBA & 3.65 & 6.30 \\
PFBA & 3.34 & 8.55 \\
Bromide & 4.39 & 9.20 \\
Nitrate & 4.09 & 9.60 \\
\hline
\end{tabular}

The large number of samples run during each event required a new batch of mobile phase. For each new mobile phase, standard samples were run so that a new calibration curve would be generated. The standards for the calibration curve were made from four individual solution mixtures of $1,2,5$, or $10 \mathrm{ppm}$ of both PFBA and DFBA and a fifth water blank. Retention times and possible interferences were checked for each new mobile phase mixture. Checking for interferences ensured that shifts in the retention times would be accounted for and noted during the analyses. Furthermore, to ensure that the calibration curves remained valid for each method, one standard was run for each 10 samples. Slight deviations in the results from the existing calibration curve were incorporated by the software, and new calibration equations were generated. Large deviations indicated problems with the column, instrumentation or mobile phase.

In one case during the second analytical event, for samples collected on day 310 , the column began to fail. This was seen as a widening of the solute peak and an inability of the software to discern a retention time. Column failure was the only logical explanation for this problem and, consequently data for samples collected on day 310 were considered invalid.

\subsubsection{Bromide, chloride, and nitrate}

Bromide, chloride, and nitrate ions were analyzed with ion chromatography (IC) apparatus at the Soil and Water testing Laboratory of The University of Arizona. All three anions were analyzed simultaneously with Dionex equipment; a 2320i ion chromatography module, a $2021 \mathrm{i}$ conductivity detector and an HPIC-AS4A analytical column (Dionex Corporation, Sunnyvale, $\mathrm{CA}$ ). The eluant used was $7.5 \mathrm{e}^{-4} \mathrm{M} \mathrm{NaHCO}_{3} / 2.2 \mathrm{e}^{-3} \mathrm{M} \mathrm{Na}_{2} \mathrm{CO}_{3}$ at a flow rate of $2.0 \mathrm{~mL} /$ minute. The Dionex chromatography module utilizes a suppressor component to remove unwanted cations from the sample stream ahead of the detector. For these samples a $0.025 \mathrm{~N} \mathrm{H}_{2} \mathrm{SO}_{4}$ regenerant was supplied to the suppressor.

A series of four standard solution mixtures (Table 5) was used to construct an initialcalibration curve at the beginning of the analysis.

A sequence of runs was used to update the calibration curve for every 10 samples; a single run of one selected standard mixture, followed by a blank (water) solution, and a duplicate of one of the previous samples. When necessary to bring an analyzes into the IC linear working range, sample dilutions were performed with Rainin EDP electronic pipettors (Rainin Instruments Co., Inc., Woburn, MA) to dilute solution with water in a test tube.

\subsection{Data Manipulation}

Neutron probe water content data were transferred via RS-232 cable from the 
Table 5. Standard solutions for bromide, chloride and nitrate analyses.

\begin{tabular}{|l|c|c|c|c|}
\hline Mixture \# & 1 & 2 & 3 & 4 \\
\hline $\mathrm{Br}(\mathrm{ppm}):$ & 0.25 & 2.5 & 6.25 & 12.5 \\
$\mathrm{Cl}(\mathrm{ppm}):$ & 0.50 & 5.0 & 12.50 & 25.0 \\
$\mathrm{NO}_{3}(\mathrm{ppm}):$ & 0.75 & 7.5 & 18.75 & 37.5 \\
\hline
\end{tabular}

probe memory to an ASCII text file on an IBM $\mathrm{XT}$ personal computer. The data was transferred from disk to an Apple Macintosh computer for further manipulation. Macro routines in a WingZ spreadsheet (Informix Software, Lenexa, KS) were used to strip the file of unwanted output lines, produce preliminary graphics, and store the data in a usable format. Tensiometer data and data from all the chemical analyses were entered by hand into WingZ spreadsheets. For final data storage Double Helix software (Odesta Corp., Northbrook, IL) was used to combine, sort and store the data in database format.

Contour figures for this report were produced with DeltaGraph Professional software (DeltaPoint Inc., Monterey, CA, version 2.0.1) which creates triangular patches between true data points. It does not convert data into grids with algorithms based on kriging or inverse distance to power $\mathbf{n}$.

Other software applications used to produce $\mathrm{X}-\mathrm{Y}$ plots and other figures and tables from data were Microsoft Excel (Microsoft Corp., Redmond, WA, version 3.0), KaleidaGraph (Synergy Software, Reading, PA, version 2.0.2), and Canvas (Deneba Systems Inc., Miami, FL, version 3.0.2).

\subsection{Discussion of Results for Experiment IIb}

\subsubsection{Soil moisture-neutron probe data}

The distribution of volumetric water contents is presented in a series of contour plots from data that is presented in Appendix A-2. Plots for day 0 show absolute water contents at the beginning of the experiment. Plots for subsequent days show water contents as differences in water content from day 0 . A final series of contour plots show absolute water contents for day 525 .

Water content contours are plotted for planes of measurement at $X_{2}=0$ meters, which lies lengthwise along the center of the irrigated plot, and $Y_{2}=2,6$, and 10 meters from the trench face, where each of these latter planes of measurement lie perpendicular to the length of the plot. The figures for $X_{2}=0$ represent a 15 $\mathrm{m}$-long transect, monitored by 11 access tubes. Each of the planes in $Y_{2}$ represent $10 \mathrm{~m}$-long transects, monitored by 11 access tubes $(12 \mathrm{~m}$ long, 13 tubes after day 191). Therefore the plot-bisecting planes have a somewhat higher sampling density and greater density of data for plotting.

Figures 22 and 23 show the water contents on the first day of the experiment, day 0 . These represent initial conditions for this experiment, following the redistribution of experiment IIaapplied water. The mean value for these 1025 individual measurements is $0.093 \mathrm{~cm}^{3} / \mathrm{cm}^{3}$. 

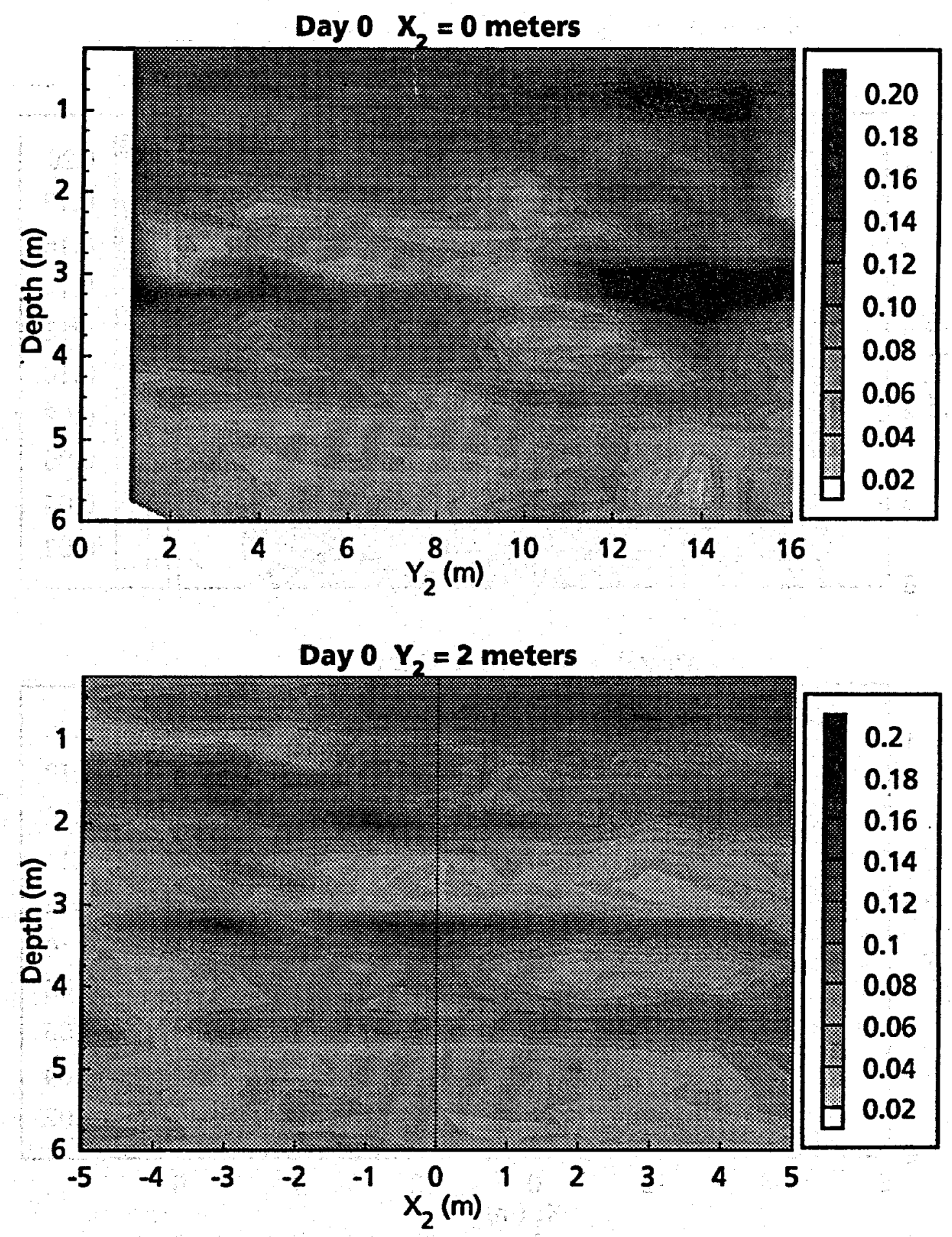

Figure 22. Experiment IIb neutron probe volumetric water content on day 0 for a plane through $X_{2}=0$ $\mathrm{m}$ along the length of plot 2 , and for a plane through $Y_{2}=2 \mathrm{~m}$ parallel to the trench face. 

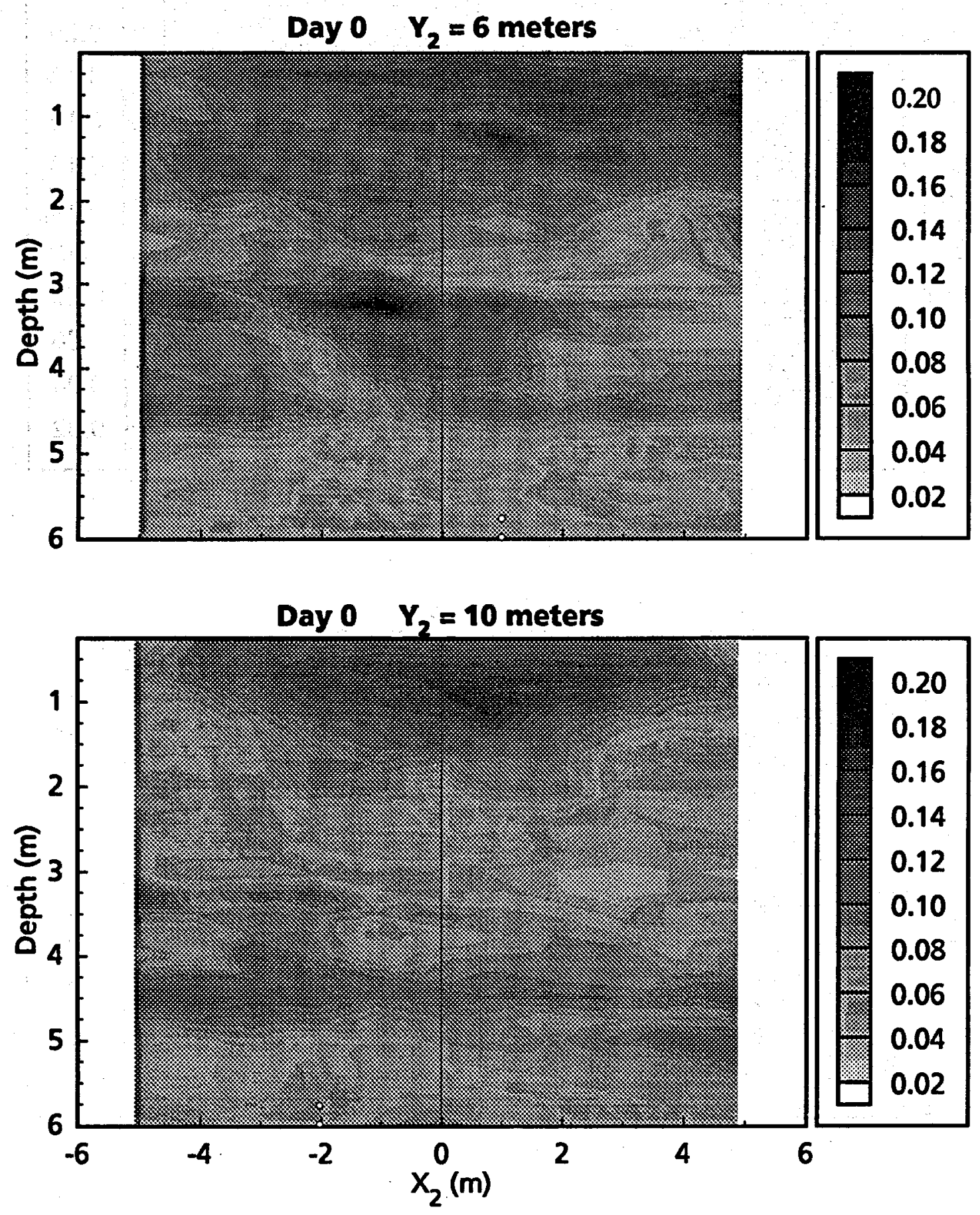

Figure 23. Experiment IIb neutron probe volumetric water content on day 0 for planes through $Y_{2}=6$ $m$ and $Y_{2}=10 \mathrm{~m}$ parallel to the trench face. 
Values range from a maximum of $0.229 \mathrm{~cm}^{3}$ $/ \mathrm{cm}^{3}$ to a minimum of $0.027 \mathrm{~cm}^{3} / \mathrm{cm}^{3}$, where both of these latter values were obtained from the same access tube at $Y_{2}=14 \mathrm{~m}$. The apparent spatial variability illustrated by these plots is a strong indication that water content is layered within the profile. Relatively consistent layers of higher moisture content are found about 3.25 and 4.5 meters deep. Additional layers, more spatially variable, are suggested above those depths. Soil texture data collected from a profile at the face of the trench $\left(X_{2}=\right.$ $-0.6 \mathrm{~m}$ ) shows an abrupt increase in clay content at the 3.31 meter mark and a similar increase in calcium carbonate at 4.61 meters (Nash, 1990; Wierenga et al, 1989) which corresponds to these high-moisture-content lenses.

In the upper half of Figure 22 a zone of relatively high moisture content is found from about $2.75 \mathrm{~m}$ to $3.5 \mathrm{~m}$ depth, beyond the north end of the irrigated plot at $Y>12$ meters. The source of this moisture is uncertain. The bulk of this moisture appears in the profile after experiment Ila irrigation, following rain events. Undetected leaks in pondliner seams at the north end of the plot are likely sources for much of this moisture. A large section of this pondliner was defective, and contained a large number of poorly sealed seams. It was replaced before experiment Ilb began. Also, near the end of IIb irrigation (day 66), a leaking union was discovered in the buried 1" PVC irrigation supply line, located about $X_{2}=13.5 \mathrm{~m}$ and $Y_{2}=0.6 \mathrm{~m}$. These combined sources contributed about 1 percent of the total water applied during experiment IIb, as measured with the neutron probe.

The next series of plots show water content differences for day 31 , about halfway through the irrigation pulse (Figures 24 and 25). Again, note that these plots show moisture as differences in water content from day 0 . By this time the leading edge of the wetting front had moved through the profile to about 3.5 to 4 meters depth, depending upon the location in $Y_{2}$.
Lateral movement in the bisecting planes was about 2 to 2.5 meters and applied water was fairly evenly distributed across the $\mathrm{X}=0$ axis.

By day 73, three days after irrigation was discontinued, the wetting front was near the 6 meter depth mark (Figures 26 and 27). In the plot at $\mathrm{X}_{2}$ meters wetting appeared somewhat more effective at $Y_{2}=6-8$ meters. In the bisecting measurement planes the wetting front had moved laterally to about $X_{2} \pm 3$ to 4 meters. The bulk of the applied and translocated water in the profile was found below 3 meters depth. In areas of soil above this depth layering of retained water is evident.

The next series of plots show water contents for day 126, 56 days after irrigation (Figures 28 and 29). The wetting front had continued to progress, though at a much slower rate. In all locations the front had passed below 6 meters, below the range of neutron probe measurement. There is further evidence of moisture layering and drying above 3 meters depth. Bisecting views of the plot show that water continued to move laterally, with more pronounced movement in portions of the profile above 3 meters depth.

The most recent data, from day 525 , show that the observed pattern of redistribution was continued (Figures 30 and 31). Downward movement of water since day 126 was almost imperceptible. However, above 3 meters a drying front is evident. This new front appeared to lag the wetting front as applied water moved laterally from the plot. At $Y_{2}=6$ meters the wetting front had moved over 5 meters from the center of the irrigated area. At the $Y_{2}=6$ and 10 meter planes of measurement soil moisture values above 3 meters depth and between $X_{2} \pm$ 2 meters had almost returned to the initial conditions of day 0 . What appears to be moisture migration from areas outside of the pondliner-covered area began to be measured downhill from the irrigated area, at $X_{2}=4$ to 6 meters. This moisture is likely the result of 

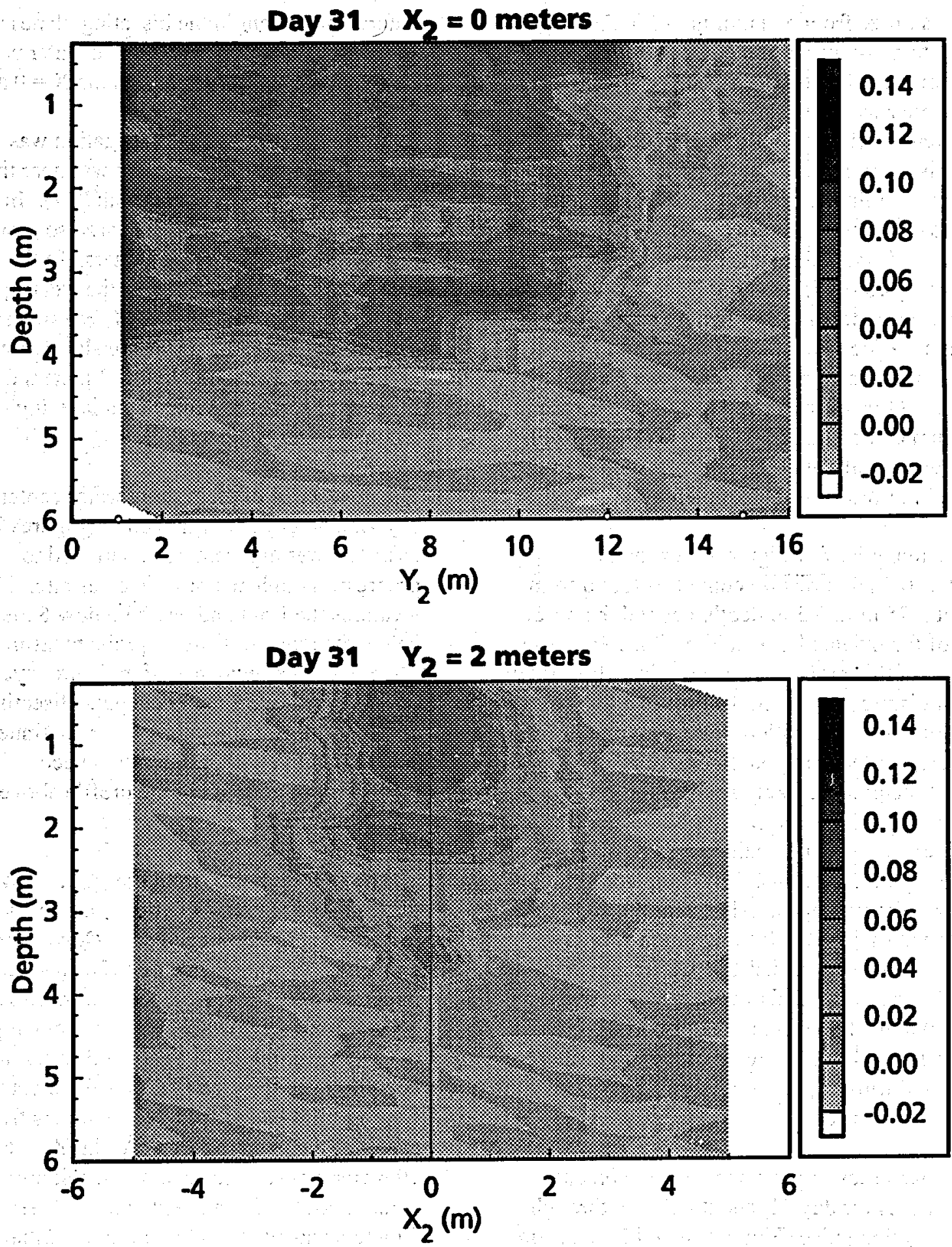

Figure 24. Experiment IIb neutron probe volumetric water content differences on day 31 for a plane through $\mathrm{X}_{2}=0 \mathrm{~m}$ along the length of plot 2 , and for a plane through $\mathrm{Y}_{2}=2 \mathrm{~m}$ parallel to the trench face. Values expressed as differences from day 0 water content. 

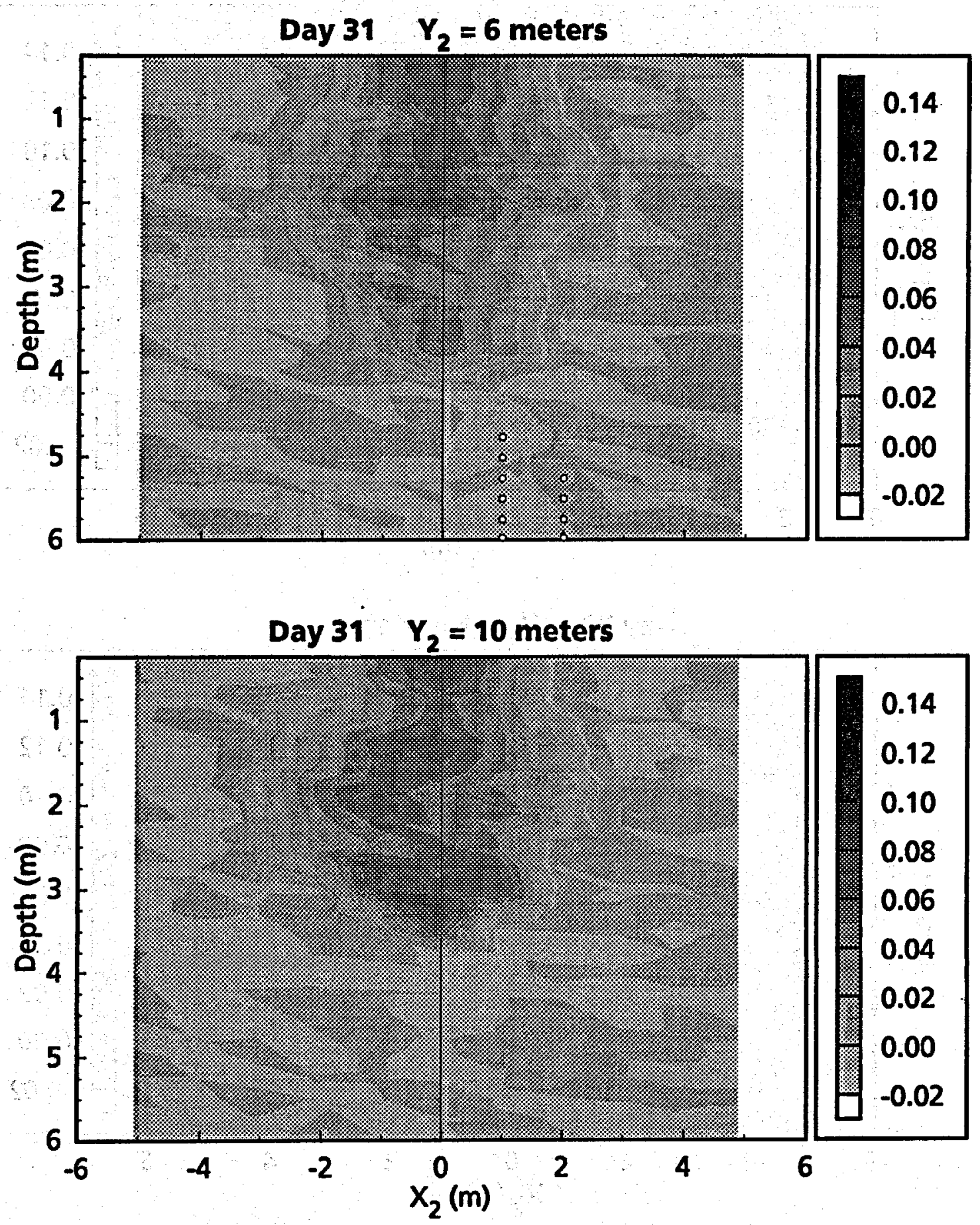

Figure 25. Experiment IIb neutron probe volumetric water content differences on day 31 for planes through $Y_{2}=6 \mathrm{~m}$ and $Y_{2}=10 \mathrm{~m}$ parallel to the trench face. Values expressed as differences from day 0 water content. Small circles in plots indicate missing data. 

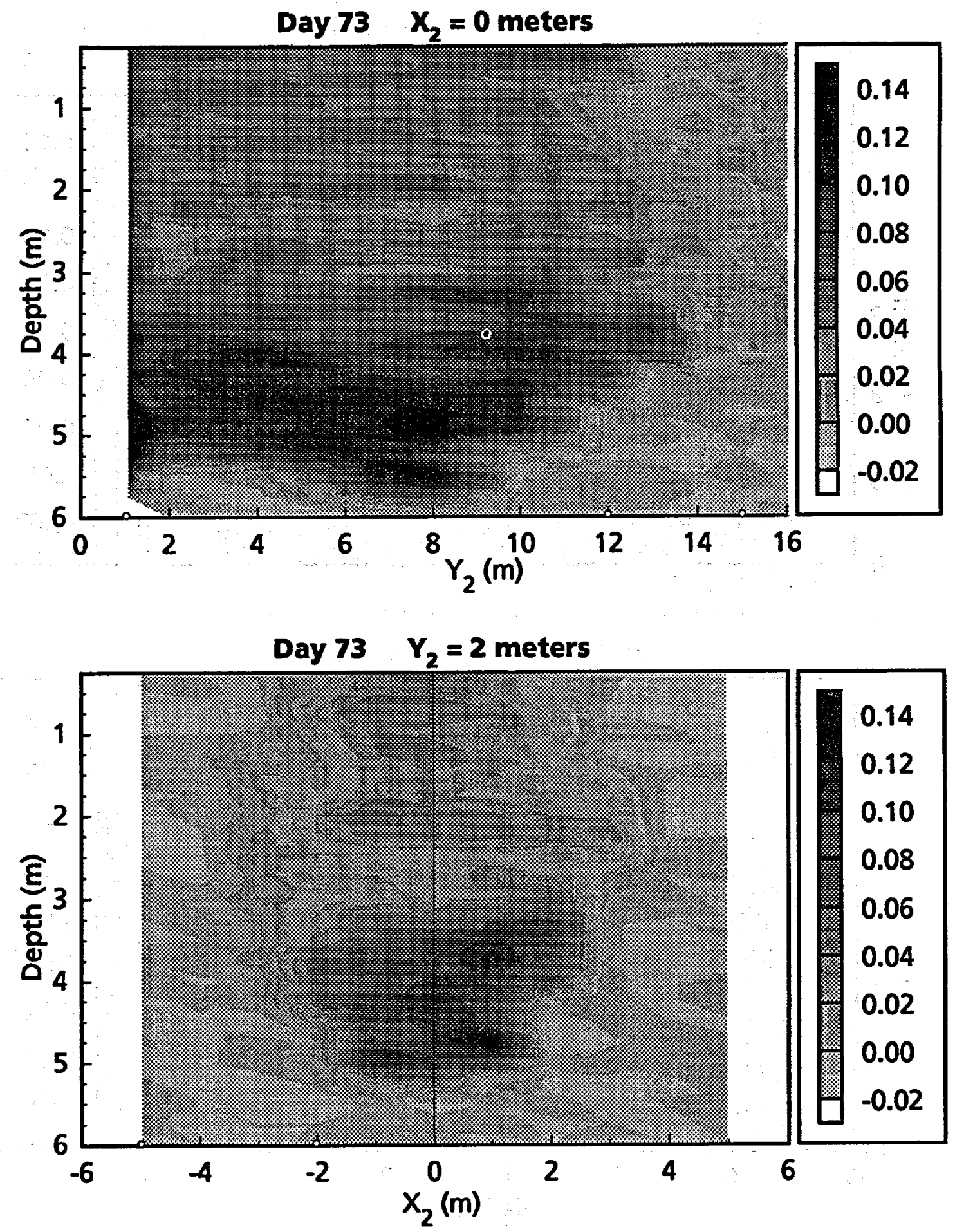

Figure 26. Experiment IIb neutron probe volumetric water content differences on day $\mathbf{7 3}$ for a plane through $X_{2}=0 \mathrm{~m}$ along the length of plot 2 , and for a plane through $Y_{2}=2 \mathrm{~m}$ parallel to the trench face. Values expressed as differences from day 0 water content. 

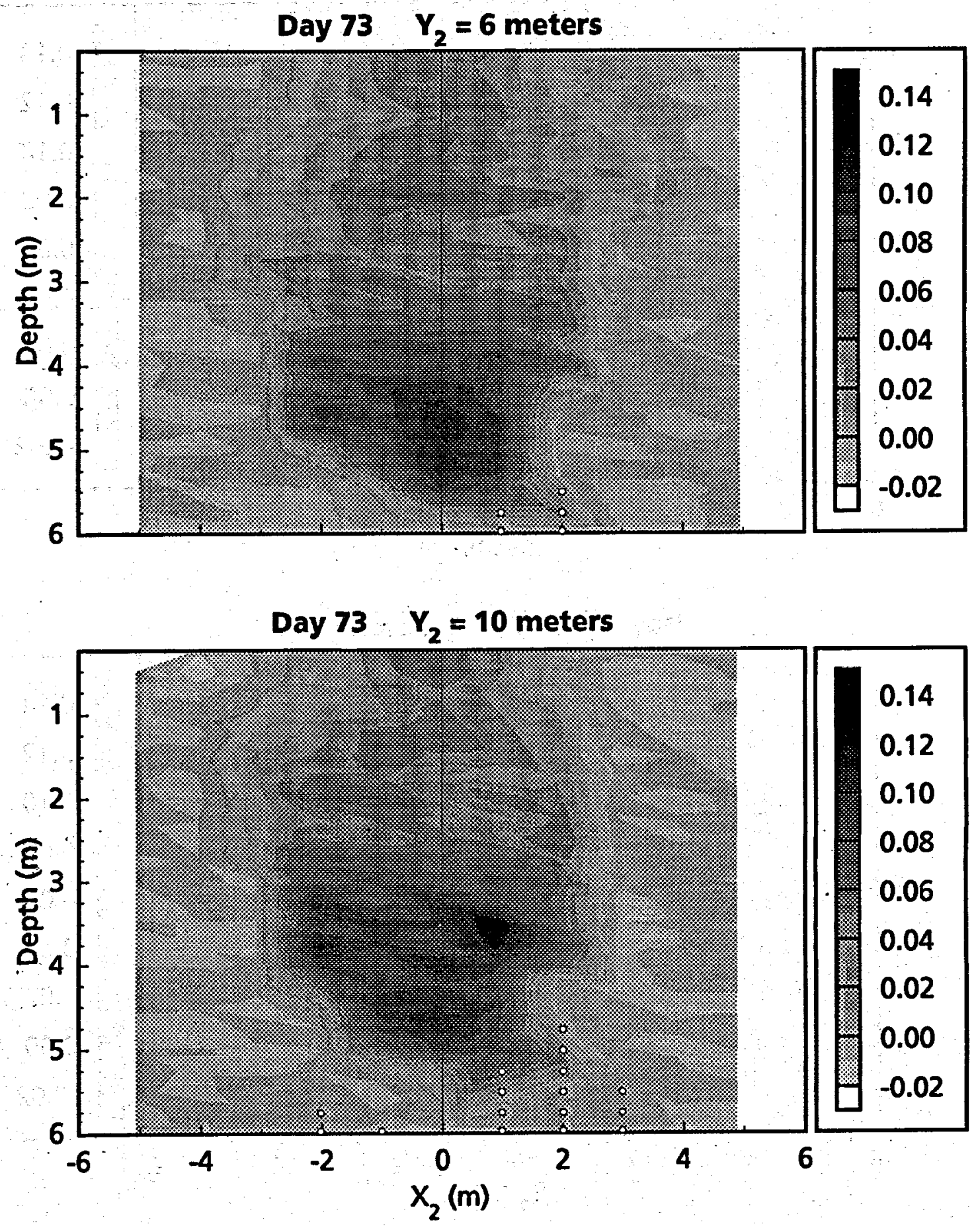

Figure 27. Experiment IIb neutron probe volumetric water content differences on day 73 for planes through $Y_{2}=6 \mathrm{~m}$ and $Y_{2}=10 \mathrm{~m}$ parallel to the trench face. Values expressed as differences from day 0 water content. Small circles in plots indicate missing data. 
Day $126 \quad X_{2}=0$ meters

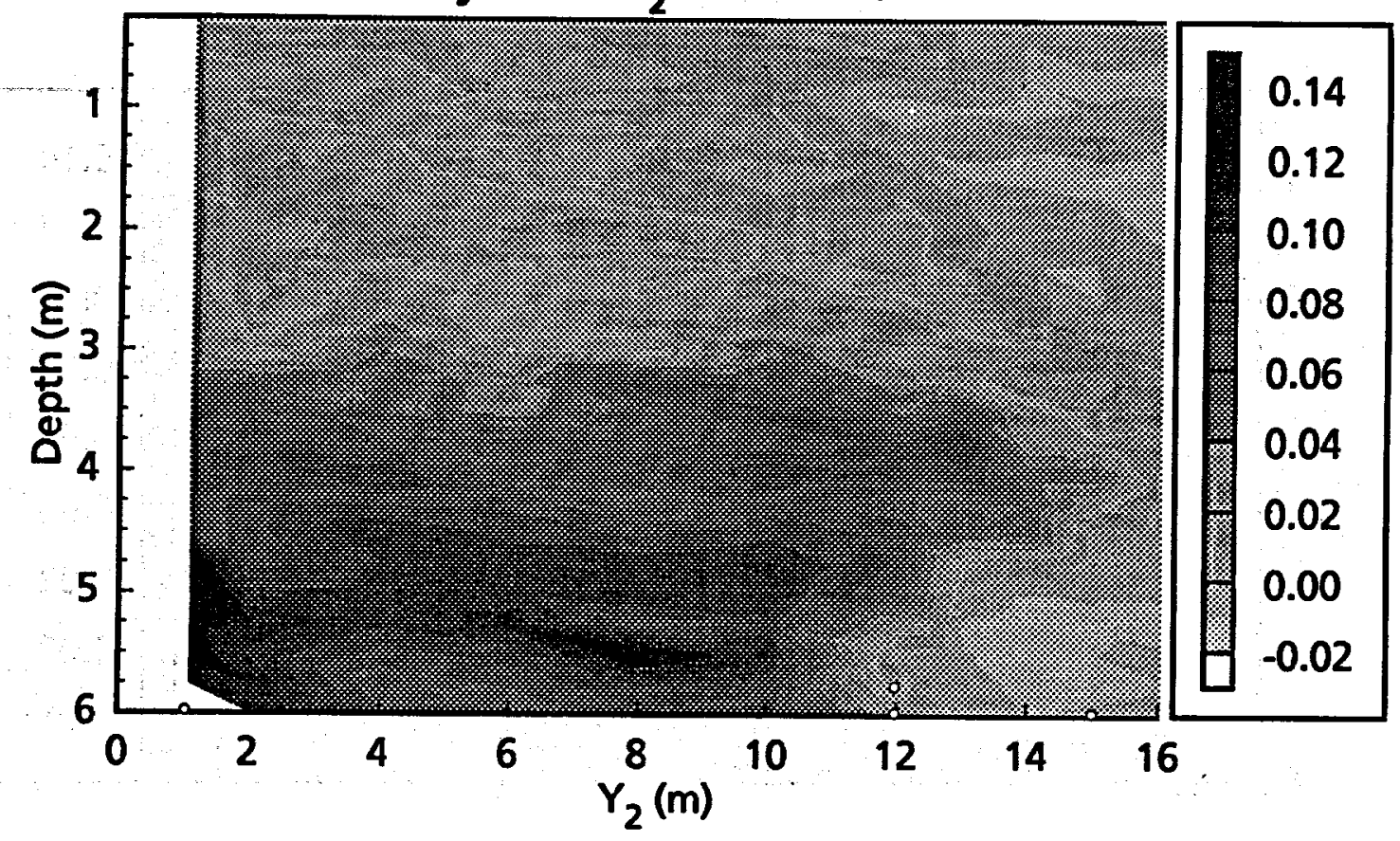

Day $126 \quad Y_{2}=2$ meters

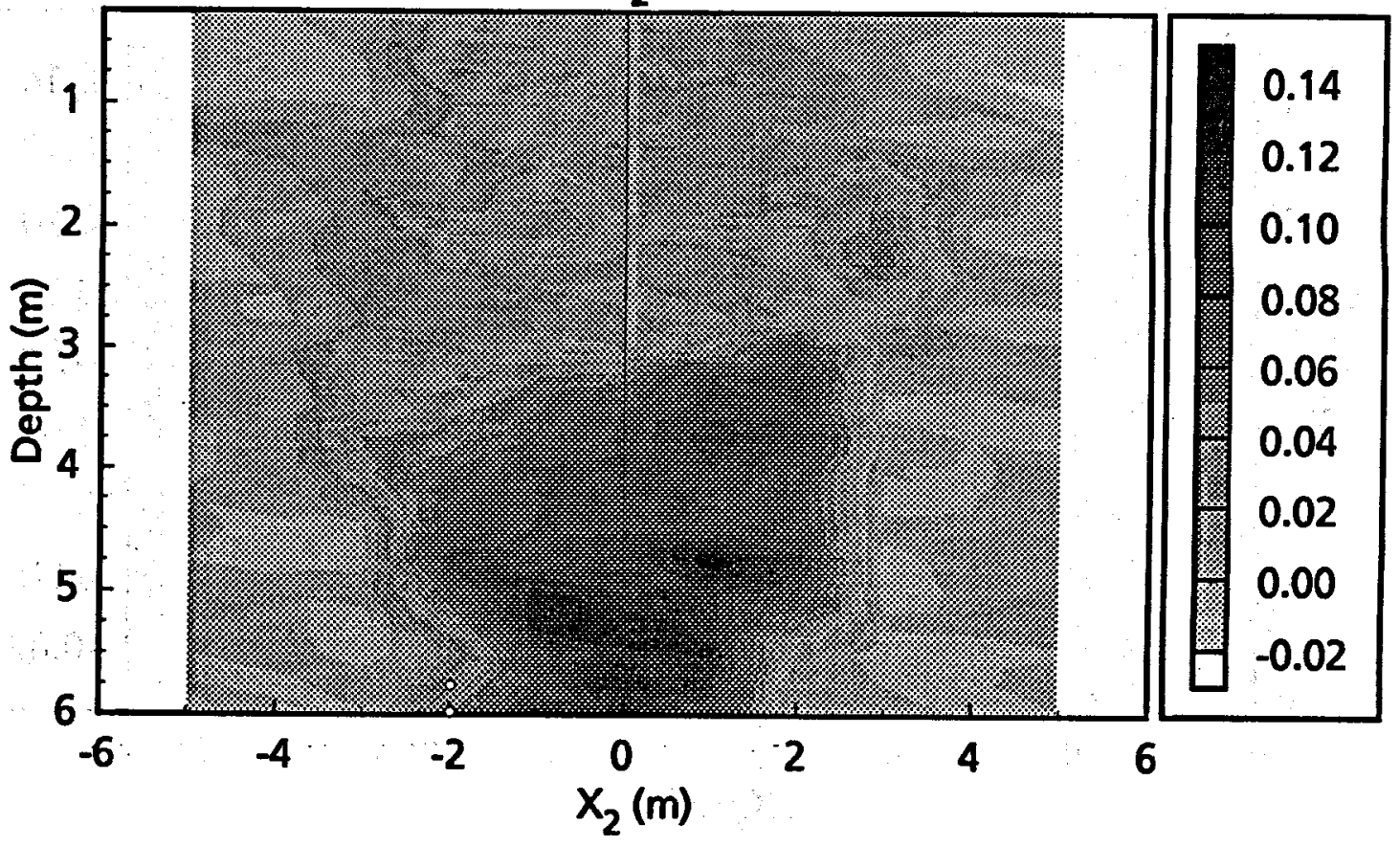

Figure 28. Experiment IIb neutron probe volumetric water content differences on day 126 for a plane through $X_{2}=0 \mathrm{~m}$ along the length of plot 2, and for a plane through $Y_{2}=2 \mathrm{~m}$ parallel to the trench face. Values expressed as differences from day 0 water content. 

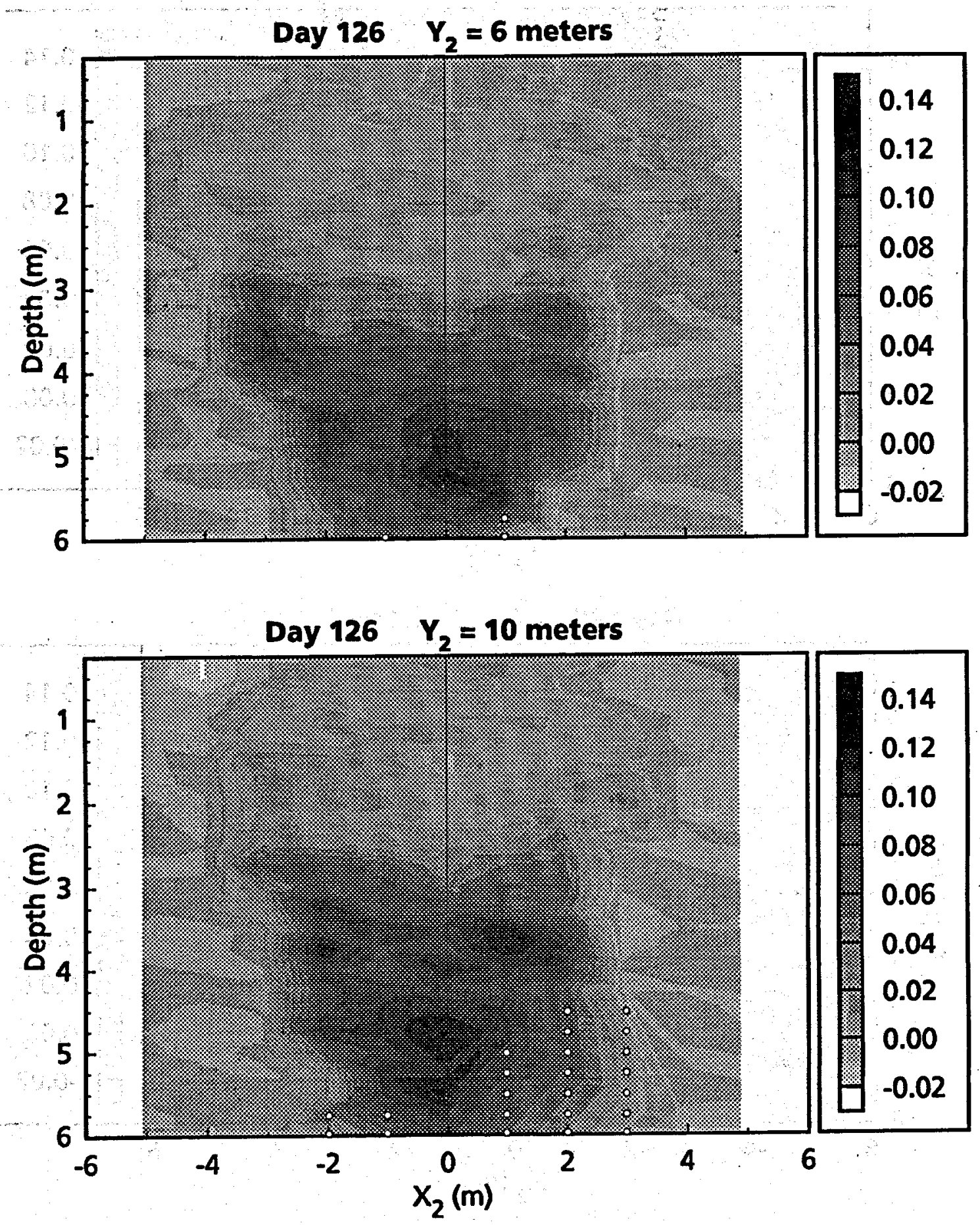

Figure 29. Experiment IIb neutron probe volumetric water content differences on day 126 for planes through $Y_{2}=6 \mathrm{~m}$ and $Y_{2}=10 \mathrm{~m}$ parallel to the trench face. Values expressed as differences from day 0 water content. Small circles in plots indicate missing data. 

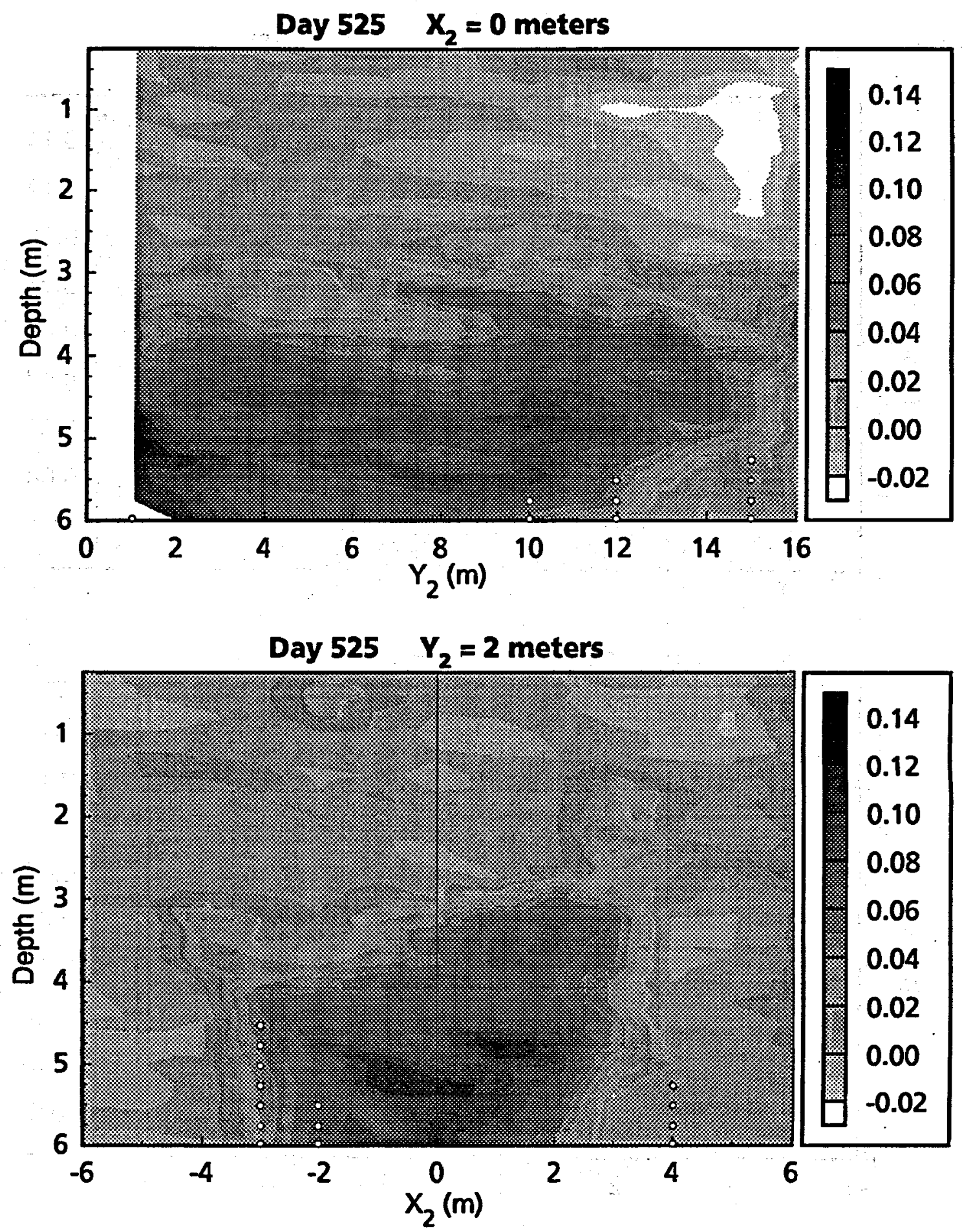

Figure 30. Experiment IIb neutron probe volumetric water content differences on day 525 for a plane through $X_{2}=0 \mathrm{~m}$ along the length of plot 2 , and for a plane through $Y_{2}=2 \mathrm{~m}$ parallel to the trench face. Values expressed as differences from day 0 water content. Small circles in plots indicate missing data. 

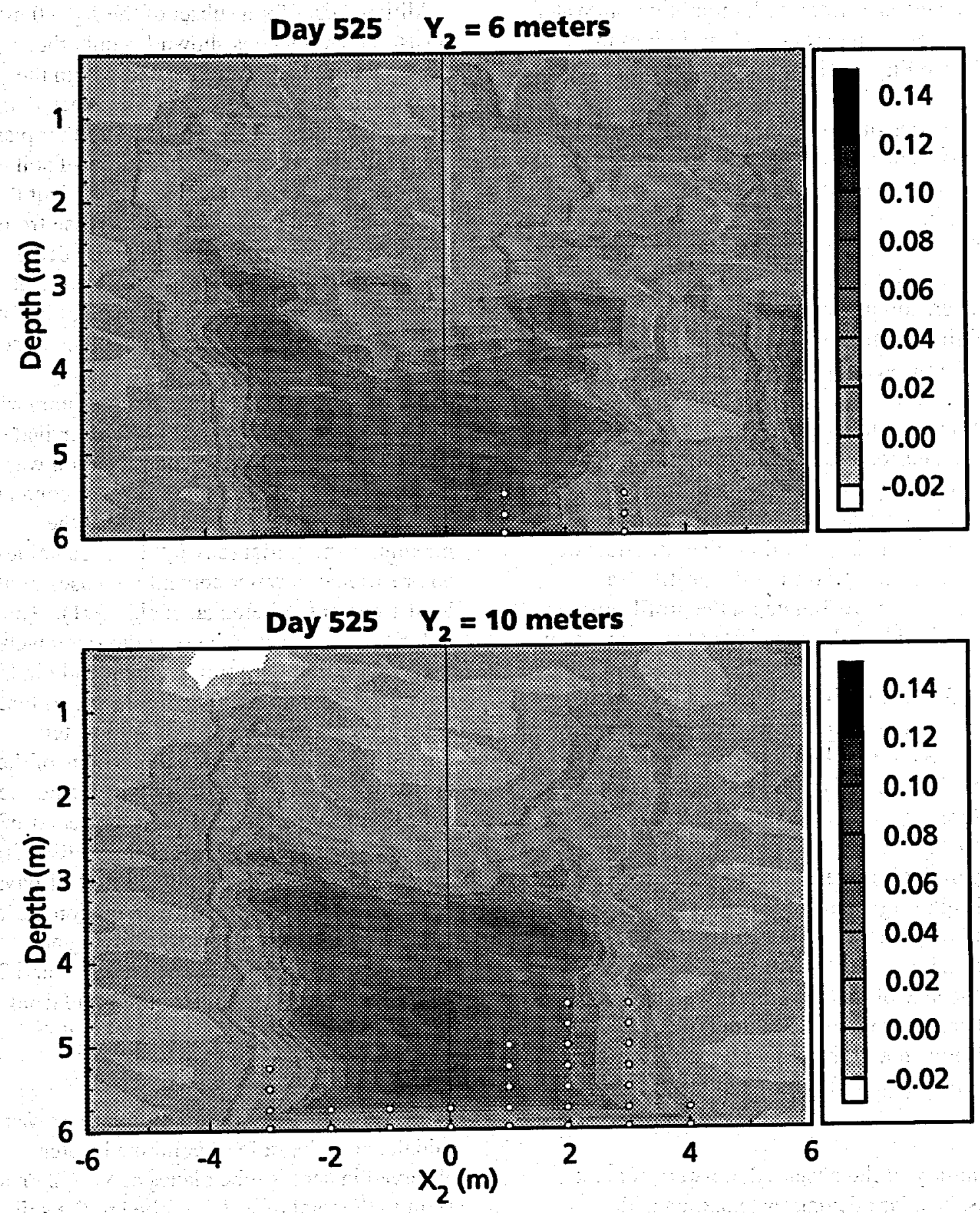

Figure 31. Experiment IIb neutron probe volumetric water content differences on day 525 for planes through $Y_{2}=6 \mathrm{~m}$ and $Y_{2}=10 \mathrm{~m}$ parallel to the trench face. Values expressed as differences from day 0 water content. Small circles in plots indicate missing data. 
runoff, where downhill sections of pondliner cover acted as a watershed, promoting rainwater entry to soil along the $X_{2}=8 \mathrm{~m}$ limit of the cover (see Figure 21).

A final series of plots show day 525 water contents as observed values, not as differences from day 0 water contents (Figures 32 and 33). When compared to "day 0 differences" plots for day 525 (see Figures 30 and 31) the location of the observed wetting plume is about the same. However, soil moisture layering and spatial variability is much more prominently displayed in the "observed" contours. In Figure 33 the lateral extent of the wetting front, about $4.5 \mathrm{~m}$ in depth, may be underestimated because of missing neutron probe readings near the bottom of the $Y_{2}=10 \mathrm{~m}$ transect. When compared to observed values at day 0 of the experiment (Figures 22 and 23) one finds that the strata of moisture-retaining areas of the profile are consistent. Above $3 \mathrm{~m}$ depth the profile appears much as it did on the first day of the experiment.

Below $3 \mathrm{~m}$ the moisture-retaining layers have higher water contents than on day 0 , especially between $X_{2} \pm 3$ to 4 meters. Perhaps the most notable feature of Figure 32 is the zone of high moisture content located at $Y_{2}>12 \mathrm{~m}$, about 2.75 to $4.25 \mathrm{~m}$ deep, that was discussed earlier. Compared to Figure 22, the location and extent of this high-moisture zone appears to have shifted only a little deeper and diminished little in the 525 elapsed days (Figure 33). During this time the size of this area increased slightly. A small area adjoining the bottom of the zone became wetter, perhaps supplying moisture to a moisture-retaining layer of soil about $4.5 \mathrm{~m}$ deep.

A summary of the observed moisture content means, standard deviations, maxima and minima for each neutron probe measurement plane of Figures 22 through 33 is given in Table 6. Note that these data are given as observed moisture contents (upper horizontal blocks in Table 6) as well as moisture content differences from day 0 (lower blocks). Additional data for a subset of the $X_{2}=0 \mathrm{~m}$ measurement plane is shown for only those access tubes which were located within the irrigated area, to $Y_{2}=10 \mathrm{~m}$. As expected, these moisture values are higher than for other planes since tubes located in dryer portions of soil are not included. From this table it is evident that mean planar moisture contents increase from days 0 through 73 and then gradually decrease through day 525. Further, the soil planes at $Y_{2}$ $=2 \mathrm{~m}$ and $6 \mathrm{~m}$ hold similar amounts of moisture compared to the dryer $Y_{2}=10 \mathrm{~m}$ plane of soil.

The progress of the wetting front is summarized for $Y_{2}=2$ meters in Figure 34. The arrival of the wetting front at a particular location was arbitrarily taken as the time for water content to increase by a value halfway between the maximum and initial (day 0 ) observed values and restricted to water content increases of at least 1 percent (Wierenga, et al., 1991). These criteria seem reasonable given the steep wetting curve for vertical water movement and random error in measurement. The figure shows that water moved, or influenced in-situ water translocation, quickly through portions of the profile which were previously wet during the irrigation of experiment IIa. At the center of the plot the downward progress of the wetting front averaged $11.3 \mathrm{~cm} \mathrm{~d}^{-1}$ during the first 31 days. From then until the end of the irrigation period the front slowed dramatically, moving an average of $4.2 \mathrm{~cm} \mathrm{~d}^{-1}$ between days 31 and 73 . In the early stages of redistribution the front again slowed to an average of $1.9 \mathrm{~cm} \mathrm{~d}^{-1}$ between days 73 and 112 .

A mass balance for neutron probe water was calculated as the ratio of combined water observed in access tube planes at $Y_{2}=2,6$, and $10 \mathrm{~m}$ to the total of water applied to the soil surface (Figure 35). Observed water contents were taken as differences from day 0 , summed across all individual observations and their representative soil volumes in $Y_{2}$. This ratio increases from day 0 to a maximum value of 

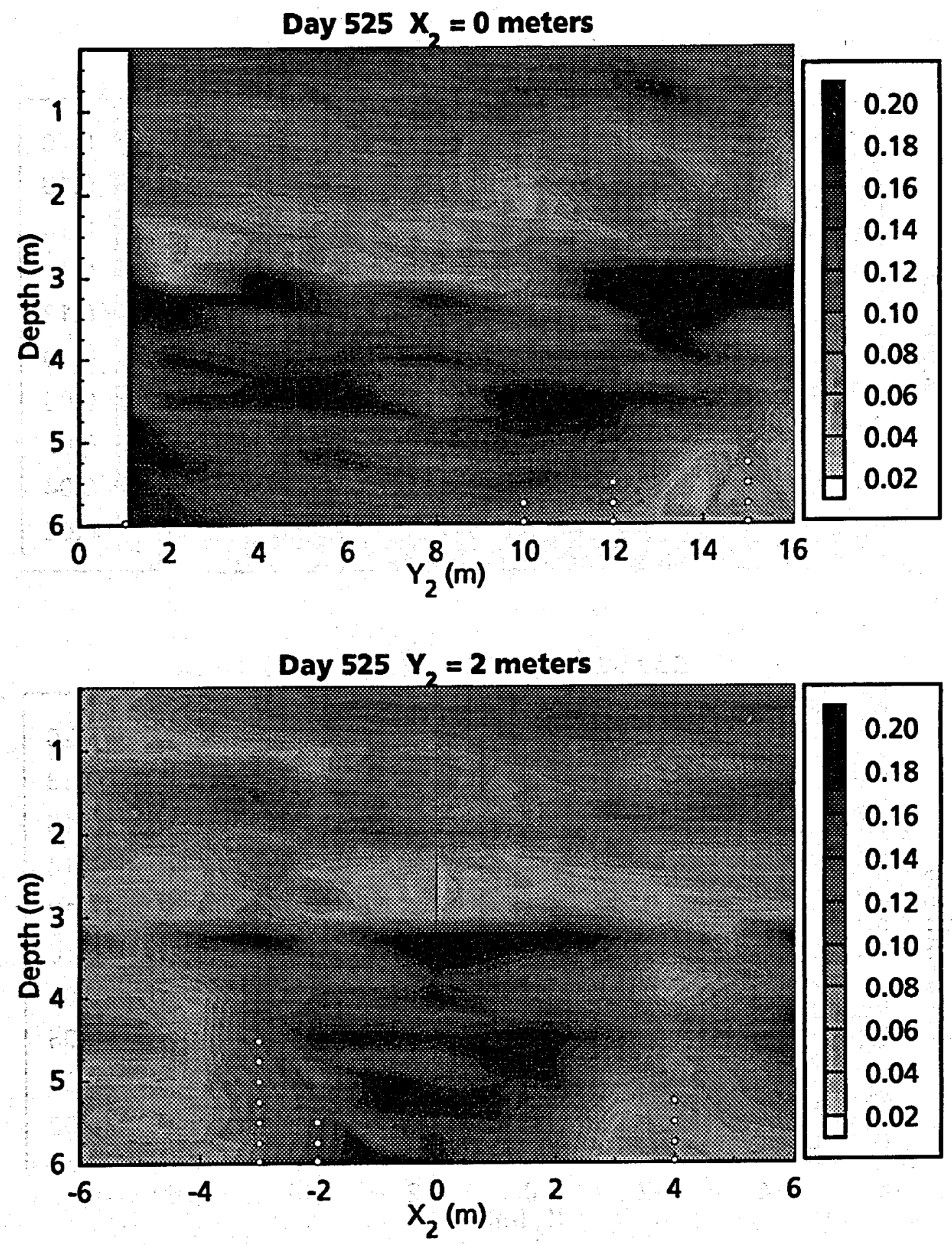

Figure 32. Experiment IIb neutron probe volumetric water content on day 525 for a plane through $\mathrm{X}_{2}=$ $0 \mathrm{~m}$ along the length of plot 2 , and for a plane through $Y_{2}=2 \mathrm{~m}$ parallel to the trench face. Small circles in plots indicate missing data. 

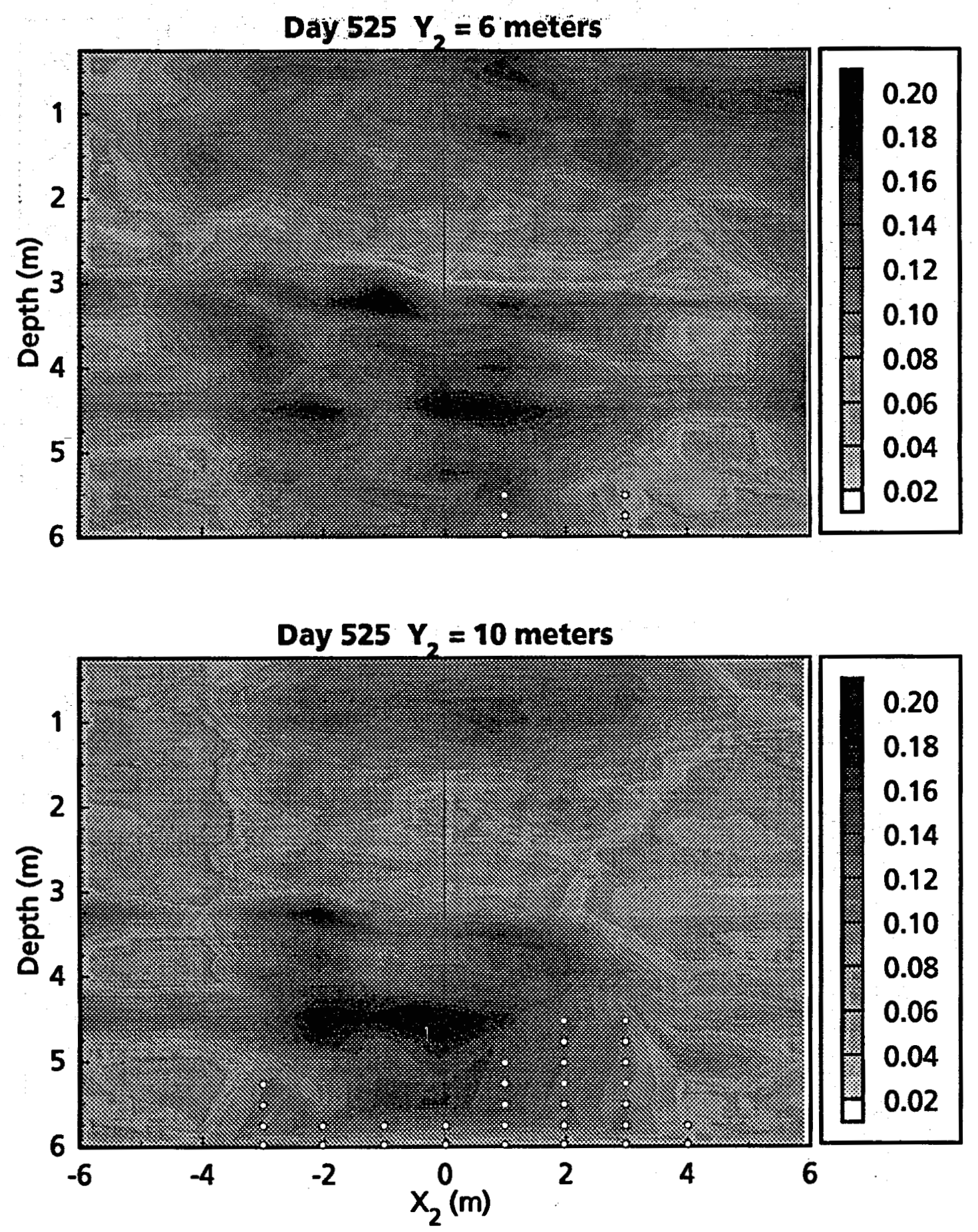

Figure 33. Experiment IIb neutron probe volumetric water content on day 525 for plane through $Y_{2}=6$ $\mathrm{m}$ and $\mathrm{Y}_{2}=10 \mathrm{~m}$ parallel to the trench face. Small circles in plots indicate missing data. 
Table 6. Mean water contents $\left(\mathrm{cm}^{3} / \mathrm{cm}^{3}\right)$ and mean water content differences $\left(\mathrm{cm}^{3} / \mathrm{cm}^{3}\right)$ for each of 4 observational planes at days 31, 72, 126, and 525 .

\begin{tabular}{|c|c|c|c|c|c|c|c|c|c|}
\hline Measurement & Day & Plane & Meters & $\begin{array}{l}\text { N- } \\
\text { obs }\end{array}$ & Mean & Stdev & $\operatorname{Max}$ & Min & $\begin{array}{l}\text { Media } \\
\mathbf{n}\end{array}$ \\
\hline $\begin{array}{l}\theta \\
\theta \\
\theta \\
\theta\end{array}$ & $\begin{array}{l}0 \\
0 \\
0 \\
0\end{array}$ & $\begin{array}{l}X \\
Y \\
Y \\
Y\end{array}$ & $\begin{array}{r}0 \\
2 \\
6 \\
10\end{array}$ & $\begin{array}{l}262 \\
263 \\
262 \\
262\end{array}$ & $\begin{array}{l}0.107 \\
0.092 \\
0.091 \\
0.082\end{array}$ & $\begin{array}{l}0.0381 \\
0.0278 \\
0.0286 \\
0.0283\end{array}$ & $\begin{array}{l}0.229 \\
0.176 \\
0.187 \\
0.170\end{array}$ & $\begin{array}{l}0.028 \\
0.035 \\
0.031 \\
0.035\end{array}$ & $\begin{array}{l}0.102 \\
0.087 \\
0.085 \\
0.076\end{array}$ \\
\hline $\begin{array}{l}\theta \\
\theta \\
\theta \\
\theta\end{array}$ & $\begin{array}{l}525 \\
525 \\
525 \\
525\end{array}$ & $\begin{array}{l}X \\
Y \\
Y \\
Y\end{array}$ & $\begin{array}{r}0 \\
2 \\
6 \\
10\end{array}$ & $\begin{array}{l}255 \\
298 \\
306 \\
282 \\
\end{array}$ & $\begin{array}{l}0.132 \\
0.113 \\
0.113 \\
0.096\end{array}$ & $\begin{array}{l}0.0403 \\
0.0372 \\
0.0349 \\
0.0369\end{array}$ & $\begin{array}{l}0.229 \\
0.207 \\
0.209 \\
0.203\end{array}$ & $\begin{array}{l}0.029 \\
0.041 \\
0.033 \\
0.037 \\
\end{array}$ & $\begin{array}{l}0.132 \\
0.112 \\
0.115 \\
0.088\end{array}$ \\
\hline $\begin{array}{l}\theta-\theta_{0} \\
\theta-\theta_{0} \\
\theta-\theta_{0} \\
\theta-\theta_{0} \\
\end{array}$ & $\begin{array}{l}31 \\
31 \\
31 \\
31 \\
\end{array}$ & $\begin{array}{l}X \\
Y \\
Y \\
Y \\
\end{array}$ & $\begin{array}{r}0 \\
2 \\
6 \\
10 \\
\end{array}$ & $\begin{array}{l}261 \\
262 \\
254 \\
255\end{array}$ & $\begin{array}{l}0.023 \\
0.008 \\
0.009 \\
0.009\end{array}$ & $\begin{array}{l}0.0275 \\
0.0180 \\
0.0173 \\
0.0205\end{array}$ & $\begin{array}{l}0.087 \\
0.072 \\
0.076 \\
0.087\end{array}$ & $\begin{array}{r}-0.014 \\
-0.011 \\
-0.008 \\
-0.007 \\
\end{array}$ & $\begin{array}{l}0.008 \\
0.001 \\
0.001 \\
0.001 \\
\end{array}$ \\
\hline $\begin{array}{l}\theta-\theta_{0} \\
\theta-\theta_{0} \\
\theta-\theta_{0} \\
\theta-\theta_{0} \\
\end{array}$ & $\begin{array}{l}73 \\
73 \\
73 \\
73 \\
\end{array}$ & $\begin{array}{l}X \\
Y \\
Y \\
Y\end{array}$ & $\begin{array}{r}0 \\
2 \\
6 \\
10 \\
\end{array}$ & $\begin{array}{l}261 \\
263 \\
259 \\
247\end{array}$ & $\begin{array}{l}0.045 \\
0.022 \\
0.024 \\
0.025 \\
\end{array}$ & $\begin{array}{l}0.0371 \\
0.0313 \\
0.0311 \\
0.0353\end{array}$ & $\begin{array}{l}0.148 \\
0.132 \\
0.116 \\
0.130 \\
\end{array}$ & $\begin{array}{l}-0.006 \\
-0.011 \\
-0.011 \\
-0.015 \\
\end{array}$ & $\begin{array}{l}0.042 \\
0.004 \\
0.007 \\
0.004 \\
\end{array}$ \\
\hline $\begin{array}{l}\theta-\theta_{0} \\
\theta-\theta_{0} \\
\theta-\theta_{0} \\
\theta-\theta_{0} \\
\end{array}$ & $\begin{array}{l}126 \\
126 \\
126 \\
126 \\
\end{array}$ & $\begin{array}{l}X \\
Y \\
Y \\
Y\end{array}$ & $\begin{array}{r}0 \\
2 \\
6 \\
6 \\
10 \\
\end{array}$ & $\begin{array}{l}260 \\
262 \\
261 \\
241 \\
\end{array}$ & $\begin{array}{l}0.037 \\
0.024 \\
0.022 \\
0.024 \\
\end{array}$ & $\begin{array}{l}0.0379 \\
0.0329 \\
0.0299 \\
0.0331\end{array}$ & $\begin{array}{l}0.157 \\
0.128 \\
0.114 \\
0.113\end{array}$ & $\begin{array}{r}-0.009 \\
-0.013 \\
-0.007 \\
-0.021\end{array}$ & $\begin{array}{l}0.022 \\
0.010 \\
0.009 \\
0.008 \\
\end{array}$ \\
\hline $\begin{array}{l}\theta-\theta_{0} \\
\theta-\theta_{0} \\
\theta-\theta_{0} \\
\theta-\theta_{0}\end{array}$ & $\begin{array}{l}525 \\
525 \\
525 \\
525\end{array}$ & $\begin{array}{l}X \\
Y \\
Y \\
Y\end{array}$ & $\begin{array}{r}0 \\
2 \\
6 \\
10\end{array}$ & $\begin{array}{l}254 \\
296 \\
305 \\
281\end{array}$ & $\begin{array}{l}0.025 \\
0.020 \\
0.022 \\
0.016\end{array}$ & $\begin{array}{l}0.0364 \\
0.0285 \\
0.0250 \\
0.0268\end{array}$ & $\begin{array}{l}0.131 \\
0.115 \\
0.090 \\
0.099\end{array}$ & $\begin{array}{r}-0.030 \\
-0.012 \\
-0.009 \\
-0.026\end{array}$ & $\begin{array}{l}0.009 \\
0.007 \\
0.012 \\
0.004\end{array}$ \\
\hline
\end{tabular}

$\theta$ is water content $\left(\mathrm{cm}^{3} / \mathrm{cm}^{3}\right)$

$\theta-\theta_{0}$ is water content difference from day $0\left(\mathrm{~cm}^{3} / \mathrm{cm}^{3}\right)$ 


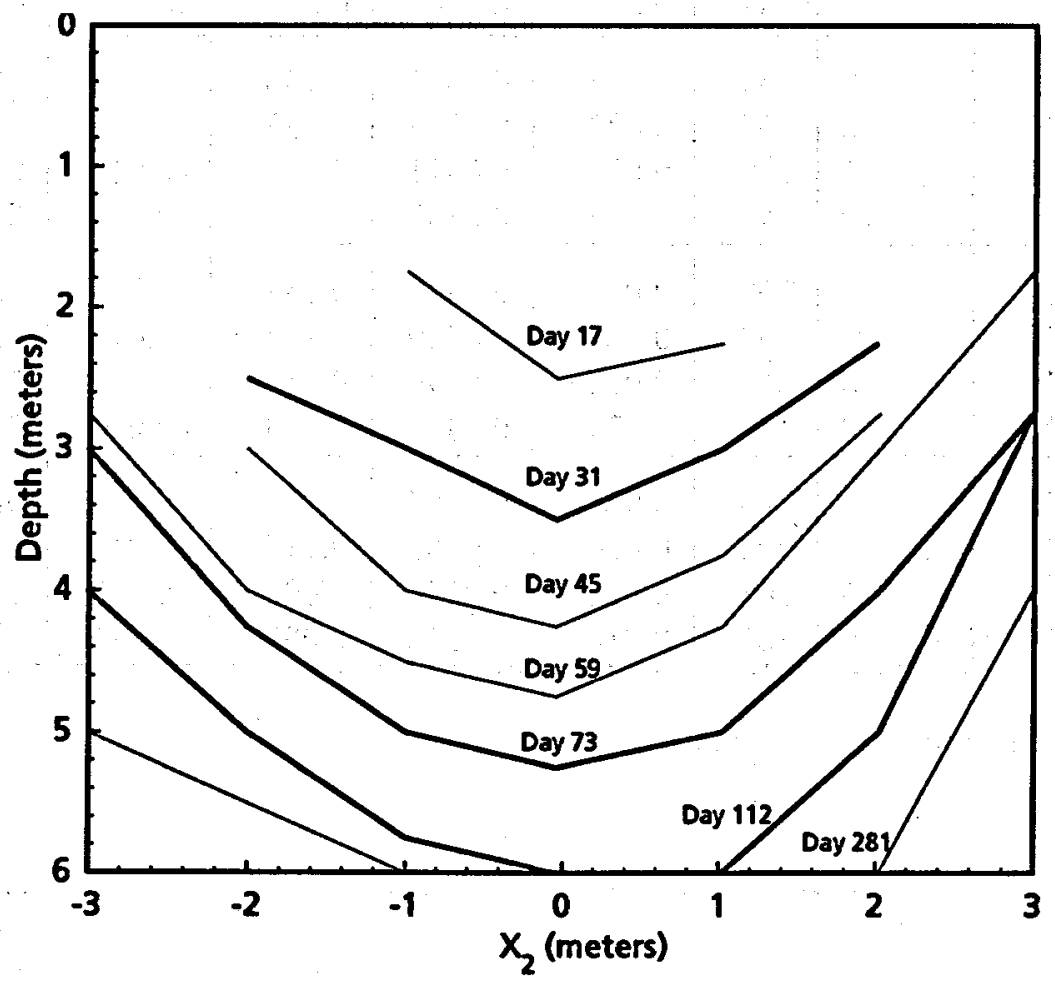

Figure 34. Experiment IIb neutron probe wetting front in a plane through $Y_{2}=2 \mathrm{~m}$ parallel to the trench face. 


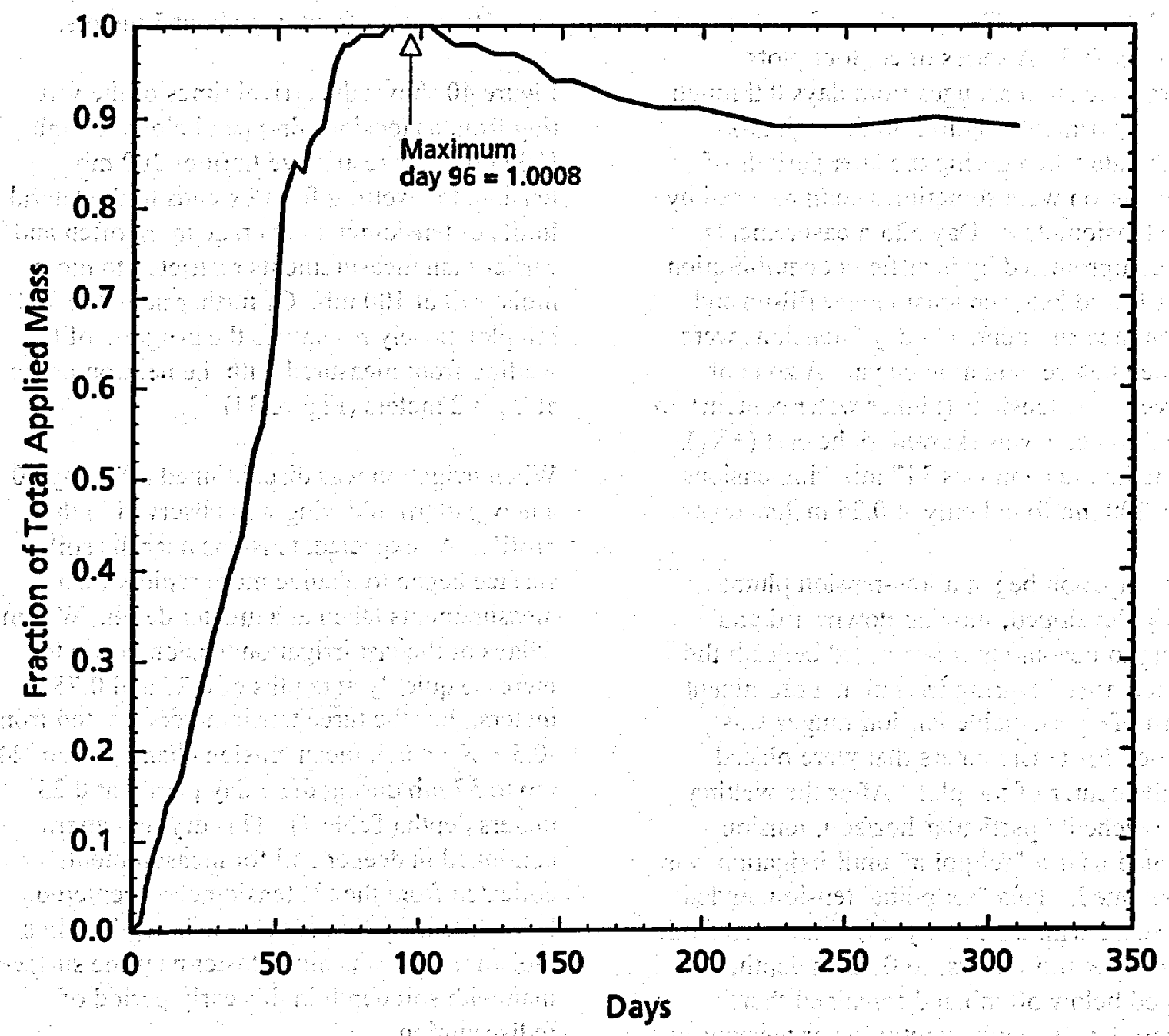

Figure 35 Experiment IIb neutron probe mass balance over time. 
1.0008 at day 96 . From day 86 through 103 the water balance was $\geq 0.99$ of applied. As water began to leave the profile, as measured to $6 \mathrm{~m}$ depth, the ratio began to decline.

\subsubsection{Soil moisture-tensions}

The wetting (and drying) changes in the profile were also monitored at the trench face, $Y_{2}=0.5$ meters, with tensiometers located from -2.11 s $\mathrm{X}_{2} \leq 2.25$ meters. These data are found in Appendix A-3. A series of contour plots illustrates tension changes from days 0 through of the experiment (Figures 36 through 38), though data taken during the later periods of redistribution were sometimes compromised by faulty tensiometers. Day 525 measurements were compromised by insufficient equilibration time allowed between tensiometer fill-up and tension measurement. On day 0 tensions were recorded before irrigation began. A zone of relatively low tensions (higher water content), to about $3 \mathrm{~m}$ deep, was skewed to the east $\left(+X_{2}\right)$. The median tension was $347 \mathrm{mb}$ with tensions below $200 \mathrm{mb}$ found only at 0.25 meters depth.

After irrigation began a low-tension plume quickly developed, moving downward and shifting to become more centered beneath the irrigated area. During irrigation a prominent pattern of quasi-stable tension ranges was observed for tensiometers that were placed near the center of the plot. After the wetting front reached a particular horizon, tension remained near a "set point" until irrigation was discontinued. This "set point" tension tended to increase with depth. By day 7 tensions from $-0.5 \leq X_{2} \leq 0.5$ meters, to $0.75 \mathrm{~m}$ depth, dropped below $50 \mathrm{mb}$ and remained there through day 70 , with slightly lower tensions at 0.25 meters. Below 0.75 meters tension never dropped below $50 \mathrm{mb}$. Tensions below $100 \mathrm{mb}$ were found as deep as 2 meters by day 14, and to 3 meters by day 33, though tensions below 4.5 meters never dipped below $100 \mathrm{mb}$.
The progress of the wetting front is summarized by tension isobars in Figure 39 and Figure 40. The first figure shows the arrival time of the wetting front measured as tension $\leq 100 \mathrm{mb}$ (Wierenga et al, 1991). A regular pattern of wetting is apparent near the center of the plot down to 4 meters depth. Laterally, this wetting front reaches the east limit of measurement only from 2 to 3 meters depth and on the west side, only at a single point, 2 meters deep. Below 4 meters this pattern of wetting is disrupted. Recall that the final penetration of the experiment IIa wetting front was about 3 meters.

Figure 40 shows the arrival times of the wetting front as tensions dropped below $300 \mathrm{mb}$. Using the less restrictive limit of $300 \mathrm{mb}$ tension, the wetting front extends to the lateral limits of tensiometer coverage more often and earlier than measurements restricted to more moist soil at $100 \mathrm{mb}$. Of further note, the 300 $\mathrm{mb}$ plot closely resembles the progress of the wetting front measured with the neutron probe at $Y_{2}=2$ meters (Figure 34).

When irrigation was discontinued after day 70 a new pattern of drying was observed in the profile. As expected, tensions near the soil surface began to change more rapidly than measurements taken at a greater depth. Within 2 days of the last irrigation tension began to increase quickly at depths of 0.25 and 0.75 .. meters. For the three tensiometers located from $-0.5 \leq \mathrm{X}_{2} \leq 0.5$, mean tension changed from 24 $\mathrm{mb}$ to $57 \mathrm{mb}$ during the 2 day period at 0.25 meters depth (Table 7). This drying pattern continued in deeper soil for measurements collected from the (3) tensiometers centered below the irrigation area at each depth. Thus moisture flow was much faster near the surface than with soil depth in this early period of redistribution.

By day 112 most of the tensions at the "previously wetted" tensiometers (tensions $\leq$ $300 \mathrm{mb}$ ) throughout the profile had dried to a dryer, but more narrow range of variability. 
Table 7. Mean tensions on days 70 and 72 for the three center tensiometer locations at each of six depths.

\begin{tabular}{||l|c|c|c|}
\hline \hline & \multicolumn{3}{|c|}{ y (mb) } \\
\hline Depth (m) & day 70 & day 72 & $\Delta(\%)$ \\
\hline 0.5 & 24 & 57 & 137 \\
0.75 & 45 & 70 & 56 \\
2 & 56 & 61 & 9 \\
3 & 56 & 62 & 10 \\
4 & 76 & 81 & 7 \\
5 & 122 & 121 & -1 \\
\hline
\end{tabular}

The median tension for all tension measurements at all depths changed from a median of $85 \mathrm{mb}$ on day $70(\mathrm{n}=96)$ to $217 \mathrm{mb}$ on day $112(n=100)$. During those 42 days of redistribution, tensiometers "wetted" by day 70 showed a decrease in variability from $\mathrm{CV}=$ $38 \%$ to $\mathrm{CV}=12 \%$. Redistribution of water caused tensions within the "wetted" portion of the profile to exhibit greater uniformity.

\subsubsection{Tritium}

Tritium was applied in two separate pulses to plot 2. In experiment IIa tritium input averaged 27,574 counts per minute and was applied during the first 11.5 days of 75.5 total days of irrigation. For experiment IIb applied tritium concentration was about 0.622 of that first pulse, at 17,142 counts per minute during days $29-44$ of 70 days of irrigation. For this discussion tritium is normalized for input of the second pulse, so $\mathrm{C} / \mathrm{C}_{\mathrm{o}}=1$ when observed counts $=17,142$. Note, however, that the experiment IIb tritium data is presented in the appendix as values normalized for the experiment IIa pulse (Appendix A-4).

Solutions were collected from solution samplers on day $\mathbf{0}$, just before initiating irrigation. These first samples were drawn in order to quantify residual plume tritium of experiment IIa. In the most shallow samplers ( 0.5 meters depth) concentrations as high as 5 percent of IIa input were found. The extent of this plume is poorly defined; because of the relatively dry soil only a limited number of solution samples could be drawn from the profile, so the contour plot (Figure 41) is represented by few observations. Within this plume the highest concentration ( 0.203 of IIa input) was found at 1 meter depth. The center of the plume was located about $\mathbf{0 . 7 5}$ meters to the east of the center of the plot and about 1 meter deep, similar to the location of the day 0 plume of low-tension values (Figure 36).

By day 33 the new experiment IIb pulse of tritium was 4 days in progress and not yet detected at the $Z_{2}=0.5$ meter row of solution samplers. The center of the older, experiment Ila plume, had moved to about 2 meters depth, though the neutron probe wetting front at $Y_{2}=2$ meters (Figure 34) and the $300 \mathrm{mb}$ tensiometer tension front (Figure 40) had moved below 3 meters. The older tritium plume shifted medially similar to the zone of lower tensions.

The bottom of the new tritium plume was well developed by day 47,3 days after the last application of new tritium. The leading edge of 


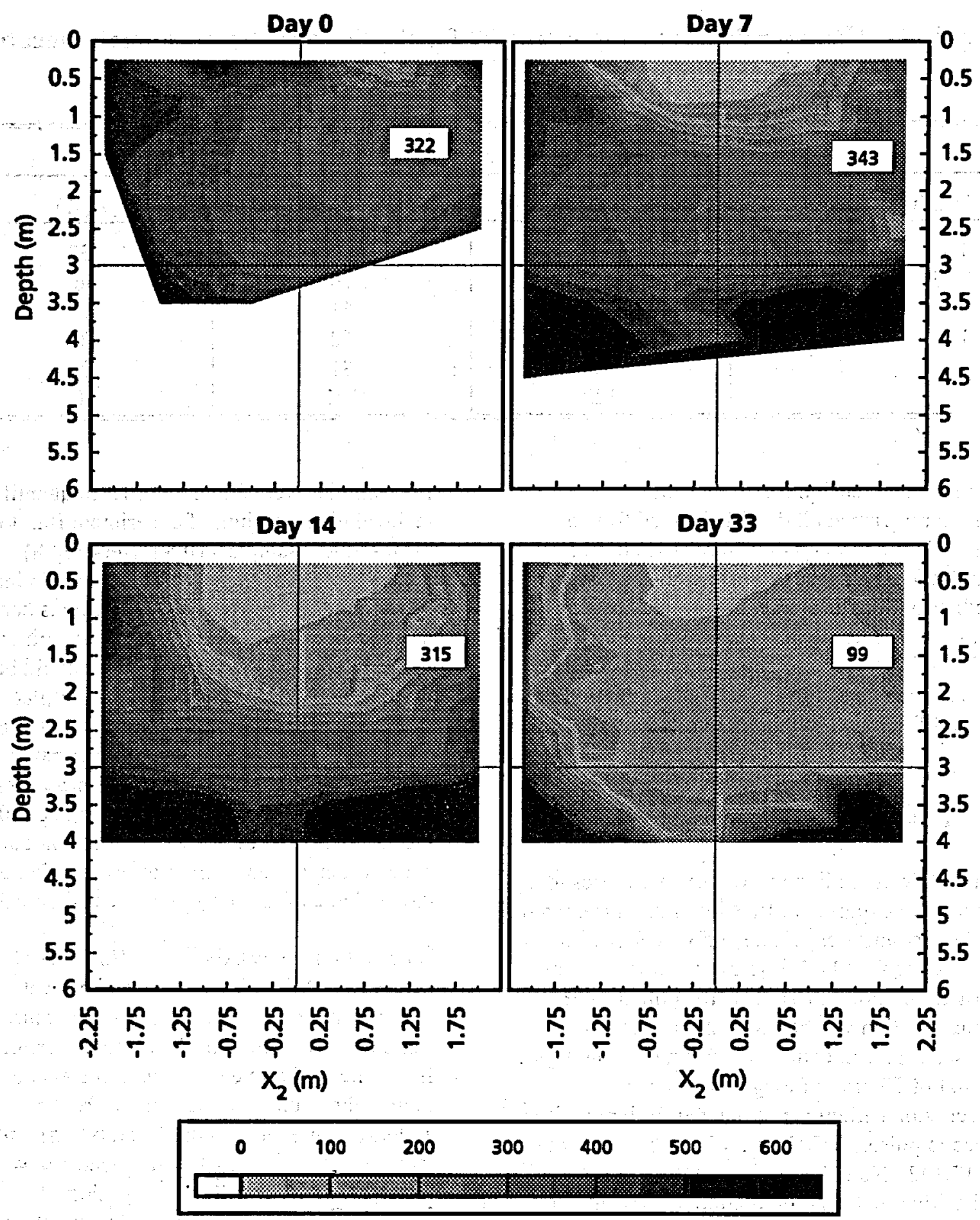

Figure 36. Experiment IIb soil tension contours at $Y_{2}=0.5 \mathrm{~m}$ from trench face for days $0,7,14$, and 33 of the experiment. Median tension values are shown within individual plots. 

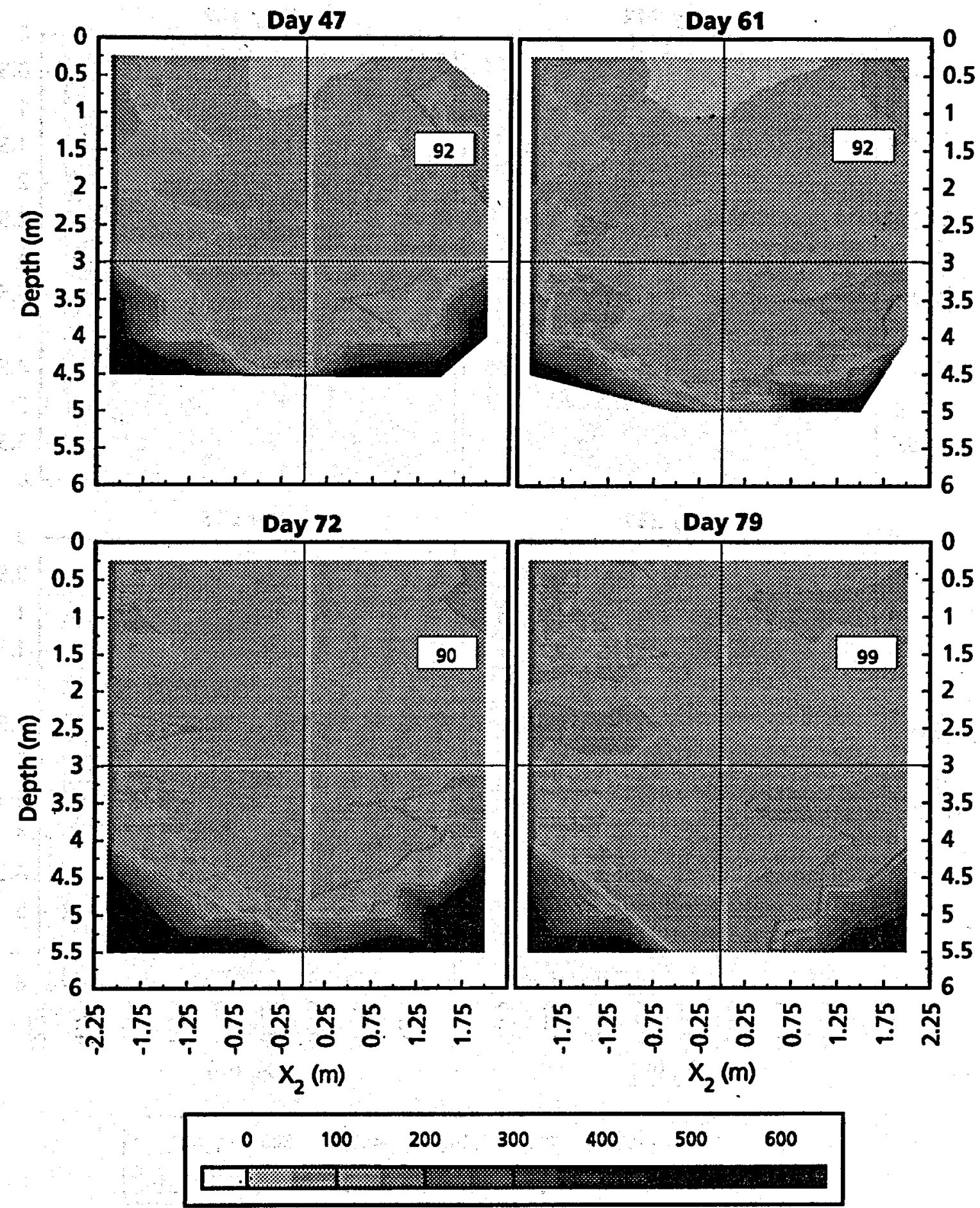

Figure 37. Experiment IIb soil tension contours at $Y_{2}=0.5 \mathrm{~m}$ from trench face for days $47,61,72$, and 79 of the experiment. Median tension values are shown within individual plots. 

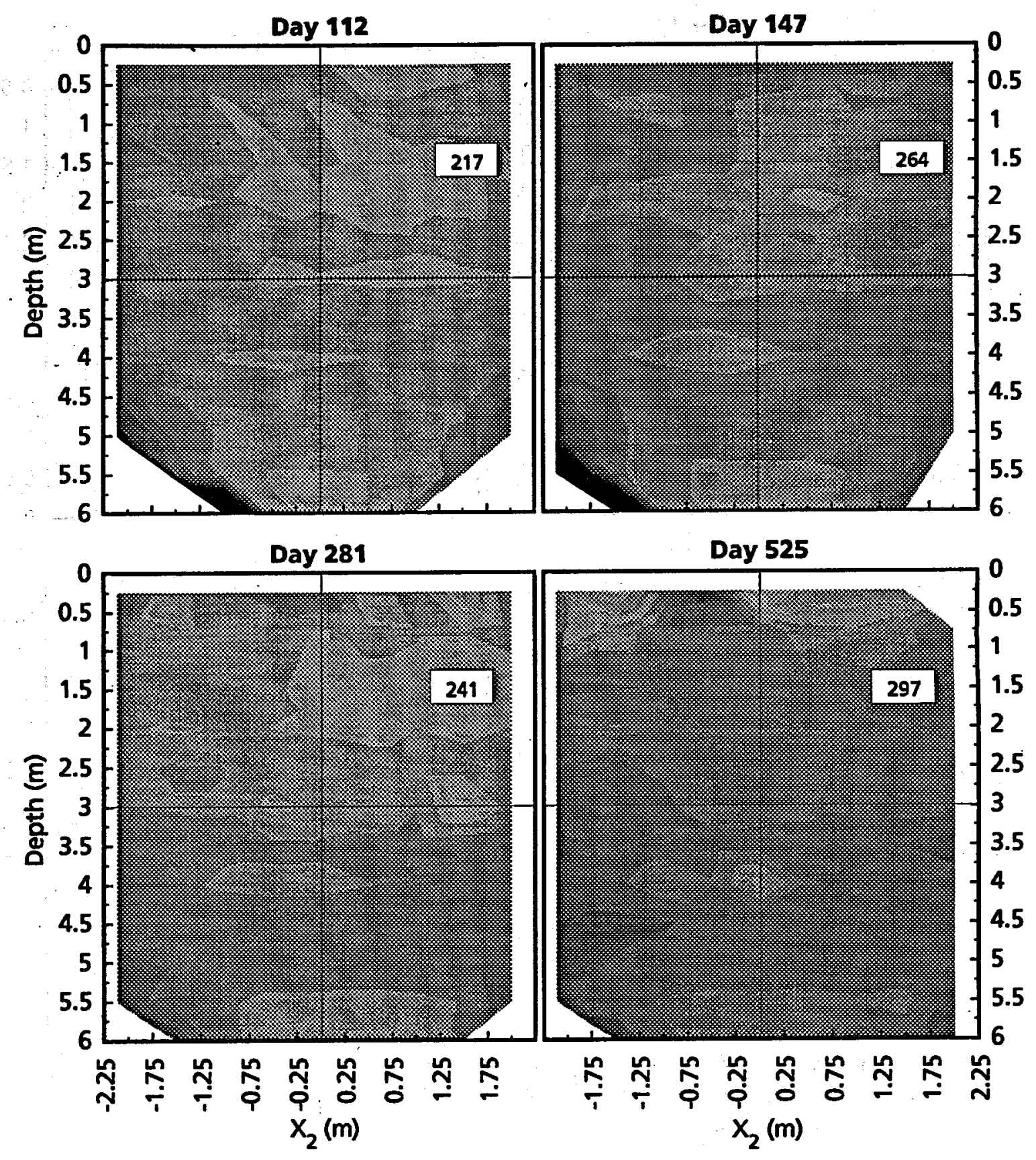

\begin{tabular}{|ccccccc|}
\hline 0 & 100 & 200 & 300 & 400 & 500 & 600 \\
\hline & & & & & \\
\hline
\end{tabular}

Figure 38. Experiment IIb soil tension contours at $\mathrm{Y}_{2}=0.5 \mathrm{~m}$ from trench face for days $112,147,281$, and 525 of the experiment. Median tension values are shown within individual plots. 


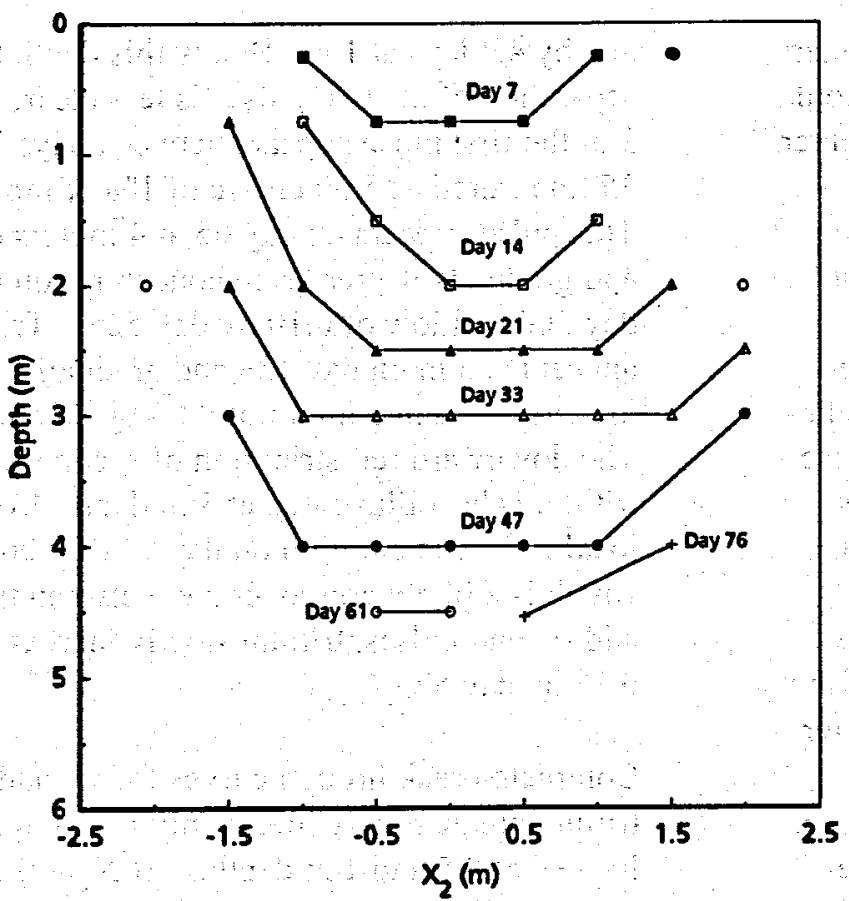

Figure 39. Experiment IIb soil tension front at $Y_{2}=0.5 \mathrm{~m}$ from trench face. Front delimited by tensions less than or equal to $100 \mathrm{mb}$.

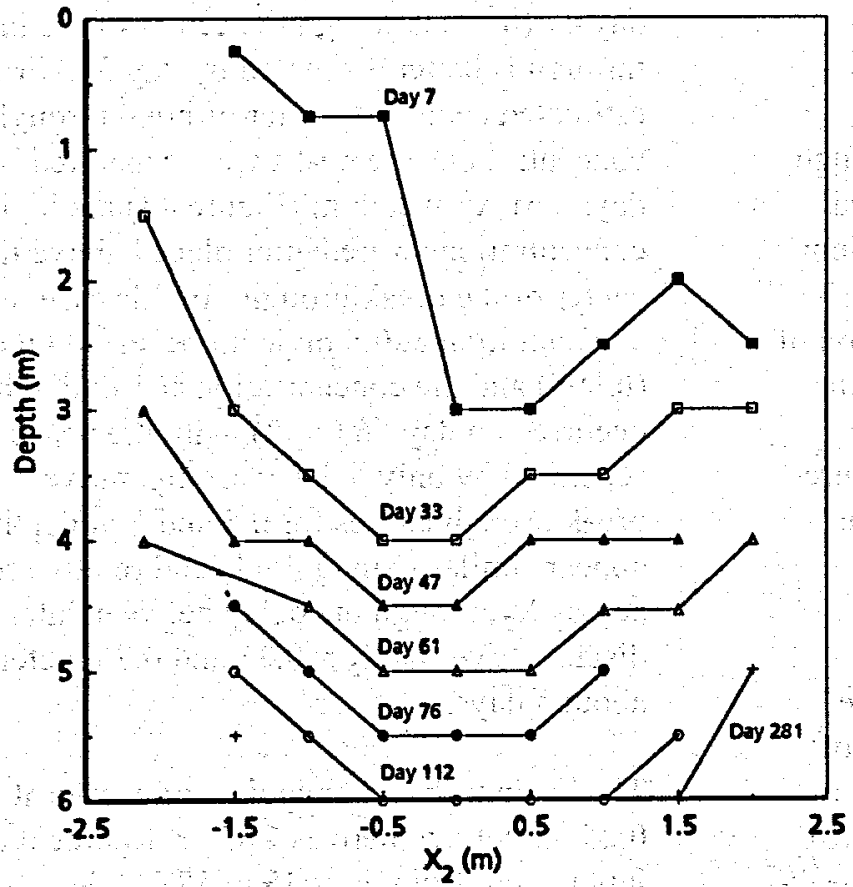

Figure 40. Experiment IIb soil tension front at $Y_{2}=0.5 \mathrm{~m}$ from trench face.

Front delimited by tensions less than or equal to $300 \mathrm{mb}$. 
the new pulse was found at samplers 1.5 meters deep. For the portion of the plume which could be observed at and below 0.5 meters, the center of mass was located east of $X_{2}=0 \mathrm{~m}$. The center of mass appeared to remain east of the center of the plot throughout the experiment. By day 70 , the new plume was readily distinguished from the old. The new plume appeared to have divided into two main bodies separated across the $X_{2}=0$ meter axis. These bodies can be distinguished in contour plots throughout the remainder of the experiment.

During redistribution the new tritium plume moved very slowly (Figure 42). The top of the plume appeared to remain fixed about 1 meter deep with some lateral migration, while the bottom of the plume moved slowly downward. Generally, lateral movement appeared more pronounced towards the top of the plume. From days 79 to 525 the leading edge of the new plume moved from about $2.5 \mathrm{~m}$ depth to almost $4 \mathrm{~m}$ while the center of the plume (mass) moved from about $2 \mathrm{~m}$ to about $2.5 \mathrm{~m}$. These observations are generally supported by breakthrough curves for individual solution samplers.

A series of plots illustrate tritium breakthrough curves across a range of soil depths for fixed locations in $X_{2}$ (Figures 43-45). Again tritium data for these plots are normalized for experiment IIb input. In general, close examination of these data shows that the first pulse of tritium (experiment IIa) is distinguishable from the second pulse tritium and is the dominant source of observed tritium in deeper portions of the profile.

Figure 43 shows breakthrough curves for solution samplers at $X_{2}=.0 .25$ meters, those nearest to the center of the plot. Remnants of the experiment IIa pulse are evident in all the curves. Only at 0.5 and $1 \mathrm{~m}$ depths are the two tritium pulses fully separated. Comparing peakto-peak concentrations during irrigation, the pulses are separated by 37 days at $0.5 \mathrm{~m}$ depth and by 42 days at $1 \mathrm{~m}$. Below this depth the separation of the two pulses is less clear. At $3 \mathrm{~m}$ the first pulse appears between days 33 and 35 (measured as an increase of $1 \%$ of input). This pulse appears on day 65 at 4 meters depth, and gradually decreases in concentration after day 200 to a low of 0.10 by day 525 . Tritium appears at $5 \mathrm{~m}$ on day 126 and gradually increases to a maximum of 0.06 by day 525 . The downward redistribution of second-pulse tritium is best illustrated at $3 \mathrm{~m}$ depth; from a local low concentration on day 93 of 0.06 , which is a likely crossover day between the first and second pulses, tritium slowly increases to 0.35 by day 525 .

Complete breakthrough curves for second-pulse tritium are generally found only for samplers located at 0.5 and $1 \mathrm{~m}$ depth. At $\mathrm{X}_{2}=0.25 \mathrm{~m}$ (Figure 43) the highest concentrations are not centered above the tritium mass. At $0.5 \mathrm{~m}$ depth the highest concentration occurs on day 42 ( 0.963 of input), though the center of the peak is better located on day $44(0.920)$. Similarly, at $1 \mathrm{~m}$ depth, the highest concentration occurs on day $47(0.841)$, though the center of the breakthrough is better estimated by day 51 . From the estimated center-to-center of breakthrough concentrations the peaks were separated by 6 days. At $X_{2}=0.75 \mathrm{~m}$ (Figure 44) the highest concentrations were better placed above the center of the breakthrough. In this case, peak concentration at $0.5 \mathrm{~m}$ depth occurred on day 51 $(0.895)$ and the concentration at $1 \mathrm{~m}$ depth occurred on day $56(.952)$, with the peaks separated by only 5 days. Comparative breakthrough curves for 0.5 and $1 \mathrm{~m}$ depths appear similar in magnitude and relative timing across $X_{2}$, though the delay between laterally distant curves at $X_{2}=0.25$ and 0.75 meters is about 5 days.

The beginning and endpoints of curves at $\mathrm{X}_{2}=$ $0.25 \mathrm{~m}$, defined here as $\mathrm{C} / \mathrm{C}_{\mathrm{o}} \leq 1$ percent of input, were from days 35 to 58 ( 23 days wide) for the $0.5 \mathrm{~m}$ depth curve and from days 40 to 70 (30 days wide) for the $1 \mathrm{~m}$ depth curve. 


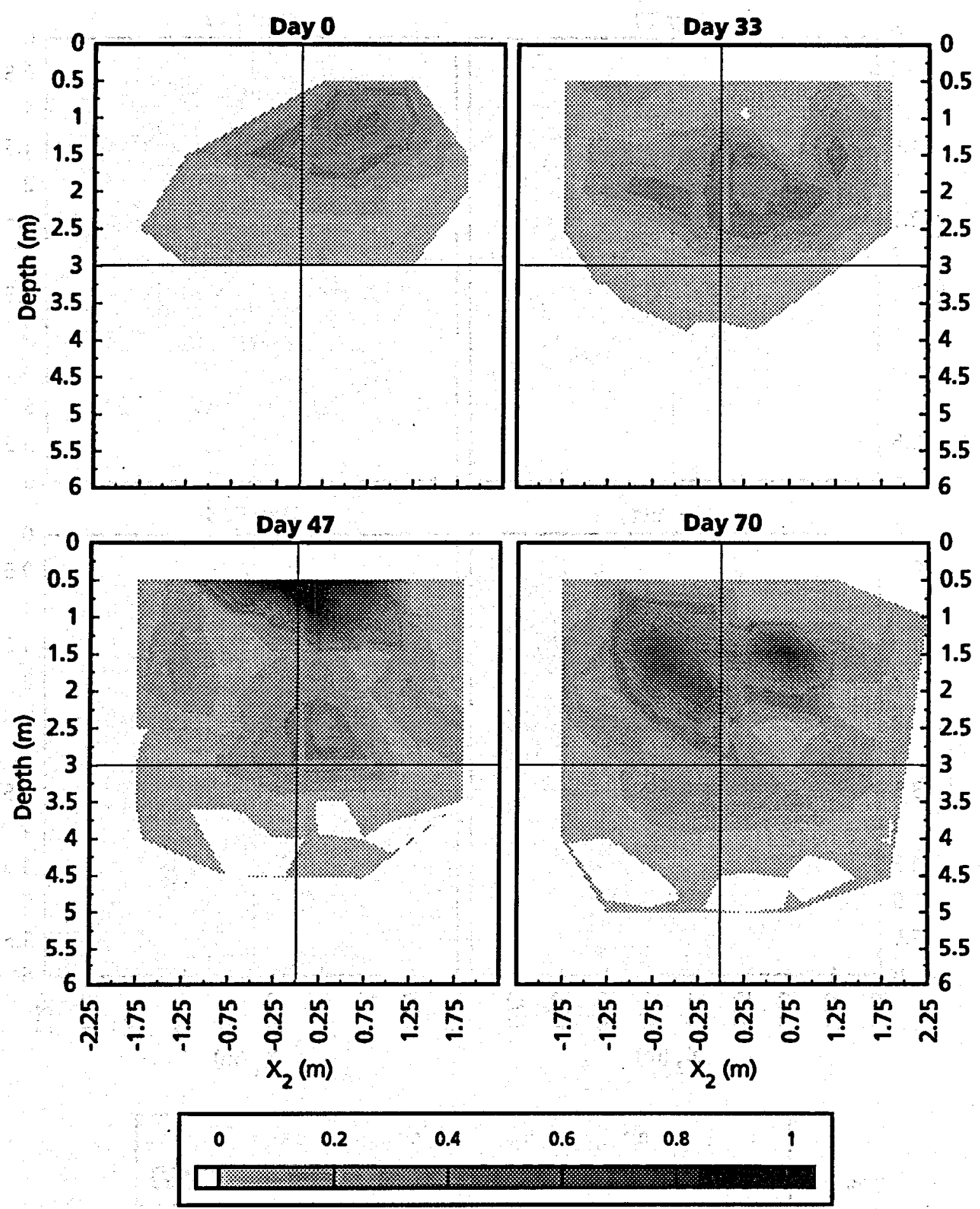

Figure 41. Experiment IIb tritium contours at $\mathrm{Y}_{2}=0.5 \mathrm{~m}$ from trench face for days $0,33,47$, and 70 of the experiment. Concentrations as $\mathrm{C} / \mathrm{C}_{0}$ normalized for experiment IIb input. 


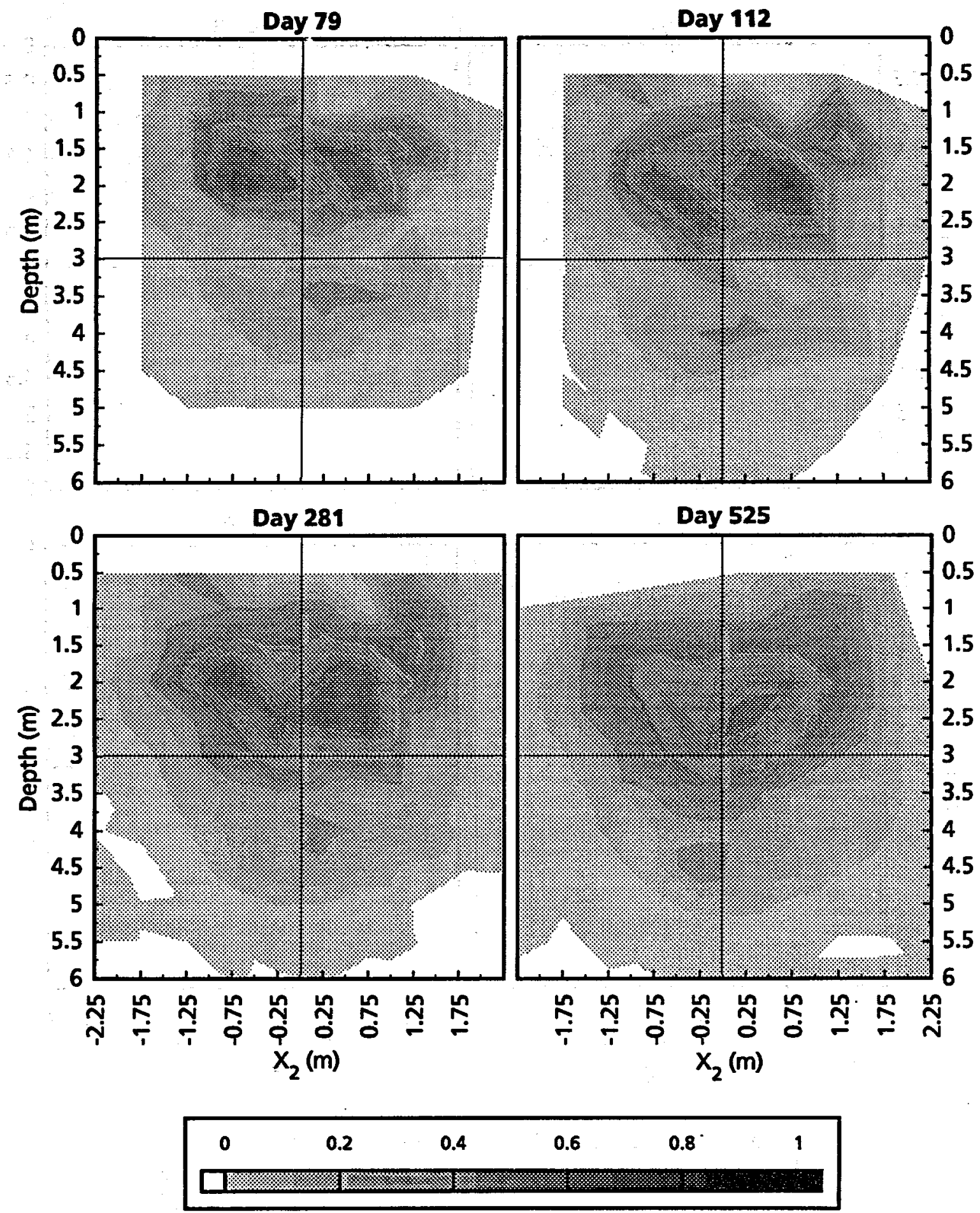

Figure 42. Experiment IIb tritium contours at $Y_{2}=0.5 \mathrm{~m}$ from trench face for days 79, 112, 281, and 525 of the experiment. Concentrations as $\mathrm{C} / \mathrm{C}_{\mathrm{o}}$ normalized for experiment IIb input. 
Respective base widths for those curves at $\mathrm{X}_{2}$ $=0.75 \mathrm{~m}$ were 28 and 38 days wide. This indicates greater plume dispersion at positions where $X_{2}=0.75 \mathrm{~m}$ than counterpart curves at $X_{2}=0.25 \mathrm{~m}$.

At $X_{2}=1.25$ meters (Figure 45) the secondpulse breakthroughs occurred after the end of irrigation. The timing of tritium arrival and the magnitude of concentration increases, is reversed of what might be expected for depths from 0.5 to 1.5 meters. Second-pulse tritium arrived about day 79 at $0.5 \mathrm{~m}$ depth, about day 63 at $1 \mathrm{~m}$ and about day 65 at $1.5 \mathrm{~m}$ depth.

These breakthrough curves indicate that there was some overlap between the first and second pulses of applied tritium, especially at $\mathrm{X}_{2}=\mathbf{0 . 2 5}$ $\mathrm{m}$ locations. Concentrations of first pulse tritium at 1:5, 2 and $3 \mathrm{~m}$ depths do not reach $\mathrm{C}_{\mathrm{o}}=0$ before beginning to increase with the appearance of the second pulse. However, this pulse overlap is not as apparent at more lateral samplers at $\mathrm{X}_{2}=0.75$ and $1.25 \mathrm{~m}$. Perhaps this effect is the result of time lags created in the vertical displacement of tritium by horizontal shifting of portions of the plume to and from the area of soil directly below irrigation drippers.

\subsubsection{Chromium, boron and PFBA}

Chromium, boron and PFBA solutes, all anions, were applied together in the first solute pulse, from days 0 to 15 . Inter-ion comparisons are difficult because the quality and quantity of data sets are quite variable. Of the three, chromium is the most complete data set (1313 observations) and is of good quality. Although boron was analyzed in the same ICP run with chromium, the boron results are unsatisfactory. Boron analysis was troubled by a drifting baseline and probable detection of interfering solutes. The causes of this poor ICP performance for this species are uncertain. During the initial ICP set-up, several different monochromat or wavelengths were used in attempts to correct these problems, but with little effect. Boron data was unacceptable and is not presented. The PFBA data set is limited by quantity, comprised of 178 total observations from solution samples collected on 5 different days. Thus, PFBA contour plots are based on relatively few observations.

The chromium data is presented in Appendix A-5. Chromium, applied as chromate ion during the first 15 days of irrigation, was first observed $\left(C / C_{0} \geq 1 \%\right)$ at $0.25 \mathrm{~m}$ depth on day 7. By day 30 the leading edge of the chromate plume was down to 1.5 meters with the highest observed concentration ( 0.55 of input) at $X_{2}=0.75$ meters (Figure 46 ). The largest portion of the plume was skewed to the east side of the plot, though this observation is based upon contour plots drawn with few horizontal data points per measurement depth.

At the end of irrigation, day 70 , the leading edge and the highest concentration of the plume coincided at approximately $4 \mathrm{~m}$ depth. By day 525 , chromate was observed as deep as 5 meters with a peak concentration at $4.5 \mathrm{~m}$. Note that chromate contour plots are based upon relatively few data points per plot and with many data values missing from the grid of solution samplers.

Chromate breakthrough curves are more instructive. Individual samplers tended to yield enough solution to complete several solute analyses on any given day of sampling. This resulted in more complete day- to-day data sets for selected samplers. Data from the more productive samplers are presented for various depths at $X_{2}=0.25,0.75$ and 1.25 meters (Figures 47, 48, and 49). Note that different time scales are used for top and bottom plots on a page. The areas beneath the chromate curves above 1.5 meters depth do not account for the total mass of chromium applied. Peak concentrations never reach 80 percent of input. This loss is probably the result of reduction of chromium (VI) to insoluble chromium (III) in 

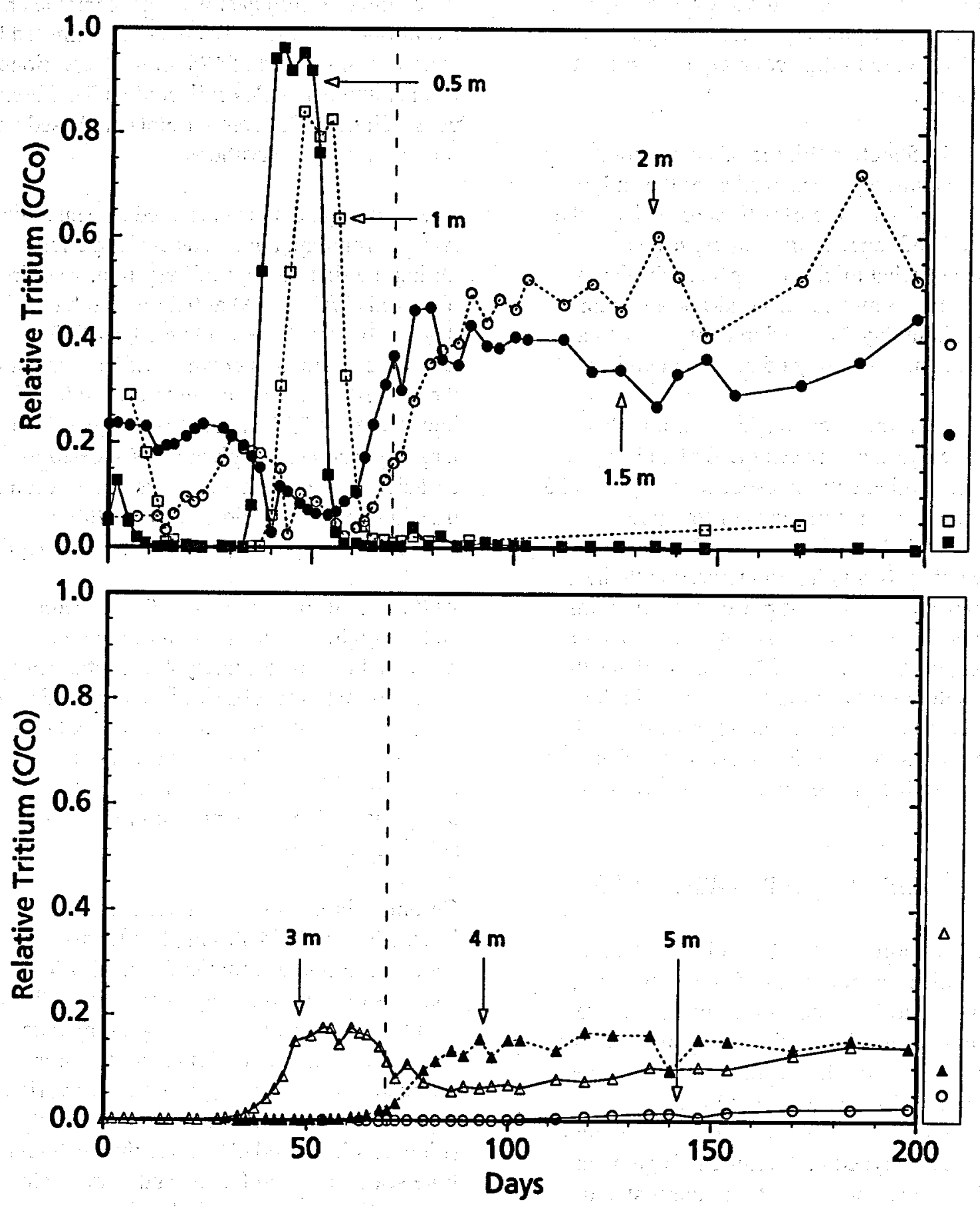

Figure 43. Experiment Ilb tritium breakthrough curves at $\mathrm{X}_{2}=0.25 \mathrm{~m}$ and samplers at various depths. Concentrations as $\mathrm{C} / \mathrm{C}_{\mathrm{o}}$ normalized for experiment IIb input. Isolated symbols boxed to the right of the plots indicate day 525 concentrations. Vertical dashed lines indicate the end of plot irrigation. 

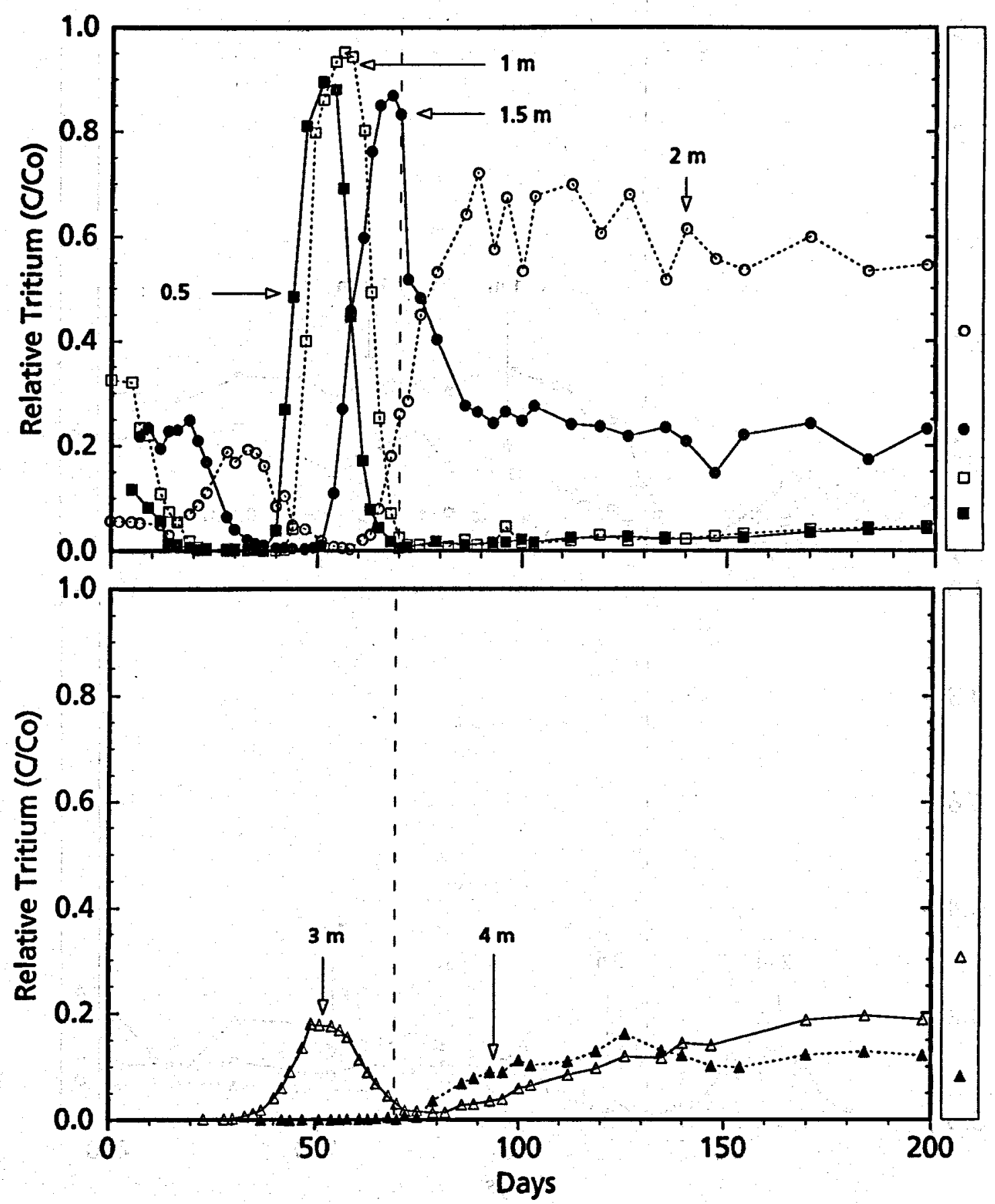

Figure 44. Experiment Ilb tritium breakthrough curves at $X_{2}=0.75 \mathrm{~m}$ and samplers at various depths. Concentrations as $\mathrm{C} / \mathrm{C}_{0}$ normalized for experiment IIb input. Isolated symbols boxed to the right of the plots indicate day 525 concentrations. Vertical dashed lines indicate the end of plot irrigation. 

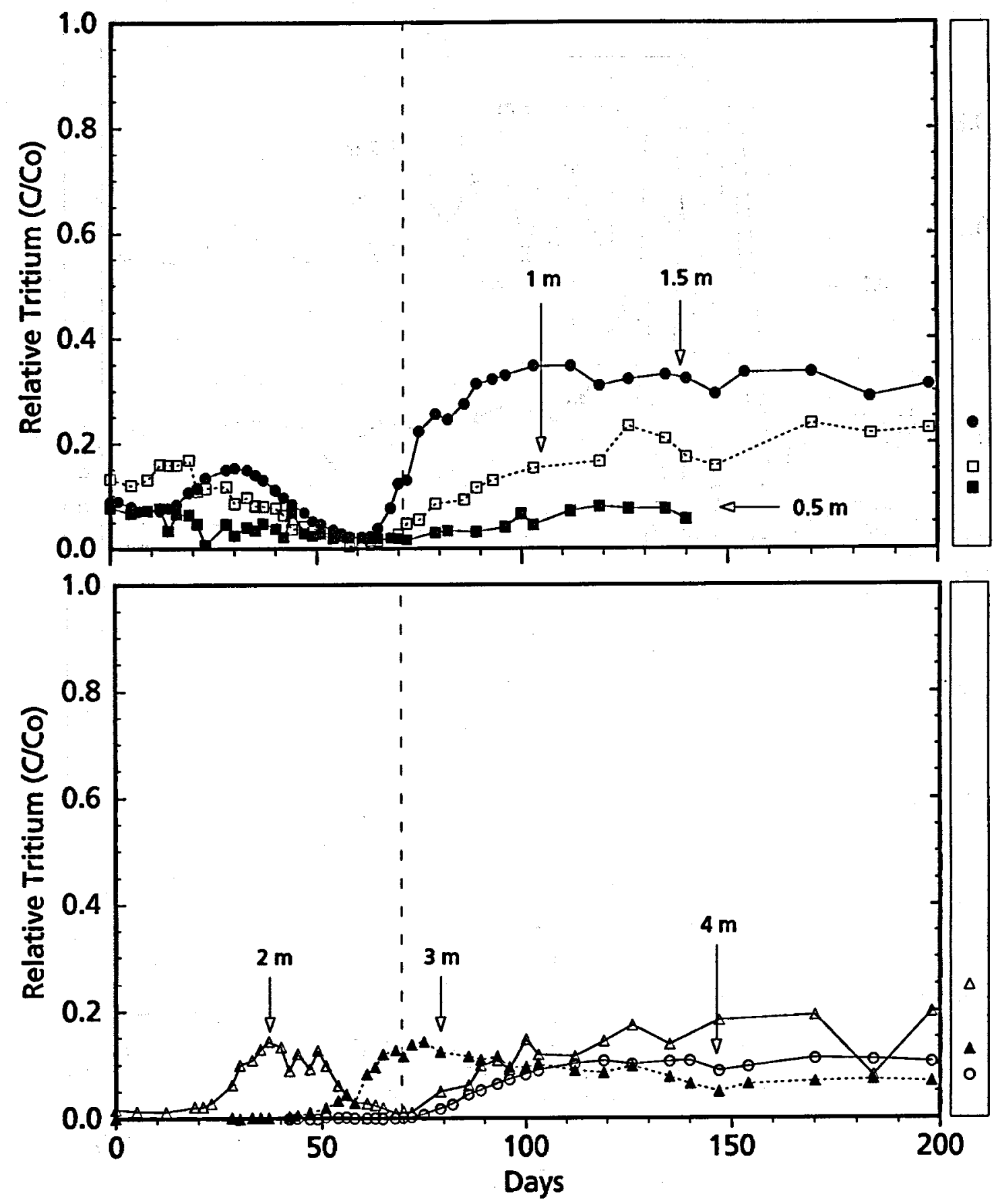

Figure 45. Experiment IIb tritium breakthrough curves at $\mathrm{X}_{2}=1.25 \mathrm{~m}$ and samplers at various depths. Concentrations as $\mathrm{C} / \mathrm{C}_{\mathrm{o}}$ normalized for experiment IIb input. Isolated symbols boxed to the right of the plots indicate day 525 concentrations. Vertical dashed lines indicate the end of plot irrigation. 
the presence of organic matter in the top $0.5 \mathrm{~m}$ of the profile.

Chromate analyses for samplers directly below the irrigation system, at $X_{2}=0.25 \mathrm{~m}$ are shown in Figure 47. By the end of irrigation breakthrough curves for samplers from 0.5 through 3 meters depth were fairly complete. By the end of the irrigation period element concentrations were increasing at $3.5 \mathrm{~m}$ depth. Breakthrough curves at $X_{2}=0.75 \mathrm{~m}$ (Figure 48) were similar in appearance, but more advanced in time than at $X_{2}=0.25 \mathrm{~m}$. For example, at those samplers located $1.5 \mathrm{~m}$ deep, chromium first appeared at $X_{2}=0.75 \mathrm{~m}$ on day 23 and at $\mathrm{X}_{2}=0.25 \mathrm{~m}$ on day 28 . Elution of chromate through samplers at $3 \mathrm{~m}$ depth was likewise more complete by the end of irrigation at $X_{2}=$ $0.75 \mathrm{~m}$ than at $0.25 \mathrm{~m}$. Interestingly, these data and supporting contour plots indicate that chromium moved more freely in areas of soil outside of the zone directly below the irrigation drippers.

At $\mathrm{X}_{2}=1.25 \mathrm{~m}$ the first appearance of chromium at $1.5 \mathrm{~m}$ depth was on day 35 , well behind samplers located more medially (Figure 49). However, at $3.5 \mathrm{~m}$ and $4 \mathrm{~m}$ depths the timing of chromium appearance and behavior are very similar to the more medial samplers. Comparisons of shallow and deep sampler data indicate that, as the chromium plume progressed deeper, lateral dispersion was significant enough to cause the chromium to migrate downward in broader plumes. This led to closer arrival times at adjacent samplers at the same depth.

During irrigation chromium appeared to progress through the profile at about the same rate as tritium, at least near the soil surface (Figure 50). The similarity of elution patterns for the two solutes is striking. At $X_{2}=0.25 \mathrm{~m}$ the chromium breakthrough at $0.5 \mathrm{~m}$ depth is centered about day 15 , estimated between measurement days 14 and 16 , rather than on day 19 where the highest concentration occurred.
For tritium this concentration peak occurred about 15 days after the first tritium was applied to the surface. For samplers located below 2 meters deep inter-solute comparisons are difficult to make since second-pulse tritium appears at those deeper solution samplers after plot irrigation ceased. At 1.5 meters depth about the same time elapsed between the first application of solute the soil surface and the arrival times $\left(C / C_{0}>0.01\right)$ of chromium and second-pulse tritium; at about 28 and 29 days respectively. Chrominum peaked on day 44 .

Second-pulse tritium concentration was yet increasing when irrigation was discontinued. Note that the time axis of Figure 50 is scaled for days after the beginning of the respective solute applications, where in fact, the solute pulse containing tritium was started 29 days after the pulse containing chromium. On this scale irrigation ended on day 70 for chromium and on day 41 for tritium.

One of the most striking features of the chromium breakthrough plots, when compared to those for tritium, is the post-irrigation elution of the chromate plume through the profile. During redistribution the chromate plume continued to elute effectively to depths of 4.5 to $5 \mathrm{~m}$ below the irrigated area (Figure 47) and at locations lateral to center (Figures 48 and 49).

By contrast, the tritium plumes developed very slowly after irrigation was discontinued (Figures 43-45). Tritium tended to remain near fixed concentrations beyond day 100 while the chromate plume continued to move. For example, at $5.0 \mathrm{~m}$ depth chromate first appeared on day 170 and then reached a high concentration of $\mathrm{C} / \mathrm{C}_{0}=0.25$ of input by day $525\left(\mathrm{X}_{2}=\right.$ $-0.25 \mathrm{~m}$ ). At the same depth tritium first appeared on day 112 and then gradually reached a high concentration of $C / C_{0}=0.06$ at the same sampler, almost certainly an observation of experiment IIa solute. It is evident that during redistribution the vertical progress of chromium was faster than for tritium. 

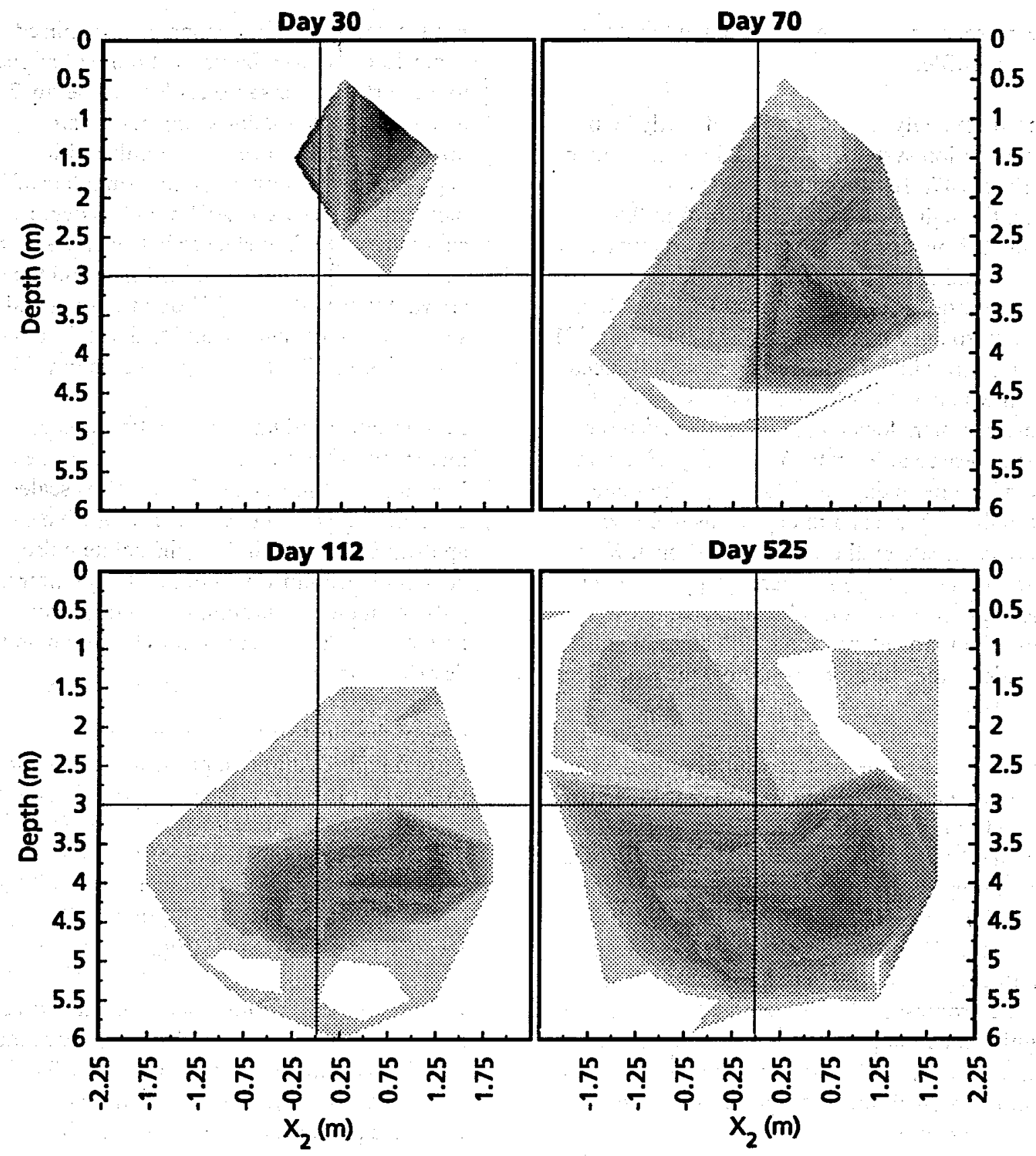

\begin{tabular}{|lllllllll|}
\hline 0 & 0.1 & 0.2 & 0.3 & 0.4 & 0.5 & 0.6 & 0.7 \\
\hline
\end{tabular}

Figure 46. Experiment IIb chromium contours at $Y_{2}=0.5 \mathrm{~m}$ from trench face for days $30,70,112$, and 525 of the experiment. Concentrations as $C / C_{0}$. 

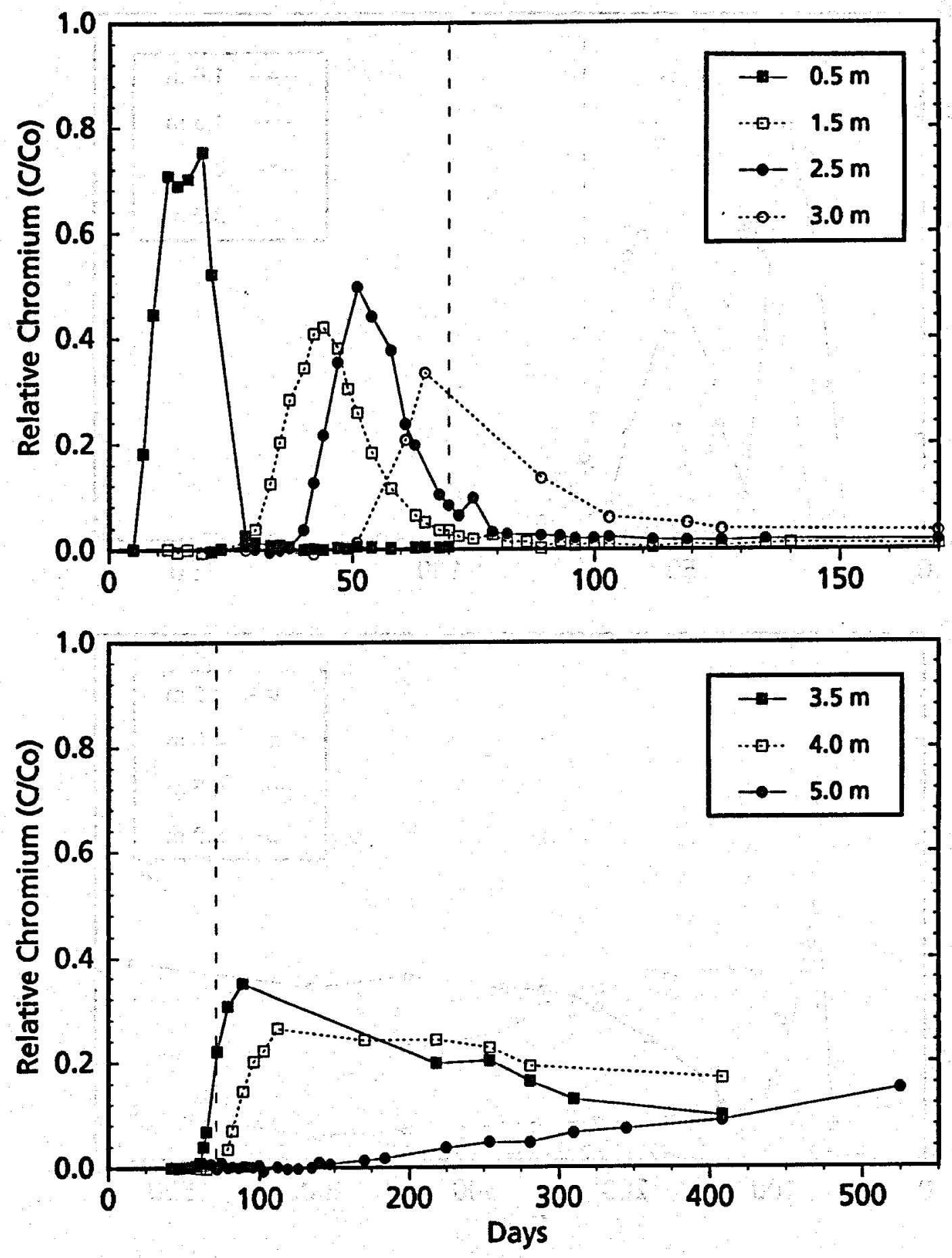

Figure 47. Experiment IIb chromium breakthrough curves at $X_{2}=0.25 \mathrm{~m}$ and samplers at various depths. Concentrations as $\mathrm{C} / \mathrm{C}_{0}$. Vertical dashed lines indicate the end of plot irrigation. 

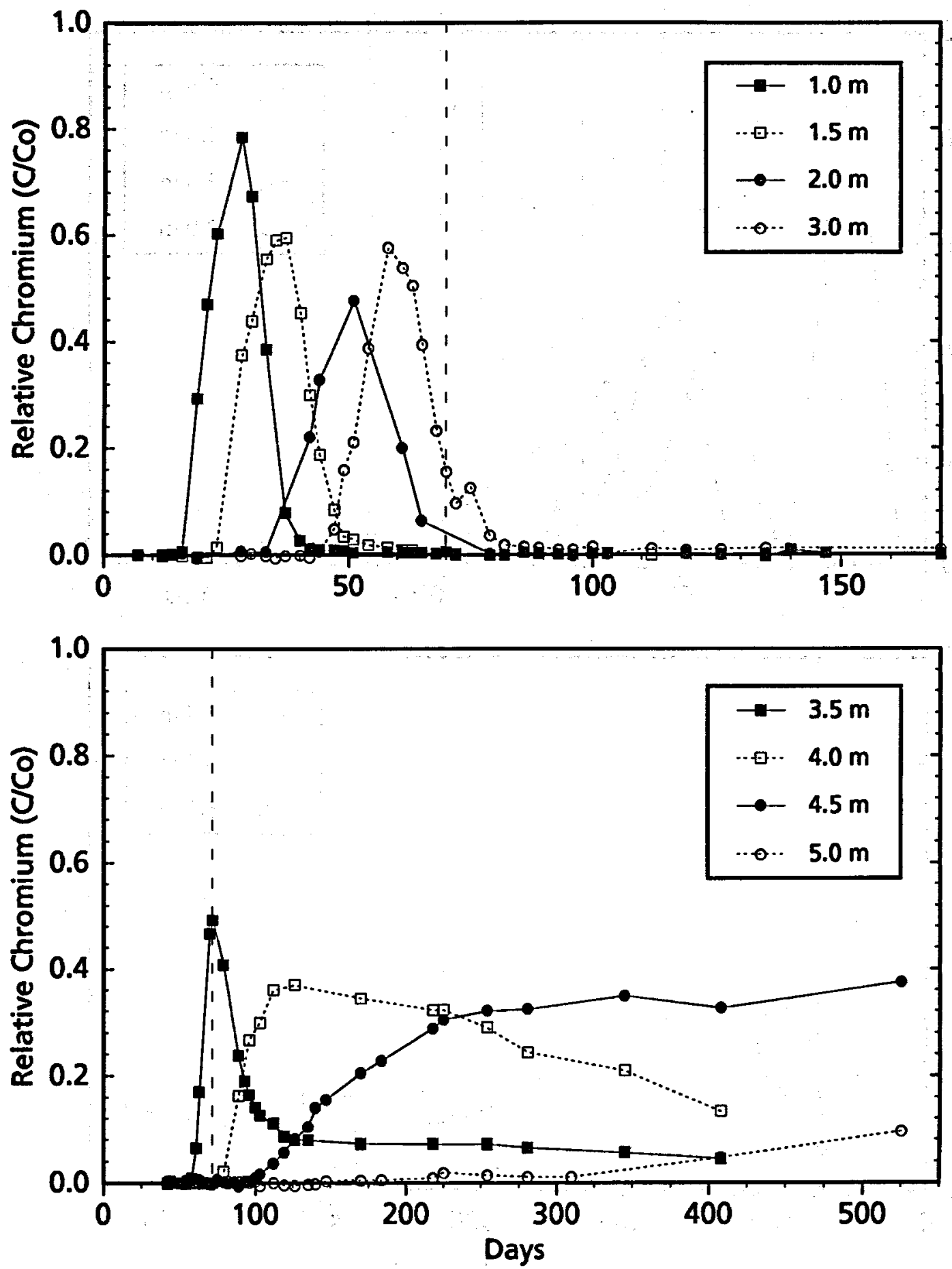

Figure 48. Experiment IIb chromium breakthrough curves at $X_{2}=0.75 \mathrm{~m}$ and samplers at various depths. Concentrations as $\mathrm{C} / \mathrm{C}_{0}$. Vertical dashed lines indicate the end of plot irrigation. 

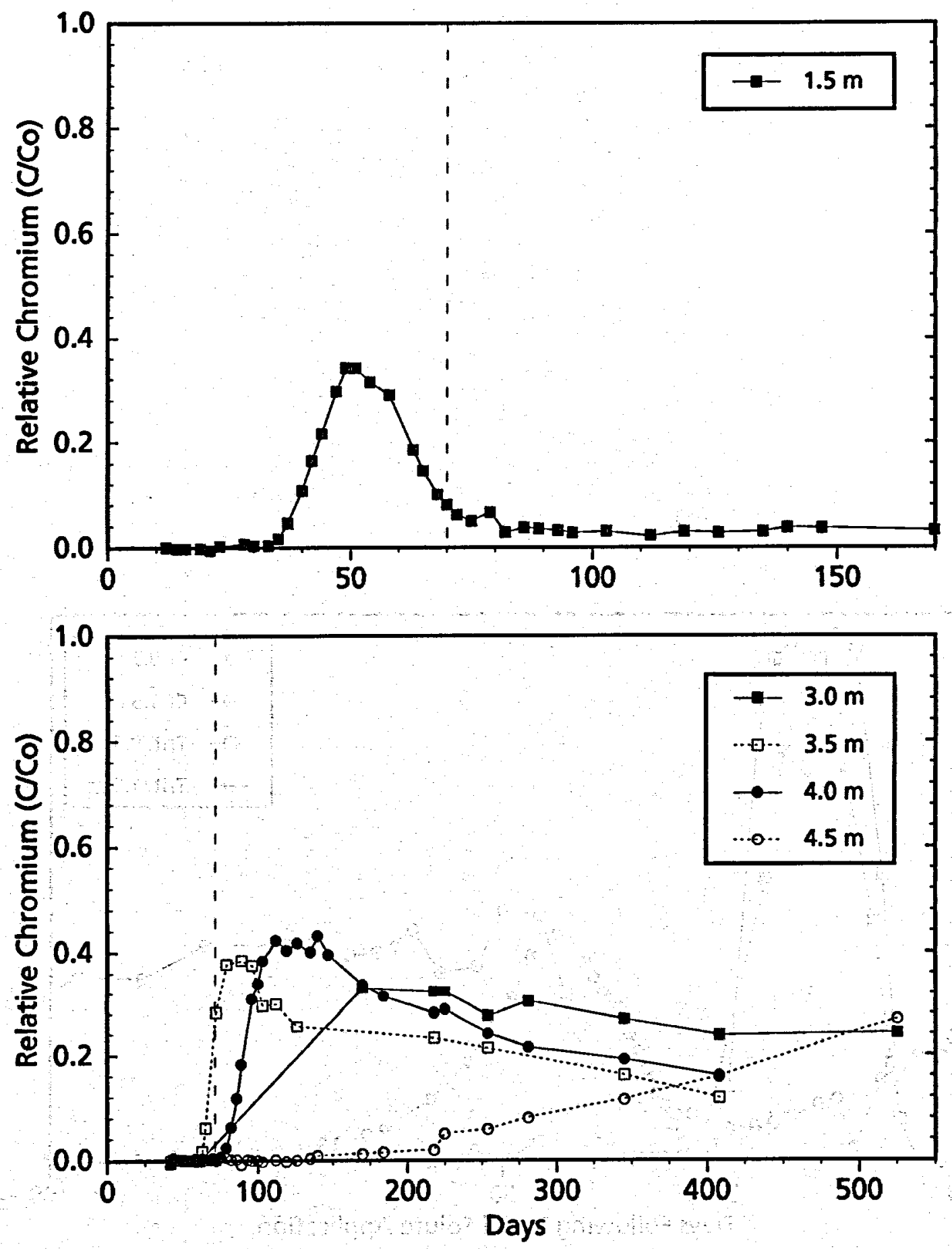

Figure 49. Experiment IIb chromium breakthrough curves at $\mathrm{X}_{2}=1.25 \mathrm{~m}$ and samplers at various depths. Concentrations as $C / C_{0}$. Vertical dashed lines indicate the end of plot irrigation. 


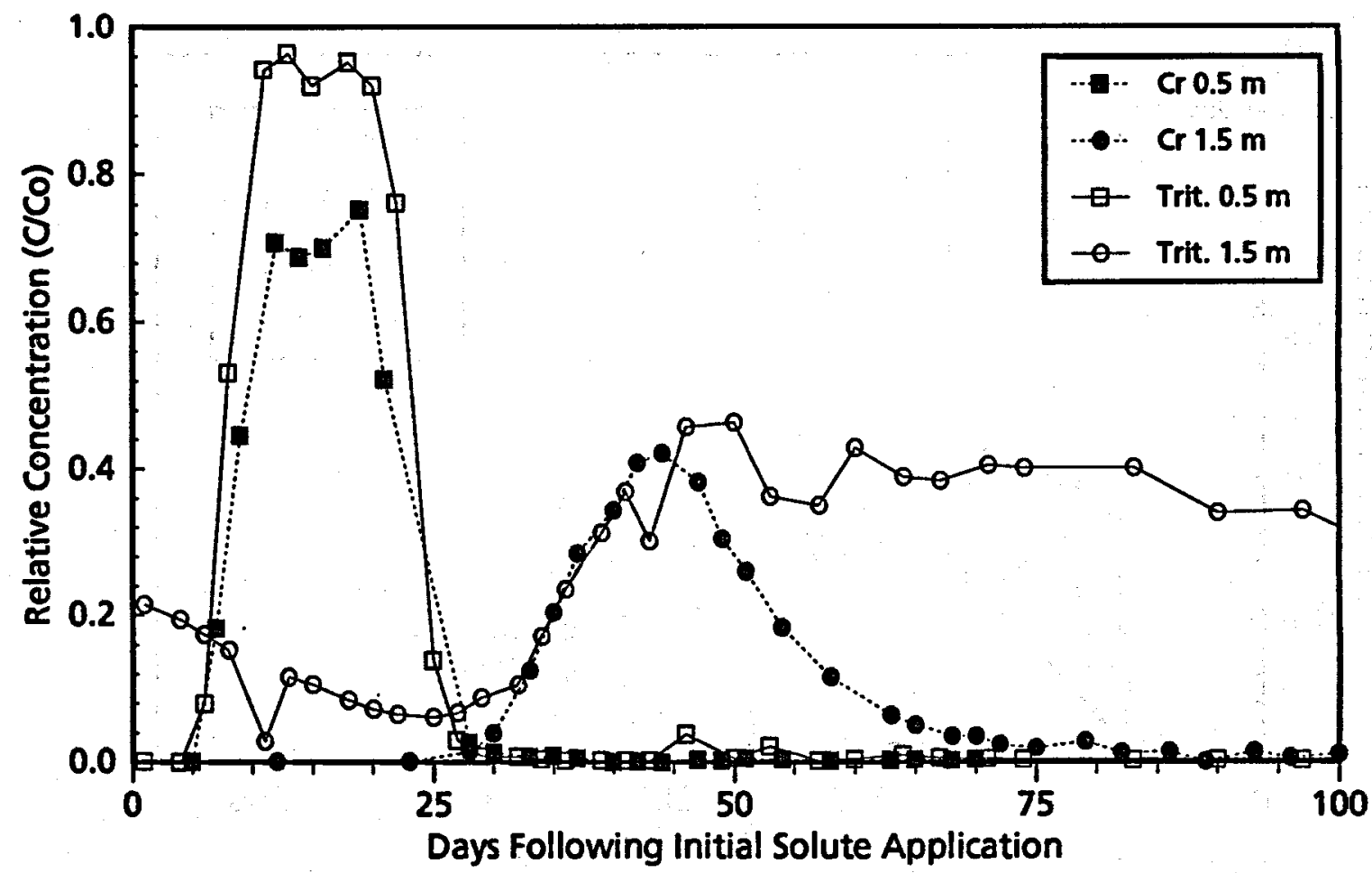

Figure 50. Experiment Ilb chromium and tritium breakthrough curves at $X_{2}=0.25 \mathrm{~m}$ and samplers at 0.5 and $1.5 \mathrm{~m}$ depths. Tritium curves shifted to the left by 29 days to normalize pulse applications at the soil surface. Concentrations expressed as $C / C_{0}$. 
This is most likely related to the fact that chromium was applied before tritium, and therefore, was already deeper in the profile at the time redistribution started.

PFBA was analyzed for days $0,14,30,47$, and 70. The progress of PFBA is given by contour plots in Figure 51. The first PFBA was found on day 14 at only three locations at $0.5 \mathrm{~m}$ depth, with a high concentration of 0.76 of input, at $\mathrm{X}_{2}=0.25 \mathrm{~m}$. By day 30 PFBA was found at locations from 1 to 2.5 meters depth with the largest portion of the plume found west of the plot centerline. On day 47 the bulk of observed PFBA was found east of the plot centerline, to 3 $m$ depth, and with a peak concentration of 0.85 of input at $X_{2}=.75$, at the $3 \mathrm{~m}$ depth. By day 70 the plume seemed to divide into several bodies with appreciable concentrations of solute stratified from $0.5 \mathrm{~m}$ through $4.5 \mathrm{~m}$ depths and evidence of lateral movement to $-1.75 \geq \mathrm{X}_{2} \geq$ 1.75. On that day the highest concentration of PFBA (0.77) was found at $X_{2}=1.25$ at the $3 \mathrm{~m}$ depth.

\subsubsection{DFBA and bromide}

DFBA data are available for days 47 and 70 only (Figure 52, Appendix A-6). On day 47 DFBA was found between 0.5 and $1.5 \mathrm{~m}$ depth and between -0.75 and $0.75 \mathrm{~m}$ in $\mathrm{X}_{2}$. Input concentration $\left(C / C_{0}=1\right)$ was attained at $0.5 \mathrm{~m}$ and $1.0 \mathrm{~m}$ depths at $\mathrm{X}_{2}=0.25 \mathrm{~m}$. The center of mass was shifted to the positive $\mathrm{X}_{2}$ direction.

By day 70 DFBA spread laterally from $\mathrm{X}_{2}=$ $-1.25 \mathrm{~m}$ to $0.75 \mathrm{~m}$ and to $3.5 \mathrm{~m}$ depth. The highest concentration was found at $2.5 \mathrm{~m}$ depth. The plume appeared to have divided into several bodies, with a center of mass shifted to the $-\mathrm{X}_{2}$ direction. However, these observations on day 70 are somewhat speculative because of the limited number of data points to represent the grid.
The evaluation of bromide is complicated by bromide presence in the profile at the beginning of the experiment and by missing values in the grid of solution samplers. In the Ila experiment bromide was applied at a relatively high concentration of $939 \mathrm{mg} / \mathrm{L}$. In experiment IIb bromide ion was applied from days 29 through 44 , at a much lower concentration, $28 \mathrm{mg} / \mathrm{L}$, or about $3 \%$ of experiment IIa input. Samples from days $0,14,30,47$, and 70 were analyzed. Figure 53 shows bromide results normalized for experiment IIa input (Appendix A-6).

Concentrations above twice those of experiment IIb input are shown as solid black.

On day 0 bromide ion is present in all five analyzed samples, to $3 \mathrm{~m}$ depth, at concentrations 0.26 to 5.23 times experiment IIb input. Likewise, bromide observed on days 14 and 30 is remnant solute applied during experiment IIa, with a high concentration on day 30 of 417 $\mathrm{mg} / \mathrm{L}$ at $2 \mathrm{~m}$ depth $\left(\mathrm{X}_{2}=0.75 \mathrm{~m}\right)$. Considering only the four solution samplers at $X_{2}=0.25$ and $0.75 \mathrm{~m}$, and from 0.5 to $1 \mathrm{~m}$ depth, bromide concentration decreased from a mean value of 0.68 on day 14 , to 0.04 on day 30 , and then increased to 1.09 by day 47 . Finally, relative concentration for these samplers decreased to 0.32 by day 70 . The separation of experiment II a and experiment IIb plumes may be discerned near the center of the plot, possibly to a depth of about $3.5 \mathrm{~m}$ by day 70 .

An unexpectedly high concentration of bromide appears at a single sampler, $1.5 \mathrm{~m}$ deep at $\mathrm{X}_{2}=$ $0.75 \mathrm{~m}$ on day $47\left(\mathrm{C} / \mathrm{C}_{\mathrm{o}}=2.51\right)$. Also, peak concentrations of experiment IIb bromide are often above 1 which indicates incomplete elution of experiment Ila bromide before the arrival of the second plume or, more likely, some concentration of solutes within sample vials by evaporation during storage (Appendix C-3). As with other solutes, overall elution appears more effective to the positive $\mathrm{X}_{2}$ 


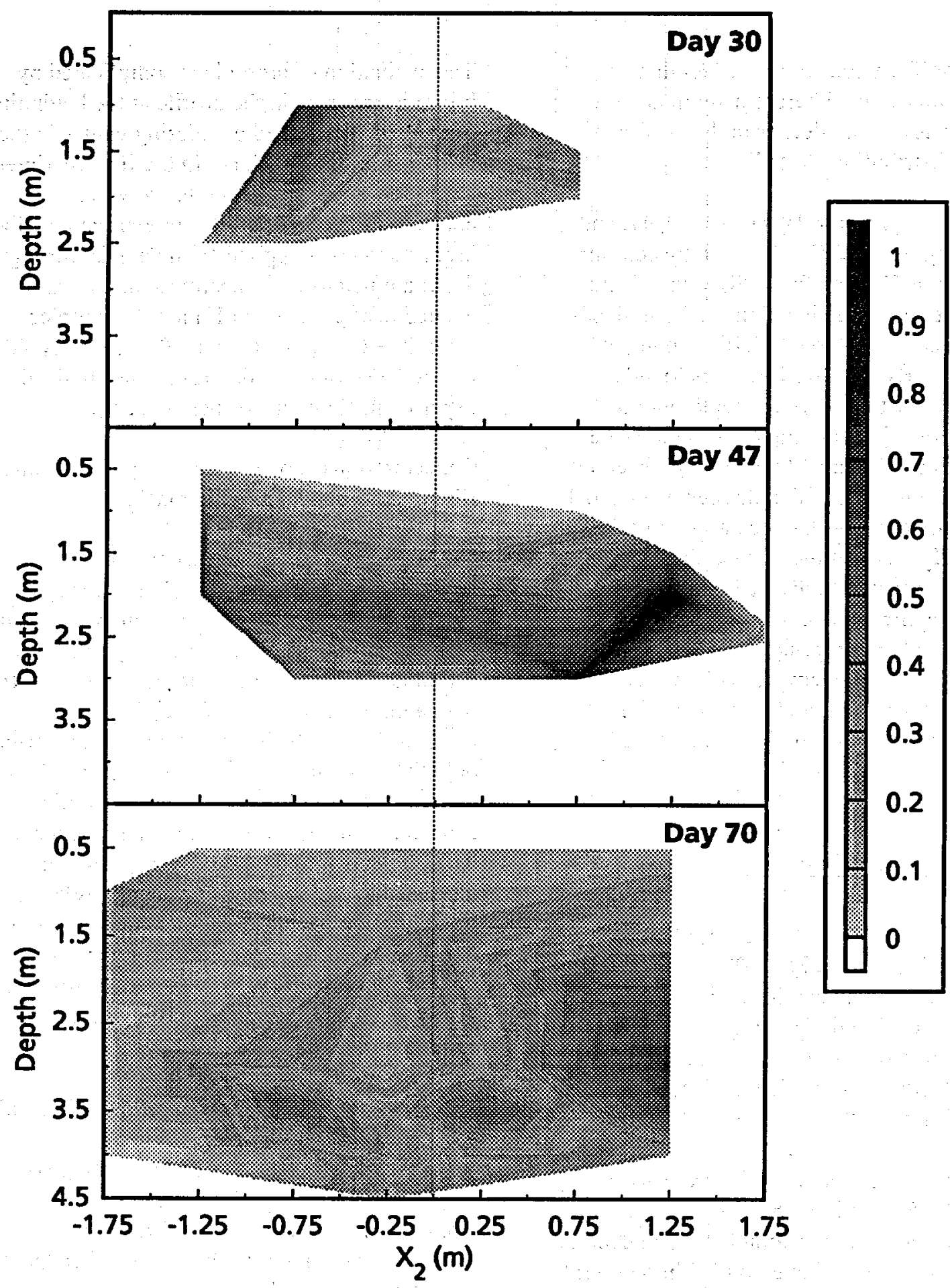

Figure 51. Experiment IIb PFBA contours at $Y_{2}=0.5 \mathrm{~m}$ from trench face for days 30,47 , and 70 of the experiment. Concentrations as $C / C_{0}$. 


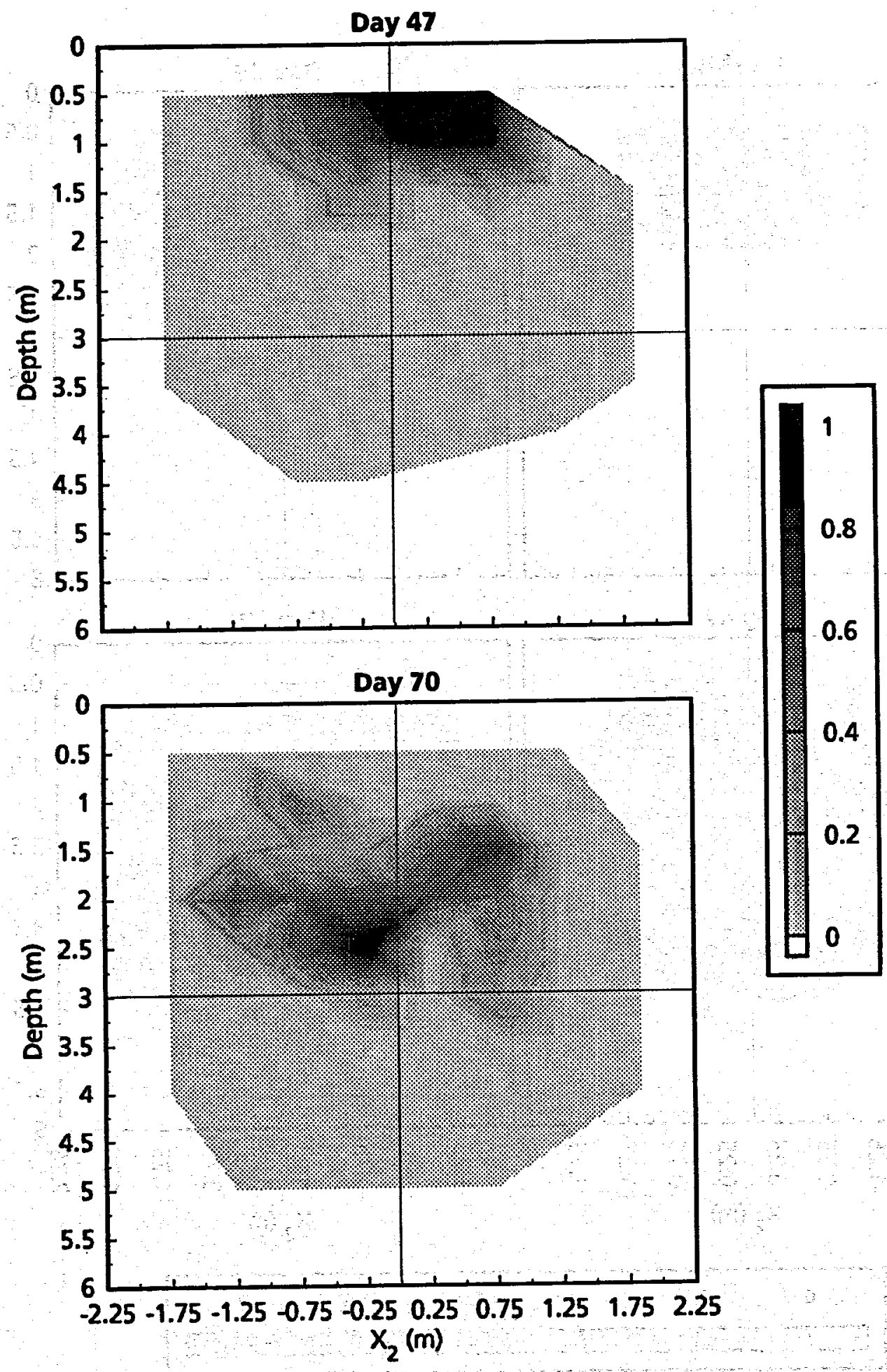

Figure 52. Experiment IIb DFBA contours at $Y_{2}=0.5 \mathrm{~m}$ from trench face for days 47 and 70 of the experiment. Concentrations as $\mathrm{C} / \mathrm{C}_{0}$. 

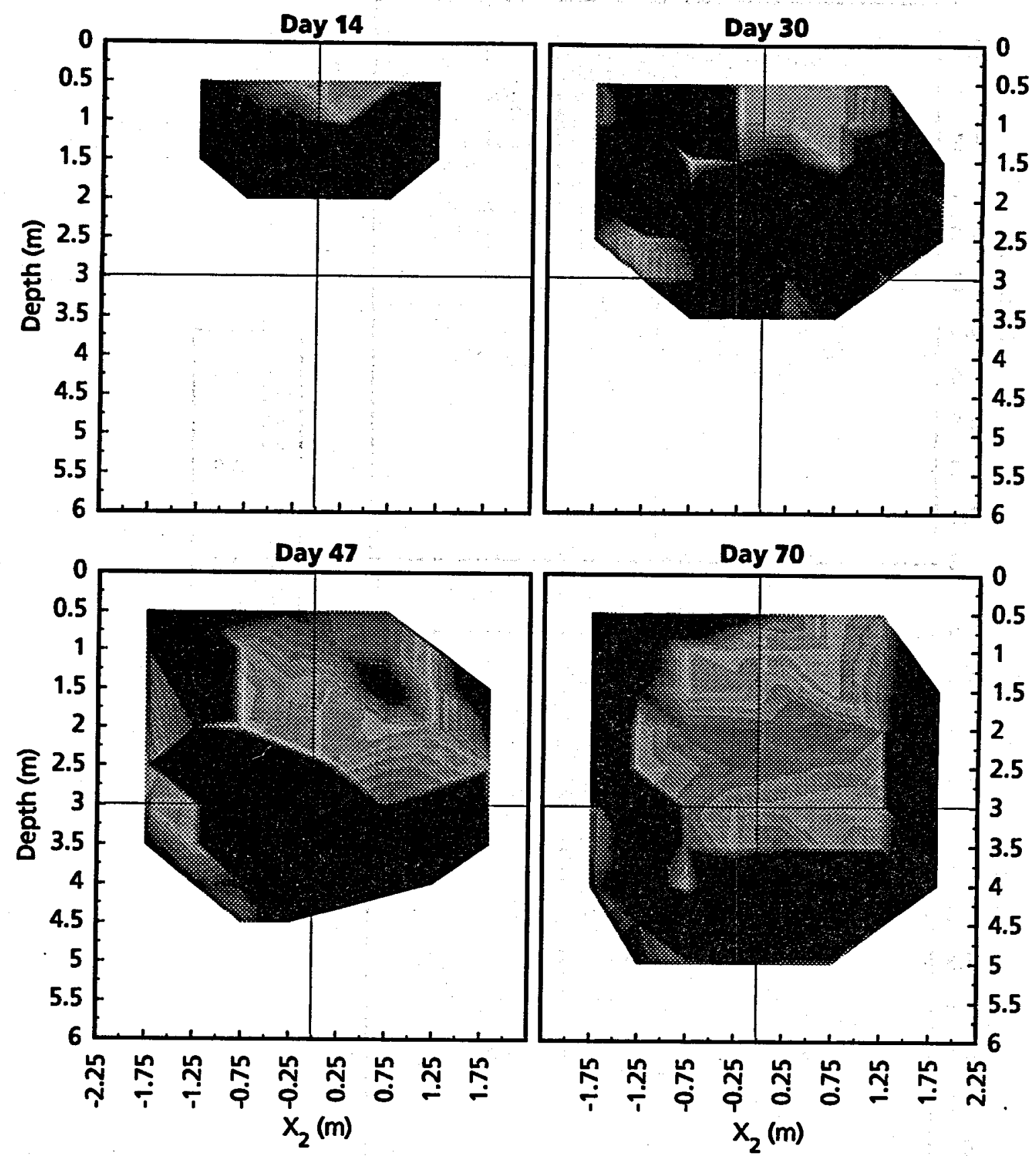

\begin{tabular}{|lllllll|}
\hline 0 & 0.4 & & 0.8 & 1.2 & 1.6 & 2 \\
\hline & & & & & &
\end{tabular}

Figure 53. Experiment IIb bromide contours at $Y_{2}=0.5 \mathrm{~m}$ from trench face for days $14,30,47$, and 70 of the experiment. Concentrations as $\mathrm{C} / \mathrm{C}_{\mathrm{o}}$ are normalized for experiment IIb application of the solute. 
direction. Other anomalous plume behavior seen in the contour plots is at least partly attributable to missing values from the sampler grid on certain measurement dates.

\subsubsection{Nitrate and chloride}

The progressive leaching of chloride and nitrate ions from the profile is illustrated by a series of contour plots in Figures 54 and 55 (data in
Appendix A-6). These plots show that the movement of these ions inversely track the progress of the applied wetting front and other applied solutes. With time the in-situ concentration of both ions in front of wetting increased to values well above what was originally present in the soil before the irrigation experiments began (Appendix C-2). The ratio of median chloride to nitrate ions concentrations (me/L) is 2.95 . 


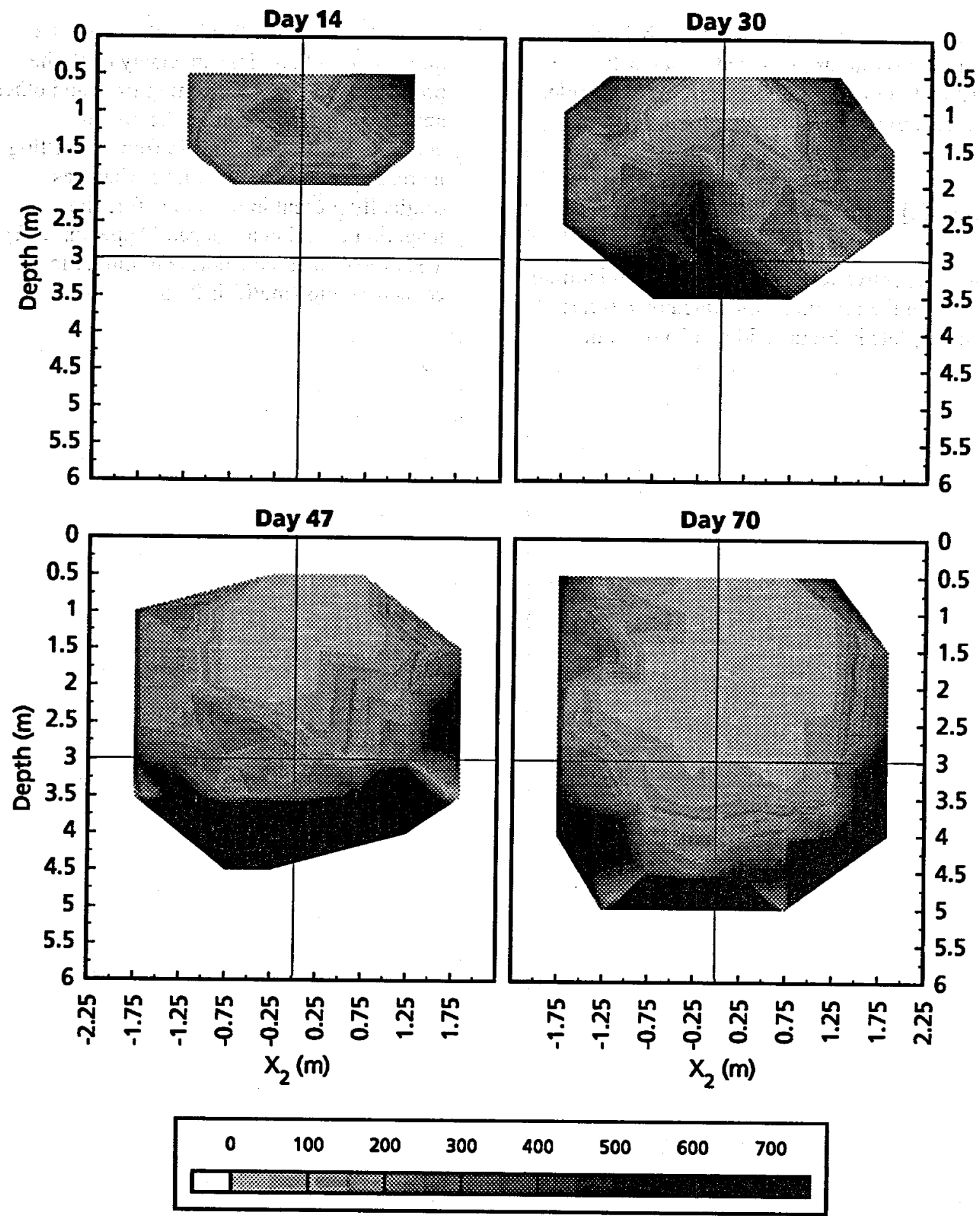

Figure 54. Experiment IIb nitrate ion contours at $Y_{2}=0.5 \mathrm{~m}$ from trench face for days $14,30,47$, and 70 of the experiment. Concentrations as $\mathrm{mg} / \mathrm{l}$ of soil solution. 

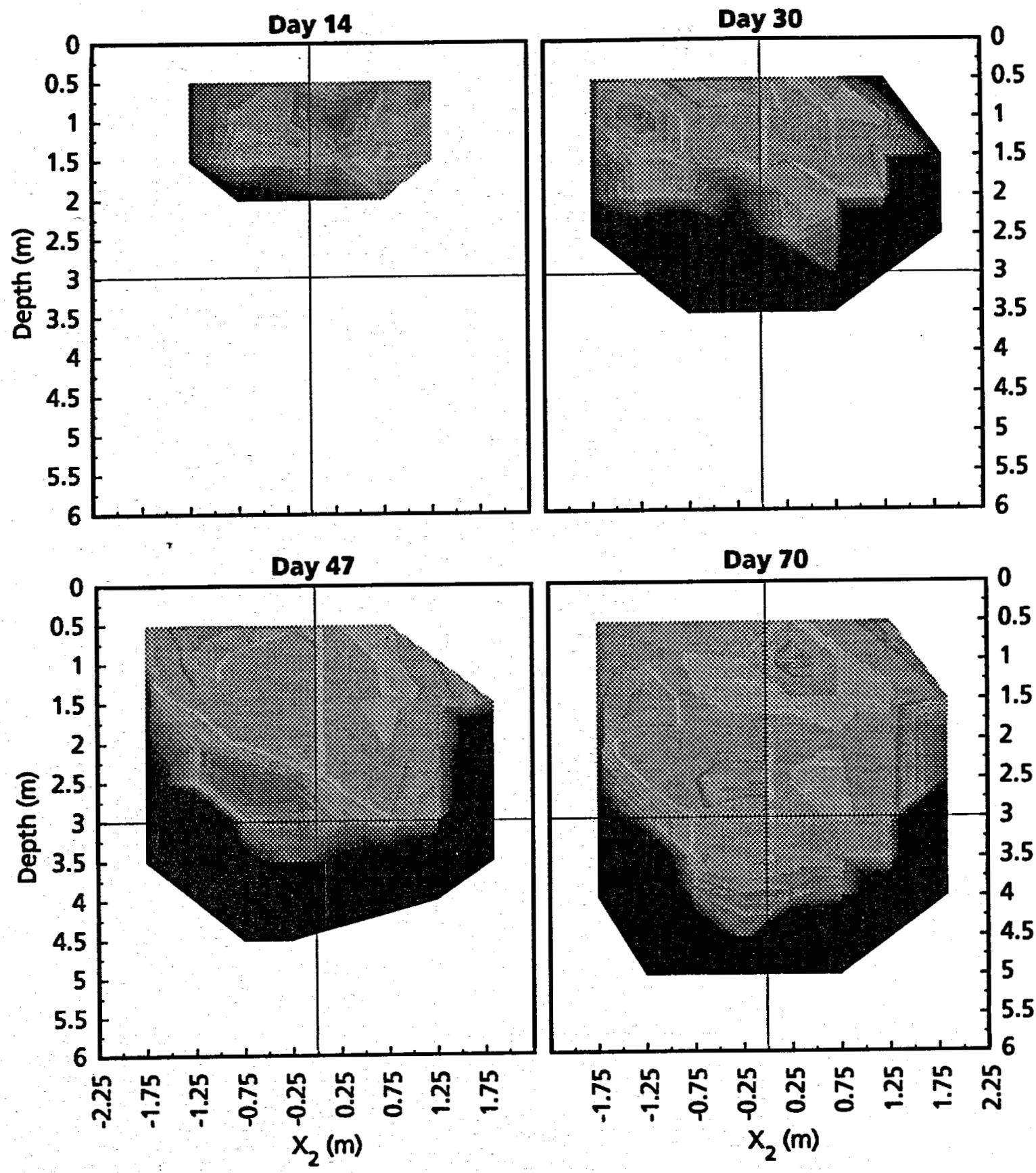

\begin{tabular}{llllllll|}
\hline 0 & 20 & 40 & 60 & 80 & 100 & 120 & 140 \\
\hline & 1 & & & & & &
\end{tabular}

Figure 55. Experiment IIb chloride ion contours at $Y_{2}=0.5 \mathrm{~m}$ from trench face for days $14,30,47$, and 70 of the experiment. Concentrations as $\mathrm{mg} / \mathrm{l}$ of soil solution. 


\section{Experiment IIb Redistribution Cores}

\subsection{Core Collection and Processing}

The second set of soil cores was collected from 24 boreholes within Plot 2 beginning on May $20,1991,222$ days after the last irrigation of experiment IIb. This second set of cores was more extensive in number and depth than cores taken after experiment IIa. Like those first cores these were also taken at $Y_{2}=5.0$ and $Y_{2}=9.0$ meters from the trench face except that the new boreholes were offset in the $\mathrm{X}_{2}$ direction by $0.25 \mathrm{~m}$ from the first set. Relative to the center of the irrigated plot, the cores were taken at $\mathrm{X}_{2}=-2.75,-2.25,-1.75,-1.25,-0.75,-0.25$, $0.25,0.75,1.25,1.75,2.25$, and 2.75 meters to a depth of about 6.4 meters (Figure 56).

The JMC Environmentalist probe (Clements Assoc., Newton, IA) used to collect these cores utilizes a lined sample tube (Kodar PETG co-polyester 6763) which takes $2.29 \mathrm{~cm}$ diameter soil cores in $3^{\prime}$ intervals of soil depth. For each core the soil probe was hammered into the soil for $3^{\prime}$ then removed from the borehole to collect the liner and sample. Seven cores were taken from each borehole to a total depth of $21^{\prime}$.

At depths below the first three foot intervals a layer of contaminant soil was often found with the desired soil core, presumably to have fallen into the open borehole when the probe was removed. This material was easily distinguished from the core of interest and was removed from the liner before further handling. Soil recovery within each 3' liner section was usually better than $80 \%$ after removing contaminant material.

The final sample handling in the field included trimming the liner material to flush with the soil core, sealing both ends with vinyl caps and then removing core sections in a protected location out of direct sunlight. At the end of the day cores were taken to the laboratory for further processing.
Each 3 ' core section was divided into four equal-length core segments. The center of each segment was assigned a depth ( $\mathrm{Z}$ meters) by proportioning the length of the recovered soil core to the column of soil being sampled ( $3^{\prime}$ ). The accuracy of this scheme assumed that the core contained a uniform representation of the 3 in-situ profile, and that compaction of the core or losses of soil to the perimeter of the sample tube occurred uniformly with depth. Each segment was capped with a vinyl cap and then weighed to the nearest $0.001 \mathrm{~g}$ on a Sartorius E2000D (Brinkman Instrument, Inc., Westbury, NY) balance, which was used for all subsequent mass measurements. Segments were then placed in refrigerated storage.

Core segments were divided into two subsamples. One sub-sample was oven-dried to determine moisture content. The other subsample was diluted with water and then extracted with a Buchner funnel apparatus to yield liquid solution samples. These solutions were analyzed for tritium, chromium, chloride, and nitrate solutes.

Any segments to be used in a particular day were taken from storage, organized on a workbench and then brought to room temperature. Each segment was reweighed to determine evaporative loss during storage. From this point forward care was taken to minimize the exposure time of the soil sample to evaporation. Soil within the liner segment was placed into a plastic beaker and then lightly crushed and mixed with a metal spatula. Since the sample size was relatively small any non-representative coarse fractions were removed, weighed separately and then discarded. Those were usually less than $1 \%$ of the total mass. At this point the sample was divided into two sub-samples, one for a determination of gravimetric water content and the other for liquid extraction. 


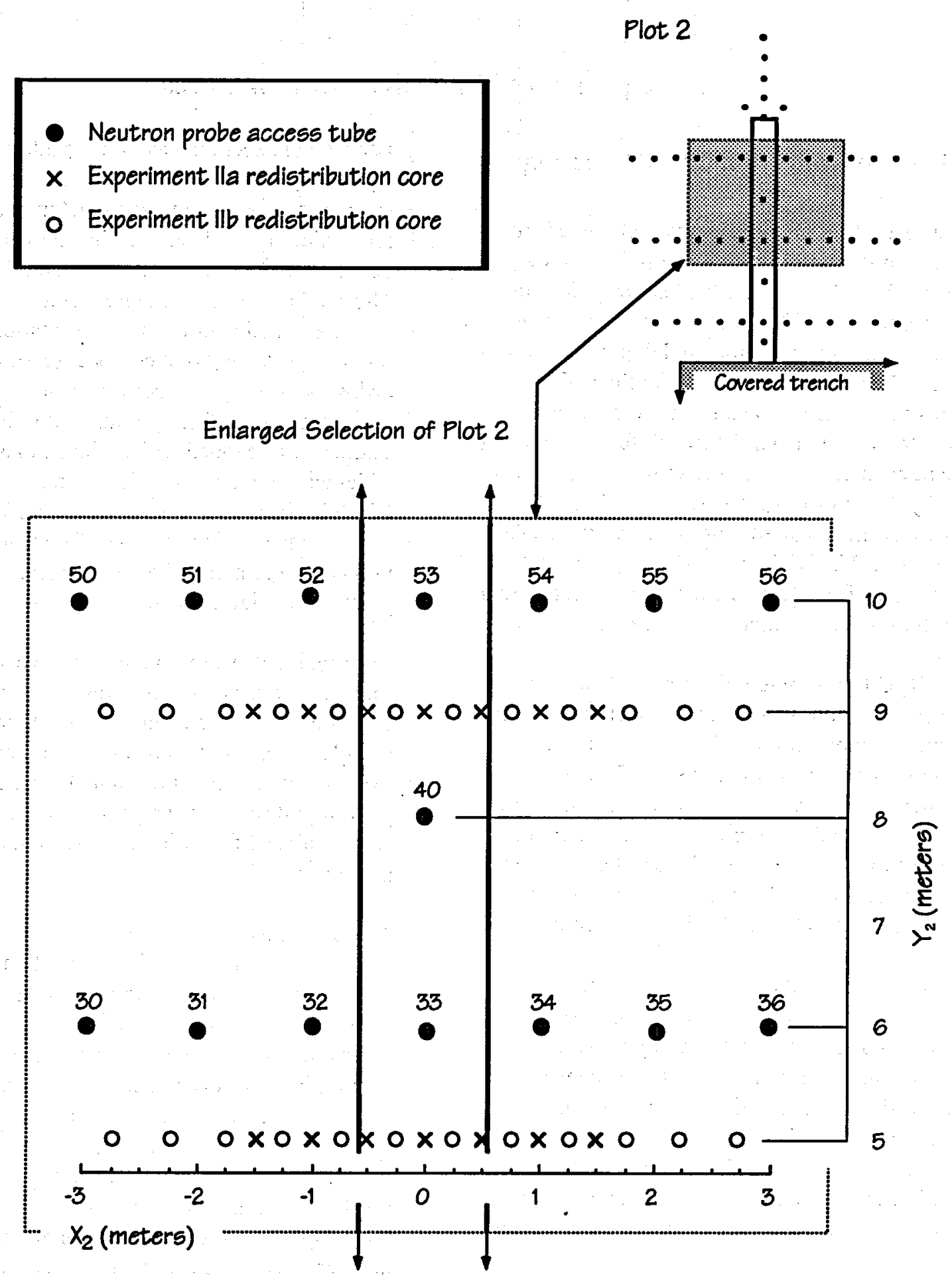

Figure 56. Location of experiment IIb soil cores relative to irrigated plot, earlier experiment IIa soil cores, and nearby neutron probe access tubes. 
An average of $36 \%$ of each fresh sample was used for moisture determination. Soil was spooned from the beaker into a tared aluminum soil can, reweighed and then placed in a $105^{\circ} \mathrm{C}$ oven for 24 hours to determine gravimetric moisture content. Samples were removed from the oven, reweighed, and then sieved through a $2 \mathrm{~mm}$ metal sieve. The fraction of material which was larger than $2 \mathrm{~mm}$ was weighed to determine the percentage of coarse materials, resulting in an average mass of $25 \%$ of the subsample (range from $2-59 \%$ ). This coarse fraction generally was quite small near the soil surface but increased with depth. All ovendried fractions were discarded after weighing.

Gravimetric water content values were converted into volumetric water units by using an estimated term for soil bulk density. This parameter was estimated from soil cores collected during the original trench characterization work, near the plane at $Y_{2}=0 \mathrm{~m}$.

The second sub-sample was moistened with distilled-deionized water to make a solution extract. The goal of dilution was to add the least amount of water necessary to extract sufficient solution to complete the chemical analyses yet provide meaningful analytical results. A-priori estimates of $6 \%$ fresh soil moisture content (by weight) and a 5 times maximum dilution of that moisture were used to calculate a ratio of $0.24 \mathrm{~g}$ added water $/ 1.0 \mathrm{~g}$ fresh soil as a target dilution rate. The subsample was weighed in its original plastic beaker. After re-taring the balance, distilleddeionized water was slowly added to the subsample to an amount equal to $26 \%$ of the fresh soil weight, on average. The dilution was varied according the sub-sample texture and moisture content as judged by the technician. The diluted samples were then stirred with a metal spatula for about one minute, covered with an acrylic plate which closely fit the rim and then allowed to stand for 20 minutes. The soil paste was briefly stirred once more during the standing period.
A ceramic Buchner funnel apparatus was used to extract the paste samples. A bank of 15 Buchner funnels was plumbed to a common vacuum source, about $425 \mathrm{mb}$ strong. Each funnel was fitted with a $5.5 \mathrm{~cm}$-diameter disk of Whatman's \#42 ashless filter paper. During filtration the Buchner funnel was covered to reduce evaporation. Filtrate was collected in a 13 dram polypropylene vials and then transferred to $20 \mathrm{ml}$ glass scintillation vials. These vials were wrapped with Parafilm ${ }^{\circ}$ before being placed in refrigerated storage.

\subsection{Methods: Sample Analyses}

For the preparation of samples for tritium analysis $0.5 \mathrm{ml}$ aliquots of sample were mixed with $5.0 \mathrm{ml}$ of Ready-Safe liquid scintillation cocktail (Beckman Instruments, Inc., Fullerton, $\mathrm{CA}$ ) in "Mini-Poly Q" $8 \mathrm{ml}$ scintillation vials (Beckman Instruments, Inc.) and run as single replications with a Beckman model LS1800 scintillator in "auto-run" mode. Raw counts were taken for 10 minutes per sample within a single-channel counting window of $0-400 \mathrm{~nm}$. Vials with less than 16 counts per minute, an approximate background count, were terminated within 1 minute of the routine.

A sequence of tritium analyses began by counting a series of five background vials and five tritium input sample vials. Background vials were prepared with $0.5 \mathrm{ml}$ aliquots of un-traced well water in place of solution sample in the scintillation vials. The input vials were prepared with $0.5 \mathrm{ml}$ aliquots of traced well water, that had been collected from the 300 . gallon solute tank on $9 / 4 / 90$. This stock of input traced water was refrigerated in a $3 \mathrm{~L}$ capped glass bottle.

Tritium data is expressed as a relative concentration, the ratio of adjusted tritium concentration of individual samples (C) to the adjusted tritium concentration applied to the plot during the irrigation of experiment IIb $\left(\mathrm{C}_{0}\right)$ : 


$$
\begin{aligned}
& \text { Relative Concentration }=\frac{C}{C_{0}} \\
& =\frac{\operatorname{Diln}\left(C^{\prime}-B k g\right)}{C_{0}^{\prime}-B k g}
\end{aligned}
$$

where Diln is the dilution factor of in-situ (fresh) soil moisture with added water, and Bkg is the mean background counts per minute for the five vials of a run. For calculation of relative concentration mean counts for the five vials of input solution were used, a value which varied slightly between analytical runs.

Tritium is also calculated on the basis of experiment IIa input concentration:

$$
\begin{aligned}
& \text { Relative Concentration }=\frac{C}{C_{0}} \\
& =\frac{\text { Diln }\left(C^{\prime}-B k g\right)}{C_{0}^{\prime}-B k g} \times 0.9379
\end{aligned}
$$

In this case $C_{0}$ is a constant value, the counts per minute of tritium applied during experiment IIa $(27,574)$. The constant 0.9379 is used to normalize counts for the equivalent use of Aquasol (Dupont New England Nuclear, Boston, MA), the scintillation cocktail used for experiment IIa. The cocktail used for the experiment IIb analyses was Ready-Safe, which has a higher counting efficiency than Aquasol (Appendix B-2).

Chloride was analyzed with a Buchler model 4-2500 chloridometer (Buchler Instruments, Inc., Ft. Lee, NJ) which utilizes timed $\mathrm{Ag} / \mathrm{AgCl}$ titration to determine chloride ion. Before using the instrument the silver electrodes were thoroughly cleaned with a commercial silver cleaner or replaced with new electrode wire, depending on the condition of the wire. The instrument was switched to high sensitivity mode, which provides a detection limit of about
$0.3 \mathrm{me} / \mathrm{l}$. Ten replicated readings of $10 \mathrm{me} / \mathrm{L}$ chloride standard were used to make a one-point calibration curve. Ten replications of background correction vials were prepared with distilled-deionized water and read. The high number of replications was used to ensure stable instrument performance before analyzing sample extracts. Sample titrations were performed with 0.10 to $1.00 \mathrm{ml}$ volume aliquots, depending on the concentration of $\mathrm{Cl}^{-}$within the sample. Samples were read as single replications.

Nitrate analysis was performed with a Technicon AutoAnalyser II (Technicon Industrial Systems, Terrytown, NY) in the New Mexico State University Soil \& Water Testing Laboratory. This instrument determines nitrate by a colorimetric procedure; a copperized cadmium column is used to reduce sample nitrate to nitrite in the presence of $\mathrm{NH}_{4} \mathrm{Cl}$. The resulting nitrite is treated with a colorizing agent to form a pink color. A colorimeter is then used to measure absorbance of the colorized solution at $540 \mathrm{~nm}$.

The sample extracts were first screened for approximate nitrate content by using nitrate test strips (EM Science Inc., Gibbstown, NJ). From these approximations the final sample dilutions to bring the samples into the working linear range $(0-10 \mathrm{mg} / \mathrm{l})$ of the AutoAnalyser were determined. Samples were diluted from $1 \times$ (full strength) to $100 \mathrm{x}$ with distilled-deionized water in dilution steps of no more than $10 x$ per dilution. The instrument was calibrated with a 1 $\mathrm{mg} / \mathrm{L} \mathrm{N}-\mathrm{NO}_{3}$ standard to produce a single-point calibration curve. The instrument was recalibrated with two replications of nitrate standard for every 10 sample extracts in a sequence of runs.

Chromium was analyzed with a Perkin-Elmer model 6000 inductively coupled plasma instrument (ICP) and a model 7500 integrated computer (Norwalk, CT). The linear working range given by Perkin-Elmer for this instrument 
is from $0.02-300 \mathrm{mg} / \mathrm{L}$ for chromium, with a detection limit of $0.004 \mathrm{mg} / \mathrm{L}$. In practice, the detection limits were found to be much higher for this machine, $0.029 \mathrm{mg} / \mathrm{L}$.

The ICP was programmed to measure spectral emission of chromium at $267.716 \mathrm{~nm}$, then switch the monochromator wavelength to $249.77 \mathrm{~nm}$ for boron. The instrument was calibrated with a series of three solutions; a standard containing $10 \mathrm{ppm}$ chromium and 10 ppm boron, a blank of distilled-deionized water and a second standard to check reproducibility. This calibration procedure was repeated after every 15 samples.

Samples were transferred to $15 \mathrm{ml}$ polystyrene conical tubes (Becton Dickinson, Lincoln Park, $\mathrm{NJ})$ and then placed in the ICP auto-sampler rack in groups of 50 samples (including calibration tubes). The run would begin after allowing the machine at least 20 minutes to warm up and produce a stable plasma field, which was confirmed by successful calibration.

\subsection{Results and Discussion}

Neutron probe volumetric water content (day 310 ) is compared to soil core volumetric water content $(\approx$ day 293$)$ in $Y_{2}$ planes separated by 1 meter. Neutron probe data from day 310 are found in Appendix A-2. Data for soil core moisture and solutes are found in Appendices A-7 and A-8.

Neutron probe moisture at $Y_{2}=6 \mathrm{~m}$ is compared to soil core moisture at $Y_{2}=5 \mathrm{~m}$ (Figure 57). Similar comparisons are made between $Y_{2}=10 \mathrm{~m}$ (probe) and $9 \mathrm{~m}$ (core) planes (Figure 58). Note that soil core sampling density was about 2 times greater than neutron probe sampling density. Within each plane neutron probe measurements were made on $1 \mathrm{~m}$ intervals in $\mathrm{X}_{2}$ and $0.25 \mathrm{~m}$ intervals in depth, or about 168 measurements per $Y_{2}$ soil core measurement plane is defined by $0.5 \mathrm{~m}$ intervals in $X_{2}$ and $0.225 \mathrm{~m}$ intervals in depth, or about 320 measurements per $Y_{2}$-plane, to $6 \mathrm{~m}$ depth. Altogether, the two soil core planes are represented by 666 measurements to a depth of $6.3 \mathrm{~m}$.

By day 310 the lateral redistribution of experiment IIb applied water (measured by neutron probe) had largely exceeded the $X_{2} \pm$ $2.75 \mathrm{~m}$ bounds of the soil cores (Figure 59). Therefore the soil cores did not fully sample the extent of lateral spread of applied water. Lateral heterogeneities between neutron probe and soil core water distributions are expected to be minimal.

In terms of vertical distribution the bulk of applied water had moved below the $3 \mathrm{~m}$ depth mark by day 310 . In absolute water content, higher overall moisture contents are found in those measurements taken below $3 \mathrm{~m}$ depth, a feature common with neutron probe and soil core measurement planes.

Though the comparative water distributions are similar the mean planar moisture contents differ. The mean neutron probe water contents were 0.113 and 0.109 at $Y_{2}=6$ and $10 \mathrm{~m}$, respectively. Mean soil core water contents were 0.121 and 0.123 at $Y_{2}=5$ and $9 \mathrm{~m}$, respectively, for an overall difference of about $1 \%$ in measured water content by volume. These differences may be due to the use of estimated terms for bulk density for the soil cores, an inaccurate calibration curve for the neutron probe or, less likely, consistent real differences between water contents at the measured planes.

The most striking feature of all the comparative moisture plots is the layering of high-moisturecontent zones in the profile. These zones are located at nearly identical depths in all Y-planes but are more clearly defined by the soil cores. 

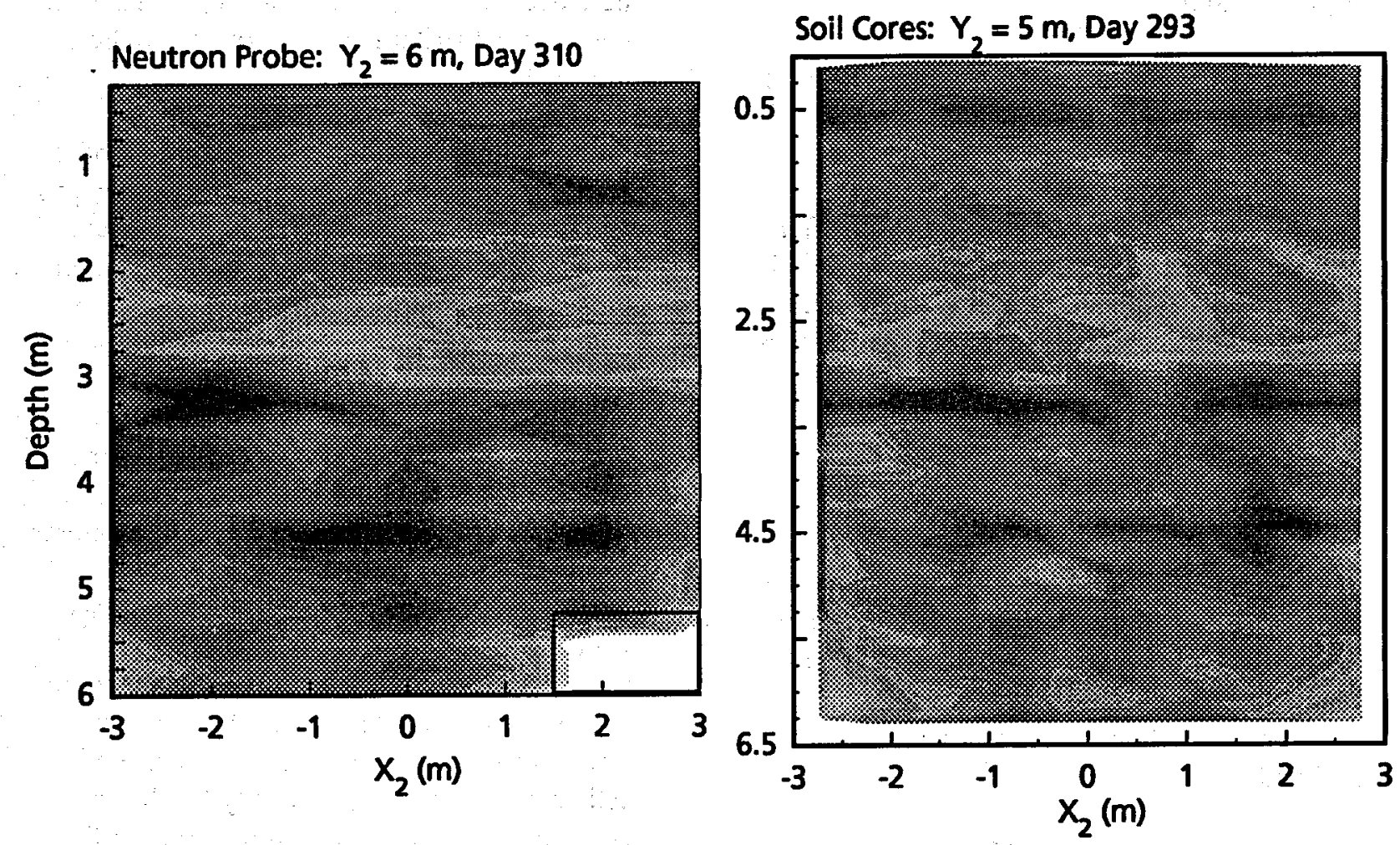

\begin{tabular}{|lllllllll|}
\hline 0.04 & 0.06 & 0.08 & 0.10 & 0.12 & 0.14 & 0.16 & 0.18 & 0.20 \\
\hline$\square$ & & & & & & & &
\end{tabular}

Figure 57. Experiment IIb redistribution cores volumetric water at $Y_{2}=5 \mathrm{~m}$ compared with neutron probe volumetric water at $Y_{2}=6 \mathrm{~m}$. 


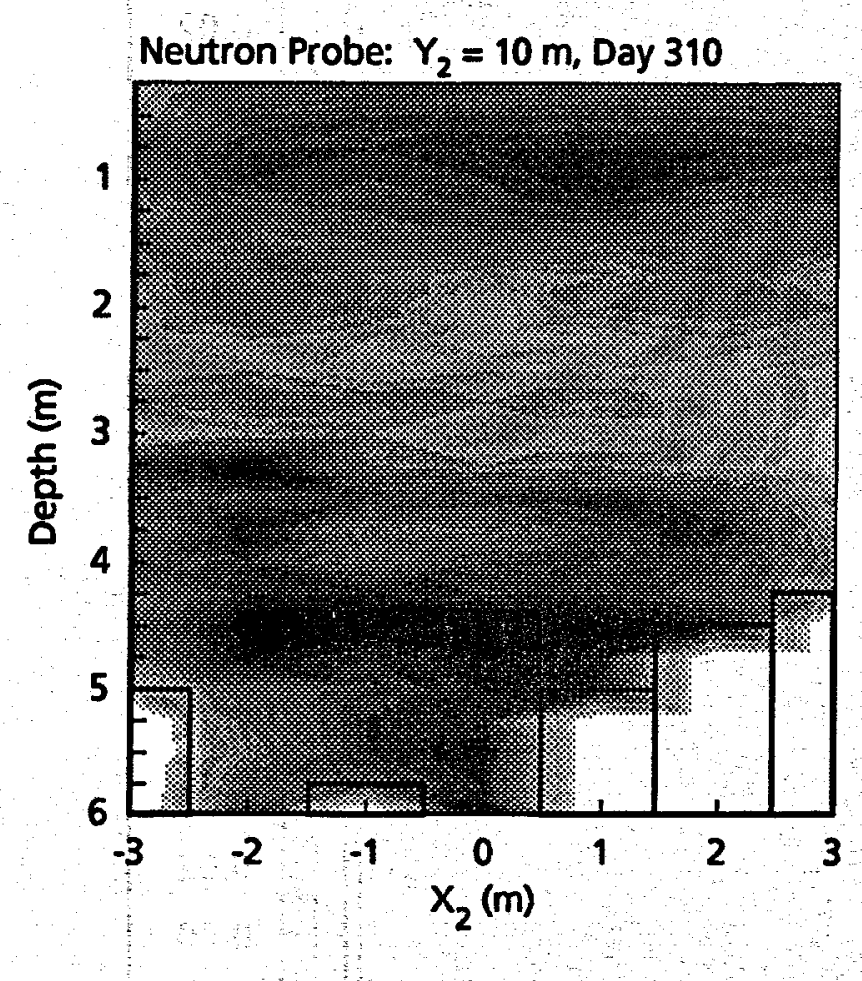

Soil Cores: $Y_{2}=9 \mathrm{~m}$, Day 293

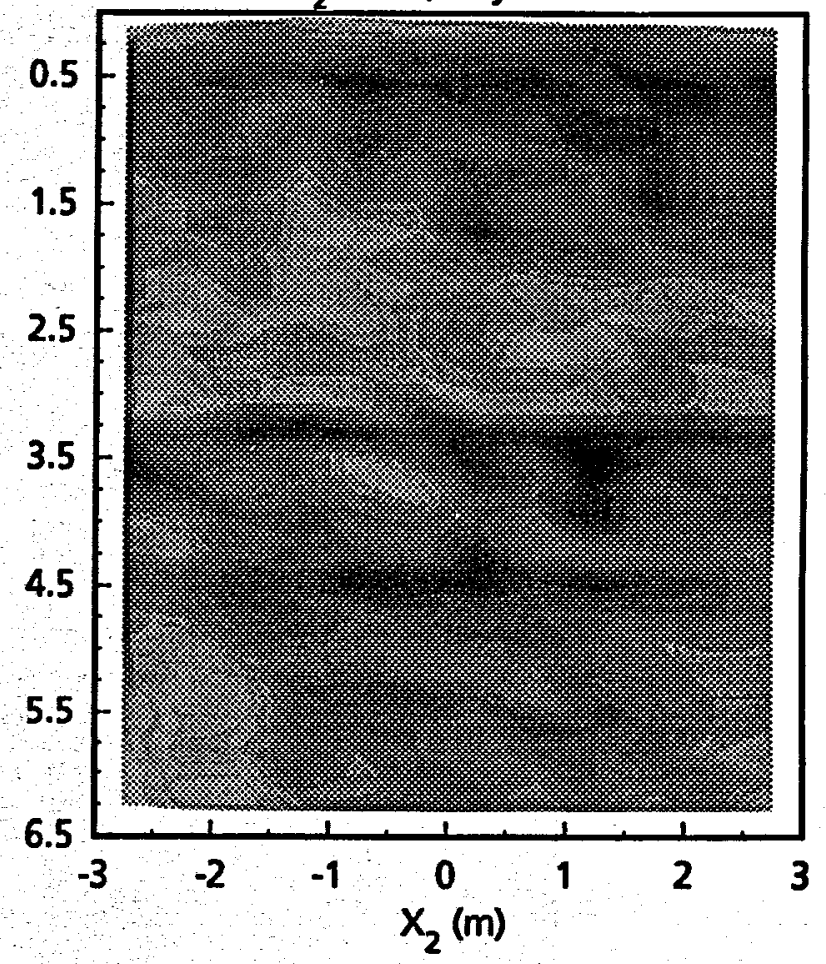

$\begin{array}{lllllllllllllllllllll}0.04 & 0.06 & 0.08 & 0.10 & 0.12 & 0.14 & 0.16 & 0.18 & 0.20\end{array}$

Figure 58. Experiment IIb redistribution cores volumetric water at $Y_{2}=9 \mathrm{~m}$ compared with neutron probe volumetric water at $Y_{2}=10 \mathrm{~m}$. 

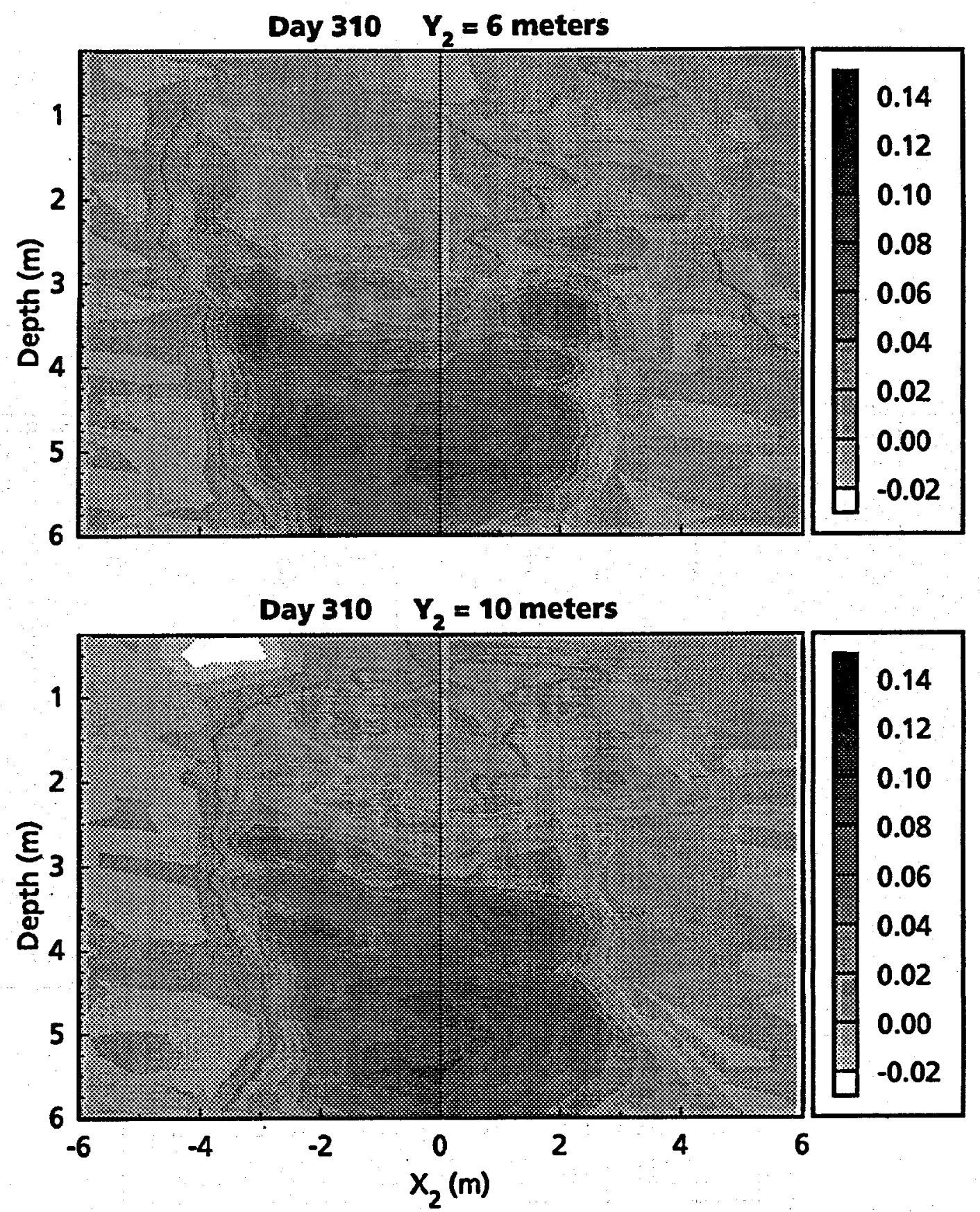

Figure 59. Experiment IIb neutron probe volumetric water content differences on day 310 for planes of access tubes at $Y_{2}=6 \mathrm{~m}$ and $Y_{2}=10 \mathrm{~m}$ from the trench face. Values expressed as differences from day 0 water content. 
This added definition is probably due to two factors: the higher sampling density in the soil cores and the non-point, "sphere of influence" measurement of moisture by the neutron probe. In addition to lenses of high-moisture-content soil at 3.25 and $4.5 \mathrm{~m}$ depth, the soil core contour plots disclose less distinct lenses about 0.5 and $1.5 \mathrm{~m}$ deep that are not evident in the neutron probe data.

As with the high-moisture lenses of soil at 3.25 and $4.5 \mathrm{~m}$ depth, which were discussed earlier in this report, the 0.5 and $1.5 \mathrm{~m}$ lenses may be associated with soil morphological properties. Soil morphology data collected from the trench face at $X_{2}=-0.6 \mathrm{~m}$ indicates a lithologic discontinuity at $1.7 \mathrm{~m}$ depth, the boundary between the Isaacks' Ranch and Jornada II soil units. About $1.5 \mathrm{~m}$ deep in the trench face the content of clay and silt abruptly decreases relative to sand. Likewise, the lens at $0.5 \mathrm{~m}$ depth may be associated with an abrupt increase of calcium carbonate content that was identified between 0.42 and $0.56 \mathrm{~m}$ deep along the trench face (Nash, 1990). The validity associations of these assume isotropic continuity of soil properties from the trench face to the planes of measurement.

Soil tritium observations are contoured in Figures 60 and 61 as the concentration of tritium relative to experiment IIb input solution. Tritium elution appeared to be more effective at $Y_{2}=9 \mathrm{~m}$ than at $5 \mathrm{~m}$. While lateral redistribution appears similar between the two measurement planes the leading edge of the combined tritium plumes (experiments IIa and IIb) occurs about $4.75 \mathrm{~m}$ deep at $5 \mathrm{~m}$ and about $5.5 \mathrm{~m}$ deep at $9 \mathrm{~m}$. For $\mathrm{Y}_{2}=5 \mathrm{~m}$ the maximum observed concentration was found at $1.9 \mathrm{~m}$ depth, $X_{2}=-0.70 \mathrm{~m}$ ( 58 percent of input concentration). At $\mathrm{Y}_{2}=9 \mathrm{~m}$ the maximum concentration was found at $2.9 \mathrm{~m}$ depth, $X_{2}=-0.25 \mathrm{~m}$ ( 55 percent $)$.

Note that the relative distribution of tritium within the two planes is in marked contrast to the distribution of first-plume tritium of soil cores collected after experiment IIa. Recovery of applied tritium mass within the two measurement planes was $81.9 \%$ at $Y_{2}=5 \mathrm{~m}$ and $101.5 \%$ at $Y_{2}=9 \mathrm{~m}$. Unlike the data used for contour plots these calculations of mass balance were adjusted for radioactive decay of tritium input through the day of sample analyses. For these calculations, the input mass and decay of experiment Ila and experiment IIb pulses were treated separately.

Separation between the first and second pulses of tritium is recognizable in the plots though there appears to be significant overlap. Without the breakthrough curve analysis presented earlier in this report the interpretation of these contour plots might have led to the conclusion that the two pulses had nearly coalesced. Note that the first-pulse of tritium, at the bottom of the plume, has relatively low activity compared to the second pulse, even though the initial concentration of tritium in the first pulse was 1.61 times more concentrated. This reduced first-pulse activity was also found in the soil core samples taken after the experiment IIa irrigation.

The results for chromium are contoured from 627 individual sample points in Figure 62 as relative concentration. The shape and extent of the chromium plumes are almost identical to the plumes of the combined tritium pulses. Chromium was most likely extracted from the soil core samples as soluble and mobile chromate ion $\left(\mathrm{CrO}_{4}\right)$, which was not appreciably retarded in this experiment.

However, the mass recovery of the chromium was much lower than for tritium. The highest relative values of $\mathrm{Cr}$ for the $\mathrm{Y}_{2}=5 \mathrm{~m}$ transect was located at $X_{2}=0.25 \mathrm{~m}$ and $4 \mathrm{~m}$ depth ( 0.215 of input concentration), and for the $Y_{2}=9 \mathrm{~m}$ transect, at $X_{2}=-0.75 \mathrm{~m}$ and $4.46 \mathrm{~m}$ depth (0.222). When the overall mass balance for chromium is calculated from only those observations where $C / C_{0} \geq 0$, the mass recovery 


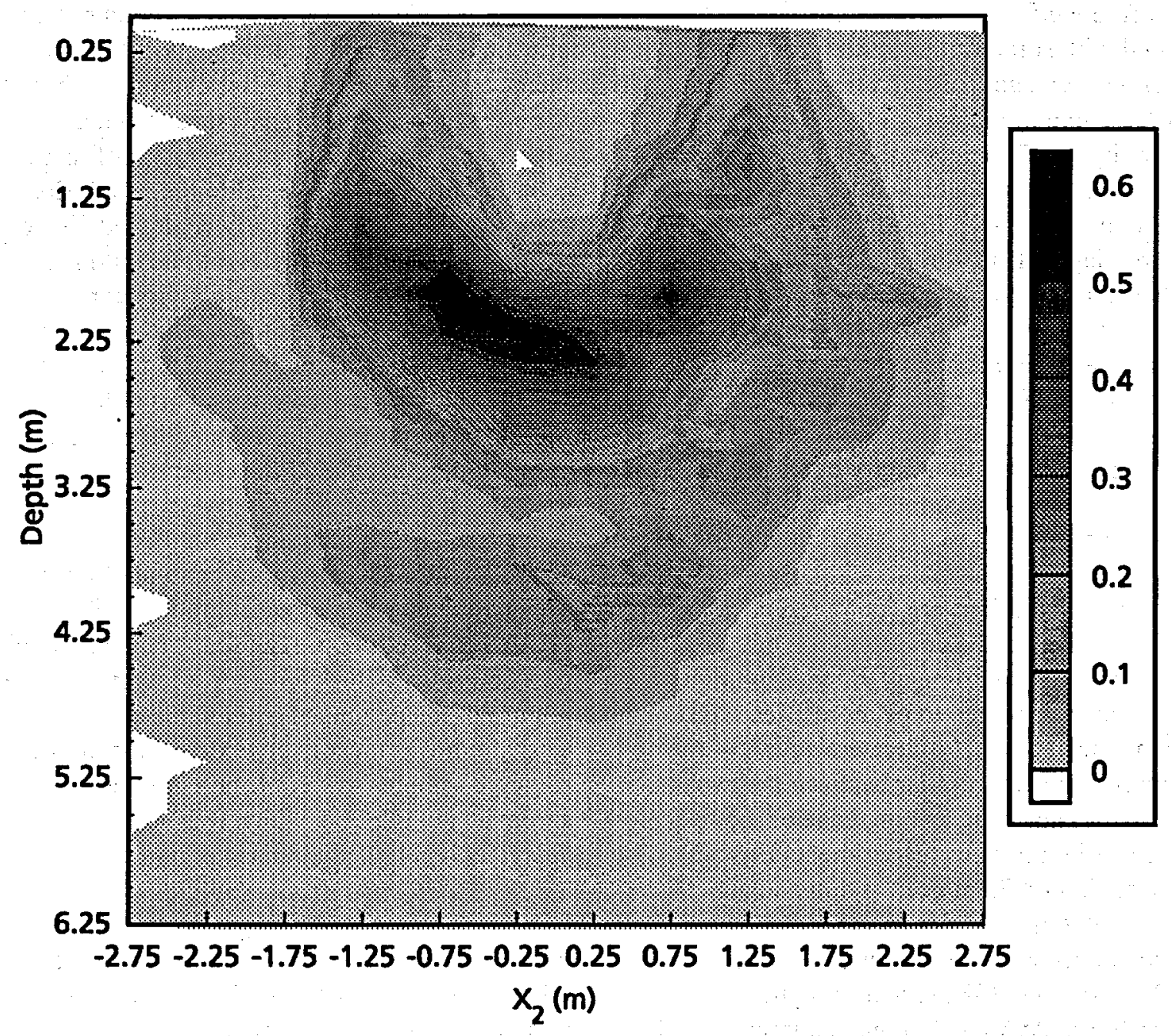

Figure 60. Experiment IIb redistribution cores tritium contours at $Y_{2}=5 \mathrm{~m}$ from the trench face. Concentration expressed as $C / C_{0}$. 


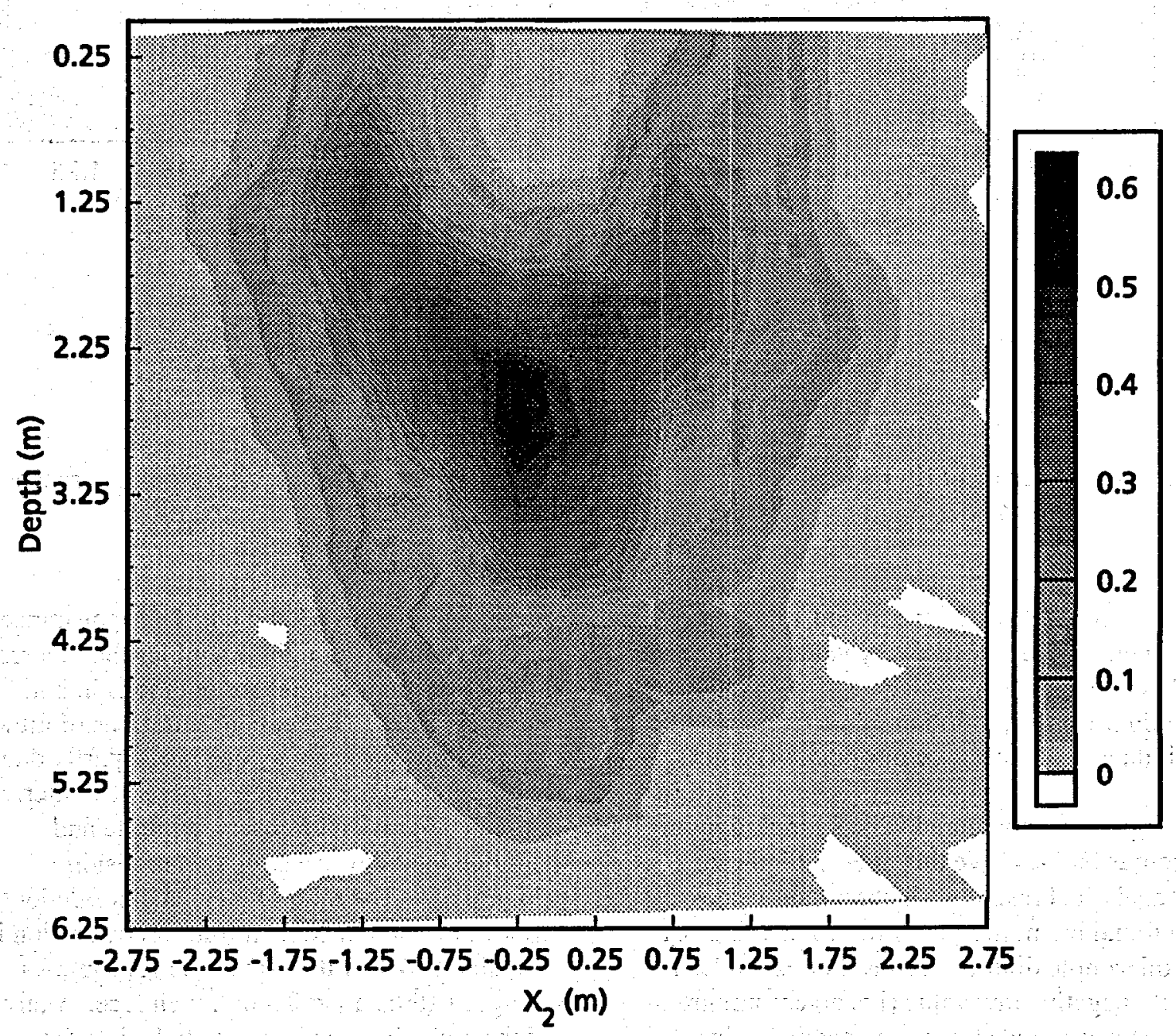

Figure 61. Experiment IIb redistribution cores tritium contours at $Y_{2}=9 \mathrm{~m}$ from the trench face. Concentration expressed as $\mathrm{C} / \mathrm{C}_{0}$. 

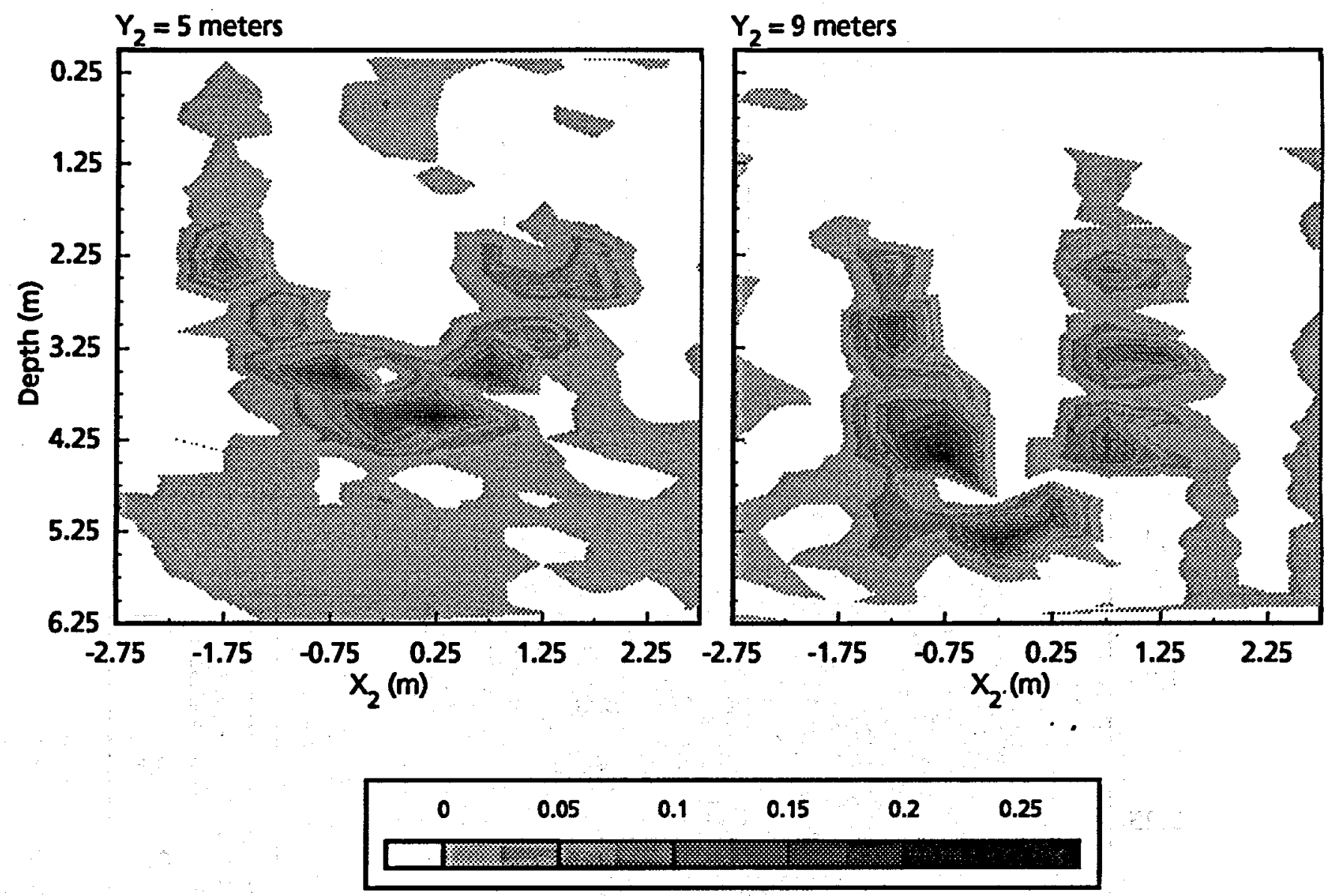

Figure 62. Experiment IIb redistribution cores chromium contours at $Y_{2}=5$ and $9 \mathrm{~m}$ from the trench face. Concentration expressed as $C / C_{0}$.

amounts to $13.9 \%$ at $\mathrm{Y}_{2}=5 \mathrm{~m}$ and $15.4 \%$ at $\mathrm{Y}_{2}=9 \mathrm{~m}$. These low recovery values may be attributable to a valence change of elemental chromium from $\mathrm{Cr}^{+6}$ to immobile $\mathrm{Cr}^{+3}$ precipitate in the presence of organic matter.

Although many contoured values for chromium are somewhat negative this does not indicate poor analytical results. All chromium values were found by multiplying raw ICP readings by the soil sample dilution factor. For example, a slightly negative raw value (i.e. no chromium present) was reduced to more negative values when multiplied by the dilution factor. The median raw negative reading was $-0.01 \mathrm{mg} / \mathrm{L}$, a value within the observed detection limit for the ICP instrument used for these analyses.

Nitrate and chloride ions are contoured per transect in Figures 63 and 64. The appearance of these plumes strongly resembles the inverse image of tritium plumes for both ions in both transect planes. Higher concentrations of nitrate near the soil surface may be at least partly due to on-going mineralization of organic matter. A paired comparison of observed nitrate and chloride ions shows a strong relationship (Figure 65). The ratio of median chloride ion concentration to median nitrate concentration is 1.6 , about $54 \%$ of the same ratio for samples analyzed at the $Y_{2}=0.5 \mathrm{~m}$ trench face. Many of the high nitrate values which deviate from the main grouping of data points are located near the soil surface. Further insights which lend to the interpretation of this data may be found in an Appendix C-2, which examines pre-irrigation levels of nitrate and chloride in the profile. 


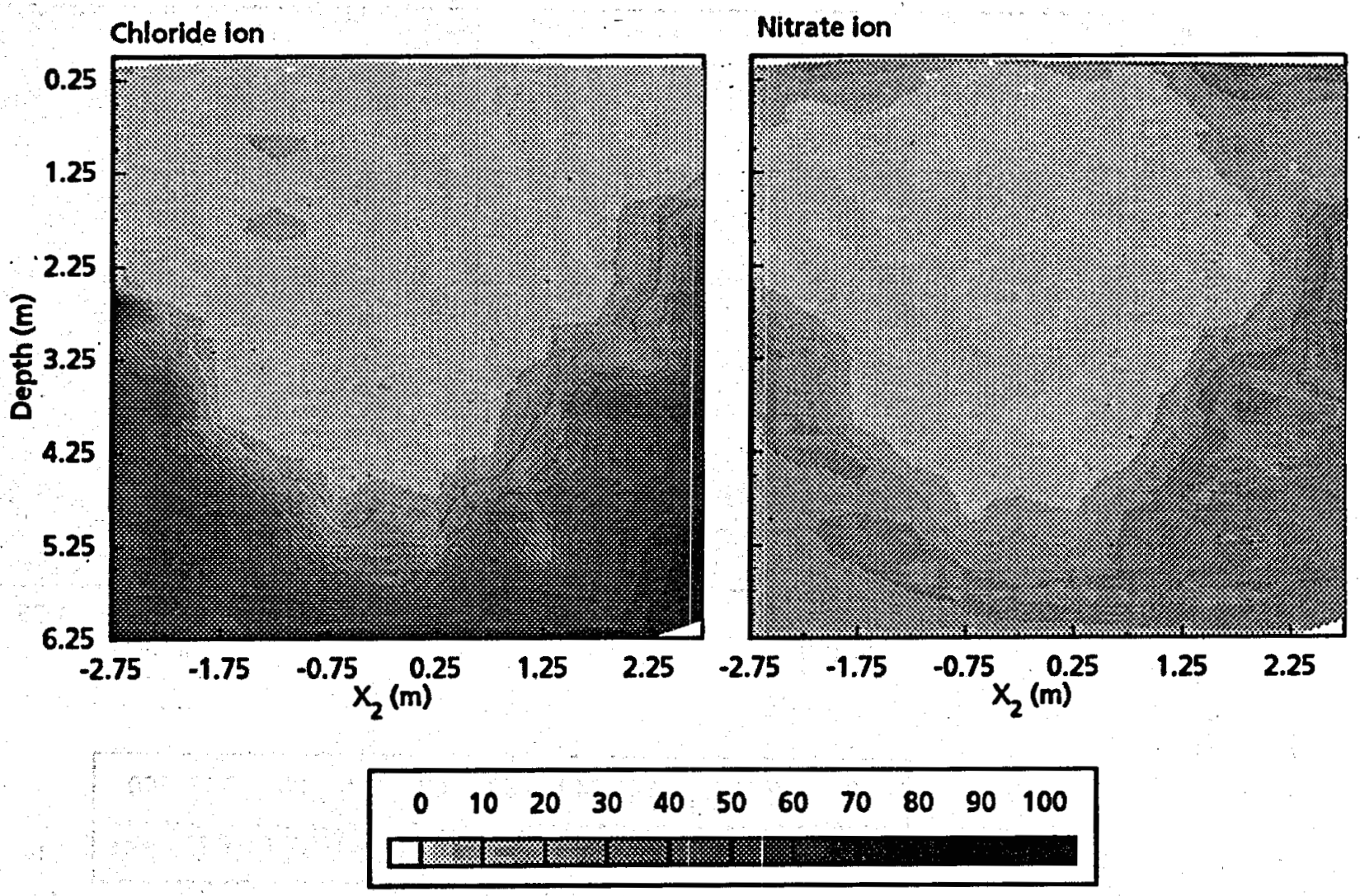

Figure 63. Experiment IIb redistribution cores chloride and nitrate contours at $Y_{2}=5 \mathrm{~m}$ from the trench face. Concentration expressed as me/L of soil solution. 

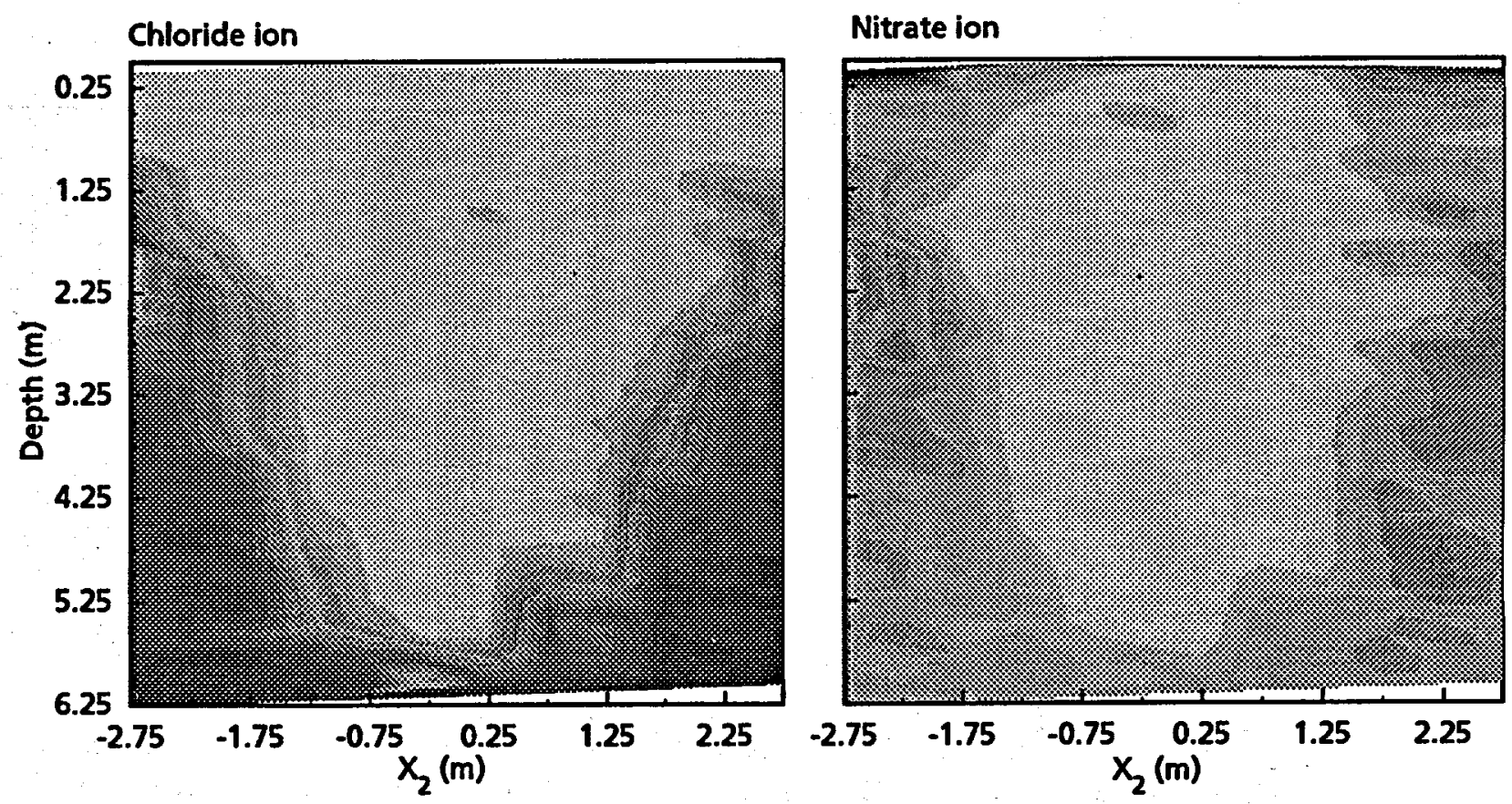

\begin{tabular}{|lllllllllll|}
\hline 0 & 10 & 20 & 30 & 40 & 50 & 60 & 70 & 80 & 90 & 100 \\
\hline & & &
\end{tabular}

Figure 64. Experiment IIb redistribution cores chloride and nitrate contours at $Y_{2}=9 \mathrm{~m}$ from the trench face. Concentration expressed as $\mathrm{me} / \mathrm{L}$ of soil solution. 


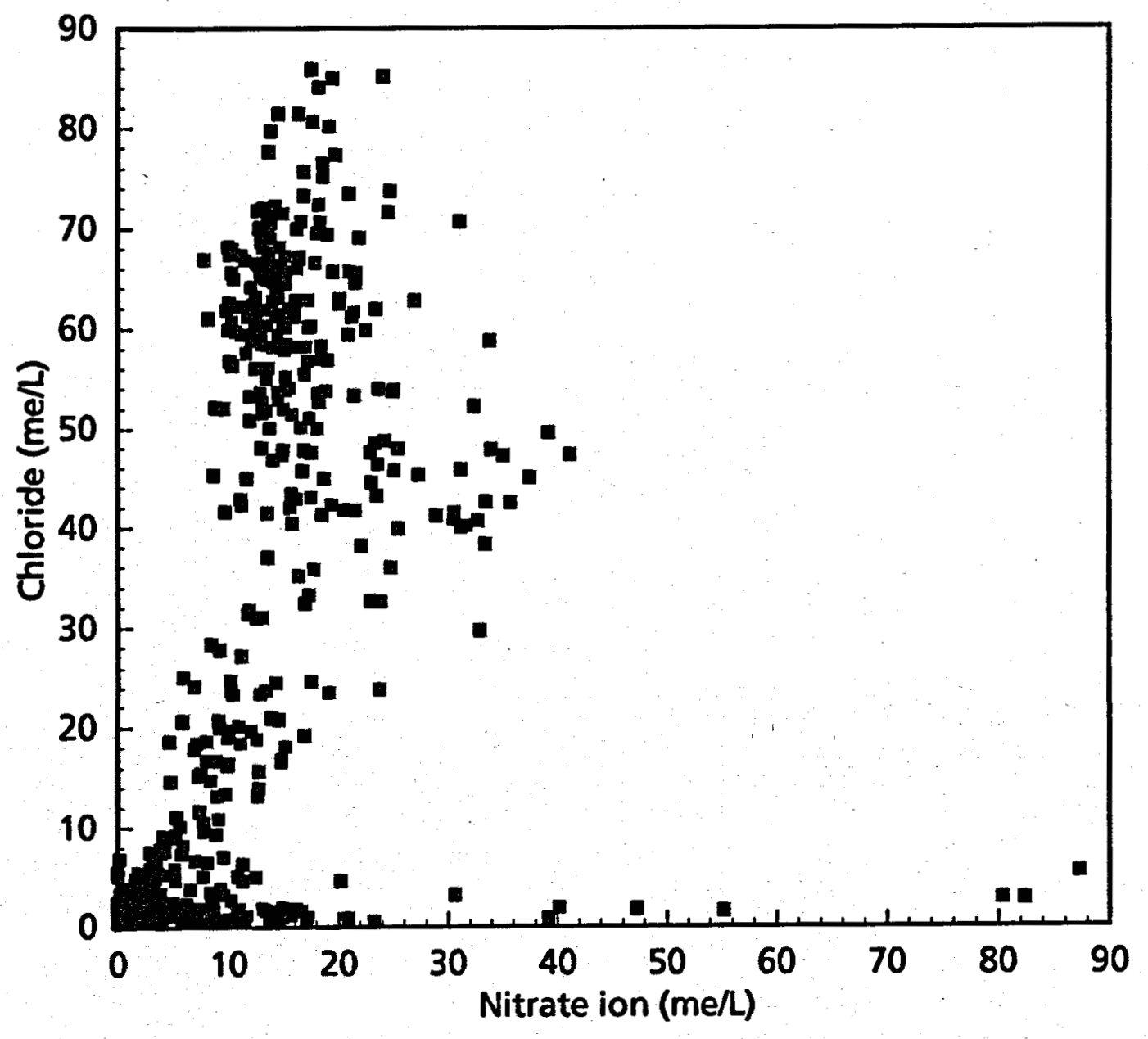

Figure 65. Experiment IIb redistribution cores chloride ion vs nitrate ion scatter plot for combined $\mathrm{Y}_{2}=5 \mathrm{~m}$ and $9 \mathrm{~m}$ observations. Concentration expressed as $\mathrm{me} / \mathrm{L}$ of soil solution. 



\section{Conclusions}

This report describes the third and final experiment conducted at the Las Cruces Trench site under this contract. The goal of this and the previous two experiments was to provide a data set that could be used by modelers and others, for checking existing and future simulation codes for soil water and solute transport. Moreover, the site characterization activities carried out for these experiments have taught us about some of the limitations in our ability to characterize deep soil profiles, and to understand how water and solute move under unsaturated conditions.

As described in Wierenga et al. (1988, 1989, 1990 , and 1991), site characterization activities at the trench consisted of extensive field sampling, with subsequent in-situ field determination of saturated hydraulic conductivity. Laboratory experiments for determining the conductivity and tension relationships were performed on undisturbed samples collected from the field. Solute transport experiments were conducted for understanding dispersion properties of the soil from the site.

Any study undertaken for characterizing a site for LLW disposal, or possibly for hazardous waste disposal, must balance the need for collecting data in-situ, with associated higher costs and less controlled field conditions, against inherent problems associated with taking soil samples into the laboratory for analysis. Though the laboratory experiments may provide a cleaner data set, applying the results to the field always leads to difficulties in describing spatial variability of a soil profile with point samples. The experiments conducted at the Las Cruces Trench site, both the characterization studies and the transient field infiltration experiments, address many of the common uncertainties associated with site characterization activities. Field saturated hydraulic conductivity results were compared to laboratory conductivities. Retention curves derived from the laboratory experiments were used in simulation models, prior to initiating Experiments IIa and IIb, to predict wetting front movement for possible flux rates. Collection of tension data from the tensiometers installed in the wall of the trench can be used in tandem with the water contents from the neutron probe readings taken less than 1.5 meters away, along the same horizontal plane. Thus, we can confirm the laboratory retention curves to actual paired values of water content and water tension collected in the field. Other relationships between field and laboratory activities can be described as well.

The Las Cruces Trench studies have provided modelers with infiltration data for simulating soil water and solute transport in the soil. The site characterization activities, conducted before the experiments began, have provided researchers with data for studying the spatial variability of soil hydraulic properties in an undisturbed soil profile. Site characterization studies conducted in preparation for license applications for LLW disposal should use the lessons learned from the trench studies. Those are 1) the need to collect data on soil hydraulic properties using more than one approach, and 2) the need to design a monitoring program that enhances the data set developed during site characterization. These lessons learned may reduce the uncertainties that arise during license review or actual site monitoring. They will also lead to a more coherent picture of potential physical processes that can be expected at the site during and after disposal of waste material. 


\section{References}

Bowman, R.S. 1984a. Analysis of soil extracts for inorganic and organic tracer anions via High-Performance Liquid Chromatography. J. Chrom. 285:467-477.

Bowman, R.S. 1984b. Evaluation of some new tracers for soil water studies. Soil Sci. Soc. Am. J. 48:987-993.

Christiansen, J.E. 1942. Irrigation by sprinkling. Calif. Agri. Exp. Sta. Bull. 670. 124 pp.

Gile, L.H. and R.B. Grossman. 1979. The Desert Project soil monograph. USDA-SCS Doc. No. PB80-135304. Nat'l. Tech. Inf. Serv., Springfield, VA.

Hills, R.G. and P.J.Wierenga. 1991. Model validation at the Las Cruces trench site. NUREG/CR-5716. U.S. Nuclear Regulatory Commission. Washington, D.C.

Hills, R.G. and P.J. Wierenga. 1993. (with contributions from S. Luis, M. Rockhold, J. Xiang, B. Scanlon and G. Wittmeyer). Intraval Phase II. Model testing of the Las Cruces Trench site. NUREG/CR-6063, U.S. Nuclear Regulatory Commission, Washington, D.C.

Klein, R.C. and E.L. Gershey. 1990. "Biodegradable" liquid scintillation counting cocktails. Health Physics 59:461-470.
Nash, M.H.H. 1990. The effect of anisotropy on selected soil properties in a deep alluvial soil. Ph.D. dissertation. Dept. Agron. and Hort. New Mexico State University, Las Cruces, New Mexico.

Wierenga, P.J., J.M. Hendrickx, M.H. Nash, J. Ludwig, and L.A. Daugherty. 1987. Variation of soil and vegetation with distance along a transect in the Chihuahuan Desert. J. of Arid Environments 13:53-63.

Wierenga, P.J., D. Bachelet, J.R. Bilskie, H. Elabd, D.B. Hudson, M. Nash, I. Porro, W.R. Strong, A. Toorman, and J. Vinson. 1988. Validation of stochastic flow and transport models for unsaturated soils. Research Report 88-SS-03. Dept. of Agronomy and Horticulture, New Mexico State University, Las Cruces.

Wierenga, P. J., D.B. Hudson, J. Vinson, M. Nash, A. Toorman, and R.G. Hills, 1989. Soil physical properties at the Las Cruces Trench Site. NUREG/CR-5441, U.S. Nuclear Regulatory Commission. Washington, D.C.

Wierenga, P. J., D.B. Hudson, R.G. Hills, I. Porro, M.R. Kirkland, and J. Vinson. 1990. Flow and transport at the Las Cruces trench site: Experiments 1 and 2. NUREG/CR-5607 U.S. Nuclear Regulatory Commission. Washington, D.C.

Wierenga, P. J., R.G. Hills, and D.B.Hudson. 1991. The Las Cruces trench site:

Characterization, experimental results, and one-dimensional flow predictions. Water Resour. Res. 27:2695-2705. 
Appendix A. Experiment IIb Data Base Tables 
Appendix A-1. Experiment IIb Water and Solute Application Record.

\begin{tabular}{|c|c|c|c|c|c|c|c|c|c|}
\hline $\begin{array}{l}\text { Timevalue } \\
\text { (deys) }\end{array}$ & $\begin{array}{l}\text { Cumulative } \\
\text { Totallzer } \\
\text { (ㄴ) }\end{array}$ & $\begin{array}{l}\text { Increment } \\
\text { Pate } \\
\text { (cm/d) }\end{array}$ & $\begin{array}{l}\text { Mean } \\
\text { Rate } \\
(\mathrm{cm} / \mathrm{d})\end{array}$ & $\begin{array}{l}\text { PFBA } \\
\text { (g) }\end{array}$ & $\begin{array}{c}B \\
\text { (g) }\end{array}$ & $\begin{array}{l}C r \\
(g)\end{array}$ & $\begin{array}{c}\text { Trit } \\
\text { (uCl) }\end{array}$ & $\begin{array}{l}\text { DFBA } \\
\text { (g) }\end{array}$ & $\begin{array}{l}B r \\
\text { (g) }\end{array}$ \\
\hline 0.13 & 32.9 & 1.82 & 1.83 & 0.40 & 0.38 & 0.16 & 0 & 0 & 0 \\
\hline 0.25 & 64.7 & 1.77 & 1.80 & 0.80 & 0.75 & 0.31 & 0 & 0 & 0 \\
\hline 0.38 & 96.5 & 1.77 & 1.79 & 1.19 & 1.12 & 0.47 & 0 & 0 & 0 \\
\hline 0.63 & 160.1 & 1.77 & 1.78 & 1.97 & 1.86 & 0.77 & 0 & 0 & 0 \\
\hline 0.75 & 181.8 & 1.77 & 1.78 & 2.36 & 2.23 & 0.93 & 0 & 0 & 0 \\
\hline 0.88 & 223.3 & 1.75 & 1.77 & 2.75 & 2.59 & 1.08 & 0 & 0 & 0 \\
\hline 1.00 & 254.7 & 1.75 & 1.77 & 3.13 & 2.96 & 1.23 & 0 & 0 & 0 \\
\hline 1.13 & 321.3 & 3.70 & 1.98 & 3.95 & 3.73 & 1.56 & 0 & 0 & 0 \\
\hline 1.88 & 513.6 & 1.78 & 1.90 & 6.32 & 5.96 & 2.49 & 0 & 0 & 0 \\
\hline 2.00 & 546.6 & 1.83 & 1.90 & 6.72 & 6.35 & 2.65 & 0 & 0 & 0 \\
\hline 2.13 & 578.7 & 1.79 & 1.89 & 7.12 & 6.72 & 2.80 & 0 & 0 & 0 \\
\hline 2.88 & 771.4 & 1.78 & 1.86 & 9.49 & 8.96 & 3.73 & 0 & 0 & 0 \\
\hline 4.00 & 1060.6 & 1.79 & 1.84 & 13.05 & 12.31 & 5.13 & 0 & 0 & 0 \\
\hline 4.75 & 1253.2 & 1.78 & 1.83 & 15.41 & 14.55 & 6.07 & 0 & 0 & 0 \\
\hline 4.88 & 1285.8 & 1.81 & 1.83 & 16.82 & 14.93 & 6.22 & 0 & 0 & 0 \\
\hline 5.00 & 1318.7 & 1.83 & 1.83 & 16.22 & 15.31 & 6.38 & 0 & 0 & 0 \\
\hline 6.13 & 1351.6 & 1.83 & 1.83 & 16.62 & 15.69 & 6.54 & 0 & 0 & 0 \\
\hline 6.00 & 1579.1 & 1.81 & 1.83 & 19.42 & 18.33 & 7.64 & 0 & 0 & 0 \\
\hline 6.13 & 1610.8 & 1.77 & 1.83 & 19.81 & 18.70 & 7.80 & 0 & 0 & 0 \\
\hline 6.88 & 1809.2 & 1.84 & 1.83 & 22.25 & 21.00 & 8.76 & 0 & 0 & 0 \\
\hline 7.00 & 1842.2 & 1.83 & 1.83 & 22.66 & 21.39 & 8.82 & 0 & 0 & 0 \\
\hline 7.13 & 1876.1 & 1.83 & 1.83 & 23.06 & 21.77 & 9.08 & 0 & 0 & 0 \\
\hline 7.25 & 1907.6 & 1.81 & 1.83 & 23.46 & 22.16 & 0.23 & 0 & 0 & 0 \\
\hline 7.88 & 2031.0 & 1.37 & 1.79 & 24.98 & 23.58 & 0.83 & 0 & 0 & 0 \\
\hline 8.00 & 2064.0 & 1.83 & 1.79 & 25.39 & 23.96 & 9.99 & 0 & 0 & 0 \\
\hline 8.13 & 2096.8 & 1.83 & 1.79 & 25.79 & 24.35 & 10.15 & 0 & 0 & 0 \\
\hline 9.00 & 2327.4 & 1.83 & 1.80 & 28.63 & 27.02 & 11.26 & 0 & 0 & 0 \\
\hline 0.13 & 2360.3 & 1.83 & 1.80 & 29.03 & 27.40 & 11.42 & 0 & 0 & 0 \\
\hline 10.00 & 2590.1 & 1.82 & 1.80 & 31.86 & 30.07 & 12.54 & 0 & 0 & 0 \\
\hline 11.88 & 3053.4 & 1.72 & 1.79 & 37.56 & 35.45 & 14.78 & 0 & 0 & 0 \\
\hline 12.00 & 3085.5 & 1.79 & 1.79 & 37.95 & 35.82 & 14.93 & 0 & 0 & 0 \\
\hline$\cdot 12.13$ & 3118.5 & 1.83 & 1.79 & 38.36 & 36.21 & 15.09 & 0 & 0 & 0 \\
\hline 13.75 & 3546.5 & 1.83 & 1.79 & 43.62 & 41.17 & 17.17 & 0 & 0 & 0 \\
\hline 13.88 & 3579.5 & 1.83 & 1.79 & 44.03 & 41.56 & 17.32 & 0 & 0 & 0 \\
\hline 14.00 & 3612.8 & 1.85 & 1.78 & 44.44 & 41.94 & 17.49 & 0 & 0 & 0 \\
\hline 15.00 & 3847.1 & 1.63 & 1.78 & 47.32 & 44.66 & 18.62 & 0 & 0 & 0 \\
\hline 15.13 & 3878.6 & 1.81 & 1.78 & 47.32 & 44.66 & 18.62 & 0 & 0 & 0 \\
\hline 15.88 & 4077.6 & 1.83 & 1.78 & 47.32 & 44.66 & 18.62 & 0 & 0 & 0 \\
\hline 16.00 & 4110.5 & 1.83 & 1.78 & 47.32 & 44.66 & 18.62 & 0 & 0 & 0 \\
\hline 16.88 & 4341.4 & 1.83 & 1.79 & 47.32 & 44.66 & 18.62 & 0 & 0 & 0 \\
\hline 17.00 & 4374.3 & 1.83 & 1.79 & 47.32 & 44.66 & 18.62 & 0 & 0 & 0 \\
\hline 17.13 & 4407.6 & 1.85 & 1.79 & 47.32 & 44.66 & 18.62 & 0 & 0 & 0 \\
\hline 19.00 & 4902.3 & 1.83 & 1.79 & 47.32 & 44.66 & 18.62 & 0 & 0 & 0 \\
\hline 19.76 & 5100.3 & 1.83 & 1.79 & 47.32 & 44.66 & 18.62 & 0 & 0 & 0 \\
\hline 20.00 & 5172.2 & 2.00 & 1.80 & 47.32 & 44.66 & 18.62 & 0 & 0 & 0 \\
\hline 20.88 & 5470.8 & 2.37 & 1.82 & 47.32 & 44.66 & 18.62 & 0 & 0 & 0 \\
\hline 21.00 & 5512.5 & 2.31 & 1.82 & 47.32 & 44.66 & 18.62 & 0 & 0 & 0 \\
\hline 21.13 & 6545.4 & 1.83 & 1.82 & 47.32 & 44.66 & 18.62 & 0 & 0 & 0 \\
\hline 21.38 & 5611.3 & 1.83 & 1.82 & 47.32 & 44.66 & 18.62 & 0 & 0 & 0 \\
\hline 23.00 & 6038.6 & 1.83 & 1.82 & 47.32 & 44.66 & 18.62 & 0 & 0 & 0 \\
\hline 23.13 & 6071.1 & 1.81 & 1.82 & 47.32 & 44.66 & 18.62 & 0 & 0 & 0 \\
\hline
\end{tabular}


Appendix A-1. Experiment IIb Water and Solute Application Record. (Continued)

\begin{tabular}{|c|c|c|c|c|c|c|c|c|c|}
\hline $\begin{array}{l}\text { Timevalue } \\
\text { (daye) }\end{array}$ & $\begin{array}{c}\text { Cumulative } \\
\text { Totallzer } \\
\text { (L) }\end{array}$ & $\begin{array}{c}\text { Increment } \\
\text { Rate } \\
\text { (cm/d) }\end{array}$ & $\begin{array}{l}\text { Mean } \\
\text { Rate } \\
(\mathrm{cm} / \mathrm{d})\end{array}$ & $\begin{array}{l}\text { PFBA } \\
(g)\end{array}$ & $\begin{array}{c}B \\
(g)\end{array}$ & $\begin{array}{l}C r \\
(\theta)\end{array}$ & $\begin{array}{l}\text { Trit } \\
\text { (uCl) }\end{array}$ & $\begin{array}{l}\text { DFBA } \\
\text { (g) }\end{array}$ & $\begin{array}{l}B r \\
(g)\end{array}$ \\
\hline 23.75 & 6234.7 & 1.82 & 1.82 & 47.32 & 44.66 & 18.62 & 0 & 0 & 0 \\
\hline 23.88 & 6267.2 & 1.81 & 1.82 & 47.32 & 44.66 & 18.62 & 0 & 0 & 0 \\
\hline 26.00 & 6819.8 & 1.81 & 1.82 & 47.32 & 44.66 & 18.62 & 0 & 0 & 0 \\
\hline 26.13 & 6852.7 & 1.83 & 1.82 & 47.32 & 44.66 & 18.62 & 0 & 0 & 0 \\
\hline 26.88 & 7048.0 & 1.81 & 1.82 & 47.32 & 44.66 & 18.62 & 0 & 0 & 0 \\
\hline 28.00 & 7341.4 & 1.81 & 1.82 & 47.32 & 44.66 & 18.62 & 0 & 0 & 0 \\
\hline 28.13 & 7374.3 & 1.83 & 1.82 & 47.32 & 44.66 & 18.62 & 0 & 0 & 0 \\
\hline 28.88 & 7569.6 & 1.81 & 1.82 & 47.32 & 44.66 & 18.62 & 0 & 0 & 0 \\
\hline 29.00 & & 1.85 & 1.82 & 47.32 & 44.66 & 18.62 & 0 & 0 & 0 \\
\hline 29.13 & & 1.81 & 1.82 & 47.32 & 44.66 & 18.62 & 3260 & 0.33 & 0.98 \\
\hline 30.00 & 7865.2 & 1.82 & 1.82 & 47.32 & 44.66 & 18.62 & 26230 & 2.62 & $7 . \overline{87}$ \\
\hline 30.13 & 789 & 1.83 & 1.82 & 47.32 & 44.66 & 18.62 & 29530 & 2.85 & 8.86 \\
\hline 32.88 & 8620.7 & 1.82 & 1.82 & 47.32 & 44.66 & 18.62 & 101780 & 10.18 & 30.53 \\
\hline 33.00 & & 1.83 & 1.82 & & 44.66 & & 105070 & 10.61 & 31.62 \\
\hline 33.13 & 8686.6 & 1.83 & 1.82 & 47.32 & 44.66 & 18.62 & 108370 & 10.84 & 32.61 \\
\hline 34.13 & 8950.0 & 1.83 & 1.82 & 47.32 & 44.66 & 18.62 & 134710 & 13.47 & 40.41 \\
\hline 35.00 & 0180.9 & 1.83 & 1.82 & 47.32 & 44.66 & 18.62 & 157800 & 16.78 & 47.34 \\
\hline 35.13 & 9214.2 & 1.85 & 1.82 & & 44.66 & & 161130 & 16.11 & 48.34 \\
\hline 37.00 & 9708.1 & 1.83 & 1.82 & 47.32 & 44.66 & 18.62 & 210520 & 21.05 & 63.16 \\
\hline 37.13 & 0741.1 & 1.8 & 1.82 & 47.32 & 44.66 & 18.62 & 213820 & 21.38 & 64.16 \\
\hline 37.88 & 9938.7 & 1.83 & 1.82 & 47 & 44.66 & 18.62 & 233580 & 23.36 & 70.07 \\
\hline 38.00 & & 1.83 & 1.82 & & 44. & & 236870 & 23.69 & 71.06 \\
\hline 40.00 & 10498.1 & 1.83 & 1.82 & 47.32 & 44.66 & 18.62 & 289520 & 28.95 & 86.86 \\
\hline 40.13 & 1.0 & 1.8 & 1.82 & 47. & 44.66 & 18. & 292810 & 29.28 & 87.84 \\
\hline 42.00 & & 1.83 & 1.82 & 47.32 & 44.66 & 18.62 & 342170 & 34.22 & 102.65 \\
\hline 42.13 & & 1.8 & 1.82 & 47.32 & 44.66 & 18.62 & 345460 & 34.65 & 103.64 \\
\hline & & 1.8 & & & 44. & 18. & 3947 & 39.47 & 118.42 \\
\hline 4.88 & 11 & 1.8 & 1.8 & 47.32 & 44.66 & 18.62 & 394740 & 39.47 & $118.4 \overline{2}$ \\
\hline 47.00 & & 1.8 & 1.82 & 47.32 & 44.66 & 18.62 & 394740 & 39.47 & 118.42 \\
\hline 47.13 & & 1.83 & 1.82 & & & 18.62 & $394 i$ & 39.47 & 118.42 \\
\hline 49.13 & 18 & 1.83 & 1.82 & 47.32 & 44.66 & 18.62 & 394740 & 39.47 & 118.42 \\
\hline .88 & & 1.84 & 1.82 & 47.32 & 4.66 & 18.62 & 394740 & 39.47 & 118.42 \\
\hline & & 1. & 1.8 & 47. & 6 & 18.62 & 384740 & 39. & 118.42 \\
\hline & & & 1.83 & 47. & & & & & 118.42 \\
\hline 4.13 & & & 1.83 & 47.32 & 44.66 & 18.62 & 394740 & 39.47 & 118.42 \\
\hline 56.00 & & & 1.83 & 47.32 & 44.66 & 18. & 394740 & 39.47 & 118.42 \\
\hline & & & & & 44.66 & 18.62 & 394740 & 39.47 & 118.42 \\
\hline .88 & & 1. & 1.83 & 47. & 1.66 & 18 & 394740 & 39.47 & 118.42 \\
\hline 3.88 & & 1. & 1.83 & 47.32 & 44.66 & 18.62 & 394740 & 39.47 & 118.42 \\
\hline & & & & & & & & 39.47 & 118.42 \\
\hline & & & & & 44.66 & 18.62 & 394740 & 39.47 & 118.42 \\
\hline & & & 1. & & & & & 39.47 & 118.42 \\
\hline 65.00 & & 1.84 & 1.83 & 47.32 & 44.66 & 18.62 & 394740 & 39.47 & 118.42 \\
\hline 65.13 & 17128.3 & 1.83 & 1.83 & 47.32 & 44.66 & 18.62 & 394740 & 39.47 & 118.42 \\
\hline & & & & & & & & & \\
\hline & & 1.83 & & 47.32 & 44.66 & 18.62 & 394740 & 39.47 & 118.42 \\
\hline & & 1.8 & & & & 18. & 394740 & 39.47 & 118.42 \\
\hline 66.25 & 17426.1 & 1.83 & 1.83 & 47.32 & 44.66 & 18.62 & 394740 & 39.47 & 118.42 \\
\hline 68.13 & 17883.7 & 1.69 & 1.82 & 47.32 & 44.66 & 18.62 & 394740 & 39.47 & 118.42 \\
\hline 70.00 & 18378.8 & 1.83 & 1.82 & 47.32 & 44.66 & 18.62 & 394740 & 39.47 & 118.42 \\
\hline
\end{tabular}


Appendix A-2. Example of Neutron Probe Soil Moisture Data.

\begin{tabular}{|c|c|c|c|c|c|c|c|c|c|c|c|}
\hline ay & Tube & $\mathrm{Z}_{2}$ & Theta & Day & Tube & $\mathrm{z}_{2}$ & Theta & Day & & $\mathrm{Z}_{2}$ & Theta \\
\hline 525 & 60 & 3.00 & 0.2018 & 525 & 71 & 3.25 & 0.1923 & 525 & 90 & 2.50 & 0.1084 \\
\hline 525 & 60 & 3.25 & 0.2099 & 525 & 71 & 3.50 & 0.1455 & 525 & 90 & 2.75 & 0.1172 \\
\hline 525 & 60 & 3.50 & & 525 & 71 & 3.75 & 0.0875 & 525 & 90 & 3.00 & .228 \\
\hline 525 & 60 & 3.75 & & 525 & 71 & 4.00 & 53 & 525 & 90 & 3.25 & .2276 \\
\hline 525 & 60 & 4.00 & 0.1513 & 525 & 71 & 4.25 & 0.0509 & 525 & 90 & 3.50 & 0.2032 \\
\hline 525 & 60 & 4.25 & 0.1421 & 525 & 71 & 4.50 & 0.0916 & 525 & 90 & 3.75 & 0.1565 \\
\hline 525 & 60 & 4.50 & 0.1824 & 525 & 71 & 4.75 & 0.1500 & 525 & 90 & 4.00 & 0.1756 \\
\hline 525 & 60 & 4.75 & 0.1744 & 525 & 71 & 5.00 & 0.0998 & 525 & 90 & & 0.145 \\
\hline 525 & 60 & 5.00 & 20 & 525 & 71 & 5.25 & 0.0768 & 525 & 90 & 50 & 0.172 \\
\hline 525 & 60 & 5.25 & 17 & 525 & 71 & 5.50 & & 525 & 90 & 4.75 & .1425 \\
\hline 525 & 70 & 0.25 & 0.1088 & 525 & 71 & 5.75 & 0.0628 & 525 & 90 & 5.00 & 0.1023 \\
\hline 525 & 70 & 0.50 & $0: 1412$ & 525 & 71 & 6.00 & 0.0716 & 525 & 90 & 5.25 & 0.0506 \\
\hline 525 & 70 & 0.75 & 0.1686 & 525 & 80 & 0.25 & 0.1060 & 525 & 90 & & 0.0313 \\
\hline 525 & 70 & 1.00 & 0.1393 & 525 & 80 & 0.50 & & 525 & 90 & 5.75 & 0.027 \\
\hline 525 & 70 & 1.25 & 1212 & 525 & 80 & 0.75 & 0.18 & 525 & 90 & 6.00 & .057 \\
\hline 525 & 70 & 1.50 & & 525 & 80 & 1.00 & & 525 & 100 & 0.25 & 017 \\
\hline 525 & 70 & 1.75 & & 525 & 80 & 1.25 & & 525 & 100 & 0.50 & 1331 \\
\hline 525 & 70 & 2.00 & 0.1286 & 525 & 80 & 1.50 & 73 & 525 & 100 & 0.75 & 0.1526 \\
\hline 525 & 70 & 2.25 & & 525 & 80 & 1.75 & & 525 & 100 & .1 .00 & 0.1427 \\
\hline 525 & 70 & 2.50 & & 525 & 80 & 2.00 & & 525 & 100 & 1.25 & 0.1384 \\
\hline 525 & 70 & 2.75 & 0.1089 & 525 & 80 & 2.25 & 0.0832 & 525 & 100 & 1.50 & 0.127 \\
\hline 25 & 70 & 3.00 & 2041 & 525 & 80 & 2.50 & & 525 & 100 & 1.75 & 129 \\
\hline 525 & 70 & 3.25 & 0.2278 & 525 & 80 & 2.75 & & 525 & 100 & 2.00 & 186 \\
\hline 525 & 70 & 3.50 & 0.1889 & 525 & 80 & 3.00 & 0.21 & 525 & 100 & 2.25 & .1085 \\
\hline 525 & 70 & 3.75 & & 525 & 80 & 3.25 & 0.2275 & 525 & 100 & 2.50 & 0.0834 \\
\hline 525 & 70 & 4.00 & 1005 & 525 & 80 & 3.50 & 0.198 & 525 & 100 & 2.75 & 0.095 \\
\hline 525 & 70 & 4.25 & & 525 & 80 & 3.75 & & 525 & 100 & 3.00 & .2016 \\
\hline 525 & 70 & 4.50 & & 525 & 80 & 4.00 & & 525 & 100 & 3.25 & 0.2222 \\
\hline 525 & 70 & 4.75 & & 525 & 80 & 4.25 & & 525 & 100 & 3.50 & 1465 \\
\hline 525 & 70 & 5.00 & & 525 & 80 & 4.50 & 0.1846 & 525 & 100 & 3.75 & 0.1126 \\
\hline 525 & 70 & 5.25 & & 525 & 80 & 4.75 & 0.1382 & 525 & 100 & 4.00 & 0.1505 \\
\hline 525 & 70 & 5.50 & & 525 & 80 & 5.00 & & 525 & 100 & 4.25 & 335 \\
\hline 525 & 70 & 5.75 & & 525 & 80 & 5.25 & & 525 & 100 & 4.50 & 468 \\
\hline 525 & 71 & 0.25 & & 525 & 80 & 5.50 & & 525 & 100 & 4.75 & 0.1308 \\
\hline 525 & 71 & 0.50 & & 525 & 80 & 5.75 & & 525 & 100 & 5.00 & 0.1052 \\
\hline 525 & 71 & 0.75 & 397 & 525 & 80 & 6.00 & 0.0642 & 525 & 110 & 0.25 & 0.1122 \\
\hline 525 & 71 & 1.00 & & 525 & 90 & 0.25 & 0.10 & 525 & 110 & 0.50 & 0.1217 \\
\hline 525 & 71 & 1.25 & & 525 & 90 & 0.50 & & 525 & 110 & 0.75 & 0.1315 \\
\hline 525 & 71 & 1.50 & & 525 & 90 & 0.75 & 0.1464 & 525 & 110 & 1.00 & 0.1110 \\
\hline 525 & 71 & 1.75 & 0.1177 & 525 & 90 & 1.00 & 0.1527 & 525 & 110 & 1.25 & 0.0896 \\
\hline 525 & 71 & 2.00 & & 525 & 90 & 1.25 & & 525 & 110 & 1.50 & 0.0666 \\
\hline 2 & 71 & 2.25 & & 525 & 90 & 1.50 & & 525 & 110 & 1.75 & 0.0625 \\
\hline 525 & 71 & 2.50 & & 525 & 90 & 1.75 & 0.0889 & 525 & 110 & 2.00 & 0.0335 \\
\hline 525 & 71 & 2.75 & 0.1024 & 525 & 90 & 2.00 & 0.1004 & 525 & 110 & 2.25 & 0.0443 \\
\hline 525 & 71 & 3.00 & 0.2133 & 525 & 90 & 2.25 & 0.1047 & 525 & 110 & 2.50 & 0.1107 \\
\hline
\end{tabular}


Appendix A-3. Experiment IIb Tensiometer Soil Moisture Data.

\begin{tabular}{|c|c|c|c|c|c|c|c|c|c|c|c|}
\hline Day & $\begin{array}{c}\text { Tens } \\
\text { D }\end{array}$ & $\begin{array}{l}\mathrm{cm}- \\
\mathrm{H}_{2} \mathrm{O}\end{array}$ & Day & $\begin{array}{l}\text { Tens } \\
\text { D }\end{array}$ & $\begin{array}{l}\mathrm{cm}- \\
\mathrm{H}_{2} \mathrm{O}\end{array}$ & Day & $\begin{array}{c}\text { Tens } \\
\mathbb{D}\end{array}$ & $\begin{array}{l}\mathrm{Cm}- \\
\mathrm{H}_{2} \mathrm{O}\end{array}$ & Day & $\begin{array}{l}\text { Tens } \\
\text { ID }\end{array}$ & $\begin{array}{c}\text { cm- } \\
\mathrm{H}_{2} \mathrm{O}\end{array}$ \\
\hline 0 & 142 & $4 \overline{2}$ & 2 & 142 & 345 & 2 & 1248 & 220 & 5 & 356 & 259 \\
\hline 0 & 144 & 253 & 2 & 144 & 114 & 2 & 1250 & 263 & 5 & 358 & 315 \\
\hline 0 & 146 & 513 & 2 & 146 & 420 & 2 & 1252 & 299 & 5 & 642 & 483 \\
\hline 0 & 148 & 405 & 2 & 148 & 19 & 2 & 1254 & 254 & 5 & 644 & 400 \\
\hline 0 & 150 & 432 & 2 & 150 & 26 & 2 & 1256 & 332 & 5 & 646 & 376 \\
\hline 0 & 152 & 190 & 2 & 152 & 37 & 2 & 1258 & 209 & 5 & 648 & 355 \\
\hline 0 & 154 & 117 & 2 & 154 & 73 & 2 & 1442 & 434 & 5 & 650 & 317 \\
\hline 0 & 156 & 249 & 2 & 156 & 106 & 2 & 1444 & 471 & 5 & 652 & 245 \\
\hline 0 & 158 & 518 & 2 & 158 & 396 & 2 & 1446 & 331 & 5 & 654 & 283 \\
\hline 0 & 344 & 493 & 2 & 342 & 393 & 2 & 1448 & 346 & 5 & 656 & 276 \\
\hline 0 & 346 & 392 & 2 & 344 & 410 & 2 & 1450 & 365 & 5 & 658 & 273 \\
\hline 0 & 348 & 346 & 2 & 346 & 274 & 2. & 1452 & 443 & 5 & 844 & 379 \\
\hline 0 & 350 & 307 & 2 & 348 & 194 & 2 & 1454 & 495 & 5 & 846 & 341 \\
\hline 0 & 352 & 278 & 2 & 350 & 241 & 2 & 1456 & 546 & 5 & 848 & 312 \\
\hline 0 & 354 & 268 & 2 & 352 & 249 & 2 & 1458 & 612 & 5 & 850 & 303 \\
\hline 0 & 356 & 288 & 2. & 354 & 250 & 2 & 1642 & 527 & 5 & 852 & 279 \\
\hline 0 & 358 & 338 & 2 & $356^{-}$ & 269 & 2 & 1644 & 509 & 5 & 854 & 293 \\
\hline 0 & 642 & 485 & 2 & 642 & 435 & 2 & 1646 & 406 & 5 & 856 & 301 \\
\hline 0 & 644 & 415 & 2 & 644 & 388 & 2 & 1648 & 369 & 5 & 858 & 360 \\
\hline 0 & 646 & 376 & 2 & 646 & 313 & 2 & 1650 & 402 & 5 & 1044 & 376 \\
\hline 0 & 648 & 329 & 2 & 648 & 301 & 2 & 1652 & 462 & 5 & 1046 & 345 \\
\hline 0 & 650 & 270 & 2 & 650 & 230 & 2 & 1654 & 548 & 5 & 1048 & 320 \\
\hline 0 & 652 & 284 & 2 & 652 & 134 & 2 & 1656 & 599 & 5 & 1050 & 319 \\
\hline 0 & 654 & 284 & 2 & 654 & 260 & 2 & 1658 & 587 & 5 & 1052 & 306 \\
\hline 0 & 656 & 271 & 2 & 656 & 241 & 2 & 1842 & 572 & 5 & 1054 & 301 \\
\hline 0 & 658 & 269 & 2 & 658 & 236 & 2 & 1848 & 602 & 5 & 1056 & 343 \\
\hline 0 & 844 & 376 & 2 & 842 & 75 & 2 & 1850 & 569 & 5 & 1058 & 149 \\
\hline 0 & 846 & 347 & 2 & 844 & 347 & 2 & 1852 & 611 & 5 & 1242 & 435 \\
\hline 0 & 848 & 322 & 2 & 846 & 321 & 2 & 1854 & 513 & 5 & 1244 & 397 \\
\hline 0 & 850 & 307 & 2 & 848 & 289 & 2 & 1856 & 551 & 5 & 1246 & 368 \\
\hline 0 & 852 & 275 & 2 & 850 & 287 & 2 & 1858 & 635 & 5 & 1248 & 323 \\
\hline 0 & 854 & 301 & 2 & 852 & 271 & 5 & 142 & 401 & 5 & 1250 & 314 \\
\hline 0 & 856 & 297 & 2 & 854 & 284 & 5 & 144 & 232 & 5 & 1252 & 306 \\
\hline 0 & 858 & 357 & 2 & 856 & 143 & 5 & 146 & 136 & 5 & 1254 & 324 \\
\hline 0 & 1044 & 377 & 2 & 858 & 295 & 5 & 148 & 53 & 5 & 1256 & 353 \\
\hline 0 & 1046 & 355 & 2 & 1042 & 67 & 5 & 150 & 17 & 5 & 1442 & 606 \\
\hline 0 & 1048 & 323 & 2 & 1044 & 361 & 5 & 152 & 19 & 5 & 1444 & 571 \\
\hline 0 & 1050 & 317 & 2 & 1046 & 325 & 5 & 154 & 32 & & 14 & \\
\hline 0 & 1052 & 315 & 2 & 1048 & 305 & 5 & 156 & 231 & 5 & 1448 & 415 \\
\hline 0 & 1054 & 301 & 2 & 1050 & 222 & 5 & 158 & 371 & 5 & 1450 & 478 \\
\hline 0 & 1058 & 455 & 2 & 1052 & 296 & 5 & 342 & 536 & 5 & 1452 & 547 \\
\hline 0 & 1248 & 321 & 2 & 1054 & 287 & 5 & 344 & 493 & 5 & 1454 & 626 \\
\hline 0 & 1250 & 311 & 2 & 1056 & 254 & 5 & 346 & 353 & 5 & 1456 & 716 \\
\hline 0 & 1252 & 301 & 2 & 1058 & 382 & 5 & 348 & 33 & 5 & 1642 & 646 \\
\hline 0 & 1444 & 590 & 2 & 1242 & 337 & 5 & 350 & 50 & 5 & 1644 & 634 \\
\hline 0 & 1446 & 485 & 2 & 1244 & 362 & 5 & 352 & 56 & 5 & 1646. & 537 \\
\hline 0 & 1448 & 456 & 2 & 1246 & 307. & 5 & 354 & 180 & 5 & 1648 & 485 \\
\hline
\end{tabular}


Appendix A-4. Experiment IIb Tritium Data.

Day Sampl. Rel. Day Sampl. Rel Day Sampl. Rel. Day Sampl. Rel.

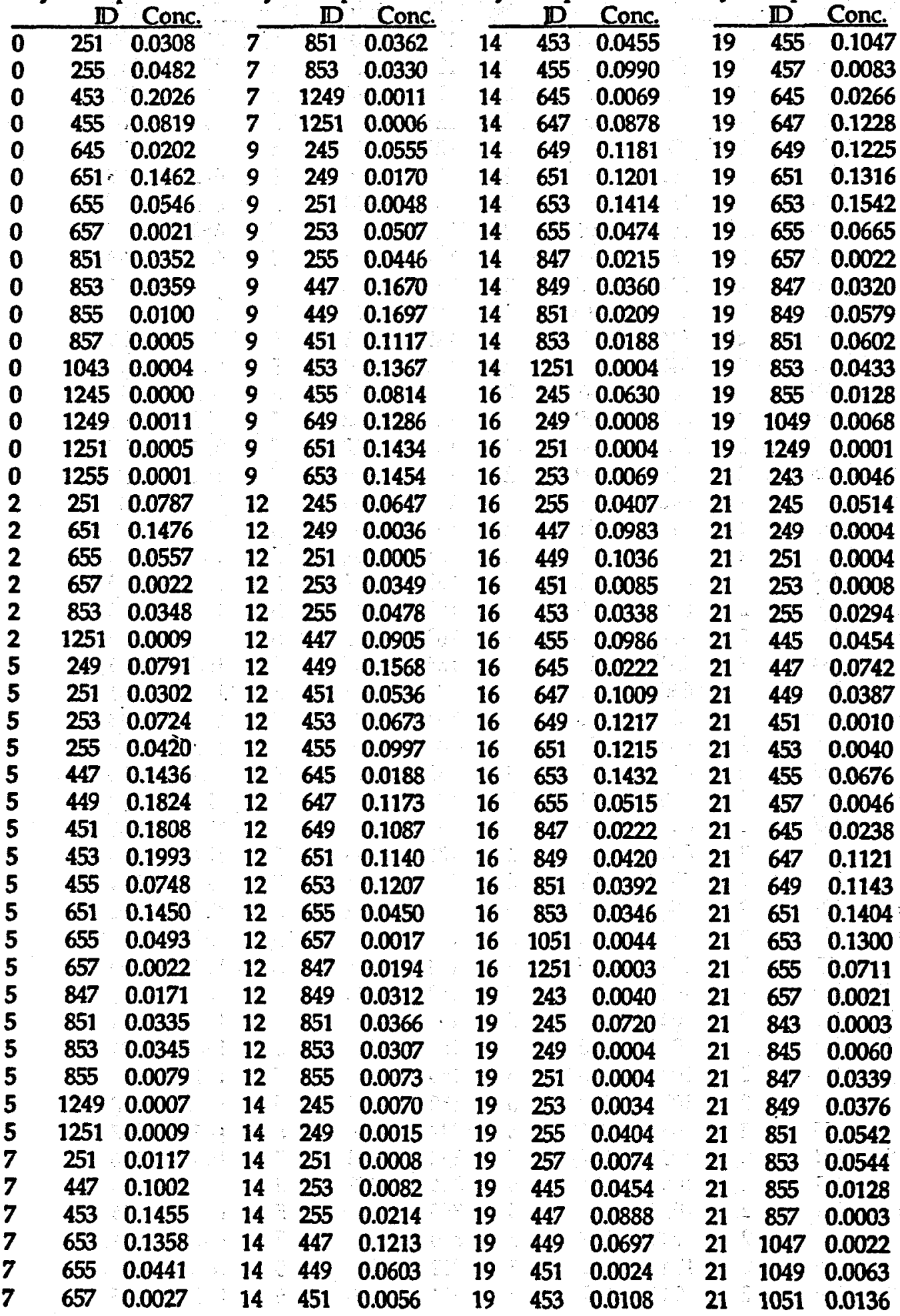


Appendix A-5. Experiment IIb Chromium Data.

Day Sampl. Rel. Day Sampl. Rel.

\begin{tabular}{|c|c|c|c|c|c|c|c|c|c|c|c|}
\hline & $\mathbb{D}$ & Conc. & & D & Conc. & & ID & Conc & & D & Con \\
\hline 5 & 251 & 0.0006 & 23 & 649 & 0.0745 & 37 & 655 & 0.0457 & 44 & 1051 & 0.2168 \\
\hline 7 & 251 & 0.1818 & 23 & 651 & 0.0006 & 37 & 1051 & 0.0028 & & 1247 & 0.0000 \\
\hline 7 & 447 & -0.0018 & 23 & 653 & 0.0135 & 37 & 1253 & -0.0018 & & 1253 & 0.0057 \\
\hline 7 & 453 & 0.0000 & 23 & 655 & 0.0022 & 40 & 251 & 0.0000 & & 1445 & 0.0022 \\
\hline 9 & 251 & 0.4459 & 23 & 1051 & 0.0006 & 40 & 453 & 0.0267 & & 1451 & 0.0000 \\
\hline 12 & 251 & 0.7079 & 28 & 251 & 0.0261 & 40 & 455 & -0.0020 & 4 & 1453 & 0.0022 \\
\hline 12 & 253 & 0.0000 & 28 & 453 & 0.7838 & 40 & 647 & 0.5311 & 44 & 1455 & -0.0002 \\
\hline 2 & 447 & -0.0032 & 28 & 649 & 0.2917 & 40 & 649 & 0.2412 & & 1653 & 0.0026 \\
\hline 2 & 453 & -0.0026 & & 651 & 0.0141 & 40 & 651 & 0.3432 & & 1655 & 0.0030 \\
\hline 2 & 649 & -0.0004 & & 653 & 0.3745 & 40 & 653 & 0.4533 & 47 & 251 & 0.0032 \\
\hline 2 & 651 & 0.0010 & & 655 & 0.0063 & 40 & 655 & 0.1083 & 47 & 443 & -0.0018 \\
\hline 2 & 653 & 0.0000 & 2 & 853 & 0.0059 & 40 & 857 & 0.0085 & 7 & 453 & 0.0087 \\
\hline 12 & 655 & 0.0000 & 8 & 1051 & 0.0063 & 40 & 1051 & 0.0368 & 7 & 647 & 0.3305 \\
\hline 4 & 251 & 0.6889 & 8 & 1247 & 0.0115 & 40 & 1247 & -0.0004 & 47 & 649 & 0.1422 \\
\hline 4 & 449 & -0.0014 & & 1251 & 0.0002 & 40 & 1253 & -0.0004 & 4 & 651 & 0.3816 \\
\hline & 453 & 0.0012 & 28 & 1253 & -0.0020 & 42 & 251 & & 7 & 653 & \\
\hline 4 & 649 & -0.0022 & 30 & 251 & 0.0135 & 42 & 443 & -0.0018 & 47 & 655 & 0.2962 \\
\hline 4 & 651 & -0.0042 & 30 & 453 & 0.6733 & 42 & 447 & 0.1970 & 47 & 1051 & 0.3543 \\
\hline 14 & 653 & 0.0000 & 30 & 649 & 0.2929 & 42 & 453 & 0.0117 & 47 & 1247 & -0.0016 \\
\hline 4 & 655 & -0.0020 & 30 & 651 & 0.0384 & 42 & 649 & 0.1909 & 47 & 1253 & 0.0485 \\
\hline 6 & 251 & 0.7010 & 30 & 653 & 0.4384 & 42 & 651 & 0.4081 & 47 & 1451 & -0.0020 \\
\hline & 449 & 0.0010 & 30 & 655 & 0.0026 & 42 & 653 & 0.3000 & 47 & 1453 & -0.0008 \\
\hline & 453 & 0.0057 & 30 & 1051 & 0.0010 & 42 & 655 & 0.1651 & 47 & 1455 & 0.0012 \\
\hline & 647 & -0.0002 & 30 & 1253 & 0.0028 & 42 & 853 & 0.2204 & 47 & 1457 & 0.0028 \\
\hline & 649 & 0.0000 & 3 & 251 & 0.0073 & 42 & 1051 & 0.1257 & 47 & 1655 & 0.0004 \\
\hline & 651 & -0.0006 & 3 & 453 & 0.38 & 42 & 1245 & -0.0061 & 47 & 1849 & 0.0022 \\
\hline & 653 & -0.002 & 3 & 649 & 0.32 & 42 & 1247 & -0.0 & 49 & 251 & 0.0018 \\
\hline & 655 & -0.0020 & & 651 & 0.12 & 42 & 1251 & & 49 & 453 & \\
\hline & 851 & -0.0028 & 3 & 653 & 0.5549 & & 1253 & -0.0 & 49 & 647 & \\
\hline & 853 & 0.0016 & 3. & 655 & 0.0026 & 42 & 1255 & -0.0059 & 49 & 649 & 0.1305 \\
\hline & 251 & 0.7527 & 3 & 853 & 0.0046 & 42 & 1445 & 0.0014 & 49 & 651 & 0.3040 \\
\hline & 253 & 0.5901 & 3 & $1051^{\prime}$ & -0.0048 & 42 & 1447 & -0.0022 & 49 & 653 & 0.0345 \\
\hline & 447. & -0.0051 & 3 & 1247 & -0.0020 & 42 & 1449 & -0.0028 & 49 & 655 & 0.3410 \\
\hline & 453 & 0.2933 & 3 & 1253 & -0.0020 & 42 & 1451 & -0.0004 & 49 & 1253 & 0.1588 \\
\hline & 647 & -0.0065 & 35 & 243 & 0.0000 & 42 & 1453 & -0.0018 & 49 & 1457. & 0.0032 \\
\hline & 649 & -0.0048 & 35 & 251 & 0.0079 & 42 & 1455 & -0.0016 & 49 & 1655 & 0.0000 \\
\hline & 651 & -0.0053 & 35 & 253 & 0.1780 & 42 & 1653 & -0.0002 & 51 & 251 & 0.0053 \\
\hline & 653 & -0.0044 & 3 & 649 & 0.3297 & 44 & 251 & 0.0002 & 51 & 453 & 0.0036 \\
\hline & 655 & -0.0026 & & 651 & 0.2036 & 44 & 253 & 0.0036 & & 649 & 0.1234 \\
\hline & 853 & -0.0065 & & 653 & 0.58 & 44 & 443 & -0.0042 & & 651 & 0.2588 \\
\hline & 251 & 0.5216 & & 655 & & 44 & 447 & 0.12 & & 653 & 0.0289 \\
\hline & 453 & 0.4689 & & 1051 & & 44 & 453 & 0.00 & & 655 & 0.3416 \\
\hline & 649 & 0.0063 & & 1253 & -0.0 & 44 & 647 & 0.4590 & & 853 & 0.4758 \\
\hline & 651 & -0.0040 & & 251 & 0.00 & 44 & 649 & 0.1667 & & 1051 & 0.4962 \\
\hline & 653 & -0.0059 & 37 & 453 & 0.0788 & 44 & 651 & 0.4206 & & 1245 & -0.0040 \\
\hline & 655 & -0.0059 & 37 & 649 & 0.3299 & 44 & 653 & 0.1875 & & 1247. & -0.0010 \\
\hline & 1051 & -0.0040 & 37 & 651 & 0.2840 & 4 & 655 & 0.2164 & & 1251 & 0.0139 \\
\hline & 453 & 0.6024 & 37 & 653 & 0.5943 & 44 & 853 & 0.3287 & 51 & 1253 & 0.2115 \\
\hline
\end{tabular}


Appendix A-6. Solute Concentrations: PFBA, DFBA, Br, NO3, and Cl.

\begin{tabular}{|c|c|c|c|c|c|c|}
\hline \multirow{2}{*}{$\begin{array}{c}\text { Exp Ilb } \\
\text { Day }\end{array}$} & \multirow{2}{*}{$\begin{array}{c}\text { Sampler } \\
\text { D }\end{array}$} & \multicolumn{3}{|c|}{ Relative Conc. - . } & \multicolumn{2}{|c|}{-- Solution ppm -- } \\
\hline & & PFBA & DFBA & Bromide & Nitrate & Chloride \\
\hline 0 & 251 & 0.000 & & 0.014 & 718.2 & 58.3 \\
\hline 0 & 453 & 0.000 & & 0.154 & 190.9 & 39.1 \\
\hline 0 & 655 & 0.000 & & 0.136 & 167.7 & 109.0 \\
\hline 0 & 853 & 0.000 & & 0.154 & 143.2 & 71.1 \\
\hline 0 & 1251 & 0.000 & & 0.008 & 414.9 & 266.0 \\
\hline 14 & 245 & 0.000 & & 0.058 & 98.0 & 82.8 \\
\hline 14 & 249 & 0.247 & & 0.005 & 101.8 & 43.6 \\
\hline 14 & 251 & 0.756 & & 0.006 & 23.0 & 19.8 \\
\hline 14 & 253 & 0.476 & & 0.007 & & 48.7 \\
\hline 14 & 255 & 0.000 & & 0.071 & 646.0 & 43.0 \\
\hline 14 & 447 & 0.000 & & 0.064 & 194.8 & 41.0 \\
\hline 14 & 449 & 0.000 & & 0.057 & 390.5 & 46.5 \\
\hline 14 & 451 & 0.000 & & 0.011 & 146.7 & 79.4 \\
\hline 14 & 645 & 0.000 & & 0.078 & 140.6 & 80. \\
\hline 14 & 647 & 0.000 & & 0.273 & 188.9 & 26. \\
\hline 14 & 649 & 0.000 & & 0.196 & 183.0 & 34.8 \\
\hline 14 & 651 & 0.000 & & 0.301 & 186.1 & 44. \\
\hline 14 & 653 & 0.000 & & 0.301 & 170.5 & 25.5 \\
\hline 14 & 655 & 0.000 & & 0.284 & 307.7 & 29.5 \\
\hline 14 & 847 & 0.000 & & 0.207 & 151.2 & 170.0 \\
\hline 14 & 851 & 0.000 & & 0.260 & 362.0 & 106.0 \\
\hline 14 & 853 & 0.000 & & 0.447 & 351.1 & 50.6 \\
\hline 30 & 243 & 0.000 & & 0.008 & & 32.2 \\
\hline 30 & 245 & 0.000 & & 0.210 & 495.0 & 25. \\
\hline 30 & 249 & 0.000 & & 0.002 & 45.1 & 50. \\
\hline 30 & 251 & 0.000 & & 0.000 & 19.3 & 19.3 \\
\hline 30 & 253 & 0.000 & & 0.001 & 26.5 & 21. \\
\hline 30 & 255 & 0.000 & & 0.031 & 607.9 & 178.0 \\
\hline 30 & 443 & 0.000 & & 0.005 & 287.5 & 37. \\
\hline 30 & 445 & 0.000 & & 0.110 & 376.9 & 62. \\
\hline 30 & 447 & 0.648 & & 0.340 & 33.3 & 23. \\
\hline 30 & 449 & 0.041 & & 0.006 & 41.2 & 22. \\
\hline 30 & 451 & 0.025 & & 0.003 & 25.7 & 26. \\
\hline 30 & 453 & 0.000 & & 0.000 & 17.9 & 19. \\
\hline 30 & 455 & 0.000 & & 0.035 & & 14.6 \\
\hline 30 & 645 & 0.000 & & 0.266 & 208.7 & 33.2 \\
\hline 30 & 647 & 0.644 & & 0.007 & 85.6 & 24. \\
\hline 30 & 649 & 0.076 & & 0.048 & 112.1 & 22 \\
\hline 30 & 651 & 0.245 & & 0.062 & 202.3 & 29.0 \\
\hline 30 & 653 & 0.397 & & 0.003 & 48.0 & 19. \\
\hline
\end{tabular}




\section{Appendix A-7. Experiment IIb Redistribution Cores (Baseline Data).}

\begin{tabular}{|c|c|c|c|c|c|c|c|c|c|c|c|c|c|c|}
\hline $\mathbb{D}$ & $\begin{array}{r}\text { Seg } \\
\quad \\
\end{array}$ & $\begin{array}{l}Y \\
(m)\end{array}$ & $\begin{array}{c}X \\
(m)\end{array}$ & $\begin{array}{c}z \\
(m)\end{array}$ & $\begin{array}{l}\text { Extr. } \\
\text { Date }\end{array}$ & $\begin{array}{c}\text { Orlg. } \\
\text { Mass } \\
(g)\end{array}$ & $\begin{array}{c}\text { Post-Stg } \\
\text { Mass } \\
\text { (g) }\end{array}$ & $\begin{array}{l}\text { Evap. } \\
\text { Loss } \\
\text { (g) }\end{array}$ & $\begin{array}{c}\text { Soll } \\
\text { Mass } \\
\text { (g) }\end{array}$ & $\begin{array}{c}\text { Discard } \\
\text { (g) }\end{array}$ & $\begin{array}{c}\text { Repres. } \\
\text { Mass } \\
\text { (g) }\end{array}$ & $\begin{array}{l}\text { Gravimet } \\
\text { Tare } \\
\text { (g) }\end{array}$ & $\begin{array}{c}\text { c Water } \\
+ \text { Freshs } \\
\text { (a) }\end{array}$ & $\begin{array}{l}\text { leas. } \\
\text { +ODrys } \\
\text { (g) }\end{array}$ \\
\hline 1 & 1 & 5 & -2.75 & 0.11 & 2-Jul-91 & 134.864 & 134.352 & 0.512 & 119.268 & 0 & 119.268 & 62.676 & 101.890 & 99.402 \\
\hline 1 & 2 & 5 & -2.75 & 0.34 & 2-Jul-91 & 130.083 & 129.914 & 0.169 & 114.067 & 0 & 114.067 & 60.449 & 94.107 & 91.449 \\
\hline 1 & 3 & 5 & -2.75 & 0.57 & 2-Jul-91 & 131.479 & 131.308 & 0.171 & 115.457 & 0 & 115.457 & 62.975 & 104.202 & 100.432 \\
\hline 1 & 4 & 5 & -2.75 & 0.80 & 2-Jul-91 & 133.560 & 133.394 & 0.166 & 118.351 & 0 & 118.351 & 62.757 & 102.931 & 100.627 \\
\hline 2 & 1 & 5 & -2.75 & 1.03 & 2-Jul-91 & 126.654 & 126.480 & 0.174 & 111.408 & 2.336 & 109.072 & 63.532 & 103.241 & 100.840 \\
\hline 2 & 2 & 5 & -2.75 & 1.26 & 2-Jul-91 & 129.818 & 129.647 & 0.171 & 115.956 & 0 & 115.956 & 59.691 & 98.871 & 96.549 \\
\hline 2 & 3 & 5 & -2.75 & 1.49 & & 132.426 & 132.242 & 0.184 & 118.540 & 0 & & & 100.056 & 97.169 \\
\hline 2 & 4 & 5 & -2.75 & 1.71 & ul-91 & 138.540 & 138.363 & 0.177 & 124.574 & 0 & & & & 113.090 \\
\hline 3 & 1 & 5 & -2.75 & 1.94 & Ul-91 & 131.423 & 131.2 & & 117.595 & 0 & & & 102. & \\
\hline 3 & 2 & 5 & -2.75 & 2.17 & 91 & 145.077 & & & & 0 & 113.844 & & 102. & 100.105 \\
\hline 3 & 3 & 5 & -2.75 & 2.40 & $1-91$ & 143.781 & .608 & 0.1 & & 0 & .649 & & 101.732 & 99. \\
\hline 3 & 4 & 5 & -2.75 & 2.63 & $2-5$ & 138.692 & 3.515 & 0.177 & 15 & 0 & & & 104. & 101. \\
\hline 4 & 1 & 5 & -2.75 & 2.86 & $2-j$ & 140.774 & 140 & 0.171 & & 1.497 & & & & \\
\hline 4 & 2 & 5 & -2.75 & 3.09 & & 993 & & & & 0 & & & & \\
\hline 4 & 3 & 5 & -2.75 & 3.31 & & 136.784 & & 0.8 & & 0 & & & 107. & 103. \\
\hline 4 & 4 & 5 & -2.75 & 3.54 & & 141.085 & & 0.1 & 85 & 1.615 & & & & 98. \\
\hline 5 & 1. & 5 & -2.75 & 3.77 & & & & & & 0 & & & 109. & 107.584 \\
\hline 5 & 2 & 5 & -2.75 & 4.00 & & & & & & 0 & & & & 95.624 \\
\hline 5 & 3 & 5 & -2.75 & 4.23 & -91 & 131.223 & & & & 0 & & & & \\
\hline 5 & 4 & 5 & -2.75 & 4.46 & .91 & 126.078 & 125 & & & 0 & & & 102 & 100 \\
\hline 6 & 1 & 5 & -2.75 & 4.69 & & 117.344 & & & & 2.080 & & & 100 & 98 \\
\hline 6 & 2 & 5 & -2.75 & 4.81 & & 138.403 & & 0.188 & 88 & 2.289 & & & 101. & -99.703 \\
\hline 6 & 3 & 5 & -2.75 & 5.14 & & 151.374 & & & & 1.567 & & & 95. & 94.090 \\
\hline 6 & 4 & 5 & -2.75 & 5.37 & $1-91$ & 133.723 & .531 & & 120.306 & 0 & & & 113. & 112.212 \\
\hline 7 & 1 & 5 & -2.75 & 5.64 & -91 & 129.953 & 129.724 & 0.2 & 13 & 2.095 & & & 107. & 106.283 \\
\hline 7 & 2 & 5 & -2.75 & 5.94 & $3-J$ & & & 0.249 & & 0.892 & & & & 115.196 \\
\hline 7 & 3 & 5 & -2.75 & 6.25 & $\mid-91$ & 120.555 & 120.353 & 0.202 & 108.077 & 0 & 108.077 & & 111.207 & 109.728 \\
\hline 8 & 1 & 5 & -2.25 & 0.11 & 3-Jul-91 & 117.799 & 117.620 & 0.179 & 103.821 & 0 & 103.821 & & 91.113 & 89.277 \\
\hline 8 & 2 & 5 & -2.25 & 0.34 & $3-J u l-91$ & 124.862 & 124.666 & 0.196 & 734 & 0.852 & 9.882 & & 98. & 95.628 \\
\hline 8 & 3 & 5 & -2.25 & 0.57 & 3-Jul-91 & 124.148 & 123.964 & 0.184 & 109 & 0 & 9.781 & & 100.894 & 97.421 \\
\hline 8 & 4 & 5 & -2.25 & 0.80 & 3-J & 127,203 & & 0.005 & & 0 & 113.565 & & 104.245 & 101.341 \\
\hline 9 & 1 & 5 & -2.25 & 1.03 & 3-Jul-91 & 133.505 & 133.310 & 0.195 & 118.818 & 0.839 & 117.979 & 62.496 & 98.682 & 96.317 \\
\hline 9 & 2 & 5 & -2.25 & 1.26 & 3-Jul-91 & 131.378 & 131,178 & 0.200 & 116.756 & 1.720 & 115.036 & 59.795 & 95.231 & 92.639 \\
\hline
\end{tabular}


Appendix A-8. Experiment IIb Redistribution Cores (Solute Data).

\begin{tabular}{|c|c|c|c|c|c|c|c|c|c|c|c|}
\hline D & $\begin{array}{c}\text { Seg } \\
\#\end{array}$ & $\begin{array}{l}Y 2 \\
(m)\end{array}$ & $\begin{array}{l}X 2 \\
(m)\end{array}$ & $\begin{array}{l}22 \\
(m)\end{array}$ & $\begin{array}{c}\text { Rel. } \\
\text { Tritlum }\end{array}$ & $\begin{array}{l}\text { Cr-solid } \\
\text { (ppm) }\end{array}$ & $\begin{array}{c}\text { Cr-solution } \\
\text { (ppm) }\end{array}$ & $\begin{array}{l}\text { No3-solid } \\
\text { (ppm) }\end{array}$ & $\begin{array}{c}\text { NO3-sin } \\
\text { (me/l) }\end{array}$ & $\begin{array}{l}\text { Cl-8olid } \\
\text { (ppm) }\end{array}$ & $\begin{array}{c}\text { Cl-ein } \\
(\mathrm{me} / \mathrm{l})\end{array}$ \\
\hline 1 & 1 & 5 & -2.75 & 0.11 & 0.000 & -0.005 & -0.068 & 48.3 & 9.54 & 19.5 & 7.04 \\
\hline 1 & 2 & 5 & -2.75 & 0.34 & 0.001 & -0.004 & -0.052 & 70.2 & 11.67 & 3.9 & 1.14 \\
\hline 1 & 3 & 5 & -2.75 & 0.57 & 0.000 & -0.003 & -0.048 & 62.0 & 8.68 & 7.5 & 1.85 \\
\hline 1 & 4 & 5 & -2.75 & 0.80 & -0.001 & -0.004 & -0.045 & 23.0 & 5.49 & 3.6 & 1.52 \\
\hline 2 & 1 & 5 & -2.75 & 1.03 & 0.000 & $-0,008$ & -0.128 & 17.0 & 3.81 & 5.6 & 2.25 \\
\hline 2 & 2 & 5 & -2.75 & 1.26 & 0.000 & -0.009 & -0.127 & 11.6 & 2.66 & 5.5 & 2.26 \\
\hline 2 & 3 & 5 & -2.75 & 1.49 & 0.006 & -0.011 & -0.167 & 7.7 & 1.41 & 4.4 & 1.44 \\
\hline 2 & 4 & 5 & -2.75 & 1.71 & 0.018 & -0.008 & -0.132 & 3.1 & 0.75 & 4.3 & 1.85 \\
\hline 3 & 1 & 5 & -2.75 & 1.94 & 0.017 & -0.009 & -0.121 & 12.3 & 2.76 & 8.0 & 3.20 \\
\hline 3 & 2 & 5 & -2.75 & 2.17 & 0.023 & -0.008 & -0.138 & 5.2 & 1.33 & 5.4 & 2.44 \\
\hline 3 & 3 & 5 & -2.75 & 2.40 & 0.019 & -0.004 & -0.048 & 22.9 & 5.81 & 16.4 & 7.42 \\
\hline 3 & 4 & 5 & -2.75 & 2.63 & 0.010 & -0.011 & -0.105 & 66.5 & 13.80 & 216.4 & 79.73 \\
\hline 4 & 1 & 5 & -2.75 & 2.86 & 0.006 & -0.017 & -0.283 & 85.7 & 24.03 & 332.6 & 166.65 \\
\hline 4 & 2 & 5 & -2.75 & 3.09 & 0.000 & -0.001 & -0.036 & 152.3 & 27.31 & 142.4 & 45.30 \\
\hline 4 & 3 & 5 & -2.75 & 3.31 & 0.001 & -0.001 & -0.018 & 165.2 & 24.99 & 199.9 & 53.75 \\
\hline 4 & 4 & 5 & -2.75 & 3.54 & 0.002 & -0.004 & -0.069 & 77.2 & 20.57 & N/A & N/A \\
\hline 5 & 1 & 5 & -2.75 & 3.77 & 0.002 & -0.005 & -0.087 & 38.5 & 15.03 & 86.6 & 61.36 \\
\hline 5 & 2 & 5 & -2.75 & 4.00 & -0.001 & -0.004 & -0.066 & 62.2 & 14.05 & 144.6 & 58.26 \\
\hline 5 & 3 & 5 & -2.75 & 4.23 & -0.001 & -0.002 & -0.040 & 49.8 & 13.50 & 131.7 & 64.90 \\
\hline 5 & 4 & 5 & -2.75 & 4.46 & 0.002 & -0.003 & -0.051 & 43.3 & 11.65 & 126.9 & 61.15 \\
\hline 6 & 1 & 5 & -2.75 & 4.69 & 0.002 & -0.003 & -0.070 & 45.9 & 12.88 & 108.1 & 53.56 \\
\hline 6 & 2 & 5 & -2.75 & 4.91 & 0.000 & 0.003 & 0.063 & 46.4 & 12.53 & 126.8 & 61.16 \\
\hline 6 & 3 & 5 & -2.75 & 5.14 & -0.001 & -0.001 & -0.029 & 34.7 & 9.54 & 106.1 & 52.07 \\
\hline 6 & 4 & 5 & -2.75 & 5.37 & -0.001 & -0.003 & -0.082 & 24.3 & 9.82 & 84.3 & 61.87 \\
\hline 7 & 1 & 5 & -2.75 & 5.64 & 0.000 & -0.008 & -0.102 & 25.8 & 10.00 & 84.7 & 59.96 \\
\hline 7 & 2 & 5 & -2.75 & 5.94 & 0.002 & -0.001 & -0.016 & 25.3 & 10.37 & 90.5 & 67.94 \\
\hline 7 & 3 & 5 & -2.75 & 6.25 & 0.001 & -0.003 & -0.025 & 22.6 & 10.01 & 83.8 & 68.14 \\
\hline 8 & 1 & 5 & -2.25 & 0.11 & -0.001 & -0.007 & -0.082 & 84.2 & 17.17 & 2.8 & 1.01 \\
\hline 8 & 2 & 5 & -2.25 & 0.34 & 0.001 & -0.005 & -0.057 & 48.8 & 8.51 & 2.5 & 0.78 \\
\hline 8 & 3 & 5 & -2.25 & 0.57 & 0.001 & -0.002 & -0.024 & 17.4 & 2.48 & 7.0 & 1.75 \\
\hline 8 & 4 & 5 & -2.25 & 0.80 & 0.000 & -0.001 & -0.008 & 16.8 & 3.11 & 4.0 & 1.30 \\
\hline 9 & 1 & 5 & -2.25 & 1.03 & 0.002 & -0.005 & -0.053 & 9.2 & 1.90 & 2.0 & 0.74 \\
\hline 9 & 2 & 5 & -2.25 & 1.26 & 0.005 & -0.003 & -0.048 & 8.9 & 1.60 & 2.4 & 0.76 \\
\hline
\end{tabular}


Appendix B. Experiment IIb Extension. 
Appendix B-1. Experiment IIb Dripper Uniformity Test.

Test Date, 30 May, 1990

East Header

West Header

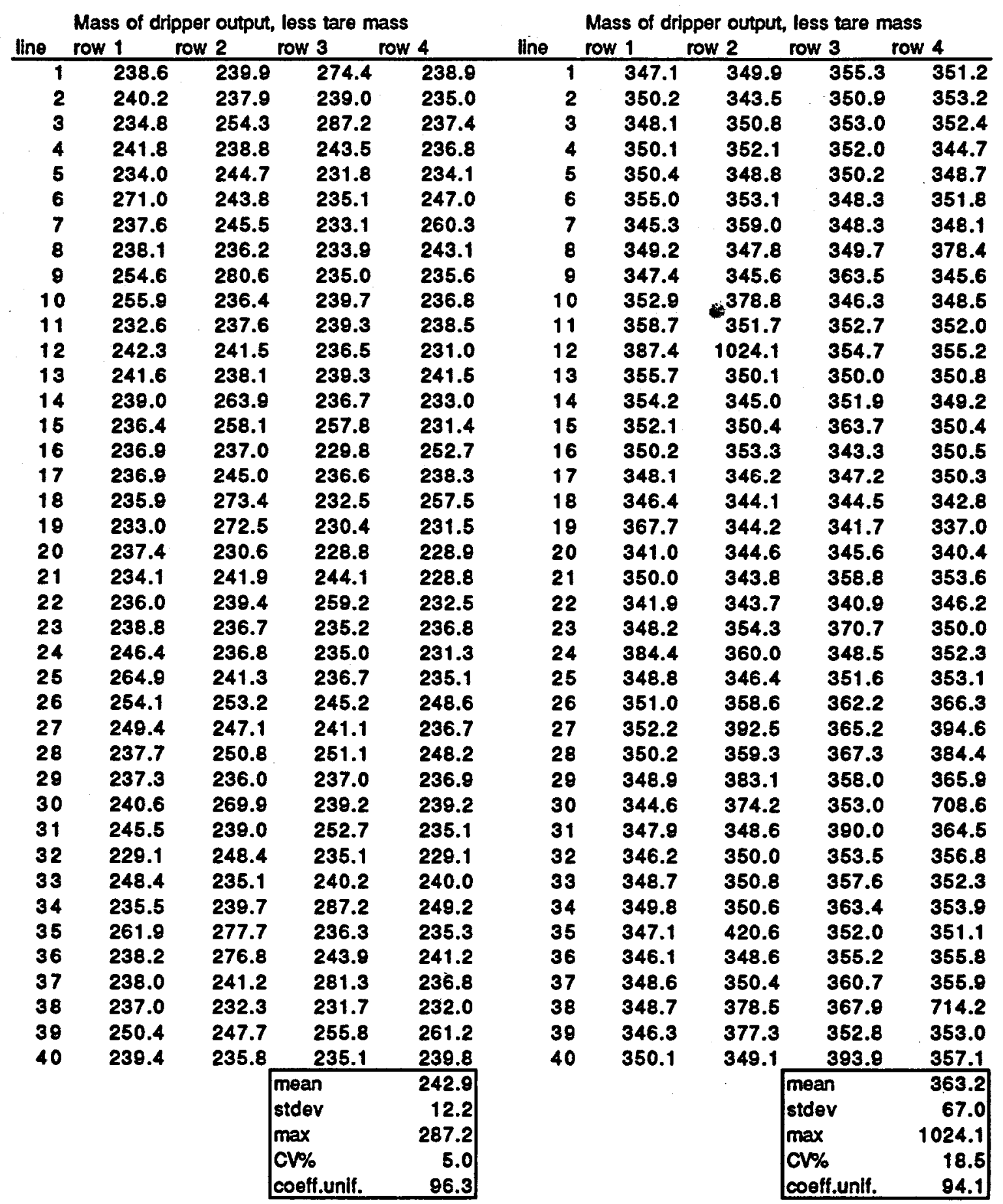


Appendix B-1. Experiment IIb Dripper Uniformity Test. (Continued)

Test Date, 16 July, 1990

Eact Header

West Header

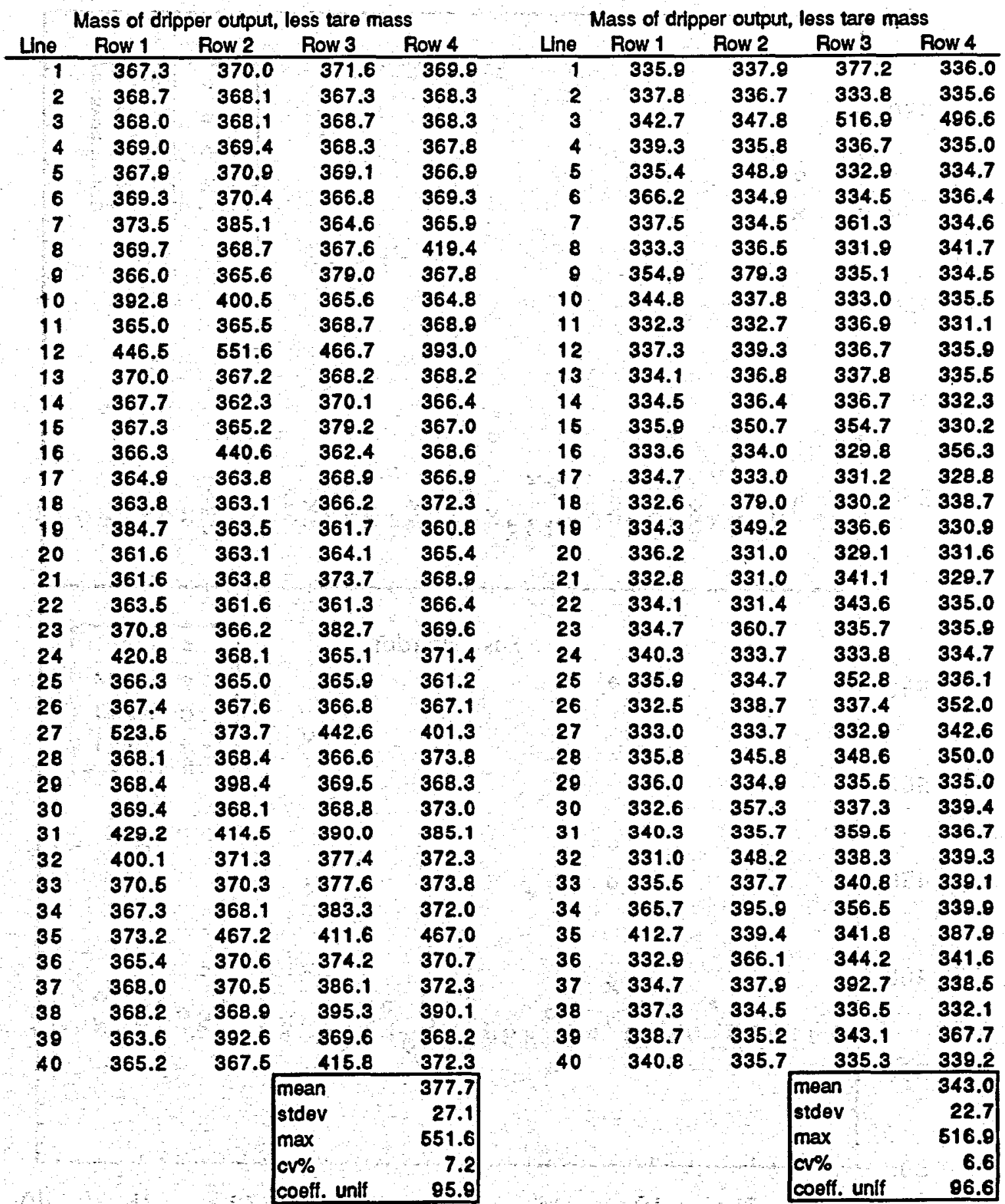


Appendix B-1. Experiment IIb Dripper Uniformity Test. (Continued)

Dripper Uniformity Test, 16 July 1990

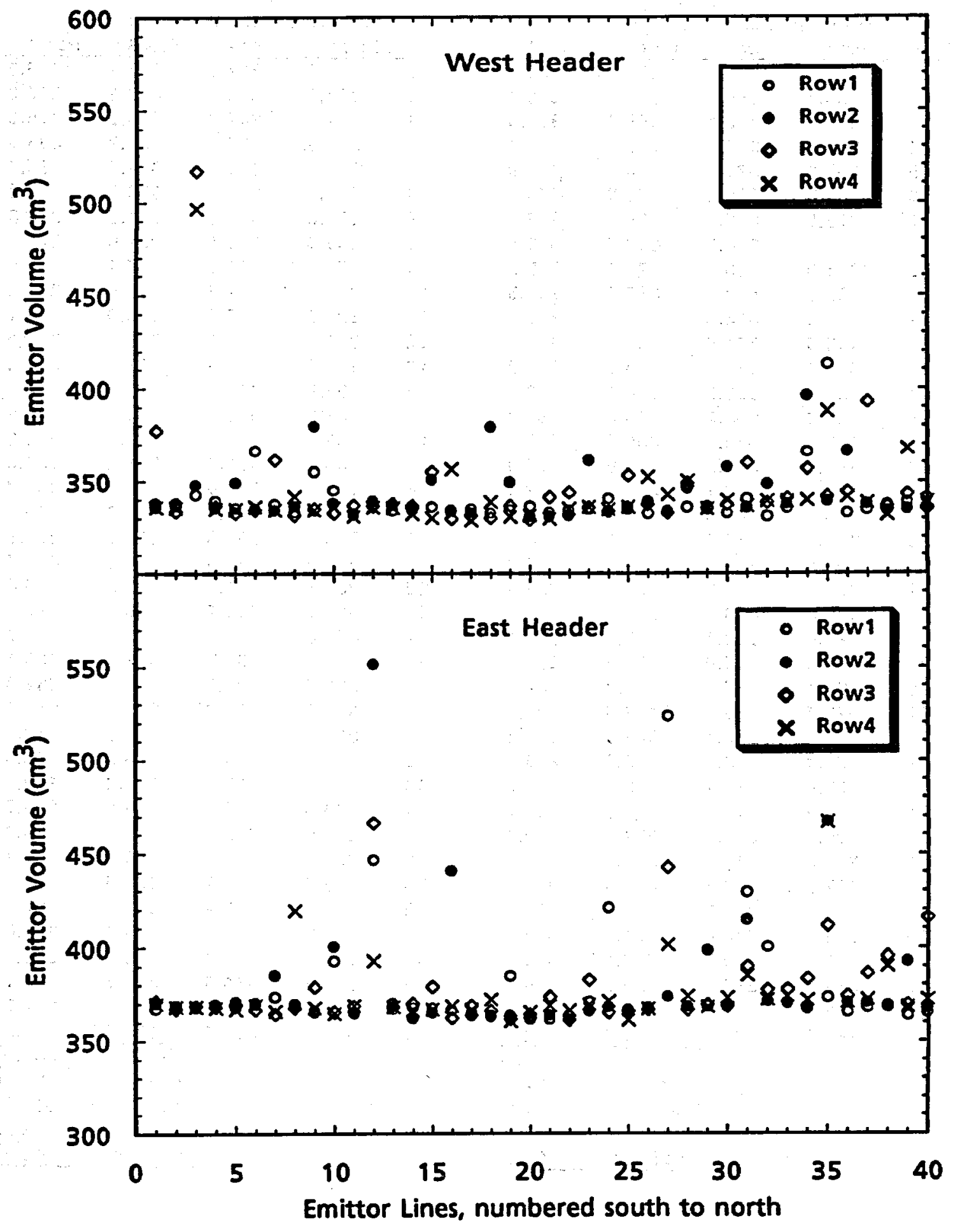


Appendix B-1. Experiment IIb Dripper Uniformity Test. (Continued)

Test Date, 1 August, 1990

Enst Header

West Header

Mass of dripper output, less tare mass

Mass of dripper output, less tare mass

\begin{tabular}{|c|c|c|c|c|c|c|c|c|c|}
\hline $\operatorname{Lin} \theta$ & Row 1 & Row 2 & Row 3 & Row 4 & Une & Row 1 & Row 2 & Row 3 & Row 4 \\
\hline 1 & $386: 0$ & 385.6 & 387.4 & 386.5 & 1 & 366.7 & 368.9 & 368.1 & 408.8 \\
\hline 2 & 398.9 & 388.8 & 387.6 & 410.4 & 2 & 368.5 & 366.0 & 366.4 & 365.7 \\
\hline 3 & 387.1 & 387.8 & 391.7 & 396.2 & 3 & 363.7 & 457.4 & 378.3 & 384.1 \\
\hline 4 & 386.1 & 384.6 & 392.0 & 381.9 & 4 & 370.9 & 365.0 & 365.7 & 370.0 \\
\hline 5 & 387.5 & 393.7 & 384.5 & 382.4 & 6 & 363.9 & 393.2 & 362.7 & 364.7 \\
\hline 6 & 384.9 & 383.9 & 382.4 & 385.1 & 6 & 411.0 & 369.6 & 362.3 & 358.6 \\
\hline 7 & 380.7 & 391.8 & 381.1 & 387.0 & 7 & 367.3 & 362.8 & 361.4 & 396.5 \\
\hline 8 & 385.3 & 385.0 & 381.9 & 425.2 & 8 & 361.7 & 365.0 & 365.3 & 364.3 \\
\hline 9 & N/A & 370.8 & 389.8 & 380.8 & $\theta$ & 420.8 & 394.8 & 364.6 & 367.0 \\
\hline 10 & 390.0 & 415.6 & 381.2 & 381.3 & 10 & 370.9 & 365.1 & 363.1 & 365.0 \\
\hline 11 & 384.3 & 381.7 & 383.2 & 380.4 & 11 & 363.0 & 362.6 & 367.9 & 365.4 \\
\hline 12 & 396.2 & 395.6 & 418.8 & 463.2 & 12 & 366.1 & 368.9 & 366.4 & 367.3 \\
\hline 13 & 389.2 & 386.1 & 384.2 & 382.4 & 13 & 360.8 & 366.0 & 362.2 & 362.4 \\
\hline 14 & 385.4 & 383.7 & 390.3 & 380.9 & 14 & 365.2 & 366.3 & 368.5 & 362.6 \\
\hline 15 & 388.6 & 390.1 & 399.6 & 385.9 & 16 & 364.6 & 381.3 & 383.8 & 358.7 \\
\hline 16 & 391.3 & 397.4 & 380.4 & 389.2 & 16 & 361.9 & 363.6 & 359.7 & 380.7 \\
\hline 17 & 395.4 & 396.5 & 392.0 & 384.4 & 17 & 361.8 & 362.3 & 363.7 & 360.8 \\
\hline 18 & 386.9 & 385.3 & 384.7 & 386.1 & 18 & 365.6 & 395.2 & 362.8 & 371.3 \\
\hline 19 & 395.7 & 383.7 & 380.3 & 377.8 & 19 & 364.4 & 381.0 & 366.6 & 360.6 \\
\hline 20 & 380.2 & 381.6 & 381.6 & 380.9 & 20 & 366.5 & 360.0 & 358.1 & 363.9 \\
\hline 21 & 382.3 & 382.0 & 389.4 & 381.0 & 21 & 363.7 & 359.4 & 368.8 & 357.2 \\
\hline 22 & 379.3 & 379.2 & 378.6 & 380.8 & 22 & 363.1 & 359.2 & 365.9 & 365.0 \\
\hline 23 & 387.6 & 383.3 & 398.3 & 384.3 & 23 & 364.6 & 378.1 & 364.5 & 365.4 \\
\hline 24 & 399.4 & 381.3 & 378.1 & 383.0 & 24 & 369.7 & 360.8 & 363.2 & 363.3 \\
\hline 25 & 385.4 & 382.5 & 383.7 & 379.9 & 25 & 363.6 & 364.9 & 386.8 & 364.6 \\
\hline 26 & 405.2 & 400.8 & 394.0 & 387.1 & 26 & 357.4 & 361.6 & 365.8 & 380.4 \\
\hline 27 & 404.9 & 397.4 & 401.0 & 400.8 & 27 & 360.1 & 367.2 & 365.0 & 372.2 \\
\hline 28 & 384.9 & 390.2 & 387.3 & 391.8 & 28 & 365.6 & 377.3 & 380.6 & 377.6 \\
\hline 29 & 387.8 & 414.9 & 390.7 & 389.3 & 29 & 365.7 & 365.8 & 366.5 & 363.7 \\
\hline 30 & 382.7 & 381.8 & 388.6 & 385.9 & 30 & 332.8 & 394.1 & 364.0 & 376.1 \\
\hline 31 & 395.7 & 412.7 & 429.6 & 411.8 & 31 & 372.3 & 368.5 & 395.5 & 368.5 \\
\hline 32 & 421.8 & 390.9 & 398.6 & 401.3 & 32 & 360.8 & 382.5 & 341.3 & 370.4 \\
\hline 33 & 389.6 & 386.6 & 398.0 & 392.1 & 33 & 359.0 & 369.8 & 373.0 & 369.0 \\
\hline 34 & 394.4 & 389.2 & 400.8 & 392.2 & 34 & 446.7 & 401.5 & 385.3 & 372.4 \\
\hline 35 & 390.4 & 408.1 & 451.4 & 396.7 & 35 & 409.8 & 372.9 & 376.7 & 373.0 \\
\hline 36 & 382.6 & 390.6 & 399.2 & 392.8 & 36 & 387.6 & 416.6 & 369.9 & 370.9 \\
\hline 37 & 387.2 & 392.9 & 406.6 & 391.0 & 37 & 369.7 & 372.1 & 400.4 & 370.8 \\
\hline 38 & 384.2 & 385.8 & 416.6 & 405.6 & 38 & 367.1 & 365.4 & 366.6 & 363.6 \\
\hline $\begin{array}{l}38 \\
40\end{array}$ & $\begin{array}{l}383.0 \\
391.8\end{array}$ & $\begin{array}{l}403.1 \\
391.6\end{array}$ & $\begin{array}{l}388.0 \\
407.0\end{array}$ & $\begin{array}{l}391.1 \\
406.4\end{array}$ & $\begin{array}{l}39 \\
40\end{array}$ & $\begin{array}{l}367.8 \\
382.9\end{array}$ & $\begin{array}{l}367.9 \\
373.1\end{array}$ & $\begin{array}{l}381.9 \\
367.4\end{array}$ & $\begin{array}{l}372.8 \\
371.2\end{array}$ \\
\hline $\begin{array}{l}3 \\
\ddots \\
\vdots\end{array}$ & 更 & & $\begin{array}{l}\text { mean } \\
\text { stdev } \\
\text { max } \\
\text { cv\% } \\
\text { coeff. unif }\end{array}$ & $\begin{array}{r}391.4 \\
12.4 \\
463.2 \\
3.2 \\
97.8\end{array}$ & & & & $\begin{array}{l}\text { mean } \\
\text { stdev } \\
\text { max } \\
\text { cr\% } \\
\text { coeff. unif }\end{array}$ & $\begin{array}{r}371.2 \\
16.4 \\
457.4 \\
4.1 \\
97.4 \\
\end{array}$ \\
\hline
\end{tabular}


Appendix B-1. Experiment IIb Dripper Uniformity Test. (Continued)

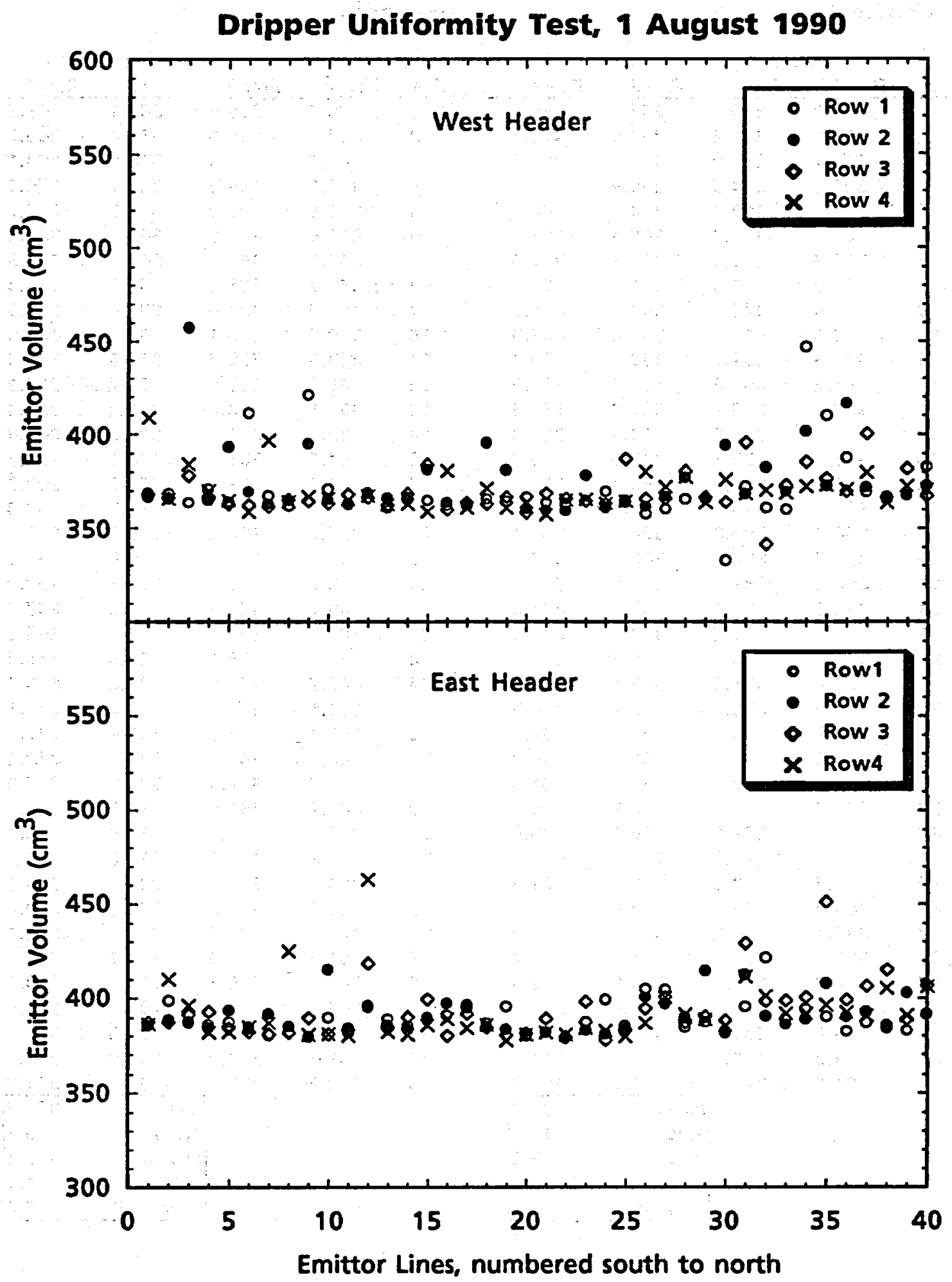


Appendix B-1. Experiment IIb Dripper Uniformity Test. (Continued)

Test Date, 24 October, 1990

East Header

West Header

\begin{tabular}{|c|c|c|c|c|c|c|c|c|c|}
\hline \multicolumn{5}{|c|}{ Mass of dripper output, less tare mass } & \multicolumn{5}{|c|}{ Mass of dripper output, less tare mass } \\
\hline Line : & Row 1 & Row 2 & Row 3 & Row 4 & Line & Row 1 & Row 2 & Row 3 & Row 4. \\
\hline 1. & 419.0 & 397.7 & 399.7 & 402.2 & 1 & 414.46 & 461.86 & 399.96 & 401.56 \\
\hline 2 & 409.9 & 399.5 & 397.7 & 401.3 & 2 & 403.26 & 402.96 & 406.26 & 403.46 \\
\hline 3 & 403.3 & 405.4 & 397.9 & 402.0 & 3 & 406.26 & 410.26 & 428.26 & 442.16 \\
\hline 4 & 405.3 & 401.1 & 401.6 & 403.4 & 4 & 403.56 & 406.96 & 401.46 & 406.06 \\
\hline 5 & 393.3 & 400.8 & 402.2 & 400.6 & 6 & 395.06 & 396.76 & 416.66 & 405.06 \\
\hline 6 & 397.8 & 401.0 & 406.2 & 404.7 & 6 & 407.26 & 398.06 & 399.06 & 406.36 \\
\hline 7 & 397.2 & 397.2 & 399.7 & 409.1 & 7 & 406.36 & 403.66 & 397.86 & 406.66 \\
\hline 8 & 402.8 & 402.1 & 404.6 & 406.4 & 8 & 412.96 & 401.46 & 403.76 & 402.66 \\
\hline$\theta$ & 398.3 & 406.7 & 397.8 & 395.8 & $\theta$ & 406.06 & 403.56 & 407.26 & 405.16 \\
\hline 10 & 407.0 & 396.1 & 445.4 & 406.4 & 10 & 406.96 & 398.26 & 403.36 & 411.26 \\
\hline 11 & 402.7 & 400.8 & 396.1 & 393.8 & 11 & 391.96 & 410.56 & 401.56 & 400.76 \\
\hline 12 & 405.8 & 415.0 & 406.1 & 403.2 & 12 & 407.36 & 403.46 & 400.26 & 400.26 \\
\hline 13 & 404.7 & 399.6 & 400.3 & 410.0 & 13 & 400.56 & 402.56 & 401.26 & 404.06 \\
\hline 14 & 399.6 & 399.6 & 392.7 & 397.0 & 14 & 402.06 & 401.26 & 404.66 & 401.96 \\
\hline 16 & 398.3 & 426.4 & 400.5 & 402.1 & 16 & 400.16 & 429.66 & 402.16 & 401.16 \\
\hline 16 & 401.5 & 395.6 & 405.3 & 400.8 & 16 & 422.36 & 396.26 & 400.96 & 399.86 \\
\hline 17 & 398.8 & 397.6 & 397.1 & 398.0 & 17 & 398.96 & 397.96 & 420.26 & 402.66 \\
\hline 18 & 405.4 & 402.8 & 393.3 & 397.1 & 18 & 395.26 & 399.06 & 416.66 & 404.46 \\
\hline 10 & 395.6 & 395.0 & 397.6 & 420.9 & 10 & 405.46 & 402.06 & 426.86 & 397.56 \\
\hline 20 & 406.3 & 399.8 & 394.3 & 395.4 & 20 & 406.46 & 396.46 & 396.16 & 406.16 \\
\hline 21 & 404.6 & 411.0 & 413.4 & 392.3 & 21 & 405.66 & 411.96 & 396.26 & 400.46 \\
\hline 22 & 398.1 & 395.1 & 403.7 & 399.1 & 22 & 404.06 & 399.06 & 451.06 & 399.66 \\
\hline 23 & 402.0 & 394.6 & 401.3 & 403.3 & 23 & 404.36 & 398.26 & 408.26 & 404.36 \\
\hline 24 & 399.6 & 393.3 & 401.1 & 389.0 & 24 & 403.86 & 413.56 & 399.36 & 398.06 \\
\hline $25^{\circ}$ & 398.3 & 399.1 & 403.8 & 402.5 & 25 & 405.06 & 408.66 & 399.16 & 428.56 \\
\hline 26 & 398.7 & 400.0 & 396.0 & 400.7 & 26 & 408.96 & 401.16 & 398.86 & 398.46 \\
\hline 27 & 510.2 & 401.8 & 415.3 & 410.5 & 27 & 415.96 & 401.36 & 409.06 & 403.46 \\
\hline 28 & 404.6 & 401.4 & 398.4 & 404.5 & 28 & 419.66 & 426.96 & 403.06 & 403.76 \\
\hline 28 & 405.2 & 404.6 & 437.7 & 403.8 & 29 & 399.96 & 401.76 & 401.56 & 403.46 \\
\hline 30 & 399.6 & 396.7 & 400 & & 30 & & 398.56 & 426.76 & 403.36 \\
\hline 31 & 413.3 & 416.7 & 411.6 & 408.4 & 31 & 401.56 & 430.56 & 402.96 & 407.26 \\
\hline $\begin{array}{l}32 \\
33\end{array}$ & $\begin{array}{l}403.5 \\
405.0\end{array}$ & $\begin{array}{l}406.3 \\
417.7\end{array}$ & $\begin{array}{l}398.3 \\
399.7\end{array}$ & $\begin{array}{l}415.5 \\
398.6\end{array}$ & $\begin{array}{l}32 \\
33\end{array}$ & $\begin{array}{l}405.96 \\
405.96\end{array}$ & $\begin{array}{l}403.16 \\
408.96\end{array}$ & $\begin{array}{l}409.86 \\
402.66\end{array}$ & $\begin{array}{l}393.66 \\
405.96\end{array}$ \\
\hline 34 & 407.5 & 419.1 & 395.6 & 401.0 & 34 & 405.06 & 404.66 & 410.26 & 409.36 \\
\hline 35 & 406.4 & 405.8 & 408.3 & 400.7 & 35 & 404.66 & 401.16 & 404.36 & 406.06 \\
\hline 36 & 397.0 & 400.5 & 400.2 & 410.6 & 36 & 402.96 & 398.56 & 418.76 & 404.86 \\
\hline 37 & 401.0 & 416.7 & 401.6 & 400.1 & 37 & 402.66 & 421.46 & 412.76 & 404.76 \\
\hline 38 & 407.8 & 410.1 & 396.7 & 401.3 & 38 & & & & 405.06 \\
\hline 39 & 400.4 & 400.5 & 414.4 & 394.3 & 38 & 405.26 & 443.36 & 400.56 & 403.46 \\
\hline 40 & 400.3 & & 398.0 & 398.5 & 40 & 423.26 & 402.86 & 417.26 & 423.76 \\
\hline & & & & 403.5 & & & & nean & 406.6 \\
\hline & & & v & 11.4 & & & & $t d \theta v$ & 10.3 \\
\hline & & & & & & & & $\max$ & 461.0 \\
\hline & & & $\begin{array}{l}\text { V\% } \\
\text { peff.unif. }\end{array}$ & $\begin{array}{r}2.8 \\
98.5\end{array}$ & & & & 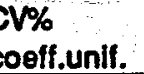 & $\begin{array}{r}2.5 \\
98.3\end{array}$ \\
\hline
\end{tabular}


Appendix B-1. Experiment IIb Dripper Uniformity Test. (Continued)

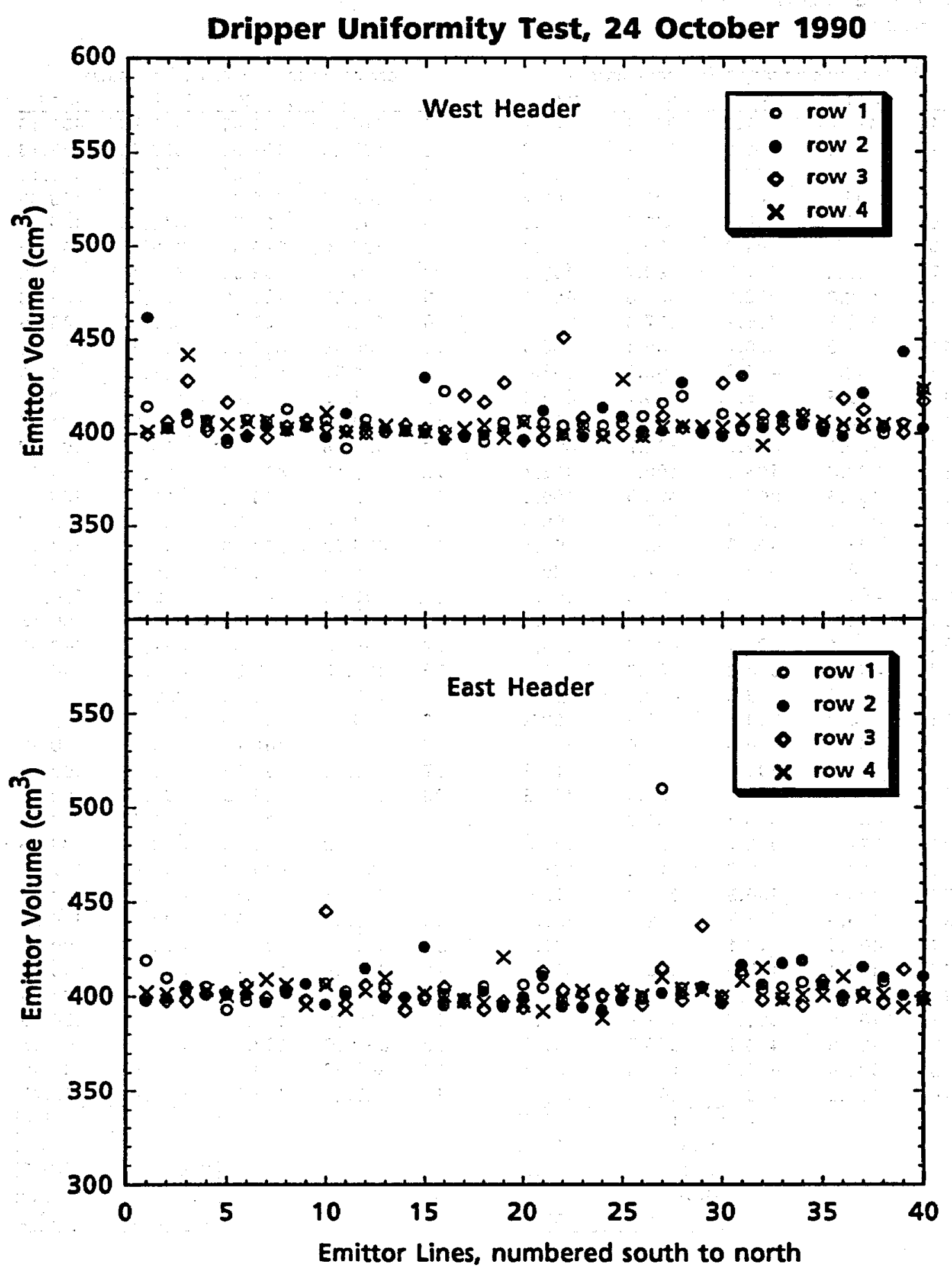




\section{Appendix B-2. Tritium Scintillation Cocktail and Aliquot Volume Adjustments.}

\section{Introduction}

Two different scintillation cocktails were used to analyze the tritium samples of experiment IIb. A unique set of counting efficiencies is associated with each cocktail where the scintillation response is a function of the cocktail formulation, the particle being counted and the liquid with which the cocktail is mixed. The cocktail used for the trench irrigation experiment IIb samples from days 0 through 184 was Aquasol universal LSC, a xylene-based product manufactured by Dupont New England Nuclear (p/n NEF-934, Boston, MA). After day 184 samples were prepared with Ready-Safe LSC (Beckman Instruments Inc, Fullerton, CA), a newer cocktail formulation with significantly higher counting efficiency than xylene based cocktails (Klein and Gershey, 1990). Ready-Safe was less expensive, more safely handled, and proved to mix more readily with aqueous sample to form a uniform gel than did Aquasol.

The amount of sample mixed with cocktail for experiment IIb tritium analyses was reduced when insufficient sample had been collected in the field to use the normal $0.5 \mathrm{ml}$ aliquot size.
This volume varied from 0.1 to $0.5 \mathrm{ml}$ by steps of $0.1 \mathrm{ml}$. The quenching characteristic of a scintillation gel is sensitive to the proportions of sample and cocktail. As sample aliquot size is reduced the resulting counts are disproportionately increased. Therefore, a vial counted with $0.1 \mathrm{ml}$ of solution added to cocktail produces more than $1 / 5=20 \%$ of the counts as when $0.5 \mathrm{ml}$ of solution is used.

Two experiments were performed to elucidate the characteristics of quenching and counting efficiency of the two cocktails used in experiments IIa and IIb. The first experiment was done early in experiment IIb, before the switch from Aquasol cocktail was contemplated. Its purpose was to identify the character of quenching with different sample aliquot sizes. The second experiment was done just prior to the first use of Ready-Safe. Its purpose was not only to identify quenching characteristics of both cocktails with various aliquot sizes but to identify the differences in cocktail-specific counting efficiencies. These data produced simple normalization factors to allow all experiment IIb counting data to be expressed as equivalent counts of Aquasol-cocktail gels at a normal sample aliquot volume of $0.5 \mathrm{ml}$. 


\section{Appendix B-2. Tritium Scintillation Cocktail and Aliquot Volume Adjustments. (Continued)}

\section{Experiment 1: Aquasol}

Tritium was used at three different concentrations. The most concentrated solution, about 0.2 $\mathrm{mCi} / \mathrm{L}$, was diluted serially to produce the other two solutions, 0.02 and $0.002 \mathrm{mCi} / \mathrm{L}$, respectively. Since relative concentrations were sought rather than absolute tritium activity no attempt was made to correct observed counts for decay or known standards. Five aliquot volumes were used; $0.1,0.2,0.3,0.4$ and $0.5 \mathrm{ml}$. Each aliquot was used with each of the three solutions for seven replicate vials of each combination. The treatments for this experiment were therefore:

$$
\begin{aligned}
& \text { Concentration (3) x Aliquot (5) } \\
& \times \text { Replication (7) }=105, n
\end{aligned}
$$

Tritium was analyzed with a Beckman LS- 1800 programmable liquid scintillation counter. Individual replications of sample were prepared for scintillation by pipetting the designated aliquot $(0-100 \mu 1$ adjustable Eppendorf pipettor or $500 \mu \mathrm{l}$ Eppendorf pipettor, Brinkman Instruments, Inc., Westbury, NY) to $5 \mathrm{ml}$ of scintillation cocktail in $8 \mathrm{ml}$ Beckman Mini Poly-Q vials. Each vial was capped and placed in a rack with other vials. When the entire rack was filled it was vigorously shaken to mix the vials contents to form the desired gel. Vials were then transferred to a counting rack compatible with the counter. The counter was programmed to run in auto-count mode, with a single channel window of $0-400 \mathrm{~nm}$ and no internal standard. Counting time per vial was set to a maximum of 10 minutes. At the beginning of a run a series of ten background vials was counted. These vials were prepared with $0.5 \mathrm{ml}$ aliquots of un-traced distilled-deionized water in place of treatment solution in the vials.

The results are given in Table 1 . The observed counts for the $0.5 \mathrm{ml}$ aliquot treatment at the highest concentration of tritium are used to anchor the expected counts for the remainder of the treatments. Therefore, expected counts for $0.1 \mathrm{ml}$ aliquot at the lowest concentration would be the baseline anchor counts $x \mathbf{0 . 0 1}$ dilution $x$ $1 / 5$ aliquot ratio. This treatment assumed that counts derived from tritium are linear across the entire range of measurement and that the dilutions of full-strength tritium were exact when making the medium-and lowconcentration solutions.

Figure 1 illustrates the linear relationship between observed counts of $0.2,0.02$, and 0.002 $\mathrm{mCi} / \mathrm{L}$ solutions at the same aliquot size and the predicted counts for each treatment. Each data point represents the mean of seven replications, less background counts. The intercepts for the regression models were forced through $\mathrm{Y}=\mathbf{0}$. The strong linear nature of the scintillation response to tritium concentration is evident in the high values for individual determination coefficients. As the aliquot size is reduced the slope of the prediction equation increases, which indicates less quenching of $\beta$-emissions as the activity of tritium is decreased relative to scintillation cocktail. These data indicate that this characteristic was highly predictable. 


\section{Appendix B-2. Tritium Scintillation Cocktail and Aliquot Volume Adjustments. (Continued)}

Experiment 1: Aquasol. Data expressed as counts per minute

\begin{tabular}{|c|c|c|c|c|c|c|c|c|c|c|c|c|c|c|c|}
\hline \multirow{2}{*}{$\begin{array}{l}\text { Tritium Conc. } \\
\text { Aliquot (mi) }\end{array}$} & \multicolumn{4}{|c|}{$0.2 \mathrm{mCl}$} & \multicolumn{4}{|c|}{$0.02 \mathrm{mCl}$} & \multicolumn{5}{|c|}{$0.002 \mathrm{mcl}$} & \multirow[b]{2}{*}{0.2} & \multirow[b]{2}{*}{0.1} \\
\hline & 0.5 & 0.4 & 0.3 & 0.2 & 0.1 & 0.5 & 0.4 & $0.3 \cdots$ & 0.2 & 0.1 & 0.5 & 0.4 & 0.3 & & \\
\hline - Rep. & 37239 & 30647 & 23622 & 16761 & 8819 & 3785 & 3081 & 2444 & 1678 & 922 & 394 & 321 & 252 & 192 & 100 \\
\hline & 37452 & 30801 & 24060 & 16610 & 8970 & 3780 & 3076 & 2407 & 1668 & 805 & 388 & 319 & 255 & 179 & 102 \\
\hline & 37074 & 30807 & 23816 & 16669 & 8893 & 3777 & 3099 & 2416 & 1685 & 898 & 378 & 323 & 254 & 175 & 103 \\
\hline & 37563 & 30536 & 24055 & 16613 & 8872 & 3735 & 3137 & 2409 & 1669 & 920 & 389 & 333 & 259 & 180 & 104 \\
\hline & 37179 & 30750 & 23778 & 16629 & 8848 & 3780 & 3107 & 2404 & 1695 & 915 & 390 & 326 & 247 & 183 & 101 \\
\hline & 37253 & 31048 & 23802 & 16779 & 8866 & 3724 & 3091 & 2415 & 1673 & 224 & 407 & 331 & 248 & 177 & 98 \\
\hline & 37413 & 30541 & 23796 & 164 & 8927 & 3778 & 3094 & 240 & 1686 & 888 & 387 & 324 & 252 & 178 & 103 \\
\hline Mean Counts & 37310 & 30733 & 23847 & & 8885 & & 3098 & 2415 & 1678 & 910 & 390 & 325 & 252 & 181 & 102 \\
\hline Std Dov & 17123 & 179.20 & 157.91 & 105.86 & 50.51 & 24.97 & 20.17 & 13.61 & 9.99 & 13.65 & 8.77 & 5.12 & 4.12 & 5.62 & 2.07 \\
\hline CV $\%$ & 0.46 & 0.58 & 0.66 & $\quad 0.64$ & 0.57 & 0.66 & 0.65 & 0.56 & 0.59 & 1.50 & 2.25 & 1.57 & 1.63 & 3.11 & 2.04 \\
\hline $\begin{array}{l}\text { Expected Counts } \\
\text { Error }\end{array}$ & $\begin{array}{l}37310 \\
0.000\end{array}$ & $\begin{array}{r}29848 \\
1.030\end{array}$ & $\begin{array}{r}22386 \\
1.065\end{array}$ & $\begin{array}{r}14924 \\
1.115\end{array}$ & $\begin{array}{l}7462 \\
1.191\end{array}$ & $\begin{array}{l}3731 \\
1.009\end{array}$ & $\begin{array}{l}2985 \\
1.038\end{array}$ & $\begin{array}{l}2239 \\
1.079\end{array}$ & $\begin{array}{l}1492 \\
1.125\end{array}$ & $\begin{array}{r}746 \\
1.220\end{array}$ & $\begin{array}{r}373 \\
1.047\end{array}$ & $\begin{array}{r}298 \\
1.090\end{array}$ & $\begin{array}{r}224 \\
1.128\end{array}$ & $\begin{array}{r}149 \\
1.210\end{array}$ & $\begin{array}{r}75 \\
1.362\end{array}$ \\
\hline
\end{tabular}

Treatments: Cocktail (1) $\times$ Tritium concentration (3) $\times$ Aliquot size (5) $\times$ Replication (7) Cocktail . NEN Aquasol Universal LSC Tritium concentration $0.2,0.02,0.002 \mathrm{mcin}$ Aliquot size $\quad 0.5,0.4,0.3,0.2,0.1 \mathrm{ml}$ 
Appendix B-2. Tritium Scintillation Cocktail and Aliquot Volume Adjustments. (Continued)

Experiment 1: Predicted v Observed Counts for Tritium in Aquasol Cocktail

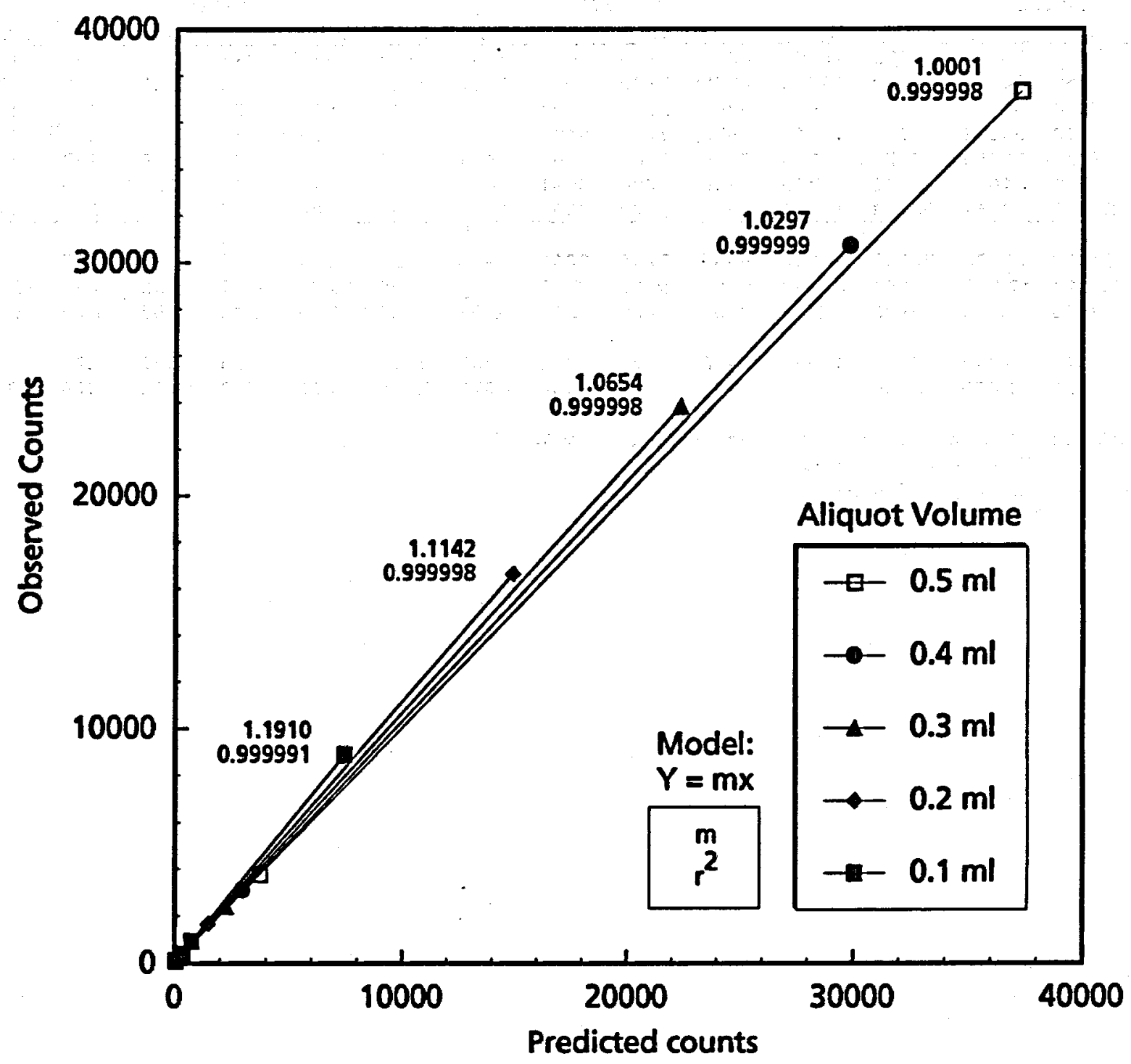




\section{Appendix B-2. Tritium Scintillation Cocktail and Aliquot Volume Adjustments (Continued)}

\section{Experiment 2: Aquasol and Ready-Safe Cocktails}

The procedures for experiment 1 were expanded to include treatments with a new cocktail, Ready-Safe. The number of replications was reduced to 4 . The mechanics and tritium solutions used in experiment 2 remained the same. The new treatment regime was:

Concentration (3) $x$ Aliquot (5) $x$ Replication (4) $x$ Cocktail $(2)=120, n$.

The results are given in Table 2. The observed counts for $0.5 \mathrm{ml}$ aliquot volume at the highest. concentration of tritium in Aquasol cocktail are used to anchor the expected counts for the remainder of the treatments. Therefore, expected counts for $0.1 \mathrm{ml}$ aliquot at the lowest concentration would be the anchored baseline counts $\times 0.01$ dilution $\times 1 / 5$ aliquot ratio. The same assumptions as in experiment 1 were in force. As before, the variability about the treatment means was relatively low and did not suffer here from fewer replications.

Figure 2 illustrates the linear relationship between observed counts of $0.2,0.02$, and 0.002
$\mathrm{mCi} / \mathrm{L}$ solutions at the same aliquot size and the counts for each treatment. Each data point represents the mean of four replications, less background counts. The intercepts for the linear regression models were forced through $Y=0$. The highly linear nature of the scintillation response to tritium concentration was again evident, this time for both cocktails. With Ready Safe, the margin of improved counting efficiency over Aquasol tended to decrease as the aliquot size was reduced, from about 6-7 percent improved performance over Aquasol at $0.5,0.4$, and $0.3 \mathrm{ml}$ aliquot sizes, to about $3 \%$ at $0.2 \mathrm{ml}$ and less than $1 \%$ at $0.1 \mathrm{ml}$. These data indicate that the quenching characteristic was again highly predictable for both cocktails.

This data was used to make a simple correction factor for experiment IIb scintillation counts for tritium. This factor was used to normalize all count data for $0.5 \mathrm{ml}$ aliquot volumes in Aquasol cocktail. Since the results for experiment 1 and experiment 2 Aquasol treatments were so similar, the experiment 2 data were used for all correction factors. These volume/cocktail correction factors, Kvc, (Table 3), were found by taking the inverse of each slope parameter from the individual regression models.

Table 3. Background adjusted correction factors

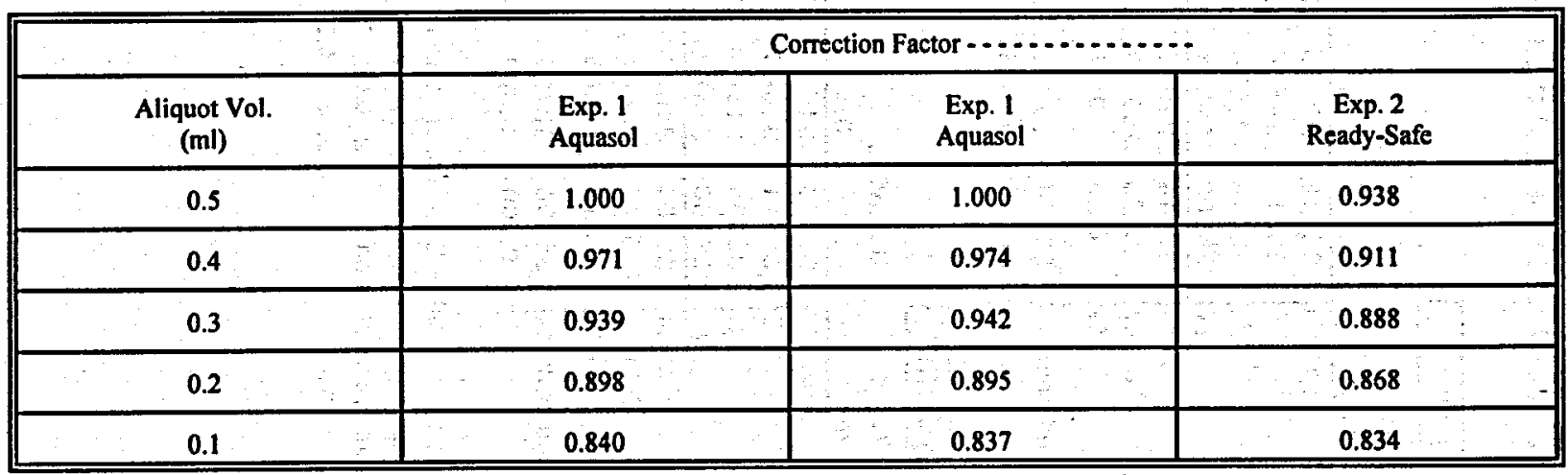




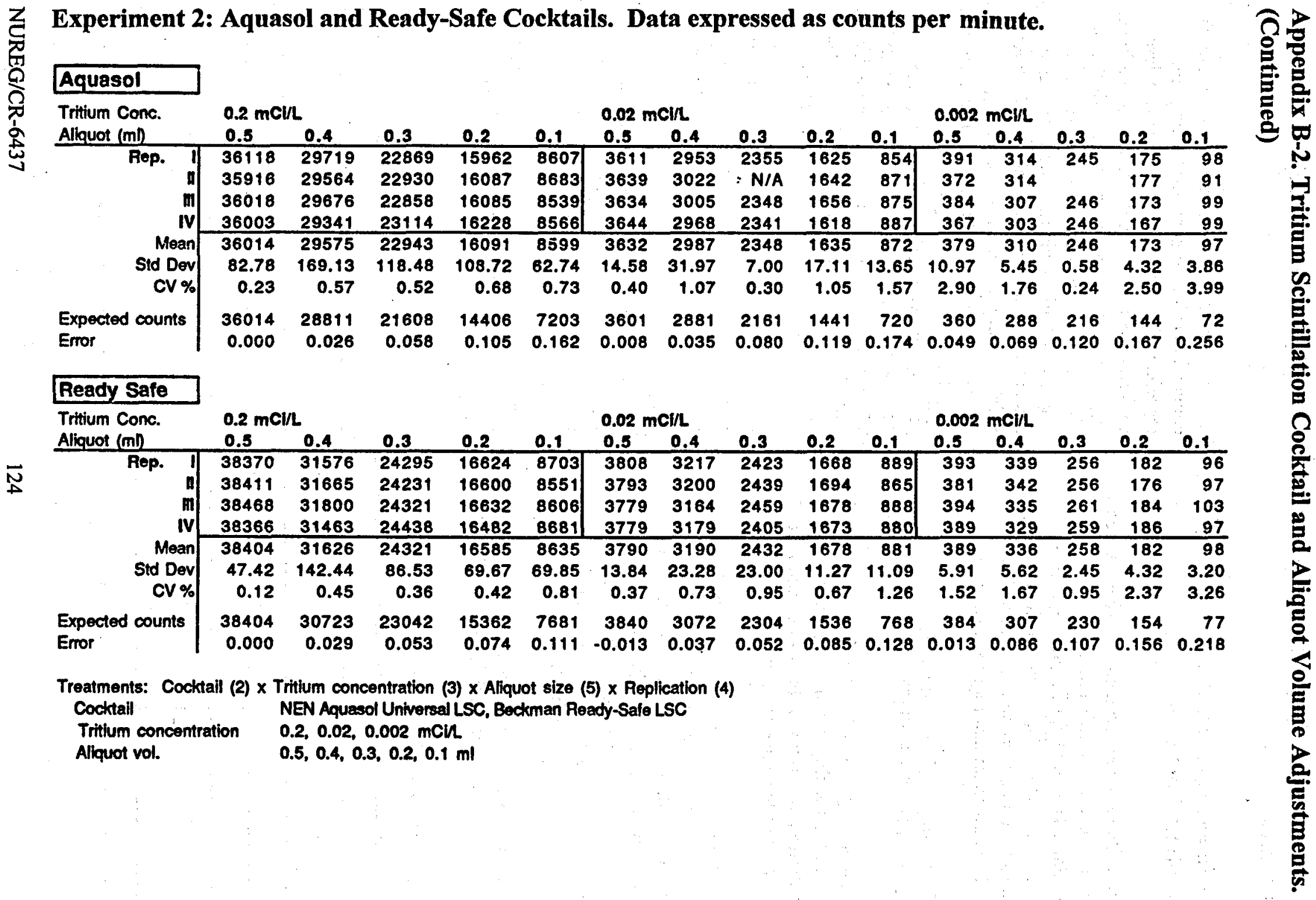


Appendix B-2. Tritium Scintillation Cocktail and Aliquot Volume Adjustments. (Continued)

Experiment 2: Predicted v Observed Counts for Tritium in Aquasol and Ready-Safe Cocktails.

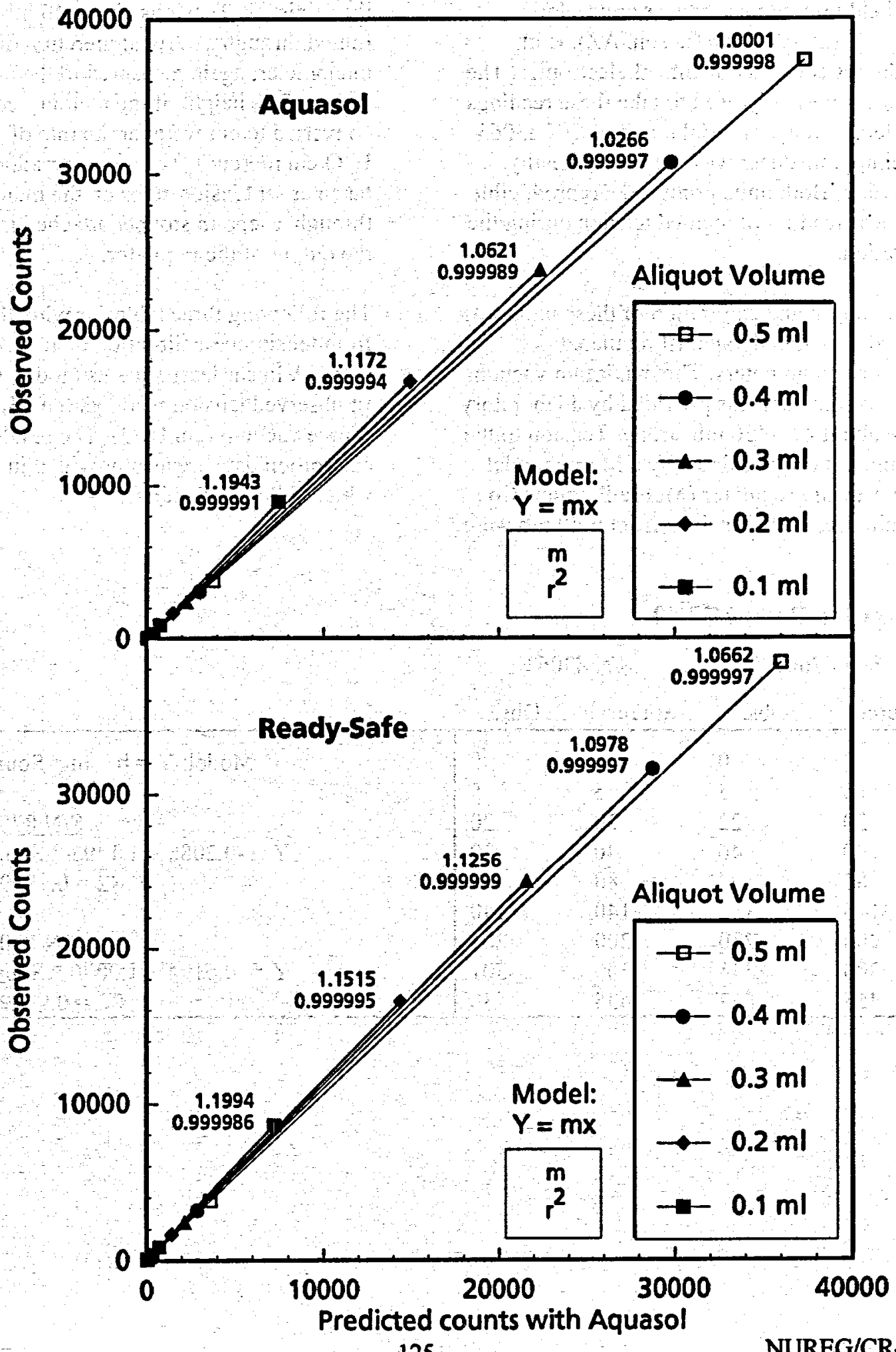




\section{Appendix B-3. Tensimeter Calibration.}

Soil tensions during experiment IIb were measured to the nearest millibar with one of two hand-held pressure transducer units (Soil Measurement Systems, Tucson, AZ). Both transducers contained identical electronics. The transducer normally used to take these readings was identified by its serial number, S/N 83063. A backup transducer was used occasionally, $\mathrm{S} / \mathrm{N}$ 87662. Both units produced a reproducible and linear reading of applied tension during the calibration.

The operation and calibration of these units was checked periodically against regulated laboratory manometers. The maximum vacuum to the manometers was provided by a laboratory source about $425-450 \mathrm{mb}$ strong. Tension to the manometers was regulated by a Moore model 44-20 pressure regulator (Moore Products Co., Springhouse, PA). Tensions from $0-40 \mathrm{mb}$ were routed through a vertical water-filled manometer, measured to the nearest $0.1 \mathrm{~cm}$ at the miniscus. Tensions above $40 \mathrm{mb}$ were routed through a vertical mercury-filled manometer, again measured to the nearest 0.1 $\mathrm{cm}$ hg. The height of the mercury column was converted to $\mathrm{cm}$ water at the rate of $13.6 \mathrm{~cm}$ $\mathrm{H}_{2} \mathrm{O} / \mathrm{cm}$ mercury. Tensimeter readings were taken at set tension steps on the manometer through a septum stopper attached indirectly to the output of the regulator:

The following three tables provide the results from tensimeter calibration on three separate dates. A linear least-squares model was used to fit observed tension readings $\left(\mathrm{cm} \mathrm{H}_{2} \mathrm{O}\right)$ to source vacuum ( $\mathrm{cm} \mathrm{H}_{2} \mathrm{O}$ ). The results of the last calibration date are also provided in a plot which follows the tables.

\section{Calibration Date: 7/27/90}

S/N 87662

S/N 83063

\begin{tabular}{|c|c|c|c|c|}
\hline Source & Obs. & Source & Obs. & \\
\hline 0 & 0 & 0 & 0 & Model: $Y=b+m *$ Source \\
\hline 5 & 5 & 5 & 5 & \\
\hline 20 & 22 & 20. & 20 & S/N 87662 \\
\hline 40 & 46 & 40 & 39 & $Y=-0.2085+1.1493 *$ Source \\
\hline 85 & 97 & 80 & 79 & $R 2=0.999985$ \\
\hline 140 & 162 & 140 & 140 & \\
\hline 200 & 230 & 200 & 201 & S/N 83064 \\
\hline 300 & 345 & 300 & 301 & $Y=-0.6194+1.0070 *$ Source \\
\hline 455 & 522 & 435 & 438 & $R 2=0.999984$ \\
\hline
\end{tabular}


Appendix B-3. Tensimeter Calibration. (Continued)

Calibration Date: 10/9/90

S/N 87662

S/N 83064

Source Obs. Source Obs.

\begin{tabular}{rrrr|r}
\hline 0 & 0 & 0 & 0 & Model: $\mathrm{Y}=\mathrm{b}+\mathrm{m}^{\text {* source }}$ \\
5 & 5 & 5 & 5 & \\
21 & 24 & 20 & 21 & $\mathrm{~S} / \mathrm{N} 87662$ \\
41 & 48 & 40 & 40 & $\mathrm{Y}=-0.6020+1.1512$ * Source \\
80 & 91 & 80 & 80 & $\mathrm{R}^{2}=0.999965$ \\
120 & 136 & 120 & 119 & \\
200 & 229 & 200 & 200 & $\mathrm{~S} / \mathrm{N} 83064$ \\
301 & 345 & 310 & 309 & $Y=-0.1288+1.0018$. Source \\
425 & 490 & 440 & 442 & $\mathrm{R}^{2}=0.99997$
\end{tabular}

Calibration Date: $12 / 12 / 90$

S/N 87662

S/N 83064

Source Obs. Source

\begin{tabular}{rrrr|r}
0 & 0 & 0 & 4 & Model: $Y=b+m$ * source \\
4 & 4 & 10 & 10 & \\
20 & 22 & 20 & 21 & S/N 87662 \\
40 & 44 & 40 & 40 & \\
80 & 91 & 80 & 79 & $Y=-0.8767+1.1444$ * Source \\
136 & 153 & 54 & 54 & $R^{2}=0.99998$ \\
204 & 234 & 136 & 132 & \\
272 & 310 & 204 & 207 & S/N 83064 \\
408 & 466 & 272 & 273 & $Y=-0.2634+1.0029 *$ Source \\
476 & 544 & 408 & 410 & $R^{2}=0.99989$ \\
& & 474 & 474 &
\end{tabular}


Appendix B-3. Tensimeter Calibration. (Continued)

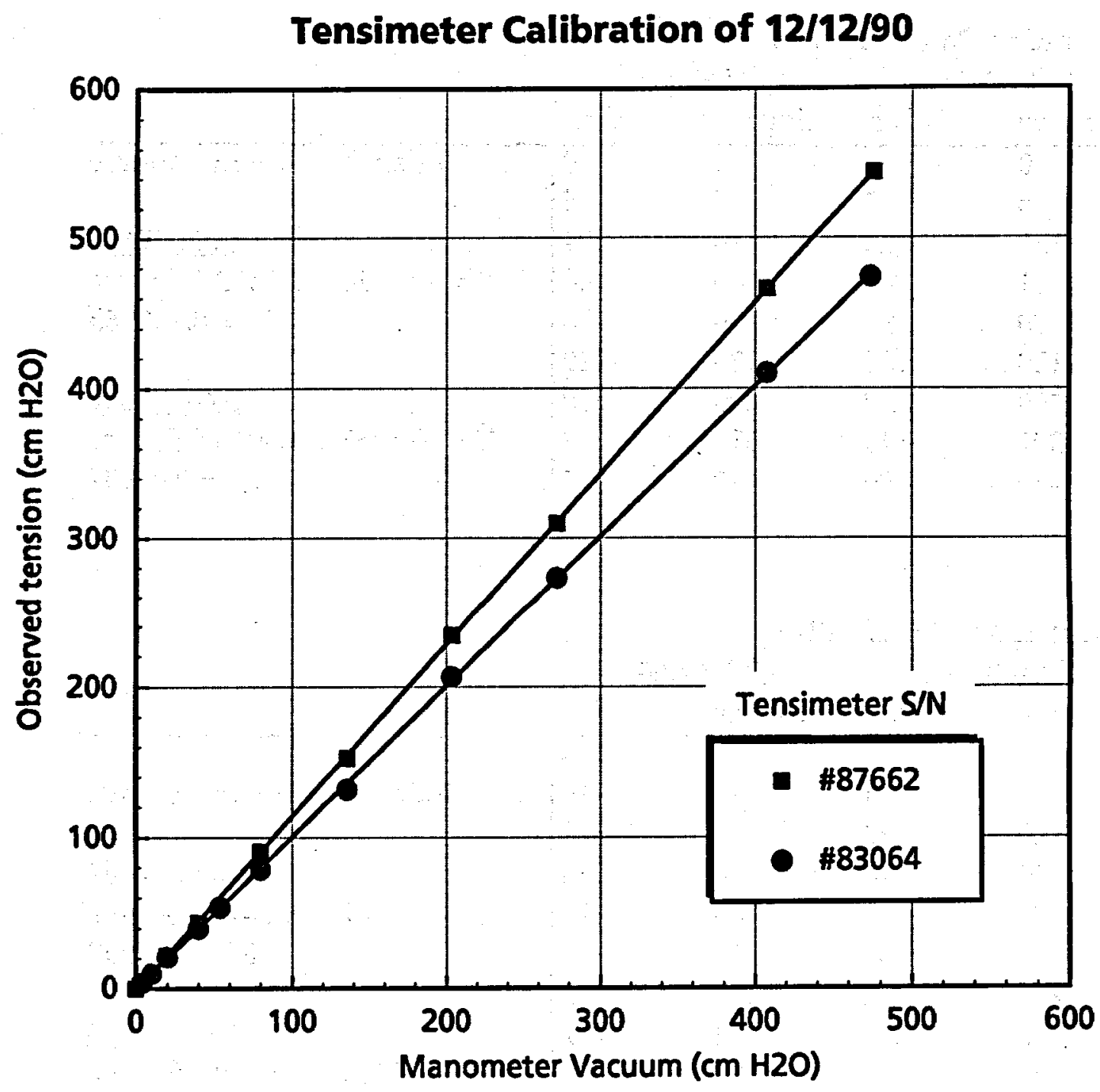




\section{Appendix B-4. Comparison of NMSU and University of Arizona Laboratory Results.}

A small selection of samples from the Experiment IIb soil cores liquid extracts was sent to the Soil, Water and Plant Analyses Laboratory of the University of Arizona to verify earlier results obtained by technicians at New Mexico State University. Ten samples (identified by core section and segment number) had been analyzed at NMSU for nitrate, chloride, chromium and boron by methods described in section 5.2 of this report.
At the University of Arizona laboratory nitrate and chloride were analyzed by ion chromatography methods. Corresponding methods at NMSU were by spectrophotometric autoanalyzer and by chloridometer tritration, respectively. Chromium and Boron were analyzed at the University of Arizona by inductively coupled plasma emission methods like at NMSU. The results of these comparative analyses are provided in the following tables. 
Appendix B-4. Comparison of NMSU and University of Arizona Laboratory Results. (Continued)

\begin{tabular}{crrrr}
$\begin{array}{l}\text { New Mexico State University, (ppm) } \\
\text { Sample ID }\end{array}$ & \multicolumn{1}{c}{ Nitrate } & \multicolumn{1}{c}{ Chloride } & \multicolumn{1}{c}{ Boron } & Chromium \\
\hline $33-1$ & 0.3 & 11.7 & 4.776 & 0.071 \\
$33-2$ & 5.9 & 16.1 & 4.052 & 0.064 \\
$35-1$ & 225.3 & 406.1 & 5.187 & 0.005 \\
$35-2$ & 241.2 & 518.5 & 4.505 & 0.007 \\
$35-3$ & 232.4 & 587.6 & 3.957 & 0.015 \\
$35-4$ & 201.5 & 608.5 & 4.894 & 0.010 \\
$36-1$ & 14.3 & 4.7 & 4.471 & 0.003 \\
$36-2$ & 16.4 & 6.5 & 4.372 & 0.001 \\
$36-3$ & 8.1 & 5.0 & 3.836 & 0.035 \\
$36-4$ & 5.2 & 8.0 & 4.318 & 0.027
\end{tabular}

University of Arizona, (ppm)

\begin{tabular}{crrrr} 
Sample ID & \multicolumn{1}{c}{ Nitrate } & \multicolumn{1}{c}{ Chloride } & \multicolumn{1}{c}{ Boron } & Chromium \\
\hline $33-1$ & 1.2 & 7.1 & 5.120 & 0.094 \\
$33-2$ & 4.3 & 9.1 & 4.520 & 0.084 \\
$35-1$ & 247.0 & 396.0 & 5.500 & -0.002 \\
$35-2$ & 264.0 & 516.0 & 4.830 & -0.002 \\
$35-3$ & 241.0 & 588.0 & 4.150 & -0.002 \\
$35-4$ & 220.0 & 597.0 & 5.190 & -0.002 \\
$36-1$ & 15.9 & 6.1 & 5.130 & 0.003 \\
$36-2$ & 20.3 & 7.2 & 4.900 & -0.002 \\
$36-3$ & 12.8 & 6.5 & 4.170 & 0.019 \\
$36-4$ & 6.4 & 8.3 & 4.670 & 0.015
\end{tabular}

Difference between measurements, U/A - NMSU, (ppm)

\begin{tabular}{crrrr} 
Sample ID & \multicolumn{1}{c}{ Nitrate } & \multicolumn{1}{c}{ Chloride } & \multicolumn{1}{c}{ Boron } & \multicolumn{1}{c}{ Chromium } \\
\hline $33-1$ & 0.9 & -4.6 & 0.344 & 0.023 \\
$33-2$ & -1.6 & -7.0 & 0.468 & 0.020 \\
$35-1$ & 21.7 & -10.1 & 0.313 & -0.007 \\
$35-2$ & 22.8 & -2.5 & 0.325 & -0.009 \\
$35-3$ & 8.6 & 0.4 & 0.193 & -0.017 \\
$35-4$ & 18.5 & -11.5 & 0.296 & -0.012 \\
$36-1$ & 1.6 & 1.4 & 0.659 & 0.000 \\
$36-2$ & 3.9 & 0.7 & 0.528 & -0.003 \\
$36-3$ & 4.7 & 1.5 & 0.334 & -0.016 \\
$36-4$ & 1.2 & 0.3 & 0.352 & -0.012
\end{tabular}


Appendix B-5. Experiment IIb Forms for field data collection. NRC TRENCH EXPERIMENT Ib - PUMPHOUSE RECORD

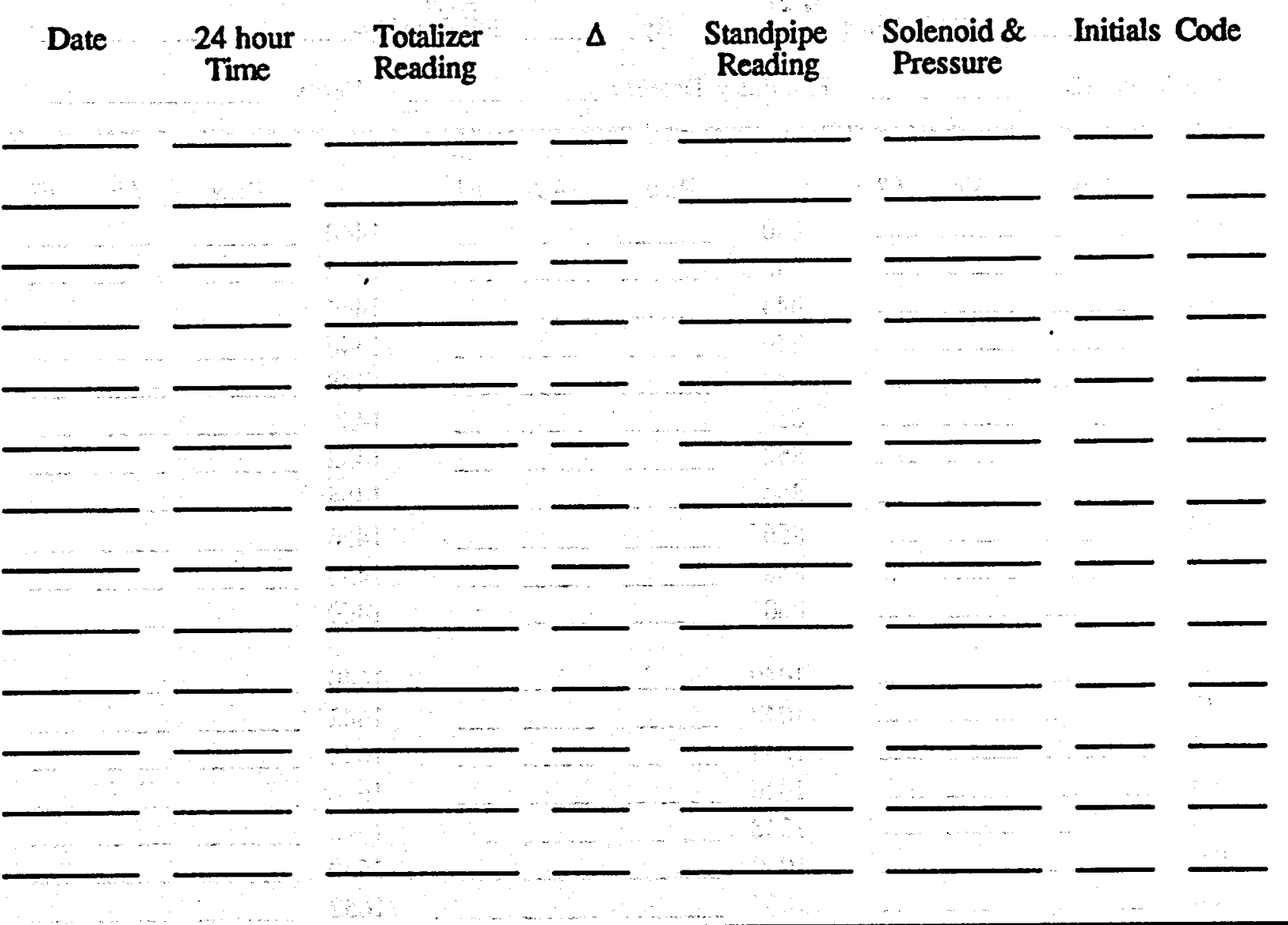

\section{Comments:}


Appendix B-5. Experiment IIb Forms for field data collection. (Continued)

NRC TRENCH EXPERIMENT IIb - TENSIOMETER READINGS

Date $24 \mathrm{hr}$ Time

By Fill Date/By

Tensimeter ID File Entry Date/By Verify

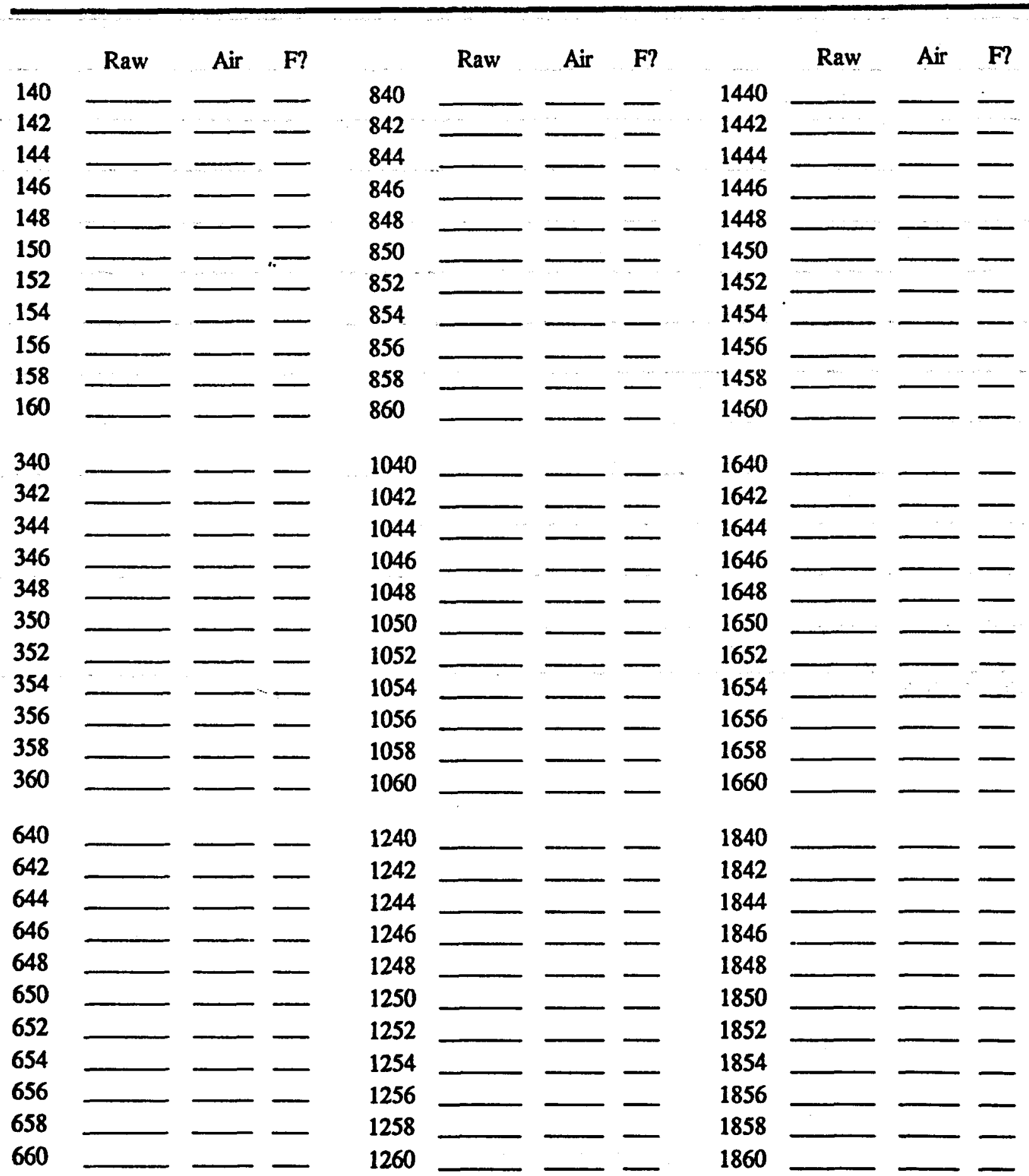


Appendix B-5. Experiment IIb Forms for field data collection. (Continued) NRC TRENCH EXPERIMENT Ib - TENSIOMETER READINGS (continued)

\begin{tabular}{|c|c|c|c|c|c|c|c|c|c|c|}
\hline & Raw & Air & F? & & Raw & Air & F? & & Raw & Air \\
\hline 2040 & & 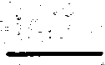 & 3 & 2240 & $-i$ & 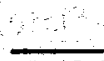 & $\cdots$ & 2440 & & \\
\hline )42 & $\because$ & 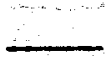 & - & 2242 & & 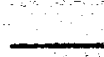 & - & 2442 & & \\
\hline 2044 & - & - & - & 2244 & & + & 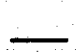 & 2444 & & \\
\hline 046 & $\ldots$ & 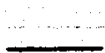 & $\therefore$ & 2246 & & $\ldots$ & - & 2446 & $\cdots$ & - \\
\hline 048 & $\cdots$ & $\ldots$ & 3 & 2248 & & 2 & 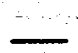 & 2448 & - & 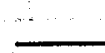 \\
\hline 050 & $\cdots$ & $\ldots$ & 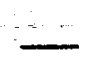 & 2250 & 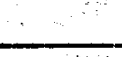 & 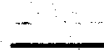 & 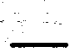 & 2450 & & - \\
\hline 052 & $\ldots$ & $\ldots$ & - & 2252 & 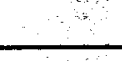 & 1 & - & 2452 & & - \\
\hline 554 & $\ldots$ & 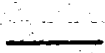 & - & 2254 & 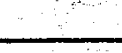 & $\ldots$ & - & 2454 & & 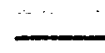 \\
\hline 056 & $\ldots$ & $-\ldots$ & 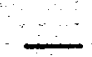 & 2256 & & 0 & + & 2456 & - & + \\
\hline 058 & $\ldots$ & 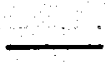 & 2 & 2258 & & $\ldots$ & 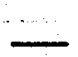 & 2458 & $\cdots$ & $\ldots$ \\
\hline 60 & $0-1$ & $\ldots$ & - & 2260 & 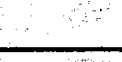 & $\ldots$ & 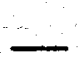 & 2460 & & $\ldots$ \\
\hline
\end{tabular}

Comments: 
Appendix B-5. Experiment IIb Forms for field data collection. (Continued) NRC TRENCH EXPERIMENT IIb - SOLUTION SAMPLE COLLECTION \& VOLUME

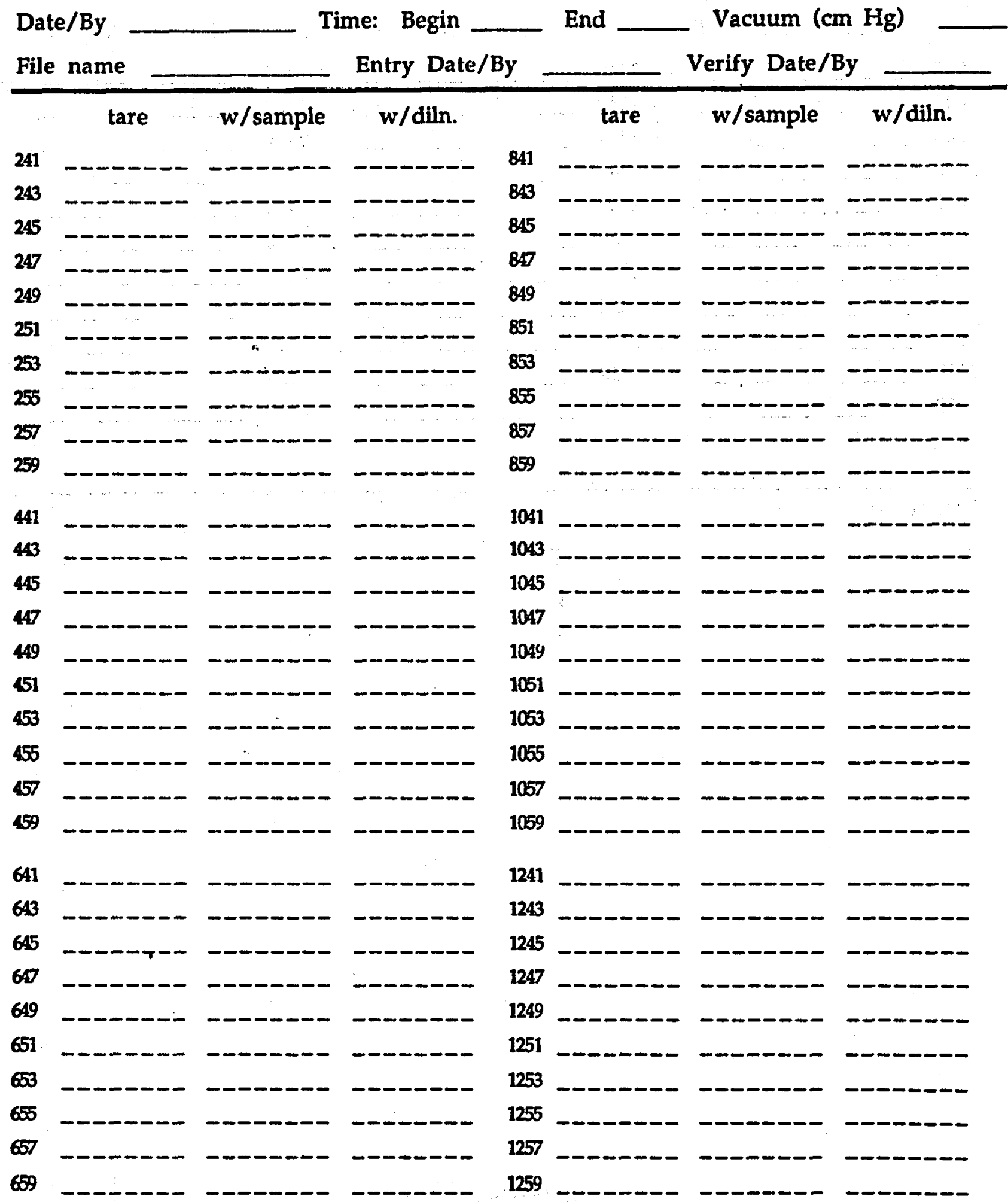


Appendix B-5. Experiment IIb Forms for field data collection. (Continued)

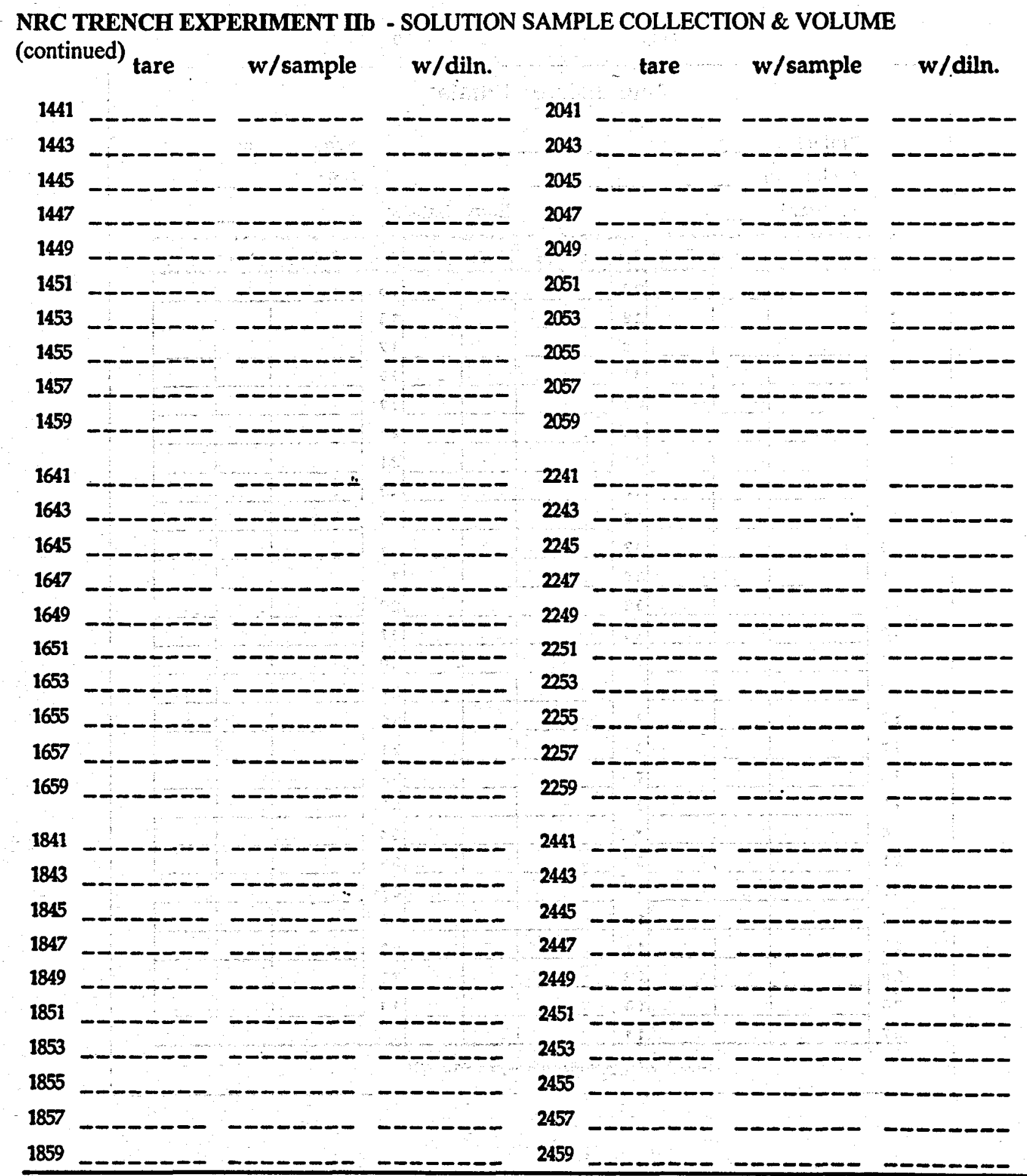

Code Explanation \& Comments: 
Appendix B-5. Experiment IIb Forms for field data collection. (Continued)

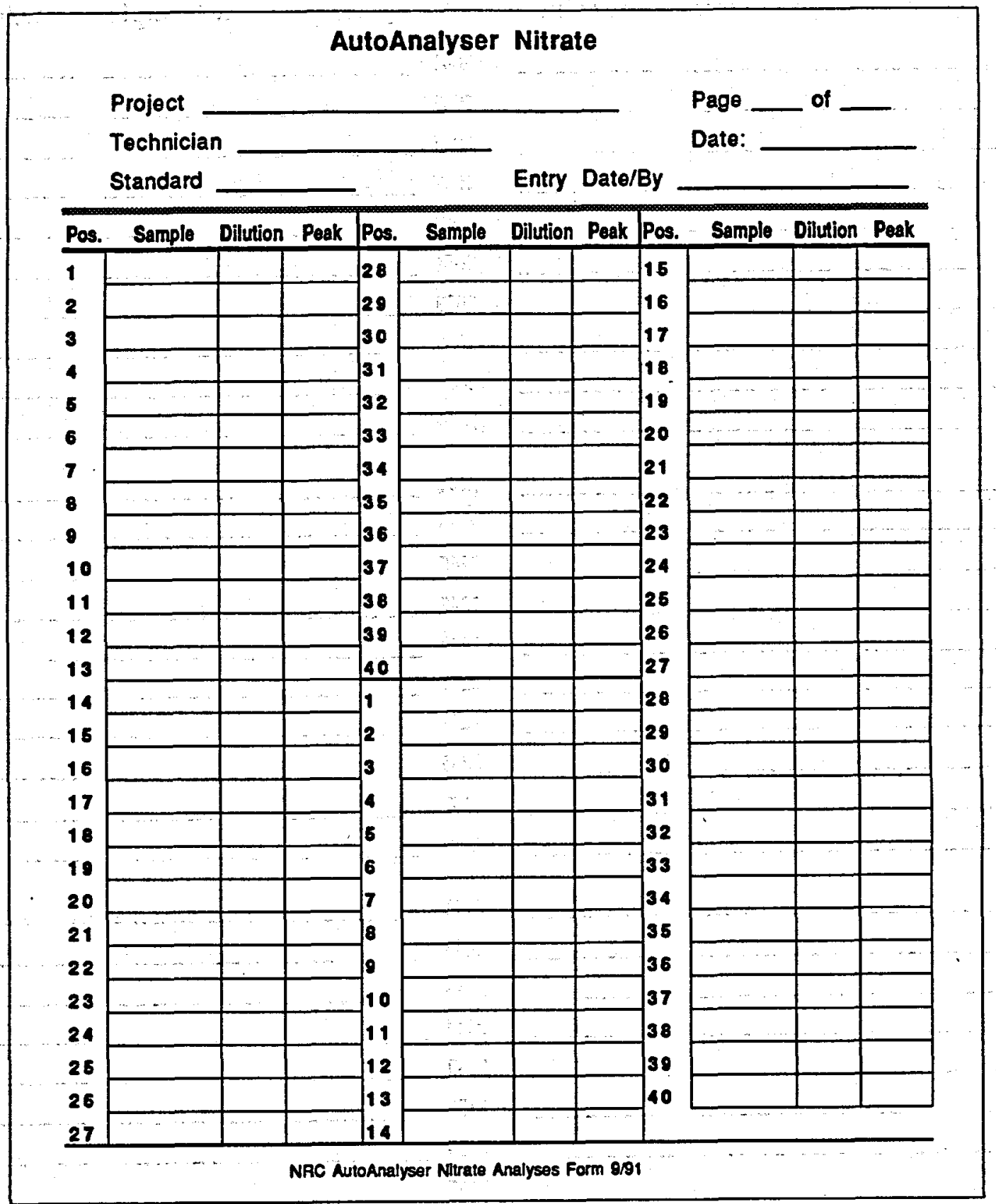


Appendix B-5. Experiment IIb Forms for field data collection. (Continued)

CHLORIDOMETER CHLORIDE

Project

Date

File: Name
Time

Entry Date/By
By

Verify Date/By

$-------2-$

Normal chloridometer mode:

Low

High

Background (me/1):

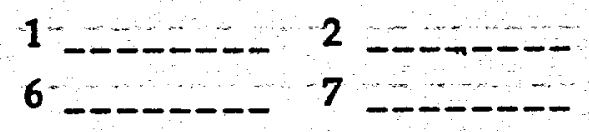

Mean

Sample ID \# A me/l

Comments:
Std Dev

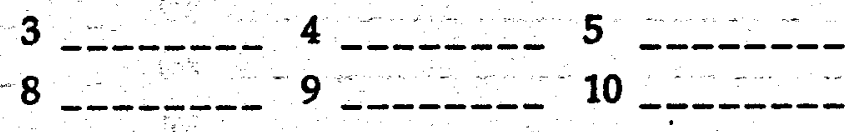

CV

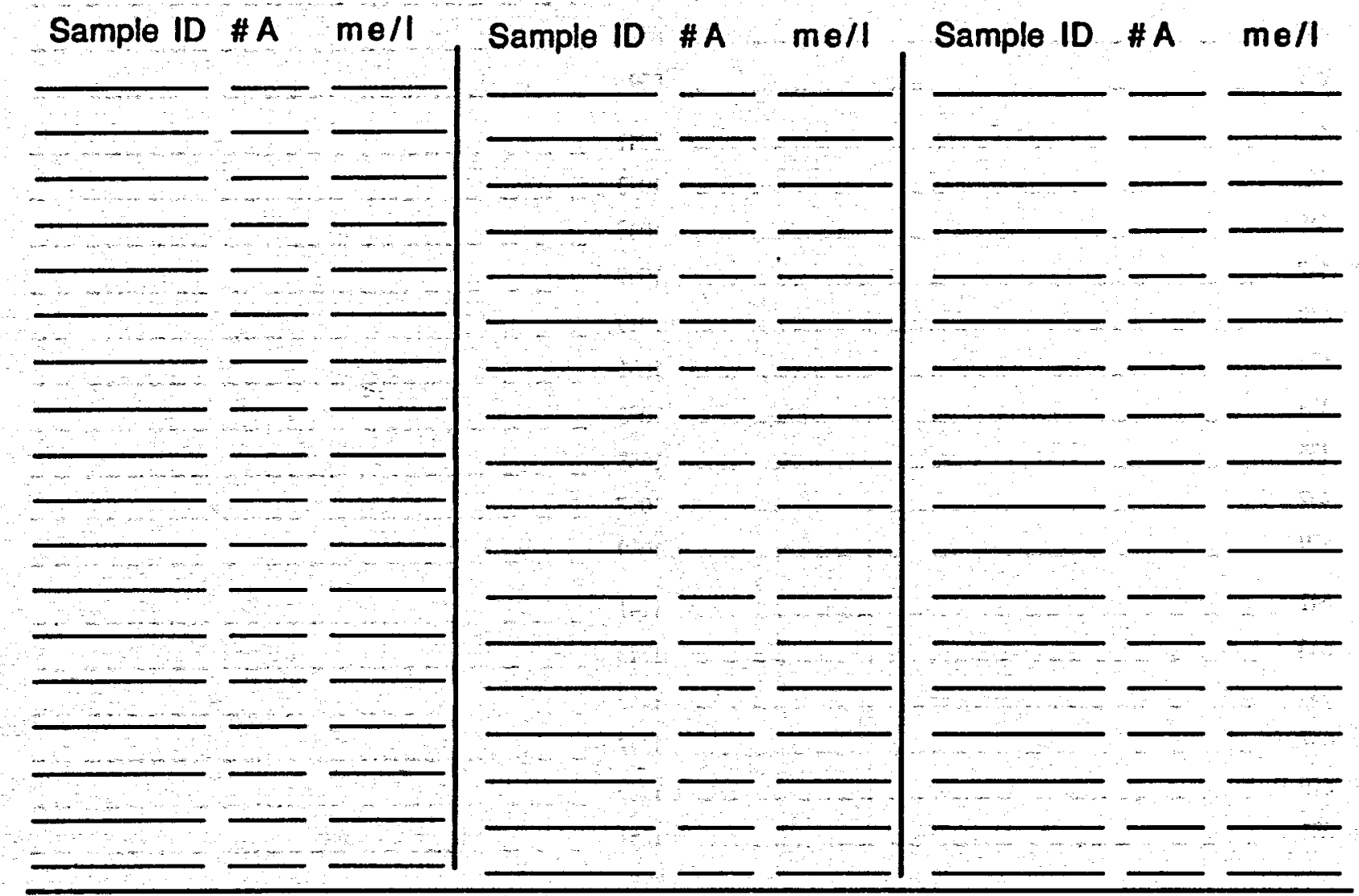


Appendix B-5. Experiment IIb Forms for field data collection. (Continued)

NRC TRENCH EXPERIMENT IIb - TRITIUM REPEAT ANALYSES

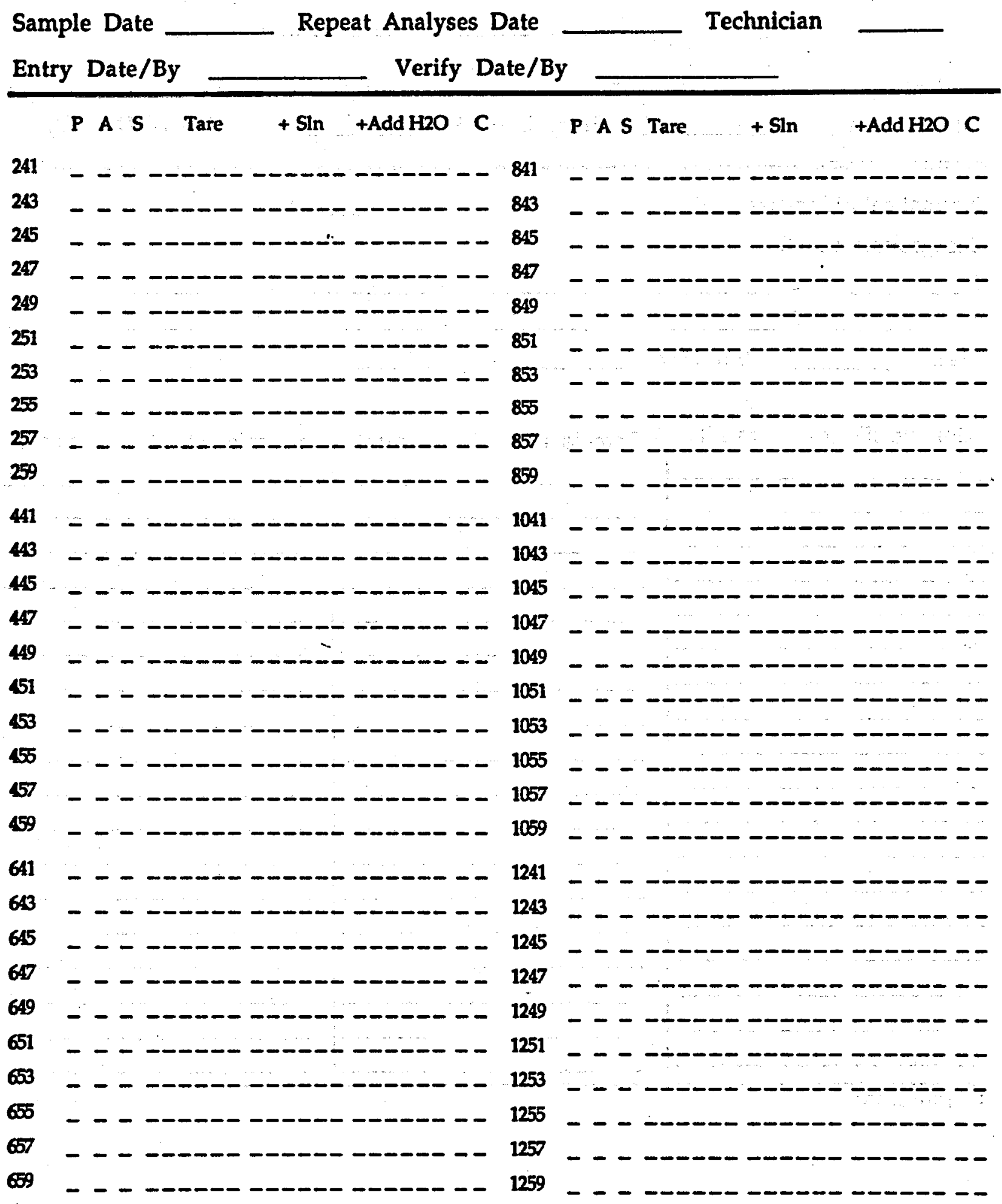




\section{Appendix B-5. Experiment IIb Forms for field data collection. (Continued)}

\section{NRC TRENCH EXPERIMENT IIb - TRITIUM REPEAT ANALYSES (continued)}
P A S Tare
$+\operatorname{Sin}$
+Add H2O C

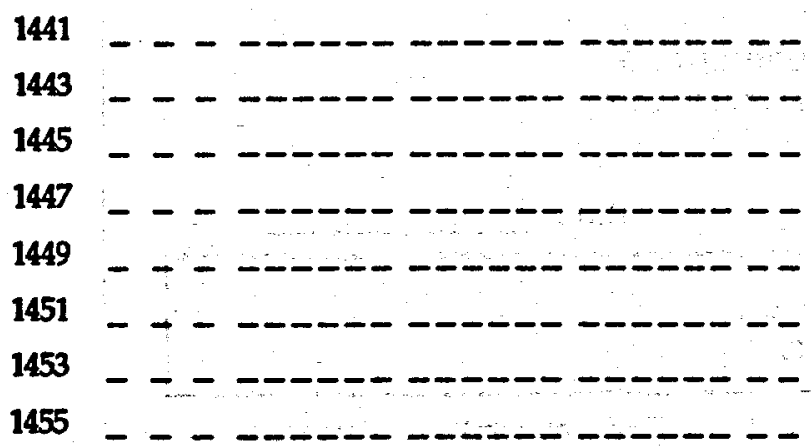

1457

1459

1641

1643

1645

1647

1649

1651

1653

1655

1657

1659

1841

1843

1845

1847

1849

1851

1853

1855

1857

1859

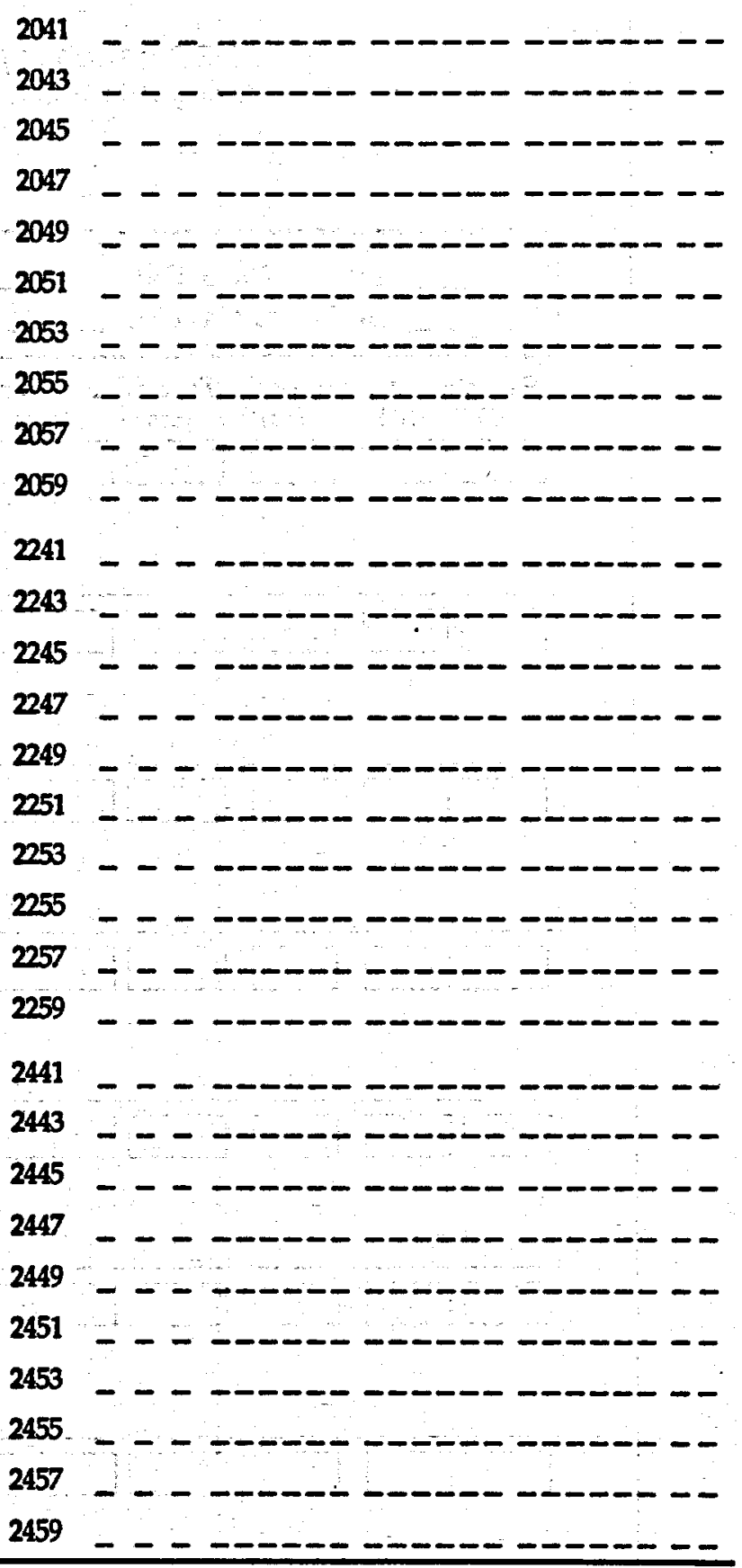

Code Explanation \& Comments: 
Appendix B-5. Experiment IIb Forms for field data collection. (Continued)

NRC TRENCH EXPERIMENT IIb

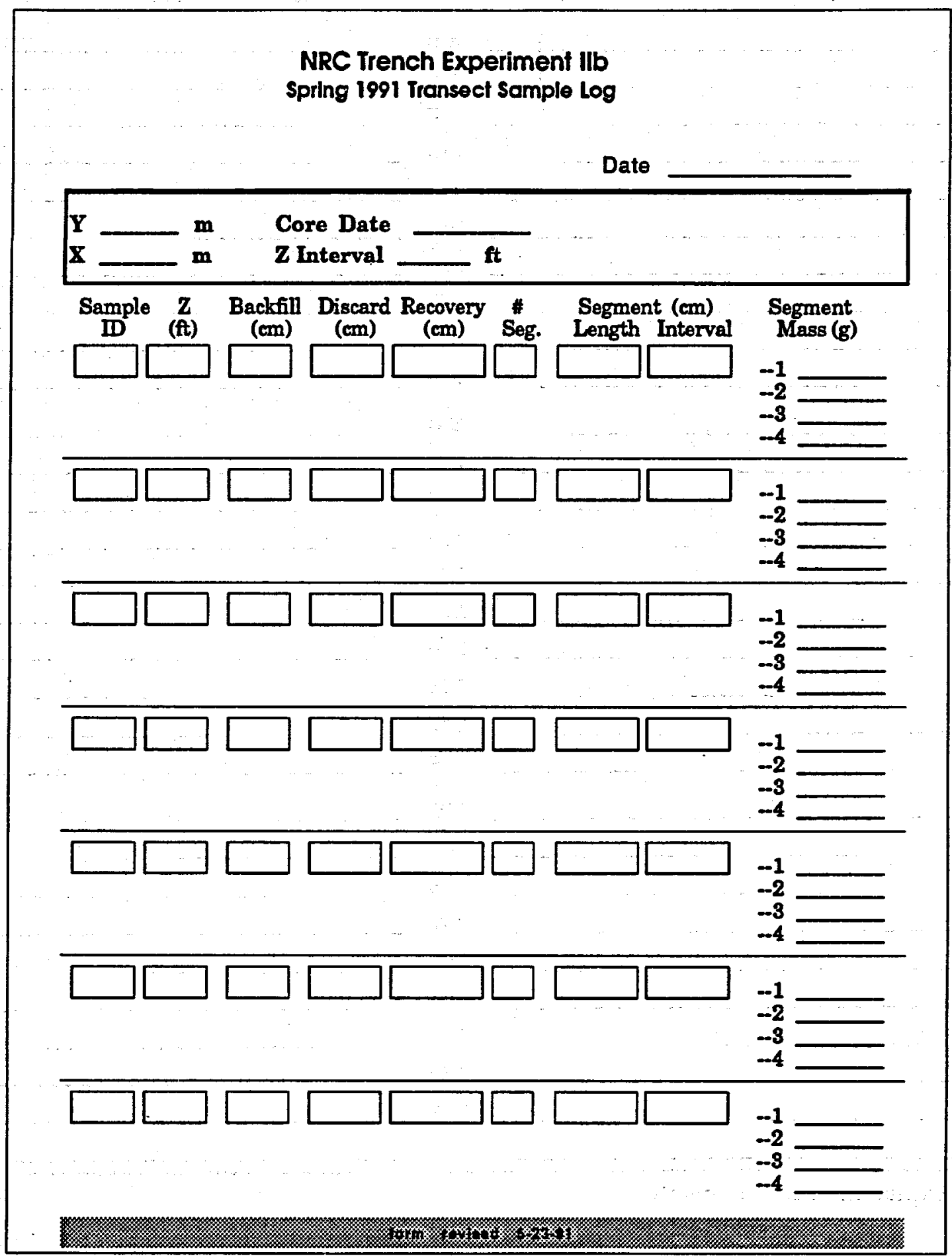


Appendix B-5. Experiment IIb Forms for field data collection. (Continued)

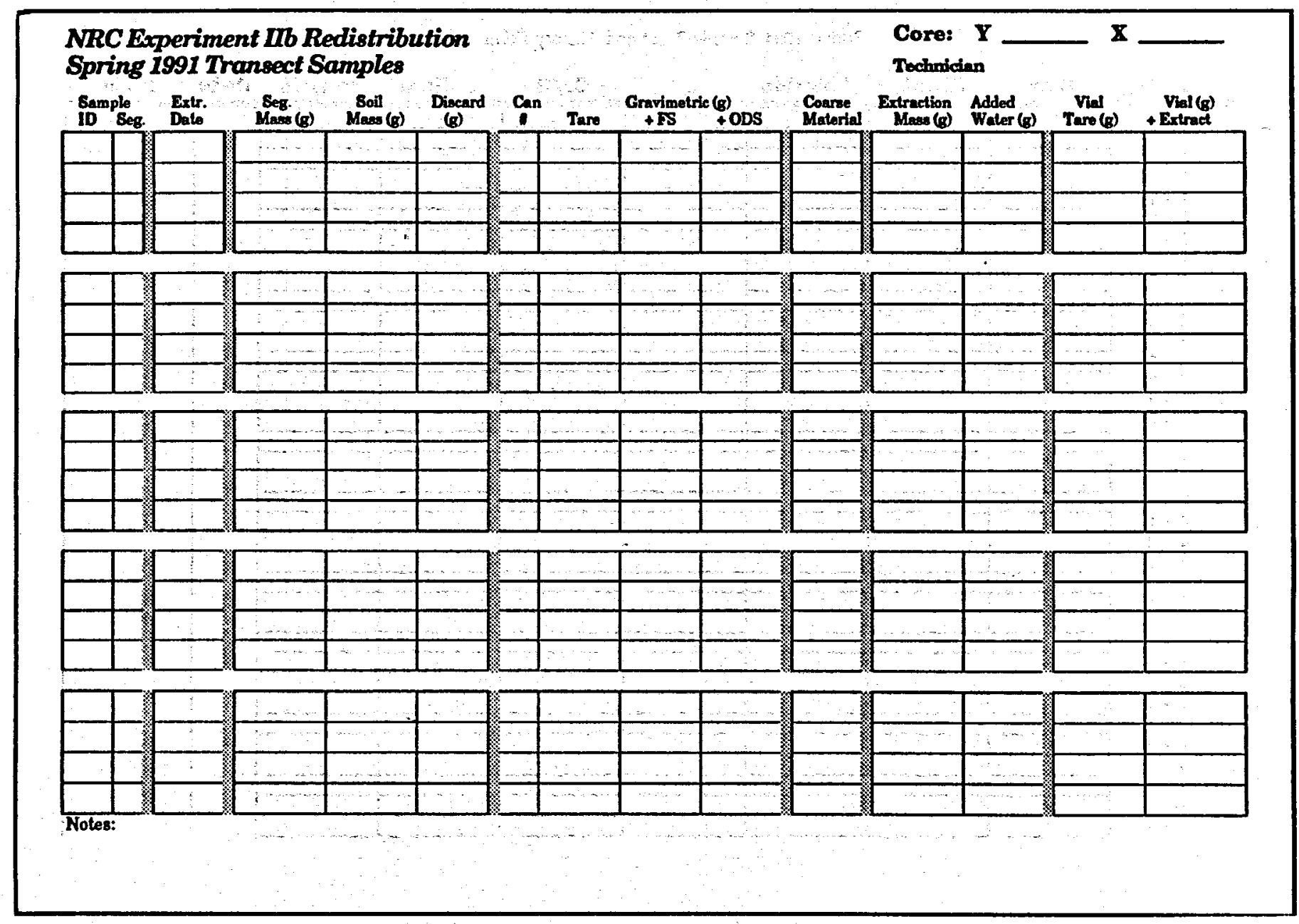


Appendix B-5. Experiment IIb Forms for field data collection. (Continued)

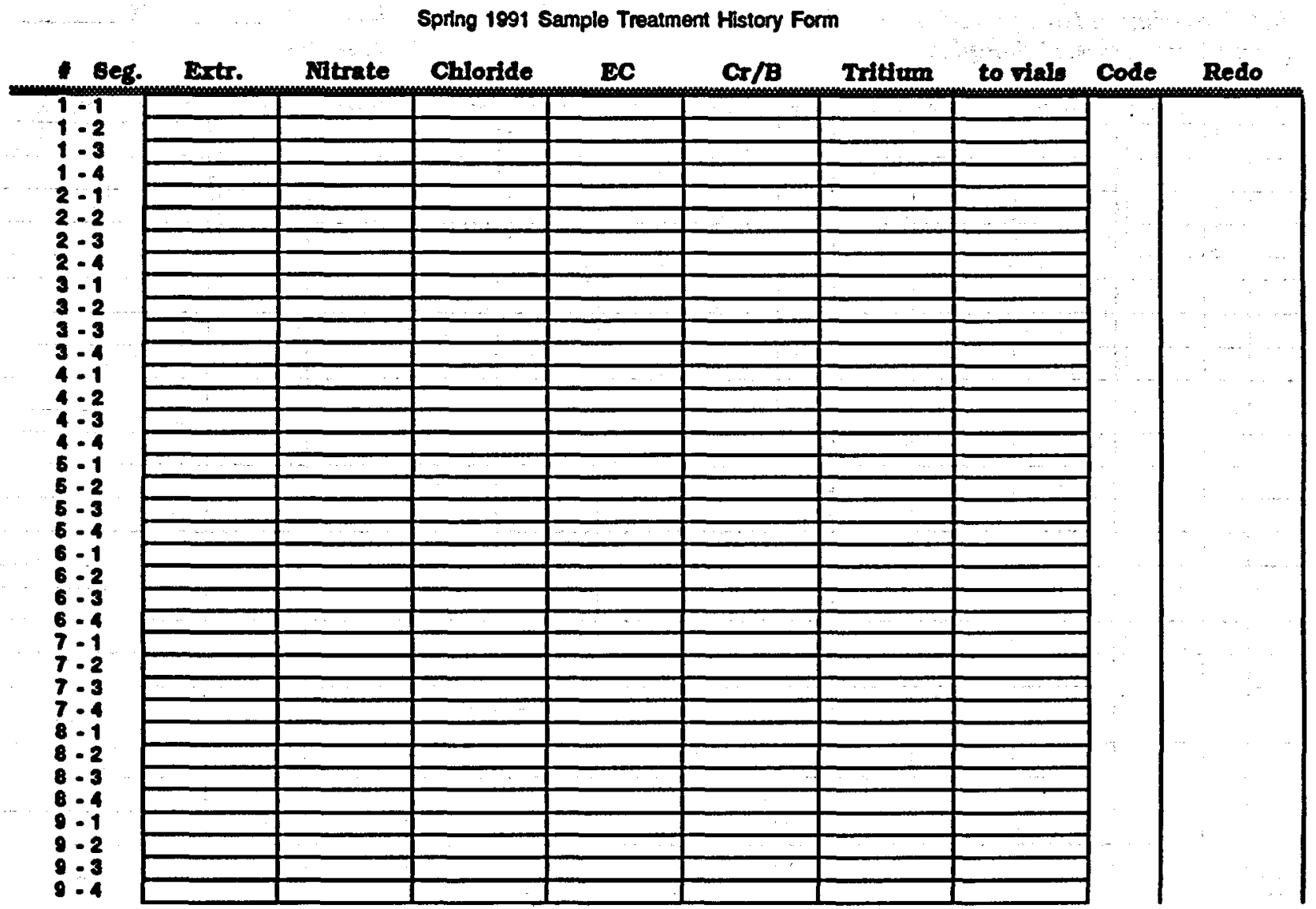




\section{Appendix B-6. Experiment IIb Sample pH, Day 225.}

The samples collected on day 225 (3/14/91) from the Experiment IIb solution samplers were evaluated for approximate $\mathrm{pH}$ in the field. Narrow range $\mathrm{pH}$ indicator strips (ColorpHast strips, EM Science, Gibbstown, NJ), pH 6.510.0 were dipped into the fresh solution of each sampler, allowed to develop $\mathrm{pH}$-dependant color changes for about 1 minute and then compared to a color chart to determine $\mathrm{pH}$. The color comparisons were made in natural light conditions. The semi-quantitative nature of these strips allows $\mathrm{pH}$ resolution of 0.2 units between $\mathrm{pH}=6.8-7.8$ and 0.3 units above and below those limits. Some visual interpolations between those units were made when evaluating these samples. The following table lists the results for each solution sample collected on that day.

\begin{tabular}{|c|c|c|c|c|c|c|c|c|c|c|c|}
\hline$x_{2}$ & $Y_{2}$ & $z_{2}$ & pH & $x_{2}$ & $Y_{2}$ & $z_{2}$ & pH & $x_{2}$ & $\mathbf{Y}_{2}$ & $\mathbf{Z}_{2}$ & pH \\
\hline-2.26 & 0.5 & 0.5 & 7.4 & 1.84 & 0.5 & 2.0 & 75 & -0.25 & 0.5 & 4.0 & 8.2 \\
\hline-1.75 & 0.5 & 0.5 & 75 & 2.24 & 0.5 & 2.0 & 7.6 & 0.25 & 0.5 & 4.0 & $8 A$ \\
\hline-1.25 & 0.5 & 0.5 & 7.3 & -2.25 & 0.5 & 2.5 & 8.0 & 0.75 & 0.5 & 4.0 & $8 . A$ \\
\hline-0.25 & 0.5 & 0.5 & 7.6 & -1.75 & 0.5 & 2.5 & 8.0 & 1.25 & 0.5 & 4.0 & 82 \\
\hline 0.25 & 0.5 & 0.5 & 7.6 & -1.25 & 0.5 & 2.5 & 8.1 & 1.84 & 0.5 & 4.0 & 8.1 \\
\hline 0.75 & 0.5 & 0.5 & 72 & -0.75 & 0.5 & 25 & 7.6 & -1.75 & 0.5 & 4.5 & 75 \\
\hline 1.25 & 0.5 & 0.5 & 6.9 & 0.25 & 0.5 & 25 & 77 & -1.25 & 0.5 & 4.5 & 73 \\
\hline 1.84 & 0.5 & 0.5 & 7.3 & 1.25 & 0.5 & 25 & 77 & -0.75 & 0.5 & 4.5 & 8.3 \\
\hline 2.23 & 0.5 & 0.5 & 7.2 & 1.84 & 0.5 & 25 & 7.6 & -0.25 & 0.5 & 4.5 & 8.1 \\
\hline-2.26 & 0.5 & 1.0 & 6.6 & 2.24 & 0.5 & 2.5 & 7.6 & 0.25 & 0.5 & 4.5 & 8.4 \\
\hline-1.75 & 0.5 & 1.0 & 7.6 & -2.26 & 0.5 & 3.0 & 8.3 & 0.75 & 0.5 & 4.5 & 8.1 \\
\hline-0.75 & 0.5 & 1.0 & 7.3 & -1.75 & 0.5 & 3.0 & 8.1 & 1.25 & 0.5 & 4.5 & 8.1 \\
\hline-0.25 & 0.5 & 1.0 & 7.1 & -1.25 & 0.5 & 3.0 & 7.9 & 1.84 & 0.5 & 4.5 & 8.1 \\
\hline 0.75 & 0.5 & 1.0 & 7.6 & -0.75 & 0.5 & 3.0 & 8.4 & 2.24 & 0.5 & 4.6 & 8.1 \\
\hline 1.25 & 0.5 & 1.0 & 7.4 & -0.25 & 0.5 & 3.0 & 82 & -1.75 & 0.5 & 5.0 & 72 \\
\hline 1.84 & 0.5 & 1.0 & 7.5 & 0.25 & 0.5 & 3.0 & 8.1 & -1.25 & 0.5 & 5.0 & 75 \\
\hline 224 & 0.5 & 1.0 & 7.4 & 0.75 & 0.5 & 3.0 & 8.3 & -0.75 & 0.5 & 5.0 & 7.6 \\
\hline-2.25 & 0.5 & 1.5 & 7.7 & 1.25 & 0.5 & 3.0 & 8.4 & -0.25 & 0.5 & 5.0 & 8.2 \\
\hline-1.75 & 0.5 & 1.5 & 7.8 & 1.84 & 0.5 & 3.0 & 82 & 0.25 & 0.5 & 5.0 & 78 \\
\hline-1.25 & 0.5 & 1.5 & 7.7 & 2.24 & 0.5 & 3.0 & 7.6 & 0.75 & 0.5 & 5.0 & 7.7 \\
\hline-0.75 & 0.5 & 1.5 & 7.6 & -2.25 & 0.5 & 3.5 & 7.7 & 1.25 & 0.5 & 5.0 & 7.6 \\
\hline-0.25 & 0.5 & 1.5 & 7.5 & -1.75 & 0.5 & 3.5 & 7.7 & -1.25 & 0.5 & 5.5 & 75 \\
\hline 0.25 & 0.5 & 1.5 & 7.9 & -1.25 & 0.5 & 3.5 & 78 & -0.75 & 0.5 & 5.5 & $7 A$ \\
\hline 0.75 & 0.5 & 1.5 & 7.6 & -0.75 & 0.5 & 3.5 & 8.3 & -0.25 & 0.5 & 5.5 & 76 \\
\hline 125 & 0.5 & 1.5 & 7.7 & -0.25 & 0.5 & 3.5 & 8.1 & 0.25 & 0.5 & 5.5 & 7.6 \\
\hline 1.84 & 0.5 & 1.5 & 7.6 & 0.25 & 0.5 & 3.5 & 8.7 & 0.75 & 0.5 & 5.5 & 7.5 \\
\hline 224 & 0.5 & 1.5 & 7.5 & 0.75 & 0.5 & 3.5 & 8.4 & 1.25 & 0.5 & 5.5 & 73 \\
\hline-1.75 & 0.5 & 2.0 & 8.1 & 1.25 & 0.5 & 3.5 & 8.5 & -1.25 & 0.5 & 6.0 & 73 \\
\hline-0.75 & 0.5 & 2.0 & 7.8 & 1.84 & 0.5 & 3.5 & 7.6 & -0.75 & 0.5 & 6.0 & 73 \\
\hline-0.25 & 0.5 & 2.0 & 7.7 & -2.25 & 0.5 & 4.0 & 78 & -0.25 & 0.5 & 6.0 & 7.4 \\
\hline 0.25 & 0.5 & 2.0 & 7.1 & -1.75 & 0.5 & 4.0 & 8.1 & 0.25 & 0.5 & 6.0 & $7 A$ \\
\hline 0.75 & 0.5 & 2.0 & 7.7 & -1.25 & 0.5 & 4.0 & 8.1 & & & & \\
\hline 1.25 & 0.5 & 2.0 & 7.6 & -0.75 & 0.5 & 4.0 & 8.4 & & & & \\
\hline
\end{tabular}





\section{Appendix C. Miscellaneous Appendices.}

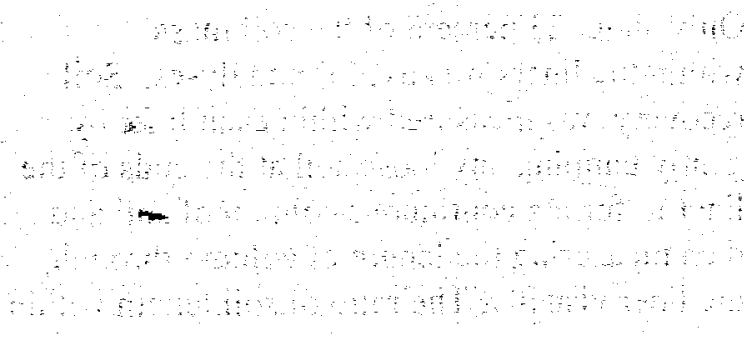




\section{Appendix C-1. Experiment I Redistribution Cores II and III.}

\section{Introduction}

On April 6, 1989, 594 days after the last irrigation of plot I, a $14.6 \mathrm{~m}$ deep borehole was made near the center of the $4 \mathrm{~m}$ by $9 \mathrm{~m}$ irrigated area $\left(X_{1}=0.46 \mathrm{~m}, Y_{1}=4.67 \mathrm{~m}\right)$. A 2" diameter split-spoon sampler was used to collect continuous soil cores in 2' intervals. These samples were analyzed for water content, tritium, chloride ion, electrical conductivity and soil morphological features. The results of that work is discussed by Vinson, et al, 1989.

Another pair of cores was collected from plot I nearly a year later, on March 16, 1990, 938 days after the last irrigation. The purpose of these cores was to supplement the data collected from the center-of-plot borehole with borehole data from the edge and 2 meters outside of the irrigated area, while remaining near the center of the plot with respect to the Y-plane. These core samples were analyzed for volumetric water content, tritium, chloride ion and nitrate ion. The locations of these new boreholes are:

\begin{tabular}{|cccc|}
\hline & $\mathrm{X}_{1}(\mathrm{~m})$ & $\mathrm{Y}_{1}(\mathrm{~m})$ & $\mathrm{Z}_{\text {total }}(\mathrm{m})$ \\
BoreholeII & 2.02 & 4.95 & 15.22 \\
& & & \\
Borehole III & 4.00 & 5.00 & 15.19 \\
\hline
\end{tabular}

The split-spoon apparatus used for the first "center" borehole was able to penetrate below about 8 meters depth with great difficulty. Also, the analyses of the soil cores and solution extracts revealed that some sample cross contamination across borehole depths had probably occurred, most likely during the periods when the split-spoon sampler was pulled from the borehole to recover a core segment or pushed back into the borehole to regain a previous depth. In an effort to correct these previous difficulties a different drilling method was used to produce the Borehole II and Borehole III samples that we discussed here.

\section{Borehole Drilling}

A 6" hollow-stem CME rotary drill apparatus mounted on a drill truck (Southwest Engineering, Inc., Las Cruces, New Mexico) was used. The sampling tube, designed to collect uncontaminated cores, was lined with $3^{\prime \prime}$ OD. acrylic tubing ( $1 / 8^{\prime \prime}$ wall thickness) in $5^{\prime}$ lengths ( 2 pieces, 2.5 feet long, joined with tape). A total of twenty $2.5^{\prime}$ long core sections were collected from each borehole. A technician was stationed to monitor and measure drilling depths each time the sampling tube was pulled from the borehole. As each acrylic liner was extracted from the sample tube it was permanently marked to note sample depth and orientation, sealed at either end with several layers of Parafilm ${ }^{\oplus}$ paraffin wrap (American Can, Greenwich, CT), capped with acrylic end plates, and then protected from accidental disturbance with additional seals of cloth duct tape. As a precaution each segment was kept in a horizontal position to reduce any vertical displacement of materials within the liner. The core segments were kept out of direct sunlight while in the field. At the end of the field operations the cores were taken to the laboratory for refrigerated storage.

\section{Sample Preparation}

Only about 25 percent of the soil mass within the liners was used for analyses. Soil recovery was measured within each liner by gently tamping any loose soil at the ends of the liner to form a continuous column of soil and then measuring the length of column through the liner visually. The ratio of soil length within 


\section{Appendix C-1. Experiment I Redistribution Cores II and III. (Continued)}

the liner to the depth of soil drilled to fill the liner was used to assign depths to, core subsamples.

Á section of an individual core was selected for sampling and its assigned depth was based on the cumulative borehole depth at the top of the liner section plus the proportioned length of

the soil core and measured to the center of the sample. On average, core samples were centered at $0.35 \mathrm{~m}$ intervals along the depth of the boreholes. An average of $498 \mathrm{~g}$ of fresh soil was taken for each sub-sample. About half of this was used to determine oven-dry gravimetric moisture and the other half for making solution extracts. Because of the large sample size the samples were not pre-screened for nonrepresentative sample fractions.

Gravimetric moisture was measured by placing a core sub-sample in a tared aluminum soil can, measuring the can mass to the nearest $0.01 \mathrm{~g}$ on a Sartorius E2000D balance (Brinkman Instru- : ment, Inc., Westbury, NY), and then drying the sample in a laboratory convective oven at $105^{\circ}$ $C$ for 36-48 hours before re-weighing the can. A period longer than 24 hours was necessary to ensure equilibrium moisture contents $(<0.01$ $\mathrm{g} / 12 \mathrm{hr}$. mass change) for the large sample masses.

Volumetric water content was estimated from gravimetric water content by using soil bulk density data from the original trench characterization work (Wierenga et al., 1988, 1989, $1990)$. A grand mean value $\left(1.67 \mathrm{Mg} / \mathrm{m}^{3}\right)$ of all bulk density values of cores used for site characterization, those collected from the north face of the trench during excavation, was universally used to calculate borehole sample volumetric water content.

Solution extracts were made by mixing fresh soil samples with distilled-deionized water. A plastic beaker was tared to the nearest $0.01 \mathrm{~g}$ on the Sartorius balance. The fresh soil was added, re-weighed with the beaker, and then the balance was tared once again. Water was then added to the beaker, an amount equal to about 25 percent of the fresh weight of the soil before recording a final mass of the beaker and soil paste. The amount of diluent water added was varied, depending upon the moisture contained in the sub-sample and its texture. The goal of sample dilution was to add the least amount of water necessary to enable extraction of a sufficiently large sample to complete the chemical analyses, about $20 \mathrm{ml}$. Drier soil core sections and those with heavier textures (i.e., some samples with very high clay content) required more water.

The samples were then vigorously stirred with a metal spatula for about one minute. The beakers were covered with an acrylic plate which closely fit the beaker rim and allowed to stand for 20 minutes. The soil paste was briefly stirred once more during the standing period.

A Buchner funnel apparatus was used to extract the paste samples. A bank of 15 Buchner funnels was plumbed to a common vacuum source, about $425 \mathrm{mb}$ strong. Each ceramic funnel was fit with a $5.5 \mathrm{~cm}$-diameter disk of Whatman's \#42 ashless filter paper. During filtration the rim of a paste filled Buchner funnel was covered to reduce evaporation. The effluent was collected in clean 13 dram polypropylene vials which were capped and then wrapped with Parafilm ${ }^{\oplus}$ before being placed in refrigerated storage.

\section{Sample Analyses}

The extracts were analyzed for tritium, chloride ion and nitrate ion. For tritium $0.5 \mathrm{ml}$ aliquots of sample were mixed with $5.0 \mathrm{ml}$ of Aquasol universal liquid scintillation cocktail (Dupont New England Nuclear, Boston, MA) in 


\section{Appendix C-1. Experiment I Redistribution Cores II and III. (Continued)}

"Mini-Q" $8 \mathrm{ml}$ scintillation vials (Beckman Instruments, Inc., Fullerton, $\mathrm{CA}$ ) and run as single replications with a Beckman model LS1800 scintillator in "auto-run" mode. Raw counts were taken for $\mathbf{2 0}$ minutes per sample within a single-channel counting window of $0-400 \mathrm{~nm}$. No quenching factors were used in the counting routine. Tritium background was taken from vials prepared with untraced well water in place of extract.

Chloride was analyzed with a Buchler model 4-2500 chloridometer (Buchler Instruments, Inc., Ft. Lee, NJ) which utilizes timed $\mathrm{Ag} / \mathrm{AgCl}$ titrimetry to determine chloride ion. Before using the instrument the silver electrodes were thoroughly cleaned with a commercial silver cleaner or re placed. The instrument was switched to high sensitivity mode, which provides a detection limit of about $0.3 \mathrm{me} / \mathrm{l}$. Ten replicated readings each of $10 \mathrm{me} / \mathrm{l}$ and $60 \mathrm{me} / \mathrm{l}$ chloride standards were used to make a twopoint calibration curve. Ten replications of background correction vials were prepared with distilled-deionized water and read. The high number of replications was used to ensure stable instrument performance before analyzing extracts. Sample titrations were performed with 0.10 to $1.00 \mathrm{ml}$ volume aliquots, on the $\mathrm{Cl}^{-}$ concentration within the sample. Samples were read as single replications.

Nitrate analyses was performed with a Technicon AutoAnalyser II in the NMSU Soil \& Water Testing Laboratory. This instrument determines nitrate by a colorimetric procedure; copperized cadmium column is used to reduce sample nitrate to nitrite in the presence of $\mathrm{NH}_{4} \mathrm{Cl}$. The resulting nitrite is treated with a

colorizing agent to form a pink color. A colorimeter is then used to measure absorbance of the colorized solution at $540 \mathrm{~nm}$.

The sample extracts were first screened for approximate nitrate content by using nitrate test strips (EM Science Inc., Gibbstown, NJ). From these approximations the final sample dilutions to bring the samples into the working linear range $(0-10 \mathrm{mg} / \mathrm{l})$ of the AutoAnalyser were determined. Samples were diluted from $1 x$ (full strength) to $100 \mathrm{x}$ with distilled-de ionized water in dilution steps of no more than 10x per dilution. The instrument was calibrated to a single point curve with a $1 \mathrm{mg} / 1 \mathrm{~N}-\mathrm{NO}_{3}$ standard. The instrument was re-calibrated with two replications of nitrate standard for every 10 sample extracts in a sequence of run. 


\section{Appendix C-1. Experiment I Redistribution Cores II and III (Continued).}

\section{Results \& Discussion}

Figure $\mathrm{C}-1.1$ shows the depth-distribution of volumetric water content and includes data from the earlier borehole at $X_{1}=0$ meters for comparison (mean $0.135 \mathrm{~cm}^{3} / \mathrm{cm}^{3}$ ). Water content was quite variable about a means of $0.138 \mathrm{~cm}^{3} / \mathrm{cm}^{3}$ at $\mathrm{X}_{1}=2$ meters and 0.127 $\mathrm{cm}^{3} / \mathrm{cm}^{3}$ at $X_{1}=4$ meters with no clear trends or distinct wetting front of applied Experiment $I$ water in evidence. Lower water contents, below: 10 meters depth, are responsible for the relatively low 4 meter-mean value. High water contents between about $81 / 2$ and $91 / 2$ meters correspond to the abrupt appearance of a clay textured lens of soil that is common to all three boreholes at that depth.

Scintillation counts for tritium applied to the irrigation area (Co) were adjusted for a 2.91 year period of decay between day 0 of Experiment I and April 23, 1990, the day that tritium samples were counted for this experiment:

$$
N(t)=N_{0}(1 / 2)^{t / 1 / 2}
$$

where

$N(t)=$ decay adjusted Co

$\mathrm{N}_{\mathrm{o}}=$ original Co, 5/27/87, 7037 counts

$\mathrm{t}$ = elapsed time, years

$t^{1 / 2}=$ tritium half - life, 12.3 years

This resulted in a calculated value of 0.1713 half-lives of tritium decay during the period. This value was used to adjust calculations for a mass balance of tritium recovered from the borehole located at $Y_{1}=2$ meters. Based upon an original applied tritium pulse, $1.76 \mathrm{~cm} / \mathrm{d}$ applied for the first 10 days of Experiment I, about $7.22 \mathrm{~cm}$ of tritium was recovered from the borehole, about 41.1 percent. This compares to a 50.2 percent recovery from the borehole near the center of the plot, taken almost a year earlier
(Vinson et al., 1989). No tritium was found in samples collected at $Y_{1}=4$ meters.

Tritium data is presented as the observed concentration $\mathrm{C}$ relative to input tritium (Co). In the 2 meter borehole the first appearance of tritium occurs about 4 meters in depth, peaks at the 8.55 meter mark, at $\mathrm{C} / \mathrm{Co}=17.1 \%$ and does not occur below about 10 meters in depth (Figure C-12). Compared to the 0-meter borehole, the leading front of tritium in the 2-meter borehole reached about the same depth but with the mien of great dispersion. This may be due to delayed lateral movement of tritium from the center of the irrigated area to the periphery.

Nitrate and chloride ion profiles are quite similar in appearance within the same borehole (Figures C-1.3 and C-1.4). At $\mathrm{Y}_{1}=2$ meters the ions appear to have been effectively eluted from the profile to about 10 meters depth. From there the ion concentrations increase dramatically, to nearly the depth where the tritium plume becomes extinct. The concentrations of both ions peak at a depth of 11.53 meters.

At $Y_{1}=4$ meters the anions have been eluted to about 4 meters depth. They show a much more irregular concentration profile, with relatively high concentrations from 4 meters to about 13 meters depth where they begin to co-vary with ion concentrations in the 2 meter borehole. Cumulative ion masses within the whole profile are much higher than in the 2 meter borehole which again may be due to salt additions from areas within the irrigated plot.

A curious spike in nitrate concentration occurs near the soil surface in both boreholes. This may be due to mineralization of soil organic matter over time in the moist soil environment unsaturated by the plot moisture barrier. 
Appendix C-1. Experiment I Redistribution Cores II and III. (Continued)

Plot 1 Redistribution Cores - Volumetric Water Content

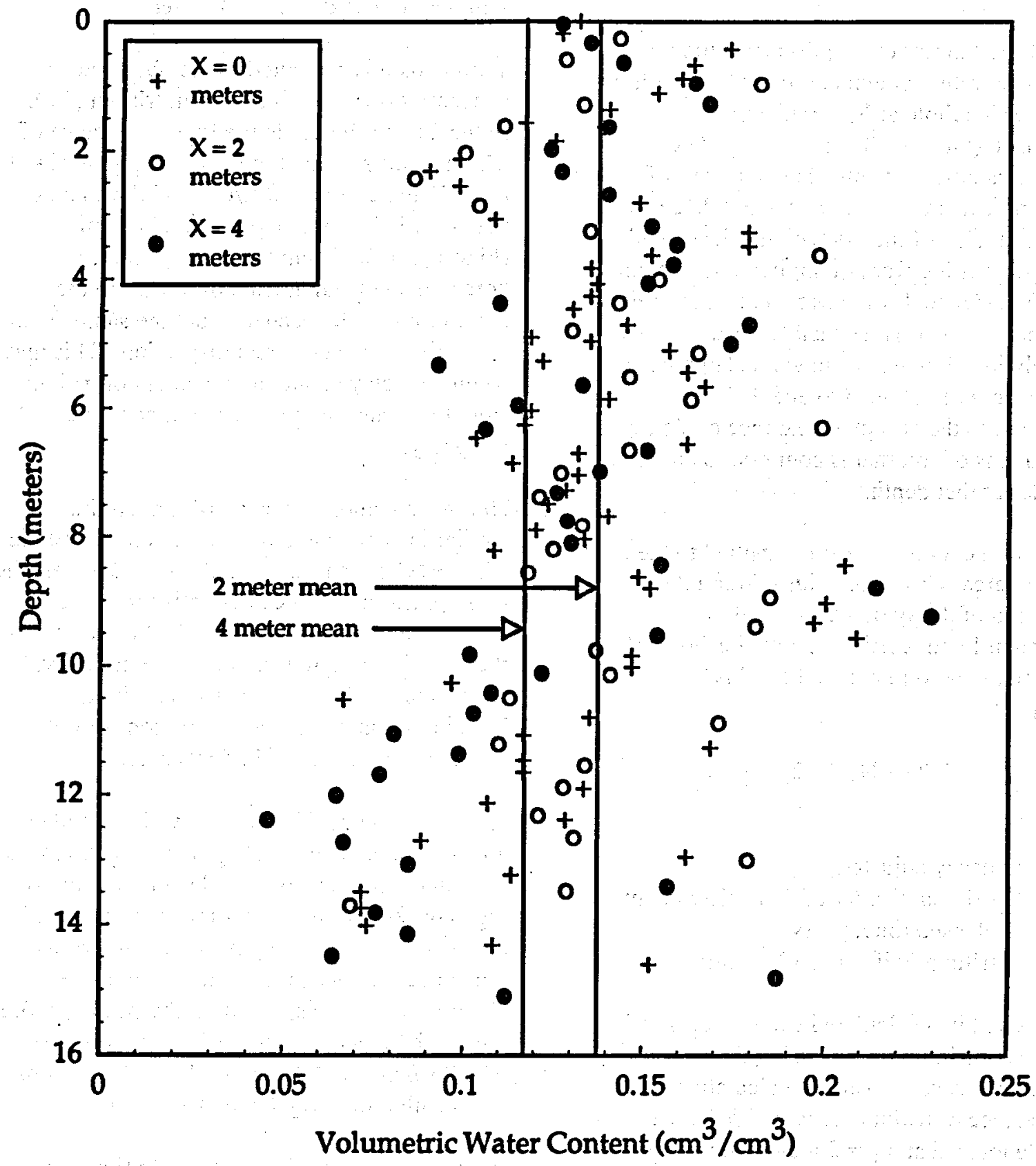


Appendix C-1. Experiment I Redistribution Cores II and III. (Continued)

Plot 1 Redistribution Cores - Tritium distribution

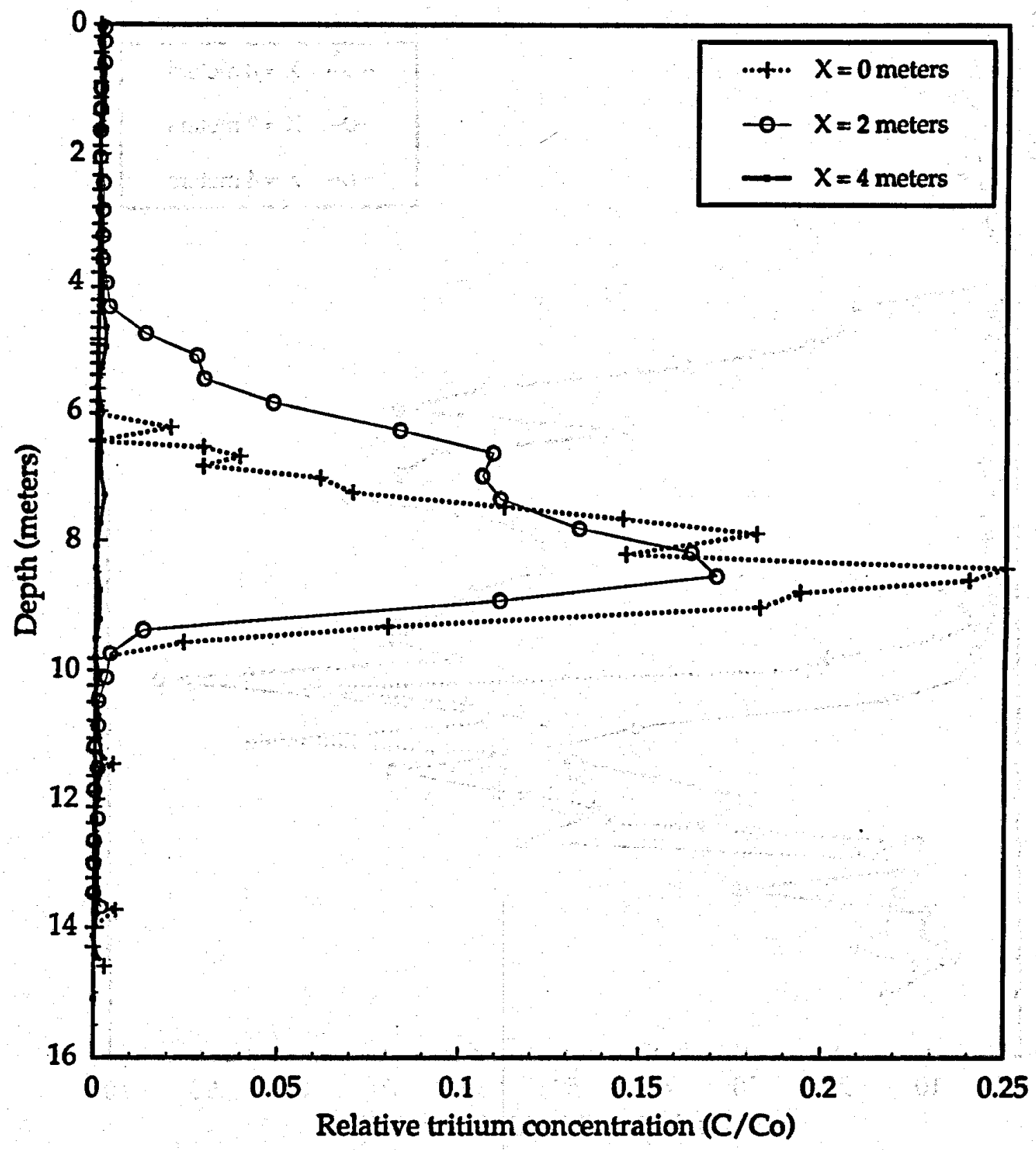


Appendix C-1. Experiment I Redistribution Cores II and III. (Continued)

Plot 1 Redistribution Cores - Chloride distribution

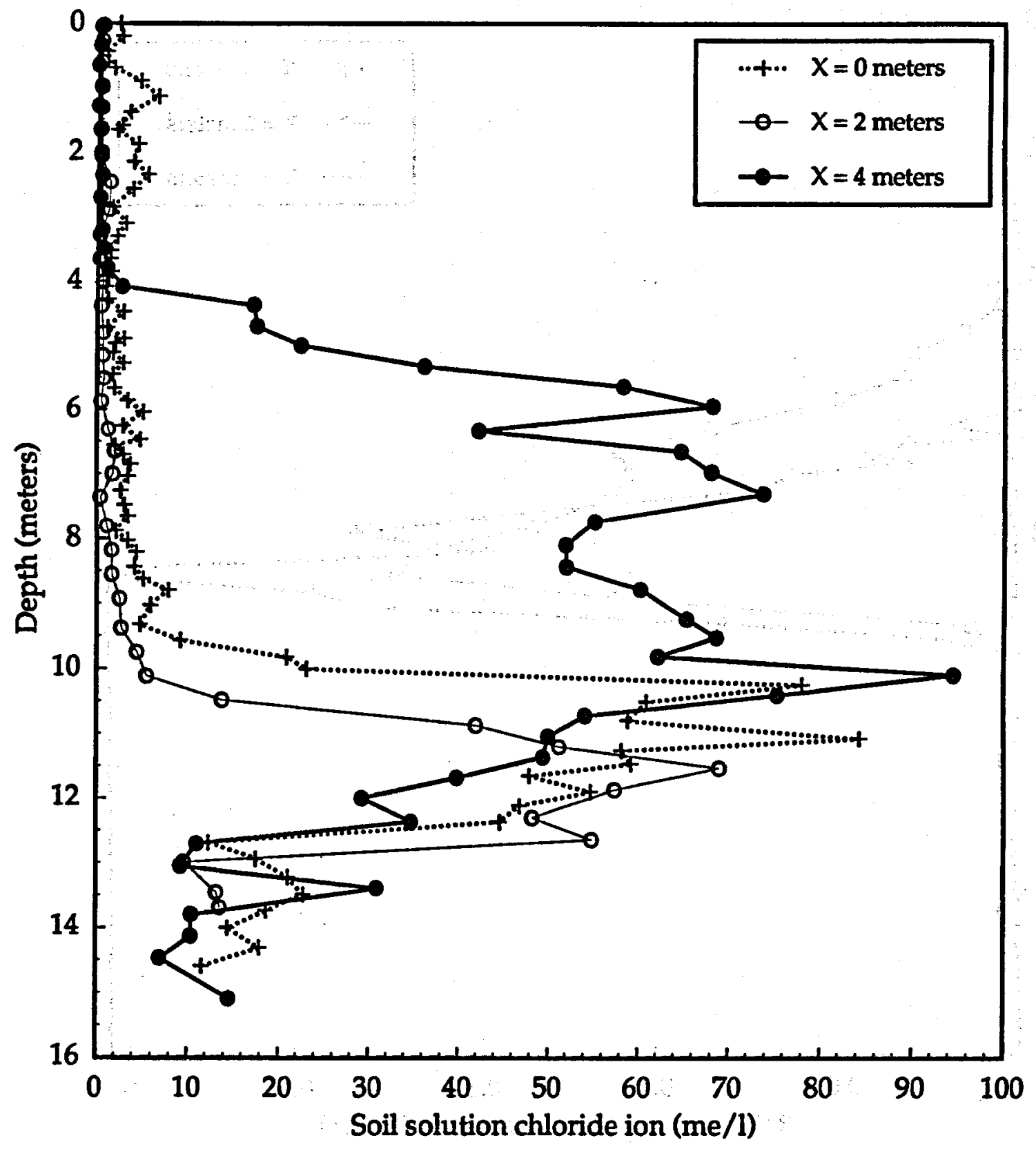


Appendix C-1. Experiment I Redistribution Cores II and III. (Continued)

Plot 1 Redistribution Cores - Nitrate distribution

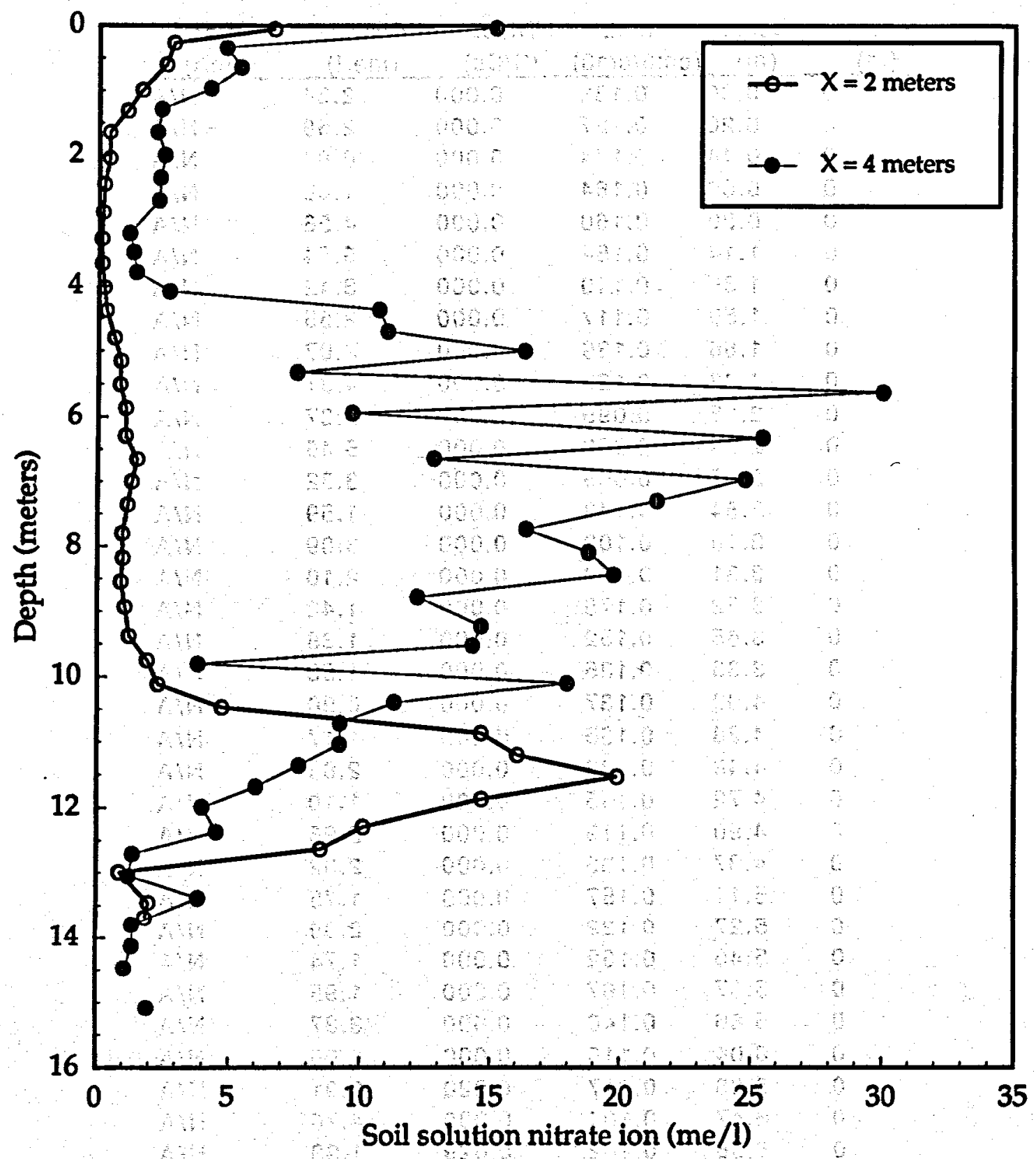


Appendix C-1. Experiment I Redistribution Cores II and III. (Continued)

Experiment I Redistribution Cores - Solute Data and Volumetric Water Content

\begin{tabular}{|c|c|c|c|c|c|}
\hline $\begin{array}{c}Y \\
(m)\end{array}$ & $\begin{array}{l}\text { Depth } \\
\text { (m) }\end{array}$ & $\begin{array}{l}\text { Theta } \\
(\mathrm{cm} 3 / \mathrm{cm} 3)\end{array}$ & $\begin{array}{l}\text { Tritium } \\
(\mathrm{C} / \mathrm{Co})\end{array}$ & $\begin{array}{l}\text { Chloride } \\
\text { (me/l) }\end{array}$ & $\begin{array}{l}\text { Nitrate } \\
(\mathrm{me} / \mathrm{l})\end{array}$ \\
\hline 0 & 0.00 & 0.132 & 0.000 & 2.34 & N/A \\
\hline 0 & 0.20 & 0.127 & 0.000 & 2.58 & N/A \\
\hline 0 & 0.44 & 0.174 & 0.000 & 0.84 & N/A \\
\hline 0 & 0.69 & 0.164 & 0.000 & 1.68 & N/A \\
\hline 0 & 0.90 & 0.160 & 0.000 & 4.56 & N/A \\
\hline 0 & 1.14 & 0.154 & 0.000 & 6.54 & N/A \\
\hline 0 & 1.39 & 0.140 & 0.000 & 3.42 & N/A \\
\hline 0 & 1.59 & 0.117 & 0.000 & 2.55 & $N / A$ \\
\hline 0 & 1.66 & 0.139 & 0.000 & 2.07 & N/A \\
\hline 0 & 1.88 & 0.125 & 0.000 & 4.31 & N/A \\
\hline 0 & 2.15 & 0.099 & 0.000 & 3.87 & N/A \\
\hline 0 & 2.34 & 0.090 & 0.000 & 5.49 & N/A \\
\hline 0 & 2.57 & 0.099 & 0.000 & 3.82 & $N / A$ \\
\hline 0 & 2.84 & 0.149 & 0.000 & 1.59 & N/A \\
\hline 0 & 3.10 & 0.109 & 0.000 & 3.09 & N/A \\
\hline 0 & 3.31 & 0.179 & 0.000 & 2.10 & N/A \\
\hline 0 & 3.52 & 0.179 & 0.000 & 1.42 & N/A \\
\hline 0 & 3.65 & 0.152 & 0.000 & 1.38 & N/A \\
\hline 0 & 3.85 & 0.135 & 0.000 & 1.53 & N/A \\
\hline 0 & 4.09 & 0.137 & 0.000 & 0.90 & N/A \\
\hline 0 & 4.28 & 0.135 & 0.000 & 1.17 & N/A \\
\hline 0 & 4.48 & 0.130 & 0.000 & 2.89 & N/A \\
\hline 0 & 4.72 & 0.145 & 0.000 & 1.10 & N/A \\
\hline 0 & 4.90 & 0.119 & 0.000 & 2.96 & N/A \\
\hline 0 & 4.97 & 0.135 & 0.000 & 2.02 & N/A \\
\hline 0 & 5.11 & 0.157 & 0.000 & 1.75 & N/A \\
\hline 0 & 5.27 & 0.122 & 0.000 & 2.89 & N/A \\
\hline 0 & 5.45 & 0.162 & 0.000 & 1.74 & N/A \\
\hline 0 & 5.67 & 0.167 & 0.000 & 1.95 & N/A \\
\hline 0 & 5.86 & 0.140 & 0.000 & 3.37 & $N / A$ \\
\hline 0 & 6.04 & 0.119 & 0.000 & 5.06 & $N / A$ \\
\hline 0 & 6.26 & 0.117 & 0.020 & 2.91 & N/A \\
\hline 0 & 6.47 & 0.104 & 0.000 & 4.76 & N/A \\
\hline 0 & 6.56 & 0.162 & 0.029 & 1.99 & N/A \\
\hline 0 & 6.70 & 0.132 & 0.039 & 2.94 & N/A \\
\hline 0 & 6.85 & 0.114 & 0.029 & 3.67 & $\mathbf{N} / \mathbf{A}$ \\
\hline 0 & 7.03 & 0.132 & 0.061 & 3.44 & N/A \\
\hline 0 & 7.26 & 0.129 & 0.070 & 2.61 & N/A \\
\hline 0 & 7.48 & 0.124 & 0.112 & 3.07 & N/A \\
\hline 0 & 7.66 & 0.140 & 0.145 & 3.43 & N/A \\
\hline 0 & 7.89 & 0.120 & 0.182 & 2.12 & $N / A$ \\
\hline
\end{tabular}


Appendix C-1. Experiment I Redistribution Cores II and III. (Continued)

Experiment I Redistribution Cores - Solute Data and Volumetric Water Content

\begin{tabular}{|c|c|c|c|c|c|c|}
\hline $\begin{array}{l}Y \\
\text { (m) }\end{array}$ & & $\begin{array}{l}\text { Depth } \\
\text { (m) }\end{array}$ & $\begin{array}{l}\text { Theta } \\
(\mathrm{cm} 3 / \mathrm{cm} 3)\end{array}$ & $\begin{array}{l}\text { Tritium } \\
(\mathrm{C} / \mathrm{Co})\end{array}$ & $\begin{array}{l}\text { Chloride } \\
\text { (me/I) }\end{array}$ & $\begin{array}{l}\text { Nitrate } \\
(\mathrm{me} / \mathrm{l})\end{array}$ \\
\hline & 0 & 8.03 & 0.134 & 0.019 & 3.40 & $N / A$ \\
\hline 8 & 0 & 8.21 & 0.109 & 0.146 & 4.36 & $N / A$ \\
\hline & 0 & 8.44 & 0.205 & 0.250 & 4.17 & N/A \\
\hline$\therefore$ & 0 & 8.62 & 0.149 & 0.240 & 5.11 & $N / A$ \\
\hline$\because$ & 0 & 8.80 & 0.152 & 0.194 & 7.87 & $N / A$ \\
\hline$\because$ & 0 & 9.03 & 0.200 & 0.183 & 5.92 & $N / A$ \\
\hline 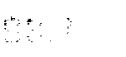 & 0 & 9.33 & 0.197 & 0.080 & 4.77 & N/A \\
\hline : & 0 & 9.57 & 0.209 & 0.024 & 9.22 & N/A \\
\hline 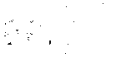 & 0 & 9.83 & 0.147 & 0.000 & 20.89 & N/A \\
\hline$\therefore$ & 0 & 10.01 & 0.147 & 0.000 & 23.12 & N/A \\
\hline & 0 & 10.25 & 0.097 & 0.000 & 78.02 & N/A \\
\hline 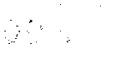 & 0 & 10.51 & 0.067 & 0.000 & 60.94 & N/A \\
\hline$\because$ & 0 & 10.79 & 0.135 & 0.000 & 58.81 & N/A \\
\hline & 0 & 11.07 & 0.117 & 0.000 & 84.29 & N/A \\
\hline$\%$ & 0 & 11.26 & 0.169 & 0.000 & 58.13 & N/A \\
\hline & 0 & 11.46 & 0.117 & 0.005 & 59.24 & $N / A$ \\
\hline & 0 & 11.65 & 0.117 & 0.000 & 47.94 & N/A \\
\hline 8 & 0 & 11.90 & 0.134 & 0.000 & 54.76 & N/A \\
\hline & 0 & 12.12 & 0.107 & 0.000 & 46.81 & N/A \\
\hline & 0 & 12.37 & 0.129 & 0.000 & 44.65 & N/A \\
\hline & 0 & 12.69 & 0.089 & 0.000 & 12.30 & N/A \\
\hline & 0 & 12.94 & 0.162 & 0.000 & 17.50 & N/A \\
\hline$\cdots$ & 0 & 13.22 & 0.114 & 0.000 & 21.09 & N/A \\
\hline & 0 & 13.49 & 0.072 & 0.000 & 22.77 & N/A \\
\hline & 0 & 13.74 & 0.072 & 0.006 & 18.64 & N/A \\
\hline & 0 & 14.01 & 0.073 & 0.000 & 14.45 & N/A \\
\hline$\ldots$ & 0 & 14.31 & 0.109 & 0.000 & 17.92 & N/A \\
\hline$\ldots$, & 0 & 14.60 & 0.152 & 0.003 & 11.60 & N/A \\
\hline$\vdots$ & 2 & 0.05 & 0.127 & 0.001 & 0.35 & 6.65 \\
\hline & 2 & 0.27 & 0.143 & 0.001 & 0.41 & 2.84 \\
\hline 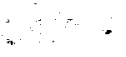 & 2 & 0.60 & 0.128 & 0.001 & 0.34 & 2.55 \\
\hline & 2 & 0.99 & 0.182 & 0.000 & 0.35 & 1.64 \\
\hline & 2 & 1.31 & 0.133 & 0.000 & 0.31 & 1.09 \\
\hline 8 & 2 & 1.64 & 0.111 & 0.000 & 0.29 & 0.41 \\
\hline & 2 & 2.05 & 0.100 & 0.000 & 0.30 & 0.40 \\
\hline & 2 & 2.45 & 0.086 & 0.001 & 1.33 & 0.21 \\
\hline 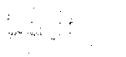 & 2 & 2.88 & 0.104 & 0.001 & 1.19 & 0.17 \\
\hline & 2 & 3.28 & 0.135 & 0.001 & 0.24 & 0.13 \\
\hline & 2 & 3.65 & 0.198 & 0.001 & 0.28 & 0.14 \\
\hline & 2 & 4.02 & 0.154 & 0.002 & 0.55 & 0.22 \\
\hline & 2 & 4.38 & 0.143 & 0.003 & 0.43 & 0.32 \\
\hline
\end{tabular}


Appendix C-1. Experiment I Redistribution Cores II and III. (Continued)

Experiment I Redistribution Cores - Solute Data and Volumetric Water Content

\begin{tabular}{|c|c|c|c|c|c|c|}
\hline $\begin{array}{l}Y \\
(m)\end{array}$ & & $\begin{array}{l}\text { Depth } \\
\text { (m) }\end{array}$ & $\begin{array}{l}\text { Theta } \\
(\mathrm{cm} 3 / \mathrm{cm} 3)\end{array}$ & $\begin{array}{l}\text { Tritium } \\
(\mathrm{C} / \mathrm{Co})\end{array}$ & $\begin{array}{l}\text { Chloride } \\
\text { (me/l) }\end{array}$ & $\begin{array}{l}\text { Nitrate } \\
\text { (me/I) }\end{array}$ \\
\hline$\therefore$ & 2 & 4.80 & 0.130 & 0.013 & 0.63 & 0.63 \\
\hline & 2 & 5.15 & 0.165 & 0.027 & 0.64 & 0.88 \\
\hline & 2 & 5.51 & 0.146 & 0.029 & 0.77 & 0.86 \\
\hline & 2 & 5.87 & 0.163 & 0.048 & 0.54 & 1.06 \\
\hline & 2 & 6.30 & 0.199 & 0.083 & 1.28 & 1.07 \\
\hline & 2 & 6.65 & 0.146 & 0.109 & 2.04 & 1.51 \\
\hline & 2 & 7.00 & 0.127 & 0.106 & 1.76 & 1.32 \\
\hline & 2 & 7.36 & 0.121 & 0.111 & 0.48 & 1.16 \\
\hline & 2 & 7.81 & 0.133 & 0.133 & 1.13 & 0.96 \\
\hline & 2 & 8.18 & 0.125 & 0.164 & 1.65 & 0.99 \\
\hline & 2 & 8.55 & $0: 118$ & 0.171 & 1.62 & 0.91 \\
\hline & 2 & 8.93 & 0.185 & 0.111 & 2.54 & 1.06 \\
\hline & 2 & 9.38 & 0.181 & 0.013 & 2.75 & 1.22 \\
\hline & 2 & 9.75 & 0.137 & 0.004 & 4.40 & 1.91 \\
\hline & 2 & 10.12 & 0.141 & 0.003 & 5.52 & 2.30 \\
\hline & 2 & 10.48 & 0.113 & 0.001 & 13.79 & 4.75 \\
\hline & 2 & 10.87 & 0.171 & 0.001 & 41.96 & 14.67 \\
\hline & 2 & 11.20 & 0.110 & 0.000 & 51.25 & 16.06 \\
\hline & 2 & 11.53 & 0.134 & 0.001 & 68.95 & 19.91 \\
\hline & 2 & 11.87 & 0.128 & 0.000 & 57.40 & 14.68 \\
\hline & 2 & 12.30 & 0.121 & 0.001 & 48.26 & 10.14 \\
\hline & 2 & 12.64 & 0.131 & 0.000 & 54.88 & 8.49 \\
\hline & 2 & 12.99 & 0.179 & 0.000 & 9.67 & 0.88 \\
\hline & 2 & 13.46 & 0.129 & 0.000 & 13.20 & 1.97 \\
\hline & 2 & 13.69 & 0.069 & 0.002 & 13.55 & 1.84 \\
\hline & 4 & 0.04 & 0.127 & 0.001 & 0.52 & 15.17 \\
\hline & 4 & 0.34 & 0.135 & 0.000 & 0.18 & 4.82 \\
\hline & 4 & 0.65 & 0.144 & 0.001 & 0.03 & 5.38 \\
\hline & 4 & 0.97 & 0.164 & 0.001 & 0.29 & 4.23 \\
\hline & 4 & 1.29 & 0.168 & 0.001 & 0.02 & 2.38 \\
\hline & 4 & 1.65 & 0.140 & 0.000 & 0.20 & 2.23 \\
\hline . & 4 & 2.00 & 0.124 & 0.000 & 0.30 & 2.51 \\
\hline & 4 & 2.35 & 0.127 & 0.000 & 0.45 & 2.33 \\
\hline & 4 & 2.70 & 0.140 & 0.000 & 0.23 & 2.28 \\
\hline & 4 & 3.20 & 0.152 & 0.001 & 0.49 & 1.18 \\
\hline & 4 & 3.49 & 0.159 & 0.000 & 0.66 & 1.34 \\
\hline & 4 & 3.79 & 0.158 & 0.001 & 1.06 & 1.44 \\
\hline & 4 & 4.08 & 0.151 & 0.001 & 2.71 & 2.70 \\
\hline & 4 & 4.38 & 0.110 & 0.001 & 17.23 & 10.71 \\
\hline & 4 & 4.71 & 0.179 & 0.002 & 17.61 & 11.05 \\
\hline & 4 & 5.01 & 0.174 & 0.002 & 22.51 & 16.33 \\
\hline
\end{tabular}


Appendix C-1. Experiment I Redistribution Cores II and III. (Continued)

Experiment I Redistribution Cores - Solute Data and Volumetric Water Content

\begin{tabular}{|c|c|c|c|c|c|c|}
\hline $\begin{array}{l}Y \\
(m)\end{array}$ & & $\begin{array}{l}\text { Depth } \\
\text { (m) }\end{array}$ & $\begin{array}{c}\text { Theta } \\
(\mathrm{cm} 3 / \mathrm{cm} 3)\end{array}$ & $\begin{array}{l}\text { Tritium } \\
\text { (C/Co) }\end{array}$ & $\begin{array}{c}\text { Chloride } \\
\text { (me/l) }\end{array}$ & $\begin{array}{l}\text { Nitrate } \\
\text { (mell) }\end{array}$ \\
\hline & 4 & 5.33 & 0.093 & 0.001 & 36.22 & 7.58 \\
\hline & 4 & 5.64 & 0.133 & 0.000 & 58.32 & 30.04 \\
\hline & 4 & 5.95 & 0.115 & 0.001 & 68.16 & 9.70 \\
\hline & 4 & 6.33 & 0.106 & 0.001 & 42.23 & 25.48 \\
\hline & 4 & 6.65 & 0.151 & 0.001 & 64.70 & 12.84 \\
\hline & 4 & 6.97 & 0.138 & 0.001 & 68.02 & 24.82 \\
\hline & 4 & 7.30 & 0.126 & 0.002 & 73.75 & 21.43 \\
\hline & 4 & 7.74 & 0.129 & 0.001 & 55.23 & 16.40 \\
\hline & 4 & 8.09 & 0.130 & 0.000 & 51.99 & 18.79 \\
\hline & 4 & 8.43 & 0.155 & 0.000 & 52.03 & 19.78 \\
\hline & 4 & 8.78 & 0.214 & 0.001 & 60.29 & 12.22 \\
\hline & 4 & 9.23 & 0.229 & 0.001 & 65.37 & 14.67 \\
\hline & 4 & 9.52 & 0.154 & 0.000 & 68.69 & 14.33 \\
\hline & 4 & 9.81 & 0.102 & 0.000 & 62.20 & 3.83 \\
\hline & 4 & 10.10 & 0.122 & 0.001 & 94.56 & 17.98 \\
\hline & 4 & 10.40 & 0.108 & 0.000 & 75.37 & 11.33 \\
\hline & 4 & 10.72 & 0.103 & 0.001 & 54.16 & 9.26 \\
\hline & 4 & 11.04 & 0.081 & 0.001 & 50.06 & 9.22 \\
\hline & 4 & 11.36 & 0.099 & 0.002 & 49.46 & 7.67 \\
\hline & 4 & 11.68 & 0.077 & 0.001 & 39.88 & 6.05 \\
\hline & 4 & 12.00 & 0.065 & 0.001 & 29.34 & 4.00 \\
\hline & 4 & 12.37 & 0.046 & 0.000 & 34.72 & 4.54 \\
\hline & 4 & 12.71 & 0.067 & 0.001 & 11.06 & 1.39 \\
\hline & 4 & 13.05 & 0.085 & 0.001 & 9.28 & 1.23 \\
\hline & 4 & 13.39 & 0.157 & 0.001 & 30.94 & 3.85 \\
\hline & 4 & 13.80 & 0.076 & 0.001 & 10.49 & 1.37 \\
\hline & 4 & 14.13 & 0.085 & 0.000 & 10.42 & 1.37 \\
\hline & 4 & 14.47 & 0.064 & 0.001 & 7.00 & 1.10 \\
\hline & 4 & 14.80 & 0.187 & N/A & N/A & $N / A$ \\
\hline & 4 & 15.09 & 0.112 & 0.000 & 14.58 & 1.93 \\
\hline
\end{tabular}

Note: Data for core located at $Y=0$ meters are reported by Vinson et al. (1989), included here for comparison with later cores (2 $\mathrm{m}$ and $4 \mathrm{~m}$ ) 
Appendix C-1. Experiment I Redistribution Cores II and III. (Continued)

Redistribution Cores II \& III of 3/16/90 - Segment Drill Depth \& Core Recovery

Core \#1, $\mathrm{X} 1=2.02 \mathrm{~m}, 3 / 16 / 90$

\begin{tabular}{|c|c|c|c|c|c|}
\hline $\begin{array}{c}\text { Core } \\
\text { Section\# }\end{array}$ & $\begin{array}{l}\text { Cumul. } \\
\text { Sampler } \\
\text { Depth } \\
\text { (cm) }\end{array}$ & $\begin{array}{c}\text { Increment } \\
\text { Sampler } \\
\text { Depth } \\
\text { (cm) }\end{array}$ & $\begin{array}{c}\text { Recovery } \\
\text { Core length } \\
\text { (less waste) } \\
\text { (cm) }\end{array}$ & $\begin{array}{l}\text { +Cutting tip } \\
\text { Add 2" } \\
\text { (cm) }\end{array}$ & $\begin{array}{l}\text { Scale Ratio } \\
\text { (depth/ } \\
\text { recov.) }\end{array}$ \\
\hline 1,2 & 142.0 & 142.0 & 117.3 & 122.38 & 1.160 \\
\hline 3,4 & 307.3 & 165.3 & 97.8 & 102.88 & 1.607 \\
\hline 5,6 & 454.7 & 147.3 & 149.5 & .58 & 0.953 \\
\hline 7,8 & 605.8 & 151.2 & 123.5 & 128.58 & 1.176 \\
\hline 9,10 & 754.4 & 148.5 & 101.7 & 106.78 & 1.391 \\
\hline 11,12 & 913.1 & 158.8 & 100.8 & 105.88 & 1.499 \\
\hline 13,14 & 1061.7 & 148.6 & 138.5 & 143.58 & 1.035 \\
\hline 15,16 & 1214.1 & 152.4 & 112.8 & 117.88 & 1.293 \\
\hline & 1369.1 & 154.9 & 102.9 & & 1.435 \\
\hline 19,20 & 1521.5 & & & & \\
\hline & & $\begin{array}{r}152.15 \\
6.150\end{array}$ & $\begin{array}{r}115.4 \\
17.261\end{array}$ & $\begin{array}{r}120.5 \\
17.261\end{array}$ & $\begin{array}{l}1.289 \\
0.207\end{array}$ \\
\hline
\end{tabular}

Core \#2, X1 = $4.00 \mathrm{~m}, 3 / 16 / 90$

\begin{tabular}{|c|c|c|c|c|c|}
\hline $\begin{array}{l}\text { Core } \\
\text { Section\# }\end{array}$ & $\begin{array}{l}\text { Cumul. } \\
\text { Sampler } \\
\text { Depth } \\
\text { (cm) }\end{array}$ & $\begin{array}{l}\text { Increment } \\
\text { Sampler } \\
\text { Depth } \\
\text { (cm) }\end{array}$ & $\begin{array}{l}\text { Recovery } \\
\text { Core length } \\
\text { (less waste) } \\
\text { (cm) }\end{array}$ & $\begin{array}{l}\text { +Cutting tip } \\
\text { Add } 2^{n \prime} \\
\text { (cm) }\end{array}$ & $\begin{array}{l}\text { Scale Ratio } \\
\text { (depth/ } \\
\text { recov.) }\end{array}$ \\
\hline 1,2 & 132.1 & 142.0 & 114.8 & 119.88 & 1.185 \\
\hline 3,4 & 299.7 . & 167.6 & 119.5 & 124.58 & 1.346 \\
\hline 5,6 & 452.1 & 152.4 & 117.9 & 122.98 & 1.239 \\
\hline 7,8 & 604.5 & 152.4 & 123.4 & 128.48 & 1.186 \\
\hline 9,10 & 756.9 & 152.4 & 121.7 & 126.78 & 1.202 \\
\hline 11,12 & 909.3 & 152.4 & 114.9 & 119.98 & 1.270 \\
\hline 13,14 & 1064.3 & 154.9 & 114.7 & 119.78 & 1.294 \\
\hline 15,16 & 1216.7 & 152.4 & 113.5 & 118.58 & 1.285 \\
\hline 17,18 & 1369.1 & 152.4 & 120.1 & 125.18 & 1.217 \\
\hline \multirow[t]{3}{*}{$19,20^{*}$} & 1518.9 & 149.9 & N/A & N/A & N/A \\
\hline & & 152.88 & & 122.91 & 1.247 \\
\hline & & 5.920 & 3.540 & 3.540 & 0.055 \\
\hline
\end{tabular}

"Core 2-20 sent to Tucson, unavailable for processing 
Experiment I Redistribution Cores II \& III of 3/16/90 - Supporting Data

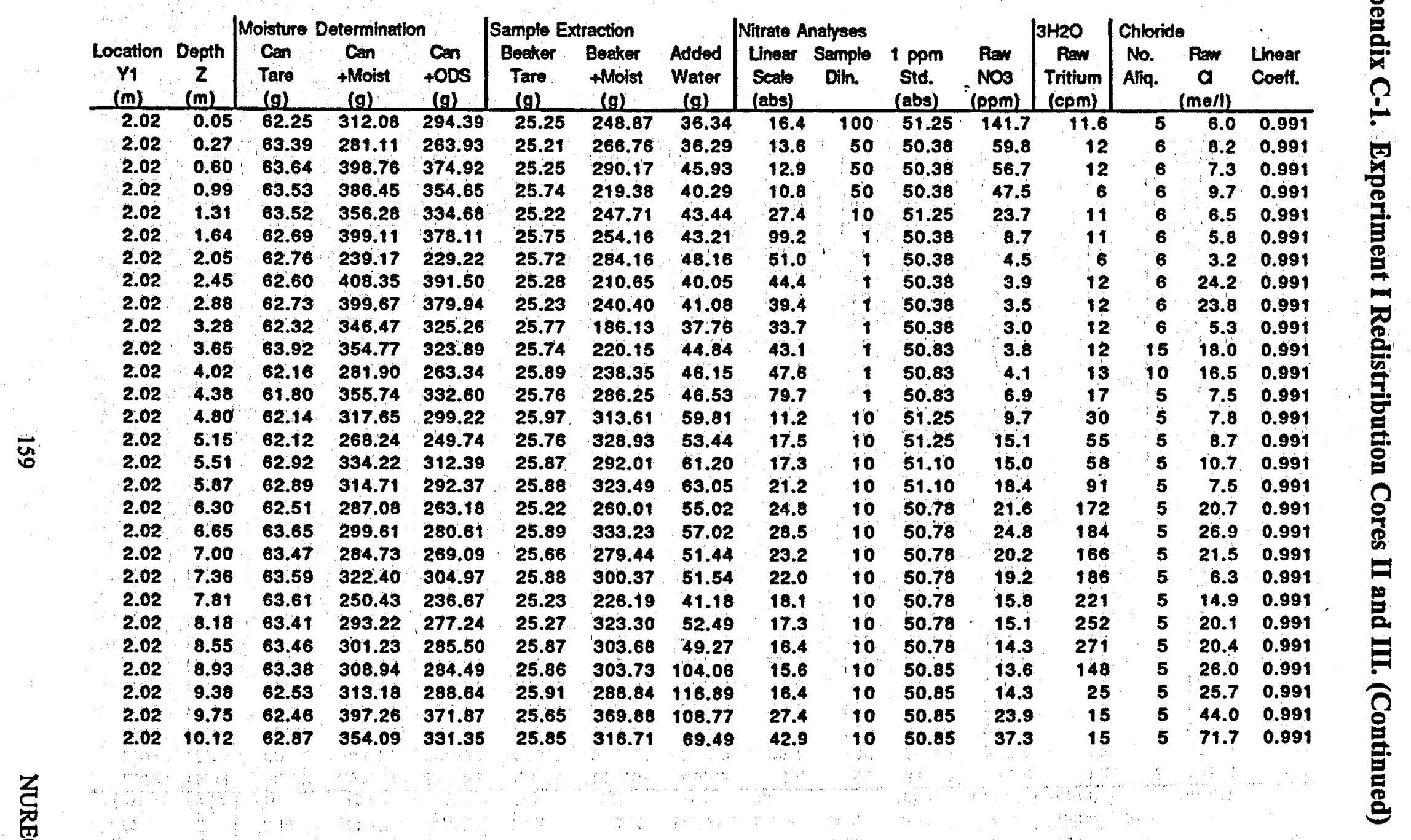




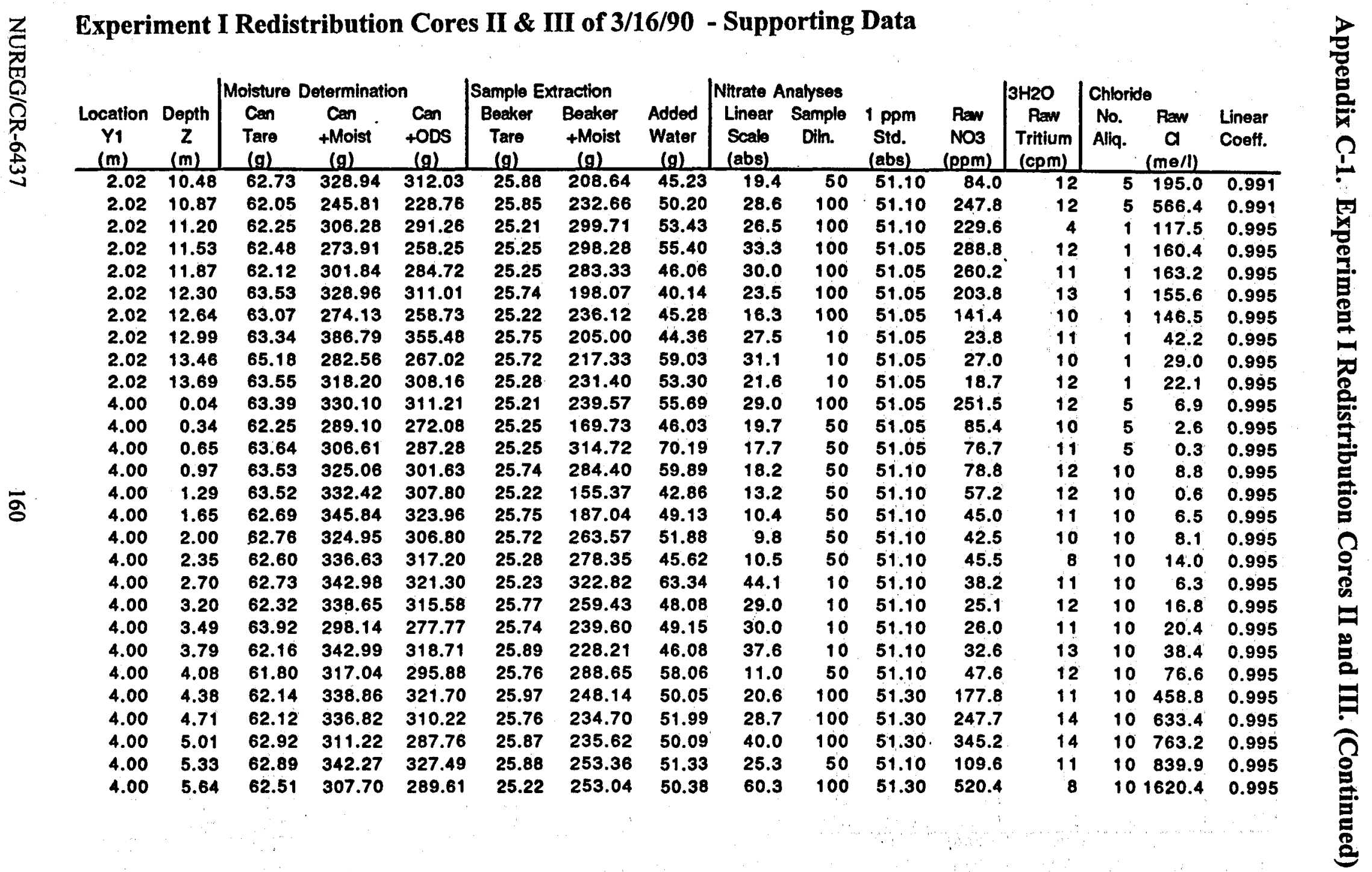


Experiment I Redistribution Cores II \& III of 3/16/90 - Supporting Data

\begin{tabular}{|c|c|c|c|c|c|c|c|c|c|c|c|c|c|c|c|}
\hline $\begin{array}{c}\text { Location } \\
Y_{1} \\
\text { (m) }\end{array}$ & $\begin{array}{c}\text { Depth } \\
Z \\
\text { (m) }\end{array}$ & $\begin{array}{l}\text { Moisture } \\
\text { Can } \\
\text { Tare } \\
\text { (g) }\end{array}$ & $\begin{array}{l}\text { Can } \\
+ \text { Moist } \\
\text { (g) }\end{array}$ & $\begin{array}{l}\text { Can } \\
\text { +ODS } \\
\text { (g) }\end{array}$ & $\begin{array}{l}\text { Sample E } \\
\text { Beaker } \\
\text { Tare } \\
\text { (g) }\end{array}$ & $\begin{array}{l}\text { Baction } \\
\text { Beaker } \\
\text { +Moist } \\
\text { (g) }\end{array}$ & $\begin{array}{l}\text { Added } \\
\text { Water } \\
\text { (g) }\end{array}$ & $\begin{array}{l}\text { Nitrate An } \\
\text { Linear } \\
\text { Scale } \\
\text { (abs) }\end{array}$ & $\begin{array}{c}\text { nalyses } \\
\text { Sample } \\
\text { Diln. }\end{array}$ & $\begin{array}{l}1 \text { ppm } \\
\text { Std. } \\
\text { (abs) }\end{array}$ & $\begin{array}{c}\text { Raw } \\
\text { NO3 } \\
\text { (ppm) }\end{array}$ & $\begin{array}{c}3 \mathrm{H} 2 \mathrm{O} \\
\text { Raw } \\
\text { Tritium } \\
\text { (cpm) }\end{array}$ & $\begin{array}{l}\text { Chloride } \\
\text { No. } \\
\text { Aliq. }\end{array}$ & $\begin{array}{c}\text { Raw } \\
\alpha \\
(\mathrm{me} / 1)\end{array}$ & $\begin{array}{l}\text { Lineat } \\
\text { Coeff. }\end{array}$ \\
\hline 4.00 & 5.95 & 63.65 & 304.37 & 288.82 & 25.89 & 301.30 & 60.04 & 30.1 & 50 & 51.10 & 130.4 & 12 & 1 & 147.0 & 0.995 \\
\hline 4.00 & 6.33 & 63.47 & 336.78 & 320.53 & 25.66 & 260.22 & 54.93 & 43.8 & 100 & 51.30 & 378.0 & 12 & 1 & 100.5 & 0.995 \\
\hline 4.00 & 6.65 & 63.59 & 294.78 & 275.65 & 25.88 & 259.46 & 54.84 & 50.5 & 50 & 50.95 & 219.4 & 12 & 1 & 177.3 & 0.995 \\
\hline 4.00 & 6.97 & 63.61 & 304.70 & 286.30 & 25.23 & 287.85 & 59.92 & 44.5 & 100 & 51.30 & 384.0 & 11 & 1 & 168.8 & 0.995 \\
\hline 4.00 & 7.30 & 63.41 & 316.33 & 298.58 & 25.27 & 337.26 & 77.81 & 30.3 & 100 & 51.25 & 261.7 & 12 & 1 & 144.5 & 0.995 \\
\hline 4.00 & 7.74 & 63.46 & 350.25 & 329.75 & 25.87 & 243.53 & 48.56 & 36.8 & 100 & 51.25 & 317.9 & 13 & 1 & 171.7 & 0.995 \\
\hline 4.00 & 8.09 & 63.38 & 346.29 & 325.87 & 25.86 & 259.49 & 57.36 & 37.4 & 100 & 51.25 & 323.1 & 8 & 1 & 143.4 & 0.995 \\
\hline 4.00 & B.43 & 62.53 & 307.16 & 286.35 & 25.91 & 246.60 & 49.02 & 45.0 & 100 & 51.25 & 388.7 & 4 & 1 & 164.0 & 0.995 \\
\hline 4.00 & 8.78 & 62.46 & 307.31 & 279.52 & 25.65 & 306.76 & 91.35 & 22.4 & 100 & 51.25 & 193.5 & 12 & 1 & 153.2 & 0.995 \\
\hline 4.00 & 9.23 & 62.87 & 316.55 & 286.00 & 25.85 & 315.82 & 125.66 & 22.8 & 100 & 51.25 & 196.9 & 11 & 1 & 140.8 & 0.995 \\
\hline 4.00 & 9.52 & 62.73 & 304.91 & 264.44 & 25.88 & 238.22 & 55.10 & 29.7 & 100 & 51.25 & 256.6 & 11 & 1 & 197.2 & 0.995 \\
\hline 4.00 & 9.81 & 62.05 & 337.13 & 321.28 & 25.85 & 237.21 & 44.86 & 15.0 & 50 & 51.25 & 64.8 & 11 & 1 & 168.7 & 0.995 \\
\hline 4.00 & 10.10 & 62.25 & 285.12 & 269.92 & 25.21 & 297.35 & 61.64 & 54.0 & 50 & 51.25 & 233.2 & 12 & 1 & 196.8 & 0.995 \\
\hline 4.00 & 10.40 & 62.48 & 379.75 & 360.56 & 25.25 & 252.74 & 57.38 & 42.6 & 50 & 51.15 & 184.4 & 8 & 1 & 196.8 & 0.995 \\
\hline 4.00 & 10.72 & 62.12 & 354.10 & 337.09 & 25.25 & 213.85 & 36.91 & 43.6 & 50 & 51.15 & 188.7 & 12 & 1 & 177.0 & 0.995 \\
\hline 4.00 & 11.04 & 63.53 & 362.83 & 348.98 & 25.74 & 251.40 & 48.40 & 30.5 & 50 & 51.15 & 132.0 & 11 & 1 & 114.9 & 0.995 \\
\hline 4.00 & 11.36 & 63.07 & 301.23 & 287.86 & 25.22 & 294.45 & 59.65 & 21.1 & 50 & 51.15 & 91.3 & 12 & 1 & 94.4 & 0.995 \\
\hline 4.00 & 11.68 & 63.34 & 333.64 & 321.70 & 25.75 & 256.49 & 52.70 & 16.6 & 50 & 51.15 & 71.8 & 11 & 1 & 76.0 & 0.995 \\
\hline 4.00 & 12.00 & 65.18 & 440.26 & 426.19 & 25.72 & 243.46 & 43.98 & 71.5 & 10 & 51.15 & 61.9 & 11 & 1 & 72.8 & 0.995 \\
\hline 4.00 & 12.37 & 63.55 & 292.60 & 286.40 & 25.28 & 264.98 & 47.56 & 38.4 & 10 & 51.15 & 33.2 & 2 & 1 & 40.8 & 0.995 \\
\hline 4.00 & 12.71 & 66.19 & 393.71 & 381.16 & 25.23 & 160.40 & 36.12 & 26.5 & 10 & 51.15 & 22.9 & 12 & 1 & 29.2 & 0.995 \\
\hline 4.00 & 13.05 & 62.58 & 392.92 & 376.84 & 25.77 & 200.58 & 50.35 & 22.1 & 10 & 51.15 & 19.1 & 12 & 1 & 23.2 & 0.995 \\
\hline 4.00 & 13.39 & 58.71 & 312.56 & 290.72 & 25.74 & 298.52 & 58.07 & 80.6 & 10 & 51.25 & 69.6 & 12 & 1 & 89.7 & 0.995 \\
\hline 4.00 & 13.80 & 60.11 & 349.55 & 336.92 & 25.89 & 196.91 & 41.93 & 46.9 & 5 & 50.95 & 20.4 & 11 & 1 & 25.0 & 0.995 \\
\hline 4.00 & 14.13 & 59.76 & 324.26 & 311.38 & 25.76 & 186.41 & 43.50 & 23.4 & 10 & 51.25 & 20.2 & 8 & 1 & 24.6 & 0.995 \\
\hline 4.00 & 14.47 & 63.61 & 398.21 & 385.80 & 25.97 & 219.02 & 49.01 & 16.5 & 10 & 51.25 & 14.3 & 11 & 1 & 14.5 & 0.995 \\
\hline 4.00 & 14.80 & 63.41 & 338.05 & 310.45 & 25.76 & 291.55 & 72.60 & N/A & N/A & N/A & N/A & N/A & $N / A$ & N/A & N/A \\
\hline 4.00 & 15.09 & 59.91 & 293.94 & 279.27 & 25.87 & 334.77 & 94.49 & 19.7 & $\therefore 10$ & 51.25 & 17.0 & 4 & $\therefore 1$ & 20.6 & 0.995 \\
\hline
\end{tabular}




\section{Appendix C-2. "A" Profile Pre-Leaching Chloride and Nitrate.}

\section{Introduction}

During the original trench excavation work 1986 an extensive collection of loose soil samples was taken from the soil profile along the north side. These samples were used to characterize moisture release profiles for 0-15 bars of tension (Wierenga et al, 1988 and 1989). Individual samples were usually less than $0.5 \mathrm{~kg}$ in mass and in many cases were depleted during the laboratory procedures.

After the collapse of the south trench wall during Experiment I a new shoring structure was built within the trench to prevent further damage to the intact trench walls during planned irrigation experiments. This structure prevented ready access to the trench soil facies. Therefore, before construction began, a second complete set of soil samples was collected along the north face of the trench as a sample bank for future study of pre-leaching soil conditions. This appendix reports the pre-leaching levels of chloride and nitrate in the soil profile at the " $A$ " profile (layer A) transect on the trench face, at $X=3.25 \mathrm{~m}$ from the northwest corner of the trench, from extractions made of these "banked" samples.

\section{Soil Sampling}

The second series of loose soil samples, designated as series II, were collected in March, 1988 in an attempt to duplicate the locations of the original soil samples in $\mathrm{X}$ and $\mathrm{Z}$ directions. It was not possible to duplicate original locations in Y: the original (series I) collection was made from a soil shelf which served as a walkway and sampling platform along the north side of the trench. This shelf was gradually sloughed away to access deeper locations as the trench was excavated. The series I samples were taken from the center of this shelf, which was approximately $120 \mathrm{~cm}$ wide. Series II samples were taken from the trench face itself $(Y=0 \mathrm{~m})$, approximately $\mathrm{Y}+60 \mathrm{~cm}$ from the original samples.

For the series II collection (layers A, B, and C) the interval in $\mathrm{Z}$ was slightly different from the original collection. For example, the original profile " $A$ " samples were taken in $13 \mathrm{~cm}$ increments in $\mathrm{Z}$ to the bottom of the trench (6.2 $\mathrm{m})$ for a total of 48 samples. For the series II collection of profile " $A$ ", the subset of samples considered in this appendix, 40 samples were collected on $15 \mathrm{~cm}$ intervals to a depth of $6 \mathrm{~m}$. Positions in $\mathrm{X}$ were identical to the series I collection, at $X=3.25 \mathrm{~m}\left(\mathrm{X}_{2}=-0.78 \mathrm{~m}\right)$.

About $0.8 \mathrm{~kg}$ of each sample was collected as loose soil and several representative peds. Samples were placed in pre-labeled plastic bags and stored at room temperature. Since the samples were collected from the exposed trench face they were relatively dry and did not require further drying before storage

\section{Sample Preparation}

Approximately 150 grams of each loose sample (peds excluded) were sieved on October 23, 1989. Samples were sieved through a $2 \mathrm{~mm}$ square mesh wire sieve (W.S. Tyler, Inc., Mentor, $\mathrm{OH}$ ). Fractions larger than $2 \mathrm{~mm}$ were ground gently in a mortar and pestle and then sieved again.

Approximately $25 \%$ of the mass of some samples would not pass through the sieve. This coarse material was discarded. To avoid exposing new un-weathered mineral facies no attempt was made to crush very hard clods. The 


\section{Appendix C-2. "A" Profile Pre-Leaching Chloride and Nitrate. (Continued)}

sieved samples were placed in paper bags and allowed to air dry until further processing.

On November 2, 1989 sub-samples of individual air-dry soil (35-41 $\mathrm{g}$ each) were gravimetrically diluted (Sartorius E2000D balance, Brinkman Instrument, Inc., Westbury, NY) with distilled-deionized water to approximate a 1:1 dilution. Each of these soil/water slurries was briefly stirred and then allowed to stand covered for about 20 minutes. Each slurry was then extracted on a Buchner funnel apparatus with a vacuum source about $400 \mathrm{mb}$ strong. The effluent was collected in 13 dram polypropylene vials which were capped and wrapped with Parafilm ${ }^{\circ}$ for refrigerated storage.

For each sample a second sub-sample, approximately $34-56 \mathrm{~g}$ of air dry soil, was oven-dried for 24 hours at $105^{\circ} \mathrm{C}$ to determine the residual moisture content for the calculations of sample dilution. These data were recorded to the nearest milligram.

\section{Chemical Analyses}

Chloride was analyzed with a Buchler model 4-2500 chloridometer (Buchler Instruments, Inc., Ft. Lee, NJ) which utilizes timed $\mathrm{Ag} / \mathrm{AgCl}$ titrimetry to determine solution $\mathrm{Cl}^{-}$content. Before using the instrument the silver electrodes were cleaned with a commercial silver cleaner or replaced. The instrument was switched to high sensitivity mode, which provided a detection limit of about $0.2 \mathrm{me} / \mathrm{l}$. A series of standard readings was taken with $0.1 \mathrm{ml}$ aliquots of $10 \mathrm{me} / \mathrm{L}$ and $60 \mathrm{me} / \mathrm{L} \mathrm{NaCl}$ solutions to make a two point calibration curve. Eight replications of each solution were read to ensure nominal instrument operation. Eight background correction vials were prepared with $0.1 \mathrm{ml}$ aliquots of distilled-deionized water. Sample titrations were performed with single replications of $0.10 \mathrm{ml}$ aliquots.

Nitrate analysis was performed with a Technicon AutoAnalyser II (Technicon Industrial Systems, Terrytown, NY) in the NMSU Soil \& Water Testing Laboratory. The sample extracts were first screened for approximate nitrate content by using nitrate test strips (EM Science Inc., Gibbstown, NJ). From these approximations the final dilutions to bring the samples into the working linear range (0-10 $\mathrm{mg} / \mathrm{l})$ of the AutoAnalyser were determined. Samples were diluted from $1 \mathbf{x}$ (full strength) to $100 \mathrm{x}$ with distilled-de ionized water in dilution steps of no more than $10 x$ per step. The instrument was calibrated to a single point curve with two to four replications of a $1 \mathrm{mg} / \mathrm{L} \mathrm{N} \mathrm{NO}$ standard and updated with two replications of nitrate standard for every 10 sample extracts in the sequence of runs.

\section{Data Reduction}

The chemical analysis data were manipulated to estimate the pre leaching solution levels of chloride and nitrate in the " $A$ " profile as in-situ solution contents and on a dry soil weight basis. For the soil solution values neutron probe measurements were used to estimate volumetric moisture contents within an "undisturbed" profile. The first neutron probe readings from plot II, taken on May 17,1988, provided the moisture content data. Values from a single access tube, that closest to the wall of the trench, at $X_{2}=0 \mathrm{~m}$ and $Y_{2}=1.09 \mathrm{~m}$, was used to provide the estimated moisture content for the "A" profile samples. Neutron probe soil moisture content values were linearly interpolated to match with the depths of " $A$ " profile samples. The neutron probe 


\section{Appendix C-2. "A" Profile Pre-Leaching Chloride and Nitrate (continued).}

measurements were separated from the soil samples by a linear distance of about $1.34 \mathrm{~m}$ and in time by about 3 months. An estimated term for sample bulk density was found by matching series II sample collection depths with linearly interpolated values of bulk density for the layer " $\mathrm{A}$ " soil cores, those collected during the original trench excavation work.

The raw chemical analyses data were adjusted for the dilution of samples by the amount of water added to make the extraction pastes, the air dry (initial) moisture contents and the estimated gravimetric water contents provided by neutron probe measurements and the bulk density of layer " $A$ " cores. The reduced data are presented as in-situ solution contents of ions on the day of neutron probe measurement $(\mathrm{me} / \mathrm{L})$ and as (less speculative) ion contents on the basis of oven-dry soil mass (ppm), which do not utilize the neutron probe or bulk density estimates.

\section{Discussion of Results}

The distribution of chloride and nitrate are presented as a dry soil mass proportion in Figure C-2a and as a concentration of the in-situ soil solution on the day of neutron probe measurement in Figure C-2b. Both expressions of the data are quite similar; most local ion concentration peaks and troughs occur at the same depths and pairs of chloride and nitrate ion concentrates in the same sample covary in a similar pattern. The most notable features of both plots are local peak concentrations at the $3.0 \mathrm{~m}$ and $4.5 \mathrm{~m}$ depths. These peaks correspond to abrupt increases in clay and calcium carbonate content between 3.18 and $3.31 \mathrm{~m}$ depths and increased calcium carbonate content between 4.48 and $4.61 \mathrm{~m}$ depths as observed in the original series I samples (Wierenga et al, 1989).

When chloride and nitrate ions are plotted against each other on a scatter diagram a clear relationship is evident (Figure C-2c). On this diagram data points along the positive slope generally corresponds with increasing depth. Almost all of those data which appear dispersed above the main cluster are associated with local concentration maxima for both anions (Figure C-2b).

Another interesting view of these data are presented in Figure C-2d. Here, the ratio of chloride to nitrate is quite variable to about 2 $m$ depth corresponding to the approximate depth of a lithologic discontinuity, at $1.7 \mathrm{~m}$ depth. This has been identified as the morphological boundary between the Isaacks' Ranch and Jornada II soil units (Wierenga et al, 1989 and Nash, 1990). Below $2 \mathrm{~m}$ depth the $\mathrm{Cl} / \mathrm{NO}_{3}$ ratio steadily increases. Given a long term net downward movement of solutes in the profile, these data suggest that nitrate mobility has been restricted relative chloride on the whole, or selectively added to or removed from portions of the profile by plants or biological transformation. In the absence of significant net downward movement of these ions, i.e., conditions of little recharge, the present-day ion distributions may represent stable historical concentrations following periods of profile accretion. 
Appendix C-2. "A" Profile Pre-Leaching Chloride and Nitrate. (Continued)

\begin{tabular}{|c|c|c|c|c|c|c|c|c|}
\hline $\begin{array}{l}\text { Sample } \\
\text { ID }\end{array}$ & $\begin{array}{l}\text { Depth, Z } \\
\text { (m) }\end{array}$ & $\begin{array}{l}\text { EXTRACTIC } \\
\text { Air-Dry } \\
\text { Soil } \\
\text { (g) }\end{array}$ & $\begin{array}{l}\text { N VALUES } \\
\text { Diluent } \\
\text { Added Water } \\
\text { (g) }\end{array}$ & $\begin{array}{l}\text { ODMO } \\
\text { CAN } \\
\text { TARE } \\
\text { (g) }\end{array}$ & $\begin{array}{l}\text { TURE CON } \\
\text { CAN + } \\
\text { Air-Dry } \\
\text { (g) }\end{array}$ & $\begin{array}{l}\text { ENT - } \\
\text { CAN + } \\
\text { Oven-Dry } \\
\text { (g) }\end{array}$ & $\begin{array}{c}\text { Est. } \\
\text { Layer A } \\
\text { Db } \\
(\mathrm{Mg} / \mathrm{m} 3)\end{array}$ & $\begin{array}{c}\text { Est. } \\
\text { Tube } 0 \\
\text { Theta } \\
(\mathrm{cm} 3 / \mathrm{cm} 3)\end{array}$ \\
\hline A-1 & 0.15 & 35.00 & 34.97 & 62.049 & 102.305 & 101.956 & 1.76 & 0.073 \\
\hline 2 & 0.30 & 35.14 & $\mathbf{3 5 . 2 5}$ & 63.650 & 102.511 & 102.183 & 1.64 & 0.083 \\
\hline 3 & 0.45 & 35.07 & 35.10 & 62.334 & 101.349 & 100.996 & 1.53 & 0.110 \\
\hline 4 & 0.60 & 35.85 & 35.76 & 63.344 & 105.370 & 105.038 & 1.72 & 0.112 \\
\hline 5 & 0.75 & 35.19 & 35.66 & 62.241 & 103.171 & 102.911 & 1.68 & 0.101 \\
\hline 6 & 0.90 & 35.95 & 35.78 & 59.744 & 99.643 & 99.402 & 1.88 & 0.083 \\
\hline 7 & 1.05 & 35.21 & 35.22 & 60.460 & 101.511 & 101.281 & 1.84 & 0.067 \\
\hline$\because$ & 1.20 & 35.64 & 36.85 & 62.824 & 101.102 & 100.881 & 1.79 & 0.053 \\
\hline 10 & 1.50 & 35.30 & 35.24 & 59.679 & 99.584 & 99.344 & 1.71 & 0.097 \\
\hline 11 & 1.65 & 36.46 & 36.77 & 62.128 & 100.594 & 100.399 & 1.72 & 0.090 \\
\hline 12 & 1.80 & 40.58 & 40.48 & 59.911 & -98.693 & 98.527 & 1.75 & 0.086 \\
\hline 13 & 1.95 & 40.45 & 41.14 & 62.410 & 100.852 & 100.641 & 1.79 & 0.086 \\
\hline 14 & 2.10 & 40.46 & 40.54 & 60.111 & 100.256 & 100.088 & 1.73 & 0.073 \\
\hline 15 & 2.25 & 40.39 & 40.87 & 59.643 & 104.034 & 103.728 & 1.74 & 0.054 \\
\hline 16 & 2.40 & 41.17 & 41.19 & 63.177 & 106.764 & 106.572 & 1.74 & 0.057 \\
\hline 17 & 2.55 & 41.10 & 41.21 & 63.275 & 102.274 & 102.099 & 1.77 & 0.059 \\
\hline 18 & 2.70 & 41.20 & 41.24 & 62.814 & 102.497 & 102.350 & 1.74 & 0.058 \\
\hline 19 & 2.85 & 40.72 & 40.98 & 63.438 & 103.126 & 102.855 & 1.78 & 0.060 \\
\hline 20 & 3.00 & 40.05 & 39.90 & 59.682 & 99.642 & 99.199 & 1.79 & 0.063 \\
\hline 21 & 3.15 & 40.08 & 40.09 & 65.073 & 105.931 & 105.473 & 1.75 & 0.094 \\
\hline 22 & 3.30 & 40.14 & 40.09 & 61.650 & 104.244 & 103.844 & 1.64 & 0.107 \\
\hline$\overline{23}$ & 3.45 & 40.26 & 40.24 & 62.702 & 99.471 & 99.252 & 1.70 & 0.084 \\
\hline 24 & 3.60 & 40.04 & 40.08 & 64.309 & 114.087 & 113.752 & 1.76 & 0.069 \\
\hline 25 & 3.75 & 40.06 & 40.02 & 63.024 & 100.128 & 99.903 & 1.71 & 0.059 \\
\hline 26 & 3.90 & 40.09 & 40.05 & 59.918 & 100.214 & 100.048 & 1.66 & 0.066 \\
\hline 27 & 4.05 & 40.01 & 40.19 & 63.392 & 120.728 & 120.157 & 1.67 & 0.070 \\
\hline 28 & 4.20 & 40.06 & 40.04 & 62.272 & 106.610 & 106.316 & 1.71 & 0.069 \\
\hline 29 & 4.35 & 40.08 & 40.15 & 63.421 & 103.632 & 103.372 & 1.64 & 0.065 \\
\hline 30 & 4.50 & 40.37 & 40.44 & 62.832 & 94.412 & 94.119 & 1.78 & 0.059 \\
\hline 31 & 4.65 & 40.21 & 40.25 & 63.632 & 100.239 & 100.028 & 1.66 & 0.069 \\
\hline 32 & 4.80 & 40.22 & 40.25 & 59.426 & 106.634 & 106.405 & 1.66 & 0.074 \\
\hline 33 & 4.95 & 40.09 & 40.13 & 62.511 & 100.299 & 100.093 & 1.62 & 0.069 \\
\hline 34 & 5.10 & 40.38 & 40.65 & 62.131 & 94.690 & 94.551 & 1.64 & 0.067 \\
\hline 35 & 5.25 & 40.01 & 40.13 & 59.967 & 98.904 & 98.757 & 1.73 & 0.066 \\
\hline 36 & 5.40 & 40.00 & 40.05 & 62.743 & 100.722 & 100.568 & 1.61 & 0.068 \\
\hline 37 & 5.55 & 40.19 & 40.16 & 62.467 & 97.058 & 96.939 & 1.62 & 0.070 \\
\hline 38 & 5.70 & 40.04 & 40.01 & 62.590 & 104.888 & 104.735 & 1.70 & 0.070 \\
\hline 39 & 5.85 & 40.11 & 40.26 & 60.250 & 92.331 & 92.201 & 1.68 & 0.066 \\
\hline 40 & 6.00 & 40.21 & 40.17 & 58.693 & 93.925 & 93.760 & 1.56 & 0.061 \\
\hline
\end{tabular}




\section{Appendix C-2. "A" Profile Pre-Leaching Chloride and Nitrate. (Continued)}

\begin{tabular}{|c|c|c|c|c|c|c|c|c|}
\hline \multirow[b]{2}{*}{$\begin{array}{c}\text { Sample } \\
\text { ID }\end{array}$} & \multirow[b]{2}{*}{$\begin{array}{c}\text { Depth, Z } \\
\text { (m) }\end{array}$} & \multirow[b]{2}{*}{$\begin{array}{c}\text { H2O content } \\
\text { in-situ } \\
\text { sample } \\
\text { (g) }\end{array}$} & \multirow[b]{2}{*}{$\begin{array}{l}\text { Liquid } \\
\text { Dilution } \\
\text { Factor }\end{array}$} & \multirow[b]{2}{*}{$\begin{array}{l}\text { Solid } \\
\text { Dilution } \\
\text { Factor }\end{array}$} & \multicolumn{4}{|c|}{ - CHLORIDE } \\
\hline & & & & & $\begin{array}{c}\text { Raw } \\
\text { Reading } \\
\text { (me/l) }\end{array}$ & $\begin{array}{l}\text { Raw } \\
\text { Corrected } \\
\text { (me/l) }\end{array}$ & $\begin{array}{l}\text { Dry-soil } \\
\text { Basis } \\
\text { (g/Mg) }\end{array}$ & $\begin{array}{c}\text { Soil Solution } \\
\text { basis } \\
\text { (me/1) }\end{array}$ \\
\hline$A-1$ & 0.15 & 1.44 & 25.21 & 1.02 & 4.53 & 0.26 & 9 & 6.6 \\
\hline 2 & 0.30 & 1.75 & 21.12 & 1.02 & 4.70 & 0.43 & 15 & 9.0 \\
\hline 3 & 0.45 & 250 & 15.05 & 1.02 & 4.91 & 0.63 & 23 & 9.5 \\
\hline 4 & 0.60 & 231 & 16.50 & 1.01 & 4.57 & 0.30 & 11 & 4.5 \\
\hline 5 & 0.75 & 2.10 & 18.00 & 1.03 & 4.78 & 0.51 & 18 & 9.1 \\
\hline 6 & 0.90 & 1.58 & 23.66 & 1.01 & 4.93 & 0.65 & 23 & 15.4 \\
\hline 7 & 1.05 & 1.28 & 28.61 & -1.01 & 4.47 & 0.20 & 7 & 5.8 \\
\hline 8 & 1.20 & 1.05 & 36.15 & 1.05 & 4.94 & 0.66 & 25 & 23.9 \\
\hline 10 & 1.50 & 2.00 & 18.66 & 1.01 & 6.04 & 1.74 & 62 & 32.5 \\
\hline 11 & 1.65 & 1.91 & 20.28 & 1.02 & 5.87 & 1.57 & 57 & 31.9 \\
\hline 12 & 1.80 & 1.98 & 21.44 & 1.01 & 5.69 & 1.40 & 50 & 30.0 \\
\hline 13 & 1.95 & 1.93 & 22.29 & 1.03 & 6.13 & 1.83 & 67 & 40.8 \\
\hline 14 & 2.10 & 1.71 & 24.76 & 1.01 & 5.71 & 1.42 & 51 & 35.1 \\
\hline 15 & 2.25 & 1.24 & 33.97 & 1.03 & 6.37 & 207 & 75 & 20.2 \\
\hline 16 & 2.40 & 1.35 & 31.46 & 1.01 & 7.02 & 270 & 97 & 85.1 \\
\hline 17 & 255 & 1.37 & 31.08 & 1.01 & 7.86 & 3.53 & 126 & 109.6 \\
\hline 18 & 2.70 & 1.37 & 31.01 & 1.01 & 6.72 & 241 & 86 & 74.7 \\
\hline 19 & 2.85 & 1.35 & 31.28 & 1.02 & 8.91 & 4.56 & 165 & 1426 \\
\hline 20 & 3.00 & 1.39 & 29.73 & 1.02 & 10.47 & 6.09 & 220 & 1810 \\
\hline 21 & 3.15 & 2.13 & 19.81 & 1.02 & 9.23 & 4.87 & 177 & 96.5 \\
\hline 22 & 3.30 & 2.60 & 16.41 & 1.02 & 7.85 & 3.52 & 127 & 57.7 \\
\hline 23 & 3.45 & 1.98 & 21.35 & 1.01 & 7.11 & 279 & 100 & 59.6 \\
\hline 24 & 3.60 & 1.57 & 26.49 & 1.01 & 7.95 & 3.62 & 130 & 95.8 \\
\hline 25 & 3.75 & 1.38 & 29.97 & 1.01 & 7.92 & 3.59 & 128 & 107.5 \\
\hline 26 & 3.90 & 1.59 & 26.19 & 1.01 & 7.73 & 3.40 & 121 & 89.1 \\
\hline 27 & 4.05 & 1.67 & 25.06 & 1.02 & 8.80 & 4.45 & 162 & 111.5 \\
\hline 28 & 4.20 & 1.60 & 26.01 & 1.01 & 9.04 & 4.69 & 168 & 121.8 \\
\hline 29 & 4.35 & 1.57 & 26.56 & 1.01 & 9.35 & 4.99 & 179 & 132.5 \\
\hline 30 & 4.50 & 1.33 & 31.48 & 1.02 & 13.51 & 9.07 & 328 & 285.6 \\
\hline 31 & 4.65 & 1.67 & 25.14 & 1.01 & 13.35 & 8.91 & 320 & 224.1 \\
\hline 32 & 4.80 & 1.79 & 23.52 & 1.01 & 11.94 & 7.53 & 270 & 177.1 \\
\hline 33 & 4.95 & 1.70 & 24.66 & 1.01 & 11.51 & 7.11 & 255 & 175.3 \\
\hline 34 & 5.10 & 1.64 & 25.86 & 1.02 & 14.18 & 9.73 & 350 & 251.6 \\
\hline 35 & 5.25 & 1.51 & 27.51 & 1.01 & 12.15 & 7.74 & 277 & 212.8 \\
\hline 36 & 5.40 & 1.69 & 24.69 & 1.01 & 12.33 & 7.91 & 283 & 195.4 \\
\hline 37 & 5.55 & 1.73 & 24.25 & 1.01 & 13.47 & 9.03 & 322 & 219.0 \\
\hline 38 & 5.70 & 1.63 & 25.48 & 1.01 & 12.70 & 8.28 & 295 & 210.9 \\
\hline 39 & 5.85 & 1.57 & 26.57 & 1.01 & 12.97 & 8.54 & 306 & 226.9 \\
\hline 40 & 6.00 & 1.55 & 26.90 & 1.01 & 14.17 & 9.72 & 347 & 261.5 \\
\hline
\end{tabular}

Chloride background $=42.65 * 0.1 \mathrm{me} / 1$

10 me $/ 1$ Chloride standard $=104.1 * 0.1 \mathrm{me} / 1$ 
Appendix C-2. "A" Profile Pre-Leaching Chloride and Nitrate. (Continued)

\begin{tabular}{|c|c|c|c|c|c|c|}
\hline $\begin{array}{c}\text { Sample } \\
\text { ID }\end{array}$ & $\begin{array}{c}\text { Depth, Z } \\
\text { (m) }\end{array}$ & $\begin{array}{c}\text { Raw } \\
\text { Reading } \\
\text { (scale) }\end{array}$ & $\begin{array}{l}\text { Sample } \\
\text { Dilution } \\
\text { (x) }\end{array}$ & $\begin{array}{l}\text { Raw } \\
\text { Corrected } \\
\text { (ppm) }\end{array}$ & $\begin{array}{l}\text { Dry-soil } \\
\text { Basis } \\
(\mathrm{g} / \mathrm{Mg})\end{array}$ & $\begin{array}{c}\text { Soil Solution } \\
\text { basis } \\
\text { (me/l) }\end{array}$ \\
\hline $\mathrm{A}-1$ & 0.15 & 35.00 & 10 & 31 & 31 & 12.8 \\
\hline 2 & 0.30 & 29.50 & 10 & 26 & 26 & 9.0 \\
\hline 3 & 0.45 & 14.25 & 10 & 12 & 13 & 3.1 \\
\hline 4 & 0.60 & 13.25 & 10 & 12 & 12 & 3.2 \\
\hline 5 & 0.75 & 13.75 & 10 & 12 & 12 & 3.6 \\
\hline 6 & 0.90 & 13.00 & 10 & 11 & 11. & 4.5 \\
\hline 7 & 1.05 & 44.50 & 1 & 4 & 4 & 18 \\
\hline 8 & 1.20 & 24.25 & 10 & 21 & 22 & 12.7 \\
\hline 10 & 1.50 & 45.50 & 10 & 40 & 40 & 123 \\
\hline 11 & 165 & 59.50 & 10 & 52 & 53 & 17.5 \\
\hline 12 & 1.80 & 62.50 & 10 & 55 & 55 & 19.4 \\
\hline 13 & 1.95 & 33.50 & 40 & 117 & 121 & 43.3 \\
\hline 14 & 2.10 & 89.00 & 10 & 78 & 79 & 32.0 \\
\hline 15 & 2.25 & 31.00 & 40 & 108 & 111 & 61.1 \\
\hline 16 & 2.40 & 53.50 & 40 & 187 & 189 & 97.7 \\
\hline 17 & 255 & 56.00 & 40 & 196 & 198 & 101.0 \\
\hline 18 & 2.70 & 40.00 & 40 & 140 & 141 & 72.0 \\
\hline 19 & 2.85 & 56.75 & 40 & 199 & 203 & 103.0 \\
\hline 20 & 3.00 & 69.00 & 40 & 241 & 246 & 119.0 \\
\hline 21 & 3.15 & 51.00 & 40 & 178 & 183 & 58.6 \\
\hline 22 & 3.30 & 34.50 & 40 & 121 & 123 & 32.9 \\
\hline 23 & 3.45 & 22.00 & 40 & 77 & 78 & 27.3 \\
\hline 24 & 3.60 & 29.00 & 40 & 101 & 103 & 44.6 \\
\hline 25 & 3.75 & 30.00 & 40 & 105 & 106 & 52.2 \\
\hline 26 & 3.90 & 24.00 & 40 & 84 & 85 & 36.5 \\
\hline 27 & 4.05 & 36.00 & 40 & 126 & 129 & 52.4 \\
\hline 28 & 4.20 & 33.25 & 40 & 116 & 118 & 50.2 \\
\hline 29 & 4.35 & 37.50 & 40 & 131 & 133 & 57.8 \\
\hline 30 & 4.50 & 64.25 & 40 & 225 & 229 & 117.4 \\
\hline 31 & 4.65 & 60.00 & 40 & 210 & 213 & 87.5 \\
\hline 32 & 4.80 & 50.00 & 40 & 175 & 177 & 68.3 \\
\hline 33 & 4.95 & 42.50 & 40 & 149 & 151 & 60.8 \\
\hline 34 & 5.10 & 60.75 & 40 & 213 & 216 & 91.2 \\
\hline 35 & 5.25 & 45.00 & 40 & 157 & 159 & 71.8 \\
\hline 36 & 5.40 & 46.50 & 40 & 163 & 164 & 66.6 \\
\hline 37 & 5.55 & 50.00 & 40 & 175 & 176 & 70.4 \\
\hline 38 & 5.70 & 43.50 & 40 & 152 & 153 & 64.3 \\
\hline 39 & 5.85 & 39.75 & 40 & 139 & 141 & 61.3 \\
\hline 40 & 6.00 & 46.00 & 40 & 161 & 162 & 718 \\
\hline
\end{tabular}


Appendix C-2a. "A" Profile Pre-Leaching Chloride and Nitrate.

Chloride \& Nitrate Distributions in "A" Profile

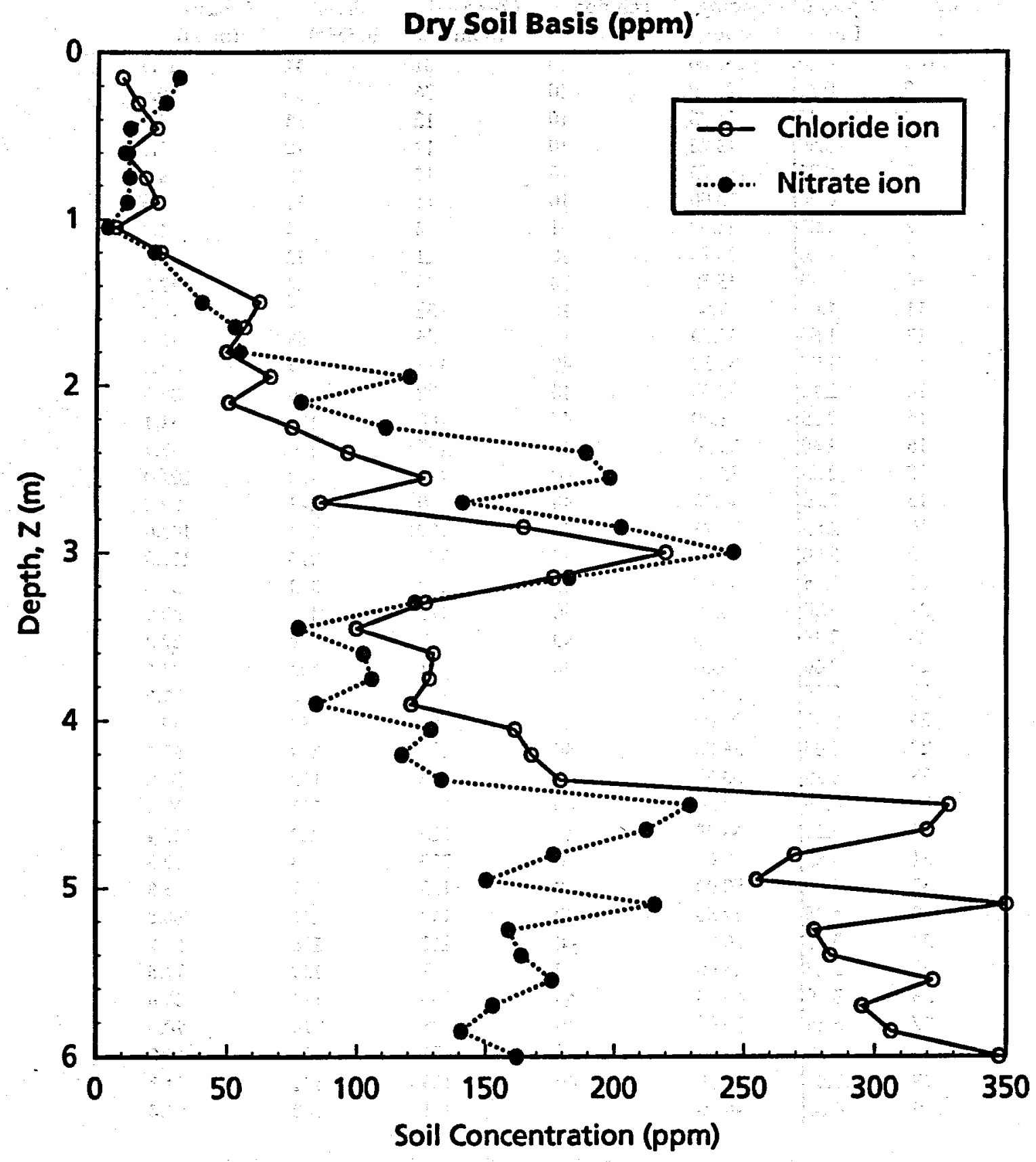


Appendix C-2b. "A" Profile Pre-Leaching Chloride and Nitrate.

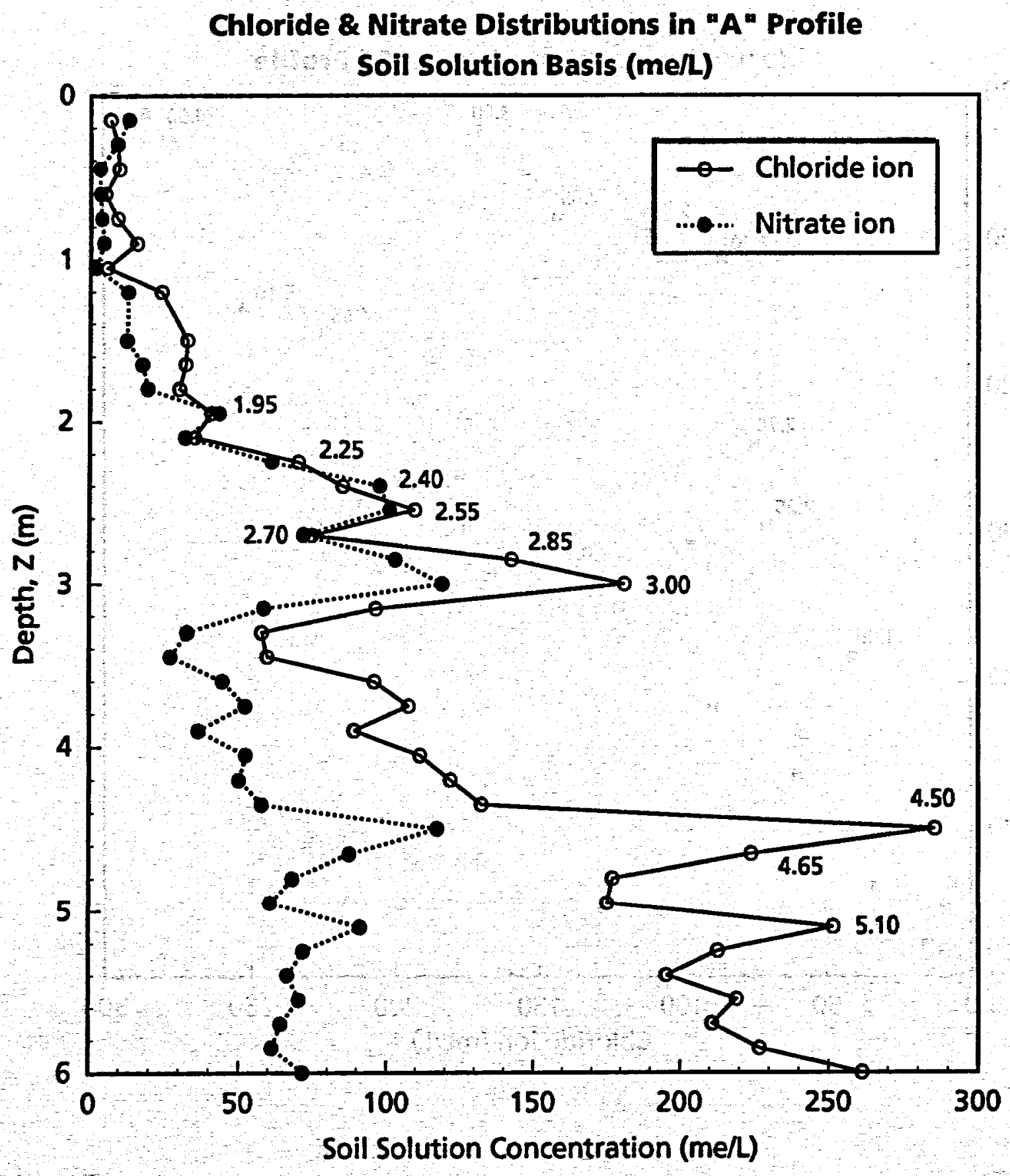


Appendix C-2c. "A" Profile Pre-Leaching Chloride and Nitrate.

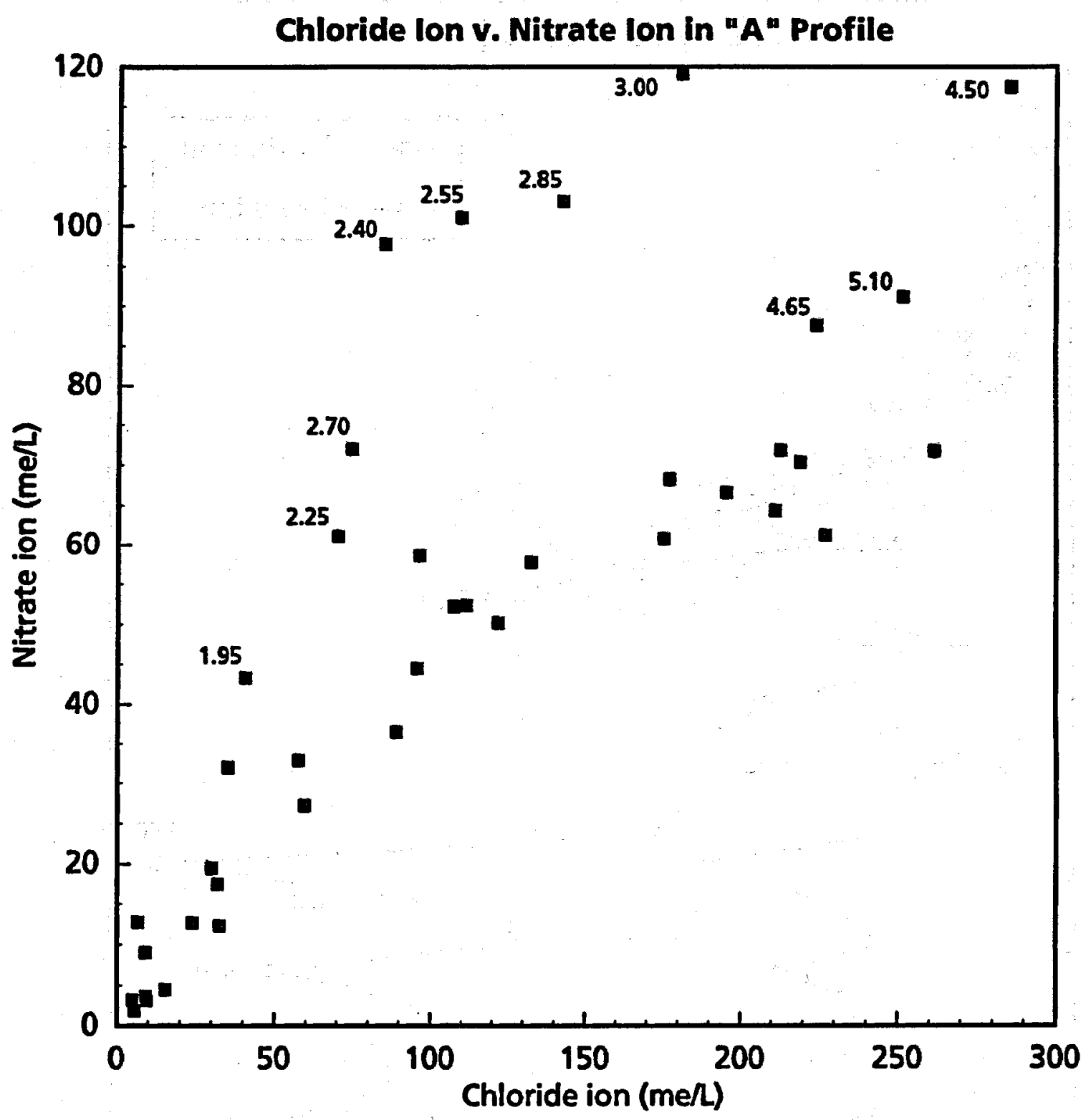


Appendix C-2d. "A" Profile Pre-Leaching Chloride and Nitrate.

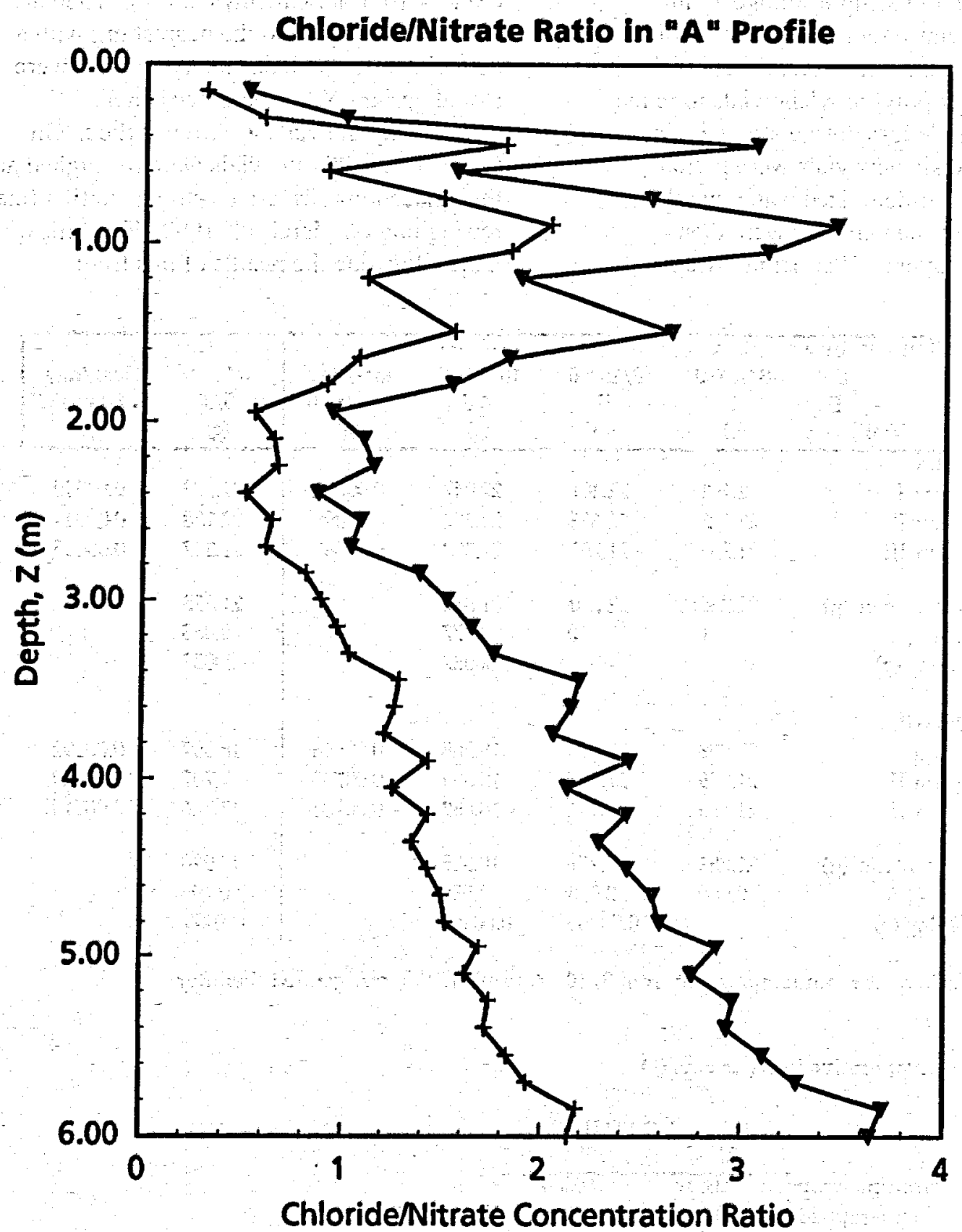

$$
\begin{aligned}
& \rightarrow \text { soil solution (me/L) } \\
& + \text { dry soil (ppm) }
\end{aligned}
$$




\section{Appendix C-3. Evaporative Loss from $20 \mathrm{ml}$ Glass Scintillation Vials.}

For long-term liquid sample storage $20 \mathrm{ml}$ capacity glass scintillation wlvials with foil lined screw-on caps were used. These vials were chosen over polypropylene vials to reduce evaporative sample loss during storage. On August 10, 1990 six new vials were partially filled with distilled-deionized water and then capped. Three of the vial caps were then wrapped with 2 layers of Paraffilm ${ }^{\star}$ wrap
(Am. Nat'l. Can, Greenwich, CT). Then all the vials were weighed to the nearest $\mathrm{mg}$ with a Sartorius E2000D balance. -All 6 vials were stored upright on a laboratory bench. Periodically the vials were reweighed. On October 17, 1991 the vials were reweighed and then placed in refrigerated storage until a final reweighing on March 11, 1992. The following tables illustrate the results of this trial:

\begin{tabular}{|c|c|c|c|c|c|c|}
\hline $\begin{array}{r}\text { Temperature } \\
\text { Date } \\
\text { Day } \\
\text { Vial Mass }\end{array}$ & $\begin{array}{c}21^{\circ} \mathrm{C} \\
8 / 10 / 90 \\
0 \\
(\mathrm{~g})\end{array}$ & $\begin{array}{c}8 / 15 / 90 \\
5 \\
(g)\end{array}$ & $\begin{array}{c}\overline{10 / 17 / 91} \\
433 \\
(\mathrm{~g})\end{array}$ & $\begin{array}{c}\text { loss/day } \\
\text { of day } 0 \\
\text { (g) }\end{array}$ & $\begin{array}{c}2^{\circ} \mathrm{C} \\
3 / 11 / 92 \\
579 \\
(g)\end{array}$ & $\begin{array}{c}\text { loss/day } \\
\text { of day } 433 \\
\text { (g) }\end{array}$ \\
\hline \multicolumn{7}{|l|}{ With Parafim } \\
\hline Replication I & 22.401 & 22.401 & 22.047 & 0.00082 & 21.989 & 0.00040 \\
\hline Replication II & 22625 & 22.625 & 22.202 & 0.00098 & 22.138 & 0.00044 \\
\hline Replication III & 21.753 & 21.753 & 21.398 & 0.00082 & 21.347 & 0.00035 \\
\hline Treatment Mean $(g)$ & 22.260 & 22.260 & 21.882 & & 21.825 & \\
\hline Mean Loss $(\mathrm{g})$ & 0 & $\mathbf{0}$ & 0.377 & & 0.058 & \\
\hline Loss per day (g) & & & 0.00087 & & 0.00039 & \\
\hline \multicolumn{7}{|l|}{ Without Parafilm } \\
\hline Replication I & 20.998 & 20.993 & 18.588 & 0.00555 & 18.307 & 0.00192 \\
\hline Replication II & 21.399 & 21.387 & 18.334 & 0.00705 & 17.707 & 0.00429 \\
\hline Replication III & 21.453 & 21.432 & 18.052 & 0.00781 & 17.746 & 0.00210 \\
\hline Treatment Mean (g) & 21.283 & 21.271 & 18.325 & & 17.920 & \\
\hline Mean Loss ( $(\mathrm{)})$ & 0.000 & 0.013 & 2.959 & & 0.405 & \\
\hline Loss per day (g) & & 0.00253 & 0.00683 & & 0.00277 & \\
\hline
\end{tabular}

All vials kept at room temperature from $8 / 10 / 90$ to $10 / 17 / 90$, refrigerated therafter

Summary: Evaporative loss per day, (g)

\begin{tabular}{r|cc} 
& \multicolumn{2}{c}{ Temperature } \\
\cline { 2 - 3 } With Parafilm wrap & $21^{\circ} \mathrm{C}$. & $2^{\circ} \mathrm{C}$. \\
\cline { 2 - 3 } Unwrapped & 0.0009 & 0.0004 \\
& 0.0068 & 0.0028
\end{tabular}


Appendix C-4. Facilities Maps of Trench Site and Ranch Headquarters.

\section{TrenCh Site Water Utilities}

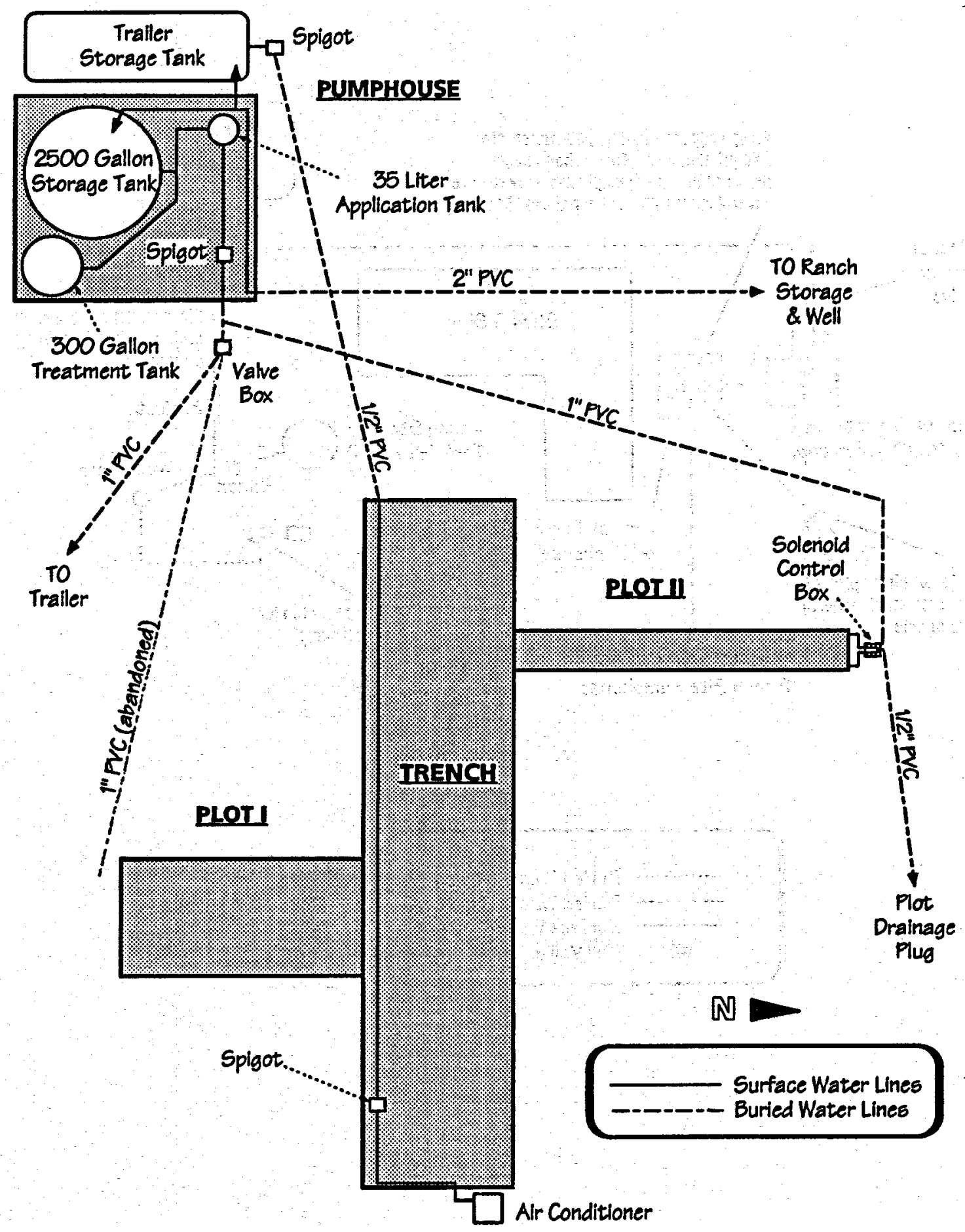




\section{Appendix C-4. Facilities Maps of Trench Site and Ranch Headquarters. (Continued)}

\section{RANCh HOUSe UtiLties}

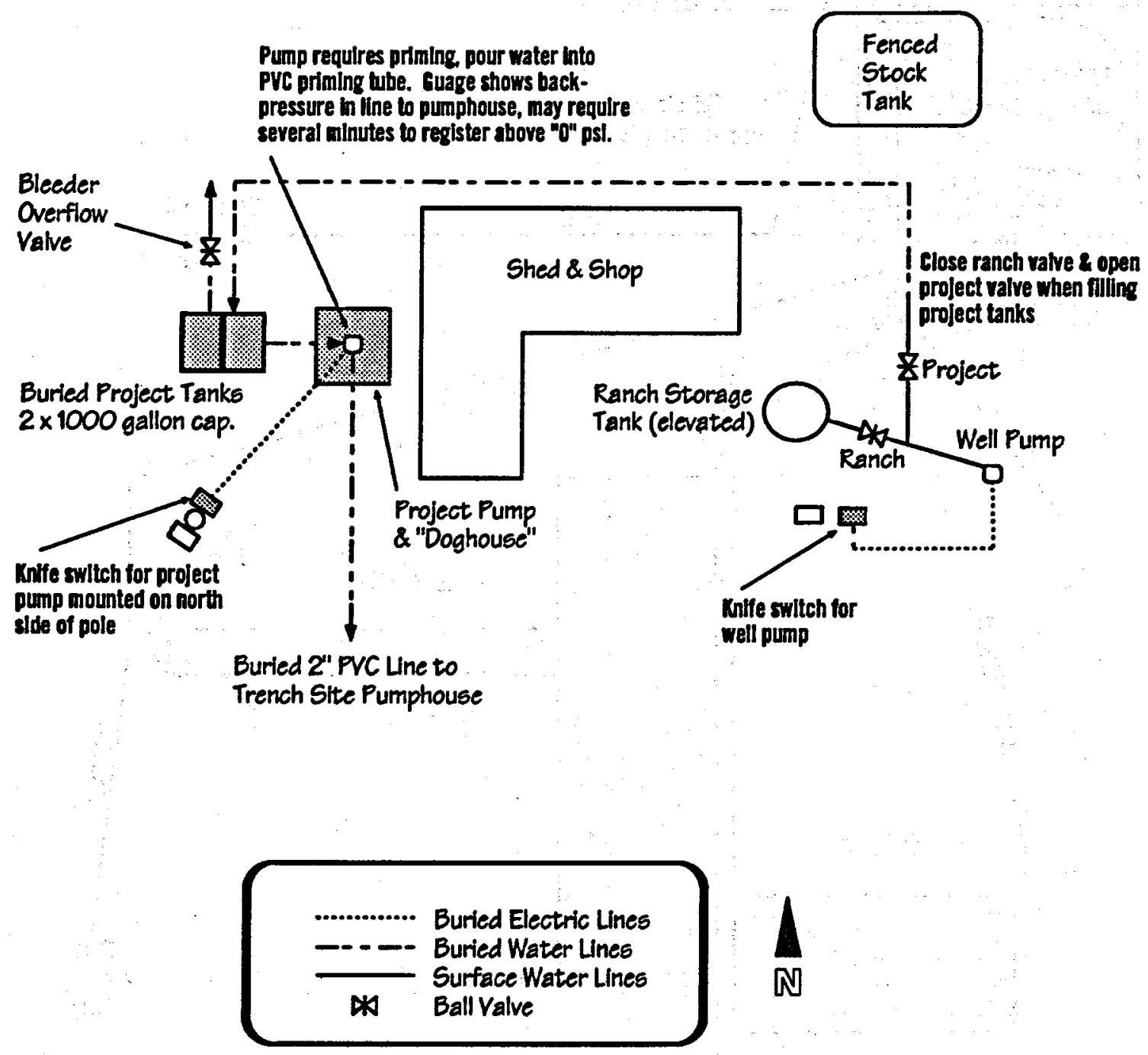




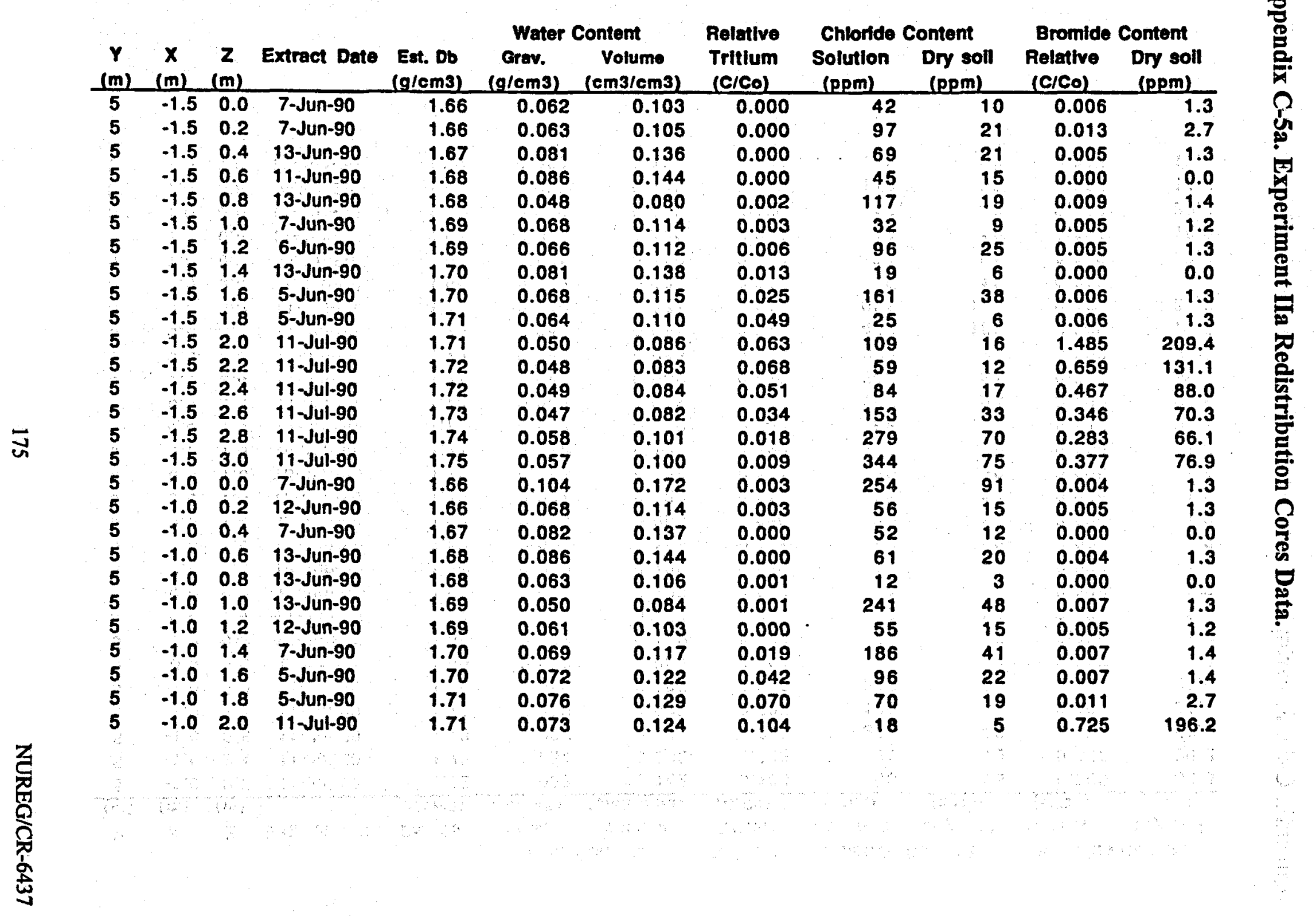




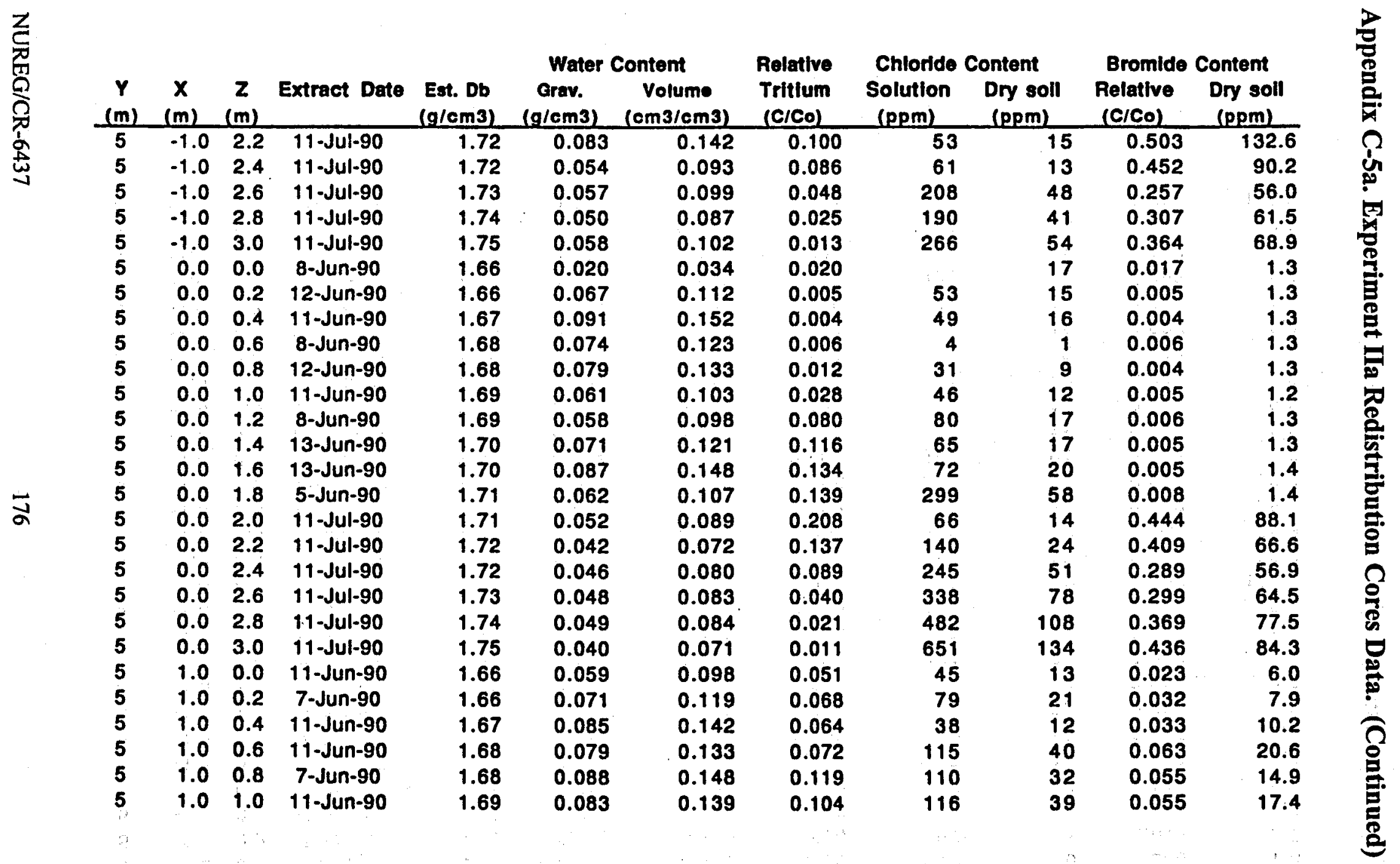




\begin{tabular}{|c|c|c|c|c|c|c|c|c|c|c|c|}
\hline $\begin{array}{c}Y \\
(m)\end{array}$ & $\begin{array}{c}x \\
(m)\end{array}$ & $\begin{array}{l}Z \\
(m)\end{array}$ & Extract Date & $\begin{array}{l}\text { Est. Db } \\
\text { (g/cm3) }\end{array}$ & $\begin{array}{c}\text { Water C } \\
\text { Grav. } \\
\text { (g/om3) }\end{array}$ & $\begin{array}{l}\text { Content } \\
\text { Volume } \\
\text { (cm3/cm3) }\end{array}$ & $\begin{array}{l}\text { Relative } \\
\text { Tritium } \\
\text { (C/Co) } \\
\end{array}$ & $\begin{array}{l}\text { Chloride C } \\
\text { Solution } \\
\text { (ppm) }\end{array}$ & $\begin{array}{l}\text { Sontent } \\
\text { Dry soil } \\
\text { (ppm) }\end{array}$ & $\begin{array}{l}\text { Bromide } \\
\text { Relative } \\
\text { (C/Co) }\end{array}$ & $\begin{array}{l}\text { Content } \\
\text { Dry soll } \\
\text { (ppm) }\end{array}$ \\
\hline 5 & 1.0 & 1.2 & 7-Jun-90 & 1.69 & 0.056 & 0.094 & 0.167 & 214 & 45 & 0.147 & 28.9 \\
\hline 5 & 1.0 & 1.4 & 13-Jun-90 & 1.70 & 0,059 & 0.100 & 0.146 & 141 & 32 & 0.276 & 59.3 \\
\hline 5 & 1.0 & 1.6 & 5-Jun-90 & 1.70 & 0.075 & 0.128 & 0.146 & 252 & 68 & 0.231 & 58.1 \\
\hline 5 & 1.0 & 1.8 & 5-Jun-90 & 1.71 & 0.066 & 0.112 & 0.162 & 135 & 32 & 0.207 & 46.2 \\
\hline 5 & 1.0 & 2.0 & 5-Jun-90 & 1.71 & 0.071 & 0.122 & 0.154 & 156 & 39 & 0.213 & 50.4 \\
\hline 5 & 1.0 & 2,2 & 11-Jul-90 & 1.72 & 0.049 & 0.085 & 0.061 & 232 & 54 & 0.258 & 56.2 \\
\hline 5 & 1.0 & 2.4 & 11-Jul-90 & 1.72 & 0.054 & 0.093 & 0.021 & 463 & 101 & 0.514 & 105.7 \\
\hline 5 & 1.0 & 2.6 & $11-J u l-90$ & 1.73 & 0.050 & 0.087 & 0.007 & 695 & 145 & 0.607 & 119.1 \\
\hline 5 & 1.0 & 2.8 & 11-Jul-90 & 1.74 & 0.045 & 0.078 & 0.004 & 882 & 160 & 0.800 & 136.3 \\
\hline 5 & 1.0 & 3.0 & 11-Jui-90 & 1.75 & 0.042 & 0.074 & 0.002 & 1070 & 197 & 0.769 & 133.1 \\
\hline 5 & 1.5 & 0.2 & 13-Jun-90 & 1.66 & 0.064 & 0.107 & 0.018 & 43 & 11 & 0.011 & 2.6 \\
\hline 5 & 1.5 & 0.4 & 11 -Jun-90 & 1.67 & 0.093 & 0.155 & 0.020 & 27 & 11 & 0.019 & 7.2 \\
\hline 5 & 1.5 & 0.6 & 7-Jun-90 & 1.68 & 0.078 & 0.131 & 0.031 & 47 & 16 & 0.047 & 14.7 \\
\hline 5 & 1.5 & 0.8 & 12-Jun-90 & 1.68 & 0.088 & 0.148 & 0.029 & 65 & 21 & 0.084 & 25.7 \\
\hline 5 & 1.5 & 1.0 & 12-Jun-90 & 1.69 & 0.060 & 0.101 & 0.027 & 176 & 44 & 0.092 & 21.4 \\
\hline 5 & 1.5 & 1.2 & 13-Jun-90 & 1.69 & 0.068 & 0.115 & 0.018 & 108 & 33 & 0.104 & 29.4 \\
\hline 5 & 1.5 & 1.4 & 11-Jun-90 & 1.70 & 0.072 & 0.123 & 0.015 & 58 & 20 & 0.092 & 29.8 \\
\hline 5 & 1.5 & 1.6 & 5-Jun-90 & 1.70 & 0.039 & 0.067 & 0.027 & 295 & 40 & 0.043 & 5.5 \\
\hline 5 & 1.5 & 1.8 & 5-Jun-90 & 1.71 & 0.069 & 0.117 & 0.013 & 331 & 74 & 0.046 & 9.7 \\
\hline 5 & 1.5 & 2.0 & 5-Jun-90 & 1.71 & 0.049 & 0.084 & 0.014 & 370 & 74 & 0.103 & 19.2 \\
\hline 5 & 1.5 & 2.2 & $11-J u l-90$ & 1.72 & 0.049 & 0.085 & 0.004 & 835 & 188 & 0.525 & 110.9 \\
\hline 5 & 1.5 & 2.6 & 11-Jul-90 & 1.73 & 0.053 & 0.091 & 0.000 & 1169 & 273 & 0.541 & 118.8 \\
\hline 5 & 1.5 & 2.8 & $11-J u l-90$ & 1.74 & 0.045 & 0.079 & 0.001 & 1307 & 289 & 0.573 & 118.9 \\
\hline 5 & 1.5 & 3.0 & $11-J u l-90$ & 1.75 & 0.038 & 0.066 & 0.001 & 1305 & 228 & 0.792 & 129.9 \\
\hline 5.1 & -0.5 & 0.0 & 12-Jun-90 & 1.66 & 0.074 & 0.122 & 0.009 & 68 & 19 & 0.010 & 2.6 \\
\hline 5.1 & -0.5 & 0.2 & 8-Jun-90 & 1.66 & 0.060 & 0.100 & 0.005 & 69 & 16 & 0.006 & 1.3 \\
\hline 5.1 & -0.5 & 0.4 & 8-Jun-90 & 1.67 & 0.087 & 0.146 & 0.002 & 138 & 38 & 0.000 & 0.0 \\
\hline Z & & 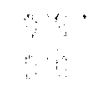 & $\ldots$ & $\cdots$ & 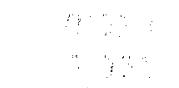 & 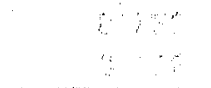 & 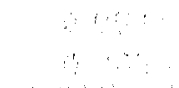 & $\because$ & $:$ & 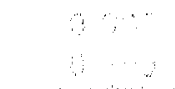 & 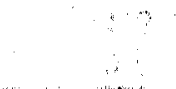 \\
\hline
\end{tabular}




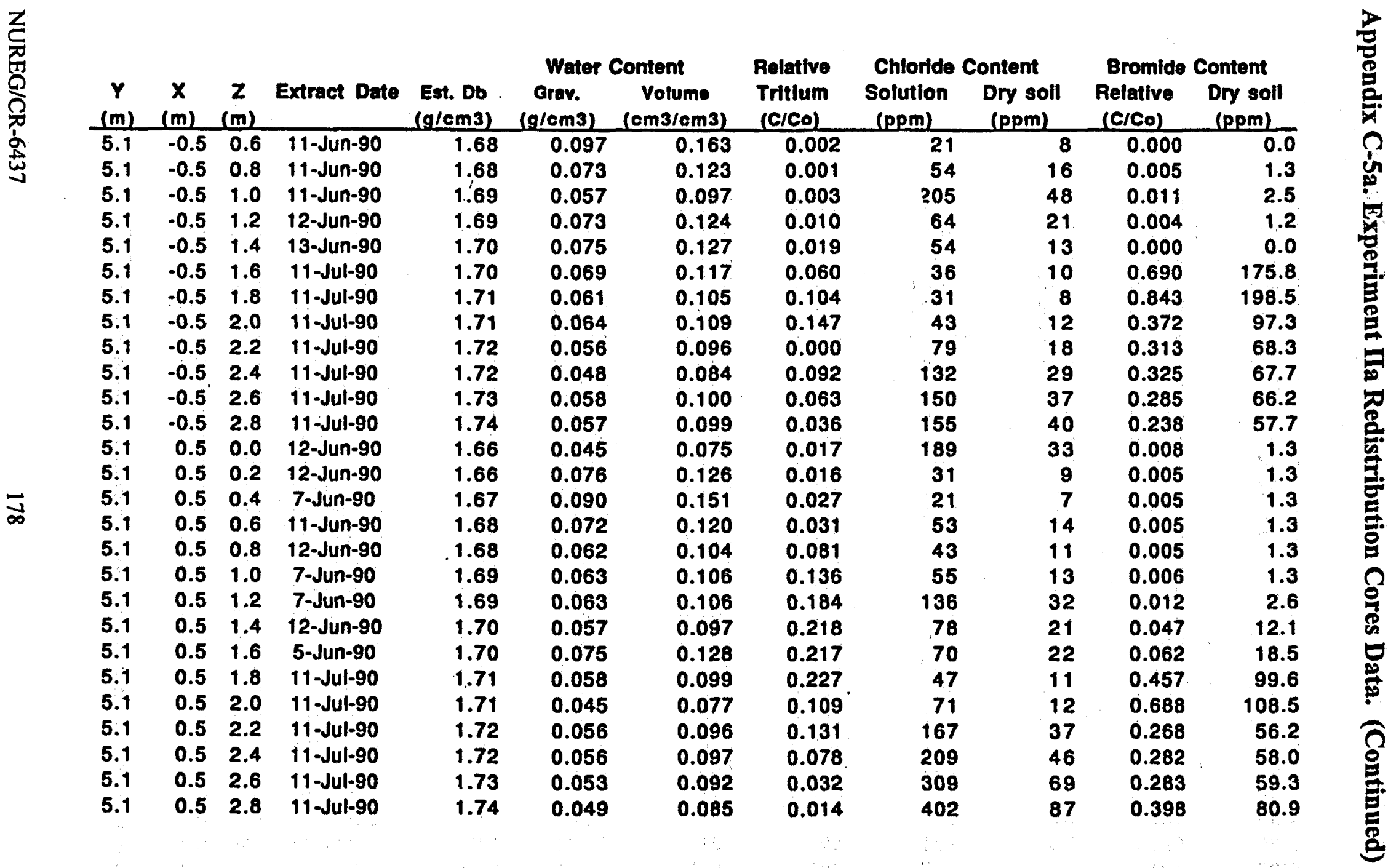




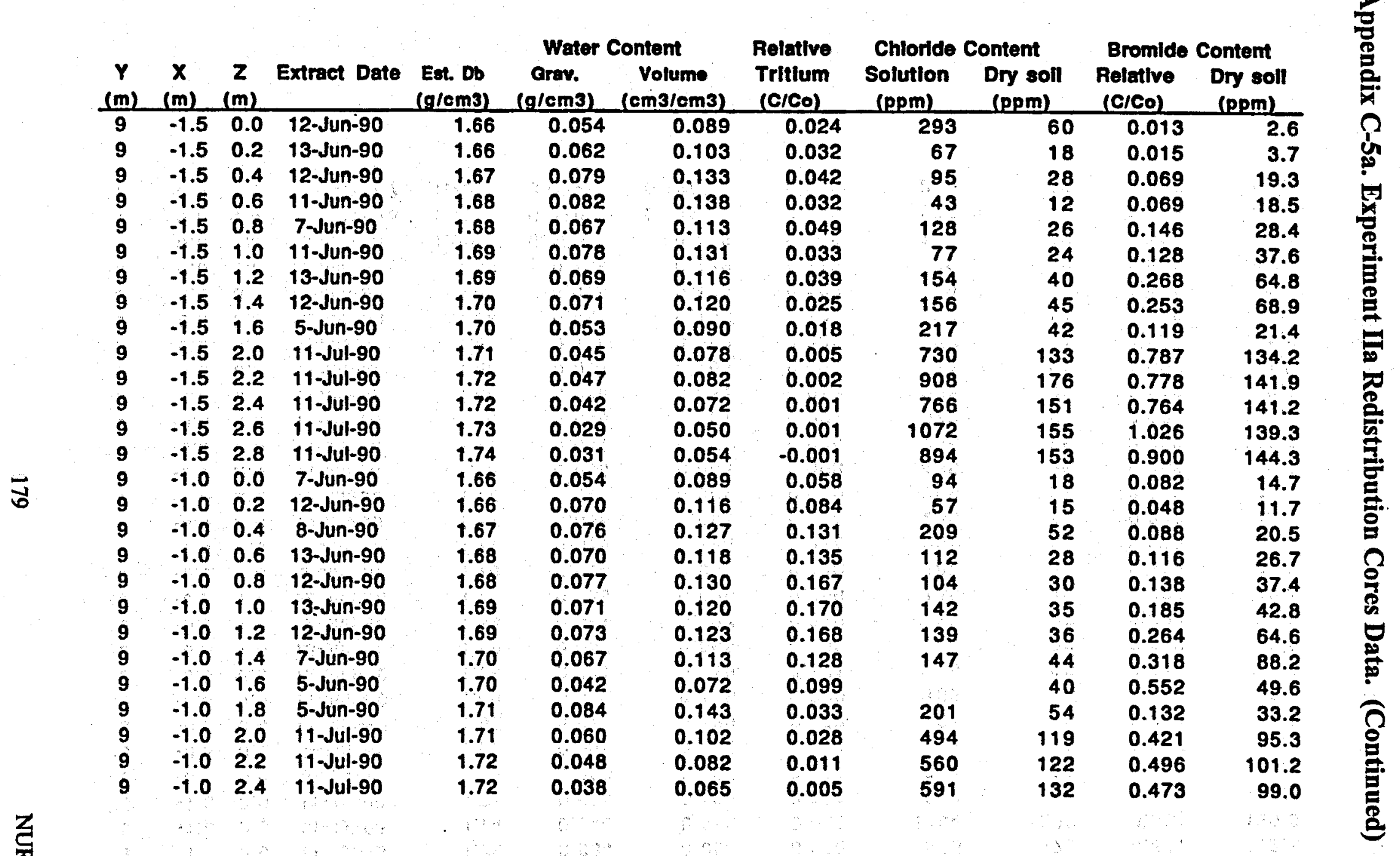




\begin{tabular}{|c|c|c|c|c|c|c|c|c|c|c|c|}
\hline \multirow[b]{2}{*}{$\begin{array}{c}Y \\
(m)\end{array}$} & \multirow[b]{2}{*}{$\begin{array}{c}x \\
(m)\end{array}$} & \multirow[b]{2}{*}{$\underset{(m)}{Z}$} & \multirow[b]{2}{*}{ Extract Date } & \multirow[b]{2}{*}{$\begin{array}{l}\text { Est. Db } \\
\text { (g/cm3) }\end{array}$} & \multicolumn{2}{|c|}{ Water Content } & \multirow{2}{*}{$\begin{array}{l}\text { Relative } \\
\text { Tritlum } \\
\text { (c/Co) }\end{array}$} & \multicolumn{2}{|c|}{ Chloride Content } & \multicolumn{2}{|c|}{ Bromide Content } \\
\hline & & & & & $\begin{array}{c}\text { Grav. } \\
\text { (g/em3) }\end{array}$ & $\begin{array}{c}\text { Volume } \\
\text { (cm3/cm3) }\end{array}$ & & $\begin{array}{c}\text { Solution } \\
\text { (ppm) }\end{array}$ & $\begin{array}{l}\text { Dry soil } \\
\text { (ppm) }\end{array}$ & $\begin{array}{l}\text { Relative } \\
\text { (C/Co) }\end{array}$ & $\begin{array}{l}\text { Dry soil } \\
\text { (ppm) }\end{array}$ \\
\hline 9 & -1.0 & 2.6 & 11-Jul-90 & 1.73 & 0.034 & 0.059 & 0.003 & 955 & 179 & 0.641 & 112.8 \\
\hline 9 & -1.0 & 2.8 & $11-J u l-90$ & 1.74 & 0.040 & 0.069 & 0.002 & 1164 & 226 & 0.661 & 120.6 \\
\hline 9 & -1.0 & 3.0 & $11 \cdot-J u l-90$ & 1.75 & 0,074 & 0.129 & 0.001 & 574 & 176 & 0.403 & 115.8 \\
\hline 9 & -0.5 & 0.0 & 8-Jun-90 & 1.66 & 0.033 & 0.055 & 0.027 & 281 & 36 & 0.055 & 6.6 \\
\hline 9 & -0.5 & 0.2 & 7-Jun-90 & 1.66 & 0.056 & 0.094 & 0.051 & 61 & 12 & 0.007 & 1.3 \\
\hline 9 & -0.5 & 0.4 & 11-Jun-90 & 1.67 & 0.077 & 0.129 & 0.072 & 61 & 19 & 0.004 & 1.3 \\
\hline 9 & -0.5 & 0.6 & 11-Jun-90 & 1.68 & 0.087 & 0.146 & 0.080 & 163 & 54 & 0.008 & 2.5 \\
\hline 9 & -0.5 & 0.8 & 12-Jun-90 & 1.68 & 0.071 & 0.119 & 0.171 & 49 & 13 & 0.010 & 2.6 \\
\hline 9 & -0.5 & 1.0 & 13-Jun-90 & 1.69 & 0.057 & 0.095 & 0.233 & 91 & 20 & 0.032 & 6.5 \\
\hline 9 & -0.5 & 1.2 & 12-Jun-90 & 1.69 & 0.081 & 0.137 & 0.236 & 77 & 27 & 0.094 & 30.6 \\
\hline 9 & -0.5 & 1.4 & 12-Jun-90 & 1.70 & 0.058 & 0.099 & 0.259 & 146 & 42 & 0.234 & 63.4 \\
\hline 9 & -0.5 & 1.6 & 5-Jun-90 & 1.70 & 0.054 & 0.092 & 0.142 & 182 & 42 & 0.312 & 67.7 \\
\hline 9 & -0.5 & 1.8 & 5-Jun-90 & 1.71 & 0.075 & 0.128 & 0.094 & 234 & 54 & 0.318 & 69.1 \\
\hline 9 & -0.5 & 2.0 & 11-Jul-90 & 1.71 & 0.056 & 0.095 & 0.044 & 235 & 58 & 0.207 & 48.2 \\
\hline 9 & -0.5 & 2.2 & 11-Jul-90 & 1.72 & 0.049 & 0.084 & 0.020 & 289 & 67 & 0.298 & 64.6 \\
\hline 9 & -0.5 & 2.4 & 11-Jul-90 & 1.72 & 0.050 & 0.086 & 0,008 & 465 & 96 & 0.456 & 88.0 \\
\hline 9 & -0.5 & 2.6 & 11-Jul-90 & 1.73 & 0.041 & 0.071 & 0.005 & 785 & 155 & 0.590 & 109.7 \\
\hline 9 & -0.5 & 2.8 & $11-J u l-90$ & 1.74 & $0: 046$ & 0.081 & 0.004 & 972 & 198 & 0.609 & 116.3 \\
\hline 9 & 0.0 & 0.0 & 7-Jun-90 & 1.66 & 0.036 & 0.061 & 0.012 & 205 & 30 & 0.019 & 2.6 \\
\hline 9 & 0.0 & 0.2 & 7-Jun-90 & 1.66 & 0.063 & 0.105 & 0.024 & 114 & 23 & 0.007 & 1.4 \\
\hline 9 & 0.0 & 0.4 & 7-Jun-90 & 1.67 & 0.118 & 0.197 & 0.031 & 27 & 11 & 0.003 & 1.3 \\
\hline 9 & 0.0 & 0.6 & 13-Jun-90 & 1.68 & 0.081 & 0.135 & 0.101 & 37 & 11 & 0.005 & 1.3 \\
\hline 9 & 0.0 & 0.8 & 11-Jun-90 & 1.68 & 0.075 & 0.126 & 0.126 & 49 & 14 & 0.010 & 2.6 \\
\hline 9 & 0.0 & 1.0 & 12-Jun-90 & 1.69 & 0.108 & 0.183 & 0.172 & 72 & 28 & 0.064 & 23.1 \\
\hline 9 & 0.0 & 1.2 & 13-Jun-90 & 1.69 & 0.068 & 0.116 & 0.194 & 135 & 32 & 0.154 & 34.7 \\
\hline 9 & 0.0 & 1.4 & 31575 & 1.70 & 0.070 & 0.119 & 0.185 & 185 & 49 & 0.195 & 49.0 \\
\hline 9 & 0.0 & 1.6 & 31567 & 1.70 & 0.074 & 0.126 & 0.143 & 204 & 52 & 0.227 & 53.9 \\
\hline
\end{tabular}




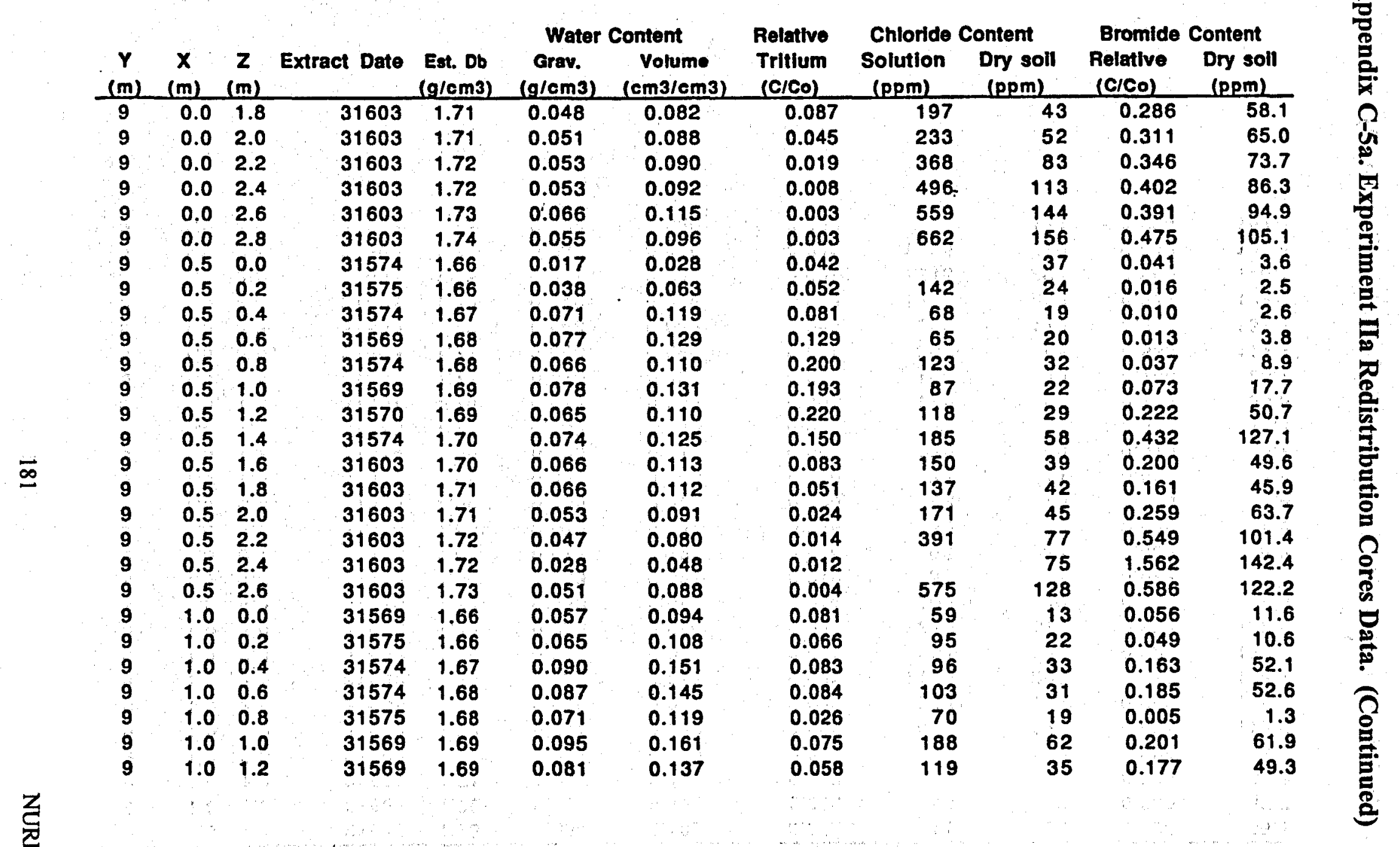




\begin{tabular}{|c|c|c|c|c|c|c|c|c|c|c|c|}
\hline $\begin{array}{c}Y \\
(\mathrm{~m})\end{array}$ & $\begin{array}{c}x \\
\text { (m) }\end{array}$ & $\begin{array}{c}z \\
(m)\end{array}$ & Extract Date & $\begin{array}{c}\text { Est. Db } \\
\text { (g/cm3) }\end{array}$ & $\begin{array}{l}\text { Water } \\
\text { Grav. } \\
\text { (g/cm3) }\end{array}$ & $\begin{array}{l}\text { Contemt } \\
\text { Volume } \\
\text { (cm3/cm3) }\end{array}$ & $\begin{array}{l}\text { Relative } \\
\text { Tritlum } \\
\text { (C/Co) }\end{array}$ & $\begin{array}{l}\text { Chloride } \\
\text { Solution } \\
\text { (ppm) }\end{array}$ & $\begin{array}{l}\text { Oontent } \\
\text { Dry soll } \\
\text { (ppm) }\end{array}$ & $\begin{array}{l}\text { Bromide } \\
\text { Relative } \\
\text { (C/Co) }\end{array}$ & $\begin{array}{l}\text { Content } \\
\text { Dry soll } \\
\text { (ppm) }\end{array}$ \\
\hline 9 & 1.0 & 1.4 & 31570 & 1.70 & 0.064 & 0.108 & 0.052 & 411 & 85 & 0.334 & 65.1 \\
\hline 9 & 1.0 & 1.6 & 31567 & 1.70 & 0.104 & 0.176 & 0.023 & 140 & 42 & 0.240 & 67.7 \\
\hline 9 & 1.0 & 1.8 & 31567 & 1.71 & 0.085 & 0.144 & 0.017 & & & 0.246 & 53.8 \\
\hline 9 & 1.0 & 2.0 & 31603 & 1.71 & 0.017 & 0.029 & 0.026 & & 113 & 1.234 & 85.7 \\
\hline 9 & 1.0 & 2.2 & 31603 & 1.72 & 0.044 & 0.076 & 0.002 & 633 & 125 & 0.625 & 115.9 \\
\hline 9 & 1.0 & 2.4 & 31603 & 1.72 & 0.040 & 0.069 & 0.001 & 967. & 189 & 0.645 & 118.2 \\
\hline 9 & 1.0 & 2.6 & 31603 & 1.73 & 0.039 & 0.068 & 0.001 & 1106 & 203 & 0.746 & 128.4 \\
\hline 9 & 1.0 & 2.8 & 31603 & 1.74 & 0.035 & 0.062 & 0.001 & 1131 & 189 & 0.825 & 129.6 \\
\hline 9 & 1.0 & 3.0 & 31603 & 1.75 & 0.028 & 0.050 & 0.001 & 1218 & 174 & 0.988 & 132.9 \\
\hline 9 & 1.5 & 0.0 & 31569 & 1.66 & 0.050 & 0.083 & 0.014 & 202 & 42 & 0.046 & 8.9 \\
\hline 9 & 1.5 & 0.2 & 31573 & 1.66 & 0.053 & 0.088 & 0.019 & 57 & 13 & 0.022 & 4.9 \\
\hline 9 & 1.5 & 0.4 & 31573 & 1.67 & 0.072 & 0.121 & 0.024 & 93 & 28 & 0.022 & 6.2 \\
\hline 9 & 1.5 & 0.6 & 31574 & 1.68 & 0.079 & 0.132 & 0.018 & 66 & 22 & 0.016 & 4.9 \\
\hline 9 & 1.5 & 0.8 & 31575 & 1.68 & 0.155 & 0.260 & 0.008 & 22 & 10 & 0.003 & 1.4 \\
\hline 9 & 1.5 & 1.0 & 31573 & 1.69 & 0.085 & 0.143 & 0.010 & 31 & 11 & 0.007 & 2.4 \\
\hline 9 & 1.5 & 1.2 & 31574 & 1.69 & 0.068 & 0.115 & 0.006 & 136 & 40 & 0.013 & 3.7 \\
\hline 9 & 1.5 & 1.4 & 31573 & 1.70 & 0.082 & 0.140 & 0.004 & & & 0.042 & 17.2 \\
\hline 9 & 1.5 & 1.6 & 31567 & 1.70 & 0.078 & 0.133 & 0.004 & 176 & 44 & 0.130 & 30.5 \\
\hline 9 & 1.5 & 1.8 & 31567 & 1.71 & 0.073 & 0.125 & 0.003 & 153 & 36 & 0.108 & 23.7 \\
\hline 9 & 1.5 & 2.0 & 31603 & 1.71 & 0.058 & 0.099 & 0.001 & 390 & 84 & 0.565 & 114.1 \\
\hline 9 & 1.5 & 2.2 & 31603 & 1.72 & 0.048 & 0.082 & 0.000 & 853 & 179 & 0.664 & 131.1 \\
\hline 9 & 1.5 & 2.4 & 31603 & 1.72 & 0.040 & 0.068 & 0.000 & 1229 & 253 & 0.631 & 122.2 \\
\hline 9 & 1.5 & 2.6 & 31603 & 1.73 & 0.043 & 0.074 & 0.001 & 1215 & 207 & 0.906 & 145.1 \\
\hline 9 & 1.5 & 2.8 & 31603 & 1.74 & 0.037 & 0.065 & -0.001 & 1355 & 229 & 0.842 & 133.4 \\
\hline 9 & 1.5 & 3.0 & 31603 & 1.75 & 0.022 & 0.039 & 0.002 & & 131 & 1.834 & 157.1 \\
\hline & & & & & & & & & & & \\
\hline : & & $\because \cdots$ & & & & & $\cdots$ & & & & \\
\hline & & $\because$ & & & & & & & & $\because$ & \\
\hline
\end{tabular}


Appendix C-5b. Experiment IIa Neutron Probe Water and Tritium Contour Data.

Neutron Probe Water at $Y=6 \mathrm{~m} \&=10 \mathrm{~m}$, Days $78 \& 655$

\begin{tabular}{rrrrrrrrrr} 
Day & $Y(m)$ & $X(m)$ & $Z(m)$ & $T h e t a$ & $Y(m)$ & X $(m)$ & $Z$ & $(m)$ & Theta \\
\hline 78 & 6 & -4 & 0.25 & 0.099 & 10 & -4 & 0.25 & 0.11 \\
78 & 6 & -4 & 0.5 & 0.094 & 10 & -4 & 0.5 & 0.097 \\
78 & 6 & -4 & 0.75 & 0.077 & 10 & -4 & 0.75 & 0.077 \\
78 & 6 & -4 & 1 & 0.072 & 10 & -4 & 1 & 0.066 \\
78 & 6 & -4 & 1.25 & 0.074 & 10 & -4 & 1.25 & 0.043 \\
78 & 6 & -4 & 1.5 & 0.076 & 10 & -4 & 1.5 & 0.052 \\
78 & 6 & -4 & 1.75 & 0.069 & 10 & -4 & 1.75 & 0.053 \\
78 & 6 & -4 & 2 & 0.063 & 10 & -4 & 2 & 0.053 \\
78 & 6 & -4 & 2.25 & 0.041 & 10 & -4 & 2.25 & 0.051 \\
78 & 6 & -4 & 2.5 & 0.041 & 10 & -4 & 2.5 & 0.057 \\
78 & 6 & -4 & 2.75 & 0.053 & 10 & -4 & 2.75 & 0.055 \\
78 & 6 & -4 & 3 & 0.071 & 10 & -4 & 3 & 0.044 \\
78 & 6 & -4 & 3.25 & 0.119 & 10 & -4 & 3.25 & 0.07 \\
78 & 6 & -4 & 3.5 & 0.084 & 10 & -4 & 3.5 & 0.078 \\
78 & 6 & -4 & 3.75 & 0.081 & 10 & -4 & 3.75 & 0.052 \\
78 & 6 & -4 & 4 & 0.086 & 10 & -4 & 4 & 0.055 \\
78 & 6 & -4 & 4.25 & 0.094 & 10 & -4 & 4.25 & 0.1 \\
78 & 6 & -4 & 4.5 & 0.115 & 10 & -4 & 4.5 & 0.128 \\
78 & 6 & -4 & 4.75 & 0.079 & 10 & -4 & 4.75 & 0.094 \\
78 & 6 & -4 & 5 & 0.064 & 10 & -4 & 5 & 0.066 \\
78 & 6 & -4 & 5.25 & 0.069 & 10 & -4 & 5.25 & 0.055 \\
78 & 6 & -4 & 5.5 & 0.079 & 10 & -4 & 5.5 & 0.06 \\
78 & 6 & -4 & 5.75 & 0.065 & 10 & -4 & 5.75 & 0.072 \\
78 & 6 & -4 & 6 & 0.063 & 10 & -4 & 6 & 0.074 \\
78 & 6 & -3 & 0.25 & 0.113 & 10 & -3 & 0.25 & 0.11 \\
78 & 6 & -3 & 0.5 & 0.12 & 10 & -3 & 0.5 & 0.113 \\
78 & 6 & -3 & 0.75 & 0.099 & 10 & -3 & 0.75 & 0.101 \\
78 & 6 & -3 & 1 & 0.088 & 10 & -3 & 1 & 0.075 \\
78 & 6 & -3 & 1.25 & 0.064 & 10 & -3 & 1.25 & 0.056 \\
78 & 6 & -3 & 1.5 & 0.055 & 10 & -3 & 1.5 & 0.063 \\
78 & 6 & -3 & 1.75 & 0.06 & 10 & -3 & 1.75 & 0.066 \\
78 & 6 & -3 & 2 & 0.043 & 10 & -3 & 2 & 0.058 \\
78 & 6 & -3 & 2.25 & 0.035 & 10 & -3 & 2.25 & 0.053 \\
78 & 6 & -3 & 2.5 & 0.044 & 10 & -3 & 2.5 & 0.054 \\
78 & 6 & -3 & 2.75 & 0.05 & 10 & -3 & 2.75 & 0.056 \\
78 & 6 & -3 & 3 & 0.078 & 10 & -3 & 3 & 0.045 \\
78 & 6 & -3 & 3.25 & 0.074 & 10 & -3 & 3.25 & 0.094 \\
78 & 6 & -3 & 3.5 & 0.074 & 10 & -3 & 3.5 & 0.085 \\
78 & 6 & -3 & 3.75 & 0.086 & 10 & -3 & 3.75 & 0.088 \\
78 & 6 & -3 & 4 & 0.088 & 10 & -3 & 4 & 0.109 \\
78 & 6 & -3 & 4.25 & 0.094 & 10 & -3 & 4.25 & 0.099
\end{tabular}


Appendix C-5b. Experiment IIa Neutron Probe Water and Tritium Contour Data. (Continued)

Observed Tritium, Solution Samplers $(Y=0.5 \mathrm{~m})$, days 78 \& 429

\begin{tabular}{|c|c|c|c|c|c|c|c|}
\hline Day & $X(m)$ & $Z(m)$ & $\mathrm{C} / \mathrm{Co}$ & Day & $X(m)$ & $z(m)$. & $\mathrm{C} / \mathrm{Co}$ \\
\hline 78 & -1.25 & 0.5 & 0 & 429 & 0.75 & 0.5 & 0.105 \\
\hline 78 & -0.75 & 0.5 & 0.054 & 429 & 1.25 & 0.5 & 0.057 \\
\hline 78 & 0.25 & 0.5 & 0.011 & 429 & 0.25 & 1 & 0.198 \\
\hline 78 & 0.75 & 0.5 & 0.391 & 429 & 0.75 & 1 & 0.268 \\
\hline 78 & -0.75 & 1 & 0.07 & 429 & 1.25 & 1 & 0.084 \\
\hline 78 & -0.25 & 1 & 0.192 & 429 & -1.25 & 1.5 & 0.007 \\
\hline 78 & 0.25 & 1 & 0.627 & 429 & -0.75 & 1.5 & 0.093 \\
\hline 78 & 0.75 & 1 & 0.689 & 429 & -0.25 & 1.5 & 0.11 \\
\hline 78 & 1.25 & 1 & 0 & 429 & 0.25 & 1.5 & 0.115 \\
\hline 78 & -1.25 & 1.5 & 0 & 429 & 0.75 & 1.5 & 0.194 \\
\hline 78 & -0.75 & 1.5 & 0.001 & 429 & 1.25 & 1.5 & 0.038 \\
\hline 78 & -0.25 & 1.5 & 0 & 429 & -1.25 & 2 & 0.001 \\
\hline 78 & 0.25 & 1.5 & $\mathbf{0}$ & 429 & -0.75 & 2 & 0.01 \\
\hline 78 & 0.75 & 1.5 & 0 & 429 & -0.25 & 2 & 0.021 \\
\hline 78 & 1.25 & 1.5 & 0 & 429 & 0.25 & 2 & 0.014 \\
\hline 78 & -1.25 & 2 & 0 & 429 & 0.75 & 2 & 0.018 \\
\hline 78 & -0.75 & 2 & 0 & 429 & 1.25 & 2 & 0.003 \\
\hline 78 & -0.25 & 2 & 0 & 429 & -1.25 & 2.5 & 0 \\
\hline 78 & 0.25 & 2 & 0 & 429 & -0.25 & 2.5 & 0.003 \\
\hline 78 & 0.75 & 2 & 0.001 & 429 & 0.25 & 2.5 & 0.002 \\
\hline 78 & 0.25 & 2.5 & 0 & 429 & 0.75 & 2.5 & 0.001 \\
\hline 78 & -0.5 & 0.25 & 0.005 & 429 & 1.25 & 2.5 & 0 \\
\hline 78 & 0 & 0.25 & 0.003 & & & & \\
\hline 78 & 0.5 & 0.25 & 0.006 & & & & \\
\hline 78 & -0.5 & 0.75 & 0.506 & & & & \\
\hline 78 & 0 & 0.75 & 0.115 & & & & \\
\hline 78 & 0.5 & 0.75 & 0.205 & & & & \\
\hline
\end{tabular}




\section{Appendix C-6. Construction of Narrow-Profile Ceramic Devices.}

\section{Introduction}

This document describes the procedures used to construct small-diameter (1/4" nominal ID) ceramic devices for use as tensiometers or solution samplers. These have served as the primary ceramic devices in a variety of lysimeter experiments at the Jornada trench site of the NMSU college ranch. The small-diameter device differs from the more familiar $1 / 2$ " ID PVC device in that these are designed for installation into a vertical soil wall.

The small diameter device has a $90^{\circ}$ elbow at one end which forms a sealed chamber continuous with the straight PVC and ceramic cup. When installed elbow-up into a vertical soil face and filled with water the air bubble in the elbow is more easily accessed with the tensimeter needle.

Since first conceived several design changes have been made including changes in the cements used, the ceramic and plastic raw materials used, and the methods for fabricating and fitting parts. The current product is more durable and more dependable.

The following procedure is used for making a standard device, approximately $1.4 \mathrm{~cm}$ outside diameter and $90 \mathrm{~cm}$ long. In use, this device is inserted about $82 \mathrm{~cm}$ into soil material at a $10^{\circ}$ angle from horizontal into a pre-drilled hole.

\section{Materials}

PVC pipe: Schedule 40 clear, 1/4" nominal (OD Excelon, US Plastics Corp. (Lima, $\mathrm{OH}$ ), $\mathrm{p} / \mathrm{n} 34100$.

Ceramic cups: 1 bar High-Flow, $1.0 \mathrm{~cm}$ OD* $10 \mathrm{~cm}$ long, $0.16 \mathrm{~cm}$ wall, Soil Moisture Equip. Corp. (Santa Barbara, CA), p/n 652X04-B1M3.

Acrylic tubing: 5/8" OD, 1/2." ID, local purchase.

Copper elbows: $90^{\circ}$ plumbing, 5/8" X 1 /2", local hardware purchase.

Normal cements:

1) JB Weld 2-part epoxy aB Weld Co., Sulphur Springs, TX); local hardware purchase.

2) Clear PVC pipe cleaner and cement, local hardware purchase. Note: Not all cements are compatible with all PVC plastics: May substitute methyl-ethyl ketone (MEK) for pipe cleaner.

3) Clear 2-part ceramic epoxy, Soil Moisture Equipment Corp. (Santa Barbara, $\mathrm{CA}$ ), available in $8 \mathrm{oz}$. through 1 gallon sizes. 


\section{Appendix C-6. Construction of Narrow-Profile Ceramic Devices. (Continued)}

\section{Methods}

1) Cut the PVC pipe to length. Cut with a PVC pipe cutting tool or with a hacksaw while the pipe is clamped securely in a vice. Eighty-three $\mathrm{cm}$ is the standard length. For installation directly below the walkways in the trench add an additional $5 \mathrm{~cm}$ to length, to 88 $\mathrm{cm}$.

2) Ream about $1.5 \mathrm{~cm}$ into one end of the PVC to accept a ceramic cup. The PVC pipe and the $1 / 2$ " variable speed drill used to make the hole are mounted securely in a special jig in the laboratory to properly align the drill bit relative to the PVC pipe. It is extremely important that the hole is drilled centered and straight into the PVC. If not, the finished device is considerably weakened and the ceramic device becomes more likely to break during installation.

Use a sharp 27/64" cobalt-alloy drill bit (1.072 $\mathrm{cm}$ ) available locally from a hardware store. Special drill bits for acrylic are less effective and difficult to find in the correct diameter. Expect the drill bit to dull quickly.

Because of the heat produced during drilling, the bit will wallow a larger hole than expected. To control warp and glazing in the plastic it is important to cool the PVC and remove cuttings during the drilling. This can be accomplished by directing a stream of water from a wash bottle at the area being drilled while frequently removing the cuttings from the hole. If pressurized air is available a nozzle can be fashioned to force air through the PVC from the end opposite that being drilled. This will cool the plastic and help force cuttings out of the hole.

Proceed slowly. The drill motor should be set to about $500 \mathrm{rpm}$ (fast speed). Drilling should take about 30 seconds for the $1.5 \mathrm{~cm}$-deep hole.
3) Lathe the other end of the PVC to accept the $1 / 2$ " end of the copper elbow. Use a sharp bit to remove about $0.010^{\prime \prime}$ to $0.020^{\prime \prime}$ from the outside diameter of the pipe, about $2 \mathrm{~cm}$ into the length of the PVC. The amount of material removed will vary since the ID of copper elbows varies from lot to lot. Start by taking a small amount of material first. Keep an elbow handy to check the fit on the PVC. It should fit snugly.

4) Cut acrylic tubing to $6 \mathrm{~cm}$ lengths. This can be done with an acrylic cutting tool or, carefully, with a hacksaw (and a sharp blade). Debur the cut surfaces with a de-burring tool or knife. Bevel the inside edge of one end to about $30^{\circ}$ with a routing bit. A beveled surface will accept a rubber septum stopper more easily. Chuck the acrylic carefully since this material is easily fractured.

5) Cement the un-beveled end of the acrylic tubing into the copper elbow. Mix the JB Weld epoxy components with equal parts by weight. Apply a thin layer of epoxy to both surfaces to be bonded and insert the acrylic into the elbow. A small bead of cement should form around the outside rim of the joint. Too little cement will not seal the joint. Note: Other epoxy cements, e.g. that sold by Soil Moisture Equipment Corp., have an unfavorable oxidation reaction with copper over time which renders the bond useless. Most other epoxies are somewhat water soluble and will soften with time.

6) Seal the pores of the open end of the ceramic cup with epoxy and heat treat. By pre-sealing the pores of the cup it can be cemented to the PVC pipe without forming seal-breaking air pockets. Note: Before handling cups, wrap all but the last $3 \mathrm{~cm}$ of surface in tissue and tape in place. This will protect the surface of the cup from absorbing oils from the skin. Use Soil Moisture Equipment Corp. 2-part 


\section{Appendix C-6. Construction of Narrow-Profile Ceramic Devices. (Continued)}

epoxy cement mixed as equal parts by volume. With a foam brush apply an even thin coat of cement to the last $1 \mathrm{~cm}$ of outside cup surface. Allow the cement to be absorbed by the pores of the cup for about 15 minutes and reapply if necessary. Allow to dry for 24 hours. Heat the cup at $110 \mathrm{~F}$ to $150 \mathrm{~F}$ for about 4 hours.

7) Cement the copper elbow to the PVC. Use equal parts by weight of the two JB Weld components. Apply a uniform coat of cement to the inside surface of the $5 / 8$ " end of the elbow and to the outside lathed surface of the PVC pipe. A good joint will form a bead of cement about the rim of the elbow.

8) Cement the ceramic cup into the PVC. Quickly dip the open end of the cup and the reamed end of the PVC pipe into the PVC pipe cleaner (or MEK). Apply a layer of PVC cement to the outside surface of the end of the cup and to the inside surface of the PVC. Insert the cup into the PVC with a twisting motion. Ensure that the cup is firmly seated straight into the pipe and that no air bubble are visible through the PVC. If any bubbles can be seen it will be necessary to re-seat the cup with more cement. Care must be taken not to use too much cement.
Excess cement and a sloughed layer of PVC may block the opening of the cup when the cup is pushed into the pipe. Ensure that the cup extends straight out from the PVC. Allow the cup to dry for at least 48 hours.

9) Test the ceramic device for leaks to about $450 \mathrm{~cm}$ water head. Though the high-flow cups are rated for a bubble pressure of 1 bar, regions within individual cups may be less restrictive. Most cups will hold at least $450 \mathrm{~cm}$ pressure. Remove the protective tissue from the cup and immerse the entire cup-end of the device into water. Allow the pores to fill with water for about $1 / 2$ to 1 minute. Seal the acrylic end of the device with an appropriate $(13 \times 20)$ rubber septum stopper. With a regulated supply of pressurized air apply $32-36 \mathrm{~cm} \mathrm{Hg}$ pressure into the sealed system. This can be accomplished with a 26 gauge needle, connected to the pressure source and pushed through the septum. Hold the cup end of the device under water and carefully watch for the formation of air bubbles about the cemented joint. Slowly rotate the cup to observe all cemented surfaces. It may take $\mathbf{3 0}$ seconds or more for slow leaks to appear. In like fashion, place the copper elbow end of the device under water and observe for bubbles. 


\section{Appendix C-7. NRC Trench Wall Ceramic Device Installation.}

\section{Introduction}

Installation methods for trench wall devices have evolved through several improvements. A $1 / 2$ " standard drill and drill bit was used to complete the hole drilling. Since the OD of the PVC tubing is $0.540^{\prime \prime}$ it was necessary to use a great deal of force to push the ceramic device to its' final position. It was often necessary to jet some water into the drilled hole and ream this "mudhole" many times with a masonry bit to soften the trench wall soil for insertion of the device. In hard soil 2 persons working all day could expect to complete the installation of 5 to 7 devices in a day.

The present method of installation is a great improvement. A hammer drill facilitates much faster and easier drilling. A $1 / 2$ " bit is used to drill a pilot hole in the soil. This hole is finished with a $9116 "$ bit to enlarge all but the final $8 \mathrm{~cm}$ of hole length for easier insertion of the device. A mud slurry using finely sieved native materials is used to create a more reliable soil/ceramic contact. With these improvements two persons can now usually install 15 to 20 devices in a day, regardless of soil hardness.

\section{Materials}

Makita 3/4", 6A hammer drill, 2 speed, reversible, local purchase

Black and Decker $1 / 2$ " variable speed drill, reversible, heavy duty

Bosch $1 / 2$ " carbide masonry bit, 18" medium speed parabolic flutes, machined to chuck into $1 / 2$ " straight shank drill, silver soldered to $1 / 2$ " steel bar to a total length of $105 \mathrm{~cm}$ (campus PSL machine shop for adding extension to bit), local purchase
9/16" carbide masonry bit, 16" medium speed standard flutes, 18 " length overall, silver soldered to $1 / 2$ " steel bar to a total length of $105 \mathrm{~cm}$ (campus PSL), Hayden Twist Drill \& Tool, Warren, MI, p/n 161-18

9/16" carbide masonry bit, 20" high speed shallow flutes, extended to $105 \mathrm{~cm}$ by campus PSL, local purchase

9/16" wood bit, 8" overall length, medium speed aggressive flutes, local purchase

Protractor for setting and maintaining $10^{\circ}$ hole angle during drilling, local purchase

Shop-Vac with $1 / 2$ " OD and 3/8" OD acrylic tubing "straws" for vacuuming cuttings from drilled holes, local purchase

Native soil materials, air dried, chipped (NMSU Soil and Water Testing Lab) and sieved through 80 mesh screen. Note: For soils at the trench an 80 mesh sieve is the maximum, any larger opening size will make mud slurry difficult to push through a $3 / 8$ " slurry "straw"

Distilled-deionized water for making mud slurry

3/8" OD acrylic tubing $x 1 \mathrm{~m}$ long, connected to approx. $20 \mathrm{~cm}$ of Tygon tubing for making a mud slurry "straw", local purchase

$3 / 8^{\prime \prime} \times 1 / 2$ " ax-handle wedges, for securing devices to wooden wall of trench, local purchase 


\section{Appendix C-7. NRC Trench Wall Ceramic Device Installation. (Continued)}

\section{Procedure}

Drilling a hole through the wood and the pilot hole through soil requires two persons. One person operates the drill while the second measures the drill bit angle and monitors that the bit is maintained at that angle throughout the drilling process. When the pilot hole is completed $(1 / 2$ " bit to the bottom of the hole one person can finish the drilling and installation of ceramic devices.

First drill through the wood structure with a $9 / 16$ " wood drill. The hammer drill is not required for this. Use a protractor to set and monitor a $10^{\circ}$ angle several times during the drilling. Place the protractor directly on the flutes of the drill and take measurements while the drill is stopped.

The pilot hole is drilled with a $1 / 2$ " masonry bit to the bottom of the hole. It is very important that the bit angle is monitored closely, possibly 5 or 6 pauses to measure with the protractor, for at least the first one-half of the length of this hole. As the drilled hole becomes deeper corrections of the drilling angle become more difficult. Use the hammer drill set to high speed with hammering action turned on. It is common for the drill bit to become lodged in the hole as cuttings collect behind the flutes of the bit. At these times it is important not to load the drill motor when the bit will not turn. Reverse the direction of the bit to dislodge it and begin again. When the bit begins to labor it is best to ream the hole to carry cuttings out. Sometimes it is necessary to switch drill speed to "low" to increase torque. When the pilot hole is drilled to length use the 3/8" vacuum straw and Shop-Vac to remove cuttings from the hole. For an $86 \mathrm{~cm}$ length hole the straw should be inserted no further than $78 \mathrm{~cm}$ to leave native cuttings in the portion of the hole where the ceramic cup will seat.

Moisture Equipment Corp. 2-part epoxy cement mixed as equal parts by volume. With a foam brush apply an even thin coat of cement to the last $1 \mathrm{~cm}$ of outside cup surface. Allow the cement to be absorbed by the pores of the cup for about 15 minutes and reapply if necessary. Allow to dry for 24 hours. Heat the cup at $110 \mathrm{~F}$ to $150 \mathrm{~F}$ for about 4 hours.

The pilot hole is finished with the deep/medium speed fluted 9/16" masonry bit. For an $86 \mathrm{~cm}$ pilot hole this bit is not used any deeper than $\mathbf{7 8}$ $\mathrm{cm}$ to avoid over-reaming the hole for the diameter of the ceramic. The larger diameter hole which is made with this bit allows easier installation of ceramic devices. The hammer drill is used in the same manner as with the $1 / 2$ " bit. It is not necessary to use the protractor. When the $9 / 16^{\prime \prime}$ hole is completed the $1 / 2$ " acrylic straw and Shop-Vac are used to remove cuttings from the hole. Again, do not vacuum to the end of the hole.

Usually it is necessary to alternate the $1 / 2$ " bit (to the end of the hole, $86 \mathrm{~cm}$ ) with the 9/16" bit (to $78 \mathrm{~cm}$ deep) several times before the hole is finished. The standard drill is usually powerful enough to use at this point. Between each bit change it is helpful to vacuum the cuttings. The hole is finished when the ceramic device can easily be inserted into position without undue torque (< approx. $10 \mathrm{ft}$. lbs.).

A mud slurry is made from 80 mesh-sieved native soil materials taken from about the same depth in the trench at which the ceramic cup is to be placed. In a wide mouth plastic bottle mix approx. 3 parts water with 5 parts dry sieved soil (by weight), cap and shake the bottle vigorously. The slurry should have viscosity 


\section{Appendix C-7. NRC Trench Wall Ceramic Device Installation. (Continued)}

approximating honey. Use the acrylic slurry straw to draw $15 \mathrm{ml}$ of slurry (mark position on tubing for $15 \mathrm{ml}$ ) into the straw. Crimp the Tygon tubing and push the straw to the end of the hole. Blow through the Tygon and slowly withdraw the straw approx. $5 \mathrm{~cm}$ to place the mud in the last $5 \mathrm{~cm}$ of the hole. Promptly insert the ceramic device into the hole and seat the cup in the mud.

When the cup is seated in the mud is important that the mud/cup interface is not disturbed.
Since the PVC tubing is about $1 / 2$ " diameter and is placed within a $9 / 16$ " diameter hole the device should be protected from accidental disturbance. Use an ax handle wedge to shim the PVC tubing against the wood. Insert the wedge between the PVC and wood under the tubing and gently hammer the wedge into place. When finished it should not be possible to twist the device by hand. The wedge should not be hammered flush with the wood but instead protrude from the wood so that it may be easily pulled with pliers in case the device needs to be removed. 
Appendix C-7. NRC Trench Wall Ceramic Device Installation. (Continued)

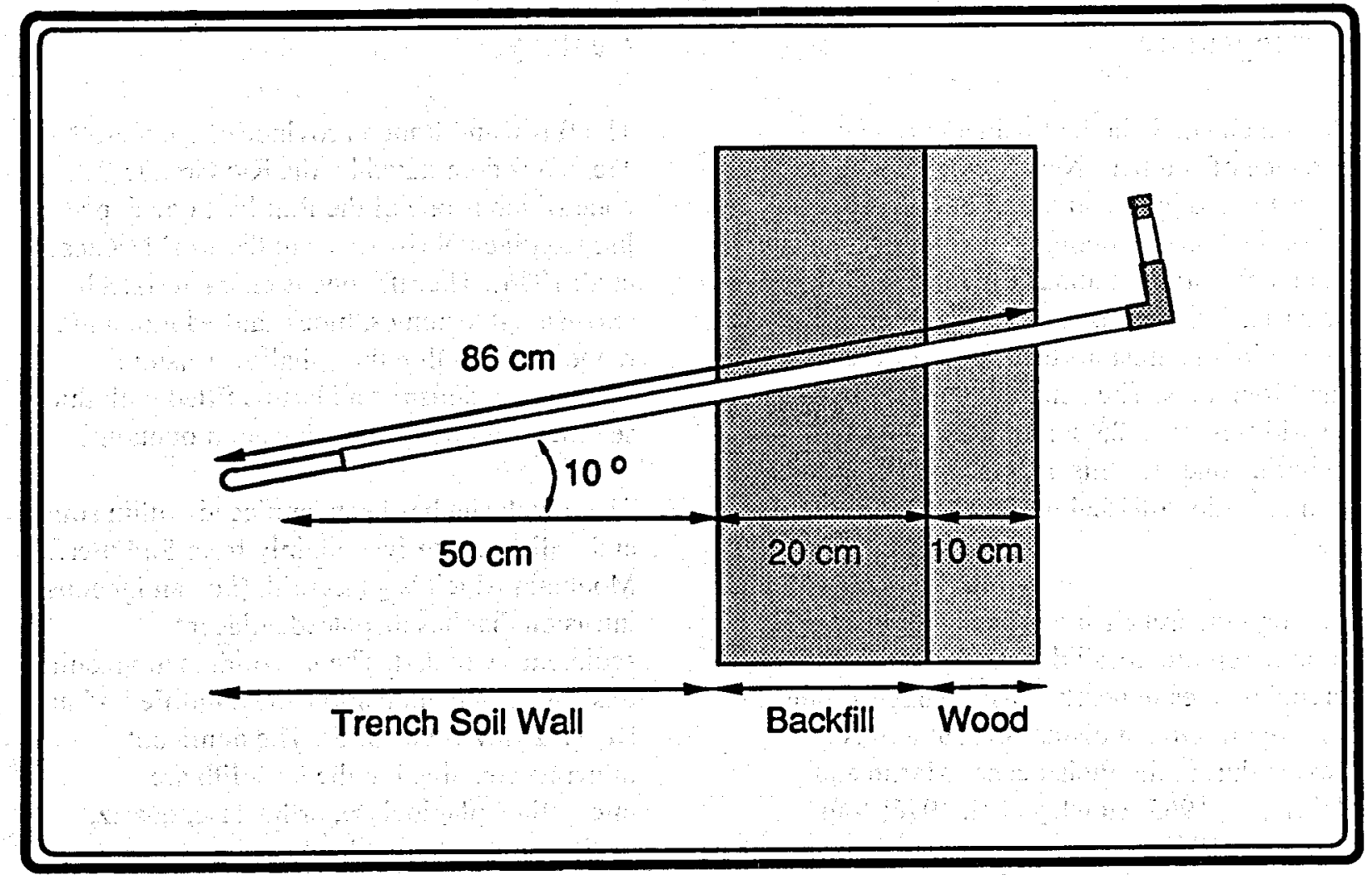




\section{Appendix C-8. Soils and Geomorphology at the Las Cruces Trench Site.}

\section{Physiography}

The trench site is in the Basin and Range Province of southern New Mexico which is characterized by broad desert basins between discontinuous mountain ranges. The trench site is at an elevation of about $1357 \mathrm{~m}$ ( $4450 \mathrm{ft}$.) on the eastern Piedmont slope of Summerford Mountain, the most northern peak of the Dona Ana Mountains. The general boundary between the arid (less than $25 \mathrm{~cm}$ rain per year) and semiarid zones for this area is considered to be at an elevation of $1524 \mathrm{~m}(5000 \mathrm{ft}$.) (Gile et al, 1981).

Although the trench site is presently in an arid climatic regime, fossil data and evidence of perennial lakes in nearby basins suggest times of more effective moisture in southern New Mexico during the Pleistocene (Martin and Mehringer, 1965; Hawley et al, 1976; Van Devender, 1977). Times of more effective moisture (i.e., more precipitation and lower evaporation) probably correspond to glacial advances in the Northern Hemisphere. For example, Reeves (1973) described a cool-moist interval (the Tahoka pluvial) on the southern High Plains that dates from 15,000 to 22,500 years B.P., which is roughly the time of the last full glacial advance, the Wisconsin. Climatic conditions in this region prior to the last full glacial are poorly documented (Gile et al, 1981).

\section{Geology}

The Basin and Range Province of southern New Mexico is dominated by the Rio Grande Rift zone which is one of the four best developed intracontinental rift zones in the world (Kues et al, 1986). The rift zone is characterized by extensional tectonics, block faulted mountain ranges, crustal thinning, shallow seismic activity, volcanism, and basins filled with thick sediments eroded from adjacent mountains.

The trench site has been emplaced within soils and sediments derived mainly from Summerford Mountain which is a laccolith (i.e., an igneous intrusion that has displaced adjacent sedimentary rocks). The laccolith is monzonitic to syenitic in composition and solidified $35 \mathrm{~m} . \mathrm{y}$. BP. (Hawley et al, 1975). The dominant minerals contained in the laccolith are microcline, plagioclase, orthoclase, quartz, augite, biotite, horneblende, sphene, apatite, magnetite, and zircon (Hurlbut and Klein, 1977).

\section{Soils and Geomorphology}

The trench site extends downward through the Isaacks' Ranch and Jornada II deposits into what can probably be classified as the Camp Rice Formation, Jornada I surface (L.H. Gile, personal communication). Their approximate ages are 8,000-15,000 years, 25,000-150,000 years, and $250,000-400,000$ years, respectively.

Prepared by H. Curtis Monger, Department of Agronomy and Horticulture, New Mexico State University, Las Cruces, New Mexico 


\section{Appendix C-8. Soils and Geomorphology at the Las Cruces Trench Site. (Continued)}

The Jornada I surface is exposed approximately $3.1 \mathrm{~m}$ below the top of the trench. Soils of the Jornada I surface reflect a very long time of landscape stability (Gile et al, 1981). These soils are generally underlain by a sequence of buried soils within the Camp Rice Formation. The trench site contains at least two buried soils beneath the Jornada I surface which can be identified by separate calcic horizons. A well. defined argillic horizon marks the top of the Jornada I surface; the A horizon appears to have been truncated when overlying material was deposited.

The Jornada II surface is exposed approximately $1.4 \mathrm{~m}$ below the top of the trench. The Jornada II deposit is nearly continuous within the trench except where it has been truncated on the north and south walls by an arroyo associated with the Isaacks' Ranch deposit. The Jornada II surface can be identified by its stage III (Gile et al, 1966) calcic horizon. Apparently, the A and B horizons associated with the Jornada II soil were truncated by the deposition of Isaacks' Ranch.

Isaacks' Ranch was deposited after a period of landscape stability and soil formation represented by the Jornada II surface (Gile et al, 1981). The deposition of Isaacks' Ranch probably accompanied a climatic change, as did the deposition of Jornada II, Jornada I, and the buried soils associated with the Camp Rice Formation. During times of more effective moisture plants cover the landscape and prevent erosion. Soil formation is more effective during these wetter conditions because more water is available for leaching. When the climate again became arid, plant cover must have decreased while erosion increased, especially during summer thunderstorms.

Each of the deposits exhibit a fining-upward sequence (i.e., coarser particles at depth grading upward to finer particles). The deposits are lithologically similar (Gile et al, 1981) except that Isaacks' Ranch contains less carbonate (stage II), reflecting its younger age, than the underlying deposits which contain stage III carbonate accumulation.

\section{Geochemistry}

Gile et al (1981) reported that the general soil mineral composition of the Desert Project is dominated by quartz, feldspars, and biotite in the sand and silt fraction. The clay mineralogy of soils in the Desert Project as reported by Gile et al. (1981) contain kaolinite, mica and small amounts of chlorite and montmorillonite.

Monger et al (1987) studied the mineralogy of soils in the southern portion of the Jornada Basin derived from similar parent material as the soils at the trench site and found that quartz, $\mathrm{K}$-feldspar, plagioclase, and authigenic calcite dominated the bulk ( $<2 \mathrm{~mm}$ ) mineralogy with minor amounts of biotites and opaques. They found the clay mineralogy was dominated by quartz, kaolinite, illite, and authigenic calcite with minor amounts of feldspars, chlorite, and smectites.

Based on the above discussion of soil mineralogy the chemical species most likely to be found in the soil solution are $\mathrm{Ca}++\mathrm{K}+, \mathrm{Na}$, $\mathrm{Mg}++, \mathrm{Si}(\mathrm{OH} 4), \mathrm{Fe}+++, \mathrm{Al}+++, \mathrm{H}+, \mathrm{OH}-$, and C03--. Because of the highly reactive nature of $\mathrm{Fe}+++$ and $\mathrm{Al}+++$, these species should quickly precipitate as oxides of, in the case of $\mathrm{Al}+++$, be incorporated into clay mineral structures. 


\section{Appendix C-8. Soils and Geomorphology at the Las Cruces Trench Site. (Continued)}

\section{References}

Gile, L.H., F.F. Peterson, and R.B. Grossman. 1966. Morphological and genetic sequences of carbonate accumulation in desert soils. Soil Sci. 101:347-360.

Gile, L.H., J.W. Hawley, and R.B. Grossman. 1981. Soils and geomorphology in the Basin and Range area of Southern New Mexico-Guidebook to the Desert Project. New Mexico Bureau of Mines and Mineral Resources. Memoir 39. p. 75.

Hawley, J.W., W.S. Seager, and R.E. Clemons. 1975. Third day road log from Las Cruces to North Mesilla Valley, Cedar Hills, San Diego Mountains, and Rincon areas. New Mexico Geol. Soc. Guidebook, 26th Field Conf., Las Cruces Country. p. 37.

Hawley, J.W., G.O. Bachman, and K. Manley. 1976. Quaternary stratigraphy in the Basin and Range and Great Plains provinces, New Mexico and west Texas. In W.C. Mahaney (ed) Quaternary stratigraphy of North America. Stroudsburg, Pennsylvania, Dowden, Hutchinson, and Ross, Inc. p. 235-274.

Hurlbut, C.S. and C. Klein. 1977. Manual of mineralogy. 19th ed. John Wiley and Sons, New York. p. 459.
Kues, B.S., C.T. Smith, R.M. North, S.G. Lucas, S.A. Northrop, C.L. Balk, and R.W.

Eveleth. 1986. New Mexico's geological panorama. In D. Hsi and J. Panitz (ed.) From sundaggers to space exploration-significant scientific contributions to science and technology in New Mexico. New Mexico Journal of Science, vol. 26, no. 1. NM Academy of Science and NM Sigma Xi Chapter. p. 231.

Martin, P.S. and P.J. Mehringer. 1965.

Pleistocene pollen analysis and biogeography of the Southwest. In H.E. Wright and D.G Frey (eds.) The Quaternary of the United States. Princeton Univ. Press. p. 433-451.

Monger, H.C., L.A. Daugherty, M.O. McCurry, and R.A. Earl. 1987. Mineralogy of some Pleistocene paleosols in southern New Mexico: Implications for soil genesis in the arid Southwest during Pleistocene pluvials. Clay Minerals Society 24th Annual Meeting Abstract. p. 99.

Reeves, C.C., Jr. 1973. The full-glacial climate of the southern High Plains, west Texas. J. of Geol. 81:693-704.

Van Devender, T.R. 1977. Holocene woodlands in the southwestern deserts Science 198:189-192. 
Appendix C-9. Chronology of Las Cruces Trench Site Experimental Events.

\begin{tabular}{|c|c|c|c|c|c|c|c|c|c|}
\hline \multicolumn{10}{|c|}{ italics indicates solution sample collection days } \\
\hline \multicolumn{10}{|c|}{ boid Indicates neutron probe reading days } \\
\hline 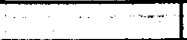 & & & & 7 & & & & & \\
\hline & \multicolumn{3}{|c|}{ Experiment days } & 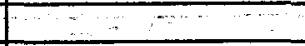 & & \multicolumn{3}{|c|}{ Experiment daye } & 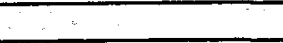 \\
\hline Date & 1 & Ula. & IIb & Event & Date - & 1 & Ha & 116 & Event \\
\hline $5 / 11 / 87$ & -16 & & & 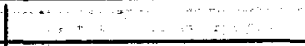 & $6 / 30 / 87$ & 34 & & & \\
\hline $5 / 12 / 87$ & -15 & & & 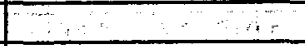 & $7 / 1 / 87$ & 35 & & & \\
\hline $5 / 13 / 87$ & -14 & $:$ & & 3 & 712187 & 36 & & & $\therefore$ \\
\hline $5 / 14 / 87$ & -13 & & & & $7 / 3 / 87$ & 37 & $\therefore$ & & 5 \\
\hline $5 / 16 / 87$ & -12 & 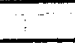 & & 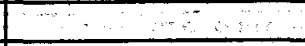 & $7 / 4 / 87$ & 38 & $\vdots$ & $\because$ & $\therefore$ \\
\hline $5 / 16 / 87$ & -11 & & & 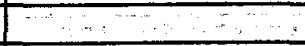 & $7 / 5 / 87$ & 39 & & & 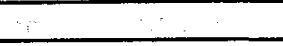 \\
\hline $5 / 17 / 87$ & -10 & & & 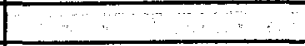 & $7 / 6 / 87$ & 40 & & & \\
\hline $5 / 18 / 87$ & -9 & ; & & 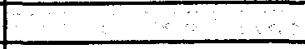 & $7 / 7 / 87$ & 41 & & $:$ & \\
\hline $5 / 19 / 87$ & .8 & & & $\therefore \therefore \therefore$ & $7 / 8 / 87$ & 42 & & & $\therefore$ \\
\hline $5 / 20 / 87$ & .7 & & & 2 & $7 / 8 / 87$ & 43 & & : & 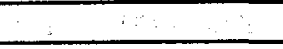 \\
\hline $5 / 21 / 87$ & -6 & & & $\therefore$ & $7 / 10 / 87$ & 44 & & & \\
\hline $5 / 22 / 87$ & -5 & & & & $7 / 11 / 87$ & 45 & & & $\therefore$ \\
\hline $5 / 23 / 87$ & -4 & & & 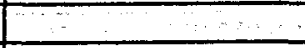 & $7 / 12 / 87$ & 46 & & 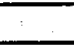 & $\therefore$ \\
\hline $5 / 24 / 87$ & -3 & & & -1 & $7 / 13 / 87$ & 47 & & & $\cdots$ \\
\hline $6 / 25 / 87$ & -2 & & & $\because \quad$ & $7 / 14 / 87$ & 48 & & & $\therefore$ \\
\hline $5 / 26 / 87$ & -1 & & & & $7 / 16 / 87$ & 49 & 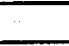 & & $\therefore$ \\
\hline $5 / 27 / 87$ & 0 & $\therefore$ & & begin Irrigation I & $7 / 16 / 87$ & 50 & 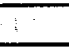 & - & \\
\hline $5 / 28 / 87$ & 1 & & & 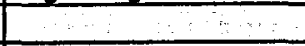 & $7 / 17 / 87$ & 51 & & 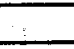 & \\
\hline $5 / 29 / 87$ & 2 & & & 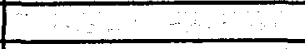 & $7 / 18 / 87$ & 52 & & $:$ & $\therefore$ \\
\hline $5 / 30 / 87$ & 3 & & & $\therefore$ & $7 / 19 / 87$ & 53 & & 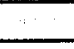 & $\because$ \\
\hline $5 / 31 / 87$ & 4 & & & 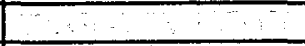 & $7 / 20 / 87$ & 54 & & 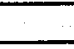 & - \\
\hline $6 / 1 / 87$ & 5 & & & & $7 / 21 / 87$ & 55 & & 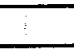 & $=$ \\
\hline $6 / 2 / 87$ & 6 & & & & $7 / 22187$ & 56 & Z & & $\therefore$ \\
\hline $6 / 3 / 87$ & 7 & & & 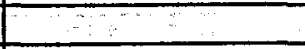 & $7 / 23 / 87$ & .57 & & $?$ & \\
\hline $6 / 4 / 87$ & 8 & & & $\ldots$ & $7 / 24 / 87$ & 58 & & 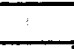 & \\
\hline $6 / 5 / 87$ & 8 & & $\therefore$ & & $7 / 25 / 87$ & 69 & & $\therefore$ & $\ldots$ \\
\hline $6 / 6 / 87$ & 10 & & & end tracer pulse l & $7 / 26 / 87$ & 60 & & $\therefore$ & $\therefore$ \\
\hline $6 / 7187$ & 11 & & & $\begin{array}{l}0 \\
\end{array}$ & $7 / 27 / 87$ & 61 & & 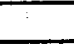 & \\
\hline $6 / 8 / 87$ & 12 & & & & $7 / 28 / 87$ & 62 & & & \\
\hline $6 / 9187$ & 13 & & & 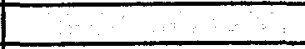 & $7 / 29 / 87$ & 63 & & & $\therefore$ \\
\hline $6 / 10 / 87$ & 14 & & 3 & $\therefore \ldots$ & $7 / 30 / 87$ & 64 & & & $\therefore$ \\
\hline $6 / 11 / 87$ & 16 & & & $\therefore \quad 3$ & $7 / 31 / 87$ & 65 & & & \\
\hline $6 / 12 / 87$ & 16 & & & 9 & $8 / 1 / 87$ & 66 & & & $\therefore$ \\
\hline $6 / 13 / 87$ & 17 & & & 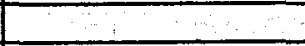 & $8 / 2 / 87$ & 67 & & & \\
\hline $6 / 14 / 87$ & 18 & & & & $8 / 3 / 87$ & 68 & & & $\vdots$ \\
\hline $6 / 15 / 87$ & 10 & & $\vdots$ & $\because$ & $8 / 4 / 87$ & 69 & & & $\cdots$ \\
\hline $6 / 16 / 87$ & 20 & & & $\therefore$ & $8 / 5187$ & 70 & & & 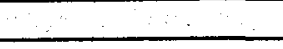 \\
\hline $6 / 17 / 87$ & 21 & & & 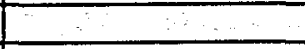 & $8 / 6 / 87$ & 71 & & & $\therefore$ \\
\hline $6 / 18 / 87$ & 22 & & & $\therefore \therefore: \quad:$ & $8 / 7 / 87$ & 72 & & $\therefore$ & 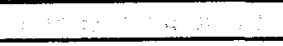 \\
\hline $6 / 18 / 87$ & 23 & & & $8 \quad \therefore$ & $8 / 8 / 87$ & 73 & & 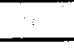 & $\therefore$ \\
\hline $6 / 20 / 87$ & 24 & & & $\therefore$ & $8 / 9187$ & 74 & & & \\
\hline $6121 / 87$ & 26 & & & 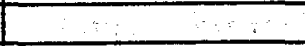 & $8 / 10 / 87$ & 75 & & & \\
\hline $6 / 22 / 87$ & 26 & & & $\therefore$ & $8 / 11 / 87$ & 76 & & 4 & $\therefore$ \\
\hline $6 / 23 / 87$ & 27 & & & $\therefore$ & $8 / 12 / 87$ & 77 & & & $\therefore$ \\
\hline $6 / 24 / 87$ & 28 & & & $=$ & $8 / 13 / 87$ & 78 & & & \\
\hline $6 / 25 / 87$ & 28 & & & $\ldots \ldots$ & $8 / 14 / 87$ & 79 & & 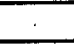 & \\
\hline $6 / 26 / 87$ & 30 & & $\because$ & & $8 / 15 / 87$ & 80 & & & \\
\hline $6 / 27 / 87$ & 31 & & & . & $8 / 16 / 87$ & 81 & $\vdots$ & & 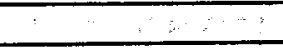 \\
\hline $6 / 28 / 87$ & 32 & & 1 & & $8 / 17 / 87$ & 82 & & & $\therefore$ \\
\hline $6 / 29 / 87$ & 33 & & & & $8 / 18 / 87$ & 83 & & & \\
\hline
\end{tabular}


Appendix C-9. Chronology of Las Cruces Trench Site Experimental Events.

\begin{tabular}{|c|c|c|c|c|c|c|c|c|c|}
\hline \multicolumn{10}{|c|}{ Italics indicates solution sample collection days } \\
\hline \multicolumn{10}{|c|}{ bold Indicates neutron probe reading days } \\
\hline & & & & & & & & & 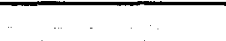 \\
\hline & \multicolumn{3}{|c|}{ Experiment days } & & $\ldots \ldots \ldots$ & \multicolumn{3}{|c|}{ Experiment days } & \\
\hline Date & 1 & Ita & $\mathrm{Hb}$ & Event & Date & 1 & Ha & Ilb & …... Event \\
\hline $8 / 19 / 87$ & 84 & & & $\ldots \ldots$ & $10 / 8 / 87$ & 134 & & & $\begin{array}{cc}2+2 \\
\end{array}$ \\
\hline $8 / 20 / 87$ & 85 & & & $\ldots$ & $10 / 9 / 87$ & 135 & & & $\cdots$ \\
\hline $8 / 21 / 87$ & 86 & & & trench wall collapse & $10 / 10 / 87$ & 136 & & $\ldots$ & \\
\hline $8 / 22 / 87$ & 87 & & & (end I irrigation) & $10 / 11 / 87$ & 137 & & & \\
\hline $8 / 23 / 87$ & 88 & & & $\therefore \quad \therefore$ & $10 / 12 / 87$ & 138 & $\infty$ & & \\
\hline $8 / 24 / 87$ & 89 & & & $\ldots$ & $10 / 13 / 87$ & 139 & & & \\
\hline $8 / 25 / 87$ & 90 & & & $\div$ & $10 / 14 / 87$ & 140 & & & \\
\hline $8 / 26 / 87$ & 91 & & & $\therefore$ & $10 / 15 / 87$ & 141 & & & \\
\hline $8 / 27 / 87$ & 92 & & & & $10 / 16 / 87$ & 142 & & & $\therefore$ \\
\hline $8 / 28 / 87$ & 93 & & & $\therefore$ & $10 / 17 / 87$ & 143 & & & \\
\hline $8 / 29 / 87$ & 94 & & & 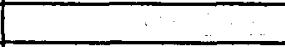 & $10 / 18 / 87$ & 144 & & & \\
\hline $8 / 30 / 87$ & 95 & & & & $10 / 19 / 87$ & 145 & & & \\
\hline $8 / 31 / 87$ & 96 & & & & $10 / 20 / 87$ & 146 & & & \\
\hline $9 / 1 / 87$ & 97 & & & $\therefore$ & $10 / 21 / 87$ & 147 & & & \\
\hline $8 / 2 / 87$ & 98 & & & & $10 / 22 / 87$ & 148 & & & \\
\hline $9 / 3 / 87$ & 99 & & & & $10 / 23 / 87$ & 149 & & & 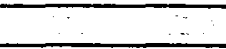 \\
\hline $9 / 4 / 87$ & 100 & & & $=$ & $10 / 24 / 87$ & 150 & & & $\ldots$ \\
\hline $9 / 5 / 87$ & 101 & & & & $10 / 25 / 87$ & 151 & & & \\
\hline $9 / 6 / 87$ & 102 & & & & $10 / 26 / 87$ & 152 & & & 3 \\
\hline $9 / 7 / 87$ & 103 & & & & $10 / 27 / 87$ & 153 & & & \\
\hline $9 / 8 / 87$ & 104 & & & & $10 / 28 / 87$ & 154 & & & \\
\hline $9 / 9 / 87$ & 105 & & & & $10 / 29 / 87$ & 155 & & & \\
\hline $9 / 10 / 87$ & 106 & & & & $10 / 30 / 87$ & 156 & & & \\
\hline $8 / 11 / 87$ & 107 & & & & $10 / 31 / 87$ & 157 & & & \\
\hline $9 / 12 / 87$ & 108 & & & $\therefore$ & $11 / 1 / 87$ & 158 & & & \\
\hline $9 / 13 / 87$ & 109 & & & & $11 / 2 / 87$ & 159 & & & \\
\hline $9 / 14 / 87$ & 110 & & & & $11 / 3 / 87$ & 160 & & & 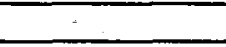 \\
\hline $9 / 15 / 87$ & 111 & & & & $11 / 4 / 87$ & 161 & & & \\
\hline $9 / 16 / 87$ & 112 & & & & $11 / 5 / 87$ & 162 & & & 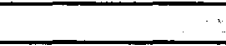 \\
\hline $9 / 17 / 87$ & 113 & & & & $11 / 6 / 87$ & 163 & & & \\
\hline $9 / 18 / 87$ & 114 & & & & $11 / 7 / 87$ & 164 & & & \\
\hline $9 / 19 / 87$ & 115 & & & & $11 / 8 / 87$ & 165 & & & \\
\hline 9120187 & 116 & & & & $11 / 9 / 87$ & 166 & & & \\
\hline $9 / 21 / 87$ & 117 & & & & $11 / 10 / 87$ & 167 & & & \\
\hline $9 / 22 / 87$ & 118 & & & & $11 / 11 / 87$ & 168 & & & \\
\hline $9 / 23 / 87$ & 119 & & & & $11 / 12 / 87$ & 169 & & & \\
\hline $9 / 24 / 87$ & 120 & & & + & $11 / 13 / 87$ & 170 & & & \\
\hline $9 / 25 / 87$ & 121 & & & & $11 / 14 / 87$ & 171 & & & \\
\hline $9 / 26 / 87$ & 122 & & & & $11 / 15 / 87$ & 172 & & & \\
\hline $9 / 27 / 87$ & 123 & & & & $11 / 16 / 87$ & 173 & & & \\
\hline $9 / 28 / 87$ & 124 & & & & $11 / 17 / 87$ & 174 & & & 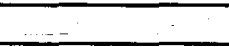 \\
\hline $9 / 29 / 87$ & 125 & & & & $11 / 18 / 87$ & 175 & & & \\
\hline $9 / 30 / 87$ & 126 & & & & $11 / 19 / 87$ & 176 & & & \\
\hline $10 / 1 / 87$ & 127 & & & & $11 / 20 / 87$ & 177 & & & \\
\hline $10 / 2 / 87$ & 128 & & & & $11 / 21 / 87$ & 178 & & & \\
\hline $10 / 3 / 87$ & 129 & & & $\therefore$ & $11 / 22 / 87$ & 179 & & & \\
\hline $10 / 4 / 87$ & 130 & & & & $11 / 23 / 87$ & 180 & & & \\
\hline $10 / 5 / 87$ & 131 & & & 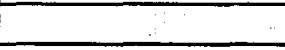 & $11 / 24 / 87$ & 181 & & & 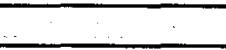 \\
\hline $10 / 6 / 87$ & 132 & & & & $11 / 25 / 87$ & 182 & & & \\
\hline $10 / 7 / 87$ & 133 & & & & $11 / 26 / 87$ & 183 & & & \\
\hline
\end{tabular}


Appendix C-9. Chronology of Las Cruces Trench Site Experimental Events.

\begin{tabular}{|c|c|c|c|c|c|c|c|c|c|}
\hline \multicolumn{10}{|c|}{ italies indicates solution sample collection days } \\
\hline \multicolumn{10}{|c|}{ bold Indicates neutron probe reading days } \\
\hline & & & & \multirow[b]{3}{*}{ Event } & \multirow[b]{3}{*}{ Date } & & & & \\
\hline & \multicolumn{3}{|c|}{ Experiment days } & & & \multicolumn{3}{|c|}{ Experiment days } & \multirow[b]{2}{*}{ Event } \\
\hline Date & 1 & Ila & III & & & 1 & Ha & 116 & \\
\hline $19 / 27 / 87$ & 184 & & & $2 \mathrm{~s}$ & $1 / 16 / 88$ & 234 & & & \\
\hline $11 / 28 / 87$ & 185 & & & & $1 / 17 / 88$ & 235 & & & \\
\hline $11 / 29 / 87$ & 186 & & & $\ldots$ & $1 / 18 / 88$ & 236 & & & \\
\hline $11 / 30 / 87$ & 187 & & & $E$ & $1 / 19 / 88$ & 237 & & & $\therefore$ \\
\hline $12 / 1 / 87$ & 188 & & 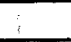 & $\therefore$ & $1 / 20 / 88$ & 238 & & & \\
\hline $12 / 2 / 87$ & 189 & & & & $1 / 21 / 88$ & 239 & & & \\
\hline $12 / 3 / 87$ & 190 & 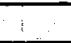 & 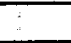 & $=$ & $1 / 22 / 88$ & 240 & & & \\
\hline $12 / 4 / 87$ & 181 & & & 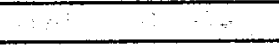 & $1 / 23 / 88$ & 241 & & & \\
\hline $12 / 5 / 87$ & 192 & & & & $1 / 24 / 88$ & 242 & & & \\
\hline $12 / 6 / 87$ & 193 & & 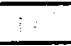 & $\therefore$ & $1 / 25 / 88$ & 243 & & & \\
\hline $12 / 7 / 87$ & 194 & & 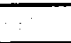 & & $1 / 26 / 88$ & 244 & & & \\
\hline $12 / 8 / 87$ & 195 & & & & $1 / 27 / 88$ & 245 & & & \\
\hline $12 / 9 / 87$ & 196 & & 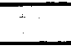 & & $1 / 28 / 88$ & 246 & & & \\
\hline $12 / 10 / 87$ & 187 & & & 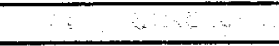 & $1 / 29 / 88$ & 247 & & & \\
\hline $12 / 11 / 87$ & 198 & & & & $1 / 30 / 88$ & 248 & & & \\
\hline $12 / 12 / 87$ & 199 & & & - & $1 / 31 / 88$ & 249 & & & \\
\hline $12 / 13 / 87$ & 200 & & & & $2 / 1 / 88$ & 250 & & & \\
\hline $12 / 14 / 87$ & 201 & & & $\ldots$ & $2 / 2 / 88$ & 251 & & & \\
\hline $12 / 15 / 87$ & 202 & & & $\therefore$ & $2 / 3 / 88$ & 252 & & & \\
\hline $12 / 16 / 87$ & 203 & & & 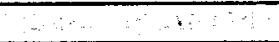 & $2 / 4 / 88$ & 253 & & & \\
\hline $12 / 17 / 87$ & 204 & & & $\cdots$ & $2 / 5 / 88$ & 254 & & & \\
\hline $12 / 18 / 87$ & 205 & & & 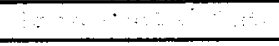 & $2 / 6 / 88$ & 255 & & & \\
\hline $12 / 18 / 87$ & 206 & & & -2 & $2 / 7 / 88$ & 256 & & & \\
\hline $12 / 20 / 87$ & 207 & & & 7 & $2 / 8 / 88$ & 257 & & & \\
\hline $12 / 21 / 87$ & 208 & & & & $2 / 8 / 88$ & 258 & & & \\
\hline $12 / 22187$ & 200 & & & & $2 / 10 / 88$ & 259 & & & \\
\hline $12 / 23 / 87$ & 210 & & & & $2 / 11 / 88$ & 260 & & & \\
\hline $12 / 24 / 87$ & 211 & & & & $2 / 12 / 88$ & 261 & & & \\
\hline $12 / 25 / 87$ & 212 & & & & $2 / 13 / 88$ & 262 & & & \\
\hline $12 / 26 / 87$ & 213 & & 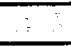 & $\therefore$ & $2 / 14 / 88$ & 263 & & & \\
\hline $12 / 27 / 87$ & 214 & & & & $2 / 15 / 88$ & 264 & & & \\
\hline $12 / 28 / 87$ & 215 & & 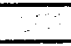 & 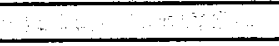 & $2 / 16 / 88$ & 265 & & & \\
\hline $12 / 29 / 87$ & 216 & & 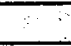 & & $2 / 17 / 88$ & 266 & & & \\
\hline $12 / 30 / 87$ & 217 & & $=$ & $a$ & $2 / 18 / 88$ & 267 & & & \\
\hline $12 / 31 / 87$ & 218 & & 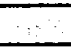 & $\therefore$ & $2 / 19 / 88$ & 268 & & & \\
\hline $1 / 1 / 88$ & 210 & & & & $2 / 20 / 88$ & 260 & & & \\
\hline $1 / 2 / 88$ & 220 & & 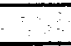 & & $2 / 21 / 88$ & 270 & & & \\
\hline $1 / 3 / 88$ & 221 & & 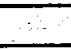 & & $2 / 22 / 88$ & 271 & & & \\
\hline $1 / 4 / 88$ & 222 & & & 8 & $2 / 23 / 88$ & 272 & & & \\
\hline $1 / 5 / 88$ & 223 & & $\therefore$ & & $2 / 24 / 88$ & 273 & & & \\
\hline $1 / 6 / 88$ & 224 & & & & $2 / 25 / 88$ & 274 & & & \\
\hline $1 / 7 / 88$ & 225 & & & 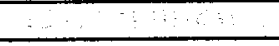 & $2 / 26 / 88$ & 275 & & & \\
\hline $1 / 8 / 88$ & 226 & & & & $2 / 27 / 88$ & 276 & & & \\
\hline $1 / 8 / 88$ & 227 & & & 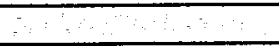 & $2 / 28 / 88$ & 277 & & & \\
\hline $1 / 10 / 88$ & 228 & & $\therefore$ & 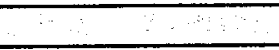 & $2 / 29 / 88$ & 278 & & & $\therefore$ \\
\hline $1 / 11 / 88$ & 229 & & & 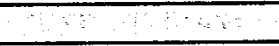 & $3 / 1 / 88$ & 279 & & & \\
\hline $1 / 12 / 88$ & 230 & & & 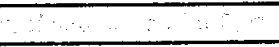 & $-3 / 2 / 88$ & 280 & & & \\
\hline $1 / 13 / 88$ & 231 & & & 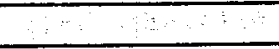 & $3 / 3 / 88$ & 281 & & & \\
\hline $1 / 14 / 88$ & 232 & & & & $3 / 4 / 88$ & 282 & & & \\
\hline $1 / 15 / 88$ & 233 & & & & $3 / 5 / 88$ & 283 & & & \\
\hline
\end{tabular}


Appendix C-9. Chronology of Las Cruces Trench Site Experimental Events.

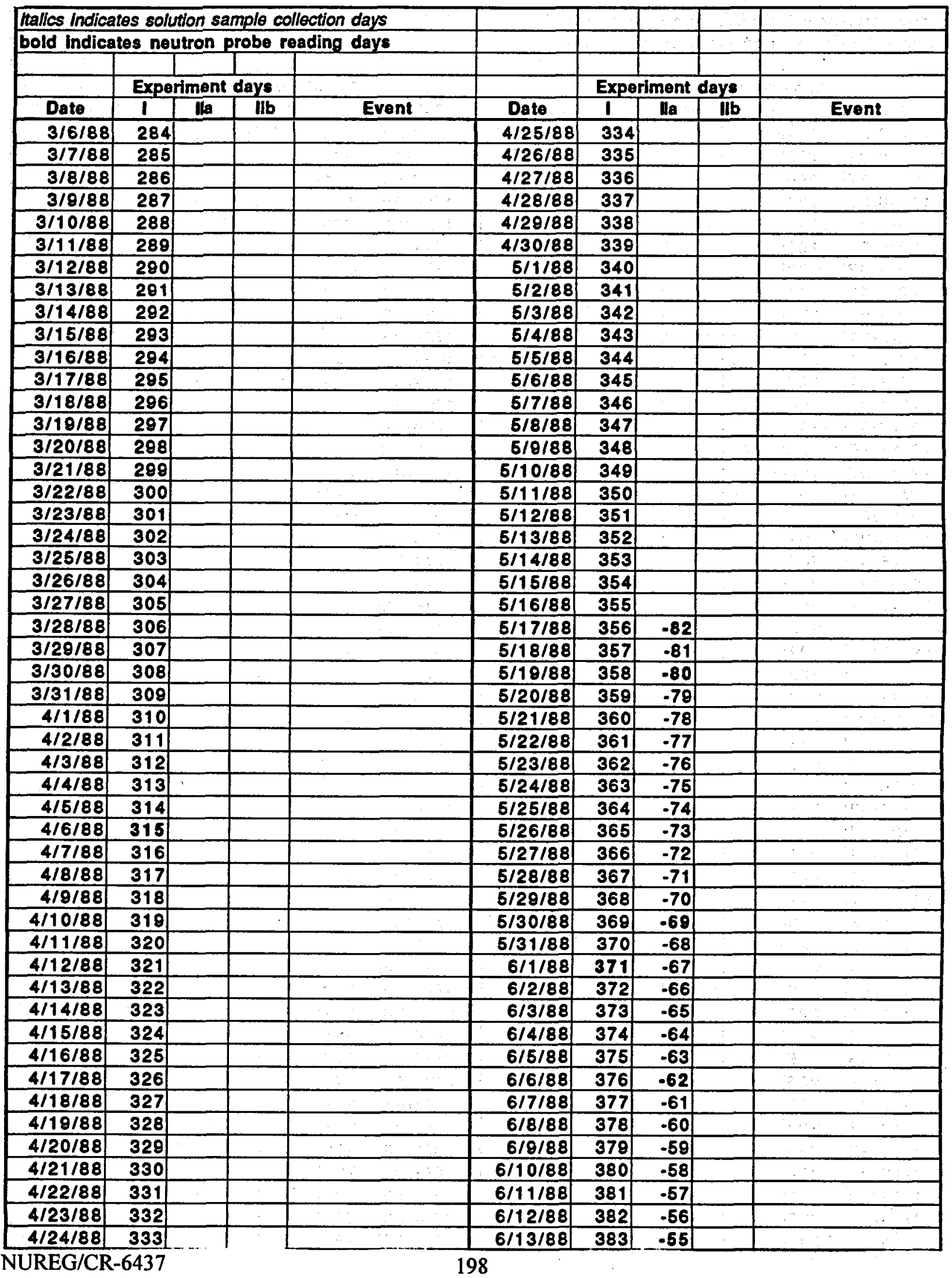


Appendix C-9. Chronology of Las Cruces Trench Site Experimental Events.

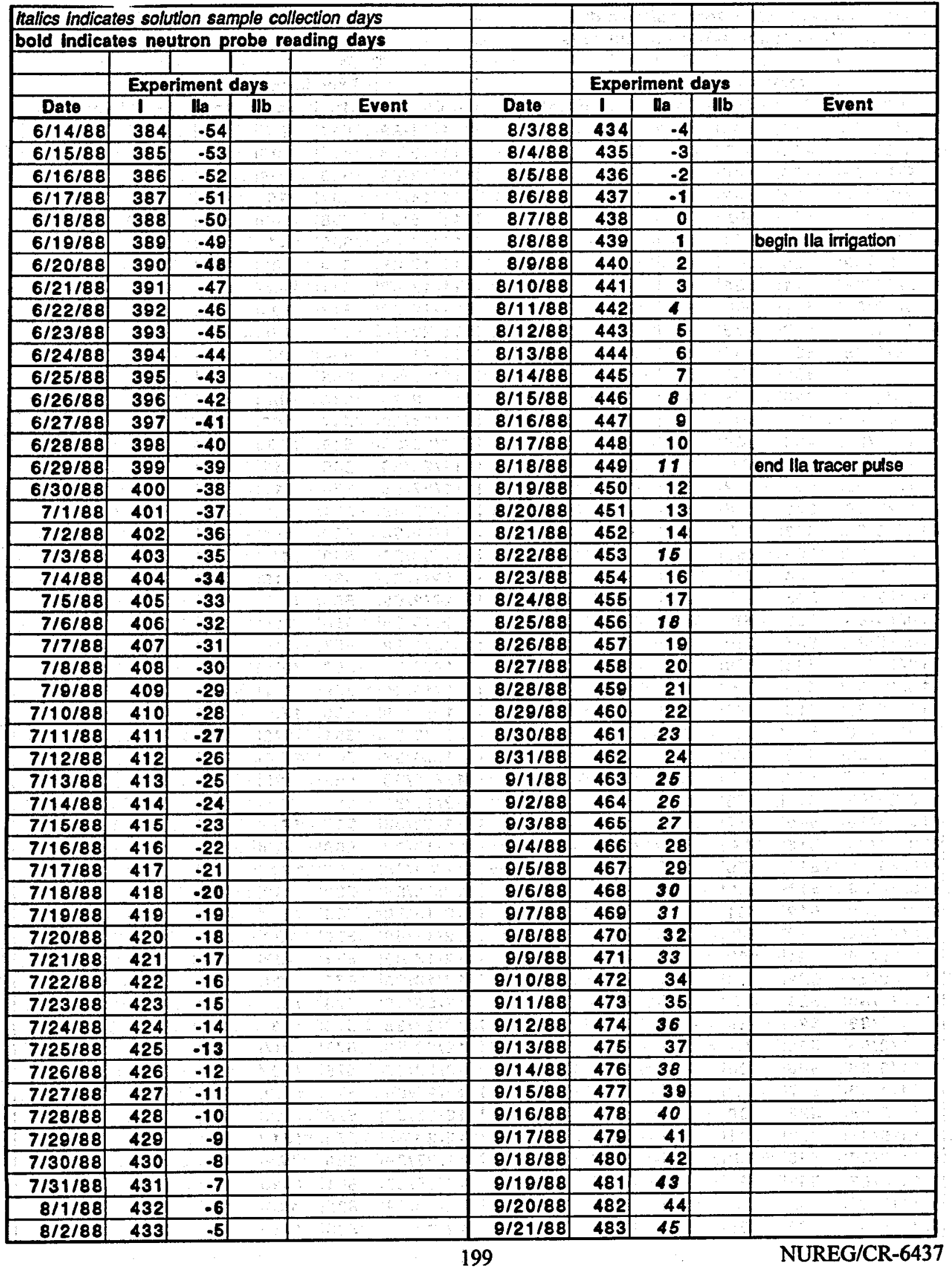


Appendix C-9. Chronology of Las Cruces Trench Site Experimental Events.

\begin{tabular}{|c|c|c|c|c|c|c|c|c|c|}
\hline \multicolumn{10}{|c|}{ hallics indicates solution sample collection days } \\
\hline \multicolumn{10}{|c|}{ bold indicates neutron probe reading days } \\
\hline & & & & & & & & & \\
\hline & \multicolumn{3}{|c|}{ Experiment days } & & & \multicolumn{3}{|c|}{ Experiment days } & \\
\hline Date & 1 & Ila & IIb & Event & Date & 1 & lla & 116 & Event \\
\hline $8 / 22 / 88$ & 484 & 46 & & & $11 / 11 / 88$ & 534 & 96 & & \\
\hline $9 / 23 / 88$ & 485 & 47 & & & $11 / 12 / 88$ & 535 & 97 & & \\
\hline $8 / 24 / 88$ & 486 & 48 & & & $11 / 13 / 88$ & 536 & 98 & & \\
\hline $9 / 25 / 88$ & 487 & 49 & & & $11 / 14 / 88$ & 537 & 99 & & \\
\hline $9 / 26 / 88$ & 488 & 50 & & $\because$ & $11 / 15 / 88$ & 538 & 100 & & \\
\hline $9 / 27 / 88$ & 489 & 51 & & & $11 / 16 / 88$ & 530 & 101 & & \\
\hline $9 / 28 / 88$ & 490 & 52 & & & $11 / 17 / 88$ & 540 & 102 & & 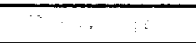 \\
\hline $9 / 29 / 88$ & 491 & 53 & & & $11 / 18 / 88$ & 541 & 103 & & \\
\hline $9 / 30 / 88$ & 492 & 54 & & & $11 / 18 / 88$ & 542 & 104 & & \\
\hline $10 / 1 / 88$ & 493 & 55 & & & $11 / 20 / 88$ & 543 & 105 & & \\
\hline $10 / 2 / 88$ & 494 & 56 & & & $11 / 21 / 88$ & 544 & 106 & & \\
\hline $10 / 3 / 88$ & 495 & 57 & & & $11 / 22 / 88$ & 545 & 107 & & \\
\hline $10 / 4 / 88$ & 486 & 58 & & & $11 / 23 / 88$ & 546 & 108 & & \\
\hline $10 / 5 / 88$ & 497 & 59 & & & $11 / 24 / 88$ & 547 & 109 & & \\
\hline $10 / 6 / 88$ & 498 & 60 & & & $11 / 25 / 88$ & 548 & 110 & & \\
\hline $10 / 7 / 88$ & 499 & 61 & & & $11 / 26 / 88$ & 549 & 111 & & \\
\hline $10 / 8 / 88$ & 500 & 62 & & & $11 / 27 / 88$ & 550 & 112 & & \\
\hline $10 / 9 / 88$ & 501 & 63 & & & $11 / 28 / 88$ & 551 & 113 & & \\
\hline $10 / 10 / 88$ & 502 & 64 & & & $11 / 29 / 88$ & 552 & 114 & & \\
\hline $10 / 11 / 88$ & 503 & 65 & & & $11 / 30 / 88$ & 553 & 115 & & \\
\hline $10 / 12 / 88$ & 504 & 66 & & & $12 / 1 / 88$ & 554 & 116 & & \\
\hline $10 / 13 / 88$ & 505 & 67 & & & $12 / 2 / 88$ & 555 & 117 & & \\
\hline $10 / 14 / 88$ & 506 & 68 & & & $12 / 3 / 88$ & 556 & 118 & & \\
\hline $10 / 15 / 88$ & 507 & 69 & & & $12 / 4 / 88$ & 557 & 119 & & \\
\hline $10 / 16 / 88$ & 508 & 70 & & & $12 / 5 / 88$ & 558 & 120 & & \\
\hline $10 / 17 / 88$ & 509 & 71 & & & $12 / 6 / 88$ & 559 & 121 & & \\
\hline $10 / 18 / 88$ & 510 & 72 & & & $12 / 7 / 88$ & 560 & 122 & & \\
\hline $10 / 19 / 88$ & 511 & 73 & & & $12 / 8 / 88$ & 561 & 123 & & \\
\hline $10 / 20 / 88$ & 512 & 74 & . & & $12 / 9 / 88$ & 562 & 124 & & \\
\hline $10 / 21 / 88$ & 513 & 75 & & end lla irrigation & $12 / 10 / 88$ & 563 & 125 & & \\
\hline $10 / 22 / 88$ & 514 & 76 & & & $12 / 11 / 88$ & 564 & 126 & & \\
\hline $10 / 23 / 88$ & 515 & 77 & & & $12 / 12 / 88$ & 565 & 127 & & \\
\hline $10 / 24 / 88$ & 516 & 78 & & ef probe & $12 / 13 / 88$ & 566 & 128 & & \\
\hline $10 / 25 / 88$ & 517 & 79 & & & $12 / 14 / 88$ & 567 & 129 & & \\
\hline $10 / 26 / 88$ & 518 & 80 & & & $12 / 15 / 88$ & 568 & 130 & & \\
\hline $10 / 27 / 88$ & 519 & 81 & & & $12 / 16 / 88$ & 569 & 131 & & \\
\hline $10 / 28 / 88$ & 520 & 82 & & & $12 / 17 / 88$ & 570 & 132 & & \\
\hline $10 / 29 / 88$ & 521 & 83 & & & $12 / 18 / 88$ & 571 & 133 & & \\
\hline $10 / 30 / 88$ & 522 & 84 & & & $12 / 19 / 88$ & 572 & 134 & & \\
\hline $10 / 31 / 88$ & 523 & 85 & & & $12 / 20 / 88$ & 573 & 135 & & \\
\hline $11 / 1 / 88$ & 524 & 86 & & & $12 / 21 / 88$ & 574 & 136 & & \\
\hline $11 / 2 / 88$ & 525 & 87 & & & $12 / 22 / 88$ & 575 & 137 & & \\
\hline $11 / 3 / 88$ & 526 & 88 & & & $12 / 23 / 88$ & 576 & 138 & & \\
\hline $11 / 4 / 88$ & 527 & 89 & & & $12 / 24 / 88$ & 577 & 139 & & \\
\hline $11 / 5 / 88$ & 528 & 90 & & & $12 / 25 / 88$ & 578 & 140 & & \\
\hline $11 / 6 / 88$ & 529 & 81 & & & $12 / 26 / 88$ & 579 & 141 & & \\
\hline $11 / 7 / 88$ & 530 & 92 & & & $12 / 27 / 88$ & 580 & 142 & & \\
\hline $11 / 8 / 88$ & 531 & 93 & & & $12 / 28 / 88$ & 581 & 143 & & \\
\hline $11 / 9 / 88$ & 532 & 94 & & & $12 / 29 / 88$ & 582 & 144 & & \\
\hline $11 / 10 / 88$ & 533 & 95 & & & $12 / 30 / 88$ & 583 & 145 & & \\
\hline
\end{tabular}


Appendix C-9. Chronology of Las Cruces Trench Site Experimental Events.

\begin{tabular}{|c|c|c|c|c|c|c|c|c|c|}
\hline \multicolumn{5}{|c|}{ halics indicates solution sample collection days } & & & $\therefore$ & & \\
\hline \multicolumn{10}{|c|}{ bold Indicates noutron probe reading days } \\
\hline & & & & & & & & & \\
\hline & \multicolumn{3}{|c|}{ Experiment days } & & & \multicolumn{3}{|c|}{ Experiment days } & \\
\hline Date & 1 & Ila & ilb & Event & Date & 1 & Ma & illb & Event \\
\hline $12 / 31 / 88$ & 584 & 146 & & $\therefore$ & $2 / 19 / 89$ & 634 & 196 & $\cdots$ & 3 \\
\hline $1 / 1 / 89$ & 585 & 147 & & $\therefore$ & $2 / 20 / 89$ & 635 & 197 & & $\bar{c}$ \\
\hline $1 / 2 / 89$ & 586 & 148 & & 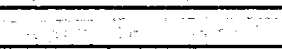 & $2 / 21 / 89$ & 636 & 198 & & \\
\hline $1 / 3 / 80$ & 587 & 149 & & & $2 / 22 / 89$ & 637 & 199 & & \\
\hline $1 / 4 / 89$ & 588 & 150 & & & $2 / 23 / 89$ & 638 & 200 & & $\because$ \\
\hline $1 / 5 / 80$ & 589 & 151 & & & $2 / 24 / 89$ & 639 & 201 & 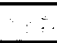 & 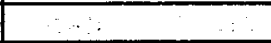 \\
\hline $1 / 6 / 89$ & 580 & 152 & & & $2 / 25 / 89$ & 640 & 202 & & $=$ \\
\hline $1 / 7 / 89$ & 591 & 153 & & & $2 / 26 / 89$ & 641 & 203 & 2 & \\
\hline $1 / 8 / 80$ & 592 & 154 & & & $2 / 27 / 89$ & 642 & 204 & & 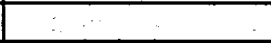 \\
\hline $1 / 9 / 89$ & 593 & 155 & . & $\cdots$ & $2 / 28 / 8 \theta$ & 643 & 206 & & \\
\hline $1 / 10 / 89$ & 594 & 156 & & & $3 / 1 / 80$ & 644 & 206 & & \\
\hline $1 / 11 / 89$ & 595 & 157 & & & $3 / 2 / 89$ & 645 & 207 & & \\
\hline $1 / 12 / 80$ & 596 & 158 & $\pi$ & & $3 / 3 / 89$ & 646 & 208 & & \\
\hline $1 / 13 / 89$ & 597 & 159 & - & $\therefore$ & $3 / 4 / 89$ & 647 & 209 & & $\therefore$ \\
\hline $1 / 14 / 89$ & 598 & 160 & 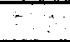 & $\because$ & $3 / 5 / 89$ & 648 & 210 & & 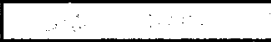 \\
\hline $1 / 15 / 89$ & 599 & 161 & & $\therefore$ & $3 / 6 / 89$ & 648 & 211 & & $\therefore$ \\
\hline $1 / 16 / 89$ & 600 & 162 & 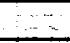 & & $3 / 7 / 89$ & 650 & 212 & & 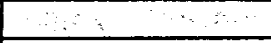 \\
\hline $1 / 17 / 89$ & 601 & 163 & & & $3 / 8 / 89$ & 651 & 213 & 8 & \\
\hline $1 / 18 / 89$ & 602 & 164 & & 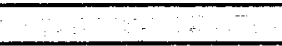 & $3 / 9 / 89$ & 652 & 214 & & \\
\hline $1 / 19 / 89$ & 603 & 165 & & - & $3 / 10 / 89$ & 653 & 215 & & \\
\hline $1 / 20 / 89$ & 604 & 166 & & 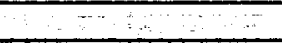 & $3 / 11 / 89$ & 654 & 216 & & \\
\hline $1 / 21 / 80$ & 605 & 167 & 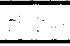 & 1 & $3 / 12 / 89$ & 655 & 217 & & $\vdots$ \\
\hline $1 / 22 / 80$ & 606 & 168 & & $\ldots$ & $3 / 13 / 89$ & 656 & 218 & & \\
\hline $1 / 23 / 89$ & 607 & 169 & 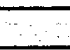 & 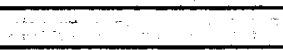 & $3 / 14 / 89$ & 657 & 219 & & \\
\hline $1 / 24 / 89$ & 608 & 170 & 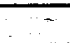 & $\ldots$ & $3 / 15 / 89$ & 658 & 220 & & i. \\
\hline $1 / 25 / 89$ & 609 & 171 & & & $3 / 16 / 89$ & 659 & 221 & & \\
\hline $1 / 26 / 89$ & 610 & 172 & 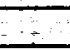 & & $3 / 17 / 89$ & 660 & 222 & & \\
\hline $1 / 27 / 8 \theta$ & 611 & 173 & & & $3 / 18 / 89$ & 661 & 223 & & \\
\hline $1 / 28 / 89$ & 612 & 174 & & $a$ & $3 / 19 / 89$ & 662 & 224 & & \\
\hline $1 / 29 / 89$ & 613 & 176 & & 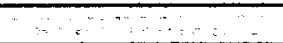 & $3 / 20 / 89$ & 663 & 225 & & \\
\hline $1 / 30 / 8 \theta$ & 614 & 176 & & & $3 / 21 / 89$ & 664 & 226 & & \\
\hline $1 / 31 / 89$ & 615 & 177 & & $x=$ & $3 / 22 / 89$ & 665 & 227 & & \\
\hline $2 / 1 / 89$ & 616 & 178 & $\therefore$ & 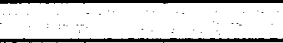 & $3 / 23 / 89$ & 666 & 228 & & $\therefore$ \\
\hline 212180 & 617 & 179 & 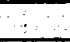 & & $3 / 24 / 89$ & 667 & 228 & & $=$ \\
\hline $2 / 3 / 89$ & 618 & 180 & & & $3 / 25 / 89$ & 668 & 230 & & \\
\hline $2 / 4 / 8 \theta$ & 619 & 181 & & & $3 / 26 / 89$ & 669 & 231 & & \\
\hline $2 / 5 / 80$ & 620 & 182 & & & $3 / 27 / 89$ & 670 & 232 & & \\
\hline $2 / 6 / 80$ & 621 & 183 & & 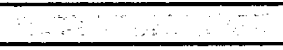 & $3 / 28 / 89$ & 671 & 233 & & \\
\hline $217 / 89$ & 622 & 184 & & & $3 / 29 / 89$ & 672 & 234 & & \\
\hline $2 / 8 / 89$ & 623 & 185 & & $\ldots$ & $3 / 30 / 89$ & 673 & 235 & & \\
\hline $2 / 8 / 89$ & 624 & 186 & & & $3 / 31 / 89$ & 674 & 236 & & \\
\hline $2 / 10 / 8 \theta$ & 625 & 187 & & $=$ & $4 / 1 / 89$ & 675 & 237 & & \\
\hline $2 / 11 / 80$ & 626 & 188 & & $\therefore$ & $4 / 2 / 89$ & 676 & 238 & & \\
\hline $2 / 12 / 89$ & 627 & 180 & & & $4 / 3 / 89$ & 677 & 239 & & \\
\hline $2 / 13 / 89$ & 628 & 190 & & & $4 / 4 / 89$ & 678 & 240 & & 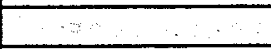 \\
\hline $2 / 14 / 89$ & 629 & 191 & & & $4 / 5 / 89$ & 670 & 241 & & \\
\hline $2 / 15 / 8 \theta$ & 630 & 192 & & & $4 / 6189$ & 680 & 242 & & Exp I deep core *1 \\
\hline $2 / 16 / 8 \theta$ & 631 & 193 & & 28 & $4 / 7 / 89$ & 681 & 243 & & \\
\hline $2 / 17 / 89$ & 632 & 194 & & & $4 / 8 / 89$ & 682 & 244 & & \\
\hline $2 / 18 / 8 \theta$ & 633 & 195 & & & $419 / 89$ & 683 & 245 & & \\
\hline
\end{tabular}


Appendix C-9. Chronology of Las Cruces Trench Site Experimental Events.

\begin{tabular}{|c|c|c|c|c|c|c|c|c|c|}
\hline \multirow{2}{*}{\multicolumn{10}{|c|}{$\begin{array}{l}\text { Ltalics indicates solution sample collection days } \\
\text { bold indicates noutron probe reading days }\end{array}$}} \\
\hline & & & & & & & & & \\
\hline & \multicolumn{3}{|c|}{ Experiment days } & & & & Experiment days & & \\
\hline Date & 1 & Hla & ilb & Event & Date & 1 & Ha & IIb & Event \\
\hline $4 / 10 / 80$ & 684 & 246 & & 6 now access & $5 / 30 / 89$ & 734 & 296 & & \\
\hline $4 / 11 / 89$ & 685 & 247 & & tubes, to $\$ 43$ & $5 / 31 / 89$ & 735 & 297 & & E \\
\hline $4 / 12 / 89$ & 686 & 248 & & & $6 / 1 / 89$ & 736 & 298 & & - \\
\hline $4 / 13 / 89$ & 687 & 249 & & & $6 / 2 / 89$ & 737 & 299 & & \\
\hline $4 / 14 / 89$ & 688 & 250 & & & $6 / 3 / 89$ & 738 & 300 & & \\
\hline $4 / 15 / 89$ & 689 & 251 & & & $6 / 4 / 89$ & 739 & 301 & & \\
\hline $4 / 16 / 89$ & 690 & 252 & & & $6 / 5 / 80$ & 740 & 302 & & \\
\hline $4 / 17 / 88$ & 691 & 253 & & & $6 / 6 / 89$ & 741 & 303 & & \\
\hline $4 / 18 / 89$ & 692 & 254 & & & $6 / 7 / 89$ & 742 & 304 & & \\
\hline $4 / 19 / 89$ & 693 & 255 & & & $6 / 8189$ & 743 & 305 & & \\
\hline $4 / 20 / 89$ & 694 & 256 & & & $6 / 9 / 89$ & 744 & 306 & & \\
\hline $4 / 21 / 89$ & 695 & 257 & & & $6 / 10 / 80$ & 745 & 307 & & \\
\hline $4 / 22 / 88$ & 696 & 258 & & & $6 / 11 / 89$ & 746 & 308 & & \\
\hline $4 / 23 / 89$ & 697 & 259 & & & $6 / 12 / 89$ & 747 & 309 & & \\
\hline $4 / 24 / 89$ & 698 & 260 & & & $6 / 13 / 89$ & 748 & 310 & & \\
\hline $4 / 25 / 89$ & 699 & 261 & & & $6 / 14 / 89$ & 748 & 311 & & \\
\hline $4 / 26 / 89$ & 700 & 262 & & & $6 / 15 / 89$ & 750 & 312 & & \\
\hline $4 / 27 / 89$ & 701 & 263 & & & $6 / 16 / 89$ & 751 & 313 & & \\
\hline $4 / 28 / 89$ & 702 & 264 & & & $6 / 17 / 89$ & 752 & 314 & & \\
\hline $4 / 29 / 89$ & 703 & 265 & & & $6 / 18 / 80$ & 753 & 315 & & \\
\hline $4 / 30 / 80$ & 704 & 266 & & & $6 / 19 / 80$ & 754 & 316 & & \\
\hline $5 / 1 / 89$ & 705 & 267 & & & $6 / 20188$ & 755 & 317 & & \\
\hline $5 / 2 / 89$ & 706 & 268 & & & $6 / 21 / 88$ & 756 & 318 & & \\
\hline $6 / 3 / 89$ & 707 & 269 & & & $6 / 22 / 89$ & 757 & 310 & & \\
\hline $5 / 4 / 89$ & 708 & 270 & & & $6 / 23 / 89$ & 758 & 320 & & \\
\hline $5 / 5 / 89$ & 709 & 271 & & & $6 / 24 / 89$ & 759 & 321 & & \\
\hline $5 / 6 / 89$ & 710 & 272 & & & $6 / 25 / 89$ & 760 & 322 & & \\
\hline $5 / 7 / 89$ & 711 & 273 & & & $6 / 26 / 89$ & 761 & 323 & & \\
\hline $5 / 8 / 89$ & 712 & 274 & & & $6 / 27 / 89$ & 762 & 324 & & \\
\hline $5 / 9 / 89$ & 713 & 275 & & & $6 / 28 / 89$ & 763 & 325 & & \\
\hline $5 / 10 / 89$ & 714 & 276 & & & $6 / 29 / 89$ & 764 & 326 & & \\
\hline $5 / 11 / 89$ & 715 & 277 & & & $6 / 30 / 89$ & 765 & 327 & & \\
\hline $5 / 12 / 89$ & 716 & 278 & & & $7 / 1 / 88$ & 766 & 328 & & \\
\hline $5 / 13 / 89$ & 717 & 279 & & & $7 / 2 / 89$ & 767 & 329 & & \\
\hline $5 / 14 / 89$ & 718 & 280 & & & $7 / 3 / 80$ & 768 & 330 & & \\
\hline $5 / 15 / 89$ & 719 & 281 & & & $7 / 4 / 89$ & 769 & 331 & & \\
\hline $5 / 16 / 80$ & 720 & 282 & & & $7 / 5 / 89$ & 770 & 332 & & \\
\hline $6 / 17 / 89$ & 721 & 283 & & & $7 / 6 / 89$ & 771 & 333 & & \\
\hline $6 / 18 / 89$ & 722 & 284 & & & $7 / 7 / 89$ & 772 & 334 & & \\
\hline $5 / 18 / 89$ & 723 & 285 & & & $7 / 8 / 89$ & 773 & 335 & & \\
\hline $5 / 20 / 89$ & 724 & 286 & & & $7 / 9189$ & 774 & 336 & & \\
\hline $5 / 21 / 80$ & 725 & 287 & & & $7 / 10 / 89$ & 775 & 337 & & \\
\hline $5 / 22 / 89$ & 726 & 288 & & & $7 / 11 / 89$ & 776 & 338 & & \\
\hline $5 / 23 / 89$ & 727 & 289 & & i & $7 / 12 / 80$ & 777 & 339 & & \\
\hline $5 / 24 / 89$ & 728 & 290 & & & $7 / 13 / 89$ & 778 & 340 & & \\
\hline $5 / 25 / 89$ & 728 & 291 & & & $7 / 14 / 80$ & 779 & 341 & & \\
\hline $5 / 26 / 89$ & 730 & 292 & & & $7 / 15 / 89$ & 780 & 342 & & \\
\hline $5 / 27 / 89$ & 731 & 293 & & & $7 / 16 / 89$ & 781 & 343 & & \\
\hline $5 / 28 / 89$ & 732 & 294 & & & $7 / 17 / 89$ & .782 & 344 & & \\
\hline $5 / 29 / 89$ & 733 & 295 & & & $7 / 18 / 89$ & 783 & 345 & & \\
\hline NUREG/CI & -6437 & & & & & & & & \\
\hline
\end{tabular}


Appendix C-9. Chronology of Las Cruces Trench Site Experimental Events.

\begin{tabular}{|c|c|c|c|c|c|c|c|c|c|}
\hline \multicolumn{5}{|c|}{ Ihalles Indicates solution sample collection days } & & & & & \\
\hline \multicolumn{10}{|c|}{ bold indicates neutron probe reading days } \\
\hline & & & & & & & & & \\
\hline & \multicolumn{3}{|c|}{ Experiment days } & & & \multicolumn{3}{|c|}{ Exporiment days } & - \\
\hline Date... & 1 & Ila & $\| \mathbf{i b}$ & Event & Date & 1 & $\mathrm{Ila}$ & $\mathrm{IIb}$ & Event \\
\hline $7 / 19 / 89$ & 784 & 346 & - & + & $9 / 7 / 89$ & 834 & 396 & & \\
\hline $7 / 20 / 88$ & 785 & 347 & & & $9 / 8 / 89$ & 835 & 397 & & \\
\hline $7 / 21 / 89$ & 786 & 348 & & 5 & $9 / 8 / 89$ & 836 & 398 & & $\therefore$ \\
\hline $7 / 22 / 89$ & 787 & 349 & & 5 & $9 / 10 / 89$ & 837 & 399 & & \\
\hline $7 / 23 / 89$ & 788 & 350 & & & $9 / 11 / 89$ & 838 & 400 & & \\
\hline $7 / 24 / 89$ & 789 & 351 & & & $9 / 12 / 89$ & 839 & 401 & & \\
\hline $7 / 25 / 89$ & 790 & 352 & & & $9 / 13 / 88$ & 840 & 402 & & \\
\hline $7 / 26 / 89$ & 791 & 353 & & 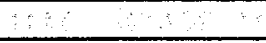 & $8 / 14 / 89$ & 841 & 403 & & \\
\hline $7 / 27 / 88$ & 792 & 354 & & ? & $8 / 15 / 89$ & 842 & 404 & & \\
\hline $7 / 28 / 89$ & 793 & 355 & & 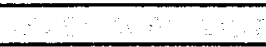 & $9 / 16 / 89$ & 843 & 405 & & \\
\hline $7 / 29 / 89$ & 794 & 356 & & & $9 / 17 / 89$ & 844 & 406 & & \\
\hline $7 / 30 / 89$ & 795 & 357 & 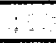 & & $9 / 18 / 89$ & 845 & 407 & & \\
\hline $7 / 31 / 89$ & 796 & 358 & & & $8 / 19 / 8 \theta$ & 846 & 408 & & \\
\hline $8 / 1 / 89$ & 797 & 359 & & $\because$ & $9 / 20 / 89$ & 847 & 409 & & \\
\hline 812189 & 788 & 360 & & 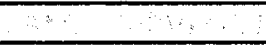 & $9 / 21 / 89$ & 848 & 410 & & \\
\hline $8 / 3 / 89$ & 798 & 361 & & 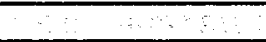 & $8 / 22 / 89$ & 849 & 411 & & \\
\hline $8 / 4 / 80$ & 800 & 362 & & 2 & $8 / 23 / 8 \theta$ & 850 & 412 & & \\
\hline $8 / 5 / 80$ & 801 & 363 & & $\cdots$ & $9 / 24 / 89$ & 851 & 413 & & \\
\hline $8 / 6180$ & 802 & 364 & 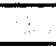 & 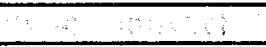 & $9 / 25 / 89$ & 852 & 414 & & 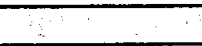 \\
\hline $8 / 7 / 89$ & 803 & 365 & 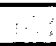 & 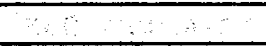 & $8 / 26 / 8 \theta$ & 853 & 416 & & \\
\hline $8 / 8 / 89$ & 804 & 366 & & & $9 / 27 / 8 \theta$ & 854 & 416 & & \\
\hline $8 / 8 / 89$ & 805 & 367 & 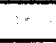 & 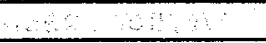 & $9 / 28 / 89$ & 855 & 417 & & \\
\hline $8 / 10 / 89$ & 806 & 368 & & 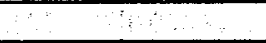 & $9 / 29 / 89$ & 856 & 418 & & \\
\hline $8 / 11 / 80$ & 807 & 369 & 3 & 4 & $9 / 30 / 8 \theta$ & 857 & 410 & & \\
\hline $8 / 12 / 80$ & 808 & 370 & & 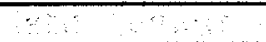 & $10 / 1 / 8 \theta$ & 858 & 420 & & \\
\hline $8 / 13 / 89$ & 809 & 371 & & 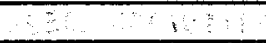 & $10 / 2 / 8 \theta$ & 859 & 421 & & \\
\hline $8 / 14 / 80$ & 810 & 372 & & $\because$ & $10 / 3 / 89$ & 860 & 422 & & $\because$ \\
\hline $8 / 15 / 89$ & 811 & 373 & & & $10 / 4 / 88$ & 861 & 423 & & \\
\hline $8 / 16 / 89$ & 812 & 374 & 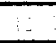 & का & $10 / 5 / 89$ & 862 & 424 & & \\
\hline $8 / 17 / 89$ & 813 & 375 & & & $10 / 6 / 8 \theta$ & 863 & 425 & & \\
\hline $8 / 18 / 89$ & 814 & 376 & & & $10 / 7 / 89$ & 864 & 426 & & \\
\hline $8 / 18 / 89$ & 815 & 377 & & 3 & $10 / 8 / 89$ & 865 & 427 & & \\
\hline $8 / 20 / 89$ & 816 & 378 & & $\quad \ldots$ & $10 / 9 / 80$ & 866 & 428 & & \\
\hline $8 / 21 / 89$ & 817 & 379 & & $\therefore \quad$ & $10 / 10 / 89$ & 867 & 429 & & \\
\hline $8 / 22 / 89$ & 818 & 380 & 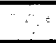 & & $10 / 11 / 88$ & 868 & 430 & & \\
\hline $8 / 23 / 89$ & 819 & 381 & & & $10 / 12 / 89$ & 869 & 431 & & \\
\hline $8 / 24 / 89$ & 820 & 382 & & 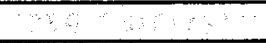 & $10 / 13 / 89$ & 870 & 432 & & \\
\hline $8 / 25 / 89$ & 821 & 383 & & 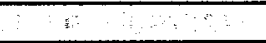 & $10 / 14 / 89$ & 871 & 433 & & \\
\hline $8 / 26 / 89$ & 822 & 384 & & & $10 / 15 / 89$ & 872 & 434 & & \\
\hline $8 / 27 / 89$ & 823 & 385 & & & $10 / 16 / 89$ & 873 & 435 & & \\
\hline $8 / 28 / 89$ & 824 & 386 & 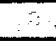 & 3 & $10 / 17 / 89$ & 874 & 436 & & \\
\hline $8 / 29 / 89$ & 825 & 387 & 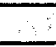 & 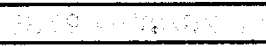 & $10 / 18 / 89$ & 875 & 437 & & \\
\hline $8 / 30 / 89$ & 826 & 388 & $\Rightarrow$ & 8 & $10 / 19 / 89$ & 876 & 438 & & $\vdots$ \\
\hline $8 / 31 / 8 \theta$ & 827 & 388 & $\bar{x}$ & & $10 / 20 / 89$ & 877 & 439 & & \\
\hline $9 / 1 / 89$ & 828 & 390 & & 8 & $10 / 21 / 89$ & 878 & 440 & & \\
\hline $9 / 2 / 89$ & 829 & 391 & & 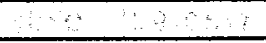 & $10 / 22 / 89$ & 879 & 441 & & \\
\hline $9 / 3 / 89$ & 830 & 392 & & $\ldots$ & $10 / 23 / 89$ & 880 & 442 & & \\
\hline $9 / 4 / 89$ & 831 & 393 & & & $10 / 24 / 89$ & 881 & 443 & & \\
\hline $9 / 5 / 89$ & 832 & 394 & $\therefore$ & & $10.25 / 89$ & 882 & 444 & & \\
\hline $9 / 6 / 89$ & 833 & 395 & & 3 & $10 / 26 / 89$ & 883 & 445 & & \\
\hline
\end{tabular}


Appendix C-9. Chronology of Las Cruces Trench Site Experimental Events.

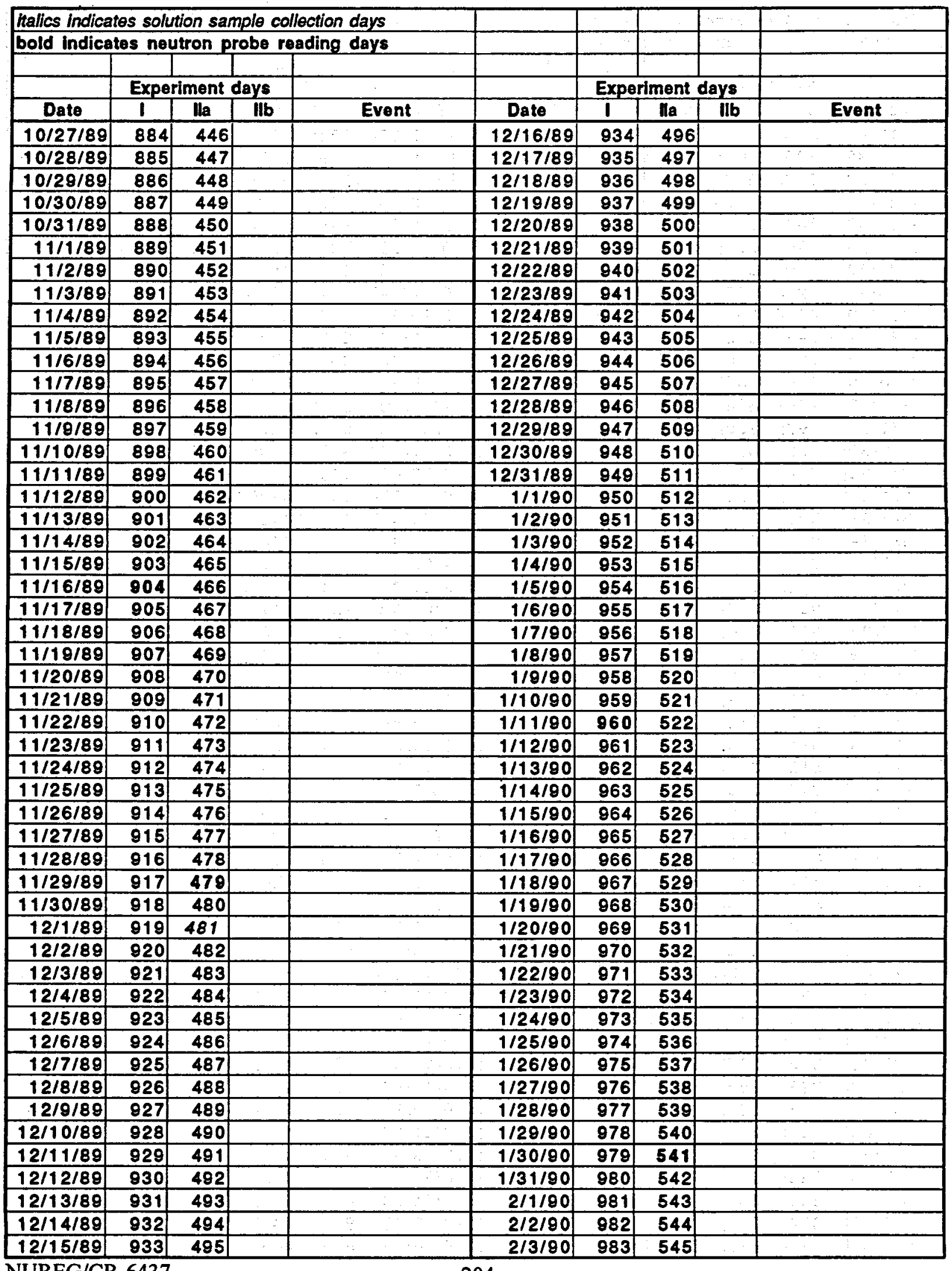

NUREG/CR-6437 
Appendix C-9. Chronology of Las Cruces Trench Site Experimental Events.

\begin{tabular}{|c|c|c|c|c|c|c|c|c|c|}
\hline \multicolumn{10}{|c|}{ halics Indicates solution sample collection days } \\
\hline \multicolumn{10}{|c|}{ bold Indicates noutron probe reading days } \\
\hline & & & & & & & & & \\
\hline & \multicolumn{3}{|c|}{ Experiment days } & & & \multicolumn{3}{|c|}{ Experiment days } & \\
\hline Date & 1 & Ila & 116 & Event & Date & 1 & lla & $11 b$ & Event \\
\hline $2 / 4 / 90$ & 984 & 546 & & & $3 / 26 / 90$ & 1034 & 596 & & \\
\hline $2 / 5 / 90$ & 985 & 547 & & $\cdots$ & $3 / 27 / 90$ & 1035 & 597 & & \\
\hline $2 / 6 / 90$ & 886 & 548 & & & $3 / 28 / 90$ & 1036 & 598 & & \\
\hline $2 / 7 / 90$ & 887 & 549 & & & $3 / 29 / 90$ & 1037 & 599 & & \\
\hline $2 / 8 / 90$ & 988 & 550 & & & $3 / 30 / 80$ & 1038 & 600 & & \\
\hline $2 / 9 / 90$ & 989 & 551 & $\cdots$ & & $3 / 31 / 90$ & 1039 & 601 & & \\
\hline $2 / 10 / 90$ & 990 & 552 & & & $4 / 1 / 90$ & 1040 & 602 & & \\
\hline $2 / 11 / 90$ & 991 & 553 & & & $4 / 2 / 90$ & 1041 & 603 & & \\
\hline $2 / 12 / 90$ & 992 & 554 & & 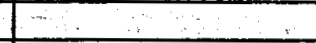 & $4 / 3 / 90$ & 1042 & 604 & & \\
\hline $2 / 13 / 90$ & 993 & 555 & . & 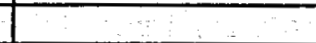 & $4 / 4 / 90$ & 1043 & 605 & & \\
\hline $2 / 14 / 90$ & 994 & 556 & & 4 & $4 / 5 / 90$ & 1044 & 606 & & \\
\hline $2 / 15 / 90$ & 995 & 657 & & & $4 / 6 / 90$ & 1045 & 607 & & \\
\hline $2 / 16 / 80$ & 996 & 558 & & & $4 / 7 / 90$ & 1046 & 608 & & \\
\hline $2 / 17 / 90$ & 997 & 559 & & 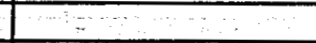 & $4 / 8 / 90$ & 1047 & 609 & & \\
\hline $2 / 18 / 90$ & 898 & 560 & & 9 & $4 / 9 / 90$ & 1048 & 610 & & \\
\hline $2 / 19 / 90$ & 999 & 561 & & $\cdots$ & $4 / 10 / 90$ & 1049 & 611 & & \\
\hline $2 / 20 / 80$ & 1000 & 562 & & $=$ & $4 / 11 / 90$ & 1050 & 612 & & \\
\hline $2 / 21 / 90$ & 1001 & 563 & & & $4 / 12 / 80$ & 1051 & 613 & 3 & \\
\hline $2 / 22 / 90$ & 1002 & 564 & & 0 & $4 / 13 / 80$ & 1052 & 614 & & \\
\hline $2123 / 90$ & 1003 & 565 & & & $4 / 14 / 90$ & 1053 & 615 & & \\
\hline $2 / 24100$ & 1004 & 566 & & & $4 / 15 / 90$ & 1054 & 616 & & \\
\hline $2 / 25 / 90$ & 1005 & 567 & & & $4 / 16 / 90$ & 1055 & 617 & & \\
\hline $2 / 26 / 90$ & 1006 & 568 & & & $4 / 17 / 90$ & 1056 & 618 & & \\
\hline $2 / 27190$ & 1007 & 569 & 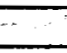 & & $4 / 18 / 90$ & 1057 & 610 & & \\
\hline $2 / 28 / 90$ & 1008 & 570 & & & $4 / 18 / 90$ & 1058 & 620 & & \\
\hline $3 / 1 / 90$ & 1009 & 571 & & & $4 / 20 / 80$ & 1059 & 621 & & \\
\hline $3 / 2 / 90$ & 1010 & 572 & & & $4 / 21 / 90$ & 1060 & 622 & i & \\
\hline $3 / 3 / 90$ & 1011 & 573 & & $\ldots$ & $4 / 22 / 90$ & 1061 & 623 & & \\
\hline $3 / 4 / 80$ & 1012 & 574 & & & $4 / 23 / 90$ & 1062 & 624 & & \\
\hline $3 / 5 / 90$ & 1013 & 576 & & & $4 / 24 / 80$ & 1063 & 625 & & \\
\hline $3 / 6 / 90$ & 1014 & 576 & & & $4 / 25 / 90$ & 1064 & 626 & & \\
\hline $3 / 7 / 90$ & 1015 & 577 & & & $4 / 26 / 90$ & 1065 & 627 & & \\
\hline $3 / 8 / 90$ & 1016 & 578 & & & $4 / 27 / 90$ & 1066 & 628 & & \\
\hline $3 / 8 / 90$ & 1017 & 579 & - & & $4 / 28 / 90$ & 1067 & 629 & & \\
\hline $3 / 10 / 80$ & 1018 & 580 & & & $4 / 29 / 90$ & 1068 & 630 & & \\
\hline $3 / 11 / 90$ & 1019 & 581 & & & $4 / 30 / 90$ & 1069 & 631 & & \\
\hline $3 / 12 / 80$ & 1020 & 682 & & $\approx$ & $5 / 1 / 90$ & 1070 & 632 & & \\
\hline $3 / 13 / 90$ & 1021 & 583 & & & $5 / 2 / 90$ & 1071 & 633 & & \\
\hline $3 / 14 / 90$ & 1022 & 584 & & & $5 / 3 / 90$ & 1072 & 634 & & \\
\hline $3 / 15 / 90$ & 1023 & 585 & & & $5 / 4 / 90$ & 1073 & 635 & & \\
\hline $3 / 16 / 90$ & 1024 & 586 & & Exp I deep cores $\$ 2,3$ & $5 / 6 / 90$ & 1074 & 636 & & $\cdots$ \\
\hline $3 / 17 / 90$ & 1025 & 687 & & & $5 / 6 / 90$ & 1076 & 637 & & \\
\hline $3 / 18 / 90$ & 1026 & 588 & & & $5 / 7 / 90$ & 1076 & 638 & & \\
\hline $3 / 19 / 90$ & 1027 & 589 & & & $5 / 8 / 90$ & 1077 & 639 & & \\
\hline $3 / 20 / 90$ & 1028 & 590 & & & $5 / 9 / 90$ & 1078 & 640 & & \\
\hline $3 / 21 / 80$ & 1029 & 591 & & & $5 / 10 / 80$ & 1079 & 641 & & $\cdots$ \\
\hline $3 / 22 / 90$ & 1030 & 592 & & & $5 / 11 / 90$ & 1080 & 642 & & \\
\hline $3 / 23 / 90$ & 1031 & 593 & 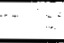 & & $5 / 12 / 80$ & 1081 & 643 & & E \\
\hline $3 / 24 / 90$ & 1032 & 594 & & & $5 / 13 / 90$ & 1082 & 644 & & \\
\hline $3 / 25 / 90$ & 1033 & 595 & & & $5 / 14 / 90$ & 1083 & 645 & & \\
\hline
\end{tabular}


Appendix C-9. Chronology of Las Cruces Trench Site Experimental Events.

\begin{tabular}{|c|c|c|c|c|c|c|c|c|c|}
\hline \multicolumn{5}{|c|}{ italics indicates solution sample collection days } & $: \cdots$ & & & & \\
\hline \multicolumn{5}{|c|}{ bold indicates noutron -probe reading days } & $\ldots \ldots \ldots$ & & $\ldots . .$. & & \\
\hline$\ldots$ & & & & $\ldots$ & $\ldots \ldots$ & $\cdots$ & & & 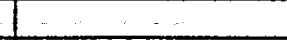 \\
\hline & \multicolumn{3}{|c|}{ Experiment days } & $\ldots$ & & \multicolumn{3}{|c|}{ Experiment days } & \multirow{2}{*}{ Event } \\
\hline Date: & 1 & . IIa & $11 \mathrm{~b}$ & Event & Date & 1 & lla & $\| \mathrm{b}$ & \\
\hline $5 / 15 / 90$ & 1084 & 646 & & & $7 / 4 / 90$ & 1134 & 696 & & $\ldots$ \\
\hline $5 / 16 / 90$ & 1085 & 647 & & & $7 / 5 / 90$ & 1135 & 697 & & $\ldots$ \\
\hline $5 / 17 / 80$ & 1086 & 648 & & $\cdots$ & $7 / 6 / 90$ & 1136 & 698 & & $\cdots$ \\
\hline $5 / 18 / 90$ & 1087 & 649 & & & $7 / 7 / 90$ & 1137 & 699 & & 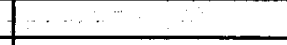 \\
\hline $5 / 19 / 90$ & 1088 & 650 & & $\cdots$ & $7 / 8 / 80$ & 1138 & 700 & & \\
\hline $5 / 20 / 90$ & 1089 & 651 & & & $7 / 8190$ & 1139 & 701 & $\ldots$ & Exp lla soil cores \\
\hline $5 / 21 / 90$ & 1090 & 652 & & $\ldots$ & $7 / 10 / 90$ & 1140 & 702 & $\ldots$ & (To 3 m deep) \\
\hline $5 / 22 / 90$ & 1091 & 653 & $\ldots$ & $\therefore$ & $7 / 11 / 90$ & 1141 & 703 & & $\begin{array}{r} \\
\end{array}$ \\
\hline $5 / 23 / 90$ & 1092 & 654 & & $\cdots$ & $7 / 12 / 90$ & 1142 & 704 & & \\
\hline $5 / 24 / 90$ & 1093 & 655 & & & $7 / 13 / 80$ & 1143 & 705 & & \\
\hline $5 / 25 / 90$ & 1094 & 656 & & & $7 / 14 / 90$ & 1144 & 706 & & \\
\hline $5 / 26 / 90$ & 1095 & 657 & & & $7 / 15 / 90$ & 1145 & 707 & & $\cdots$ \\
\hline $5 / 27 / 90$ & 1096 & 658 & & & $7 / 16 / 90$ & 1146 & 708 & & $\therefore \div$ \\
\hline $5 / 28 / 90$ & 1097 & 659 & & & $7 / 17 / 90$ & 1147 & 709 & & \\
\hline $5 / 29 / 90$ & 1098 & 660 & & \begin{tabular}{|c|} 
\\
\end{tabular} & $7 / 18 / 90$ & 1148 & 710 & & $2 \ldots$ \\
\hline $5 / 30 / 90$ & 1099 & 661 & & Exp lla soll cores & $7 / 19 / 90$ & 1140 & 711 & & $\therefore$ \\
\hline $5 / 31 / 90$ & 1100 & 662 & & (To $1.6 \mathrm{~m}$ de日p) & $7 / 20 / 90$ & 1150 & 712 & & $\ldots \ldots$ \\
\hline $6 / 1 / 90$ & 1101 & 663 & & $\therefore$ & $7 / 21 / 80$ & 1151 & 713 & & \\
\hline $6 / 2 / 90$ & 1102 & 664 & & & $7 / 22 / 90$ & 1152 & 714 & & \\
\hline $6 / 3 / 90$ & 1103 & 665 & & $\therefore$ & $7 / 23 / 90$ & 1153 & 715 & & \\
\hline $6 / 4 / 90$ & 1104 & 666 & & & $7 / 24 / 90$ & 1154 & 716 & & . \\
\hline $6 / 5 / 90$ & 1105 & 667 & & $\cdots$ & $7 / 25 / 90$ & 1155 & 717 & & $\ldots$ \\
\hline $6 / 6 / 90$ & 1106 & 668 & & & $7 / 26 / 00$ & 1156 & 718 & & $\ldots$ \\
\hline $6 / 7 / 90$ & 1107 & 669 & & & $7 / 27 / 90$ & 1157 & 719 & - & 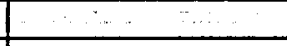 \\
\hline $6 / 8 / 90$ & 1108 & 670 & & & $7 / 28 / 90$ & 1158 & 720 & & \\
\hline $6 / 8 / 90$ & 1109 & 671 & $\therefore$ & & $7 / 29 / 90$ & 1159 & 721 & & \\
\hline $6 / 10 / 90$ & 1110 & 672 & & & $7 / 30 / 90$ & 1160 & 722 & & - \\
\hline $6 / 11 / 90$ & 1111 & 673 & $\cdots$ & & $7 / 31 / 90$ & 1161 & 723 & & \\
\hline $6 / 12 / 90$ & 1112 & 674 & & & $8 / 1 / 90$ & 1162 & 724 & 0 & begin llb irrigation \\
\hline $6 / 13 / 90$ & 1113 & 675 & & & $8 / 2 / 90$ & 1163 & 725 & 1 & \\
\hline $6 / 14 / 90$ & 1114 & 676 & & $\therefore$ & $8 / 3 / 90$ & 1164 & 726 & $\ldots 2$ & \\
\hline $6 / 15 / 00$ & 1115 & 677 & $\cdots$ & 2. & $8 / 4 / 90$ & 1165 & 727 & 3 & \\
\hline $6 / 16 / 90$ & 1116 & 678 & & & $8 / 5 / 90$ & 1166 & 728 & 4 & \\
\hline $6 / 17 / 90$ & 1117 & 679 & & & $8 / 6 / 90$ & 1167 & $72 \theta$ & 5 & $\cdots$ \\
\hline $6 / 18 / 80$ & 1118 & 680 & & $\cdots$ & $8 / 7190$ & 1168 & 730 & 6 & \\
\hline $6 / 19 / 90$ & 1119 & 681 & & & $8 / 8 / 90$ & 1169 & 731 & 7 & \\
\hline $6 / 20 / 90$ & 1120 & 682 & & & $8 / 9 / 90$ & 1170 & 732 & 8 & $=$ \\
\hline $6 / 21 / 90$ & 1121 & 683 & $\ldots$ & & $8 / 10 / 00$ & 1171 & 733 & 9 & $\ldots$ \\
\hline $6 / 22 / 90$ & 1122 & 684 & & $\therefore$ & $8 / 11 / 90$ & 1172 & 734 & 10 & \\
\hline $6 / 23 / 90$ & 1123 & 685 & & & $8 / 12 / 90$ & 1173 & 735 & 11 & . \\
\hline $6 / 24 / 90$ & 1124 & 686 & & & $8 / 13 / 90$ & 1174 & 736 & 12 & $\therefore$ \\
\hline $6 / 25 / 90$ & 1125 & 687 & & & $8 / 14 / 80$ & 1175 & 737 & 13 & - \\
\hline 6126190 & 1126 & 688 & & & $8 / 16 / 90$ & 1176 & 738 & 14 & $+\cdots$ \\
\hline $6 / 27 / 90$ & 1127 & 689 & $\because$ & & $8 / 16 / 80$ & 1177 & 739 & 16 & end tracer pulse \\
\hline $6 / 28 / 90$ & 1128 & 690 & & $\ldots$ & $8 / 17 / 90$ & 1178 & 740 & 16 & \begin{tabular}{|lll} 
& $\cdots$ & \\
\end{tabular} \\
\hline $6 / 29 / 90$ & 1129 & 691 & & & $8 / 18 / 90$ & 1179 & 741 & 17 & $\begin{array}{ll}\ldots \ldots \ldots \\
\ldots \ldots\end{array}$ \\
\hline $6 / 30 / 90$ & 1130 & 692 & - & $\therefore$ & $8 / 18 / 90$ & 1180 & 742 & -18 & $\cdots$ \\
\hline $7 / 1 / 90$ & 1131 & 693 & & $\ldots$ & $8 / 20 / 90$ & 1181 & 743 & 10 & $2 \ldots:$ \\
\hline $7 / 2 / 90$ & 1132 & 694 & & $\therefore \quad \ldots$ & $8 / 21 / 90$ & 1182 & 744 & 20 & $\therefore$ \\
\hline $7 / 3 / 90$ & 1133 & 695 & & 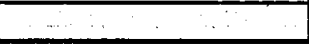 & $8 / 22 / 90$ & 1183 & 745 & 21 & -.. \\
\hline
\end{tabular}


Appendix C-9. Chronology of Las Cruces Trench Site Experimental Events.

\begin{tabular}{|c|c|c|c|c|c|c|c|c|c|}
\hline \multicolumn{10}{|c|}{ hallics indicates solution sample collection days } \\
\hline \multicolumn{10}{|c|}{ bold Indicates neutron probe reading days } \\
\hline & & & & & & & & & \\
\hline & \multicolumn{3}{|c|}{ Experiment days } & & & \multicolumn{3}{|c|}{ Experiment days } & \\
\hline Date & $T$ & Ila & Itb & Event & Date & 1 & Ua & IIb & Event \\
\hline $8 / 23 / 90$ & 1184 & 746 & 22 & & $10 / 13 / 90$ & 1235 & 797 & 73 & \\
\hline $8 / 24 / 90$ & 1185 & 747 & 23 & & $10 / 15 / 90$ & 1237 & 799 & 75 & \\
\hline $8 / 25 / 90$ & 1186 & 748 & 24 & - & $10 / 19 / 90$ & 1241 & 803 & 79 & \\
\hline $8 / 26 / 80$ & 1187 & 749 & 25 & & $10 / 22 / 90$ & 1244 & 806 & 82 & \\
\hline $8 / 27 / 90$ & 1188 & 750 & 26 & $\bar{i}$ & $10 / 26 / 90$ & 1248 & 810 & 86 & \\
\hline $8 / 28 / 90$ & 1189 & 751 & 27 & 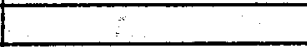 & $10 / 29 / 90$ & 1251 & 813 & 89 & \\
\hline $8 / 28 / 90$ & 1190 & 762 & 28 & & $11 / 2 / 90$ & 1255 & 817 & 93 & \\
\hline $8 / 30 / 00$ & 1191 & 753 & 29 & begin tracer pulse $\# 2$ & $11 / 5 / 90$ & 1258 & 820 & 96 & \\
\hline $8 / 31 / 90$ & 1192 & 754 & 30 & & $11 / 8 / 90$ & 1262 & 824 & 100 & \\
\hline $8 / 1 / 90$ & 1193 & 755 & 31 & & $11 / 12 / 90$ & 1265 & 827 & 103 & \\
\hline $8 / 2 / 90$ & 1194 & 756 & 32 & 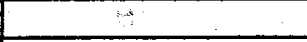 & $11 / 21 / 80$ & 1274 & 836 & 112 & \\
\hline $9 / 3 / 90$ & 1195 & 757 & 33 & $\ldots$ & $11 / 28 / 90$ & 1281 & 843 & 110 & \\
\hline $9 / 4 / 90$ & 1196 & 768 & 34 & 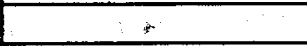 & $12 / 5 / 90$ & 1288 & 850 & 126 & \\
\hline $9 / 5 / 90$ & 1197 & 759 & 35 & $=$ & $12 / 12 / 90$ & 1295 & 857 & 133 & \\
\hline $8 / 6 / 90$ & 1188 & 760 & 36 & & $12 / 14 / 80$ & 1297 & 859 & 135 & \\
\hline $8 / 7 / 90$ & 1189 & 761 & 37 & 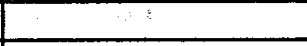 & $12 / 18 / 80$ & 1302 & 864 & 140 & \\
\hline $9 / 8 / 90$ & 1200 & 762 & 38 & $\therefore$ & $12 / 26 / 90$ & 1309 & 871 & 147 & \\
\hline $8 / 9 / 90$ & 1201 & 763 & 39 & $=1$ & $1 / 2 / 91$ & 1316 & 878 & 154 & \\
\hline $8 / 10 / 90$ & 1202 & 764 & 40 & $m$ & $1 / 18 / 91$ & 1332 & 894 & 170 & \\
\hline$\theta / 11 / 90$ & 1203 & 765 & 41 & . & $2 / 1 / 91$ & 1346 & 808 & 184 & \\
\hline $8 / 12 / 00$ & 1204 & 766 & 42 & & $2 / 7 / 91$ & 1352 & 816 & 190 & add 6 access tubes \\
\hline $8 / 13 / 90$ & 1205 & 767 & 43 & & $2 / 8 / 91$ & 1353 & 817 & 191 & add 6 access tubes \\
\hline $8 / 14 / 90$ & 1206 & 768 & 44 & end tracer pulse $\# 2$ & $2 / 15 / 91$ & 1360 & 922 & 198 & \\
\hline $9 / 15 / 00$ & 1207 & 269 & 45 & & $3 / 1 / 81$ & 1374 & 836 & 212 & \\
\hline $9 / 16 / 90$ & 1208 & 770 & 46 & 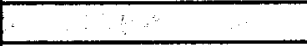 & $3 / 7 / 91$ & 1380 & 942 & 218 & \\
\hline $8 / 17 / 90$ & 1209 & 771 & 47 & $\therefore$ & $3 / 14 / 91$ & 1387 & 949 & 225 & \\
\hline $9 / 18 / 90$ & 1210 & 772 & 48 & & $4 / 11 / 91$ & 1415 & 977 & 253 & $=$ \\
\hline $9 / 19 / 90$ & 1211 & 773 & 49 & & $4 / 12 / 91$ & 1416 & 878 & 254 & \\
\hline $9 / 20 / 90$ & 1212 & 774 & 50 & & $5 / 9 / 91$ & 1443 & 1005 & 281 & \\
\hline $8 / 21 / 90$ & 1213 & 775 & 51 & & $5 / 21 / 91$ & 1455 & 1017 & 293 & Exp. Ilb soll cores \\
\hline $8 / 22 / 90$ & 1214 & 776 & 52 & & $6 / 7 / 91$ & 1472 & 1034 & 310 & \\
\hline $8 / 23190$ & 1216 & 777 & 53 & & $7 / 12 / 81$ & 1507 & 1069 & 345 & \\
\hline $8 / 24 / 80$ & 1216 & 778 & 54 & & $0 / 13 / 91$ & 1570 & 1132 & 408 & \\
\hline $8 / 25 / 00$ & 1217 & 779 & 55 & & $8 / 20 / 91$ & 1577 & 1139 & 416 & \\
\hline $9 / 26 / 90$ & 1218 & 780 & 56 & & $1 / 8 / 92$ & 1687 & 1249 & 525 & \\
\hline $9 / 27 / 90$ & 1219 & 781 & 57 & & & & & & \\
\hline $8 / 28 / 90$ & 1220 & 782 & 58 & & & & & & \\
\hline $9 / 29 / 90$ & 1221 & 783 & 59 & & & 1 & & & \\
\hline $9 / 30 / 90$ & 1222 & 784 & 60 & & & $\therefore$ & & & $=$ \\
\hline $10 / 1 / 90$ & 1223 & 785 & 61 & & & & & & \\
\hline $10 / 2190$ & 1224 & 786 & 62 & & & & & & \\
\hline $10 / 3 / 90$ & 1225 & 787 & 63 & & & $\cdots$ & - & & \\
\hline $10 / 4 / 90$ & 1226 & 788 & 64 & & & & & & $=$ \\
\hline $10 / 5 / 80$ & 1227 & 789 & 65 & & & 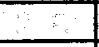 & 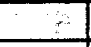 & & \\
\hline $10 / 6 / 90$ & 1228 & 790 & 66 & & & & & & \\
\hline $10 / 7 / 80$ & 1229 & 791 & 67 & & & & & & \\
\hline $10 / 8 / 90$ & 1230 & 792 & 68 & & & & & & \\
\hline $10 / 8 / 90$ & 1231 & 793 & 69 & & - & & & & \\
\hline $10 / 10 / 90$ & 1232 & 794 & 70 & end llb Irrigation & & & & & \\
\hline $10 / 12 / 90$ & 1234 & 796 & 72 & & & & & & \\
\hline
\end{tabular}


Appendix C-10. Location of Experimental Assets.

All measures described in meters

\begin{tabular}{|c|c|c|c|c|}
\hline \multicolumn{5}{|c|}{ Irrigation Plot I Corners } \\
\hline Corner & $x_{1}$ & $Y_{1}$ & $x$ & $Y$ \\
\hline $\mathbf{S E}$ & -1.98 & 8.98 & 15.14 & -13.79 \\
\hline NE & -1.98 & 0.00 & 15.16 & -4.81 \\
\hline Sw & 1.98 & 8.98 & 11.18 & -13.78 \\
\hline NW & 1.98 & 0.00 & 11.20 & -4.80 \\
\hline
\end{tabular}

\begin{tabular}{|c|c|c|c|}
\hline \multicolumn{4}{|c|}{ Experiment Ilb Soll Cores } \\
\hline No. & $\mathbf{Y} \mathbf{2}$ & $x_{2}$ & $\mathbf{Z}$ \\
\hline 1 & 5 & -2.75 & 6.25 \\
\hline 2 & 5 & -2.25 & 6.29 \\
\hline 3 & 5 & -1.75 & 6.21 \\
\hline 4 & 5 & -1.25 & 6.21 \\
\hline 5 & 5 & -0.70 & 6.29 \\
\hline 6 & 5 & -0.25 & 6.29 \\
\hline 7 & 5 & 0.25 & 6.29 \\
\hline 8 & 5 & 0.75 & 6.29 \\
\hline 9 & 5 & 1.25 & 6.29 \\
\hline 10 & 5 & 1.75 & 6.29 \\
\hline 11 & 5 & 2.25 & 6.25 \\
\hline 12 & 5 & 2.75 & 6.29 \\
\hline 13 & 9 & -2.75 & 6.25 \\
\hline 14 & 9 & -2.25 & 6.29 \\
\hline 15 & 9 & -1.75 & 6.26 \\
\hline 16 & 9 & -1.25 & 6.21 \\
\hline 17 & 9 & -0.75 & 6.29 \\
\hline 18 & 9 & -0.25 & 6.29 \\
\hline 19 & 9 & 0.25 & 6.29 \\
\hline 20 & 9 & 0.75 & 6.29 \\
\hline 21 & 9 & 1.25 & 6.29 \\
\hline 22 & 9 & 1.75 & 6.29 \\
\hline 23 & 9 & 2.25 & 6.29 \\
\hline 24 & $\mathbf{9}$ & 2.75 & 6.29 \\
\hline
\end{tabular}

\begin{tabular}{rrrr}
\multicolumn{4}{c}{ Experiment I Deep Cores } \\
No. & $X_{1}$ & $Y_{1}$ & $Z$ \\
\hline 1 & -0.46 & 4.67 & 14.60 \\
2 & 2.02 & 4.95 & 15.22 \\
3 & 4.00 & 5.00 & 15.19
\end{tabular}

\begin{tabular}{rrrr}
\multicolumn{5}{c}{$\begin{array}{l}\text { Experiment lla Soll Cores } \\
\text { No. }\end{array}$} & Y2 & X2 & Z \\
\hline 1 & 5 & -1.5 & 3.0 \\
2 & 5 & -1.0 & 3.0 \\
3 & 5.09 & -0.5 & 2.8 \\
4 & 5 & 0.0 & 3.0 \\
5 & 5.09 & 0.5 & 2.8 \\
6 & 5 & 1.0 & 3.0 \\
7 & 5 & 1.5 & 3.0 \\
8 & 9 & -1.5 & 2.8 \\
9 & 9 & -1.0 & 3.0 \\
10 & 9 & -0.5 & 2.8 \\
11 & 9 & 0.0 & 2.8 \\
12 & 9 & 0.5 & 2.6 \\
13 & 9 & 1.0 & 3.0 \\
14 & 9 & 1.5 & 3.0
\end{tabular}


Appendix C-10. Location of Experimental Assets. (Continued) Plot II Neutron Probe Access Tubes (described in meters)

\begin{tabular}{|c|c|c|c|c|}
\hline e No. & $\times 2$ & $\mathbf{Y 2}$ & $\mathbf{X}$ & \\
\hline 0 & 0.00 & 1.09 & 4.03 & 1.09 \\
\hline 7 & -5.98 & .2 .02 & -1.95 & 2.02 \\
\hline 8 & -4.98 & 2.03 & -0.96 & 2.03 \\
\hline 9 & -3.97 & 2.03 & 0.06 & 2.03 \\
\hline 10 & -3.01 & 2.02 & 1.02 & 2.02 \\
\hline 11 & -2.01 & 2.03 & 2.01 & 2.03 \\
\hline 12 & -1.00 & 2.04 & 3.03 & 2.04 \\
\hline 13 & -0.04 & 2.03 & 3.98 & 2.03 \\
\hline 14 & 1.02 & 1.99 & 5.04 & 1.99 \\
\hline 15 & 2.02 & 2.02 & 6.05 & 2.02 \\
\hline 16 & 2.99 & 2.02 & 7.01 & 2.02 \\
\hline 17 & 3.97 & 2.04 & 7.99 & 2.04 \\
\hline 18 & 4.97 & 2.05 & 9.00 & 2.05 \\
\hline 19 & 6.00 & 2.05 & 10.03 & 2.05 \\
\hline 20 & -0.01 & 4.03 & 4.02 & 4.03 \\
\hline 27 & -6.03 & 6.09 & -2.00 & 6.09 \\
\hline 28 & -5.00 & 6.05 & -0.97 & 6.05 \\
\hline 29 & -3.99 & 6.04 & 0.03 & 6.04 \\
\hline 30 & -3.02 & 6.05 & 1.01 & 6.05 \\
\hline 31 & -2.06 & 6.02 & 1.97 & 6.02 \\
\hline 32 & -1.04 & 6.05 & 2.99 & 6.05 \\
\hline 33 & -0.04 & 6.03 & 3.99 & 6.03 \\
\hline 34 & 0.95 & 6.05 & 4.98 & 6.05 \\
\hline 35 & 1.95 & 6.03 & 5.97 & 6.03 \\
\hline 36 & 2.95 & 6.05 & 6.98 & 6.05 \\
\hline 37 & 3.94 & 6.07 & 7.96 & 6.07 \\
\hline 38 & 4.93 & 6.07 & 8.96 & 6.07 \\
\hline 39 & 5.94 & 6.11 & 9.97 & 6.11 \\
\hline 40 & -0.08 & 8.03 & 3.94 & 8.03 \\
\hline 47 & -6.06 & 10.03 & -2.03 & 10.03 \\
\hline 48 & -5.06 & 10.02 & -1.03 & 10.02 \\
\hline 49 & -4.06 & 10.08 & -0.03 & 10.08 \\
\hline 50 & -3.09 & 10.05 & 0.94 & 10.05 \\
\hline 51 & -2.10 & 10.01 & 1.93 & 10.01 \\
\hline 52 & -1.08 & 10.06 & 2.94 & 10.06 \\
\hline 53 & -0.08 & 10.02 & 3.94 & 10.02 \\
\hline 54 & 0.91 & 10.04 & 4.93 & 10.04 \\
\hline 55 & 1.91 & 10.04 & 5.94 & 10.04 \\
\hline 56 & 2.92 & 10.04 & 6.95 & 10.04 \\
\hline 57 & 3.88 & 10.06 & 7.91 & 10.06 \\
\hline 58 & 4.88 & 10.07 & 8.91 & 10.07 \\
\hline 59 & 5.89 & 10.10 & 9.92 & 10.10 \\
\hline 60 & -0.18 & 12.04 & 3.85 & 12.04 \\
\hline 70 & -1.01 & 12.58 & 3.02 & 12.58 \\
\hline 71 & 0.72 & 12.51 & 4.75 & 12.51 \\
\hline 80 & -0.18 & 13.04 & 3.85 & 13.04 \\
\hline 90 & -0.17 & 13.99 & 3.85 & 13.99 \\
\hline 100 & -0.14 & 15.02 & 3.89 & 15.02 \\
\hline 110 & -0.18 & 16.02 & 3.84 & 16.02 \\
\hline
\end{tabular}


Appendix C-10. Location of Experimental Assets. Continued) Plot II Tensiometers (described in meters)

\begin{tabular}{|c|c|c|c|c|c|c|}
\hline ID & $\times 2$ & Y2 & $\mathbf{z}$ & $\mathbf{X}$ & $\mathbf{Y}$ & Exp lla ID \\
\hline 142 & -2.11 & 0.5 & 0.25 & 1.92 & 0.5 & $N / A$ \\
\hline 144 & -1.5 & 0.5 & 0.25 & 2.53 & 0.5 & 'A1' \\
\hline 146 & -1 & 0.5 & 0.25 & 3.03 & 0.5 & $.13{ }^{\prime}$ \\
\hline 148 & -0.5 & 0.5 & 0.25 & 3.53 & 0.5 & $' 14^{\prime}$ \\
\hline 150 & 0 & 0.5 & 0.25 & 4.03 & 0.5 & $' 15^{\prime}$ \\
\hline 152 & 0.5 & 0.5 & 0.25 & 4.53 & 0.5 & $' 16^{\prime}$ \\
\hline 154 & 1 & 0.5 & 0.25 & 5.03 & 0.5 & $' 17^{\prime}$ \\
\hline 156 & 1.5 & 0.5 & 0.25 & 5.53 & 0.5 & 'A2' \\
\hline 158 & 2 & 0.5 & 0.25 & 6.03 & 0.5 & N/A \\
\hline 342 & -2.11 & 0.5 & 0.75 & 1.92 & 0.5 & $N / A$ \\
\hline 344 & -1.5 & 0.5 & 0.75 & 2.53 & 0.5 & 'B1' \\
\hline 346 & -1 & 0.5 & 0.75 & 3.03 & 0.5 & $' 18^{\circ}$ \\
\hline 348 & -0.5 & 0.5 & 0.75 & 3.53 & 0.5 & $' 19 '$ \\
\hline 350 & 0 & 0.5 & 0.75 & 4.03 & 0.5 & $' 20{ }^{\prime}$ \\
\hline 352 & 0.5 & 0.5 & 0.75 & 4.53 & 0.5 & $' 21{ }^{\prime}$ \\
\hline 354 & 1 & 0.5 & 0.75 & 5.03 & 0.5 & '22' \\
\hline 356 & 1.5 & 0.5 & 0.75 & 5.53 & 0.5 & 'B2' \\
\hline 358 & 2 & 0.5 & 0.75 & 6.03 & 0.5 & N/A \\
\hline 642 & -2.11 & 0.5 & 1.5 & 1.92 & 0.5 & N/A \\
\hline 644 & -1.5 & 0.5 & 1.5 & 2.53 & 0.5 & '23' \\
\hline 646 & -1 & 0.5 & 1.5 & 3.03 & 0.5 & $' 24^{\prime}$ \\
\hline 648 & -0.5 & 0.5 & 1.5 & 3.53 & 0.5 & $' 25^{\prime}$ \\
\hline 650 & 0 & 0.5 & 1.5 & 4.03 & 0.5 & $' 26^{\prime}$ \\
\hline 652 & 0.5 & 0.5 & 1.5 & 4.53 & 0.5 & $' 27{ }^{\prime}$ \\
\hline 654 & 1 & 0.5 & 1.5 & 5.03 & 0.5 & $' 28^{\prime}$ \\
\hline 656 & 1.5 & 0.5 & 1.5 & 5.53 & 0.5 & '29' \\
\hline 658 & 2 & 0.5 & 1.5 & 6.03 & 0.5 & N/A \\
\hline 842 & -2.11 & 0.5 & 2 & 1.92 & 0.5 & N/A \\
\hline 844 & -1.5 & 0.5 & 2 & 2.53 & 0.5 & $' 30^{\prime}$ \\
\hline 846 & -1 & 0.5 & 2 & 3.03 & 0.5 & '31' \\
\hline 848 & -0.5 & 0.5 & 2 & 3.53 & 0.5 & '32' \\
\hline 850 & 0 & 0.5 & 2 & 4.03 & 0.5 & '33' \\
\hline 852 & 0.5 & 0.5 & 2 & 4.53 & 0.5 & $' 34^{\prime}$ \\
\hline 854 & 1 & 0.5 & 2 & 5.03 & 0.5 & $' 35^{\prime}$ \\
\hline 856 & 1.5 & 0.5 & 2 & 5.53 & 0.5 & $' 36^{\prime}$ \\
\hline 858 & 2 & 0.5 & 2 & 6.03 & 0.5 & N/A \\
\hline 1042 & -2.11 & 0.5 & 2.5 & 1.92 & 0.5 & N/A \\
\hline 1044 & -1.5 & 0.5 & 2.5 & 2.53 & 0.5 & '37' \\
\hline 1046 & -1 & 0.5 & 2.5 & 3.03 & 0.5 & '38' \\
\hline 1048 & -0.5 & 0.5 & 2.5 & 3.53 & 0.5 & '39' \\
\hline 1050 & 0 & 0.5 & 2.5 & 4.03 & 0.5 & $' 40^{\prime}$ \\
\hline 1052 & 0.5 & 0.5 & 2.5 & 4.53 & 0.5 & '41' \\
\hline 6437 & & & 210 & & & \\
\hline
\end{tabular}


Appendix C-10. Location of Experimental Assets. (Continued)

Plot II Tensiometers (described in meters)

\begin{tabular}{|c|c|c|c|c|c|c|}
\hline $\mathbf{D}$ & $x_{2}$ & $\mathbf{Y 2}$ & $\mathbf{Z}$ & $\mathbf{x}$ & $\mathbf{Y}$ & Exp lla ID \\
\hline 1054 & 1 & 0.5 & 2.5 & 5.03 & 0.5 & $42^{\prime}$ \\
\hline 1056 & 1.5 & 0.5 & 2.5 & 5.53 & 0.5 & $43^{\prime}$ \\
\hline 1058 & 2 & 0.5 & 2.5 & 6.03 & 0.5 & N/A \\
\hline 1242 & -2.11 & 0.5 & 3 & 1.92 & 0.5 & N/A \\
\hline 1244 & -1.5 & 0.5 & 3 & 2.53 & 0.5 & $' 44^{\prime}$ \\
\hline 1246 & -1 & 0.5 & 3 & 3.03 & 0.5 & $45^{\prime}$ \\
\hline 1248 & -0.5 & 0.5 & 3 & 3.53 & 0.5 & $46^{\circ}$ \\
\hline 1250 & 0 & 0.5 & 3 & 4.03 & 0.5 & $47^{\prime}$ \\
\hline 1252 & 0.5 & 0.5 & 3 & 4.53 & 0.5 & $48^{\prime}$ \\
\hline 1254 & 1 & 0.5 & 3 & 5.03 & 0.5 & $49^{\circ}$ \\
\hline 1256 & 1.5 & 0.5 & 3 & 5.53 & 0.5 & $50^{\circ}$ \\
\hline 1258 & 2 & 0.5 & 3 & 6.03 & 0.5 & N/A \\
\hline 1442 & -2.11 & 0.5 & 3.5 & 1.92 & 0.5 & N/A \\
\hline 1444 & -1.5 & 0.5 & 3.5 & 2.53 & 0.5 & '51' \\
\hline 1446 & -1 & 0.5 & 3.5 & 3.03 & 0.5 & $' 52^{\prime}$ \\
\hline 1448 & -0.5 & 0.5 & 3.5 & 3.53 & 0.5 & $53^{\prime}$ \\
\hline 1450 & 0 & 0.5 & 3.5 & 4.03 & 0.5 & $154^{\prime}$ \\
\hline 1452 & 0.5 & 0.5 & 3.5 & 4.53 & 0.5 & $55^{\prime}$ \\
\hline 1454 & 1 & 0.5 & 3.5 & 5.03 & 0.5 & $56^{\prime}$ \\
\hline 1456 & 1.5 & 0.5 & 3.5 & 5.53 & 0.5 & $57^{\prime}$ \\
\hline 1458 & 2 & 0.5 & 3.5 & 6.03 & 0.5 & N/A \\
\hline 1642 & -2.11 & 0.5 & 4 & 1.92 & 0.5 & $N / A$ \\
\hline 1644 & -1.5 & 0.5 & 4 & 2.53 & 0.5 & N/A \\
\hline 1646 & -1 & 0.5 & 4 & 3.03 & 0.5 & N/A \\
\hline 1648 & -0.5 & 0.5 & 4 & 3.53 & 0.5 & N/A \\
\hline 1650 & 0 & 0.5 & 4 & 4.03 & 0.5 & N/A \\
\hline 1652 & 0.5 & 0.5 & 4 & 4.53 & 0.5 & N/A \\
\hline 1654 & 1 & 0.5 & 4 & 5.03 & 0.5 & N/A \\
\hline 1656 & 1.5 & 0.5 & 4 & 5.53 & 0.5 & N/A \\
\hline 1658 & 2 & 0.5 & 4 & 6.03 & 0.5 & N/A \\
\hline 1842 & -2.11 & 0.5 & 4.5 & 1.92 & 0.5 & N/A \\
\hline 1844 & -1.5 & 0.5 & 4.5 & 2.53 & 0.5 & N/A \\
\hline 1846 & -1 & 0.5 & 4.5 & 3.03 & 0.5 & N/A \\
\hline 1848 & -0.5 & 0.5 & 4.5 & 3.53 & 0.5 & N/A \\
\hline 1850 & 0 & 0.5 & 4.5 & 4.03 & 0.5 & N/A \\
\hline 1852 & 0.5 & 0.5 & 4.54 & 4.53 & 0.5 & N/A \\
\hline 1854 & 1 & 0.5 & 4.54 & 5.03 & 0.5 & N/A \\
\hline 1856 & 1.5 & 0.5 & 4.54 & 5.53 & 0.5 & N/A \\
\hline 1858 & 2 & 0.5 & 4.58 & 6.03 & 0.5 & N/A \\
\hline 2042 & -2.11 & 0.5 & 5 & 1.92 & 0.5 & N/A \\
\hline 2044 & -1.5 & 0.5 & 5 & 2.53 & 0.5 & N/A \\
\hline \multirow[t]{2}{*}{2046} & -1 & 0.5 & 5 & 3.03 & 0.5 & $N / A$ \\
\hline & & & 211 & & & NUF \\
\hline
\end{tabular}


Appendix C-10. Location of Experimental Assets. (Continued) Plot II Tensiometers (described in meters)

\begin{tabular}{rrrrrrr} 
ID & X2 & Y2 & $Z$ & $X$ & $Y$ & Exp II ID \\
\hline 2048 & -0.5 & 0.5 & 5 & 3.53 & 0.5 & N/A \\
2050 & 0 & 0.5 & 5 & 4.03 & 0.5 & N/A \\
2052 & 0.5 & 0.5 & 5 & 4.53 & 0.5 & N/A \\
2054 & 1 & 0.5 & 5 & 5.03 & 0.5 & N/A \\
2056 & 1.5 & 0.5 & 5 & 5.53 & 0.5 & N/A \\
2058 & 2 & 0.5 & 5 & 6.03 & 0.5 & N/A \\
2242 & -2.11 & 0.5 & 5.5 & 1.92 & 0.5 & N/A \\
2244 & -1.5 & 0.5 & 5.5 & 2.53 & 0.5 & N/A \\
2246 & -1 & 0.5 & 5.5 & 3.03 & 0.5 & N/A \\
2248 & -0.5 & 0.5 & 5.5 & 3.53 & 0.5 & N/A \\
2250 & 0 & 0.5 & 5.5 & 4.03 & 0.5 & N/A \\
2252 & 0.5 & 0.5 & 5.5 & 4.53 & 0.5 & N/A \\
2254 & 1 & 0.5 & 5.5 & 5.03 & 0.5 & N/A \\
2256 & 1.5 & 0.5 & 5.5 & 5.53 & 0.5 & N/A \\
2258 & 2 & 0.5 & 5.5 & 6.03 & 0.5 & N/A \\
2442 & -2.11 & 0.5 & 6 & 1.92 & 0.5 & N/A \\
2444 & -1.5 & 0.5 & 6 & 2.53 & 0.5 & N/A \\
2446 & -1 & 0.5 & 6 & 3.03 & 0.5 & N/A \\
2448 & -0.5 & 0.5 & 6 & 3.53 & 0.5 & N/A \\
2450 & 0 & 0.5 & 6 & 4.03 & 0.5 & N/A \\
2452 & 0.5 & 0.5 & 6 & 4.53 & 0.5 & N/A \\
2454 & 1 & 0.5 & 6 & 5.03 & 0.5 & N/A \\
2456 & 1.5 & 0.5 & 6 & 5.53 & 0.5 & N/A \\
2458 & 2 & 0.5 & 6 & 6.03 & 0.5 & N/A
\end{tabular}


Appendix C-10. Location of Experimental Assets. (Continued)

Plot II Solution Samplers (described in meters)

\begin{tabular}{|c|c|c|c|c|c|c|}
\hline ID & $\times 2$ & Y2 & $\mathbf{z}$ & $\mathbf{x}$ & $\mathbf{Y}$ & Exp lla ID \\
\hline 241 & -2.26 & 0.5 & 0.5 & 1.77 & 0.5 & N/A \\
\hline 243 & -1.75 & 0.5 & 0.5 & 2.28 & 0.5 & $N / A$ \\
\hline 245 & -1.25 & 0.5 & 0.5 & 2.78 & 0.5 & 11 \\
\hline 247 & -0.75 & 0.5 & 0.5 & 3.28 & 0.5 & $' 2 '$ \\
\hline 248 & -0.25 & 0.5 & 0.5 & 3.78 & 0.5 & $3^{\prime}$ \\
\hline 251 & 0.25 & 0.5 & 0.5 & 4.28 & 0.5 & '4' \\
\hline 253 & 0.75 & 0.5 & 0.5 & 4.78 & 0.5 & '5! \\
\hline 255 & 1.25 & 0.5 & 0.5 & 5.28 & 0.5 & '6' \\
\hline 257 & 1.84 & 0.5 & 0.5 & 5.87 & 0.5 & $' 43^{\prime}$ \\
\hline 258 & 2.23 & 0.5 & 0.5 & 6.26 & 0.5 & N/A \\
\hline 441 & -2.26 & 0.5 & 1 & 1.77 & 0.5 & N/A \\
\hline 443 & -1.75 & 0.5 & 1 & 2.28 & 0.5 & N/A \\
\hline 445 & -1.25 & 0.5 & 1 & 2.78 & 0.5 & $' 7 '$ \\
\hline 447 & -0.75 & 0.5 & 1 & 3.28 & 0.5 & $' 8 '$ \\
\hline $449^{\circ}$ & -0.25 & 0.5 & 1 & 3.78 & 0.5 & $9 '$ \\
\hline $451^{\prime}$ & 0.25 & 0.5 & 1 & 4.28 & 0.5 & $110^{\circ}$ \\
\hline 453 & 0.75 & 0.5 & 1 & 4.78 & 0.5 & $111^{\prime}$ \\
\hline 455 & 1.25 & 0.5 & 1 & 5.28 & 0.5 & $.12^{\circ}$ \\
\hline 457 & 1.84 & 0.5 & 1 & 5.87 & 0.5 & $' 44^{\prime}$ \\
\hline 459 & 2.24 & 0.5 & 1 & 6.27 & 0.5 & N/A \\
\hline 641 & -2.25 & 0.5 & 1.5 & 1.78 & 0.5 & N/A \\
\hline 643 & -1.75 & 0.5 & 1.5 & 2.28 & 0.5 & N/A \\
\hline 645 & -1.25 & 0.5 & 1.5 & 2.78 & 0.5 & $' 13$ ' \\
\hline 647 & -0.75 & 0.5 & 1.5 & 3.28 & 0.5 & $' 14^{\prime}$ \\
\hline 649 & -0.25 & 0.5 & 1.5 & 3.78 & 0.5 & 115 \\
\hline 651 & 0.25 & 0.5 & 1.5 & 4.28 & 0.5 & $' 16^{\prime}$ \\
\hline 653 & 0.75 & 0.5 & 1.5 & 4.78 & 0.5 & 917 \\
\hline 655 & 1.25 & 0.5 & 1.5 & 5.28 & 0.5 & $188^{\circ}$ \\
\hline 657 & 1.84 & 0.5 & 1.5 & 5.87 & 0.5 & $' 45^{\prime}$ \\
\hline 659 & 2.24 & 0.5 & 1.5 & 6.27 & 0.5 & N/A \\
\hline 841 & -2.25 & 0.5 & 2 & 1.78 & 0.5 & N/A \\
\hline 843 & -1.75 & 0.5 & 2 & 2.28 & 0.5 & N/A \\
\hline 845 & -1.25 & 0.5 & 2 & 2.78 & 0.5 & $119^{\prime}$ \\
\hline 847 & -0.75 & 0.5 & 2 & 3.28 & 0.5 & $' 20^{\prime}$ \\
\hline 849 & -0.25 & 0.5 & 2 & 3.78 & 0.5 & '21' \\
\hline 851 & 0.25 & 0.5 & 2 & 4.28 & 0.5 & $' 22^{\prime}$ \\
\hline 853 & 0.75 & 0.5 & 2 & 4.78 & 0.5 & $23^{\prime}$ \\
\hline 855 & 1.25 & 0.5 & 2 & 5.28 & 0.5 & $24{ }^{\prime}:$ \\
\hline 857 & 1.84 & 0.5 & 2 & 5.87 & 0.5 & $' 46^{\prime}$ \\
\hline 859 & 2.24 & 0.5 & 2 & 6.27 & 0.5 & N/A \\
\hline
\end{tabular}


Appendix C-10. Location of Experimental Assets. (Continued)

Plot II Solution Samplers (described in meters)

\begin{tabular}{|c|c|c|c|c|c|c|}
\hline 10 & $\times 2$ & Y2 & $\mathbf{Z}$ & $\mathbf{x}$ & $\mathbf{Y}$ & Exp lla ID \\
\hline 1041 & -2.25 & 0.5 & 2.5 & 1.78 & 0.5 & $N / A$ \\
\hline 1043 & -1.75 & 0.5 & 2.5 & 2.28 & 0.5 & N/A \\
\hline 1045 & -1.25 & 0.5 & 2.5 & 2.78 & 0.5 & $' 25{ }^{\prime}$ \\
\hline 1047 & -0.75 & 0.5 & 2.5 & 3.28 & 0.5 & $26{ }^{\prime}$ \\
\hline 1049 & -0.25 & 0.5 & 2.5 & 3.78 & 0.5 & $' 27^{\prime}$ \\
\hline 1051 & 0.25 & 0.5 & 2.5 & 4.28 & 0.5 & $' 28^{\prime}$ \\
\hline 1053 & 0.75 & 0.5 & 2.5 & 4.78 & 0.5 & $29 '$ \\
\hline 1055 & 1.25 & 0.5 & 2.5 & 5.28 & 0.5 & '30' \\
\hline 1057 & 1.84 & 0.5 & 2.5 & 5.87 & 0.5 & $' 47^{\prime}$ \\
\hline 1059 & 2.24 & 0.5 & 2.5 & 6.27 & 0.5 & N/A \\
\hline 1241 & -2.26 & 0.5 & 3 & 1.77 & 0.5 & N/A \\
\hline 1243 & -1.75 & 0.5 & 3 & 2.28 & 0.5 & N/A \\
\hline 1245 & -1.25 & 0.5 & 3 & 2.78 & 0.5 & '31' \\
\hline 1247 & -0.75 & 0.5 & 3 & 3.28 & 0.5 & $' 32^{\prime}$ \\
\hline 1249 & -0.25 & 0.5 & 3 & 3.78 & 0.5 & '33' \\
\hline 1251 & 0.25 & 0.5 & 3 & 4.28 & 0.5 & '34' \\
\hline 1253 & 0.75 & 0.5 & 3 & 4.78 & 0.5 & '35' \\
\hline 1255 & 1.25 & 0.5 & 3 & 5.28 & 0.5 & '36' \\
\hline 1257 & 1.84 & 0.5 & 3 & 5.87 & 0.5 & '48' \\
\hline 1259 & 2.24 & 0.5 & $\mathbf{3}$ & 6.27 & 0.5 & N/A \\
\hline 1441 & -2.25 & 0.5 & 3.5 & 1.78 & 0.5 & N/A \\
\hline 1443 & -1.75 & 0.5 & 3.5 & 2.28 & 0.5 & N/A \\
\hline 1445 & -1.25 & 0.5 & 3.5 & 2.78 & 0.5 & '37' \\
\hline 1447 & -0.75 & 0.5 & 3.5 & 3.28 & 0.5 & '38' \\
\hline 1449 & -0.25 & 0.5 & 3.5 & 3.78 & 0.5 & '39' \\
\hline 1451 & 0.25 & 0.5 & 3.5 & 4.28 & 0.5 & $' 40^{\prime}$ \\
\hline 1453 & 0.75 & 0.5 & 3.5 & 4.78 & 0.5 & '41' \\
\hline 1455 & 1.25 & 0.5 & 3.5 & 5.28 & 0.5 & '42' \\
\hline 1457 & 1.84 & 0.5 & 3.5 & 5.87 & 0.5 & $149^{\prime}$ \\
\hline 1459 & 2.25 & 0.5 & 3.5 & 6.28 & 0.5 & N/A \\
\hline 1641 & -2.25 & 0.5 & 4 & 1.78 & 0.5 & N/A \\
\hline 1643 & -1.75 & 0.5 & 4 & 2.28 & 0.5 & N/A \\
\hline 1645 & -1.25 & 0.5 & 4 & 2.78 & 0.5 & N/A \\
\hline 1647 & -0.75 & 0.5 & 4 & 3.28 & 0.5 & N/A \\
\hline 1649 & -0.25 & 0.5 & 4 & 3.78 & 0.5 & N/A \\
\hline 1651 & 0.25 & 0.5 & $\therefore 4$ & 4.28 & 0.5 & N/A \\
\hline 1653 & 0.75 & 0.5 & 4 & 4.78 & 0.5 & N/A \\
\hline 1655 & 1.25 & 0.5 & 4 & 5.28 & 0.5 & N/A \\
\hline 1657 & 1.84 & 0.5 & 4 & 5.87 & 0.5 & N/A \\
\hline 1659 & 2.26 & 0.5 & 4 & 6.29 & 0.5 & N/A \\
\hline
\end{tabular}


Appendix C-10. Location of Experimental Assets. (Continued) Plot II Solution Samplers (described in meters)

\begin{tabular}{rrrrrrr} 
ID & X2 & Y2 & $Z$ & $X$ & Y & Exp lla ID \\
\hline 1841 & -2.25 & 0.5 & 4.5 & 1.78 & 0.5 & N/A \\
1843 & -1.75 & 0.5 & 4.5 & 2.28 & 0.5 & N/A \\
1845 & -1.25 & 0.5 & 4.5 & 2.78 & 0.5 & N/A \\
1847 & -0.75 & 0.5 & 4.5 & 3.28 & 0.5 & N/A \\
1849 & -0.25 & 0.5 & 4.5 & 3.78 & 0.5 & N/A \\
1851 & 0.25 & 0.5 & 4.54 & 4.28 & 0.5 & N/A \\
1853 & 0.75 & 0.5 & 4.54 & 4.78 & 0.5 & N/A \\
1855 & 1.25 & 0.5 & 4.54 & 5.28 & 0.5 & N/A \\
1857 & 1.84 & 0.5 & 4.54 & 5.87 & 0.5 & N/A \\
1859 & 2.24 & 0.5 & 4.58 & 6.27 & 0.5 & N/A \\
2041 & -2.25 & 0.5 & 5 & 1.78 & 0.5 & N/A \\
2043 & -1.75 & 0.5 & 5 & 2.28 & 0.5 & N/A \\
2045 & -1.25 & 0.5 & 5 & 2.78 & 0.5 & N/A \\
2047 & -0.75 & 0.5 & 5 & 3.28 & 0.5 & N/A \\
2049 & -0.25 & 0.5 & 5 & 3.78 & 0.5 & N/A \\
2051 & 0.25 & 0.5 & 5 & 4.28 & 0.5 & N/A \\
2053 & 0.75 & 0.5 & 5 & 4.78 & 0.5 & N/A \\
2055 & 1.25 & 0.5 & 5 & 5.28 & 0.5 & N/A \\
2057 & 1.84 & 0.5 & 5 & 5.87 & 0.5 & N/A \\
2059 & 2.24 & 0.5 & 5 & 6.27 & 0.5 & N/A \\
2241 & -2.25 & 0.5 & 5.5 & 1.78 & 0.5 & N/A \\
2243 & -1.75 & 0.5 & 5.5 & 2.28 & 0.5 & N/A \\
2245 & -1.25 & 0.5 & 5.5 & 2.78 & 0.5 & N/A \\
2247 & -0.75 & 0.5 & 5.5 & 3.28 & 0.5 & N/A \\
2249 & -0.25 & 0.5 & 5.5 & 3.78 & 0.5 & N/A \\
2251 & 0.25 & 0.5 & 5.5 & 4.28 & 0.5 & N/A \\
2253 & 0.75 & 0.5 & 5.5 & 4.78 & 0.5 & N/A \\
2255 & 1.25 & 0.5 & 5.5 & 5.28 & 0.5 & N/A \\
2257 & 1.84 & 0.5 & 5.5 & 5.87 & 0.5 & N/A \\
2259 & 2.25 & 0.5 & 5.5 & 6.28 & 0.5 & N/A \\
2441 & -2.25 & 0.5 & 6 & 1.78 & 0.5 & N/A \\
2443 & -1.75 & 0.5 & 6 & 2.28 & 0.5 & N/A \\
2445 & -1.25 & 0.5 & 6 & 2.78 & 0.5 & N/A \\
2447 & -0.75 & 0.5 & 6 & 3.28 & 0.5 & N/A \\
2449 & -0.25 & 0.5 & 6 & 3.78 & 0.5 & N/A \\
2451 & 0.25 & 0.5 & 6 & 4.28 & 0.5 & N/A \\
2453 & 0.75 & 0.5 & 6 & 4.78 & 0.5 & N/A \\
2455 & 1.25 & 0.5 & 6 & 5.28 & 0.5 & N/A \\
2457 & 1.84 & 0.5 & 6 & 5.87 & 0.5 & N/A \\
2459 & 2.25 & 0.5 & 6 & 6.28 & 0.5 & N/A \\
& & & & & &
\end{tabular}




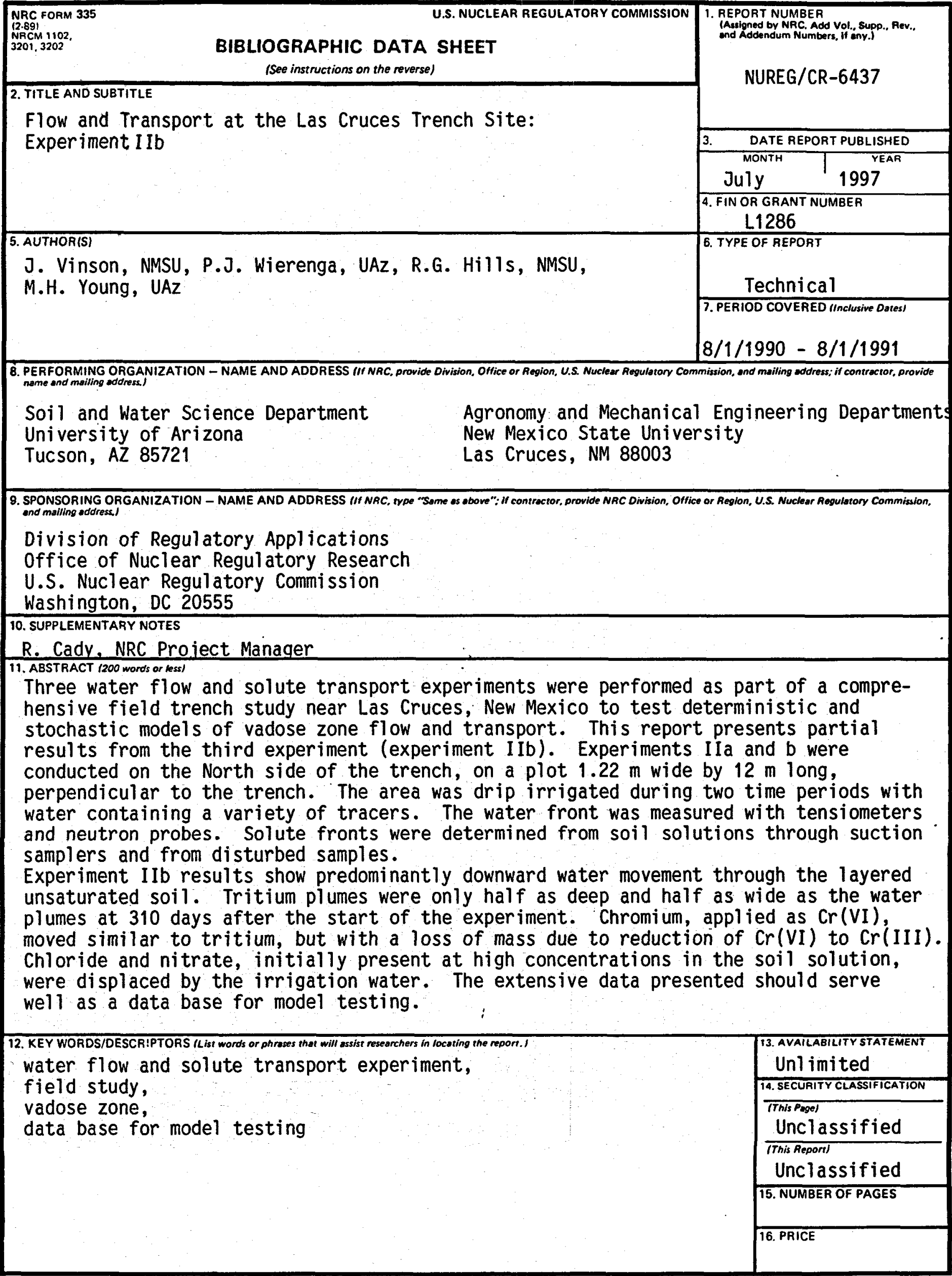




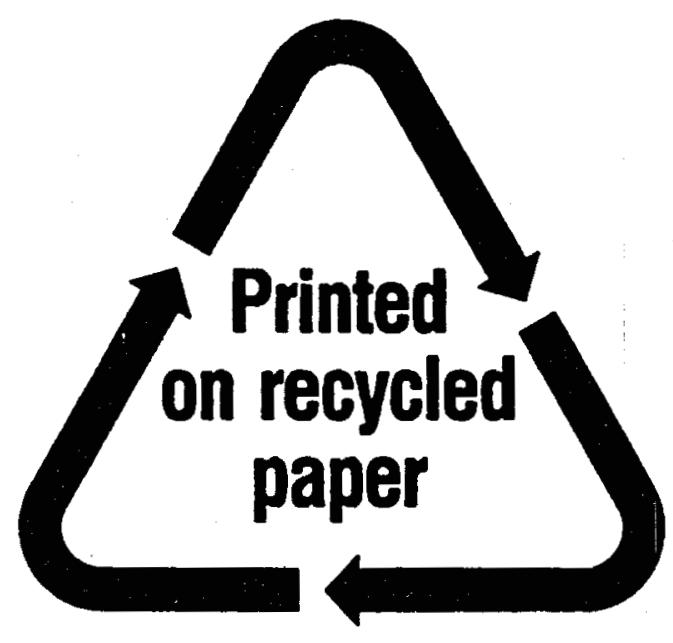

Federal Recycling Program 